\title{
Azulene-based Protecting Groups
}

by

\section{Thomas Bevan}

\author{
A thesis submitted to Victoria University of
}

Wellington in fulfilment of the requirements for the

degree of Doctor of Philosophy

2016

SCPS

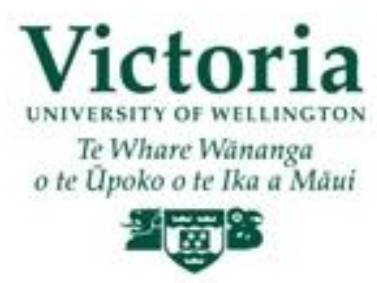




\section{Abstract}

Protecting groups form an indispensable part of modern organic synthetic chemistry. Besides the benefits of selectively passivating certain reactive functionalities, they often provide handling benefits - such as a decrease in the polarity of the compound that facilitates purification, an increase in the structural order of a compound that allows for easier crystallisation, and chromophores that enable easy visualisation on fluorescent TLC plates under UV light.

Coloured protecting groups offer additional advantages in synthetic chemistry. They expedite purification by allowing the material to be tracked visually. Phase separation and column chromatography are easier to perform, and reduce the need for the collection of large numbers of fractions, while small-scale loss of material (left behind on taps or in flasks during routine handling) and spillages are much more readily apparent. Despite these advantages, only a few coloured protecting groups have been reported in the literature.

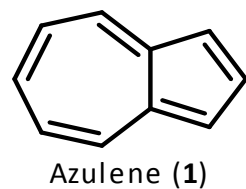

The azulenes are a class of compounds with several attractive qualities that can be exploited for use as protecting groups. They are coloured, but not overwhelmingly so. The colour is tunable through placement of electron-donating or electron-withdrawing groups at positions on the ring system, which further allows for protection/deprotection reactions to be designed that incorporate a colour change. Azulene itself is both non-polar and structurally compact, unlike many other organic chromophores such as triarylmethane dyes and carotenoids. Furthermore, azulene's ability to stabilise both positive and negative charges through resonance with tropylium and cyclopentadienide motifs allows for unusual chemistry, and therefore potentially orthogonal modes of deprotection.

Four protecting group candidates incorporating azulene were devised. The 1-azulenylmethylene amine 79 and the 1-azulenesulfonamide 82 protecting group candidates for amines had fatal flaws that were discovered early, such as a tendency to rapidly degrade in open air. The 1azulenecarboxylate protecting group candidate $\mathbf{7 4}$ for alcohols showed some promise, with a high-yielding protection reaction, but none of the deprotection conditions that were developed were sufficiently mild to be usable in a late-stage deprotection strategy on a complex target molecule. 

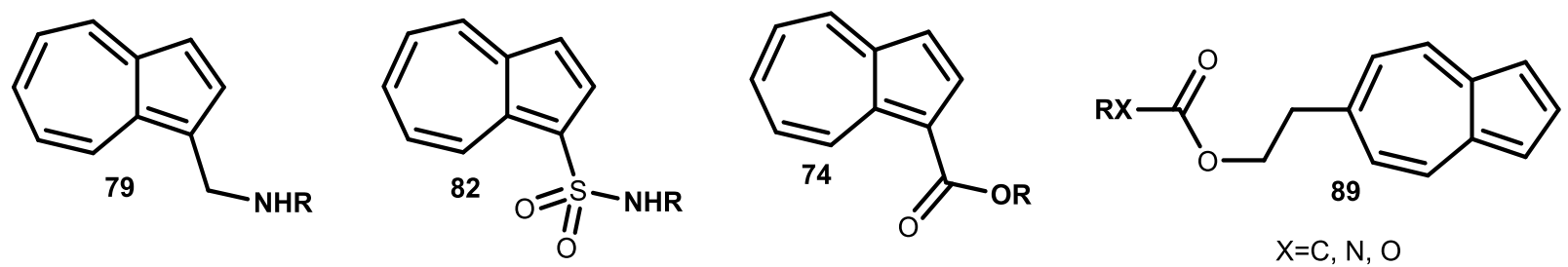

The four azulene-based protecting group candidates considered in this project

The final protecting group candidate, 6-(2-[oxycarbonyl]ethyl)azulene 89, can be used for the protection of carboxylic acids, amines and alcohols as esters, carbamates and carbonates, respectively. The substitution at the 6-position of azulene allows for deprotection through an E1cB mechanism with mild base, involving a cyclopentadienide-stabilised carbanion intermediate, in a similar fashion to the FMOC protecting group. Mild conditions for the protection of all three were found: for carboxylic acids Steglich esterification is employed, and for alcohols and amines coupling with CDI is used. A selection of mild protocols for deprotection were developed, using bases such as DBU or TBAF, or involving two-step activation-deprotection procedures.

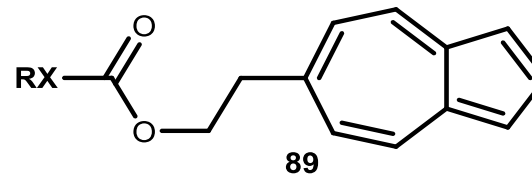

$\mathrm{X}=\mathrm{C}, \mathrm{N}, \mathrm{O}$

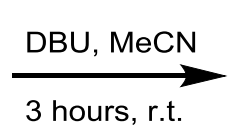

3 hours, r.t.<smiles>[R]C(=O)[O-]</smiles>
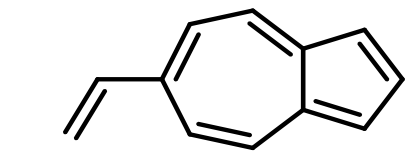

94

Deprotection of an Azul-protected substrate

Finally, the compatibility of the protecting group 89 (dubbed $A z u l$ ) with common and representative procedures in synthetic chemistry was investigated, such as with bases and with reaction conditions such as oxidations, reductions, cross-couplings, etc. Orthogonality with other common protecting groups (such as TBS, MOM, FMOC) was also explored. Some incompatibilities were found with strongly acidic conditions, high-temperature Suzuki cross-coupling reactions and Swern oxidations, but otherwise the Azul protecting group shows promise as a protecting group that expedites total synthesis through its colourful properties. 
To Normandy Somly Maestro 


\section{Acknowledgements}

First and foremost I'd like to thank my supervisor, Dr. Joanne Harvey. Joanne, you are the model of how an academic should be. I've not seen any other academic exhibit such fierce dedication towards their students. I'd like to thank my secondary supervisor A/Prof. Peter Northcote, for many invaluable discussions as the project was taking shape. A/Prof. Paul Teesdale-Spittle also deserves special mention for his many insightful comments and observations, as well as his quick wit and impeccable skills at delivering bad puns.

At this point, I thank all of my labmates, and in doing so reveal just how long I've been present in the Harvey group. Thanks go to Mark 'golden child' Bartlett, Hemi 'acetal' Cumming, Kalpani 'Hulk' Somarathne, Jingjing 'jing' Wang, Peter 'curly' Moore, Dylan 'can't punch' Davies, Claire 'flame-on' Turner, Scott 'pompeii' Riordan, Rhia 'slow down' Stone, Stephen 'ol'Mama' Tat, Loïc 'swiss cheese' Lassueur, Sarah 'glove snatcher' Brown, Amira 'breakfast for lunch' Brackovic, Sophie 'preuß' Geyrhofer, Dan 'vinegar drinker' Phipps, Helena 'asbestos fingers' Wong, Jasmin 'bubble soccer' Riesterer, Ben 'stonecutter' Durrant, José-Luis 'frenchy' Pinedo-Rivera, Chris 'boy' Orme, Hedley 'bicep' Stirrat, and finally Matt Fisk, Claire Cuyamendous and Xu Tao who I do not know well enough to grant epithets. Special thanks go to Jingjing and Sophie for their Mass-Spec services. I've seen a complete change of the guard during my stretch as $\mathrm{PhD}$ student, yet somehow the group still feels like the same supportive group I originally joined.

From outside the group, there are several people deserving of thanks. Rosie Somerville in particular for cheering me up with her boundless chemistry enthusiasm on many occasions, but also Thom Minnee, Joe Gallagher, and the majority of Martyn \& Robin's group. Thanks also go to Ian Vorster for many NMR services over the years, the mechanical workshop crew who provided us with unlimited nitrogen and dutifully fixed our continually failing diaphragm pump, and to Grant Franklin for some specialty pieces of glassware that will subsequently sit in the misc drawer to baffle many generations of chemists to come.

I'd like to thank my family. Many special thanks go to my mum, my dad, Annaliese \& Leon, all the cats and dogs (their contribution was invaluable), and my grandparents, two of whom sadly passed away over the course of my PhD.

Finally, I'd like to thank Julia and her family. Julia, you're the best thing that's happened to me. I loveyou so much. 


\section{Table of contents}

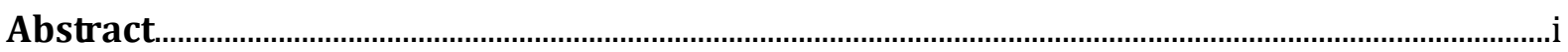

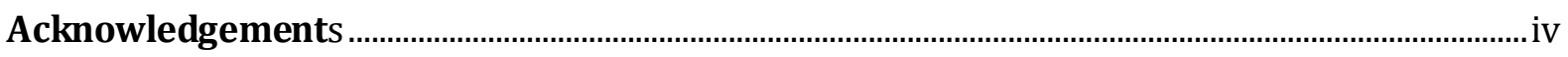

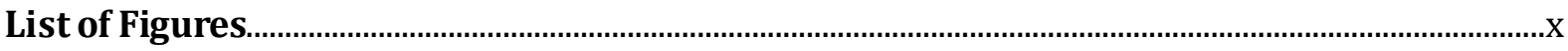

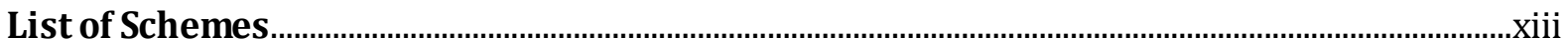

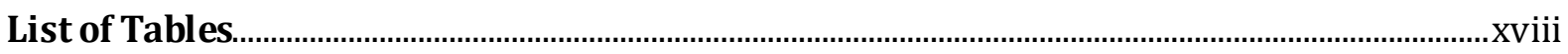

List of Abbreviations...............................................................................................................................

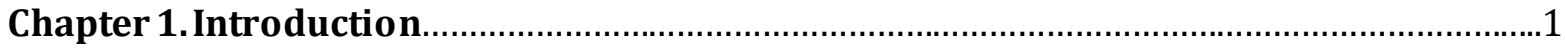

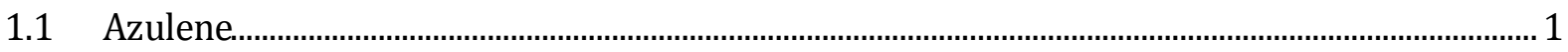

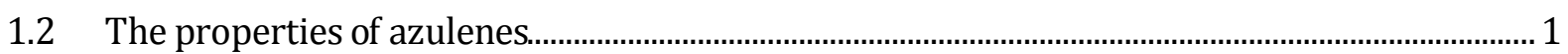

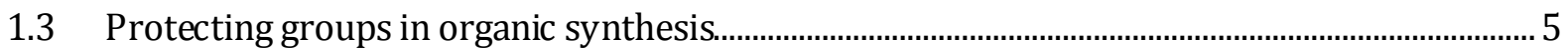

1.3.1 The requirements of a protecting group ..................................................................... 6

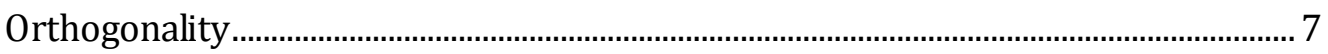

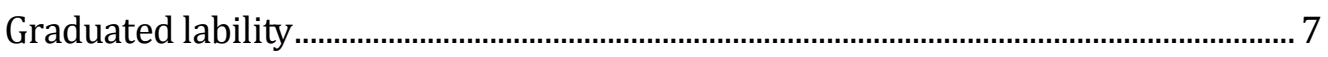

Two-step deprotection...................................................................................................... 8

1.3.2 The handling benefits of protecting groups.................................................................... 9

1.4 A brief overview of protecting group chemistry.................................................................11

1.4.1 Anatomy of a protecting group.............................................................................................11

1.4.2 Protected functionalities used in alcohol protection ......................................................12

1.4.3 Protected functionalities used in amine protection .........................................................13

1.4.4 Protected functionalities of carboxylic acids.......................................................................13

1.5 Brief overview of available protecting group bodies ........................................................................14

1.5.1 Sterics-based protecting group bodies.......................................................................... 14

1.5.2 Electron-withdrawing protecting group bodies........................................................15

1.5.3 Silyl protecting groups ....................................................................................................

1.5.4 Hydrogenation-cleavable protecting groups ..................................................................16

1.5.5 Protecting groups with an E1cB cleavage mechanism...............................................17

1.6 Research objectives........................................................................................................................

1.6.1 The advantages of a coloured protecting group ..........................................................19

1.6.2 Exploitation of the chemical properties of azulene ...................................................2

1.6.3 The ideal niche of the azulene-based protecting group ...............................................21 


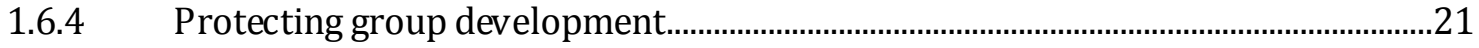

1.6.5 Approach to azulene protecting group research ............................................................21

Identification of protecting group candidates................................................................21

Characterisation of the protection and cleavage conditions.......................................22

Exploration of the protecting group in a wider synthetic context.............................22

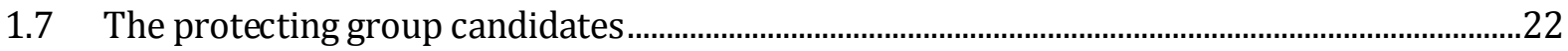

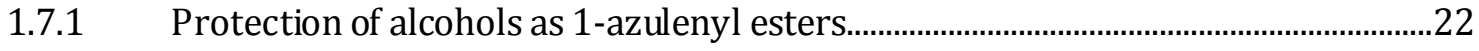

1.7.2 Protection of amines through reductive amination with 1-formylazulene..............23

1.7.3 Protection of amines as 1-azulenesulfonamides ..............................................................24

1.7.4 Protection of alcohols, amines and carboxylic acids through a $\beta$-elimination cleavable 6-(2-oxyethyl)azulene . .24

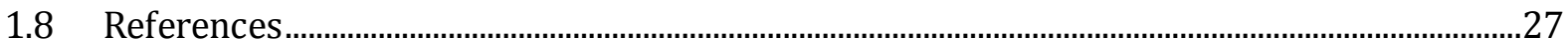

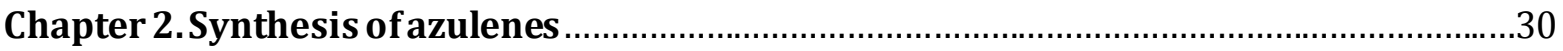

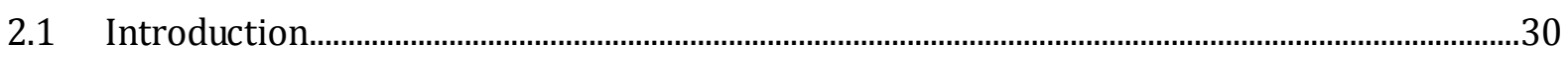

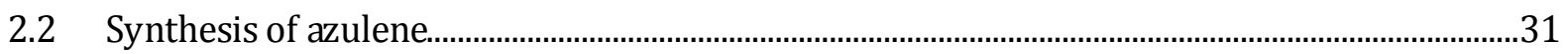

2.3 Synthesis of alkyl-substituted azulenes..............................................................................................

2.3.1 Synthesis of 4,6,8-trimethylazulene................................................................................

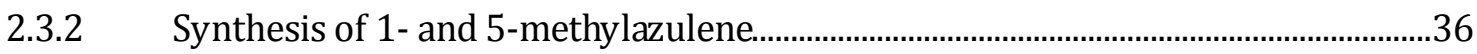

2.3.3 Synthesis of 4- and 6-methylazulene............................................................................

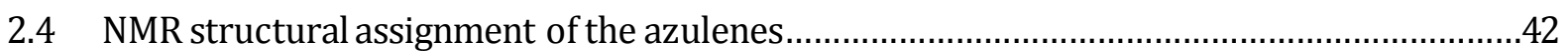

2.5 Summary of Chapter 2

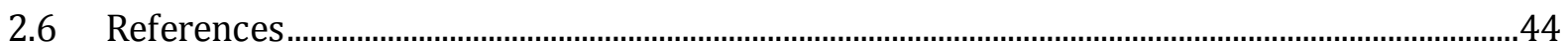

Chapter 3. 1-Substituted azulene protectinggroup candidates..............................................45

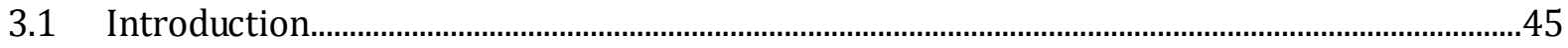

3.2 1-Azulenylmethyl protection of amines......................................................................................

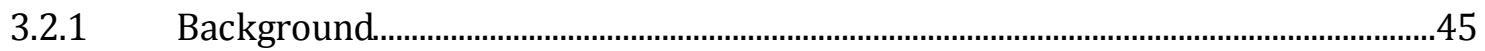

3.2.2 Initial investigation.................................................................................................

3.3 1-Azulenesulfonamide protection of amines..................................................................................4

3.4 Protection of alcohols as 1-substituted azulene esters ....................................................................48

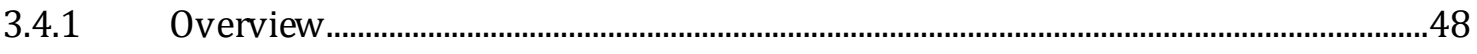

3.4.2 Protection .................................................................................................................... 49 


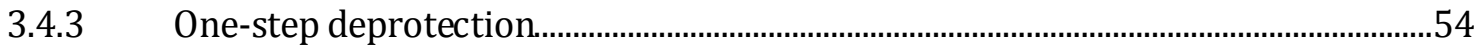

Regeneration of azulene.................................................................................................5

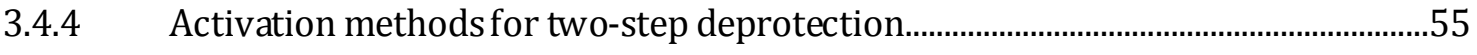

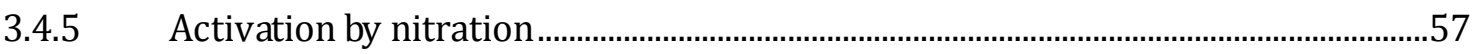

3.4.6 Other activation methods - $\pi$-complexation and breaking of aromaticity ...............61

$\pi$-Complexation with tetracyanoethylene......................................................................62

$\pi$-Complexation using an iron carbonyl complex.......................................................63

3.4.7 Substituted azulenes and 'tethered' deprotections .......................................................66

The guaiazulene ester and tethered deprotection......................................................67

The 4,6,8-trimethylazulene ester and tethered deprotection....................................70

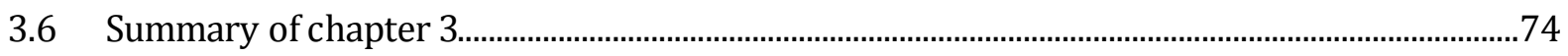

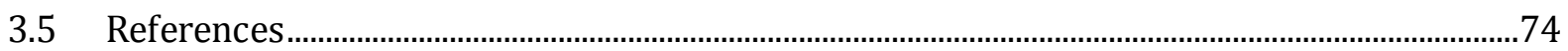

Chapter 4. Base-labile 6-substituted azulene protecting groups for carboxylic acids,

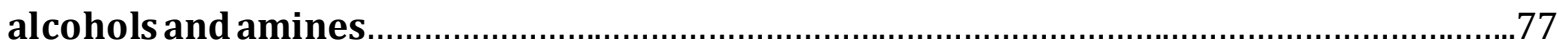

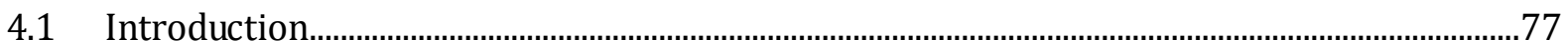

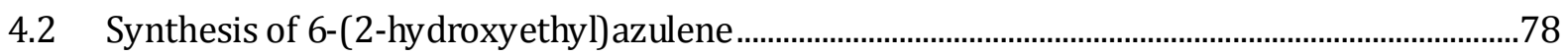

4.2.1 Investigation of the deprotonation step with methyl iodide........................................79

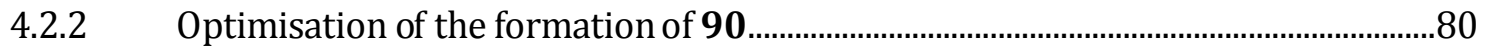

Investigation of the paraformaldehyde addition step ..................................................82

Investigation of concentration effects......................................................................... 84

Effect of dry ice/ethylene glycol bath and scaling up ................................................85

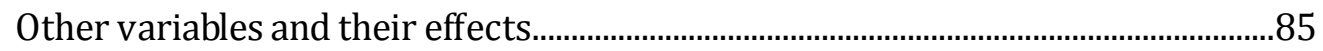

Formation of the unsymmetrical dimer 168 ………....................................................86

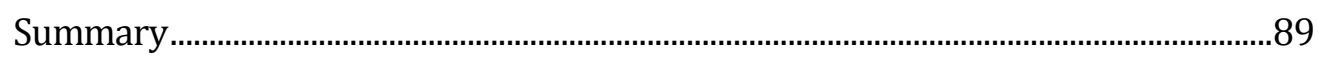

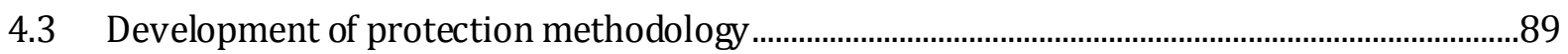

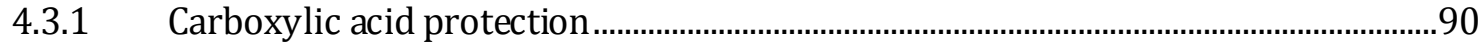

Steglich esterification ......................................................................................................91

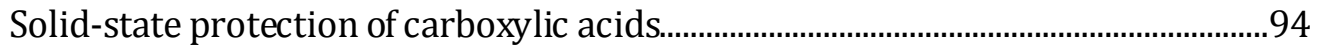

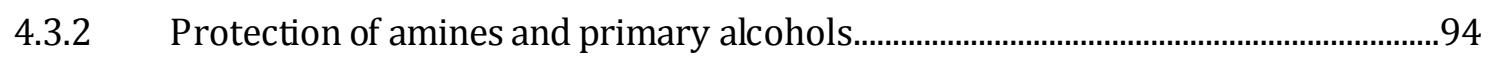

One-step protocol for alcohol protection......................................................................95

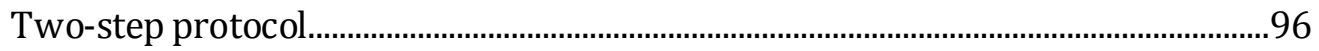


4.4 Deprotection

4.4.1 Two-step deprotection............................................................................................ 102

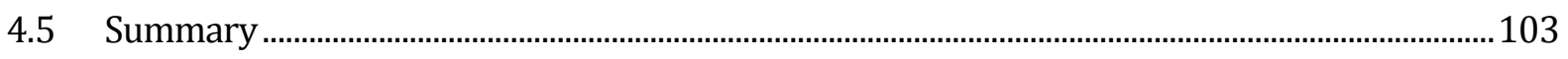

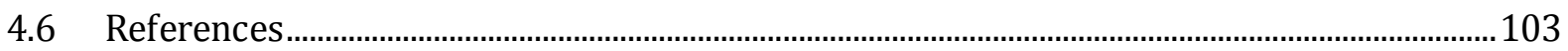

Chapter 5. Exploration of the utility of the Azul protecting group ......................................106

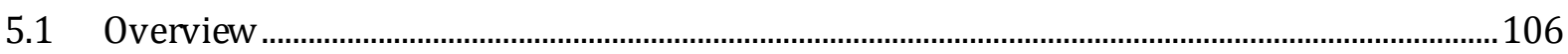

A note on quantitative NMR spectroscopy......................................................................107

5.2 Exploration of Azul deprotection conditions............................................................................108

5.2.1 Investigation of deprotection at room temperature ...................................................109

5.2.2 Investigation of deprotection at elevated temperature.............................................111

5.2.3 Two-step activation - deprotection methodology......................................................111

Addition of a methyl ketoester........................................................................................112

Addition of dimethylsulfonium chloride...................................................................114

Vilsmeier-Haack formylation....................................................................................115

5.2.4 Deprotection of other substrates...............................................................................116

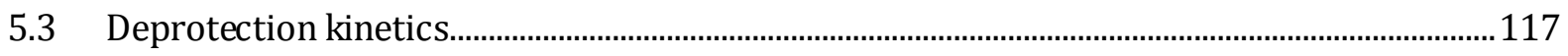

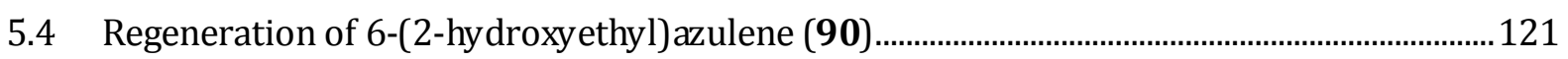

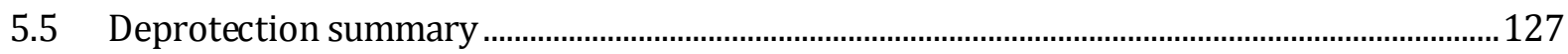

5.6 Comparisons with other protecting groups...........................................................................128

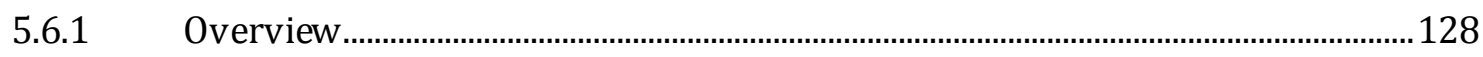

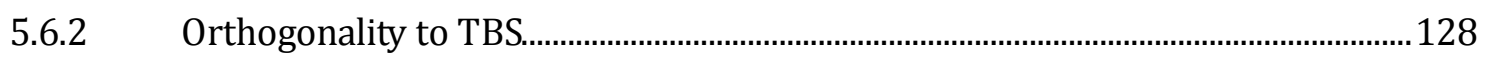

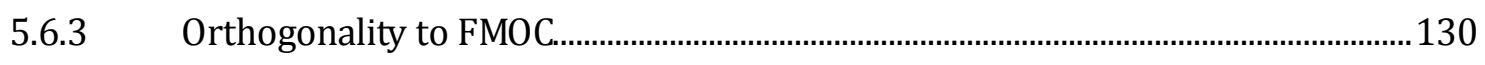

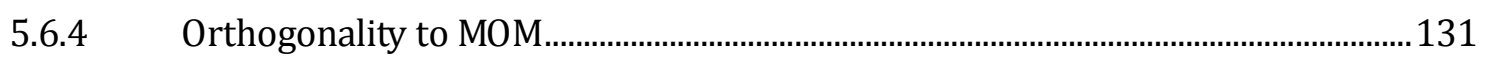

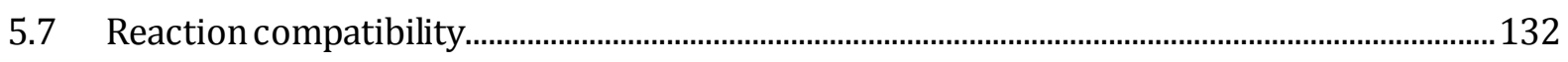

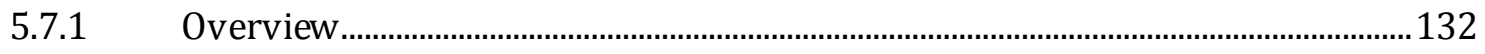

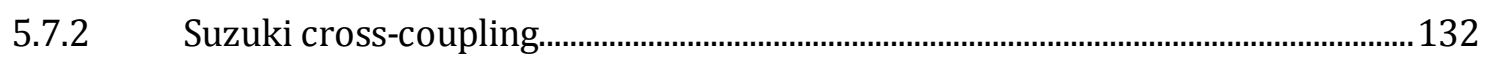

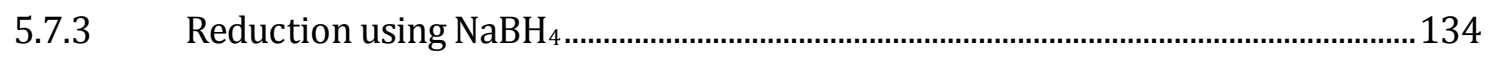

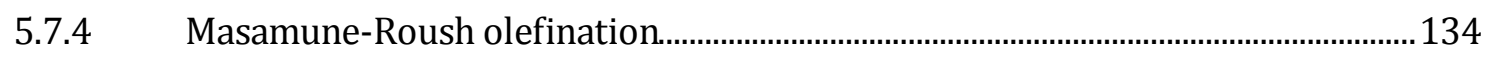

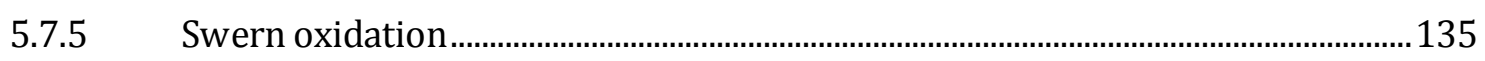

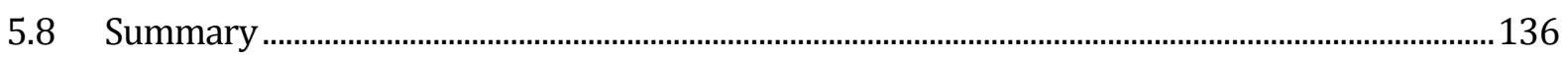




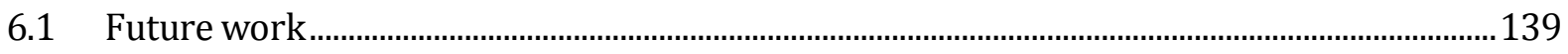

6.1.1 Further development of the Azul protecting group for carboxylic acids...............139

6.1.2 Development of the AzulOC protecting group for alcohols and amines..................141

6.1.3 Other azulene based protecting groups ……………………………………………....... 141

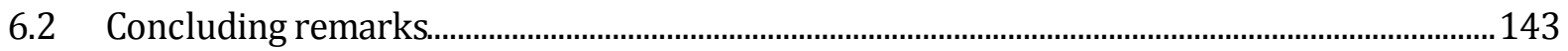

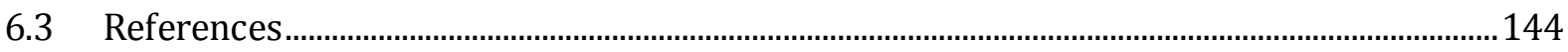

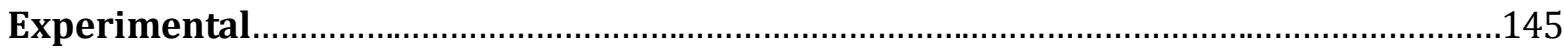

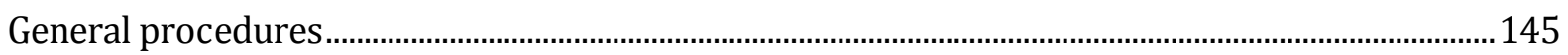

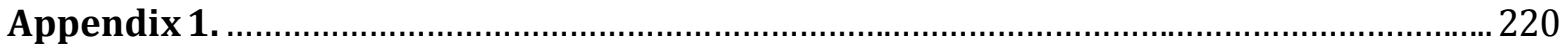

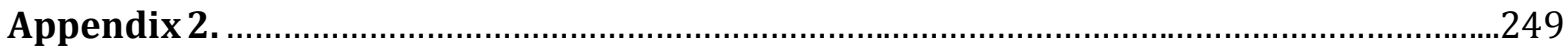




\section{List of figures}

Figure 1. Azulene

Figure 2. Naturally-occurring azulene derivatives. From left to right, guaiazulene (2), isoguaiazulene (3), chamazulene (4) and vetivazulene (5).

Figure 3. Resonance structures of azulene, illustrating the separation of charge within the molecule.

Figure 4. The frontier orbitals of naphthalene and azulene .......................................... 2

Figure 5. Conventional numbering of the azulene ring system......................................... 2

Figure 6. Stabilisation of the charged intermediates from electrophilic attack at the 1- and 3positions and nucleophilic attack at the $4-$, 6- or 8 -positions ............................................ 3

Figure 7. Stabilisation of a cation at the 1-position and an anion at the 4- and 6-positions........ 3

Figure 8. Examples of coloured azulenes. From left to right: 1,3-dinitroazulene (6), azulene1methyl ketoester (7), azulene 1-methyl ester (8), 4,6,8-trimethylazulene (9), 6-methylazulene (10), azulene (1), 5-methylazulene (11), guaiazulene (2)

Figure 9. Cartoon illustration of a role of a protecting group in the synthesis of a complex molecule, where $X$ stands for a reactive functional group 6

Figure 10. Cartoon depiction of protecting group orthogonality. Alcohol protecting groups A, B and $\mathrm{C}$ can be selectively deprotected in the presence of each other.

Figure 11. Depiction of graduated lability. Protecting group 1 is most readily deprotected, followed by 2 and finally 3

Figure 12. Example of orthogonality through the existence of two axes of graduated lability.... 8

Figure 13. Allyl and benzyl ethers are examples of UV-absorbing protecting groups................ 9

Figure 14. FMOC 16, pyrenyl 17 and dansyl 18 can be used as fluorescent protecting groups.. 10

Figure 15. Examples of coloured protecting groups 10

Figure 16. Depiction of the components of a generalised protecting group (left), and of a BOC group (right)

Figure 17. The SOB group 23 and 4-nitro-4-methyl pentanoate group $\mathbf{2 4}$ are examples of tethered protecting groups that deprotect through intramolecular nucleophilic attack. ......... 12

Figure 18. Common protected functional ities employed for alcohol protection .................... 12

Figure 19. Typical protected functionalities used in amine protection. ............................... 13

Figure 20. Carboxylic acids are protected almost exclusively as carboxylic esters.....................14

Figure 21. Examples of protecting groups with a methyl as a protecting group body ................14

Figure 22. Examples of protecting groups with a t-butyl as a protecting group body.............. 14

Figure $\mathbf{2 3 .} \mathbf{3 8}$ and $\mathbf{3 9}$ are protecting groups incorporating the bulkyadamantyl moiety, and $\mathbf{4 0}$ is the mesitoate protecting group, whose steric bulkimparts considerable stability towards aqueous base 
Figure 24. Examples of protecting group bodies incorporating electron-withdrawing groups that modify the reactivity of the protected functionality ................................................ 15

Figure 25. Examples of common silyl protecting groups.................................................... 16

Figure 26. Silyl groups incorporated onto non-ether protected functionalities...................... 16

Figure 27. Protecting groups cleavable by hydrogenation ............................................ 17

Figure 28. General depiction of a protecting group cleaved through an E1cB mechanism ....... 17

Figure 29. The PG remnant will often undergo nucleophilic attack on the vinyl moiety by leftover base

Figure 30. FMOC protecting groups 16 and $\mathbf{6 4}$ for amines and al cohols, and the Fm ester $\mathbf{6 5}$ for

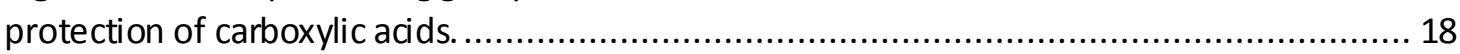

Figure 31. Further examples of protecting groups cleavable through $\beta$-elimination............... 18

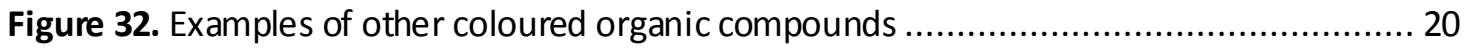

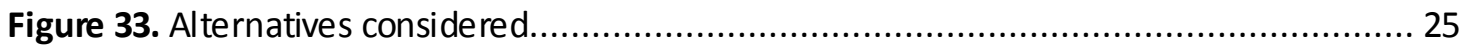

Figure 34. General form of Hafner-type syntheses of azulenes ........................................ 31

Figure 35. Methyl substituted azulenes $99, \mathbf{1 1}, \mathbf{1 0}, 9$ were targeted for synthesis ................. 34

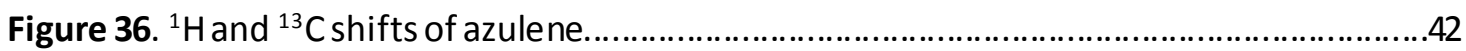

Figure 37. ${ }^{1} \mathrm{HNMR}$ spectrum and HSQC spectrum of azulene, illustrating the differences in coupling constants between the rings and the separation of the shifts of odd and even positions.

Figure 38. The three protecting group candidates incorporating a 1-substituted azulene moiety

Figure 39. Azulene (1), azulene-based by-products $\mathbf{7 5}$ and $\mathbf{1 2 3}$ isolated from protection

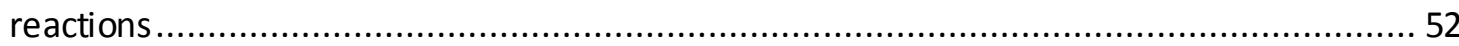

Figure 40. Proposed structure of the water-soluble orange compound .............................. 53

Figure 41. Reaction mechanism for basic hydrolysis of esters............................................ 56

Figure 42. ${ }^{1} \mathrm{H}$ NMR spectrum of the reaction mixture of $\mathbf{1 2 2}+\mathbf{1 3 6}$ with $\mathrm{KOH}, \mathrm{MeOH}$ and $\mathrm{D}_{2} \mathrm{O}$ at $\mathrm{t}=0$. Full unaltered spectrum on left, and baseline-corrected spectrum of the relevant aromatic region on right. 60

Figure 43. Graph of the saponification of the nitroazulene ester $\mathbf{1 3 6}$ over time 60

Figure 44. Logarithm of the proportion of menthyl ester 136 over time, demonstrating a linear trend......

Figure 45. Azulene diironpentacarbonyl, side on (left) and view from above (right), depicting the asymmetrical arrangement of the Fe atomsover the ring system (CO ligands omitted for clarity).

Figure 46. Azulene keto-acid chloride $\mathbf{1 5 9}$ can react with a second equivalent of azulene or oxalyl chloride, but both pathways are much slower than the initial reaction 
Figure 47. The presence of the methyl group at the 4-position is thought to force the keto-acid chloride out of plane, diminishing its electron-withdrawing effect over the azulene ring system

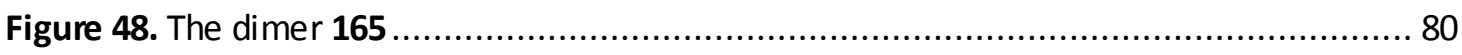

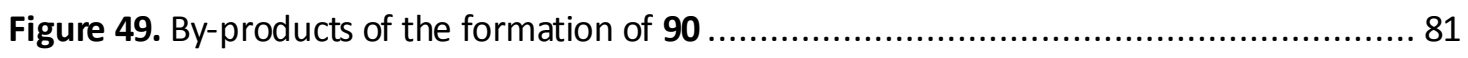

Figure 50. Equilibrium of formaldehyde with polyoxymethylene .................................... 82

Figure 51. Relationship between the excess of paraformal dehyde and the isolated yield of $\mathbf{9 0 8 3}$

Figure 52. Distribution of yields of $\mathbf{9 0}$ for the mode of addition of formal dehyde ................... 84

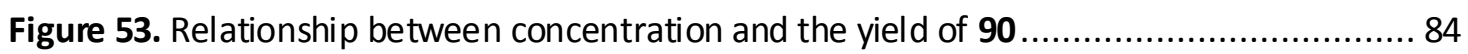

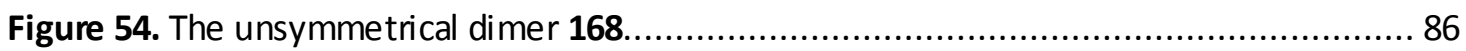

Figure 55. An expansion of the ${ }^{1} \mathrm{H}$ NMR spectrum of impure 90 , showing peaks belonging to the 4- and 2-positions with the peaks belonging to the dimer 168 masquerading as carbon satellites,

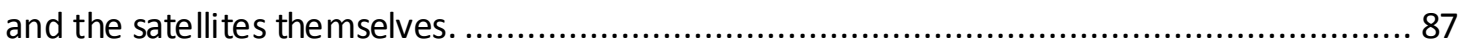

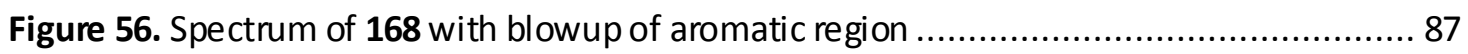

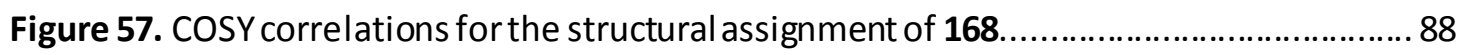

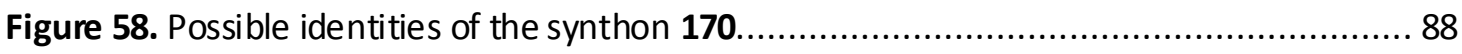

Figure 59. Cinnamate ester 178, on left, and 6-vinylazulene (94) on right, demonstrating colour change.

Figure 60. The nitro group confers additional stabilisation of the negative charge by the 6position, facilitating cleavage .102

Figure 61. The Azul protecting group for carboxylic acids, and the AzulOC protecting group for amines and alcohols. 106

Figure 62. Possible mono-and di-EWG-substituted azulene derivatives that could act as activated Azul species.

Figure 63. Absorbance spectra of protected and deprotected Azul-cyclohexanecarboxylate 183, describing the observed change in colour.

Figure 64 . Reaction rate of 183 , showing a reaction rate consistent with first order kinetics ..118

Figure 65. Correlation between the logarithm of the reaction rate and $\mathrm{pKa}$ of the leaving group

Figure 66. The elimination step - believed to be the rate-limiting step.

Figure 67. Addition of DBU causes formation of a Meisenheimer complex that is more strongly absorbing than the Azul species.

Figure 68. The postulated structure of the ol igomeric Wheland-Meisenheimer complex generated from 187

Figure 69. Resonance structure of 6-vinylazulene.

Figure 70. Possible structure of one of the products resulting from $\mathrm{CeCl}_{3}$-mediated deprotection of DMB

Figure 71. FMOC-Gly-OAzul. 
Figure 72. Azul-benzoate $\mathbf{1 8 2}$ was used as the Azul-protected substrate

Figure 73. The acidity of the $\alpha$-proton is increased through the complexation of a metal to the phosphonoacetate in the Masamune Roush olefination 135

Figure 74. Depiction of the colours encountered throughout this project 146

\section{List of Schemes}

Scheme 1. The DMOC protecting group can be deprotected through a two-step process. Substrate is bolded 9

Scheme 2. Proposed protection of alcohols as azulenyl esters

Scheme 3. The success of protecting group candidate $\mathbf{7 4}$ hinges on the ability to convert keto acid-chloride 76 to acid chloride 77.

Scheme 4. Protection of amines through reductive amination of $\mathbf{7 8}$

Scheme 5. Nucleophilic displacement of the leaving group (in this case a protonated amine) would be facilitated by the stabilisation of positive charge in the transition state.

Scheme 6. Protection and deprotection of amines as azulenesulfonamides ........................ 24

Scheme 7. The reaction discovered during my Master's research ..................................... 25

Scheme 8. The protocol for amine deprotection reported by Lindhorst et al........................ 25

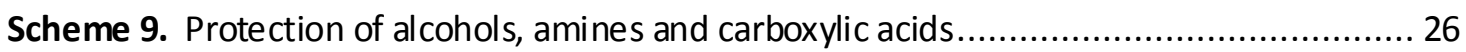

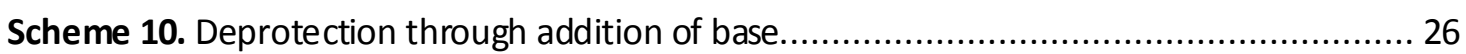

Scheme 11. Synthesis of the precursor 90 for the protecting group candidate 89 from 6-

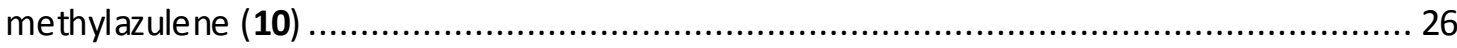

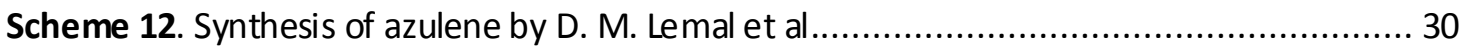

Scheme 13. Synthesis of azulene starting from cycloheptatriene by J.P. Depres et al........... 30

Scheme 14. The three steps involved in the one-pot Hafner synthesis of azulene .................. 32

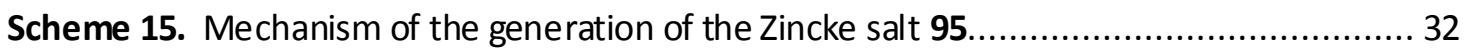

Scheme 16. Proposed mechanism of the formation of the pentamethine $96 \ldots \ldots \ldots \ldots \ldots \ldots \ldots . . . . . . . . . . .33$

Scheme 17. Proposed mechanism of the generation of azulene (1) .................................. 33

Scheme 18. Proposed mechanism of the formation of 4,6,8-trimethylazulene (9) ................. 35

Scheme 19. Proposed mechanism of formation of 2,4,6-trimethyl pyrylium tetrafluoroborate

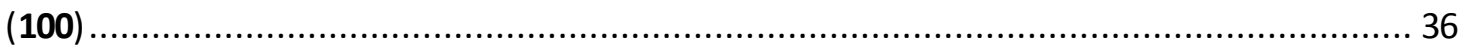

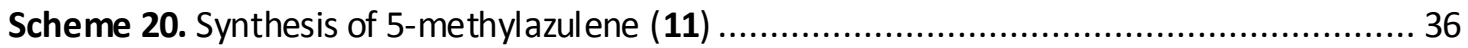

Scheme 21. Two-step synthesis of 1-methylazulene (101) through reduction of 1-formyl

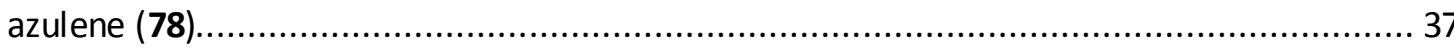

Scheme 22. Reaction of 2-, 3-, and 4-methylpyridines with DNCB................................... 37

Scheme 23. Synthesis of 4-methylazulene (99) …...................................................... 38 
Scheme 24. Literature example of an alkylated pyridinium bromide being used to synthesise the 4-methyl-7-ethylazulene (103).

Scheme 25. Literature example of the activation of the highly hindered pyridine 104 allowing the formation of the azulene 105

Scheme 26. Attempted syntheses of azulene 1 and 4-methylazul ene (99) by reacting a $\mathrm{N}$ methyl pyridinium iodide with sodium cyclopentadienide.

Scheme 27. First attempt at making 6-methylazulene (10).............................................. 40

Scheme 28. Optimised protocol for formation of 10...................................................... 41

Scheme 29. Proposed mechanism of formation of 6-p-tolylazulene (107) ........................... 42

Scheme 30. Generalised strategy for the protection and deprotection of amines with $79 \ldots . . .46$

Scheme 31. One equivalent of 108 attacks a second equivalent, releasing cyclohexylamine and methylenecyclohexylimine.

Scheme 32. A possible route to the protected substrate 79 through reductive amination of the amine with 78 . 46

Scheme 33. Investigation of protection methodology on pyrrolidine 47

Scheme 34. Generalised protection and deprotection strategy of amines with 82

Scheme 35. Proposed synthetic path to 82 from azulene.

Scheme 36. Generalised protection and deprotection strategy for alcohols as the ester $74 \ldots 48$

Scheme 37. Synthetic pathway to guaiazulene esters and ketoesters.

Scheme 38. Optimised protocol for the protection of menthol as a 1-azulenyl menthylester $\mathbf{1 2 2}$

Scheme 39. Attempted Yamaguchi esterification produced large quantities of the anhydride 123

Scheme 40. Regeneration of azulene from 75 in high yield ............................................. 55

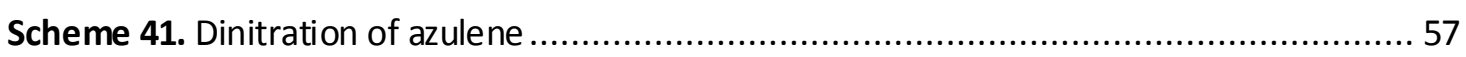

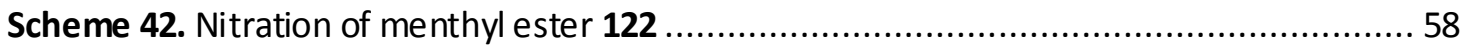

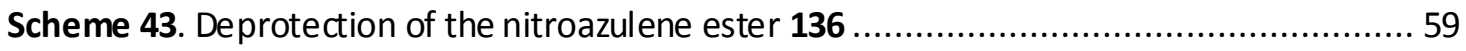

Scheme 44. Competitive deprotection of azulene ester 122 and nitroazulene ester $136 \ldots \ldots . . .60$

Scheme 45. Disruption of aromaticity through nucleophilic attack at the 4-position............... 62

Scheme 46. Possible products of nucleophilic attack on 74 by methyllithium....................... 62

Scheme 47. Possible products from TCNE reaction with 1,3-disubstituted azulenes (above) and

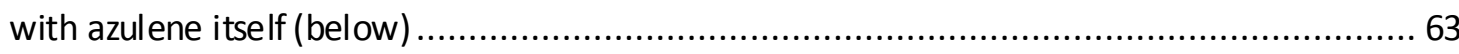

Scheme 48. Syntheses of azulene and 4,6,8-trimethylazulene iron carbonyl complexes......... 65

Scheme 49. Reaction of menthyl ester 122 with diiron nonacarbonyl generated the complex

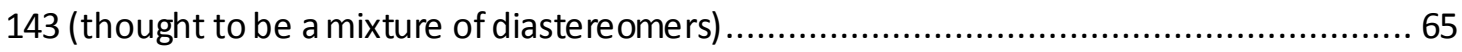

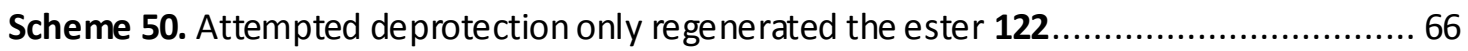


Scheme 51. Generalised depiction of tethered deprotection involving deprotonation of the 4position of $\mathbf{1 4 4}$ followed by alkoxymethylenation with paraformaldehyde to form 145 and intramolecular nucleophilic attack to release the substrate RO- and form the lactone $146 \ldots . . .66$

Scheme 52. Reaction of 4-methylazulene 85 gave the undesired isomer $147 \ldots \ldots \ldots \ldots \ldots \ldots \ldots . . . . . . . . .67$

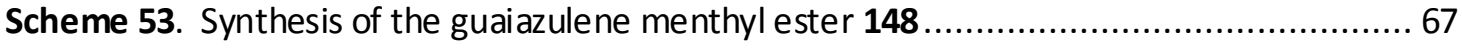

Scheme 54. Attempted one-step deprotection of the guaiazulene menthyl ester $148 \ldots \ldots \ldots . . .68$

Scheme 55. Attempted tethered deprotection using LHMDS.......................................... 69

Scheme 56. Room-temperature reaction of 148 with paraformal dehyde and DBU................69

Scheme 57. Reaction of $\mathbf{1 4 8}$ at elevated temperatures gave some deprotection and formation

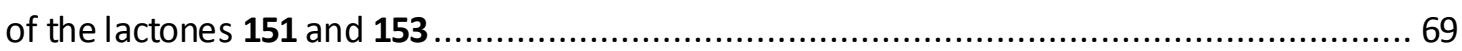

Scheme 58. Hypothesised origin of the brick-red substance 156...................................... 71

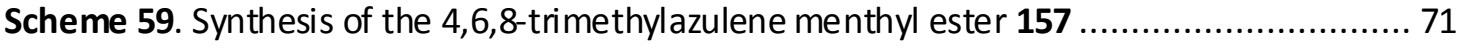

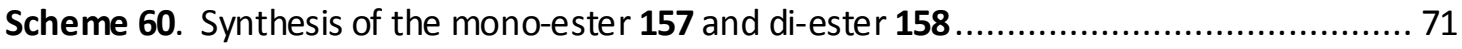

Scheme 61. One-step deprotection characteristics of the 4,6,8-trimethylazulene menthyl ester

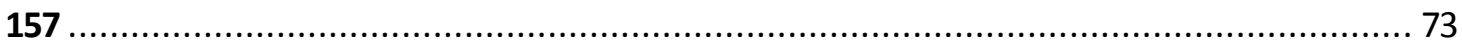

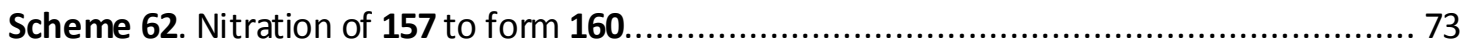

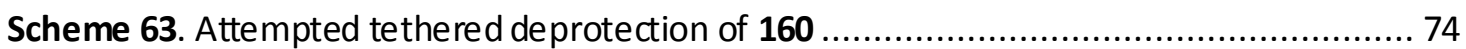

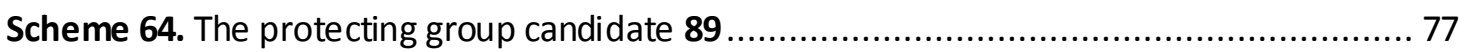

Scheme 65. General deprotection mechanism of the protecting group candidate 89 ............ 77

Scheme 66. Hydroxymethylation of guaiazulene (2) and 6-methylazulene (10).................... 78

Scheme 67. The two methods of investigating the extent of deprotection .......................... 79

Scheme 68. Attempted replication of the conditions that form 166 and 167 ...................... 81

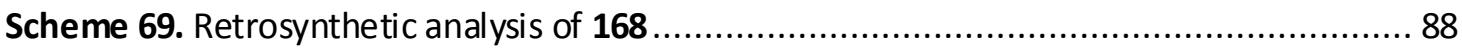

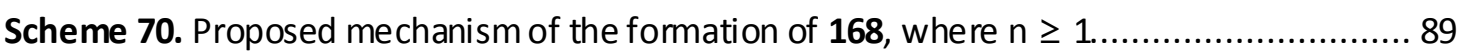

Scheme 71. Amines, alcohols and carboxylic acids were targeted as substrates for development

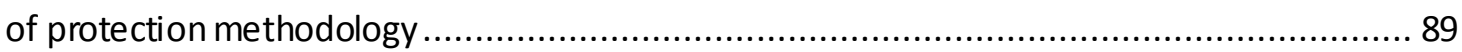

Scheme 72. General strategy of protection of alcohols, amines and carboxylic acids ............. 90

Scheme 73. Yamaguchi esterification of cinnamic acid $\mathbf{1 7 7}$ with $\mathbf{9 0}$ gave incomplete conversion

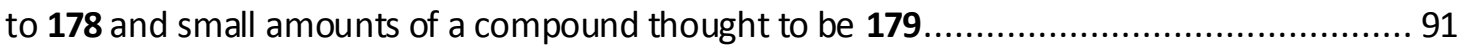

Scheme 74. Attempted coupling of 90 with cinnamyl chloride ....................................... 91

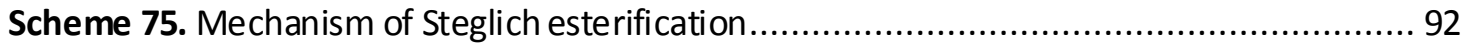

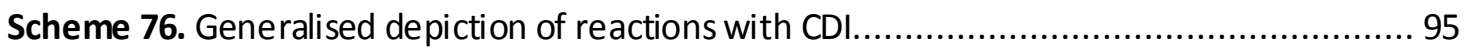

Scheme 77. Synthesis of the cydohexanemethanol carbonate 191................................... 95

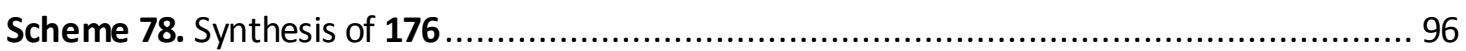

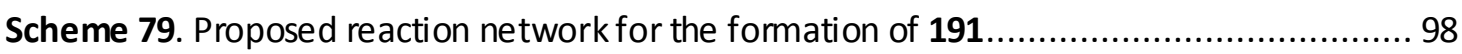


Scheme 80. Proposed reaction network responsible for the formation of the symmetric dimer

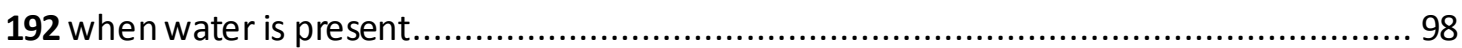

Scheme 81. Solid state formation of the cetyl carbonate 193.......................................... 99

Scheme 82. Initial deprotection conditions investigated for 178....................................101

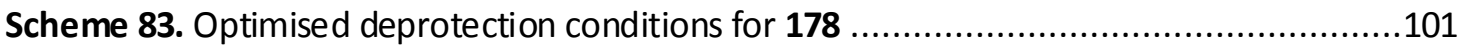

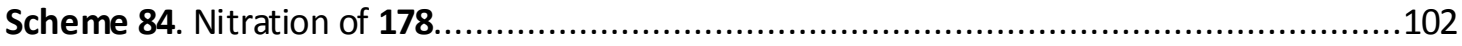

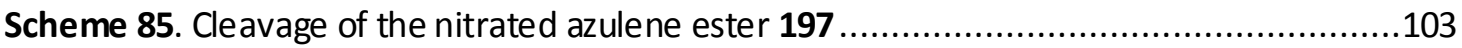

Scheme 86. Addition of a methyl ketoester to azulene .................................................113

Scheme 87. Activation of Azul cinnamate 178 to form 205 quantitatively..........................113

Scheme 88. Unsuccessful attempt at adding a second methyl ketoester to 7.......................113

Scheme 89. Addition of dimethylsulfonium to Azul can be carried out under Swernoxidation conditions.

Scheme 90. Attempted dimethylsulfonium addition-deprotection of $\mathbf{1 7 8}$ using $\mathrm{NEt}_{3}$ as a base

Scheme 91. Attempted Vilsmeier-Haack activation-deprotection

Scheme 92. Ester cleavage of trichloroacetate $\mathbf{2 1 0}$ and dichloroacetate $\mathbf{2 1 1}$ proceeds through saponification, rather than $\beta$-elimination.

Scheme 93. Formation of a coloured Meisenheimer complex from DBU and $\mathbf{1 8 7}$

Scheme 94. The piperidine adduct 195 is generated in Azul deprotections involving piperidine

Scheme 95. General strategy for regeneration of $\mathbf{9 0 .}$

Scheme 96. Mechanism for acid-catalysed alkene hydration.

Scheme 97. Mechanism for basic alkene hydration

Scheme 98. Low-temperature Suzuki cross coupling in the presence of 182 generated a small amount of 90

Scheme 99. Attempted synthesis of 95 by repeating the conditions found in Scheme 96 ......124

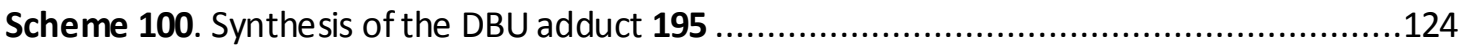

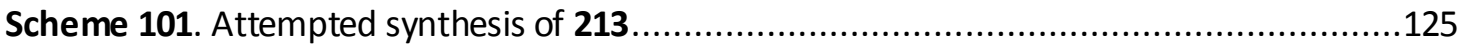

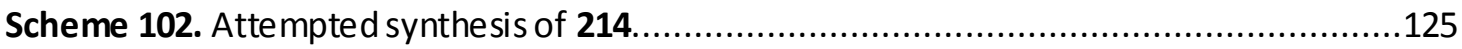

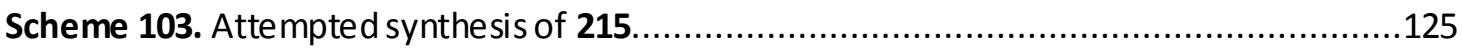

Scheme 104. The methyl ether $\mathbf{2 1 6}$ was generated from the methanolic $\mathrm{KOH}$ present in the

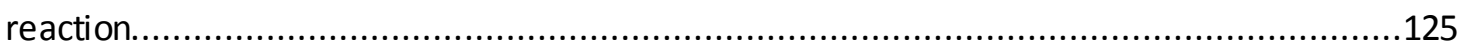

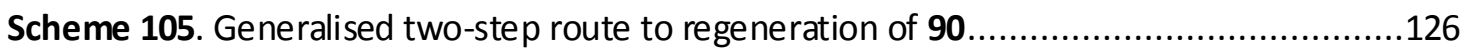

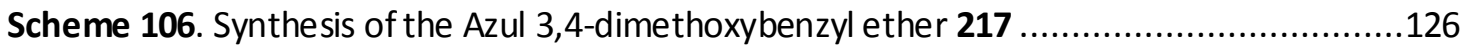

Scheme 107. Addition of DDQ resulted in rapid degradation of the azulene moiety..............126

Scheme 108. Attempted synthesis of a bis-protected glycolic acid...................................128 
Scheme 109. Various conditions for deprotection were explored on the mixture of 178 and 221

Scheme 110. Activation of FMOC-Gly-OAzul in preparation for selective Azul deprotection ...130

Scheme 111. Various conditions for deprotection were explored on the mixture of 178 and 223

Scheme 112. The reaction used to assess the compatibility of Suzuki cross coupling with Azul

Scheme 113. The reaction used to assess Azul compatibility with low-temperature Suzuki cross coupling

Scheme 114. The reaction used to assess Azul compatibility with $\mathrm{NaBH}_{4}$ reduction. 134

Scheme 115. The reaction used to assess Azul compatibility with Masamune-Roush olefination

Scheme 116. The reaction used to assess Azul compatibility with Swern oxidation .135

Scheme 117. Comparison of the reaction rates between addition of the dimethylsulfonium chloride to an al cohol and to Azul.

Scheme 118. Azul protection-olefination using Masamune-Roush..................................140

Scheme 119. Azul protection-olefination using Bestmann ylide 140

Scheme 120. Possible methods of tandem deprotection-esterification or deprotectionlactonisation 140

Scheme 121. Synthesis of the protecting group precursor 240 142

Scheme 122. Protection of $\mathbf{2 4 0}$ as $\mathbf{2 4 1}$, and its deprotection 142

Scheme 123. Generation of $\mathbf{2 4 3}$ and protection of a substrate as $\mathbf{2 4 4}$. 142 


\section{List of tables}

Table 1. Positional and electronic effect of substituents on the colour of azulene derivatives ... 4

Table 2. Reaction parametersand yields for the formation of 6-methylazulene (10) in isopropanol with sodium

Table 3. Yields of ketoesters and esters of 1,102 , and $\mathbf{9}$ when subjected to the reaction conditions above...

Table 4. Optimisation of the conditions for the formation of 1-azulenyl methyl ester.

Table 5. Optimisation of the deprotection conditions of $\mathbf{1 2 2}$

Table 6. Calculated structures and the deviations in the ir $C=0$ stretch from that of $8\left(1781 \mathrm{~cm}^{-1}\right)$

Table 7. Attempted optimisation of mono-nitration of azulene to form 1-nitroazulene $135 \ldots 58$

Table 8. Steglich esterification of cinnamic acid with $\mathbf{9 0 .}$ 92

Table 9. A selection of carboxylic acids were protected through Steglich methodology 93

Table 10. Solid state Steglich syntheses of esters 178 and 182 94

Table 11. Attempted optimisation of the formation of 191

Table 12. Optimisation of the formation of 194 . 100

Table 13. List of common mild bases in order of their basicity.... 109

Table 14. Bases and solvents and their degree of deprotection at room temperature. All reactions were performed with overnight duration, unless otherwise stated.....

Table 15. Bases and solvents and their effect on deprotection at elevated temperatures

Table 16. Exploration of the sensitivity of $\mathbf{2 0 9}$ towards various bases.

Table 17. Deprotection of Azul-protected carboxylates and Cetyl AzulOC.

Table 18. The deprotection rates and pKas of the substrates tested 118

Table 19. Attempts at synthesising 90 from 94 through direct addition of $\mathrm{H}_{2} \mathrm{O}$ or $\mathrm{OH}^{-}$

Table 20. Selective and global deprotection conditions for Azul and TBS mixtures

Table 21. Selective and global deprotection conditions for FMOC-Gly-OAzul 131

Table 22. Selective deprotection conditions for Azul and MOM mixtures 131 


\section{List of abbreviations}

Azul: $\quad$ 2-(azulen-6-yl)ethyl [protecting group]

AzulOC: $\quad$ 2-(azulen-6-yl)ethyloxycarbonyl [in carbonate or carbamate protecting groups]

CDI: $\quad N, N^{\prime}$-Carbonyldiimidazole

COSY: $\quad$ Correlation Spectroscopy

d: $\quad$ Doublet

DABCO: $\quad$ 1,4-Diazabicyclo[2.2.2]octane

DBN: $\quad$ 1,5-Diazabicyclo[3.4.0]nonane

DBU: $\quad$ 1,8-Diazabicyclo[5.4.0]undecane

DCC: $\quad N, N^{\prime}$-Dicyclohexylcarbodiimide

DDQ: $\quad$ 2,3-dichloro-5,6-dicyanoquinone

DIBAL-H: Diisobutylaluminium hydride

DIPEA: Diisopropylethylamine

DMAP: $\quad 4-N, N^{\prime}$-Dimethylaminopyridine

DMB: 3,4-Dimethoxybenzyl [protecting group]

DMP: Dess-Martin periodinane

DME: 1,2-Dimethoxyethane

EDCI: $\quad$ 1-Ethyl-3-(3-dimethylaminopropyl)carbodiimide ( $\mathrm{HCl}$ salt)

eq.: $\quad$ Equivalents

Fm: $\quad$ Fluorenyl-9-methyl [protecting group]

FMOC: $\quad$ Fluorenyl-9-methyloxycarbonyl [protecting group]

HMBC: $\quad$ Heteronuclear multiple bond correlation

HRMS: $\quad$ High-resolution mass spectrometry

HSQC: $\quad$ Heteronuclear single quantum coherence 
IR:

Infra-red [spectroscopy]

LDA: Lithium diisopropylamide

m:

Multiplet

MOM: $\quad$ Methoxymethyl [protecting group]

NMR: $\quad$ Nuclear magnetic resonance [spectroscopy]

PMB: $\quad$ para-methyoxybenzyl [protecting group]

PPTS: $\quad$ Pyridinium $p$-toluenesulfonate

q: $\quad$ Quartet

quin: Quintet

r.t. $\quad$ Room temperature $\left(\sim 20^{\circ} \mathrm{C}\right)$

RSM: $\quad$ Recovered starting material

RBF: $\quad$ Round-bottomflask

s: $\quad$ Singlet

sept: $\quad$ Septet

t: $\quad$ Triplet

TBAF: $\quad$ Tetra- $n$-butylammonium fluoride

TBDPS: $\quad$ tert-butyldiphenylsilyl [protecting group]

TBS: $\quad$ tert-butyldimethylsilyl [protecting group]

TES: $\quad$ triethylsilyl [protecting group]

TIPS: $\quad$ triisopropylsilyl [protecting group]

TMS: $\quad$ trimethylsilyl [protectinggroup]

THF: Tetrahydrofuran

TLC: Thin-layer chromatography

UV: $\quad$ Ultraviolet [spectroscopy] 


\section{Chapter 1. Introduction}

\subsection{Azulene}

The azulenes are a class of compounds, based on the parent azulene (1) (Figure 1), whose unusual properties have attracted attention from chemists ever since their discovery. These hydrocarbons exhibit interesting physical and chemical properties, including unique reactivity, and are almost always coloured. ${ }^{1}$

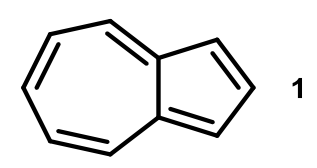

Figure 1. Azulene (1)

Several derivatives of azulene are natural products. These are usually terpenoids, and have been isolated from a variety of sources including plants and flowers, ${ }^{2}$ mushrooms ${ }^{3}$ and sea sponges. ${ }^{4}$ Guaiazulene (2) is the most common derivative, but others derivatives exist, such as isoguaiazulene (3), chamazulene (4) and vetivazulene (5) (Figure 2).
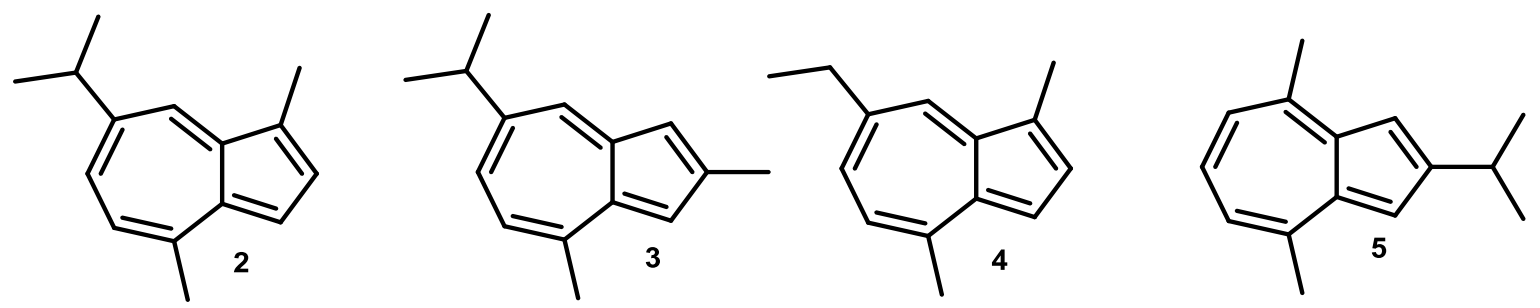

Figure 2. Na turally-occurring azulene deriva tives. From left to right, guaiazulene (2), isoguaiazulene (3), cha mazulene (4) and vetivazulene (5)

Despite their interesting properties, azulenes have found relatively few uses in chemistry and in general. Some have found applications as dyes in cosmetics, ${ }^{5}$ while others have been investigated for their biological properties. ${ }^{6}$ There is some on-going research into azulene-containing conducting polymers and photochromic substances, ${ }^{7}$ but so far only a few examples of applications of azulenes appear to exist within mainstream chemistry.

\subsection{The properties of azulenes}

The parent compound, azulene, is a fully conjugated hydrocarbon with a striking blue colour and a dipole moment of $\sim 1$ debye. ${ }^{8}$ These properties are a direct result of the 5,7-conjugated ring system. The dipole moment can be rationalised from the presence of the relatively stable cyclopentadienyl anion and tropylium cation-containing resonance structures (Figure 3). 


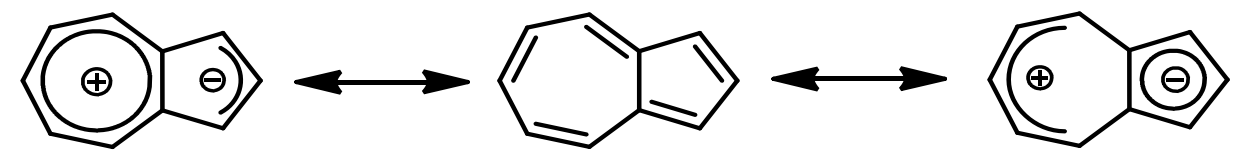

Figure 3. Resonance structures of azulene, illustrating the separation of charge within the molecule

Azulene belongs to a class of compounds called non-alternant hydrocarbons, which are conjugated compounds typified by odd-membered rings and unusual reactivity. ${ }^{9}$ This nonalternance results in the frontier orbitals being distributed in an antisymmetric manner around the ring system. Comparisons of azulene with its isomer naphthalene are usually made to illustrate the striking differences between the two and between alternant and non-alternant hydrocarbons in general (Figure 4). ${ }^{1}$

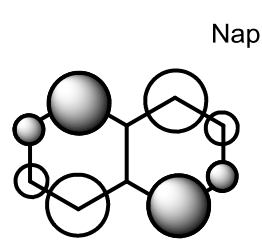

HOMO

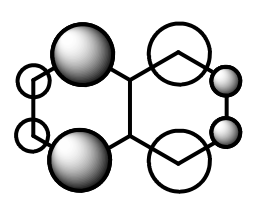

LUMO

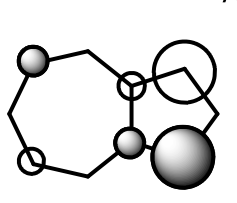

HOMO
Azulene

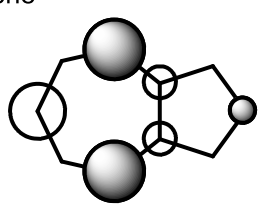

LUMO

Figure 4. The frontier orbitals of naphthalene and azulene

The electron density profile of the frontier orbitals of naphthalene exhibit a high degree of overlap with each other, whereas the electron density profile of the frontier orbitals of azulene exhibit poor overlap. This results in imbalances and localisations of electron density on azulene. The lack of overlap also creates a lack of repulsion between the HOMO and LUMO, simply because upon excitation, an electron enters an orbital where the pre-existing repulsive electron density from the HOMO is low. The HOMO-LUMO gap is reduced as a result, bringing the corresponding absorbance from ultraviolet to visible wavelengths. This poor orbital overlap and consequent small band-gap is ultimately the reason why azulene and its derivatives are coloured.

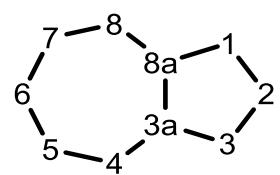

Figure 5. Conventional numbering of the azulene ring system

The localisation of electron density in azulene results in a much higher degree of reactivity when compared with naphthalene. Due to the HOMO being mainly localised on the 1- and 3-positions, these positions are highly nucleophilic. In fact, the azulenes boast the highest nucleophilicity of any neutral hydrocarbon, massively outstripping the nucleophilicity of 6-membered aromatics and fulvenes, and rivalling the nucleophilicity of heterocycles such as indoles.10 Similarly, the localisation of the LUMO at the 4-, 6-, and 8-positions means that azulenes are susceptible to 
attack by nucleophiles such as alkyllithium species and Grignard reagents. ${ }^{11}$ In contrast, the 2-, 5and 7-positions are relatively inert to either nucleophilic or electrophilic attack, and will generally undergo substitution in harsh conditions only after the more reactive centres are blocked.12

Much of this observed reactivity can be rationalised through inspection of the relevant resonance structures. For instance, electrophilic substitution on the 1- and 3-positions is promoted by the stabilising presence of the tropylium cation in the resonance structures of the intermediate in the reaction (Figure 6a). The corresponding intermediate structures that arise from electrophilic attack on other positions lack this stabilisation as they do not have access to the tropylium resonance structure. Nucleophilic attack at the 4-, 6- or 8-positions similarly results in stabilisation of the negatively-charged intermediate through access to the cyclopentadienyl anion

(Figure 6b).

a)

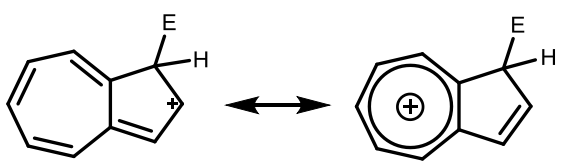

b)<smiles></smiles><smiles>N#[C+]C1C=CC2=CC=CC2=CC1</smiles>

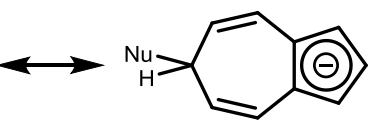

Figure 6. Sta bilisation of the charged intermediates from a) electrophilic attack at the 1- a nd 3-positions a nd b) nucleophilic attack at the 4-, 6- or 8-positions

Not only can these resonance-based arguments be used to explain the reactivity of azulene itself, they can also be used to predict the reactivity of moieties appended to the azulene ring system (Figure 7). Any full or partial positive charge directly adjacent to the 1- or 3- positions will experience dramatic stabilisation by way of the tropylium resonance structure, and similarly, full or partial negative charges adjacent to the 4-, 6-, or 8-positions will experience stabilisation through access to the cyclopentadienyl anion resonance structure. ${ }^{13}$ Judicious placements of certain functional groups on these positions can serve to promote or retard its reactivity relative to the unsubstituted counterpart, by either stabilising or destabilising a charged transition state.
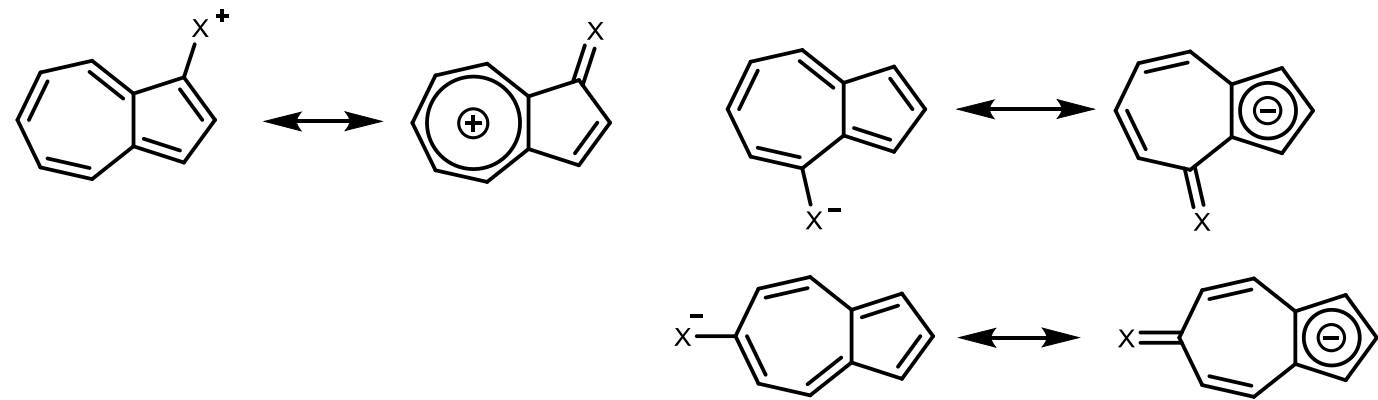

Figure 7. Stabilisation of a cation at the 1-position and an anion at the 4- and 6-positions 
Finally, a discussion on the chemistry of azulene would not be complete without a discussion of the colours of its derivatives. The blue colour of azulene itself comes from the $S_{0} \rightarrow S_{1}$ transition that absorbs light in the region around $580 \mathrm{~nm}$ - the yellow to red portion of the visible light spectrum. ${ }^{14}$ The light transmitted is primarily blue with a small amount of red, giving azulene a blue-violet colour.

The derivatives of azulene can come in a wide array of different colours, ranging usually from green to orange-red.15 Their colour can be predicted based on two different factors: the electron donating/withdrawing nature of the substituents, and their positions on the azulene ring. ${ }^{16}$ The presence of an electron donating or withdrawing group influences the relative energies of the frontier orbitals.

An electron donating group has the effect of raising the energy of the frontier orbitals, while a withdrawing group has the opposite effect. However, since there is so little overlap between the HOMO and LUMO, the electronic effects at a particular position will predominantly affect one orbital over the other. On odd numbered positions where the HOMO density is greatest, the HOMO is affected by the electronic effects of the substituents to a much greater extent than the LUMO. An EDG therefore raises the HOMO energy disproportionately and narrows the bandgap, resulting in a bathochromic shift and a corresponding greener colour. An EWG will do the opposite, lowering the HOMO energy, widening the bandgap and producing a hypsochromic shift that can result in colours from violet to orange. The converse of this is true for the even-numbered positions where the LUMO density is greatest. An EDG raises the LUMO energy while the HOMO energy remains static, widening the bandgap and resulting in a shift towards the violet. An EWG lowers the energy of the LUMO, resulting in a narrowing of the bandgap and a colour shift towards the green. As would be expected, this effect is most pronounced on the 1-, 3-, 4-, 6-, and 8- positions due to the concentration of the frontier orbitals over these positions. Colour changes also occur on the 2-, 5and 7- positions, but to a lesser degree. These effects are summed up in Table 1.

\begin{tabular}{|l|l|l|}
\hline & Electron withdrawinggroup (EWG) & $\begin{array}{l}\text { Electron donating group } \\
\text { (EDG) }\end{array}$ \\
\hline Odd position & Hypsochromic shift & Bathochromic shift \\
\hline Even position & Bathochromic shift & Hypsochromic shift \\
\hline
\end{tabular}

Table 1. Positional and electronic effect of substituents on the colour of azulene derivatives

Examples of how the placement and electronic characteristics of substituents can affect the colour of azulenes can be seen in Figure 8. Illustrative of the rules in Table 1, the compounds 8 and 9 have almost identical colours arising from opposite substitution patterns, 8 having a single 
strongly electron-withdrawing group on an odd position while 9 having three weakly electrondonating groups on even positions.
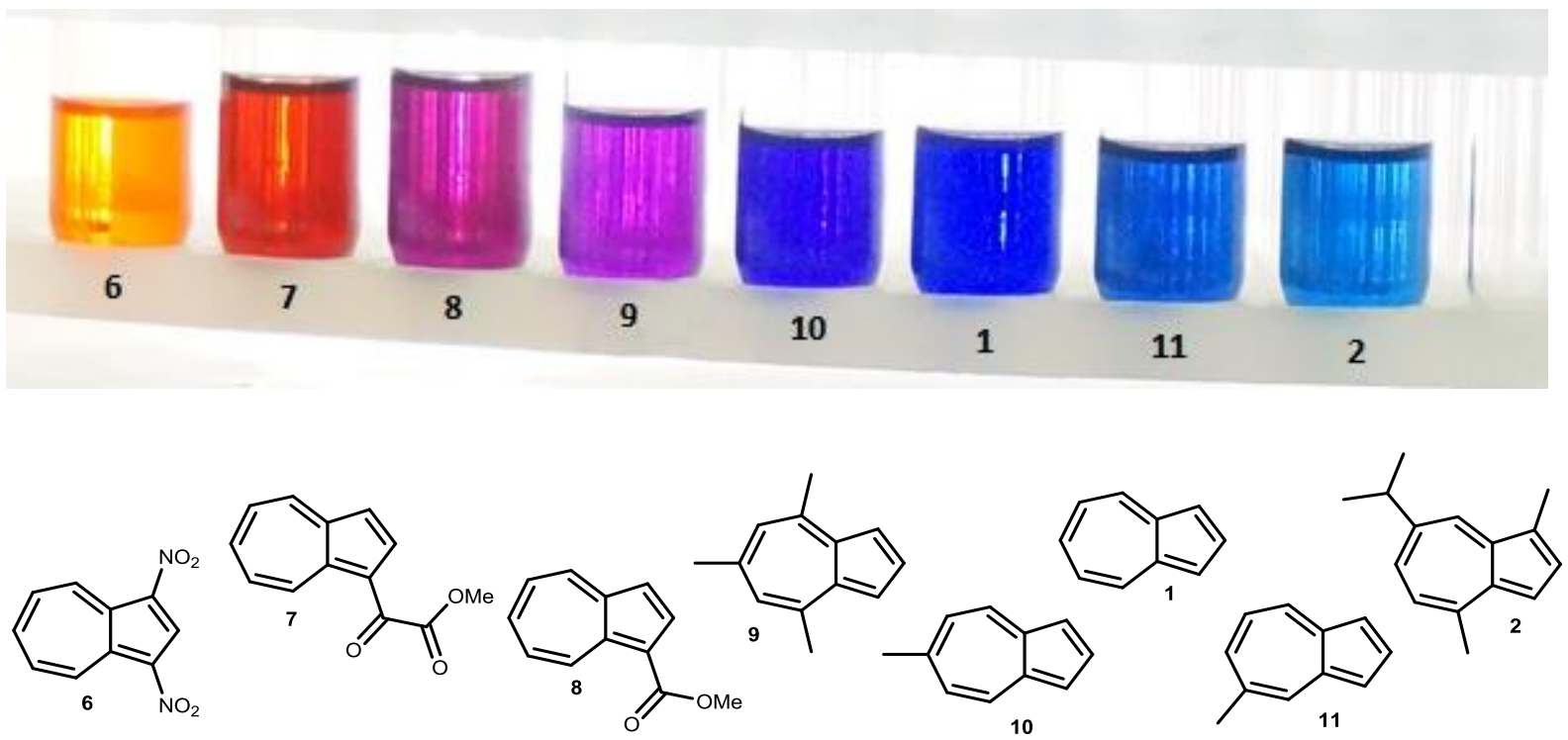

Figure 8. Exa mples of coloured a zulenes. From left to right: 1,3-dinitroazulene (6), a zulene 1-methyl ketoester (7), a zul ene 1-methyl ester (8), 4,6,8-trimethylazulene (9), 6-methylazulene (10), a zulene (1), 5-methyl azulene (11), guaiazulene (2)

\subsection{Protecting groups in organic synthesis}

The use of protecting groups is an indispensable part of organic synthesis today. Over the course of a multi-step synthesis there will often be instances where the reactivity of certain functional groups of the substrate must be masked in order to prevent unwanted reactions. Their use has a history stretching back about a century, ${ }^{17}$ although the formalisation of the concept of protection came later. ${ }^{17-18}$

Protecting group strategy plays a major role in the planning of a total synthesis, especially of larger and more complex synthetic targets which often have multiple functional groups that can react in various unwanted ways if their reactivity is not masked. Reactive groups must be passivated for the duration of the synthesis and only restored during the final deprotection steps or when a reaction is intended on these groups (Figure 9). Ideally every protected functional group should be facile to deprotection through conditions that do not disturb the rest of the molecule. 


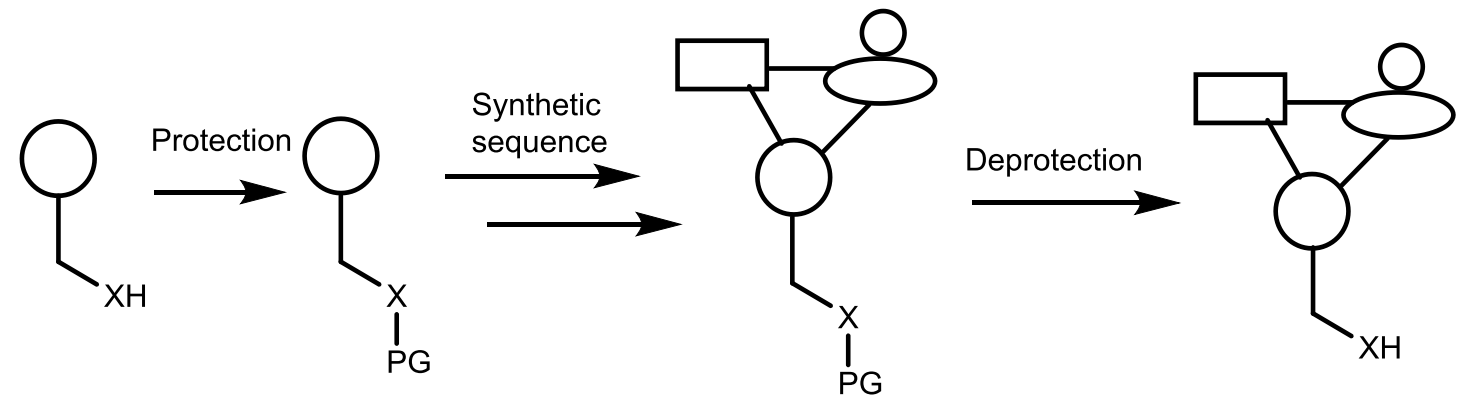

Figure 9. Ca rtoon illustration of a role of a protecting group in the synthesis of a complex molecule, where $\mathrm{X}$ stands for a reactive functional group

Functional groups that typically necessitate protection include hydroxyls, amines and carboxylic acids. These groups are often protected by default in a synthetic strategy, and are often protected as 'permanent' protecting groups (so-named because they remain for the duration of the synthesis), owing to the wide array of reaction conditions in which they can interfere. Functional groups such as carbonyls, thiols, alkynes and dienes also see protection, but these are generally as 'temporary' protecting groups (i.e. they only see use in particular circumstances where the unprotected functional group is reactive, and are generally discarded after a few steps). ${ }^{19}$

While there are examples in the literature of protecting-group-free natural product syntheses, 18 these are very much a minority. The field of protecting group-free synthesis, while promising, is still in its infancy. The synthetic targets amenable to this type of synthesis generally either do not contain many reactive functional groups, or contain functional groups that are installed late in the synthesis. Many synthetic targets are too complex or have too many reactive functional groups to be synthesised efficiently in this manner. ${ }^{20}$

\subsubsection{The requirements of a protecting group}

The ideal protecting group would be one that is completely inert to a wide range of possible reaction conditions, yet can be removed by a very specific and otherwise seldom-used set of conditions. Unfortunately, such protecting groups do not exist in reality and we must make do with protecting groups that may cleave or otherwise react in a wide variety of different conditions. One of the challenges of planning a total synthesis is the choice of protecting groups that are not only compatible with each other, but compatible with the planned transformations involved in the synthesis. The use of protecting groups is naturally associated with an increased cost, as it typically adds two steps (protection and deprotection) to a reaction sequence, which necessarily results in a loss of material over these steps and a lowering of the atom and step economy. ${ }^{18}$ Careful choice of protecting groups is necessary to avoid unintended reactivity and the unfortunate moments when one has to take a 'backwards step' and swap out a protecting group that has unforeseen incompatibilities for one that does not. 


\section{Orthogonality}

The concept of orthogonality refers to the ability of any one type of protecting group to be removed selectively, without affecting other protecting groups or functionalities present in the molecule (Figure 10). ${ }^{19}$
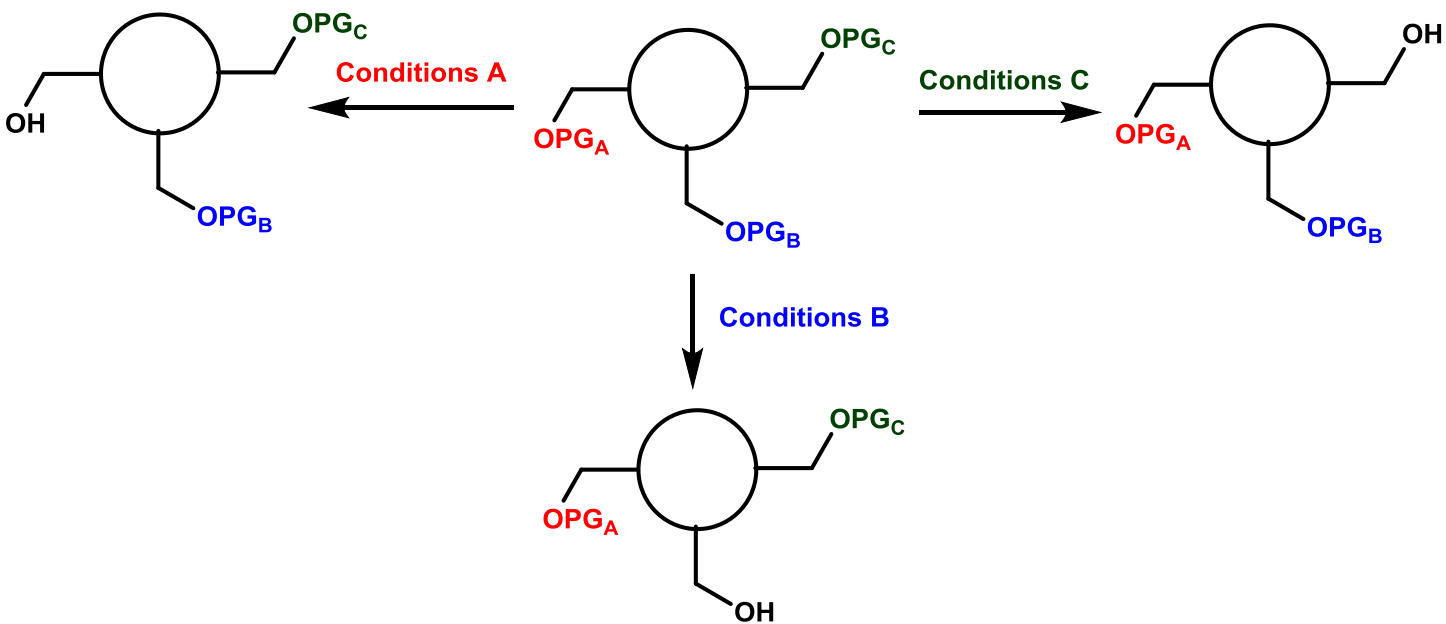

Figure 10. Cartoon depiction of protecting group orthogonality. Alcohol protecting groups A, B and C can be selectively deprotected in the presence of each other.

For larger or more complex syntheses, the number of different protecting groups employed increases, and therefore the importance of orthogonality in the choice of protecting groups increases. The synthesis of the marine natural product palytoxin by Kishi et al ${ }^{21}$ - one of the largest molecules to be synthesised to date - involved a total of 42 protecting groups of eight different types (methyl ester, acetate, benzoate, TBS ether, PMB ether, methyl ether for hemiacetal protection, acetonide and trimethylsilylethyl amide for an amine) which were cleaved over five deprotection reactions. ${ }^{21}$ The use of so many protecting groups is inevitable for such a complex target, as each fragment may be synthesised through a different set of reactions and therefore may require a different selection of protecting groups. As synthetic targets increase in size and complexity, the stringency of the requirements for orthogonality also increase.

\section{Graduated lability}

Selectivity in deprotection may arise through different protecting groups reacting with the same reagent at different rates. This is referred to as graduated lability. ${ }^{19}$ By gradually increasing the harshness of the cleavage conditions, one can cleave each protecting group in turn. These protecting groups exhibit only partial orthogonality, as the more robust protecting groups (such as $\mathrm{PG}_{3}$ in Figure 11) cannot be cleaved without also cleaving the more labile ones.

In practice, protecting groups can usually be cleaved by a variety of different conditions. Sometimes this is advantageous. For example, the silyl ether series of protecting groups (explored 
in more detail in section 1.5.3) are typically deprotected using either aqueous acid or a source of fluoride. However, while fluoride is often used to deprotect silyl ethers indiscriminately (the rate of reaction with fluoride varies by only about an order of magnitude between the different silyl ethers, making graduated lability difficult to achieve), aqueous acid can be used to selectively deprotect different silyl groups as the rate of reaction varies by about six orders of magnitude between the silyl ethers. ${ }^{22}$ If global deprotection is desired, a source of fluoride is used, otherwise if only some of the silyl protecting groups are to be removed, the appropriate strength of aqueous acid can be used.

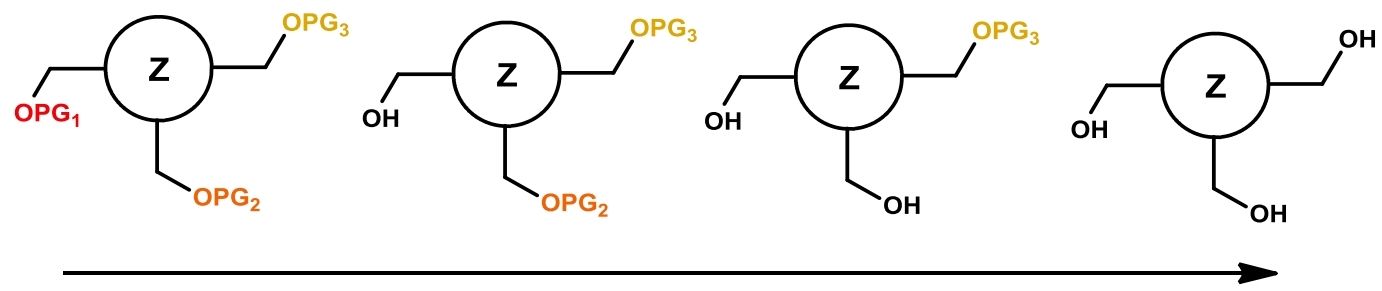

Increasing severity of deprotection conditions

Figure 11. Depiction of gra duated lability. Protecting group 1 is most readily deprotected, followed by 2 and finally 3

In another example, the benzyl (Bn) and para-methoxybenzyl (PMB) protecting groups are often used to protect alcohols (Figure 12). In most of the conditions used to cleave these protecting groups (strong aqueous acid, DDQ oxidation, catalytic hydrogenation) the PMB group is more labile than the benzyl group. However, lithium naphthalenide will cleave the benzyl group preferentially to the PMB group. ${ }^{23}$ This is an example of different and opposing regimes of graduated lability allowing much greater orthogonality to be achieved.

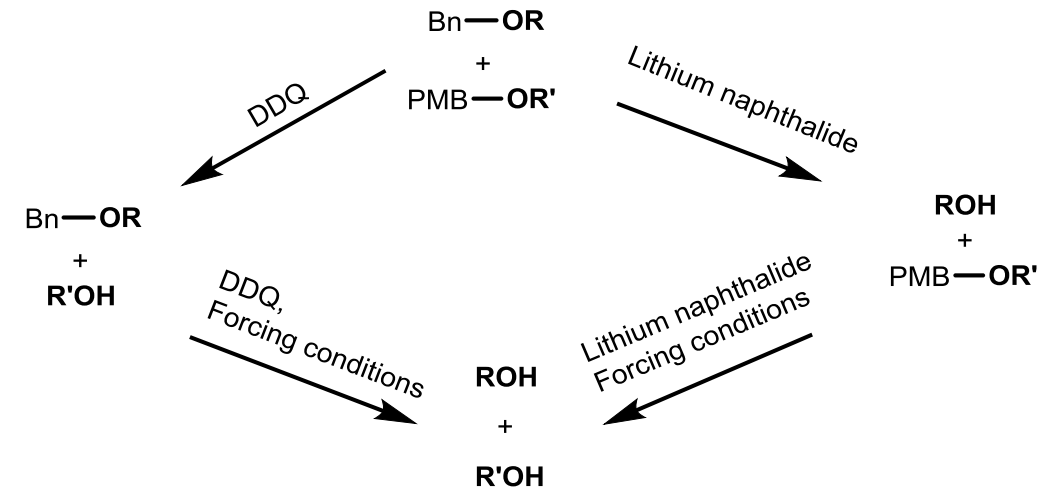

Figure 12. Example of orthogonality through the existence of two axes of graduated lability.

\section{Two-step deprotection}

There is a subclass of protecting groups called 'two step protecting groups', or sometimes 'safety catch' protecting groups. ${ }^{19}$ Deprotection typically involves an activation step followed by cleavage. The protecting group is transformed into a more labile protecting group in the first step, and this labile protecting group is then removed under mild conditions in the second step. These sequences are typically performed in one pot. One example of such a protecting group is the $(1,3-$ 
dithiane-2-yl)methoxycarbonyl (DMOC) protecting group 12 for amines ${ }^{19}$ (Scheme 1), which is activated by oxidation to the disulfone 13, and cleaved through $\beta$-elimination. The advantage a two-step protecting group has over an ordinary protecting group is that the possible pool of deprotection conditions is in principle combinatorially larger, allowing for much greater orthogonality. There are some limitations to this, though. The activation step still needs to be relatively mild and exhibit orthogonality to other protecting groups in order to achieve selective deprotection.

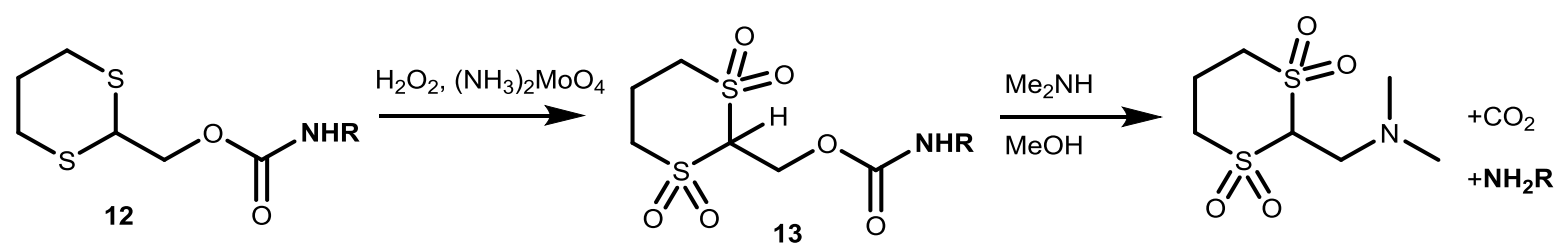

Scheme 1. The DMOC protecting group can be deprotected through a two-step process. Substrate is bolded.

\subsubsection{The handling benefits of protecting groups}

Aside from the reduction in unwanted reactivity, protecting groups confer other benefits in synthesis. Protecting groups are often less polar than their naked counterparts. This makes them easier to handle, as most organic chemistry purification techniques work best on moderately nonpolar compounds, and the increased bulk they impart to the substrate makes them less volatile and can sometimes allow for easier crystallisation. ${ }^{19}$

Another important function of protecting groups is in aiding visualisation when performing ThinLayer Chromatography (TLC), whether it be through chromophores or through specific moieties that stain vividly. Most $\mathrm{C}=\mathrm{C}$ moieties absorb strongly in the short-wave UV region, so compounds with protecting groups containing such moieties (such as allyl ether $\mathbf{1 4}$ and benzyl ether $\mathbf{1 5}$ in Figure 13) will show up as dark spots on TLC plates when irradiated by short-wave UV light.

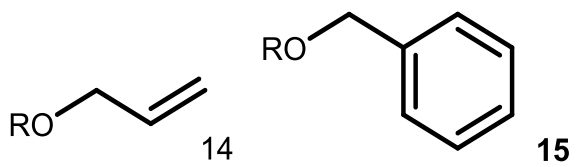

Figure 13. Allyl and benzyl ethers are examples of UV-absorbing protecting groups

Some protecting groups - such as FMOC 16 for amines, ${ }^{24}$ pyrenyl 17 for alcohols ${ }^{25}$ and dansyl 18 for amines ${ }^{26}$ - are fluorescent under short or long-wave UV light and can be easily visualised on TLC or during column chromatography with a UV lamp (Figure 14). 

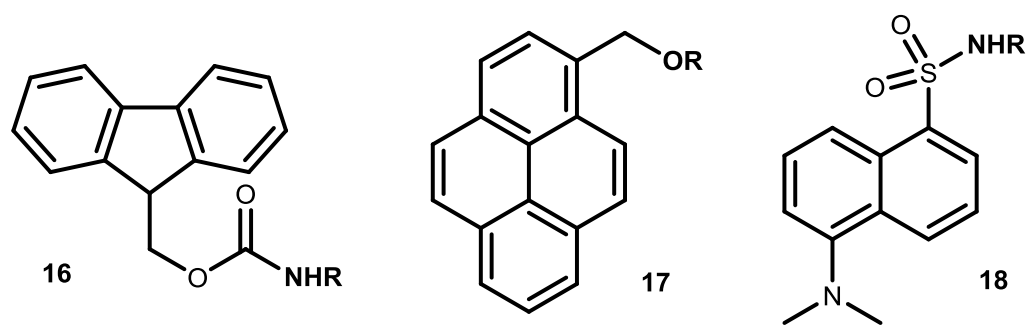

Figure 14. FMOC 16, pyrenyl 17 and dansyl 18 can be used as fluorescent protecting groups

Coloured protecting groups will often provide the best visualisation. Not only do they aid chromatography and separation techniques, their use can also slightly increase overall yield, as small spillages and other traces of the substrate remaining on glassware are readily visible and can be reclaimed. However, there are very few examples of coloured protecting groups in the literature (Figure 15 depicts the only known examples). The ferrocenylmethyl (fem) group 19 is an orange protecting group for amines, and can be introduced through reductive amination of ferrocene carboxaldehyde and removed through addition of TFA. ${ }^{27}$ Diazo groups are typically coloured, and the ( $p$-phenylazophenyl)isopropylcarbonyl (AZOC) protecting group $\mathbf{2 0}$ is used to protect hydroxyls as carbonates. It can be introduced as AZOC- $\mathrm{N}_{3}$ and cleaved with mild acid.28 The authors all note in these papers that the presence of these coloured groups allow column chromatography to be more easily performed.
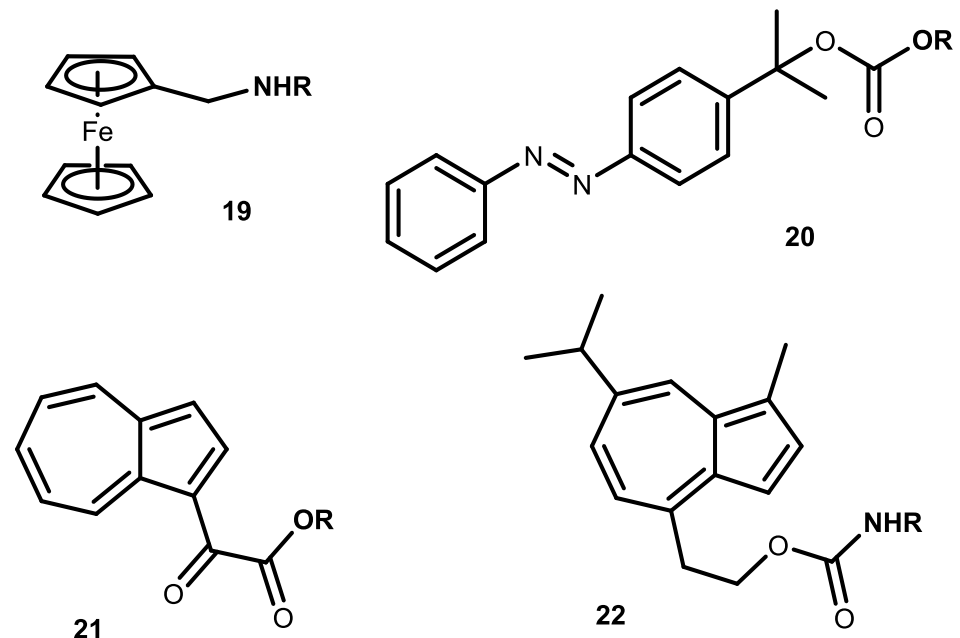

Figure 15. Examples of coloured protecting groups

There are two azulene-based coloured protecting groups in the literature. The azulene ketoester $21(\mathrm{Az})$ is a protecting group for hydroxyls and can be introduced using the corresponding ketoacid chloride and cleaved using the bidentate nucleophile 1,2-benzenediamine in refluxing acetic acid..$^{29}$ The guaiazulene-based FMOC analogue $\mathbf{2 2}$ is a protecting group for amines and can be introduced with the corresponding $N$-succinimidyl carbonate and deprotected through a $\beta$ elimination mechanism upon addition of a weak base, such as lithium thioethanolate. ${ }^{30}$ 


\subsection{A brief overview of protecting group chemistry}

When designing new protecting groups with novel function, it is imperative to have an understanding of which protecting groups are already available, their optimal deprotection conditions and their orthogonalities, and consequently, where in 'protecting group space' the gaps lie. As such, a comprehensive review of the protecting groups available to the major functional groups was undertaken. This was primarily done through the perusal of Greene \& Wuts Protective Groups in Organic Synthesis, $4^{\text {th }}$ edition. ${ }^{17}$

\subsubsection{Anatomy of a protecting group}

It is necessary at this point to define the terms that will be used for the description of various parts of the protecting group. Aside from the substrate it is attached to, a protecting group can be broadly thought of as being composed of two parts: a protected functionality - the functional group that the unprotected functionality is converted to, and a protecting group body that makes up the bulk of the protecting group (Figure 16). For many protecting groups, particularly the older and more established ones, deprotection takes place through direct cleavage of the protected functionality. There are relatively few individual protected functionalities available (such as the ester, ether, carbonate, carbamate, amide, etc) and on their own, protecting group chemistry would be rather limited if these functionalities constituted the only meaningful choice of protecting group.

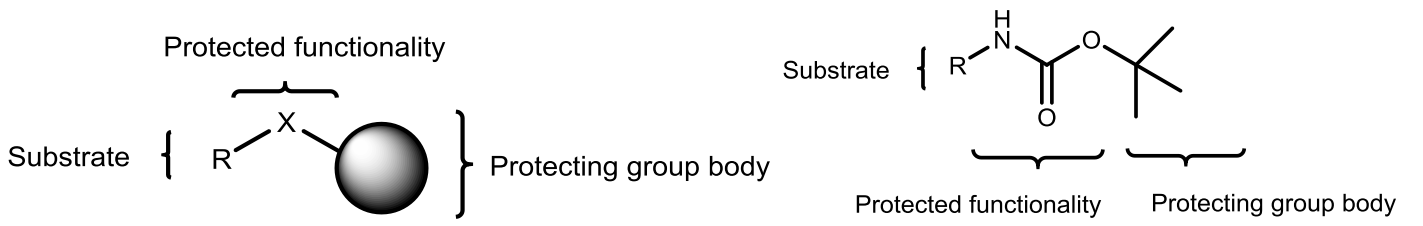

Figure 16. Depiction of the components of a generalised protecting group (left), and of a BOC group (right)

The protecting group body is the part that is responsible for much of the diversity in protecting group behaviour. Some protecting group bodies serve to modify the reactivity of the adjacent protected functionality, such as through steric bulk (examples include $t$-butyl, adamantyl and mesityl groups) or through electronic activation/deactivation (such as the trifluoromethyl and trichloromethyl groups) by incorporating electron-donating - or electron-withdrawing groups. Other protecting group bodies provide handling benefits, incorporating fluorescence, colour or rigid groups that make the substrate amenable to crystallisation. More specialised protecting groups exist that do not modify the protected functionality, but rather introduce alternate means of cleavage through properties intrinsic to their protecting group bodies. One illustrative example of this is the family of 'tethered' protecting groups (depicted in Figure 17), consisting of an electrophilic protected functionality such as an ester and an auxiliary functionality that upon some 
chemical transformation becomes nucleophilic, cleaving the protecting group through intramolecular attack. One particular example is the silyloxybutanoate (SOB) protected alcohol 23, which undergoes deprotection through addition of fluoride, cleaving the distal TBS group and generating an alkoxide that then attacks the ester to release the alcohol substrate and form a lactone by-product. Another example is the 4-nitro-4-methyl pentanoate group 24. The distal nitro group, upon reduction with zinc and ammonium chloride becomes a nucleophilic $N$ hydroxylamine which attacks the ester to form a $N$-hydroxylactam, thus releasing the substrate. ${ }^{31}$
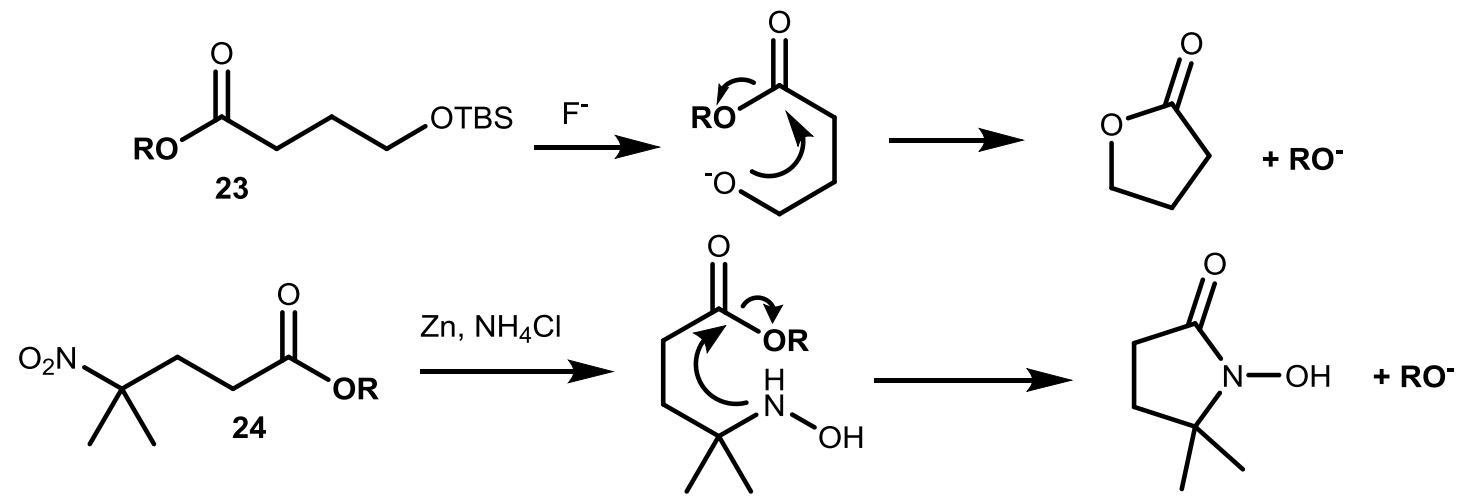

Figure 17. The SOB group 23 and 4-nitro-4-methyl pentanoate group 24 a re examples of tethered protecting groups that deprotect through intramolecular nucleophilic attack.

\subsubsection{Protected functionalities used in alcohol protection}

Alcohols are typically protected as ethers, acetals, esters or carbonates (Figure 18). Esters are labile to hydrolysis, saponification or other conditions involving nucleophilic attack on the carbonyl. As such, most of the methods for ester cleavage involve direct attack on the carbonyl. Carbonates behave similarly, but are more resistant to both acids and bases, and do not see widespread use. Ethers form perhaps the largest and most widely used pool of hydroxyl protecting groups and are typified by a diverse set of deprotection conditions. The ether functionality on its own is very difficult to break cleanly, so almost all ether-based protecting groups are deprotected through reaction of the protecting group body. Acetals rely on acidic conditions for cleavage, and the choice of protecting group body is typically made based on the stabilisation the protecting group body will confer on the positively-charged deprotection transition state.

$\mathrm{RO}$<smiles>[R]OCOC1CCCCC1</smiles><smiles>[R]OC(=O)C1CCCCC1</smiles><smiles>[R]OC(=O)OC1CCCCC1</smiles>
$\mathbf{R}=$ substrate<smiles>[R]OC1CCCCCC1</smiles>

Figure 18. Common protected functionalities employed for alcohol protection $=$ protecting group body 


\subsubsection{Protected functionalities used in amine protection}

The available selection of amine protecting groups is perhaps the largest in synthetic chemistry, due in part to the sheer breadth of chemistry involving nitrogen and the well-developed nature of peptide synthesis. In order to ensure complete protection of an amine, the amine hydrogens as well as the Lewis-basic nitrogen lone pair must be pacified. Nitrogen-containing heterocycles can behave in drastically different ways, and protecting groups that work well for aliphatic amines may be either too labile or difficult to remove for other nitrogen-containing species. ${ }^{32}$

Amines are typically encountered much more commonly in the synthesis and manipulation of amino acids, peptides and nucleic bases than in secondary metabolite synthesis, but nevertheless, nitrogen containing groups are regularly encountered in total synthesis. The requirements of the protecting groups of amines in peptide and nucleic base chemistry are often different to those in secondary metabolite synthesis due to the differences in methods and synthetic techniques between these branches of organic chemistry.

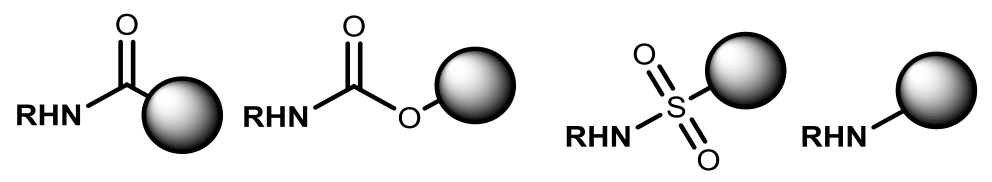

Figure 19. Typical protected functionalities used in amine protection.

There are four main types of amine protecting groups (Figure 19). Amides and carbamates are all cleavable through hydrolysis, but the conditions required are typically much more severe than those of esters and carbonates. As such, a large number of amine protecting groups are deprotected through reactions occurring on the protectinggroup body, rather than through direct attack on the protected functionality. Sulfonamides are often too stable towards hydrolysis, and are typically cleaved through single-electron reduction. These three protected functionalities all passivate the lone pair of the nitrogen through electron withdrawal. The fourth protected functionality is a higher order amine, either secondary or tertiary. These groups are analogous to ether protecting groups, and many amine analogues for these groups exist, such as silyl or benzyl amines, but they are seldom used because they do little to deactivate the lone pair on the nitrogen.

\subsubsection{Protected functionalities of carboxylic acids}

Carboxylic acids are protected almost exclusively as esters (Figure 20), and the bodies of these protecting groups are broadly similar to those of ether and carbonate protecting groups. Other protected functionalities include thioesters, oxazoles and orthoesters, but these seldom see use in synthetic applications. 


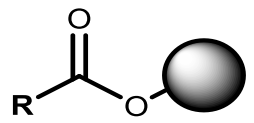

Figure 20. Carboxylic acids are protected almost exclusively as carboxylic esters.

\subsection{Brief overview of available protecting group bodies}

For the purposes of this introduction, a methyl group as the prototypical protecting group body is the baseline to which other protecting group bodies are compared. Its use is seen with practically all protected functionalities listed in section 1.4 as methyl ether $\mathbf{2 5}$, acetate $\mathbf{2 6}$, methoxymethyl ether $\mathbf{2 7}$ and methyl carbonate $\mathbf{2 8}$ for alcohols, methyl ester $\mathbf{2 9}$ for carboxylic acids, and methyl carbamate $\mathbf{3 0}$ and methanesulfonamide $\mathbf{3 1}$ for amines (Figure 21).

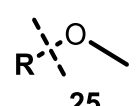

25

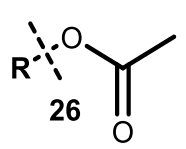<smiles>[R][C@H](C)OCOC</smiles>

27<smiles>[R]OC(=O)OC</smiles>

28

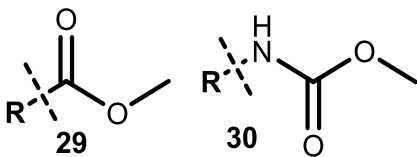

29

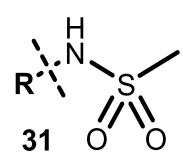

Figure 21. Examples of protecting groups with a methyl as a protecting group body

\subsubsection{Sterics-based protecting group bodies}

Increasing the steric bulk on the protecting group body serves to slow cleavage, both through steric hindrance and through the slight electron-donating effect of multiple alkyl groups in close proximity. The $t$-butyl protecting group body (Figure 22) appears in many well used protecting groups, such as pivaloate 32, $t$-butyl carbonate 33 (BOC), $t$-butyl ether 34, $t$-butoxymethyl ether 35, $t$-butyl ester 36 and the indispensable $t$-butylcarbamate group 37 (also abbreviated as BOC). In all cases, these $t$-butyl based protecting groups are more resistant to the conditions that cleave their methyl analogues. Pivaloate ester, for example, is approximately 100 times more stable to base than acetate (when introduced to $\mathrm{K}_{2} \mathrm{CO}_{3}$ in $\mathrm{MeOH}$, acetate is cleaved in 20 minutes at r.t. while pivaloate requires 2 days of heating at reflux for cleavage). Some $t$-butyl-based protecting groups ( $t$-butyl ester 36, $t$-butyl ether 34, $t$-butoxymethyl ether 35 and BOC 33, 37) have added lability in the presence of strong acid through dehydration of the $t$-butyl moiety and formation of isobutylene.<smiles>[R]OC(=O)C(C)(C)C</smiles>

32<smiles>[R]OC(=O)OC(C)(C)C</smiles>

34

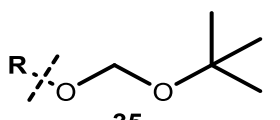

35<smiles></smiles>

36<smiles>[R]NC(=O)OC(C)(C)C</smiles>

37

Figure 22. Examples of protecting groups with a $t$-butyl as a protecting group body

Some of these $t$-butyl-based protecting groups also have adamantyl analogues (Figure 23), such as adamantoate $\mathbf{3 8}^{33}$ and adamantyl carbamate (AdOC) 39, which are slightly more sterically hindered than their $t$-butyl cousins. If even more steric hindrance is required, ortho-alkylated 
benzyl functionalities such as the mesitoate ester $\mathbf{4 0}^{34}$ can be employed. Such protecting groups push the limits of what could be considered acceptably mild cleavage conditions.
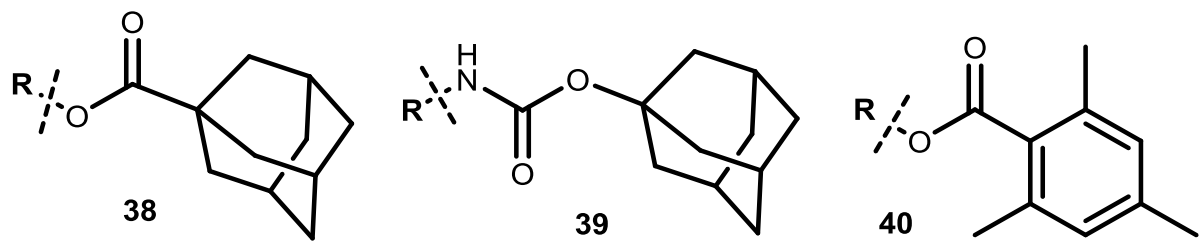

Figure 23. Protecting groups $\mathbf{3 8}$ and $\mathbf{3 9}$ incorporate the bulky a damantyl moiety, and $\mathbf{4 0}$ is the mesitoate protecting group, whose steric bulk imparts considerable stability towards aqueous base.

\subsubsection{Electron-withdrawing protecting group bodies}

Several protecting groups exist with electron-withdrawing groups that activate the protected functionality towards nucleophilic cleavage (Figure 24). These typically take the form of halidesubstituted alkyl groups. Chloroacetate $\mathbf{4 1}$ is a relatively common ester protecting group for hydroxyls, with a reaction rate towards basic hydrolysis 300 to 600 times that of acetate, which is sufficient to allow selective cleavage in the presence of acetate. Dichloroacetate $\mathbf{4 2}$ is somewhere between 7000 and 16000 times more reactive than acetate, and trichloroacetate $\mathbf{4 3}$ is between 18000 to 100000 times more reactive. 35 Trifluoromethyl-based protecting groups exist, but the extreme reactivity they impart means they see little use as esters (trifluoroacetate $\mathbf{4 4}$ is sufficiently reactive to be cleaved in water at $\mathrm{pH}$ 7). ${ }^{17}$ They are nevertheless popular as bodies for amine protecting groups, serving to mitigate the notorious stability of the amide and sulfonamide functionalities as trifluoroacetamide 45 and trifluorosulfonamide 46 respectively.<smiles>[R][X](C)(C)OC(=O)C(=O)O[Y]([R])([R])OC(=O)CCl</smiles>

42<smiles>[R]OC(=O)C(F)(F)F</smiles>

44<smiles>[R][Y10]([H])(C)C(=O)C(F)(F)F</smiles><smiles>[R]NS(=O)(=O)C(F)(F)F</smiles><smiles>[R]OCOCC</smiles>

Figure 24. Examples of protecting group bodies incorporating electron-withdrawing groups that modify the reactivity of the protected functionality

On acetal-based functionalities, electron withdrawing groups have the effect of stabilising the protecting group rather than destabilising it, as deprotection proceeds through a positivelycharged transition state. The 2,2,2-trichloroethoxymethyl ether $\mathbf{4 7}$ is an example of this, being stable to acidic conditions and requiring reduction for cleavage. 36

\subsubsection{Silyl protecting groups}

One of the most commonly used protecting groups for alcohols, silyl groups have the relatively uncommon susceptibility to the F- anion, giving them an orthogonal mode of deprotection. In 
addition, silyl groups are typically labile to acidic conditions, and some are cleavable by base. Different substitution patterns on the silicon impart considerable variation of the lability of silyl groups to acid and base. The trimethylsilyl (TMS) ether $\mathbf{4 8}$ is the most easily cleaved of the silyl ethers, owing to the lack of steric hindrance by the methyl groups. Through replacing the methyl substituents with other (typically alkyl) groups, a pantheon of different silyl protecting groups can be constructed, with the five most common ones being the ethers: TMS 48, triethylsilyl (TES) 49, triisopropylsilyl (TIPS) 50, tert-butyldimethylsilyl (TBDMS or TBS) 51, and tert-butyldiphenylsilyl (TBDPS) 52 (Figure 25). The substituents influence reactivity not only through sterics but through electronic factors too. As one progresses through the series $\mathrm{Me} \rightarrow \mathrm{Et} \rightarrow{ }^{i} \mathrm{Pr} \rightarrow{ }^{t} \mathrm{Bu}$, the electron donation of the alkyl substituents increase as well as their steric hindrance. In acidic conditions, stability increases in the order TMS $<$ TES $<$ TBDMS $<$ TIPS $<$ TBDPS, spanning a 5 million-fold increase between TMS and TBDPS. Similarly, in basic conditions a one hundred thousand-fold increase in stability is seen over the series TMS $<$ TES $<$ TBDMS $<$ TBDPS $<$ TIPS. 22 In contrast, the variation of reactivity with the F- anion is relatively flat, with TBS, TIPS and TBDPS all exhibiting reaction rates within the same order of magnitude. Only a fourfold difference exists between TIPS and TBDPS in reactivity towards F-37

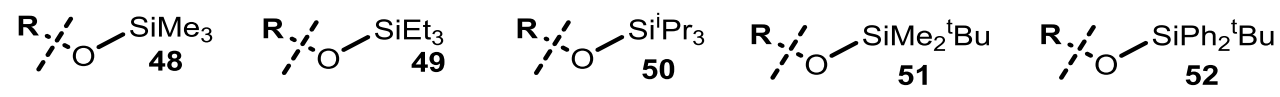

Figure 25. Examples of common silyl protecting groups.

While silyl protecting groups see the majority of their use in direct protection of alcohols as silyl ethers, they can be incorporated into the bodies of other protecting groups to give an additional mode of deprotection through addition of F-(Figure 26). The Teoc protecting group 53, used for amines and carboxylic acids, is deprotected through attack of the trimethylsilane with $\mathrm{F}$-, whose removal initiates an elimination through an E1cB mechanism. The SEM protecting group 54 operates on similar principles, while the aforementioned SOB ester $\mathbf{2 3}$ cleaves through intramolecular attack of the ester by the alkoxide resulting from the removal of the TBS group.

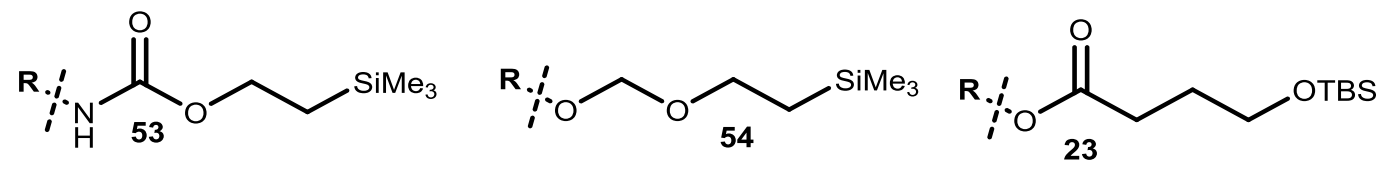

Figure 26. Silyl groups incorporated on to non-ether protected functionalities

\subsubsection{Hydrogenation-cleavable protecting groups}

Allyl and benzyl protecting group bodies are popular for their hydrogenation-based cleavage conditions. They most commonly appear as ethers, but are also well known in the carbonate and carbamate-based protecting groups AllOC 55-56 and Cbz 57-58 respectively. Trityl 59 and paramethoxybenzyl (PMB) 60 are also cleavable through hydrogenation (Figure 27). 

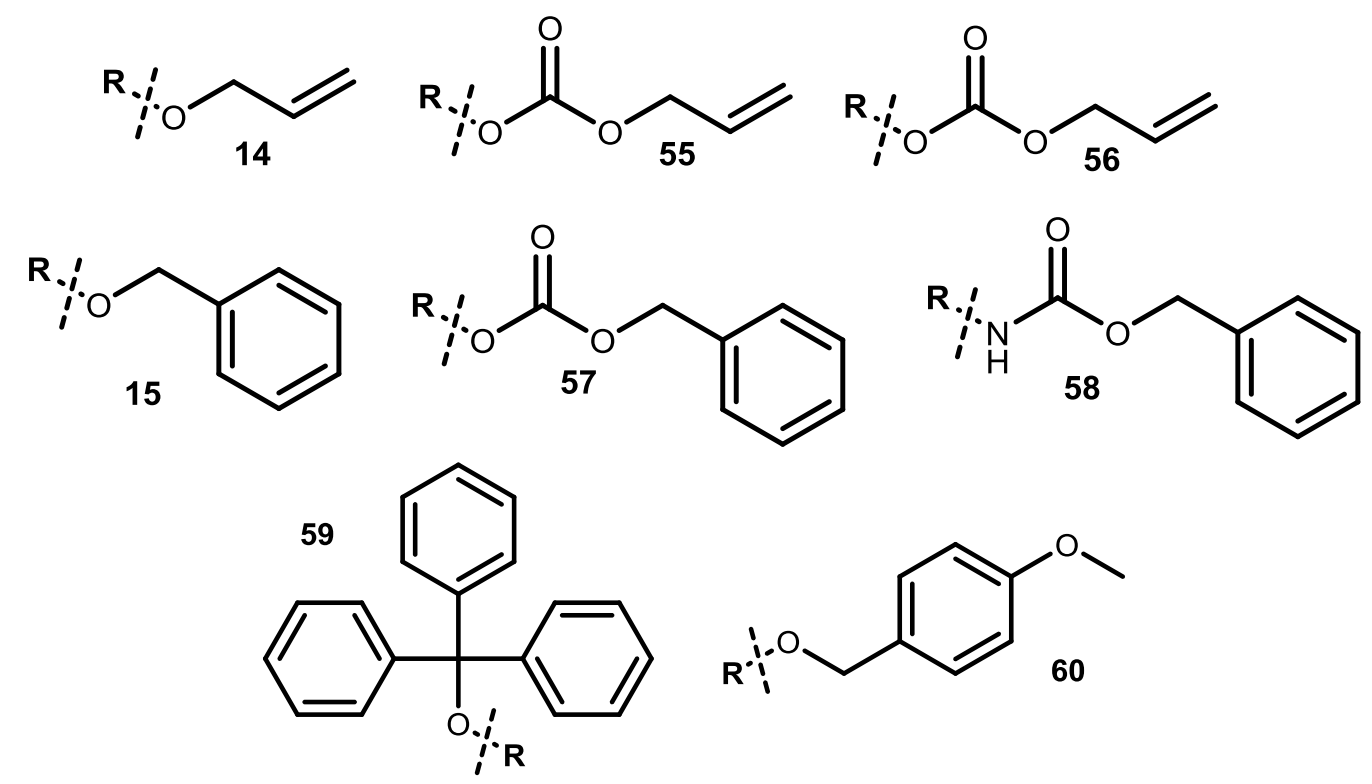

Figure 27. Protecting groups cleavable by hydrogenation

Deprotection is typically performed over Pd/C under a hydrogen atmosphere. Reaction rate can be tuned by altering the pressure of hydrogen in the reaction vessel. The lability of these protecting groups to hydrogenation vary sufficiently to allow some to be cleaved selectively in the presence of others. Trityl 59 and PMB 60 can also be cleaved through mild acid and oxidation with DDQ, respectively.

\subsubsection{Protecting groups with an E1cB cleavage mechanism}

There are many examples of protecting groups that cleave through $\beta$-elimination, the most wellknown of which is FMOC.38 The key feature of these protecting groups is the presence of an electron-withdrawing group (or a group otherwise able to stabilise negative charge) adjacent to the carbon beta- to the protected functionality that acidifies the protons on this position. Upon addition of a (typically mild) base, an equilibrium forms between the protonated species $\mathbf{6 1}$ and the deprotonated species $\mathbf{6 2}$, the latter of which then undergoes elimination through the E1cB mechanism to release the unprotected substrate and the protecting group body as the vinyl compound 63 (Figure 28).

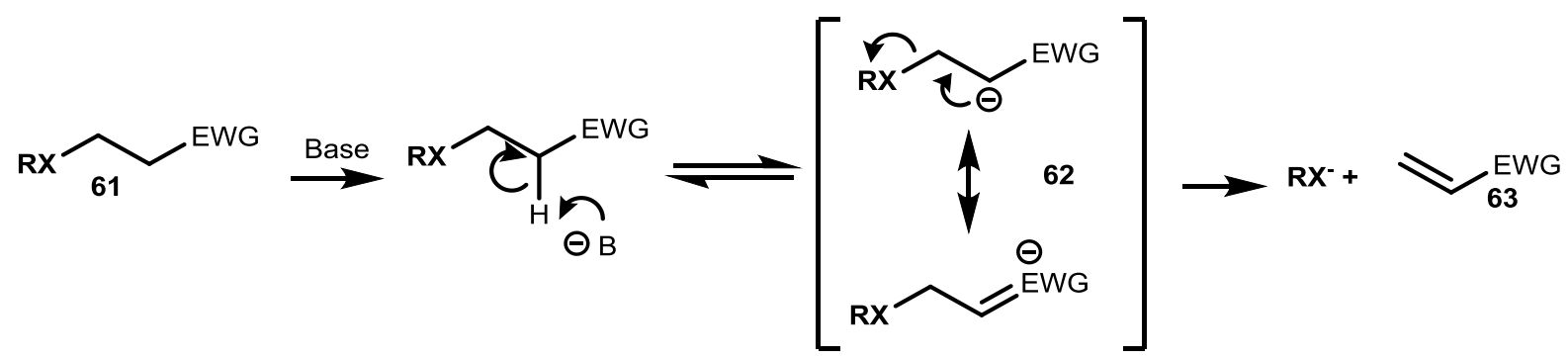

Figure 28. General depiction of a protecting group cleaved through an E1cB mechanism 
The vinyl species often undergoes subsequent attack, depending on how nucleophilic the base is (Figure 29).

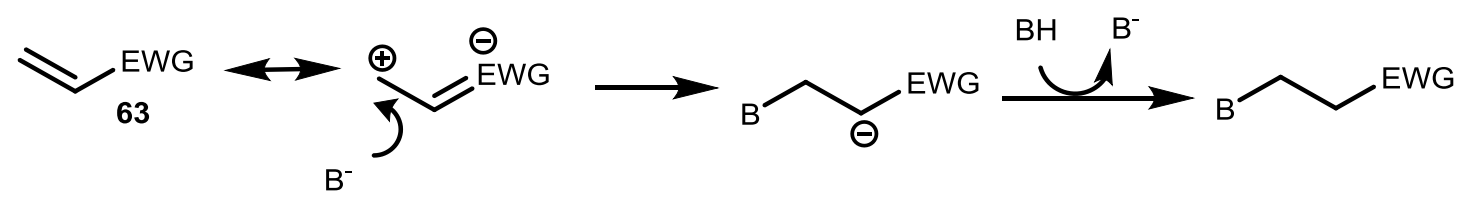

Figure 29. The PG remnant will often undergo nucleophilic attack on the vinyl moiety by leftover base.

Another requirement of $\beta$-elimination-cleavable protecting groups is that the cleaved substrate has to act as a leaving group. It does not necessarily have to be a good leaving group, but some stabilisation of the negative charge is required. This generally restricts these moieties to protected functionalities such as esters (the resulting carboxylate anion being sufficiently stable to act as a leaving group), carbonates and carbamates (whose cleavage is aided entropically by production of $\left.\mathrm{CO}_{2}\right)$.

Even so, these three protected functionalities are able to protect a large number of functional groups, from amines to alcohols and carboxylic acids. While FMOC is most often associated with amine protection (16), fluorenylmethyl carbonates 64 and esters 65 also exist (Figure 30), and due to their two-bond removal from the protected functionality, they undergo deprotection in the same way and at roughly the same rate.
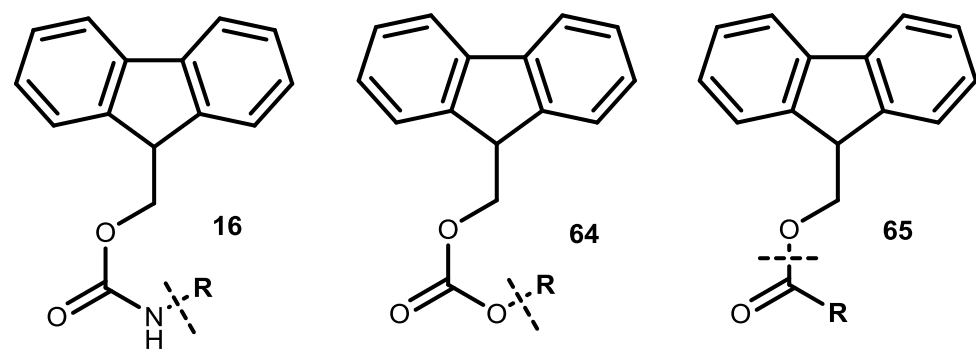

Figure 30. FMOC protecting groups $\mathbf{1 6}$ and $\mathbf{6 4}$ for a mines and alcohols, and the Fm ester $\mathbf{6 5}$ for protection of carboxylic acids.

Many other such protecting groups exist, and they typically differ from each other only through the extent of their susceptibility to mild base. Examples include Dbd-TMOC $66^{39}$ which is removable with pyridine, and 2-(p-toluenesulfonyl)ethyl carbamate $67^{40}$ and 2-(4nitrophenyl)ethyl carbamate (NpeOC) 6841, both removable with DBU (Figure 31).
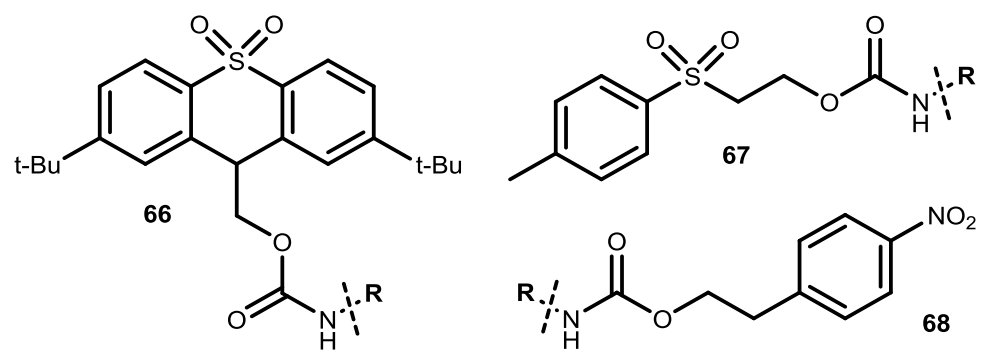

Figure 31. Further examples of protecting groups cleavable through $\beta$-elimination. 


\subsection{Research objectives}

The primary goal of this research was to develop new protecting group methodology incorporating an azulene moiety into the protecting group body. It was envisioned that the utility of this protecting group would arise from the combination of two aspects - the colour and the unusual chemical properties of azulene.

\subsubsection{The advantages of a coloured protecting group}

As mentioned earlier, azulene itself is blue, and its derivatives range from greens to purples and oranges. These colours depend on the electronic nature of the substituents around the azulene. There are multiple benefits of having a coloured protecting group present. The most immediately apparent one is in purification - the azulene-containing compound can be seen and tracked at all times. They are visible on a developing TLC, in an aqueous/organic phase separation, and their progress through a chromatographic column can be tracked visually and can obviate the need for collecting multiple fractions. Having a visibly trackable compound also increases yields by decreasing the wastage of small amounts of material during purification - from personal experience it is surprising how far some material can spread onto assorted surfaces such as in the necks of flasks, on the ends of taps, or on the inside of Pasteur pipettes while performing routine procedures, and how necessary additional rinsing steps are for maximising yields during phase separations and transferral of material between vessels. Furthermore, so-called bench extractions' and other spillages are easier to clean up, and the presence of colour allows the chemist to reclaim large proportions of the original material during such occurrences.

While an experienced chemist can be expected to have sufficiently good lab technique to minimise wastage in these ways, there are many less experienced chemists for whom this colour-based feedback would be more beneficial. In this capacity, an azulene-based protecting group could serve as a training tool for new chemists.

It bears mentioning that while azulene is often described as an 'intense' blue, the extinction coefficient of its maximum absorption is not particularly high. Azulene in acetonitrile has an extinction coefficient of $328 \mathrm{Lmol}^{-1} \mathrm{Cm}^{-1}$, which is not significantly higher than ferrocene $\left(91 \mathrm{Lmol}^{-1} \mathrm{~cm}^{-1}\right)^{42}$ and much smaller than potassium permanganate $\left(18000 \mathrm{Lmol}^{-1} \mathrm{~cm}^{-1}\right)$ and the widely-used dye Methylene blue $\left(95000 \mathrm{Lmol}^{-1} \mathrm{~cm}^{-1}\right)^{43}$. Azulene imparts sufficient colour to the substrates to allow for most synthetically useful amounts of material to be seen, but does not oversaturate and render all material effectively black. At microgram scales, chemists are not left chasing highly-coloured phantoms, and at typical synthetic scales ( $>10 \mathrm{mg}$ ), solutions do not 
become completely opaque. A good rule of thumb is that amounts that are difficult to see are difficult to weigh on a conventional chemical balance.

Azulene has distinct advantages over other colourful organic species. It is non-polar, unlike common dyes such as methylene blue 69, anthocyanidins (Cyanidin, 70), triarylmethane dyes (brilliant green, 71), or the aniline dyes (Mauvine A, 72) (Figure 32). It is also compact, unlike the carotenoids ( $\beta$-carotene, 73). Many of these dyes also have stability issues, exhibiting tautomerism at different pKa values and degradation under UV-light.<smiles></smiles><smiles>CCNc1ccc(C(c2ccccc2)c2ccc(N(CC)CC)cc2)cc1</smiles><smiles></smiles><smiles></smiles><smiles>CC1=C(/C=C/C(C)=C/C=C/C(C)=C/C=C/C=C(C)/C=C/C=C(C)/C=C/C2=C(C)CCCC2(C)C)C(C)(C)CCC1</smiles>

Figure 32. Examples of other coloured organic compounds

Finally, the chemistry of azulene leaves room for self-indicating protections and deprotections. A change in the electronic nature of a substituent results in a change of colour, and protecting groups could be engineered so that the protected functionality induces a colour change in the azulene moiety upon addition or cleavage, and thereby indicate the extent of reaction.

\subsubsection{Exploitation of the chemical properties of azulene}

There is much flexibility in the chemistry of the azulene ring system through the stabilisation of both positive and negative charges discussed in section 1.2, which enables the alteration of the reactivity of pendant protected functionalities. Furthermore, the ease of substitution at the 1- and 3 -positions and the relative ease of producing various methyl-substituted azulenes would allow for a large degree of fine-tuning of the electronic environment of the azulene ring system, as well as the possibility of two-step deprotection methods. 
Taken individually, none of these features are sufficient to warrant extensive research, but when put together, the combination of colour and deprotection flexibility could allow for the creation of a protecting group that is sufficiently synthetically useful for other chemists to find desirable.

\subsubsection{The ideal niche of the azulene-based protecting group}

In order to reap the full benefits of the presence of colour, an azulene-based protecting group would ideally be 'permanent', i.e. introduced early in the synthesis and cleaved either at a crucial late stage coupling reaction or as part of the final global deprotection. In order to accommodate this, the azulene-based protecting group must be simultaneously resistant to many of the harsher conditions employed during early-stage synthesis, and labile to a specific set of mild conditions that will not interfere with any delicate or sensitive portions of the end-stage molecule.

\subsubsection{Protecting group development}

There exists a large number of protecting groups in the literature. Greene \& Wuts lists at least one thousand protecting groups, many of which with only one example of use. In any field, it is not enough to provide an incremental improvement of a technique and expect it to see widespread adoption, as there is always a short-term cost in doing so.44 The new technique may not be sufficiently understood or may require an initial investment in time and money that offsets the projected benefits of the technique. This is especially relevant in protecting group development, as even well-established protecting groups sometimes fail in unexpected ways, so the use of a relatively new and untested protecting group is a risky prospect.

Therefore, the onus is on the developer of the protecting group to explore its chemistry in a synthetic context. The developer must investigate which reaction conditions are incompatible with the protecting group, and address any other potential pitfalls one might experience during a synthesis, in order to reduce, as much as possible, the risk a chemist takes on when using a new protecting group.

\subsubsection{Approach to azulene protecting group res earch}

The approach throughout this project towards protecting group development could be broadly described as consisting of three phases - identification, characterisation and exploration.

\section{Identification of protecting group candidates}

After sketching out an idea for an azulene protecting group on paper, the first step is to investigate whether or not the chemistry works in principle. This part is relatively quick to perform - a single 
protection and deprotection is sufficient, allowing protecting group candidates to be identified rapidly.

\section{Characterisation of the protection and cleavage conditions}

The protection and deprotection conditions need to be optimised at this step, along with the synthetic route to the protecting group precursor. If the protecting group cannot be introduced and cleaved in high yield, other chemists are unlikely to use it. For the purposes of this project, yields above $80 \%$ were arbitrarily considered to be high enough for use. During this stage, scaling up of the synthesis of the protecting group precursor is undertaken in order to produce enough material for the exhaustive testing of the next phase.

\section{Exploration of the protecting group in a wider synthetic context}

The most time-consuming step of the process is the exploration of the protecting group chemistry. This involves exploring the protection and deprotection conditions and finding alternate methods for protection/deprotection, investigating two-step deprotection, protecting and deprotecting a series of model substrates, doing comparative deprotection studies in the presence of common protecting groups, and assessing the compatibility of the protecting group with the conditions in various well-known and representative reactions. Only after the acquisition of this information can a judgment on the utility of the protecting group be made.

\subsection{The protecting group candidates}

At the start of the project, four protecting group candidates were devised. A 1-azulenyl ester for the protection of alcohols, an azulenemethylene protecting group for amines, an azulenesulfonamide protecting group for amines and a 6-(2-oxyethyl)azulene protecting group for alcohols, amines and carboxylic acids.

\subsubsection{Protection of alcohols as 1-azulenyl esters}

It was already known from previous work conducted during my master's project that guaiazulene esters could be prepared from oxalyl chloride and the corresponding alcohol. 45 This presented an attractive route to an azulene ester-based protecting group (Scheme 2). The azulene precursor could be synthesised in a single step using literature methods, and alcohol substrates to test this protecting group against are in abundant supply. Azulene could be exchanged with one of several other methylazulenes to fine-tune the reactivity. 


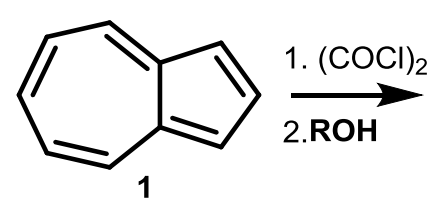

1
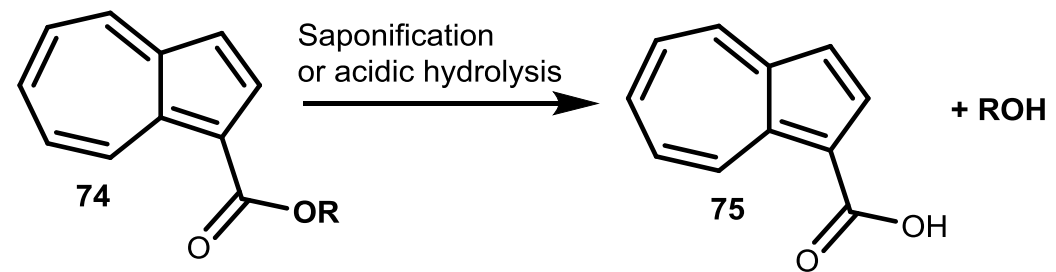

Scheme 2. Proposed protection of alcohols as azulenyl esters

The precise conditions required to generate the ester $\mathbf{7 4}$ (as opposed to the keto-ester 21, which, as discussed in section 1.3.2 mentioned previously had already been employed as a protecting group) were not known at the outset of the project, nor were the deprotection conditions, but it was assumed that the ester would be cleaved by saponification or acidic hydrolysis (Scheme 3).

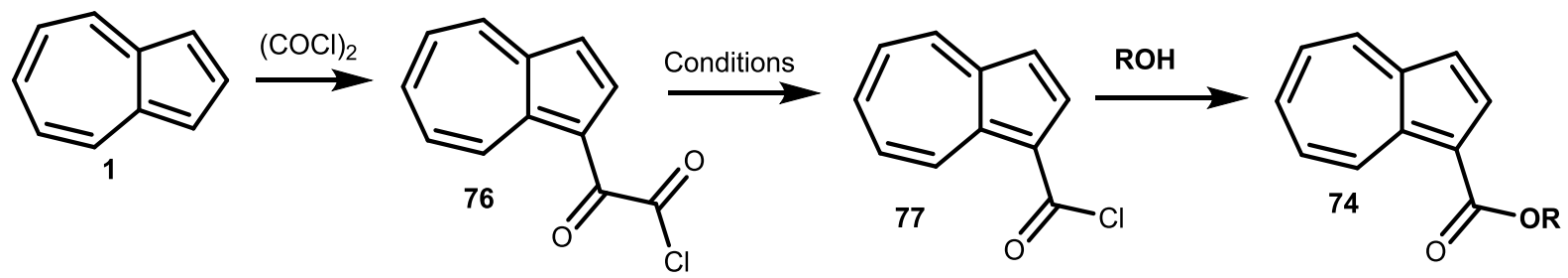

Scheme 3. The success of protecting group candidate $\mathbf{7 4}$ hinges on the ability to convert keto acid-chloride $\mathbf{7 6}$ to acid chloride 77

\subsubsection{Protection of amines through reductive amination with 1-formylazulene}

The protecting group $\mathbf{7 9}$ for amines was envisaged to be superficially analogous to the wellknown benzyl protecting group but with modified reactivity (Scheme 4). It was to be introduced through reductive amination of the amine with 1 -formylazulene $\mathbf{7 8}$, and the deprotection conditions were to utilise the stabilisation of positive charge adjacent to the 1-position in some form - either through oxidation or through nucleophilic attack at the 1-methylene position in acidic conditions (Scheme 5).

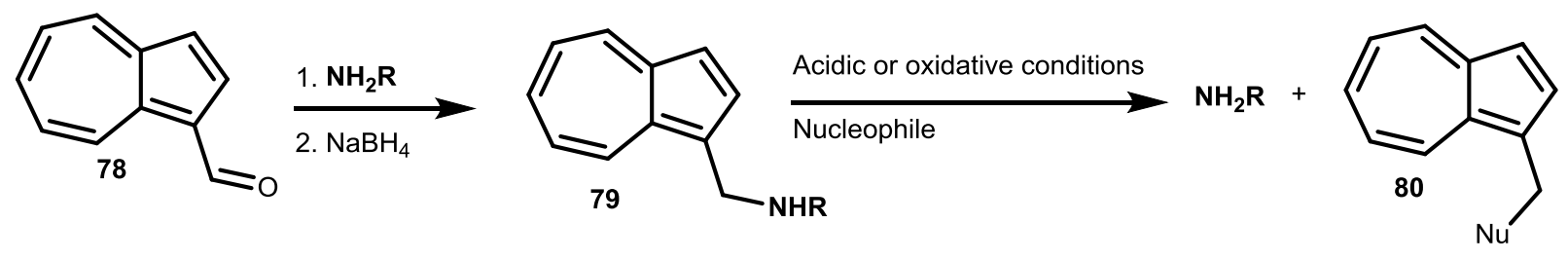

Scheme 4. Protection of amines through reductive amination of $\mathbf{7 8}$

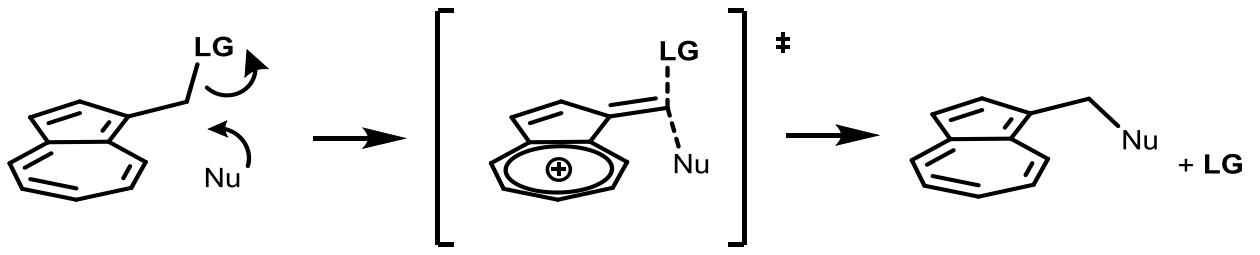


Scheme 5. Nucl eophilic displacement of the leaving group (in this case a protonated a mine) would be facilitated by the stabilisation of positive charge in the transition state

This protecting group was the only one in the list that was expected to undergo deprotection through a positively-charged transition state. Such a feature would allow two-step deprotection to be facilitated with the addition of electron donating groups, which is the opposite regime to the sensitivities of the other three protecting groups presented in this section.

\subsubsection{Protection of amines as 1-azulenesulfonamides}

Sulfonamides make for robust amine protecting groups, and an azulenesulfonamide was expected to be particularly inert, though it was expected that the reducing conditions used for sulfonamide deprotection would still function. If not, two-step deprotection was to be attempted. Protection of the amine would be performed through treatment with azulenesulfonyl chloride $\mathbf{8 1}$.

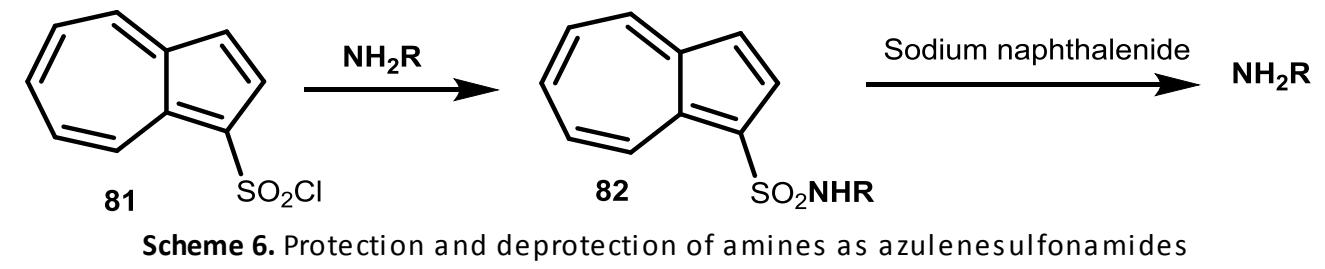

\subsubsection{Protection of alcohols, amines and carboxylic acids through a $\beta$-elimination cleavable 6-(2-oxyethyl)azulene}

The idea for a protecting group that was cleavable through $\beta$-elimination first arose during my masters' research. I proceeded to show that the guaiazulene derivative-protected acetate $\mathbf{8 3}$ could cleave in a way consistent with $\beta$-elimination (Scheme 7), but at the same time a paper was released by Aumüller and Lindhorst 30 detailing many of the same ideas (deprotection with a relatively mild base, in this case lithium thioethanolate (Scheme 8), and demonstration of a twostep deprotection method involving nitration as the activation step) while demonstrating not only its use in glycopeptides, but also its general facilitation of purification. 


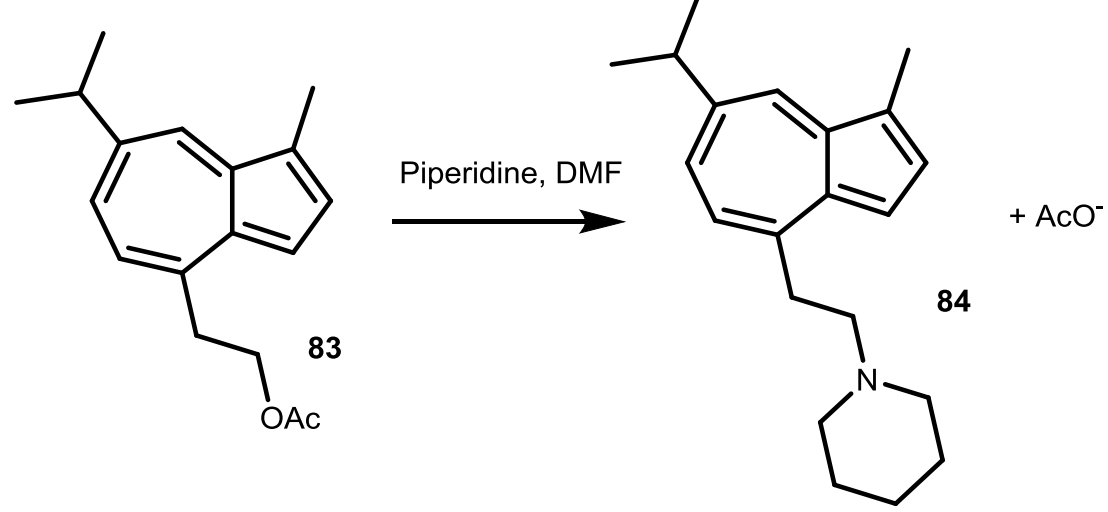

Scheme 7. The reaction discovered during my Master's research

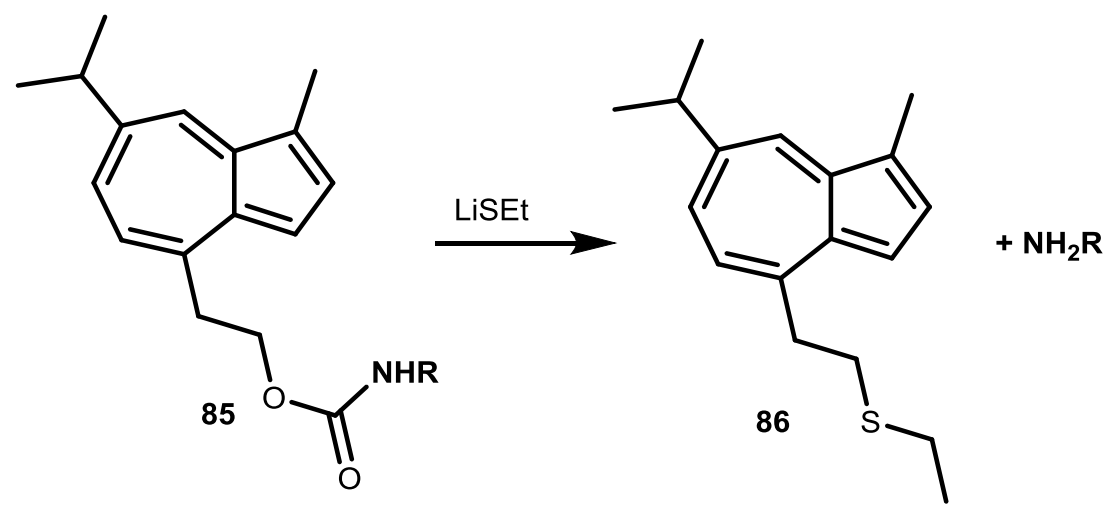

Scheme 8. The protocol for amine deprotection reported by Lindhorst et al.

Guaiazulene, however has a tendency to oxidatively polymerise, 5 and I frequently encountered problems with degradation during this research where some guaiazulene derivatives would degrade in a matter of days in open air. Needless to say, such a feature is not conducive to function as a protecting group. This lack of stability, compounded with the paper by Lindhorst et al. led us to consider alternative targets for protection (carboxylic acids and alcohols in addition to amines) as well as alternate azulene functionalities for this project, such as the 4-methylazulene, 4,6,8trimethylazulene and 6-methylazulene derivatives $\mathbf{8 7 , 8 8}$ and 89 respectively (Figure 33).
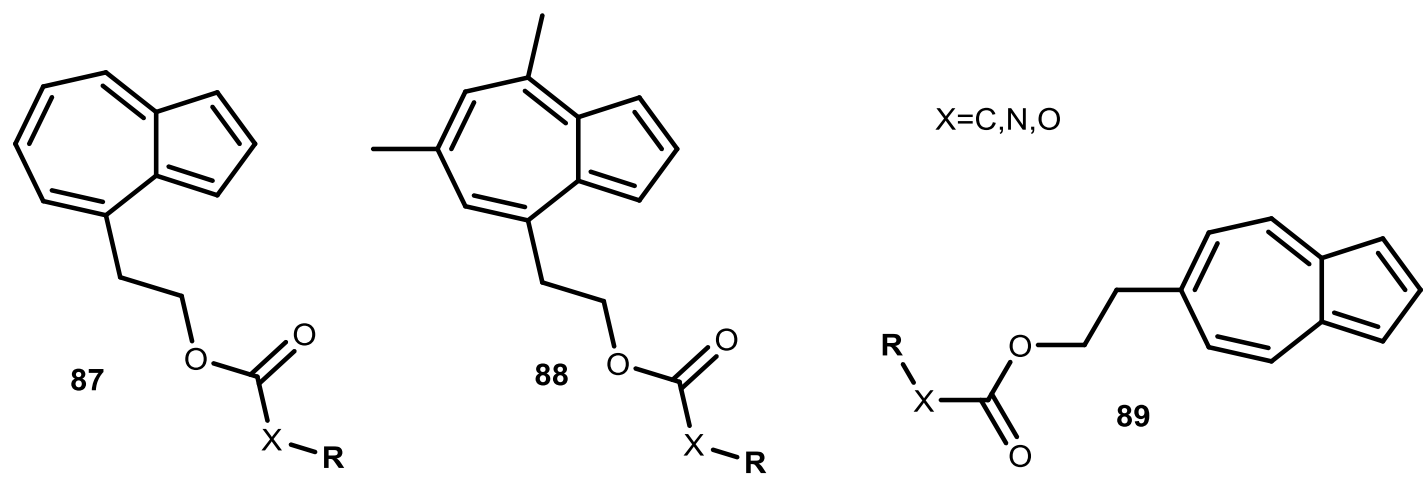

Figure 33. Alternatives considered

The 6-substituted azulene derivative $\mathbf{8 9}$ was ultimately chosen for investigation. The marginal advantage of the 6-methylazulene derivative over the others was its symmetry. The ${ }^{1} \mathrm{H}$ NMR spectrum of 6-methylazulene is much simpler than those of the other azulenes, and an unspoken 
rule of protecting groups is that they should avoid occupying too much NMR real estate. This symmetry also prevents a mixture of products forming when functionalising the 1- and 3-positions when performing two-step deprotections.

A major attraction of this protecting group candidate was the ability to protect alcohols, amines and carboxylic acids as carbonates, carbamates and esters respectively (Scheme 9). Carbonates and carbamates can be synthesised from CDI, an alcohol and the substrate alcohol/amine. Carboxylic acids could be protected through an esterification reaction.

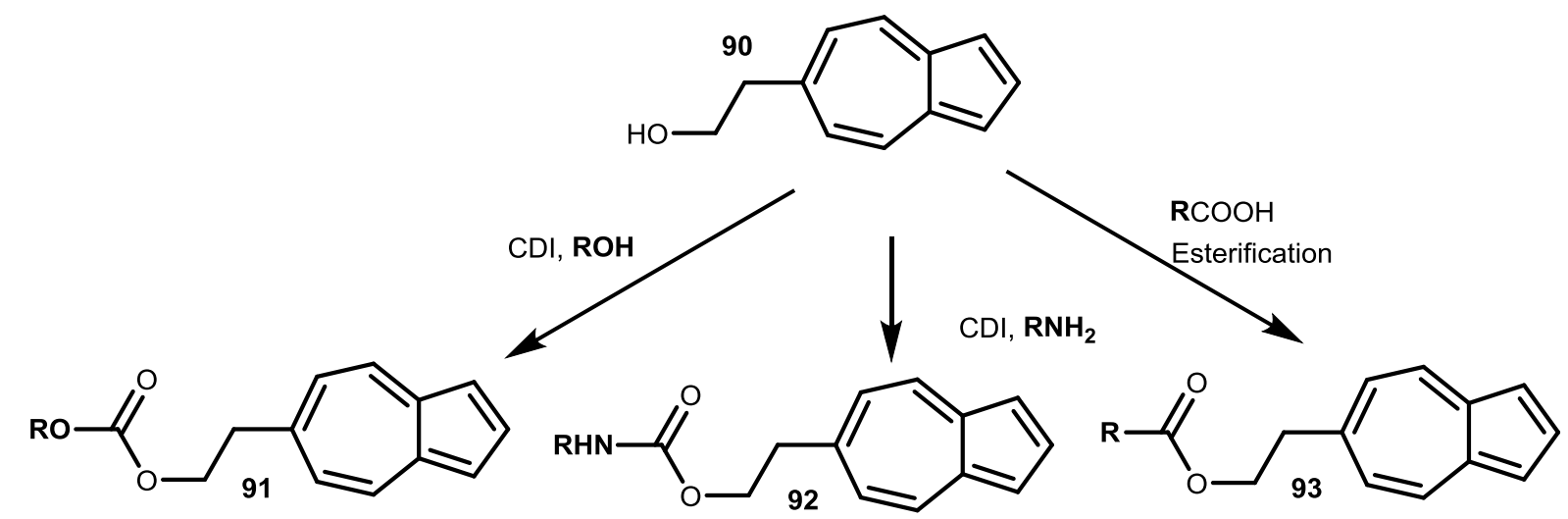

Scheme 9. Protection of alcohols, a mines and carboxylic acids

Deprotection through addition of mild base would result in generation of the vinylazulene $\mathbf{9 4}$ through $\beta$-elimination.

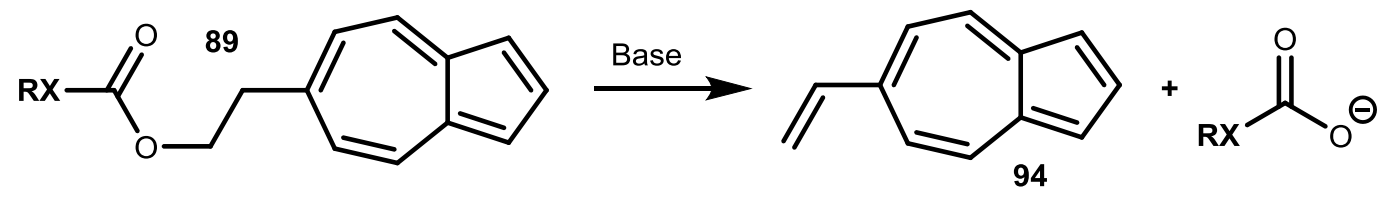

Scheme 10. Deprotection through addition of base

The precursor 6-(2-hydroxyethyl)azulene $(\mathbf{9 0})$ was to be made from 6-methylazulene (10) (Scheme 11), which no previously-existing high-yielding synthesis was available for. The lack of availability of the precursor, as well as the daunting prospect of investigating essentially three protecting groups at once meant that while this protecting group candidate was deemed to be the most promising, it would require the most work to investigate. Therefore, this protecting group candidate was investigated last.
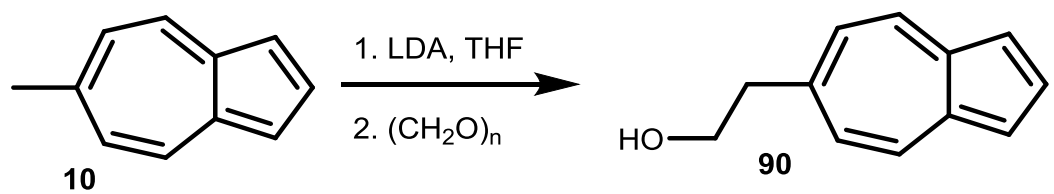

Scheme 11. Synthesis of the precursor $\mathbf{9 0}$ for the protecting group candidate $\mathbf{8 9}$ from 6-methylazulene (10) 


\subsection{References}

1. Liu, R. S., Colorful Azulene and Its Equally Colorful Derivatives. J. Chem. Ed. 2002, 79 (2), 183-185.

2. Gordon, M., The Azulenes. Chem. Rev. 1951,50,127-200.

3. Fang, L.-Z.; Shao, H.-J.; Yang, W.-Q.; Liu, J.-K., Two New Azulene Pigments from the Fruiting Bodies of the Basidiomycete Lactarius hatsudake. Helv. Chim. Acta 2006, 89, 1463-1466.

4. Attaway, D. H.; Zaborsky, O. R., Marine Biotechnology Volume 1: Pharmaceutical and Bioactive Natural Products. Springer, 1993.

5. Fiori, J.; Gotti, R.; Albini, A.; Cavrini, V., Study on the Photostability of Guaiazulene by High Performance Liquid Chromatography/Mass Spectrometry and Gas Chromatography/ Mass Spectrometry. Rapid Commun Mass Spectrom. 2008, 22, 2698-2706.

6. Kourounakis, A. P.; Rekka, E. A.; Kourounakis, P. N., Effect of Guaiazulene on Some Cytochrome P450 Activities. Implication in the Metabolic Activation and Hepatotoxicity of Paracetamol Arch. Pharm. Pharm. Med. Chem. 1997,330, 7-11.

7. Elwahy, A. H. M.; Hafner, K., Synthesis and Characterization of Novel Oligoazulenes with Mixed Ethynyl and Butadiynyl Bridges. Eur.J. Org. Chem. 2006, 3910-3916.

8. Blice-Baum, A.; Dyke, A. V.; Sigmon, I.; Salter, E. A.; Wierzbicki, A.; Pocker, Y.; Spyridis, G. T., Computational and Spectroscopic Studies Concerning the Solvatochromic Behavior of 1,3Disubstituted Azulenes. Int. J. Quant. Chem. 2006, 106, 2331-2338.

9. Zahradnik, R., Electronic Structure and Properties of Non-Alternant Hydrocarbons. Angew. Chem. Int. Ed. 1965, 4, 1039-1050.

10. Kedziorek, M.; Mayer, P.; Mayr, H., Nucleophilic Reactivities of Azulene and Fulvenes. Eur. J. Org. Chem. 2009, 1202-1206.

11. Hafner, K.; Bernhard, C.; Müller, R., Zur Kenntnis der Azulene, V. Die Nucleophile Substitution des Azulens. Liebigs Ann. 1962, 650,35-41.

12. Shoji, T.; Ito, S.; Watanabe, M.; Toyota, K.; Yasunamid, M.; Morita, N., Synthesis of 5Heteroarylazulenes: First Selective Electrophilic Substitution at the 5-position of Azulene. Tetrahedron Lett. 2007, 3009-3012.

13. Robinson, R.; Rudolf, K.; Koenig, T., Methyl Group Functionalization in 6-Methylazulene. J. Org. Chem. 1985, 50, 5871-5873.

14. Lemal, D. M.; Goldman, G. D., Synthesis of Azulene, a Blue Hydrocarbon. J. Chem. Ed. 1988, 65, 923-925.

15. Liu, R. S. H.; Muthyala, R. S.; Wang, X.-S.; Asato, A. E., Correlation of Substituent Effects and Energy Levels of the Two Lowest Excited States of the Azulenic Chromophore. Org. Lett. 2000,2,269-271. 
16. Shevyakov, S. V.; Li, H.; Muthyala, R.; Asato, A. E.; Croney, J. C.; Jameson, D. M.; Liu, R. S. H., Orbital Control of the Color and Excited State Properties of Formylated and Fluorinated Derivatives of Azulene.J. Phys. Chem. A 2003,107, 3295-3299.

17. Wuts, P. G. M.; Greene, T. W., Protective groups in organic synthesis. Wiley: 2007.

18. Young, I. S.; Baran, P. S., Protecting-Group-Free Synthesis as an Opportunity for Invention. Nature 2009, 1, 193-205.

19. Schelhaas, M.; Waldmann, H., Protecting Group Strategies in Organic Synthesis. Angew. Chem. Int. Ed. 1996,35, 2056-2083.

20. Li, J. J.; Corey, E. J., Total synthesisofnatural products. Wiley, 2012.

21. Armstrong, R. W.; Beau, J.-M.; Cheon, S. H.; Christ, W. J.; Fujioka, H.; Ham, W.-H.; Hawkins, L. D.; Jin, H.; Kang, S. H.; Kishi, Y.; Martinelli, M. J.; William W. McWhorter, J.; Mizuno, M.; Nakata, M.; Stutz, A. E.; Talamas, F.X.; Taniguchi, M.; Tino, J. A.; Ueda, K.; Uenishi, J.-i.; White, J. B.; Yonaga, M., Total Synthesis of Palytoxin Carboxylic Acid and Palytoxin Amide. J. Am. Chem. Soc. 1989, 110, 7530-7533.

22. Wuts, P. G. M.; Greene, T. W., Protective groups in organic synthesis. Wiley: 2007; 166.

23. Wuts, P. G. M.; Greene, T. W., Protective groups in organic synthesis. Wiley: 2007; p 123130.

24. Ho, P. C., HPLC of Amines as 9-Fluorenylmethyl Chloroformate Derivatives. J. Chrom Libr. 2005, 79, 471-501.

25. Yamanaa, K.; Ohashia, Y.; Nunotaa, K.; Kitamurab, M.; Nakanoa, H.; Sangena, O.; Shimidzub, T., Synthesis of Oligonucleotide Derivatives with Pyrene Group at Sugar Fragment. Tetrahedron Lett. 1991,32, 6347-6350.

26. Heise, C.; Ehrentreich-Foerster, E.; Bier, F. F., Dansyl, a Fluorescent Photoprotecting Group for Microarray Applications. J. Chem. Technol. Biotechnol. 2012.

27. Eckert, H.; Seidel, C., The Ferrocenylmethyl(fem) Group as a Highly Lipophilic and Chromophoric Group for the Masking of Peptide Bonds. Angew. Chem. Int Ed. Engl 1986, 25, 159-160.

28. Tun-Kyi, A.; Schwyzer, R., ( $p$-Phenylazophenyl)-isopropyloxycarbonyl, a New Protecting Group for Peptide Synthesis. Helv. Chim. Acta 1976, 59, 1642-1646.

29. Timmer, M. S. M.; Stocker, B. L.; Northcote, P. T.; Burkett, B. A., Az-a Colourful AzuleneDerived Protecting Group. Tetrahedron Lett. 2009, 50, 7199-7204.

30. Aumueller, I. B.; Lindhorst, T. K., Coloring Carbohydrates: Investigation of Azulene Derivatives as Blue Protecting Groups. J. Carb. Chem. 2009,28, 330-347.

31. Wuts, P. G. M.; Greene, T. W., Protective groups in organic synthesis. Wiley: 2007; p 265270.

32. Wuts, P. G. M.; Greene, T. W., Protective groups in organic synthesis. Wiley: 2007; 872. 
33. Wuts, P. G. M.; Greene, T. W., Protective groups in organic synthesis. Wiley: 2007; p 254.

34. Wuts, P. G. M.; Greene, T. W., Protective groups in organic synthesis. Wiley: 2007; p 263.

35. (a) Wuts, P. G. M.; Greene, T. W., Protective groups in organic synthesis. Wiley: 2007; p 240;

(b) Hilal, S. H., Estimation of Hydrolysis Rate Constants of Carboxylic Acid Ester and Phosphate Ester Compoundsin Aqueous Systems from Molecular Structure by SPARC. 2006.

36. Wuts, P. G. M.; Greene, T. W., Protective groups in organic synthesis. Wiley: 2007; 53.

37. Wuts, P. G. M.; Greene, T. W., Protective groups in organic synthesis. Wiley: 2007; p 198.

38. Carpino, L. A.; Han, G. Y., The 9-Fluorenylmethoxycarbonyl Amino-Protecting Group. J. Org. Chem. 1972,37, 3404-3409.

39. Carpino, L. A.; Gao, H.-S.; Ti, G.-S.; Segev, D., Thioxanthene Dioxide Based Amino-Protecting Groups Sensitive to Pyridine Bases and Dipolar Aprotic Solvents. J. Org. Chem. 1989, 54, 5887-5897.

40. Kader, A. T.; Stirling, C. J. M., Elimination-Addition. Part III. New Procedures for the Protection of Amino-groups. J. Chem. Soc. 1964, 258-266.

41. Sigmund, H.; Pfleiderer, W., Nucleosides Part LVI, Aminolysis of Carbamates of Adenosine and Cytidine. Helvetica Chimica Acta, 1994, 77, 1267-1273.

42. Vorotyntsev, M. A.; Zinovyeva, V. A.; Konev, D. V.; Picquet, M.; Gaillon, L.; Rizzi, C., Electrochemical and Spectral Properties of Ferrocene (Fc) in Ionic Liquid: 1-Butyl-3methylimidazolium Triflimide, [BMIM][NTf2]. Concentration Effects. J. Phys. Chem. B 2009, 113, 1085-1099.

43. Cenens, J.; Schoonheydt, R. A., Visible Spectroscopy of Methylene Blue on Hectorite, Laponite B, and Barasym in Aqueous Suspension. Clay and Clay Minerals 1988, 36, 214224.

44. Butler, D. L.; Sellborn, M., Barriers to Adopting Technology for Teaching and Learning. Educause Quarterly 2002.

45. Bevan, T. Investigations of azulene derivatives as self-indicating chromophores for applications in protecting group strategies and solid-phase purification. VUW, MSc thesis, 2011. 


\section{Chapter 2. Synthesis of Azulenes}

\subsection{Introduction}

From the outset of this project, it was clear that purchasing sufficient quantities of azulenesfor the proposed studies would be prohibitively expensive (as of June 2015, azulene itself can be purchased at $\$ 550 /$ gram from Sigma-Aldrich, 6-methylazulene can be purchased at $\$ 296.98 / 100$ mg from Molport and other substituted azulenes are only priced on request.)

There exist several syntheses of azulene and azulene derivatives in the literature. Many of these suffer from several drawbacks, including a relatively large number of steps, a tendency for low yields and large numbers of by-products. The synthesis of azulene by D. M. Lemal etal. ${ }^{1}$ (Scheme 12) takes place through a [4+6] cyclisation of dimethylaminofulvene and thiophene dioxide. The synthesis of azulene by J. P. Depres et al. (Scheme 13) starts from cycloheptatriene and has been additionally used to synthesise 1-, 4- and 7-alkyl-substituted azulenes. ${ }^{2}$

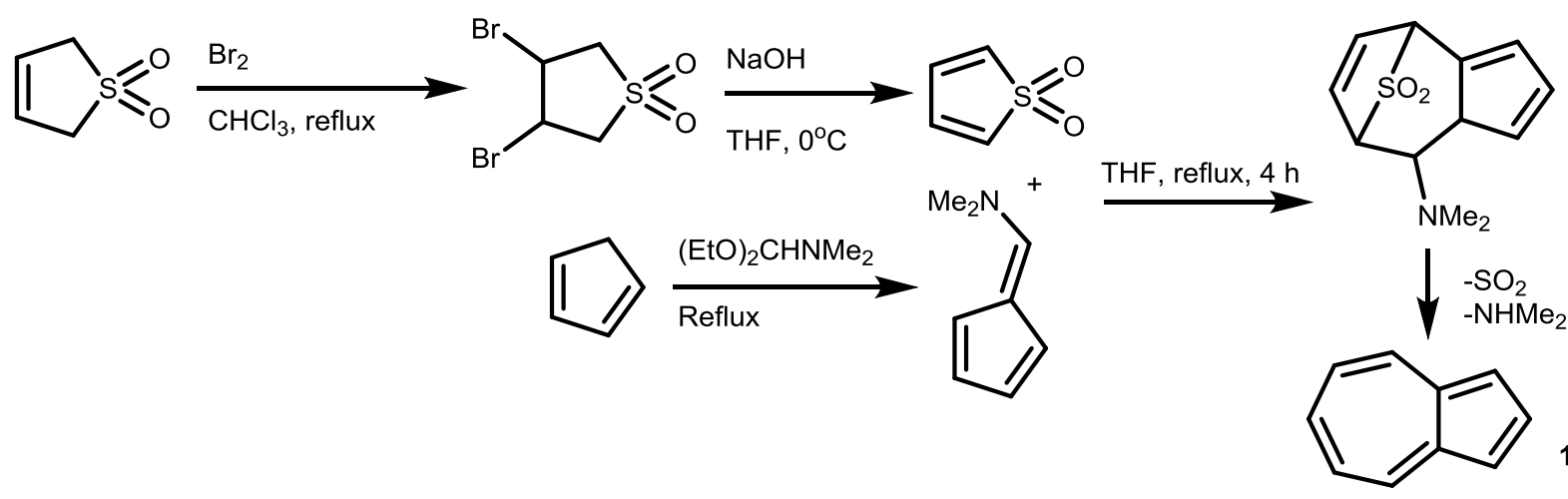

$9 \%$ overall yield over 4 steps

Scheme 12. Synthesis of azulene by D. M. Lemal et al.<smiles>C1=CCCC=CC1</smiles>

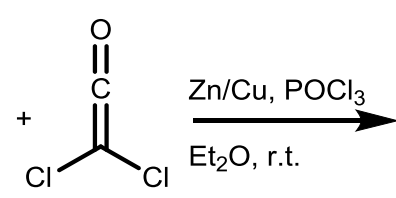<smiles>CN(C)CC1C=C[C@@H]2[C@@H](C1)C(=O)C2(Cl)Cl</smiles><smiles>O=C1C[C@H]2CC=CC=CC2=C1Cl</smiles><smiles></smiles>

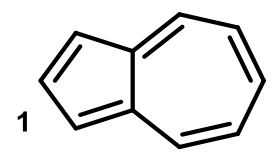

$18 \%$ overall yield over 4 steps
Polymethylhydrosiloxane, $\mathrm{Pd}(\mathrm{OAc})_{2}$, $\mathrm{dpdb}, \mathrm{K}_{3} \mathrm{PO}_{4}, \mathrm{THF}$, reflux

Scheme 13. Synthesis of azulene starting from cycloheptatriene by J. P. Depres et al. 
The Hafner approach to azulene synthesis by-passes some of the drawbacks suffered by other methods. The general form of a Hafner synthesis involves the reaction of a cyclopentadienide anion (shown in red in Figure 34) and a heterocycle such as a pyrylium or pyridinium salt (shown in blue in Figure 34). ${ }^{3}$

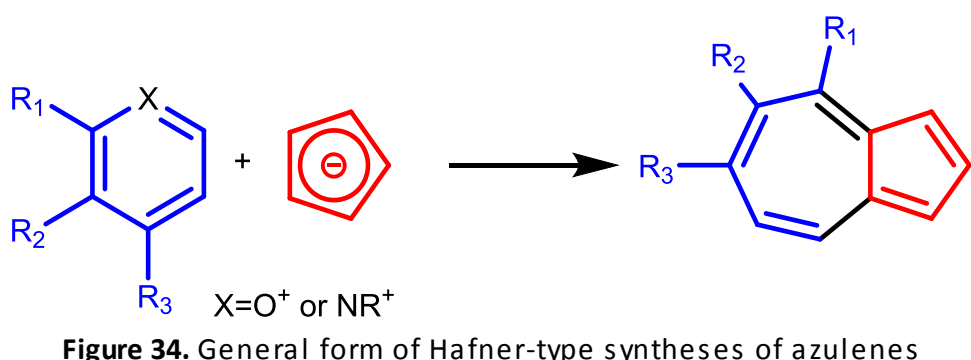

The advantages the Hafner synthesis has over other methods are multi-fold; it can be performed as a one-pot reaction using readily-available and cheap materials, (the most costly material typically being sodium metal) which offsets the typically modestyields of the azulene product that are obtained. Easy access to derivatives substituted on the 7-membered ring is provided by using the correspondingly substituted heterocyclic precursor. Furthermore, the azulene product is the most non-polar species in the reaction mixture, which facilitates separation from the large quantities of the more polar products that are produced in this reaction. For these reasons, it was decided to synthesise azulenes through this general strategy.

\subsection{Synthesis of azulene}

The Hafner synthesis of azulene reported in Organic Syntheses ${ }^{3}$ is a robust method for preparing azulene on a large scale. It is a three-step, one-pot procedure, wherein the first two steps generate the pentamethine cation $\mathbf{9 6}$ which later forms five of the seven carbons in the 7-membered ring, and the final step introduces the 5 -membered ring through addition of sodium cyclopentadienide (Scheme 14). 


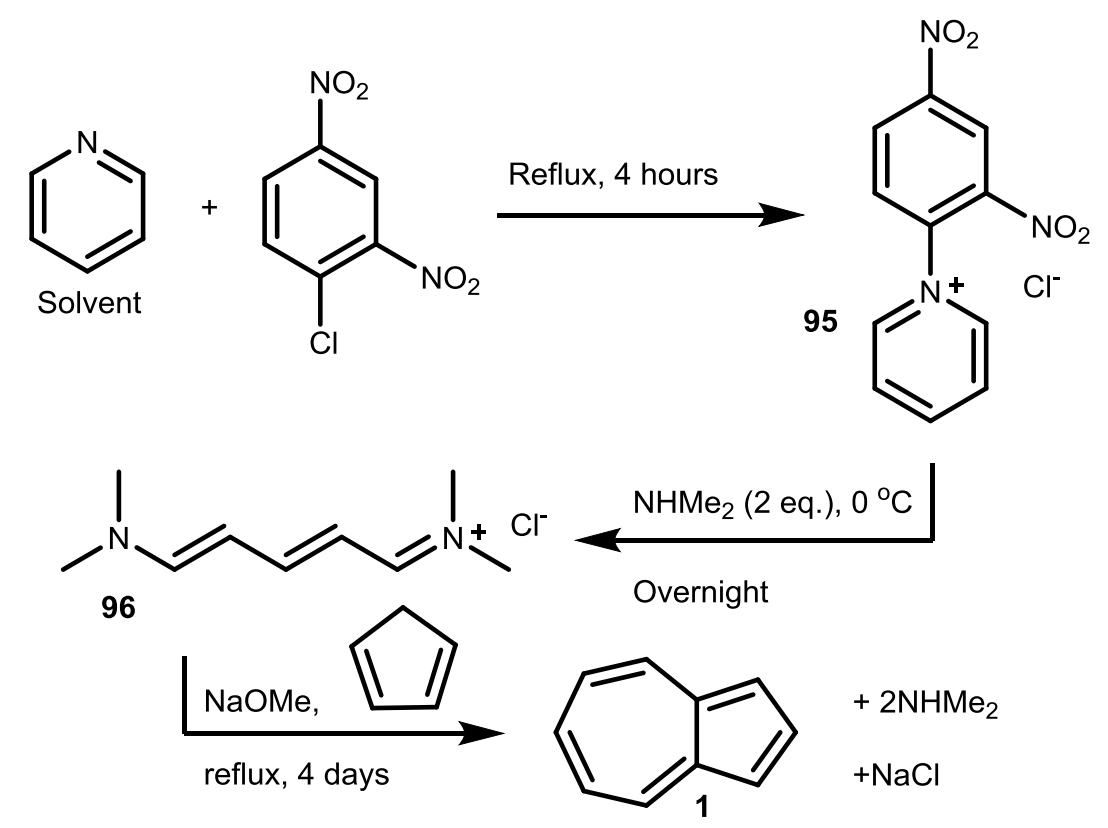

Scheme 14. The three steps involved in the one-pot Hafner synthesis of azulene

These first two steps are a subset of what is known as Zincke chemistry, discovered at the turn of the $20^{\text {th }}$ century. ${ }^{4}$ The pyridinium compound 95 , formed by the addition of 1-chloro-2,4dinitrobenzene to pyridine is known as the Zincke salt (formation depicted in Scheme 15), and has a number of synthetic uses in general organic chemistry, such as formation of $\mathrm{N}$-substituted pyridinium salts from reaction of the species 95 with a primary amine, and formation of unsaturated aldehydes from the treatment of 96 with aqueous base.

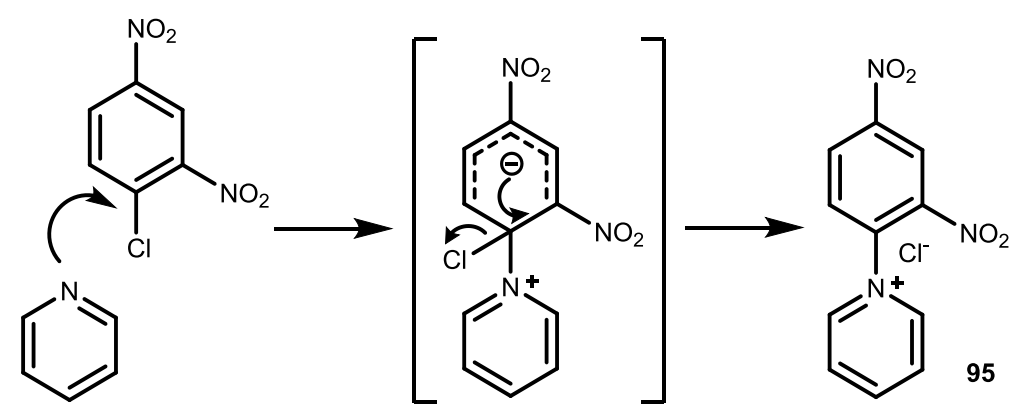

Scheme 15. Mechanism of the generation of the Zincke salt 95

In the particular case of the Hafner synthesis, the Zincke salt 95 is treated with two equivalents of dimethylamine to form the pentamethine iminium species 96 through ring-opening of the pyridinium (depicted in Scheme 16). The pentamethine 96 undergoes nucleophilic attack by the cyclopentadienide carbanion followed by elimination of dimethylamine to produce the conjugated intermediate 97 . This intermediate cyclises through a $10 \pi$ electrocyclic ring-closing process to generate 98. Finally, elimination of a second dimethylamine from $\mathbf{9 8}$ completes the mechanism, generating azulene (Scheme 17). 
<smiles>CN(C)CCCCC[n+]1ccccc1[N+](=O)[O-]</smiles><smiles>CCOc1ccc([N+](=O)[O-])cc1N1C=CC=CC1[N+]1(C)CCCC1</smiles>

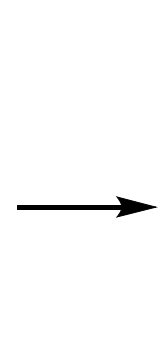

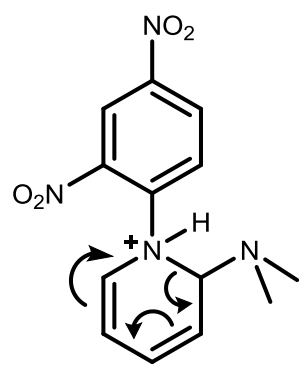<smiles>CN(C)/C=C/C=C/C=[N+](C)C</smiles>

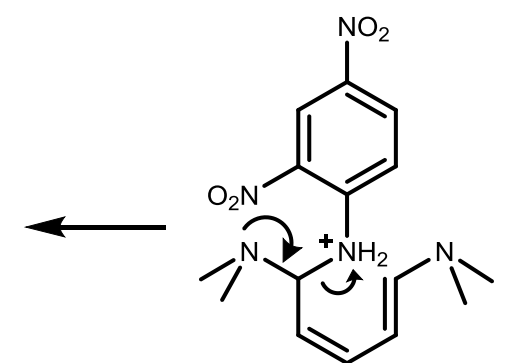

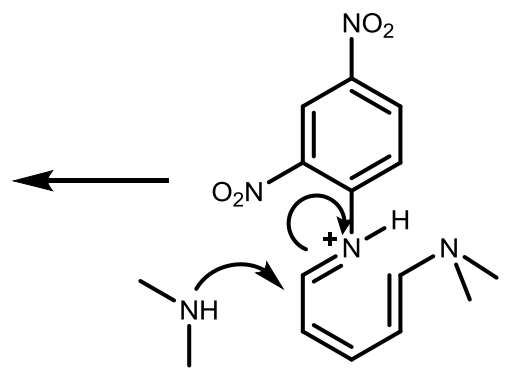

Scheme 16. Proposed mechanism of the formation of the pentamethine 96<smiles>CN(C)/C=C/C=C/C1=[N+](C)CCC1Cc1ccccc1</smiles>

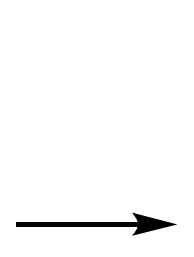

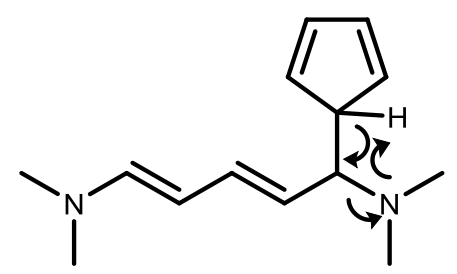<smiles>CN(C)CC12C=CC=C1C=CC=CC2(C)C</smiles>

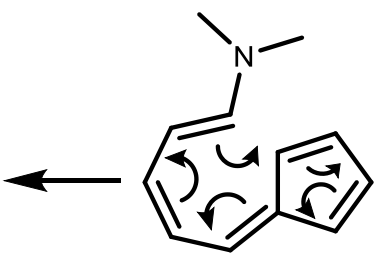<smiles>C#C</smiles><smiles>CN(C)/C=C/C=C/C=C1C=CC=C1</smiles><smiles>CC(C)Cc1cccccc2c[cH+]cc1-2</smiles>

Scheme 17. Proposed mechanism of the generation of azulene (1)

The scale of this method ( 1 mole) was too large and costly for our purposes, so various modifications were developed. Our modifications of the Hafner synthesis mainly involved a 30fold reduction in scale, which subsequently led to shorter reaction times, and the use of diethylamine instead of dimethylamine for ease of handling (the boiling point of dimethylamine is $7^{\circ} \mathrm{C}$, whereas that of diethylamine is $55^{\circ} \mathrm{C}$ ). 
The first step in the literature preparation involves addition of 1-chloro-2,4-dinitrobenzene to pyridine solvent, followed by 4 hours of heating at reflux resulting in a light brown precipitate. I found that at smaller scales this reaction would be complete at between one hour and ninety minutes of heating at reflux. After cooling to $0{ }^{\circ} \mathrm{C}$, two equivalents of diethylamine were added, and the reaction mixture rapidly became orange-red. It is important to cool the reaction mixture down to $0{ }^{\circ} \mathrm{C}$ at this stage, because at higher temperatures diethylamine would displace the pyridine instead, forming a yellow compound with a ${ }^{1} \mathrm{H}$ NMR spectrum that matches $1-(N, N-$ diethylamino)-2,4-dinitrobenzene. 5 It was not necessary for the reaction to stir for the 12 hour period described in the protocol; typically a waiting period of 30 minutes to 1 hour was used to give the same result. After this period, freshly cracked cyclopentadiene was added, followed immediately by a sodium methoxide solution prepared freshly from sodium metal in methanol. The mixture was then heated to reflux. It was found that heating at reflux overnight as opposed to the suggested four days was sufficient to give yields similar to the reported values. The yield of azulene from these reactions was typically $25-51 \%$ (compared to $51-59 \%$ recorded for the procedure in Organic Syntheses) and including the time taken for purification, the whole process could be performed in two days, a marked improvement over the 5-6 day procedure of the original paper.

Extraction of azulene from the brown-black crude mixture was a time-consuming process. Ultimately, it was found that repeated filtration using petroleum ether over a plug of silica followed by column chromatography was sufficient to obtain purified azulene.

\subsection{Synthesis of alkyl-substituted azulenes}

Several methylazulenes were targeted for synthesis, particularly those with methyl groups on the 7-membered ring, such as 99, 11, 10 and 9 (Figure 35). It was originally intended that the methyl-substituted azulenes be synthesised using the same Hafner protocol with the appropriately substituted pyridines, however the general procedure did not work in most cases, and most of these azulene derivatives had to be synthesised through different protocols.
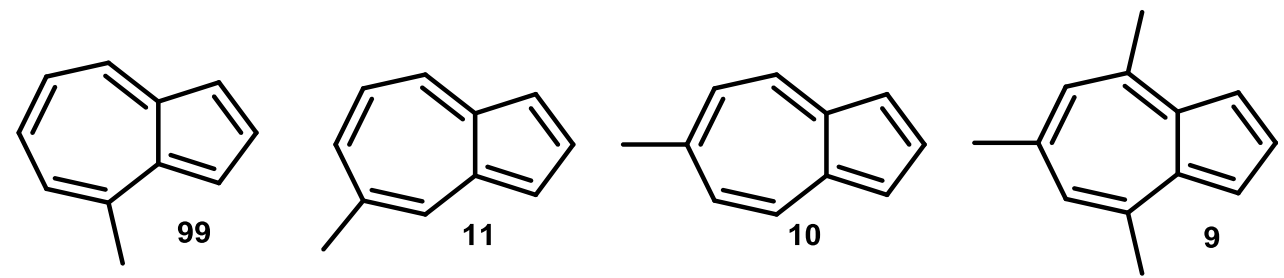

Figure 35. Methyl substituted azulenes $\mathbf{9 9}, \mathbf{1 1}, \mathbf{1 0 , 9}$ were targeted for synthesis 


\subsubsection{Synthesis of 4,6,8-trimethylazulene}

The synthesis of 4,6,8-trimethylazulene (9) is already well established, 6 and differs from the Hafner synthesis of azulene in two ways: the use of a pyrylium heterocycle rather than a pyridinium to form the 7-membered ring, and the lack of a ring-opening second step by a secondary amine (Scheme 18). The cyclopentadienide ion attacks the pyrylium ring directly, initiating the opening of the pyrylium and the subsequent $10 \pi$-electrocyclic ring-closure and loss of water to form 4,6,8-trimethylazulene.

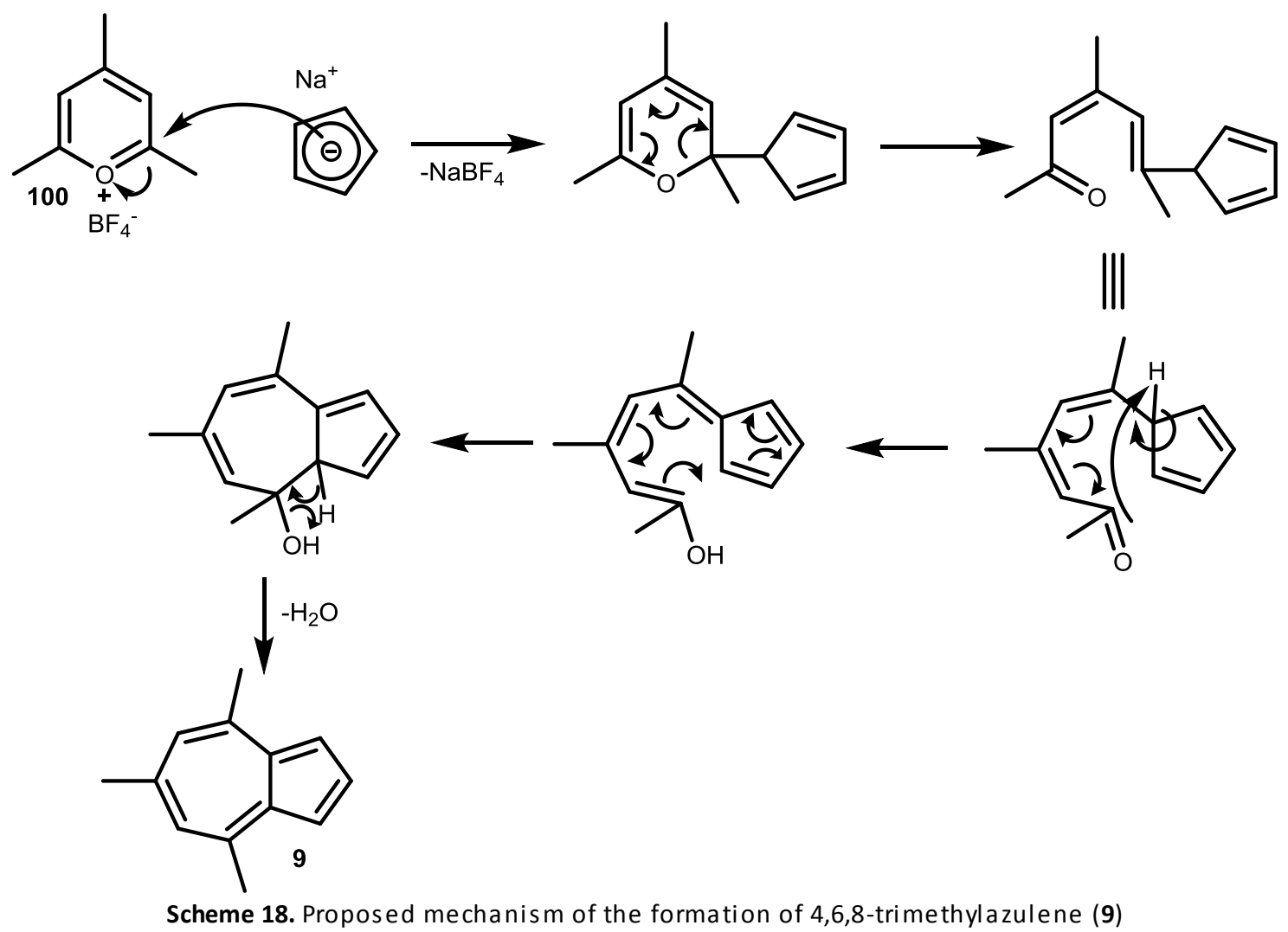

The literature protocol calls for a milder reaction temperature than that of azulene, proceeding under room temperature or slightly elevated temperatures in DMF to return yields around $50 \%{ }^{6}$ The 2,4,6-trimethylpyrylium cation can be prepared as a perchlorate or tetrafluoroborate salt. The tetrafluoroborate salt $\mathbf{1 0 0}$ is prepared through addition of tetrafluoroboric acid to tert-butanol in acetic anhydride (Scheme 19). 

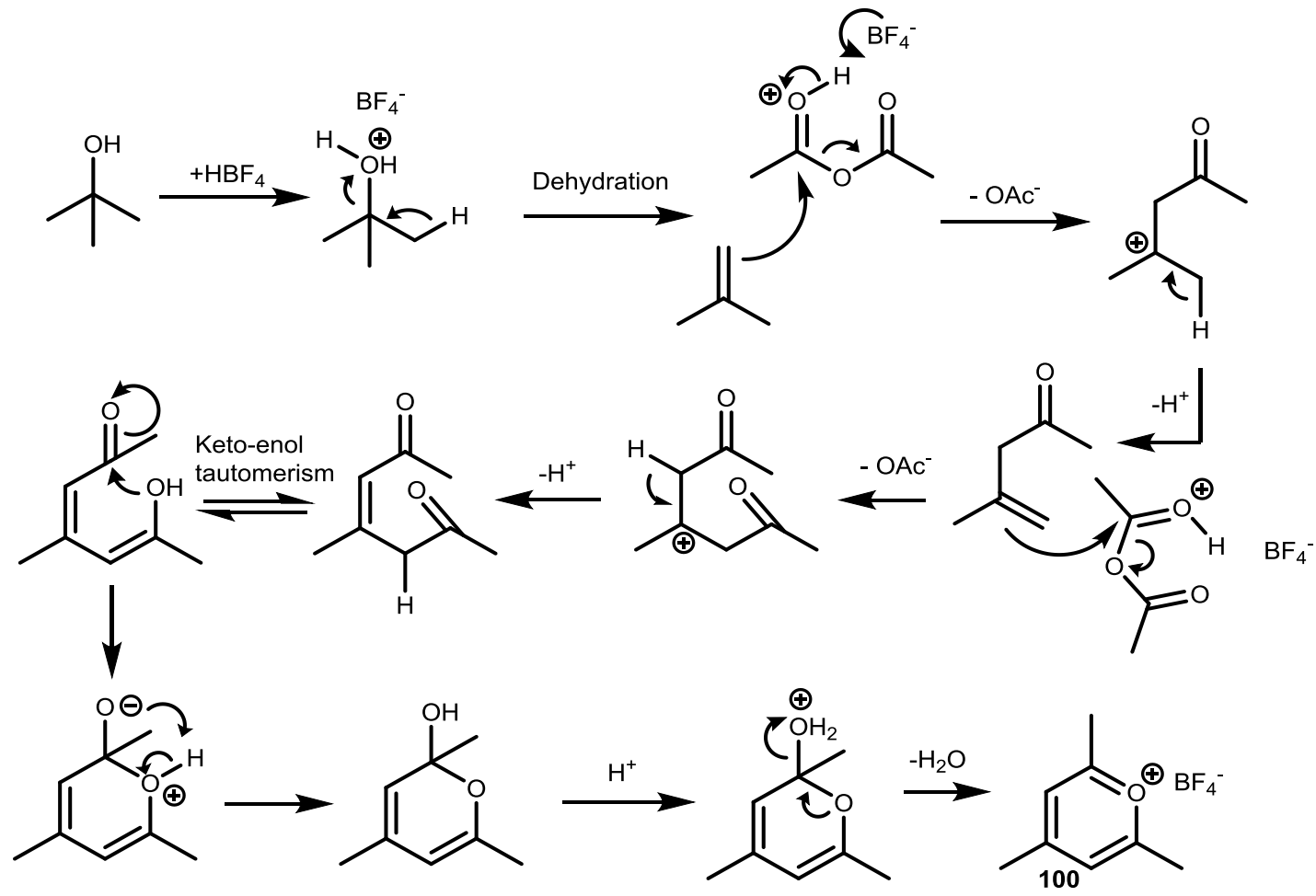

Scheme 19. Proposed mechanism of formation of 2,4,6-trimethyl pyrylium tetrafluoroborate (100)

This synthesis was attempted several times, with yields returned typically below 10\%, far lower than the $50 \%$ yield reported in the literature. ${ }^{6}$ Nevertheless, sufficient material was made to last the duration of this project, so this synthesis was never optimised.

\subsubsection{Synthesis of 1- and 5-methylazulene}

Only small amounts of 1- and 5-methylazulene were produced, as these azulenes were mainly intended for comparison purposes with azulene in terms of various reactivities.

5-Methylazulene $\mathbf{1 1}$ is one of the few methylazulenes that can be made through Zincke chemistry,7 with 3-methylpyridine used instead of pyridine (Scheme 20). The mechanism is essentially the same as that depicted in Schemes 15-17. The yield returned was only 8\%, and the reaction was only attempted once, so no further optimisation took place.
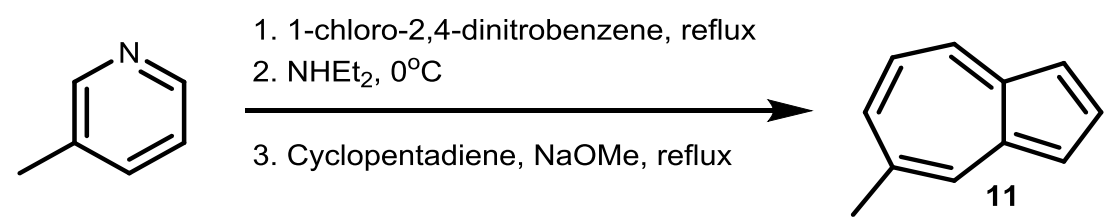

Scheme 20. Synthesis of 5-methylazulene (11)

Making 1-methylazulene directly through the Hafner method requires expensive methylcyclopentadiene, and has the disadvantage of also producing the 2-methylazulene isomer. 1-Methylazulene was instead synthesised by formylating azulene through the Vilsmeier-Haack 
method and reducing the formyl group to a methyl group with $\mathrm{LiBH}_{4}$ and $\mathrm{BF}_{3} . \mathrm{Et}_{2} \mathrm{O}$, in $40 \%$ overall yield (Scheme 21).
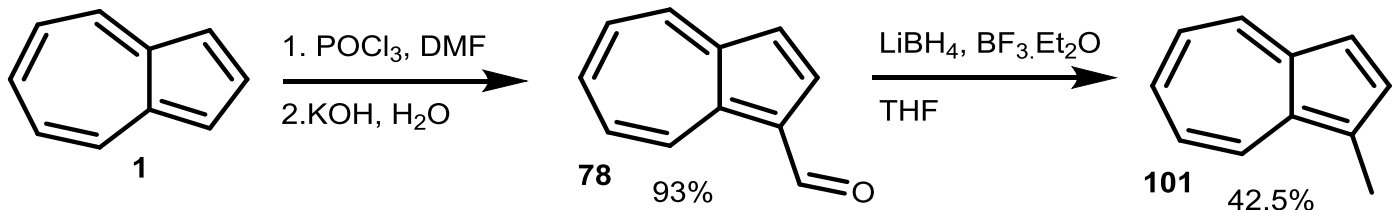

Scheme 21. Two-step synthesis of 1-methylazulene 101 through reduction of 1-formyl azulene (78)

\subsubsection{Synthesis of 4- and 6-methylazulene}

Neither the original Hafner synthesis nor the pyrylium modification is amenable to giving 4- or 6mono-substituted azulenes. Unsubstituted and partially substituted pyrylium salts do not react in the same way to cyclopentadiene as 2,4,6-trisubstituted pyrylium salts do, tending to undergo attack at the 4-position rather than the 2-position. ${ }^{8}$

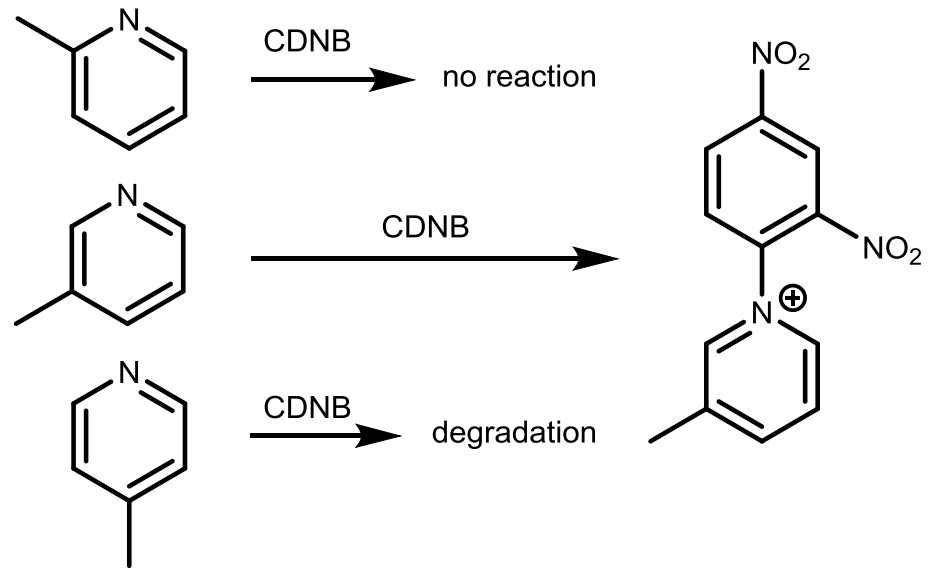<smiles>O=[N+]([O-])c1ccc(Cl)c([N+](=O)[O-])c1</smiles>

Scheme 22. Reaction of 2-, 3-, and 4-methylpyridines with CDNB

The Zincke salt also fails to form upon addition of 1-chloro-2,4-dinitrobenzene (CDNB) to 2 and 4methylpyridines (Scheme 22). In the case of 2-methylpyridine the Zincke salt completely fails to form, even under reflux at $128{ }^{\circ} \mathrm{C}$, presumably because the methyl group at the 2-position provides sufficient steric hindrance to prevent attack of the pyridine on the aryl chloride.

4-Methylpyridine, on the other hand reacts immediately with 1-chloro-2,4-dinitrobenzene at room temperature to produce a deep purple solution which, by NMR, appears to consist of at least seven species, all containing a 4-methylpyridine moiety. No explanation in the literature is given for this reactivity, apart from an oblique mention in the Collection of Czechoslovak Chemical Communications, 1947 of a 'humic' material being produced upon reaction. ${ }^{9}$ Indeed, in the Zincke chemistry literature in general, only references to 3 - and 5-substituted pyridines can be found.7, 9 
This hurdle can be circumvented for 4-methylazulene by synthesising it from azulene and methyllithium. The 4- and 8- positions are the most electrophilic, owing to the structure of the LUMO (see section 1.2), and are alkylated upon addition of MeLi. The resulting anionic species 102 must be oxidised by $p$-chloranil to obtain 4-methylazulene (99). The yield for this reaction is serviceable, at about 35\% (Scheme 23).10

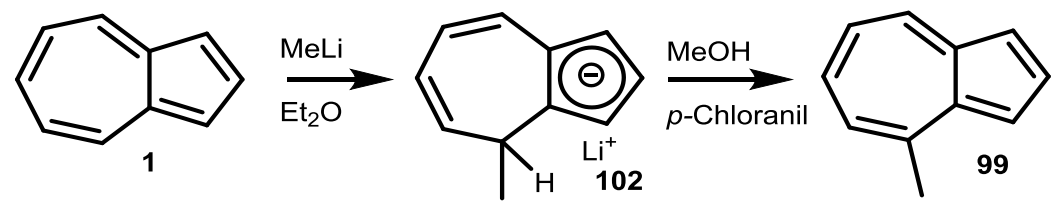

Scheme 23. Synthesis of 4-methylazulene (99)

6-Methylazulene cannot be made through this procedure, as the 6-position will only undergo nucleophilic attack once the 4- and 8- positions are blocked. Instead, examples were found in the literature of reactions using pyridinium species activated with reagents other than 1-chloro-2,4dinitrobenzene to form azulenes that were otherwise inaccessible. One example involves the activation of 2-methyl-5-ethylpyridine with $n$-butyl bromide. Attack on the resulting pyridinium bromide by sodium cyclopentadienide gives the desired 4-methyl, 7-ethylazulene. ${ }^{11}$

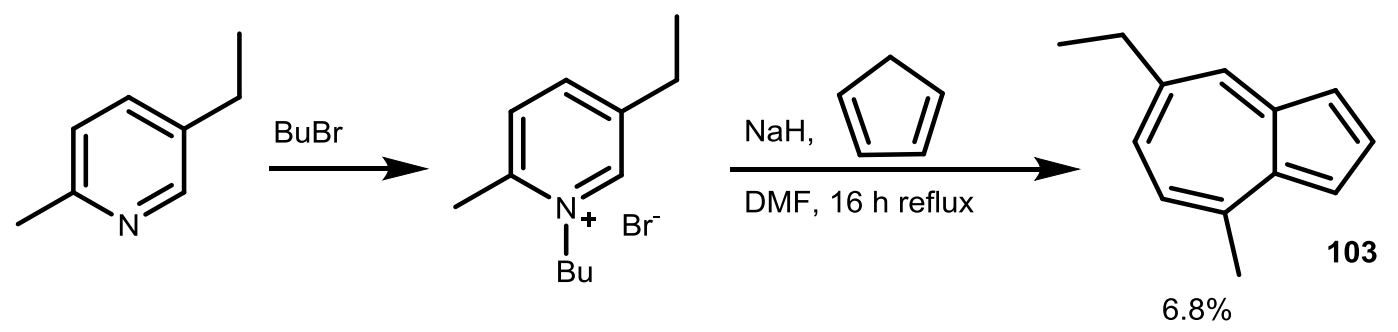

Scheme 24. Litera ture example of an alkylated pyridinium bromide being used to synthesise the 4-methyl-7-ethyla zulene (103)

Another example involves the activation of the bicyclic pyridine $\mathbf{1 0 4}$ with methyl iodide, followed by attack of sodium cyclopentadienide to produce the azulene $\mathbf{1 0 5 . 1 2}$

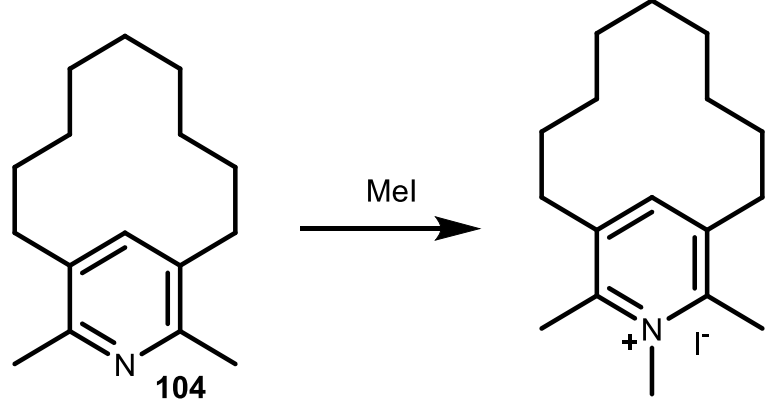

Scheme 25. Litera ture example of the activa tion of the highly hindered pyri dine $\mathbf{1 0 4}$ a llowing the formation of the a zule ne 
In both cases the yields were poor. The first returned a $6.8 \%$ yield of $\mathbf{1 0 3}$, while the second returned an abysmal $0.034 \%$ yield of $\mathbf{1 0 5}$. Nevertheless, both of these syntheses give azulenes that could not have been made through the methods that give azulene and 4,6,8-trimethylazulene.

It was decided to investigate alkyl pyridinium activation as a method for formation of azulenes, particularly 6-methylazulene. To test the chemistry on azulene itself, $N$-methylpyridinium iodide was synthesised from pyridine and methyl iodide in high (90-94\%) yield. This was allowed to react with cyclopentadiene and sodium methoxide in pyridine to give azulene in a disappointing $1 \%$ yield (Scheme 26). Attempts to make 4-methylazulene through this method fared even worse. Upon reaction of 1,2-dimethylpyridinium iodide with sodium cyclopentadienide under elevated temperatures, small traces of a bluish compound could be detected by TLC. However after heating at reflux overnight in pyridine this blue compound invariably disappeared, indicating the reaction conditions were too harsh for the survival of the 4-methylazulene product. This reaction was rerun at a temperature of $70{ }^{\circ} \mathrm{C}$ overnight, giving 4-methylazulene 99 in a $0.8 \%$ yield.<smiles>C[n+]1ccccc1</smiles><smiles>Cc1cccc[n+]1C</smiles>
$\mathrm{NaOMe}$, Pyridine, reflux overnight
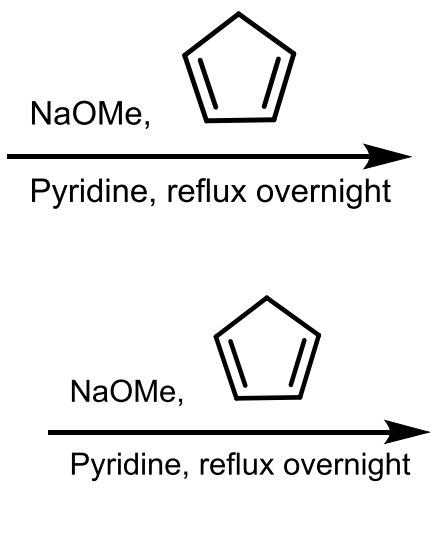
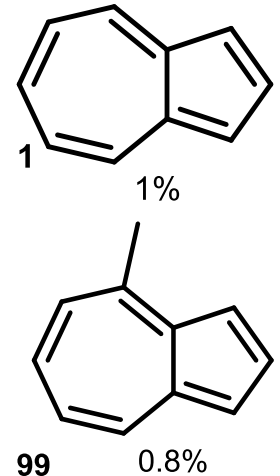

Scheme 26. Attempted syntheses of a zulene 1 and 4-methylazulene 99 by reacting a $\mathbf{N}$-methyl pyridinium iodide with sodium cyclopentadienide

Attempts to synthesise 6-methylazulene 10 fared somewhat better. The first attempt reacting 1,4dimethylpyridinium iodide with cyclopentadiene gave a $3 \%$ yield after heating at reflux overnight in pyridine (Scheme 27). For the second attempt, instead of adding sodium methoxide as a solution in methanol, a reaction vessel was prepared with freshly-made sodium methoxide as a dry solid. This was done to avoid the possibility of methanol reprotonating the cyclopentadienyl anion, a concern that later turned out to be erroneous because better yields were obtained using alcoholic solvents in place of pyridine. Nevertheless, at the time it appeared promising, as a 5.5\% yield was obtained. 


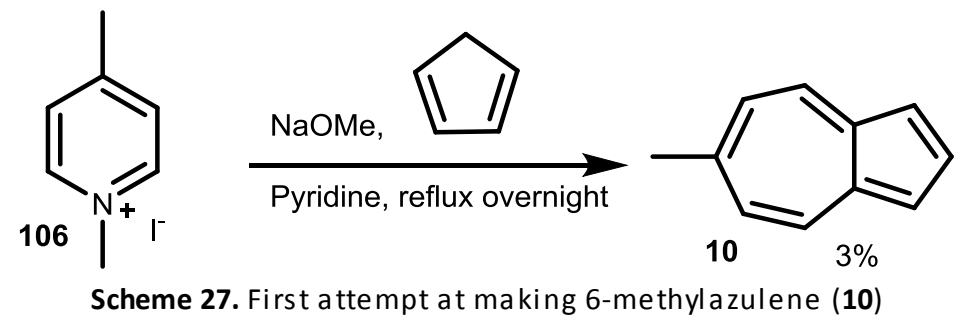

The further optimisation of this reaction was not pursued immediately, as sufficient 6methylazulene had been generated to perform some initial experiments (approximately $600 \mathrm{mg}$ ), and the chemistry was at a scale sufficiently large - and the reagents sufficiently cheap - that the very low yields were not a pressing issue. As the chemistry surrounding 6-methylazulene became more promising, it became clear that an improved synthesis of 6-methylazulene was needed.

A synthesis of 6-methylazulene was found in the literature using similar chemistry, giving a 14\% yield, the major difference being choice of solvent - ethanol, instead of pyridine. The authors described the difficulty they had with optimising this reaction. In their own words: " $A$ variety of modifications of the above method failed to improve the yield of 6-methylazulene; in fact, most attempts led to loweryieldsofproduct."13

Undeterred, a variety of different alcoholic solvents were investigated. Use of methanol gave a 3.3\% yield, whereas a 4:1 mixture of ethanol/isopropanol (distilled over sodium from drum isopropanol, which unbeknownst to us at the time consisted of $80 \%$ ethanol) gaveyields between $10 \%$ and $12 \%$. Use of pure, dry isopropanol gave better yields, between $11 \%$ and $17 \%$ (Table 2). Isopropanol reacts surprisingly slowly with sodium, requiring heating at reflux in order to go to completion. Since sodium isopropoxide is only sparingly soluble in isopropanol, care was taken to keep the reaction mixture at elevated temperatures at all times, lest the alkoxide precipitate. This was true even after the reaction had completed, and the solution had to be kept hot during the initial purification procedures. These reactions were typically performed for a duration between 80 minutes and 3 hours, using between 2 and 3 equivalents of $\mathrm{Na}$ /cyclopentadiene. A very slight positive correlation can be seen between the number of equivalents of sodium cyclopentadienide and yield, and between reaction duration and yield.

\begin{tabular}{|l|l|l|l|l|}
\hline Entry & Eq. of Na & Eq. of Cyclopentadiene & Reaction time/ min & Yield \% \\
\hline $\mathbf{1}$ & 2.03 & 2.03 & 80 & $11.1 \%$ \\
\hline $\mathbf{2}$ & 2.47 & 2.40 & 90 & $14.3 \%$ \\
\hline $\mathbf{3}$ & 2.39 & 2.40 & 190 & $16.6 \%$ \\
\hline $\mathbf{4}$ & 2.60 & 2.54 & 120 & $17.5 \%$ \\
\hline
\end{tabular}


Finally, the synthesis of 6-methylazulene was performed using potassium instead of sodium, while keeping every other variable approximately the same. Potassium is much more reactive than sodium, and addition of potassium to isopropanol required submergence in an ice bath to control the reaction rate. After heating the reaction mixture at $70{ }^{\circ} \mathrm{C}$ for 2 hours, a $35 \%$ yield of 6methylazulene was obtained, doubling my previous highest yield for this compound (entry 4, table 2) and improving on the literature yield by 2.5 fold (Scheme 28). Further experiments gave yields between $30 \%$ and $35 \%$, and indicated there is an optimal temperature somewhere between $60{ }^{\circ} \mathrm{C}$ and $80{ }^{\circ} \mathrm{C}$, above which the material begins to degrade. It is believed that through careful tuning of reaction temperature and time, higher yields ought to be possible. The reaction is amenable to scale up, and yields of up to 4.5 grams have been obtained.

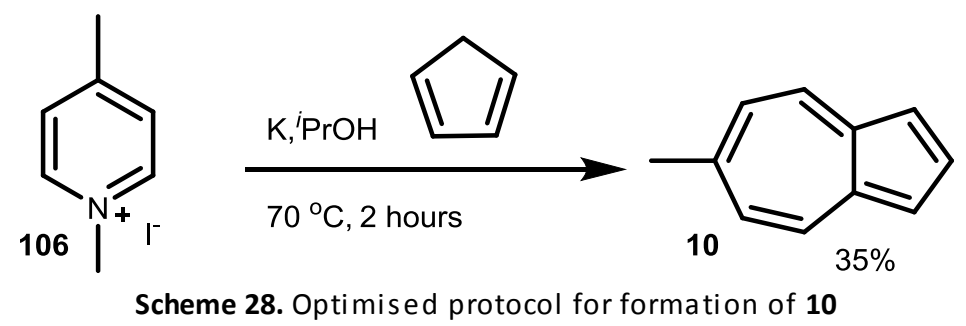

Higher yields of 10 have since been reported in the literature. In late 2015, a yield of 64\% was reported from a similar reaction involving the use of 1-butyl-4-methylpyridinium bromide instead of 106 with $\mathrm{NaH}$ as the base and DMF as the solvent. The reaction requires heating to $170{ }^{\circ} \mathrm{C}$ for 30 minutes by microwave and is not as amenable to scale-up as the protocol developed in this project. ${ }^{14}$

A curious by-product was often observed in these reactions. Present in very small $(<0.1 \%)$ yields, 6- $p$-tolylazulene 107 was present. It is believed this compound is formed through deprotonation of the product 6-methylazulene and reaction of the resulting anion with another equivalent of 1,4dimethylpyridinium chloride, via a similar sequence of sigmatropic rearrangements and eliminations (Scheme 29). 


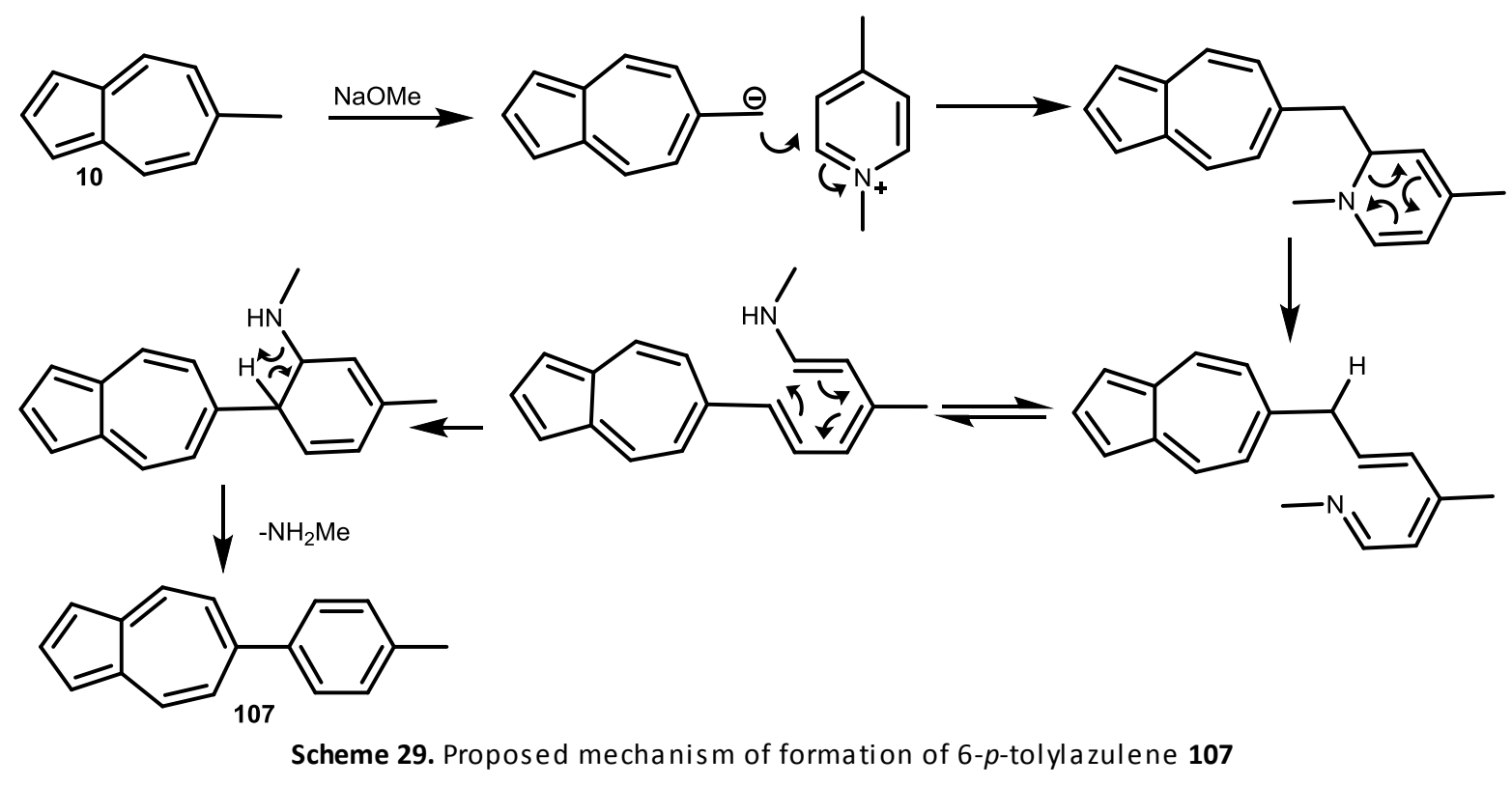

A few attempts were made at recreating this process by reacting 6-methylazulene with 1,4dimethylpyridinium iodide in the presence of the bases LDA or sodium methoxide, but no detectable amounts of 6- $p$-tolylazulene were ever found.

\subsection{NMR structural assignment of the azulenes}

The signals associated with the protons on an azulene ring system are typically well separated and have highly distinct lineshapes, allowing an organic chemist to rapidly identify the NMR shifts of individual nuclei (Figure 36).

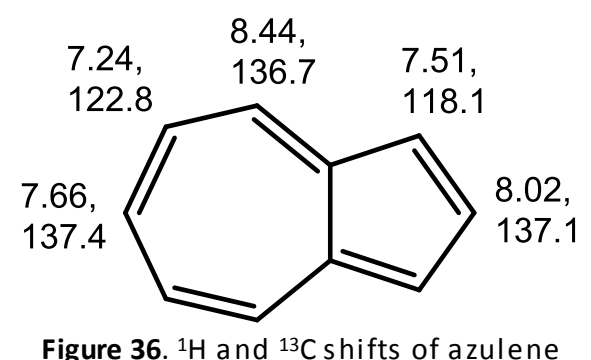

The effects of the uneven HOMO distribution are readily apparent, with the odd-numbered nuclei being highly shielded $\left(\delta_{\mathrm{H}}\right.$ of positions 1,3 and 5,7 on azulene are 7.51 and 7.24 respectively, and $\delta_{\mathrm{C}}$ of these positions are 118.1 and 122.8 ppm respectively) while the even-numbered nuclei are highly deshielded $\left(\delta_{\mathrm{H}}\right.$ of positions 2,4 and 6 are 8.02, 8.44 and $7.66 \mathrm{ppm}$ respectively, and $\delta_{\mathrm{C}}$ of these positions are 137.1, 136.7 and 137.4 ppm, respectively). This clustering of odd and even shifts can be readily seen in the HSQC of azulene (Figure 37), and is also apparent in the spectra of other azulenes. 

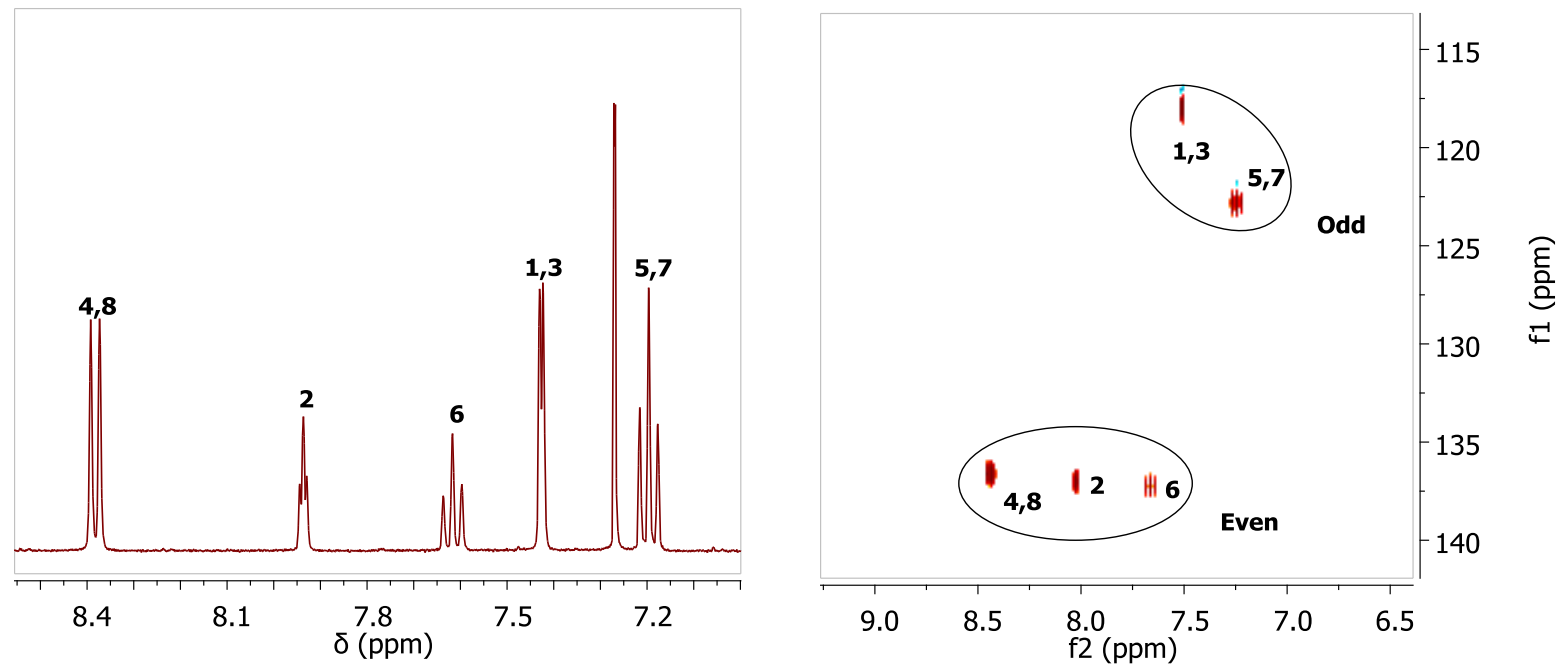

Figure 37. ${ }^{1} \mathrm{H}$ NMR s pectrum and HSQC s pectrum of a zulene, illustrating the differences in coupling constants between the rings and the separation of the shifts of odd and even positions

Protons on the 7-membered ring couple to each other with $J$-values of between 10 and $11 \mathrm{~Hz}$, whereas protons on the 5-membered ring typically have coupling constants of 4 to $5 \mathrm{~Hz}$ (Figure 37). The connectivity between protons can also be readily seen through COSY. In combination, these pieces of information were sufficient to identify the substitution pattern of azulene derivatives throughout this project.

In symmetry-breaking cases involving substitution on the 1- or 3-position with an electronwithdrawing moiety (such as almost all the 1-substituted compounds discussed in Chapter 3), the proton on the 4- or 8-position adjacent to the substitution exhibits a strong deshielding effect and the corresponding shift appear as high as 9.89 ppm, such as in the case of methyl oxo-1azulenecarboxylate (7), while the proton on the unsubstituted side of the ring will only experience a modest effect. Use of the COSY information then allows the adjacent protons on the 7-membered ring to be assigned.

\subsection{Summary of Chapter 2.}

Azulene and a series of methylazulenes were synthesised in sufficient quantities to allow for subsequent research. Sufficient quantities of azulene 1, and 4,6,8-trimethylazulene 9 were synthesised for the experiments throughout chapter 3. No optimisation of the synthetic procedures for 1-methylazulene 101, 4-methylazulene 99, 5-methylazulene 11 and 4,6,8trimethylazulene 9 was carried out, as these materials were never in large demand during this research. The synthesis of 6-methylazulene $\mathbf{1 0}$ was significantly optimised by using isopropanol as the solvent and using potassium instead of sodium, improving the yield from $3 \%$ to $35 \%$. This was sufficient to allow large quantities of 6-methylazulene to be produced, and rendered viable the E1cB-based 6-substituted azulene protecting group discussed in chapter 4 onwards. 


\subsection{References}

1. Lemal, D. M.; Goldman, G. D., Synthesis of Azulene, a Blue Hydrocarbon. J. Chem. Ed. 1988, 65, 923-925.

2. Carret, S.; Blanc, A.; Coquerel, Y.; Berthod, M.; Greene, A. E.; Depres, J.-P., Approach to the Blues: A Highly Flexible Route to the Azulenes. Angew. Chem. Int Ed.Engl. 2005, 44, 51305133.

3. Meinhardt, K.-P.; Hafner, K., Azulene. Organic Syntheses 1984, 62 (134).

4. (a) Cheng, W.-C.; Kurth, M. J., The Zincke Reaction. A Review. Org. Prep. Proc. Int. 2002, 34, 585-608; (b) Vanderwal, C. D., Reactivity and Synthesis Inspired by the Zincke RingOpening of Pyridines. J. Org. Chem. 2011, 76, 9555-9567.

5. Gale, D. J.; Rosevear, J.; Wilshire, J. F. K., The Anomalous Reactions of Some Fluoronitrobenzenes with Some N, N-Dialkylamines. Aust.J. Chem. 1995, 48, 997-1007.

6. Garst, M. E.; Hochlowski, J.; James G. Douglass, I.; Sasse, S., The Synthesis of 4,6,8Trimethylazulene.J.Chem. Ed. 1983, 60, 510-511.

7. Nguyen, T. M.; Sanchez-Salvatori, M. d. R.; Wypych, J.-C.; Marazano, C., Aminopentadiene Imines from Zincke Salts of 3-Alkylpyridines. Application to a Synthesis of Pyridinium Salts from Amino Acids. J. Org. Chem. 2007, 72, 5916-5919.

8. Foerst, W.; Birnbaum, H., Newer methods of Preparative Organic Chemistry. ACS, 1964; Vol. 3, p 397.

9. Lukes, R., 3-substituted pyridine homologs. Collection of Czechoslovak Chemical Communications 1947, 12, 263-277.

10. Hafner, K.; Bernhard, C.; Müller, R., Zur Kenntnis der Azulene, V. Die Nucleophile Substitution des Azulens. Liebigs Ann. 1962, 650,35-41.

11. Oehler, C.; Bensdorf, K.; Gust, R.; Imming, P., Chamavioline-Antiedematous, but not a constituent of Matricaria Recutita. Phytochem. Lett. 2009,2,171-175.

12. Kurokawa, S.; Jr., A. G. A., Synthesis of 4,8-Dimethyl-5,7-nonamethyleneazulene. Bull. Chem. Soc.Jpn. 1979, 52, 257-258.

13. McDonald, R. N.; Wolfe, L; Petty, H. E., Nonbenzenoid Aromatic Systems. VIII."' Buffered Acetolysis of 2-(4- and 2-(6-Azulyl)ethyl Arenesulfonates and 3-(4-Azulyl)-l-propyl Nosylate. Examples of Ar3-5 and Ar3-6 Mechanisms. J. Org. Chem. 1973,38, 1106-1113.

14. Leino, T. O.; Baumann, M.; Yli-Kauhaluoma, J.; Baxendale, I. R.; Wallén, E. A. A., Synthesis of 1,3,6-Trisubstituted Azulenes. J. Org. Chem. 2015, 80, 11513-11520. 


\section{Chapter 3. 1-Substituted azulene protecting group candidates}

\subsection{Introduction}

Of the four protecting group candidates discussed in chapter 1, three of them (the azulenylmethylamine 80, the azulenesulfonamide 82 and the azulenecarboxylate 74, Figure 38) could be accessed from azulene itself, which at the beginning of the project was much more abundant and easily accessible than the 6-(2-hydroxyethyl)azulene starting material (discussed in chapter 4). As such, these protecting group candidates were deemed the easiest to test and represented less wasted effort if they failed, so they were pursued first despite being thought less likely to succeed. Two of these three protecting group candidates (80 and $\mathbf{8 2}$, discussed in sections 3.2 and 3.3, respectively) were ruled out early when they exhibited properties deleterious to their function as protecting groups. The third, the 1-azulenyl ester discussed in section 3.4 initially showed promise, but ultimately no sufficiently mild deprotection conditions could be found for it, eliminating it as an effective protecting group.
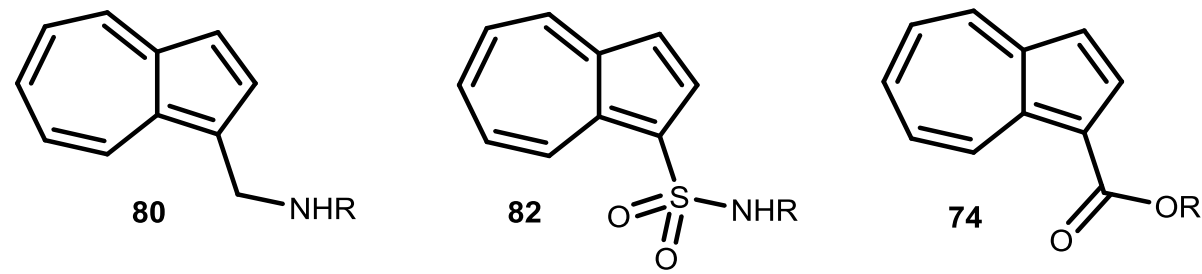

Figure 38. The three protecting group candidates incorporating a 1-substituted azulene moiety

\subsection{1-Azulenylmethyl protection of amines}

\subsubsection{Background}

One of the first protecting group candidates to be considered was the 1-azulenylmethyl group for protection of amines (Scheme 30). This candidate was intended to behave in a superficially similar way to the benzyl ether family of protecting groups, with the deprotection mechanism going through a positively-charged transition state that would be stabilised by interaction with the azulene ring. This was perceived to be a potentially distinguishing feature from the other azulenes-based protecting groups, as all the other protecting group candidates undergo deprotection through a mechanism that involves a negative charge in the transition state. 

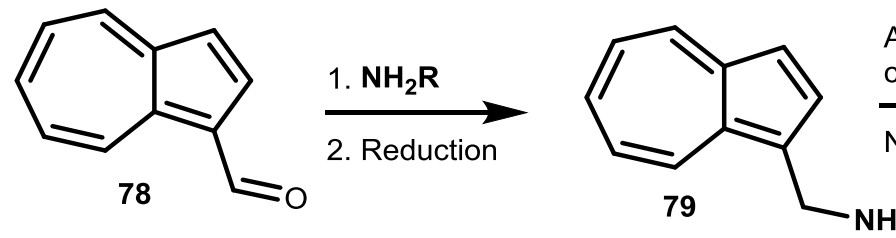

Acidic or oxidative conditions

Nucleophile

$\mathrm{NH}_{2} \mathrm{R}+$

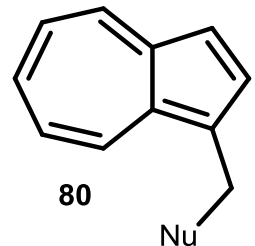

Scheme 30. Generalised strategy for the protection and deprotection of amines with $\mathbf{7 9}$

The combination of acidic/oxidative and nucleophilic deprotection conditions were inspired by an unexpected result during my Master's research (Scheme 31), where a similarly substituted guaiazulene 3-methylene cyclohexylamine 108 underwent substitution from a second equivalent when exposed to sources of $\mathrm{H}^{+}$such as silica gel, resulting in loss of the cyclohexylamine and generation of bis[guaiazulenyl]methylene 109.1 While this reactivity of the guaiazulenyl methylene 108 was considered to be a liability, it was thought that an azulene analogue, with fewer electron-donating alkyl groups, would be sufficiently inert to allow usage as a protecting group.

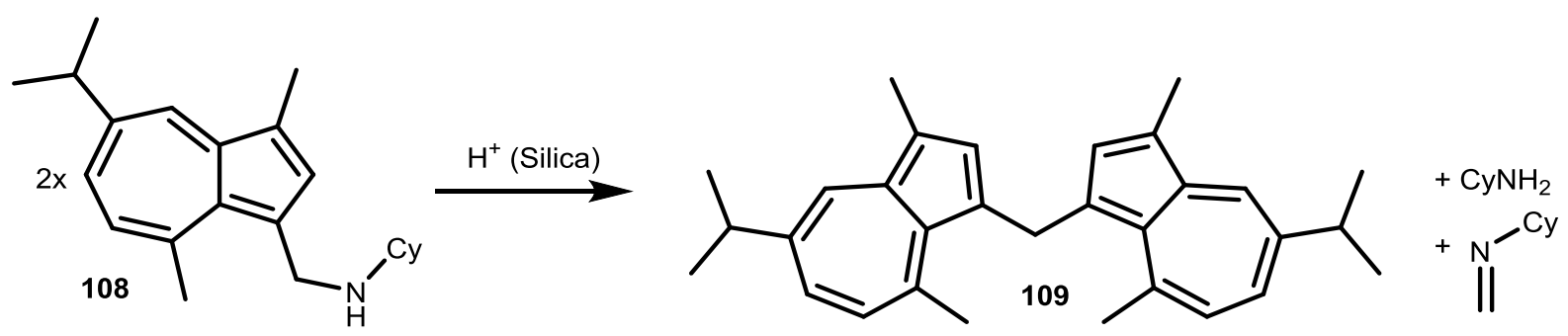

Scheme 31. One equivalent of 108 a tta cks a s econd equivalent, releasing cycl ohexylamine a nd me thylenecycl ohexyl imine

The intended protection of amines was to be carried out through reductive amination of 1-formylazulene 78, which was synthesised from azulene using Vilsmeier-Haack methodology.

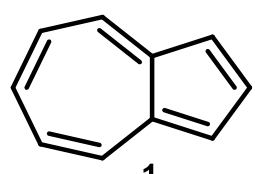

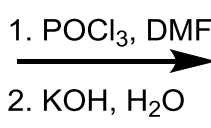

2. $\mathrm{KOH}, \mathrm{H}_{2} \mathrm{O}$
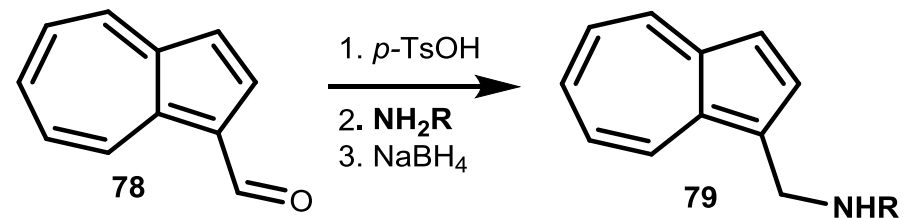

Scheme 32. A possible route to the protected substrate $\mathbf{7 9}$ through reductive amination of the amine with $\mathbf{7 8}$

\subsubsection{Initial investigation}

A reductive amination was carried out between 1-formylazulene and pyrrolidine. $p$-TsOH was added to protonate the formyl group on $\mathbf{7 8}$ followed by the addition of pyrrolidine to create the corresponding iminium. Addition of $\mathrm{NaBH}_{4}$ reduced the iminium salt to the amine $\mathbf{1 1 1}$ (Scheme 33). 

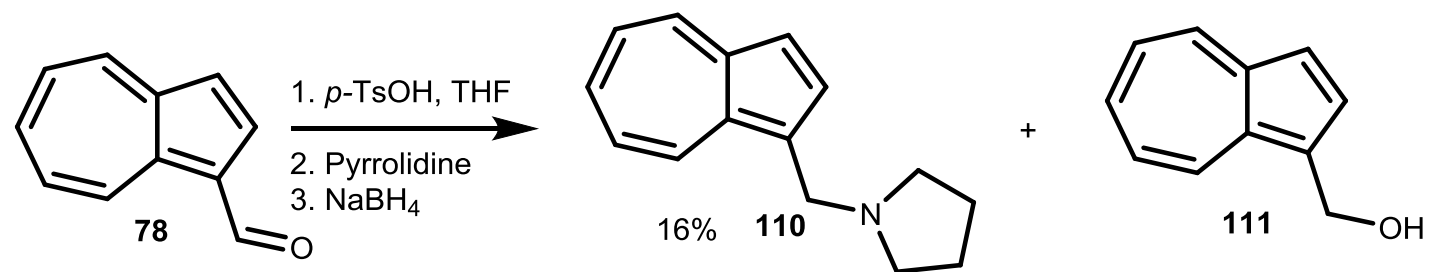

Scheme 33. Investigation of protection methodology on pyrrolidine

The yield of the desired product was 16\%, with numerous by-products also present, including what is believed to be 1-hydroxymethylazulene 111, and other compounds that were unable to be identified. This poor yield alone was not lethal to the success of the protecting group candidate, as these reactions can always be optimised further. What was lethal, however, was the discovery that these 1-substituted azulene species were highly susceptible to degradation, both in air and on silica gel, with the isolated compound degrading in a matter of days in air. It is for this reason that investigation of the 1-azulenylmethyl protecting group candidate $\mathbf{7 9}$ was not pursued further.

\subsection{1-Azulenesulfonamide protection of amines}

An azulene-based sulfonamide protecting group was intended to be introduced through addition of azulene 1-sulfonyl chloride $\mathbf{8 1}$ to the amine substrate (Scheme 34). The azulenesulfonyl chloride was to be obtained in two steps from azulene, through sulfonation with $\mathrm{SO}_{3}$.pyridine followed by treatment of the sulfonate $\mathbf{1 1 2}$ with thionyl chloride to generate the sulfonyl chloride 81 (Scheme 35).

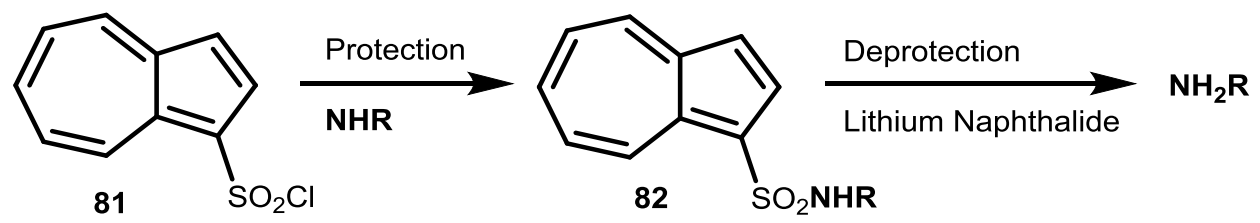

Scheme 34. Generalised protection and deprotection strategy of amines with $\mathbf{8 2}$

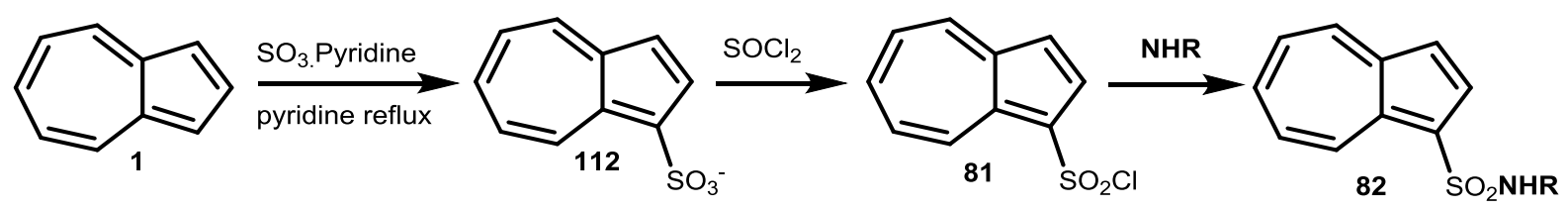

Scheme 35. Proposed synthetic path to $\mathbf{8 2}$ from azulene

Initial progress towards the sulfonamide stalled at the sulfonate stage, owing to the difficulty of isolating the highly polar 1-azulenesulfonate $\mathbf{1 1 2}$ from the aqueous phase, which contained other ionic components of the sulfonation reaction as well as what was believed to be 1,3disulfonylazulene. These difficulties in purification resulted in the investigation of this candidate being put on hold while the more successful 1-azulenyl ester was being investigated. 
Subsequently, when the difficulty of deprotecting the 1-azulenyl ester $\mathbf{7 4}$ was later discovered (as discussed in section 3.4.3), it was realised that the natural stubbornness of sulfonamides towards cleavage combined with the electron-donating properties of azulene would result in a protecting group that would be incredibly difficult to remove. As ease of deprotection was one of the requirements of the azulene protecting group, as stipulated in Chapter 1, no further investigation of this protecting group candidate was carried out.

\subsection{Protection of alcohols as 1-substituted azulene esters}

\subsubsection{Overview}

The 1-azulenyl ester (Scheme 36) was the first protecting group candidate to be seriously investigated. Protection would involve preparation of the 1-azulene acid chloride through addition of oxalyl chloride to azulene followed by loss of CO from the resultant keto-acid chloride. The alcohol-containing substrate would then be added to form an azulene-1-carboxylate ester. Deprotection would involve standard ester hydrolysis or saponification techniques.
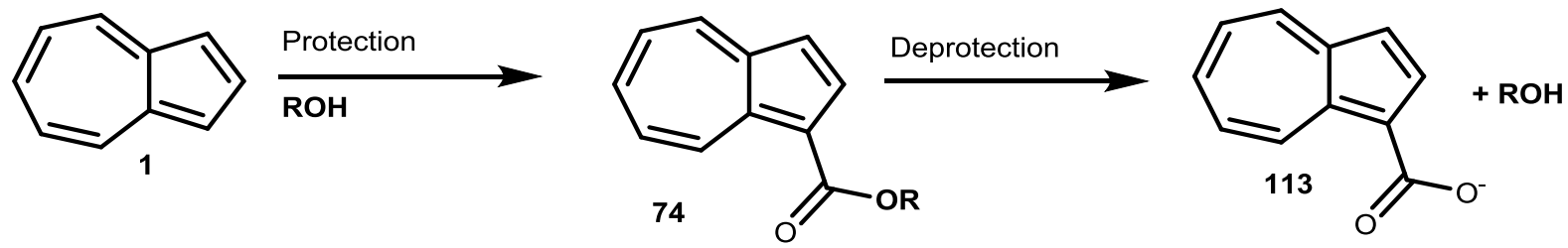

Scheme 36. Generalised protection and deprotection strategy for alcohols as the ester $\mathbf{7 4}$

Initial investigations on the 1-azulenyl ester as a protecting group showed it had a great deal of promise, with yields of the protection reaction reaching up to $90 \%$, as described in section 3.4.2. However, the conditions required for complete cleavage turned out to be much harsher than expected, as discussed in sections 3.4.3 and 3.4.4. Conditions such as refluxing $\mathrm{KOH} / \mathrm{EtOH}$ overnight were necessary for complete deprotection; many of the standard saponification or transesterification procedures did not work. This is a major drawback of the protecting group candidate, because (as stipulated in chapter 1) the deprotection conditions must be sufficiently mild to not affect the rest of the substrate, and the conditions required for deprotection would easily cleave most other esters present in the reaction mixture. In fact, the severity of these conditions exceed any described in Greene \& Wuts for the deprotection of other notoriously difficult-to-remove ester protecting groups.

In an attempt to alleviate the problems with this lack of lability, two-step deprotection procedures were attempted. Nitration of azulene and derivatives was successfully performed - albeit with unsatisfactory yields - and the increase in reactivity this offered was roughly 100 -fold, insufficient 
to make this technique synthetically useful. Complexation of azulene to an iron-carbonyl complex was also investigated, but the complex itself was more labile to base than the azulenecarboxylate ester. This work is described in sections 3.4.5 and 3.4.6.

An alternate 'tethered' 2-step deprotection was investigated (section 3.4.7) for guaiazulene and 4,6,8-trimethylazulene-based ester protecting groups. This strategy involved deprotonation of the 4-methyl position by a strong base such as KHMDS followed by addition of paraformaldehyde, with the intention of the resulting nucleophilic tethered alkoxide attacking the azulene-1carboxylate to form a 7-membered lactone and releasing the deprotected substrate. It was shown that this was indeed possible to do, but only under forcing conditions with very low yields, making the procedure unsuitable for reliable deprotection.

\subsubsection{Protection}

The protection of alcohols as azulene keto-esters by using oxalyl chloride has been demonstrated previously by Timmer, Burkett et al. ${ }^{2}$ During my Masters research, ${ }^{1}$ similar chemistry was explored with guaiazulene (Scheme 37). After addition of oxalyl chloride to guaiazulene, a species thought to be the ketoacid chloride $\mathbf{1 1 4}$ would be produced. This could be immediately reacted with an alcohol to produce the ketoester 116, or alternatively allowed to sit for 40 minutes, over which time an equivalent of carbon monoxide would be lost to produce the intermediate thought to be the acid chloride 115. Subsequent addition of an alcohol after this period would give the ester 117 in high ( $\sim 90 \%)$ yields.

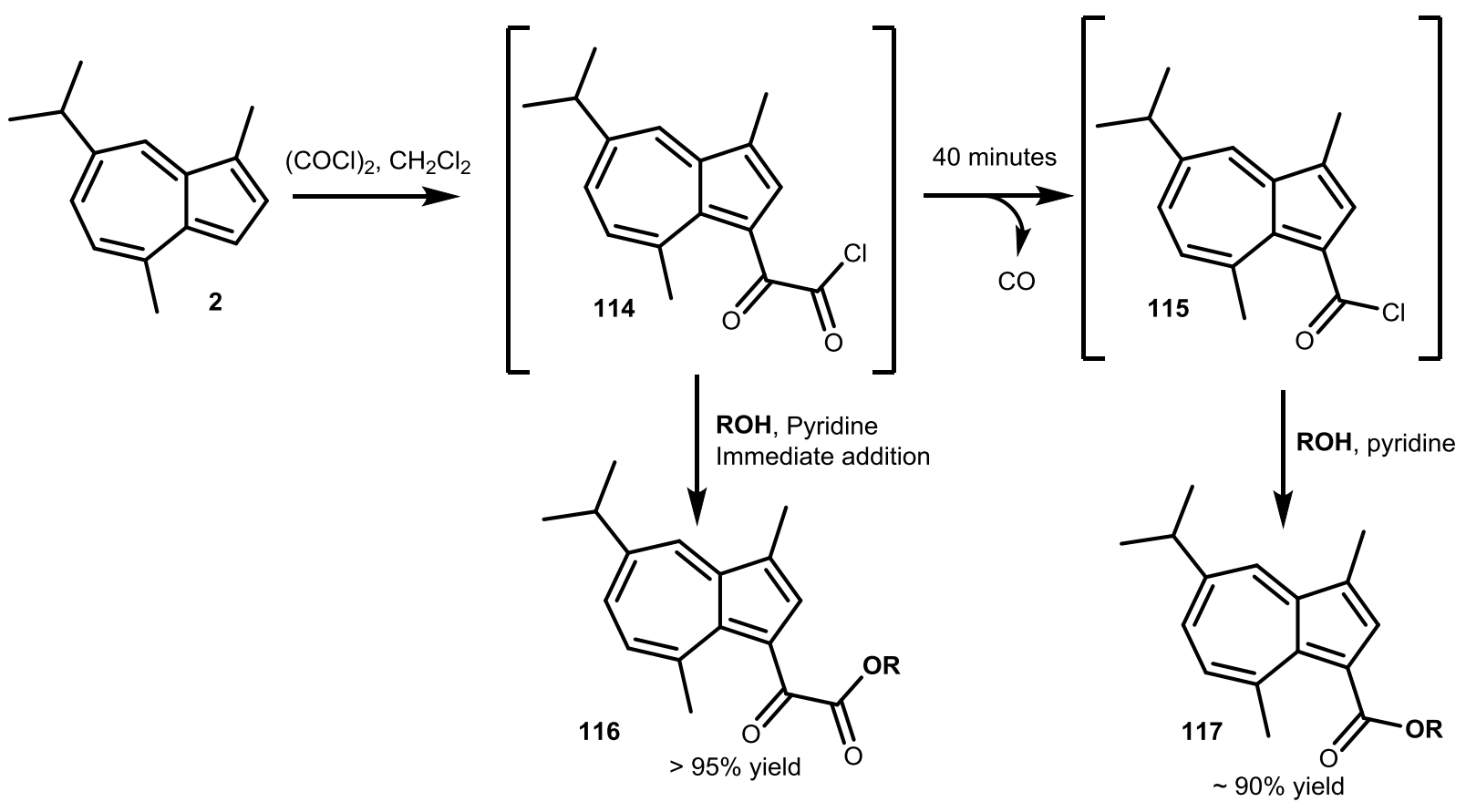

Scheme 37. Synthetic pathway to guaiazulene esters and ketoesters 
At the onset of this project, generation of the azulene acid chloride was expected to occur through a similar procedure. When azulene was treated with oxalyl chloride and left for 80 minutes before being treated with methanol, however, very little of the desired ester 8 was produced (Table 3). It was initially speculated that the presence of the electron-donating methyl on the 1-position of guaiazulene was primarily responsible for its higher reactivity. However, when subjecting 1methylazulene to the same reaction conditions, very little difference between it and azulene were observed. Much greater, though still incomplete, conversion was observed during the reaction of 4,6,8-trimethylazulene, which suggests that the conversion from ketoacid chloride to acid chloride is primarily promoted by the presence of a methyl group at the 4-position, while also being promoted slightly through the presence of electron donating groups elsewhere on the azulene ring.

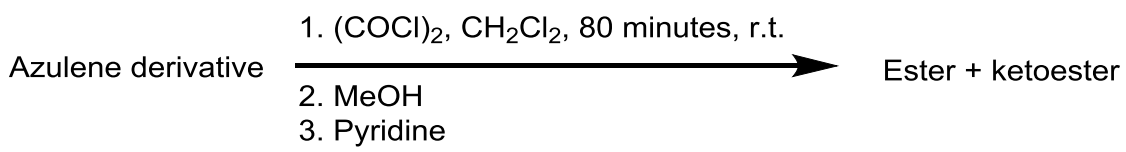

Table 3. Yields of ketoesters and esters of 1,102 , and $\mathbf{9}$ when subjected to the reaction conditions above

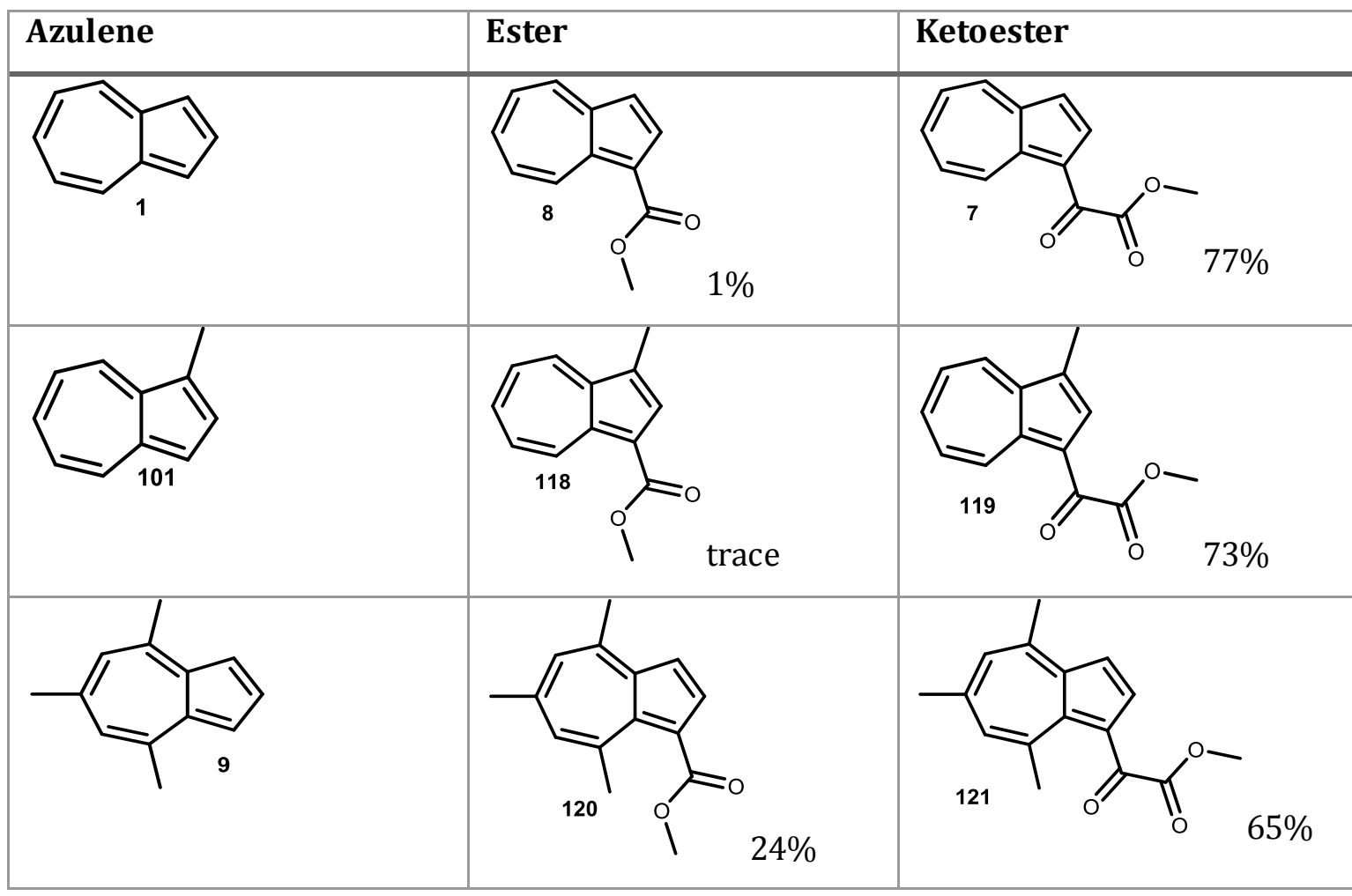

The esterification of azulene was then carried out using a variety of solvents and temperatures in order to study the conversion of ketoacid chloride to acid chloride (Table 4). For room temperature reactions, solvent choice had very little effect. Extended reaction times (overnight) gave slightly higher quantities of the ester at the cost of significant amounts of degradation. Performing the reactions at elevated temperatures gave higher quantities of $\mathbf{8}$, with yields increasing with the boiling point of the solvent $\left(\mathrm{CH}_{2} \mathrm{Cl}_{2}<\mathrm{THF}<\right.$ toluene) to $32 \%$. Finally, 
acetonitrile at reflux resulted in almost complete conversion of the ketoacid chloride to the acid chloride, and gave a pleasing $97 \%$ yield of the ester $\mathbf{8}$. This result suggests a combination of heat and stabilisation of charge by a solvent with a high dielectric constant is necessary to give complete conversion of the ketoacid chloride to the acid chloride.
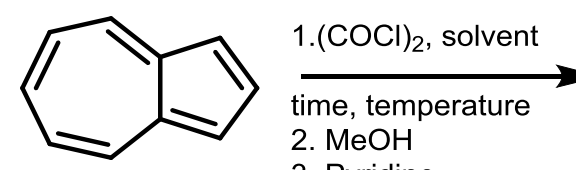

2. $\mathrm{MeOH}$

3. Pyridine

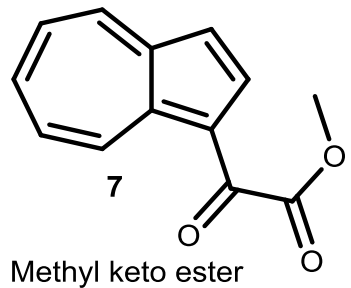

Methyl keto ester

Table 4. Optimisation of the conditions for the formation of 1-azulenyl methyl ester

\begin{tabular}{|c|c|c|c|c|c|}
\hline Entry & Solvent & Temperature & Time/minutes & $\begin{array}{l}\text { Isolated yield of } \\
\text { ester }\end{array}$ & $\begin{array}{l}\text { Isolated yield of } \\
\text { ketoester }\end{array}$ \\
\hline 1 & $\mathrm{CH}_{2} \mathrm{Cl}_{2}$ & r.t. & 80 & $1 \%$ & $77 \%$ \\
\hline 2 & $\mathrm{CH}_{2} \mathrm{Cl}_{2}$ & r.t. & 2400 (overnight) & $2.5 \%$ & $27 \%$ \\
\hline 3 & $\mathrm{CH}_{2} \mathrm{Cl}_{2}$ & reflux & 80 & $6 \%$ & $75 \%$ \\
\hline 4 & Toluene & r.t. & 80 & $0 \%$ & $73 \%$ \\
\hline 5 & Toluene & reflux & 80 & $32 \%$ & $37 \%$ \\
\hline 6 & THF & r.t. & 80 & $0 \%$ & $90 \%$ \\
\hline 7 & THF & reflux & 80 & $3 \%$ & $72 \%$ \\
\hline 8 & $\mathrm{MeCN}$ & r.t. & 80 & $3 \%$ & $92 \%$ \\
\hline 9 & $\mathrm{MeCN}$ & reflux & 80 & $97 \%$ & $2 \%$ \\
\hline
\end{tabular}

The method described in entry 9 was next tried using an appropriate alcohol as a model for the substrate to be protected. (-)-Menthol was chosen because it is a readily available model compound that is easily detectable by TLC and ${ }^{1} \mathrm{H}$ NMR spectroscopy, is solid, and is also highly non-polar for an alcohol and immiscible with water. The reactions were performed with menthol as the limiting reagent to mimic real protection protocols, employing roughly 1.1 equivalents of oxalyl chloride and 1.2 equivalents azulene. These quantities were chosen to ensure that no free oxalyl chloride was present after the addition to azulene, as unreacted oxalyl chloride would react with menthol preferentially over the 1-azulenyl acid chloride. 


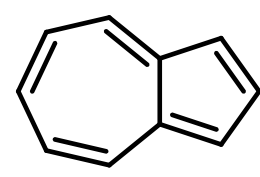

1. $(\mathrm{COCl})_{2}, \mathrm{MeCN}, 30$ min reflux

2. (-)-menthol

3. pyridine

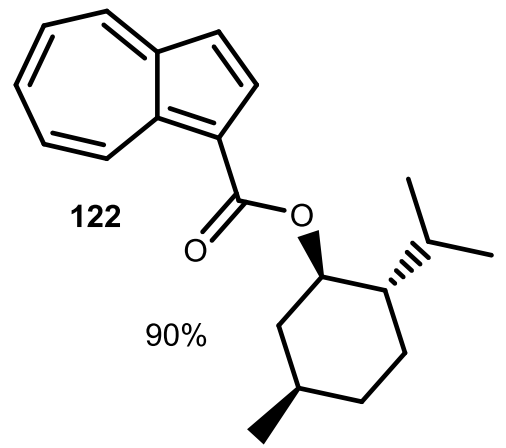

Scheme 38. Optimised protocol for the protection of menthol as a 1-azulenyl menthyl ester $\mathbf{1 2 2}$

An initial reaction using the procedure in entry 9 of Table 4 gave a yield of $80 \%$ of the desired ester 122. After some exploration of the reaction conditions, it was found that the conversion from ketoacid chloride to acid chloride required only 30 minutes to go to completion, which was indicated by a colour change from orange-red to crimson. Yields of up to $90 \%$ of $\mathbf{1 2 2}$ were obtained this way (Scheme 38). This reaction appears to be quite sensitive to conditions - several attempts have resulted in substantially lower yields, despite the amount of excess oxalyl chloride and azulene being the only variables altered. It is thought this variability stems from a high sensitivity to both moisture and to the quality of the oxalyl chloride used. Best results were typically achieved in the few days after oxalyl chloride was freshly distilled.

As the azulene was used in excess, numerous azulene by-products were usually obtained (Figure 39), including recovered azulene, azulene 1-carboxylic acid and occasionally small quantities of a stable crimson compound that was identified by HRMS to be azulene-1-carboxylic anhydride 123.

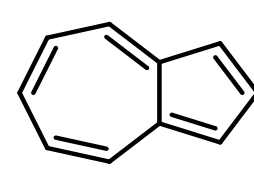

1
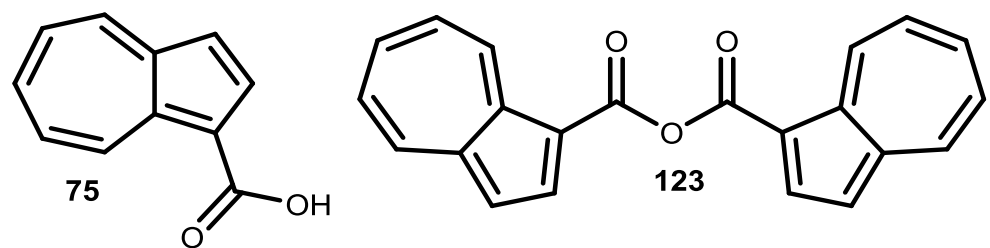

Figure 39. Azulene (1), azulene-based by-products $\mathbf{7 5}$ and $\mathbf{1 2 3}$ isolated from protection reactions

The reaction conditions for the formation of the ester $\mathbf{1 2 2}$ were deemed to be quite harsh - the reaction generates anhydrous hydrochloric acid, the presence of which at el evated temperatures could potentially lead to unwanted reactivity on a complex substrate. Typically, pyridine was added about a minute after addition of the alcohol to neutralise the reaction, although this short time period may not suffice to shield more sensitive parts of the substrate from the harsh acidic conditions. When base was omitted from the final step, significant amounts of degradation of the azulene moiety occurred, and the yield of $\mathbf{1 2 2}$ was reduced to $50 \%$.

In an attempt to mitigate the potential problem of acid sensitivity, the addition of amine bases before addition of the alcohol was trialled. DMAP was added before menthol, but only $13 \%$ of the desired product was obtained. Menthol was recovered in $62 \%$ yield, and an orange water-soluble 
by-product was observed. This is thought to be the acyl DMAP adduct 124, whose positive charge would be stabilised by both the electron-donation of the azulene moiety and resonance of the DMAP moiety (Figure 40). However, due to the difficulty of isolating this polar compound, no spectroscopic evidence for this was obtained.

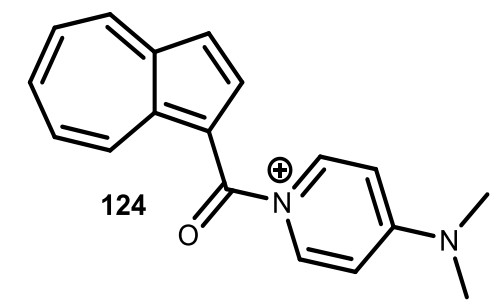

Figure 40. Proposed structure of the water-soluble orange compound

Addition of pyridine before menthol produced slightly better results. The desired ester was obtained in 34\% yield, and there was no stable water-soluble orange by-product. Due to these poor yields for both the DMAP and pyridine cases, addition of base before the alcohol was deemed to be ineffective as a way of combatting the harshly acidic conditions in the reaction.

The use of $\mathrm{AgBF}_{4}$ was briefly investigated as a means to hasten the dissociation of the azulene keto acid chloride through sequestration of the chloride ion by $\mathrm{Ag}^{+}$. A reaction was performed in acetonitrile at room temperature and $\mathrm{AgBF}_{4}$ was added immediately after oxalyl chloride and 80 minutes before the addition of menthol. A complex mixture of products was obtained from this reaction, and azulenecarboxylic acid $\mathbf{7 5}$ was obtained in $48 \%$ yield as the only identifiable product. The desired ester 122 was not present in the solution. This approach was not investigated further.

Two other esterifications were investigated as alternatives to the use of oxalyl chloride. Carbonyldiimidazole (CDI) was explored as a mild phosgene equivalent, however no observable reaction of azulene with CDI occurred, despite forcing conditions such as heating at reflux in acetonitrile for two days. A Yamaguchi esterification between the azulenecarboxylic acid $\mathbf{7 5}$ and menthol was attempted (Scheme 39), with the modification of pyridine being used instead of DMAP in the second step to avoid possible formation of $\mathbf{1 2 4}$. However, this reaction gave only $3 \%$ yield of the desired ester, with 1-azulenecarboxylic anhydride 123 being isolated in 61\% yield. Formation of symmetrical anhydride by-products is commonly associated with aliphatic carboxylic acids in Yamaguchi esterifications, ${ }^{3}$ due to aliphatic carboxylic acids being sufficiently nucleophilic to displace 2,4,6-trichlorobenzoate from the mixed anhydride intermediate. As the azulene is highly electron rich in comparison to other aromatic rings, formation of the symmetrical anhydride through the same mechanism is likely. Owing to the degree to which azulene stabilises carbonyls at the 1-position, the symmetrical anhydride $\mathbf{1 2 3}$ was rendered relatively inert, and did not participate further in the reaction. 

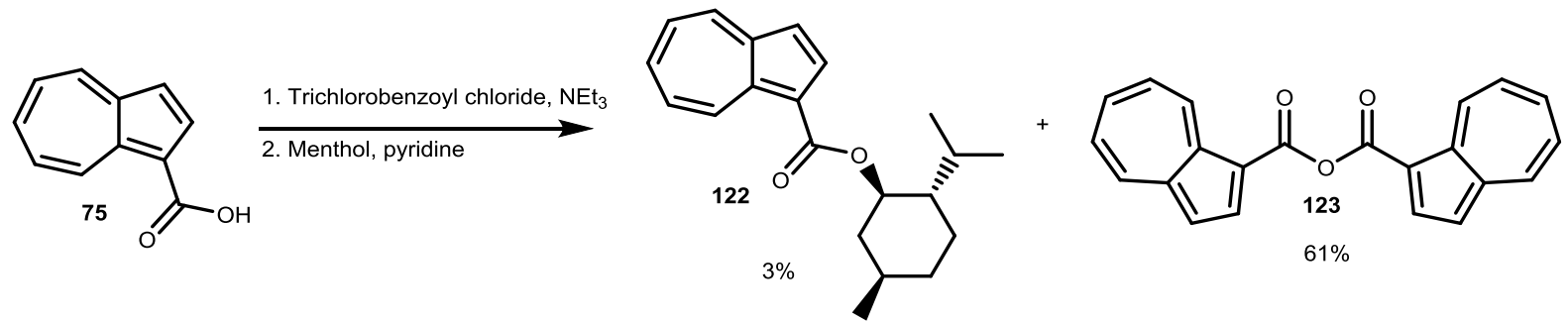

Scheme 39. Attempted Yamaguchi esterification produced large quantities of the anhydride $\mathbf{1 2 3}$

At this point, with a high-yielding protection reaction and a sufficiently large supply of menthyl ester 122 in hand, the deprotection studies commenced.

\subsubsection{One-step deprotection}

Basic saponification was the primary route investigated for one-step deprotection of these 1-azulene esters (Table 5). In order to expedite this investigation, yields were calculated through ${ }^{1} \mathrm{H}$ NMR spectroscopy by finding the total mass of the crude sample and calculating the mass fraction of free menthol by its relative integration to other identifiable components in the crude mixture. This would produce an inflated yield estimate, because it assumes all species in the crude mixture are identifiable in the NMR spectrum. Nitromethane internal standards were not used in this instance (See chapter 5.1). Isolated yields of menthol were only obtained for the two successful KOH-based reactions.

\begin{tabular}{|l|l|l|l|l|l|}
\hline Base & Solvent & Temperature & Time & $\begin{array}{l}\text { NMR } \\
\text { yield }\end{array}$ & Isolated yield \\
\hline $\mathrm{LiOH} \mathrm{0.1} \mathrm{M}$ & $1: 1 \mathrm{THF} / \mathrm{H}_{2} \mathrm{O}$ & reflux & 2 days & - & - \\
\hline $\mathrm{KOH}$ & $1: 1 \mathrm{THF} / \mathrm{H}_{2} \mathrm{O}$ & reflux & overnight & - & - \\
\hline $\mathrm{NaOMe}$ & $\mathrm{MeOH}$ & reflux & overnight & Trace & - \\
\hline $\mathrm{KOH} \mathrm{0.7} \mathrm{M}$ & EtOH & r.t. & overnight & $3 \%$ & - \\
\hline $\mathrm{LiOH} \mathrm{0.8} \mathrm{M}$ & EtOH & reflux & overnight & $20 \%$ & - \\
\hline $\mathrm{KOH} \mathrm{0.6} \mathrm{M}$ & EtOH & reflux & overnight & $94 \%$ & $42 \%$ \\
\hline $\mathrm{KOH} \mathrm{0.6} \mathrm{M}$ & $4: 1 \mathrm{EtOH} / \mathrm{PrOH}$ & reflux & overnight & $82 \%$ & $52 \%$ \\
\hline
\end{tabular}

Table 5. Optimisation of the deprotection conditions of $\mathbf{1 2 2}$

Use of $\mathrm{KOH} / \mathrm{EtOH}$ with heating at reflux overnight appeared to be the only method to produce complete deprotection. $\mathrm{LiOH} / \mathrm{EtOH}$ only gave partial deprotection, while all other reaction conditions gave either trace or no deprotection. Such strongly basic conditions are undesirable as a deprotection protocol. It is thought the disparity between the NMR and isolated yield measurements is due to menthol being slightly volatile (b.p. $212{ }^{\circ} \mathrm{C}$ ), in addition to the aforementioned calculated yield inflation. An attractive feature of this chemistry is that 
deprotection induces a colour change. The azulene 1-carboxylate salt $\mathbf{1 1 3}$ appears a bluer shade of violet in comparison to the ester starting material, and so the extent of deprotection can be judged qualitatively by its colour.

An assortment of other deprotection conditions were tried. The 1-azulenyl ester is susceptible to acidic deprotection, but only under harsh conditions. Heating the protected ester at reflux overnight in a 2:1 mixture of $\mathrm{MeOH}$ and a $10 \%$ aqueous solution of $\mathrm{HCl}$ gave only $8 \%$ deprotection and traces of azulene (1). Reduction of the ester with $\mathrm{LiAlH}_{4}$ produced a complex mixture in which only traces of menthol could be observed by TLC or NMR.

\section{Regeneration of azulene}

One approach to alleviating the concern of low atom economy that inevitably comes with protecting group usage is to develop ways of recycling the protecting group body after deprotection. ${ }^{4}$

The azulene by-product of deprotection, either azulenecarboxylic acid $\mathbf{7 5}$ or the carboxylate salt 113, is easily isolable from the deprotection reaction through an aqueous wash, and can be decarboxylated to regenerate azulene by heating in acidic media. ${ }^{5}$ To exploit this feature, a procedure was developed whereby a solution of 75 in 2:1 $\mathrm{MeOH} / 10 \% \mathrm{HCl}$ (aq.) was heated in a distillation setup (Scheme 40). Due to the volatility of azulene, it co-distilled with methanol and was collected in the receiving flask in $79 \%$ yield.

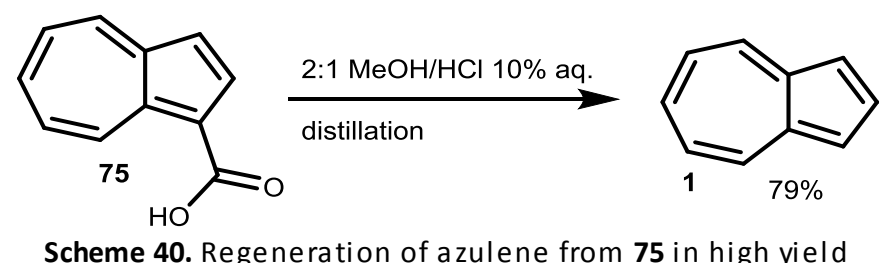

\subsubsection{Activation methods for two-step deprotection}

Due to the difficulty in finding mild deprotection conditions for the azulenyl ester protecting group, two-step activation-deprotection protocols were explored. The mechanisms of saponification and transesterification go through a negatively-charged intermediate and two negatively-charged transition states (Figure 41). Decreasing the electron-rich environment of the azulene ring through introduction of electron withdrawing groups or $\pi$-complexation will allow for greater stabilisation of this negatively charged reaction pathway, facilitating the deprotection reaction. 


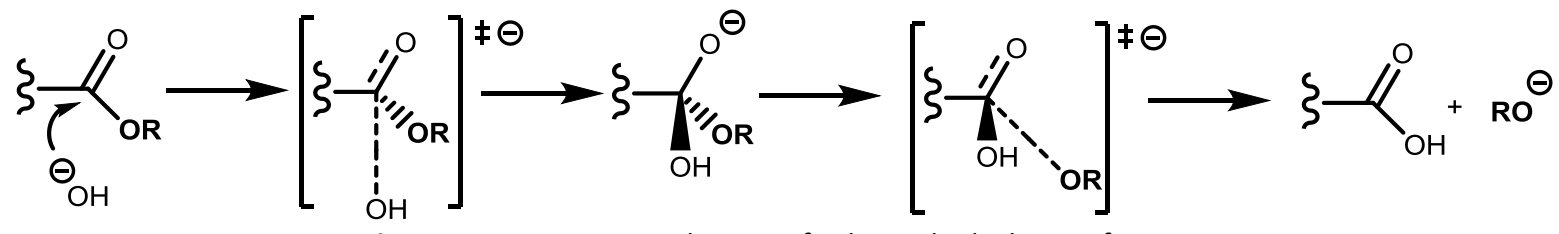

Figure 41. Reaction mechanism for basic hydrolysis of esters

Initial computational work was done to ascertain the effects of various electron withdrawing groups and $\pi$-complexing agents on the $\mathrm{C}=0$ stretching frequency of azulene-1-methyl ester. The stretching frequency of a carbonyl correlates to the reaction rate of the carbonyl towards nucleophilic attack, with higher wavenumbers typically indicating a higher reactivity. ${ }^{6}$ As such, calculations of the carbonyl stretching frequency of various azulene derivatives serves as a convenient estimation of their relative reactivity, and are computationally much cheaper than attempting to calculate the energies of both the ground and transition states.

The carbonyl stretching frequency of azulene-1-methyl ester 8 was calculated to be $1781 \mathrm{~cm}^{-1}$, and this was used as the baseline to which the stretching frequencies of the other azulene compounds in Table 6 were compared.

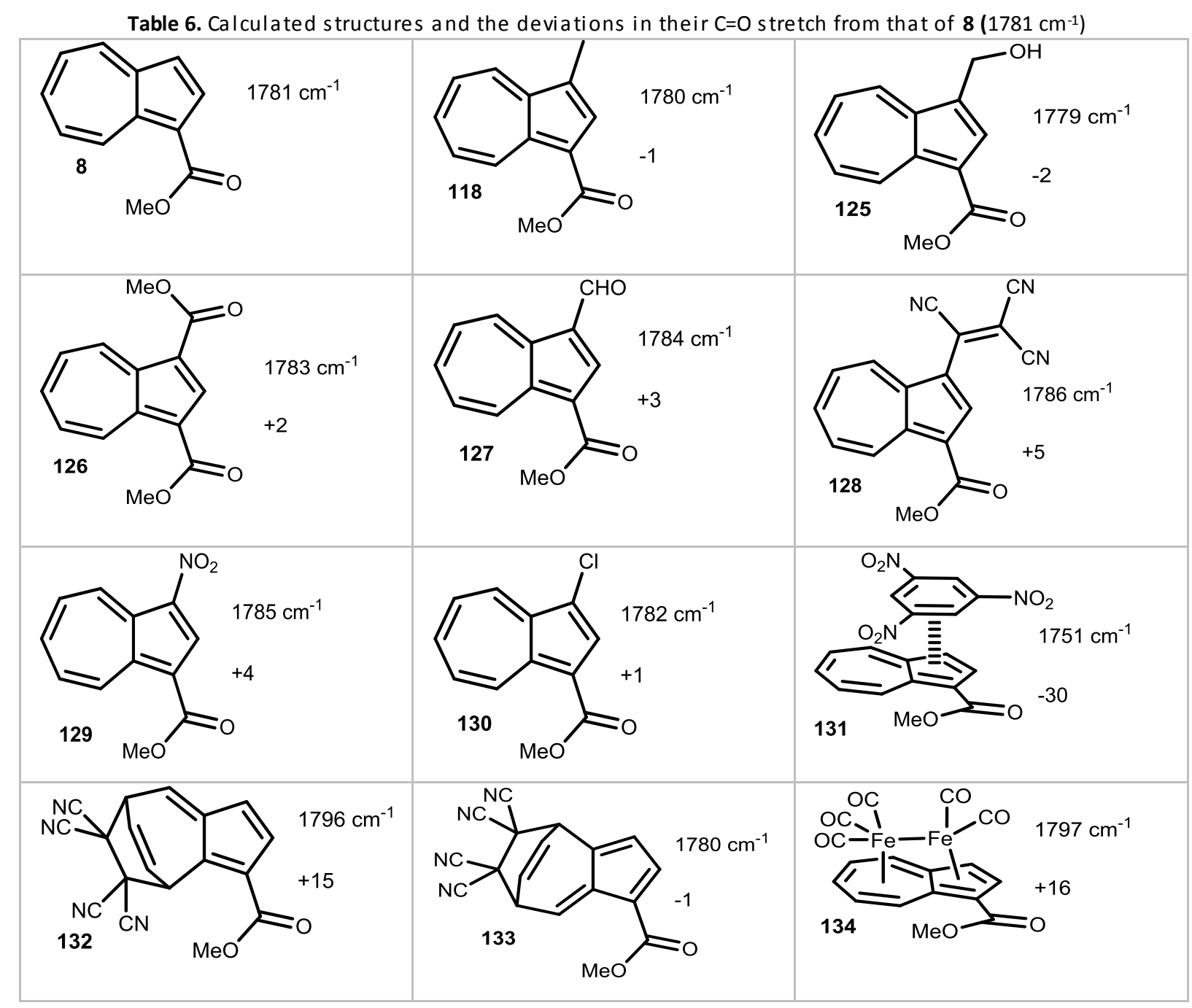


From Table 6, the three most promising potential methods of activation are nitration, TCNEbased activation and complexation with $\mathrm{Fe}_{2} \mathrm{CO}_{5}$, as they have the highest $\mathrm{C}=0$ stretches. Other substituents on the 3-position either give lower amounts of activation of the $\mathrm{C}=\mathrm{O}$ bond, or in some cases deactivate it. The result from the azulene-trinitrobenzene complex $\mathbf{1 3 1}$ appears to be anomalously low, but this may be a limitation of the level of theory employed (B3LYP/6-31G**) being unsuited for long-range interactions such as Van der Waals forces. ${ }^{7}$

A precise comparison of the validity of the measurements was not possible, as very few of the compounds shown in Table 6 were synthesised. From the related menthyl esters of compounds 8 and 129 (the compounds 122 and 136, discussed in section 3.4.5), a rough comparison can be made. The experimental value of the $\mathrm{C}=0$ stretch of 122 is $1681 \mathrm{~cm}^{-1}$, while the calculated value of the $\mathrm{C}=0$ stretch of 8 is $1781 \mathrm{~cm}^{-1}$, a difference of $100 \mathrm{~cm}^{-1}$. The experimental value for the $\mathrm{C}=0$ stretch of 136 is $1688 \mathrm{~cm}^{-1}$, which is lower than the calculated value for 129 by $97 \mathrm{~cm}^{-1}$. DFT frequency calculations are notoriously prone to overestimating the frequency of certain stretches, ${ }^{8}$ however the relative differences between unsubstituted and 1-nitro substituted species appear to be more accurate, with the calculated difference being $4 \mathrm{~cm}^{-1}$, and the experimental difference being $7 \mathrm{~cm}^{-1}$.

\subsubsection{Activation by nitration}

Addition of the nitro group at the 3-position of azulene was investigated as a way of activating the azulene towards deprotection. The nitro group is one of the most electron-withdrawing groups that can easily be introduced. ${ }^{9}$ The traditional method of nitration - using a combination of nitric acid and sulfuric acid to generate the necessary $\mathrm{NO}_{2}+$ ion - was deemed too harsh owing to the breakdown of azulene in strongly acidic conditions. Menke nitration ${ }^{10}$ circumvents this concern by using copper nitrate trihydrate in the presence of acetic anhydride to generate the active acetyl nitrate species. ${ }^{11}$ Reactions typically occur over the course of half an hour at room temperature.

Attempts at nitrating azulene itself produced low yields. It was found that nitrations in which acetic anhydride was the solvent proceeded to 1,3-dinitroazulene 6, a vivid orange compound obtained in 27\% yield (Scheme 41).

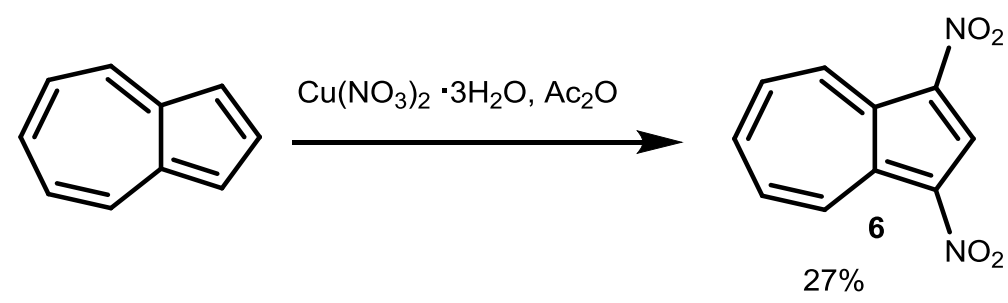

Scheme 41. Dinitration of azulene 
On the other hand, Menke nitrations in which dichloromethane was used as a co-solvent (typically 4:1 to 2:1 ratios of DCM to acetic anhydride) produced the mono-nitrated azulene product. In all cases, copper nitrate was used in 2-3 fold excess. The yields of these reactions were also poor, from $13 \%$ to $36 \%$ with large quantities of unidentifiable brown material also being produced. Addition of bases such as sodium acetate or triethylamine completely suppressed nitration (Table 7).

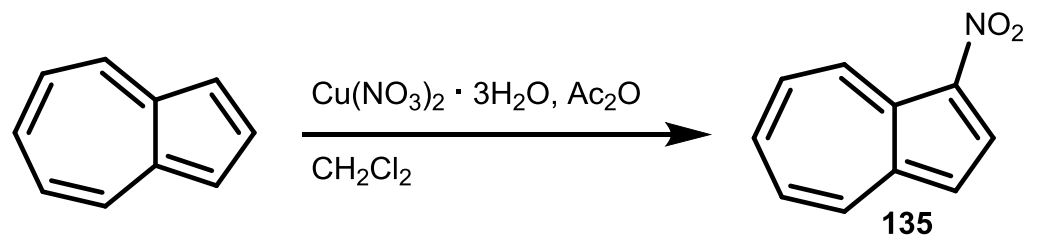

Table 7. Attempted optimisation of mono-nitration of azulene to form 1-nitroazulene (135)

\begin{tabular}{|l|l|l|}
\hline Entry & Reaction conditions & Yield $\%$ \\
\hline $\mathbf{1}$ & 1.2 eq. copper nitrate, r.t. & $13 \%$ \\
\hline $\mathbf{2}$ & 2.2 eq. copper nitrate, r.t. & $36.6 \%$ \\
\hline $\mathbf{3}$ & 1.2 eq. copper nitrate, $-\mathbf{7 8}^{\circ} \mathrm{C}$ to r.t. & $20 \%$ \\
\hline $\mathbf{4}$ & 2.5 eq. copper nitrate in $\mathrm{NEt}_{3}$ & No reaction \\
\hline $\mathbf{5}$ & 3.5 eq. copper nitrate, 2.1 eq. $\mathrm{NaOAc}$ & No reaction \\
\hline
\end{tabular}

Nitration was then attempted on the azulene menthyl ester 122 (Scheme 42). Surprisingly, this proved more amenable to nitration than azulene itself, where intuition would posit that electrondeficient azulene ester would react with relative sluggishness and result in an overall lower yield. Yields of between $30 \%$ and $52 \%$ of the product 136 were obtained. This reaction appeared to not proceed at all at $0{ }^{\circ} \mathrm{C}$ but significant degradation would occur at room temperature. Performing this reaction in a dioxane/dry ice bath $\left(10^{\circ} \mathrm{C}\right)$ gave the highest yields of $\mathbf{1 3 6}$.
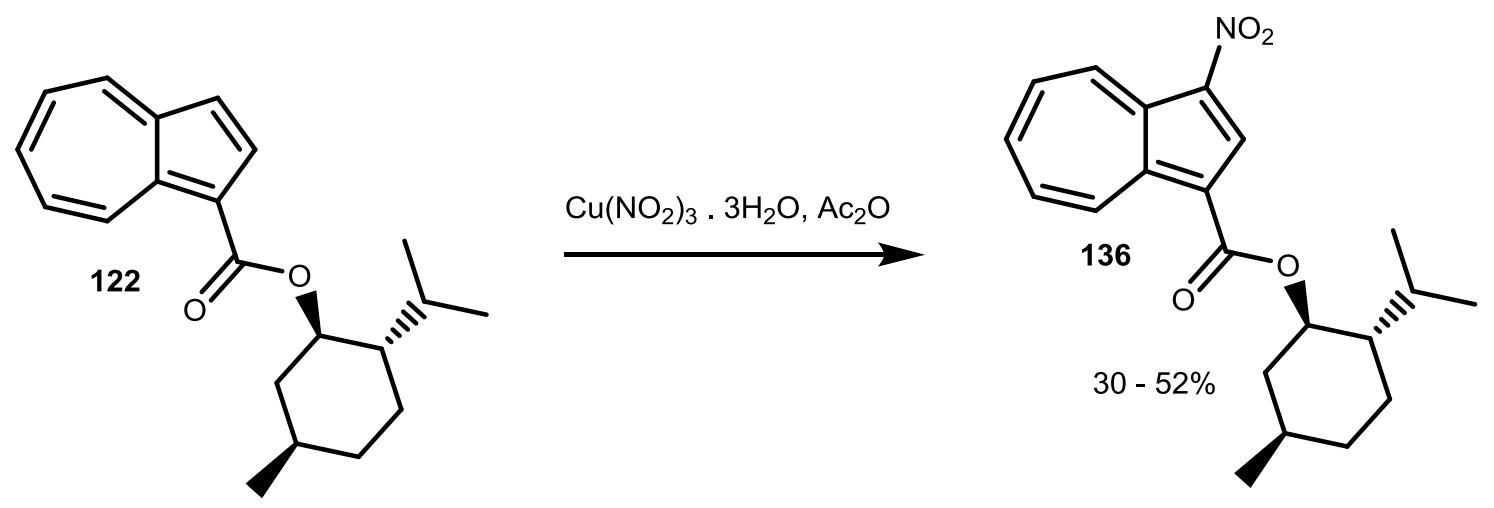

Scheme 42. Nitration of menthyl ester $\mathbf{1 2 2}$

There are literature reports that this class of acetic anhydride/ metal nitrate-based nitrations typically produce low yields. ${ }^{12}$ Nitration with tetranitromethane is reported to give higher yields, 
but tetranitromethane was deemed to be too much of a safety hazard owing to its toxic and explosive properties. This reaction was not optimised further, as sufficient nitroazulene ester 136 had been generated for further testing.

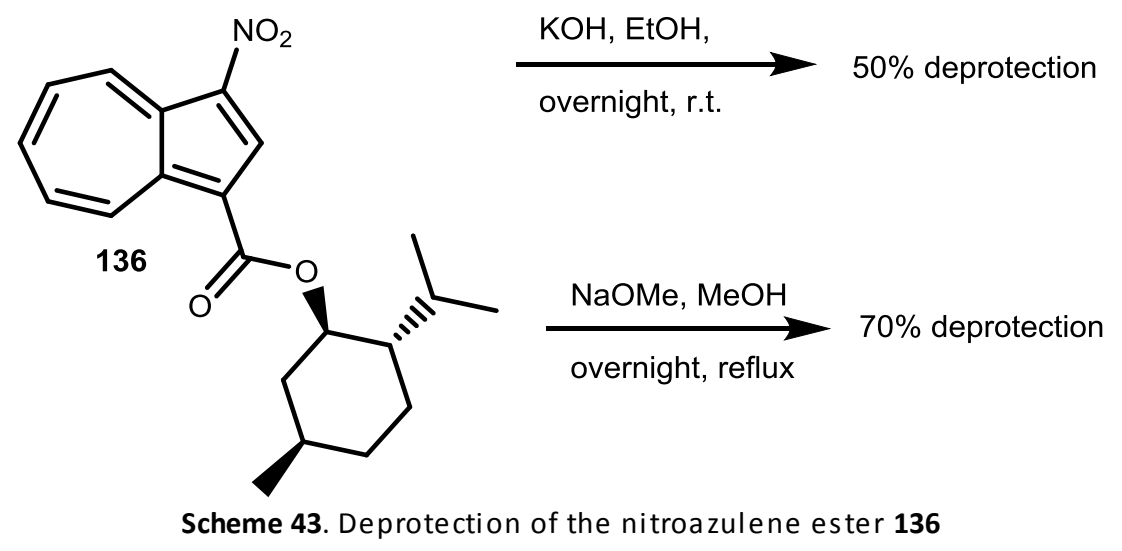

The nitroazulene ester was tested against some of the reaction conditions used to deprotect the azulene ester (Scheme 43). Again, the conversions were measured through ${ }^{1} \mathrm{H}$ NMR integration. A relatively large increase in reaction rate was observed: overnight reaction with $\mathrm{KOH} / \mathrm{EtOH}$ at room temperature led to $50 \%$ deprotection while the same conditions with the unsubstituted azulene ester gave $3 \%$ deprotection. Reaction of $\mathrm{NaOMe} / \mathrm{MeOH}$ overnight at reflux also gave $70 \%$ deprotection, whereas in the unsubstituted case these conditions gave only trace deprotection. From these results it was estimated that the nitroazulene was about one to two orders of magnitude more reactive than the unsubstituted azulene.

To obtain a more accurate picture of the relative rates of deprotection of 122 and 136, a NMR

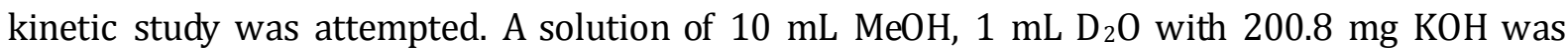
prepared, with a single drop of tetramethylsilane added as an internal standard. An aliquot of 0.5 $\mathrm{mL}$ of this solution was then added to a sample containing a 2.6:1 ratio of azulene ester $\mathbf{1 2 2}$ to nitroazulene ester 136 in $1 \mathrm{~mL}$ methanol, and this was subjected to ${ }^{1} \mathrm{H}$ NMR analysis. The total concentration of $\mathrm{KOH}$ in these samples was $0.1 \mathrm{molL}^{-1}$, with $\mathrm{KOH}$ in 14-fold excess relative to 122 . The large methyl peak belonging to methanol produced significant distortion in the NMR spectrum, but this could be corrected for with multipoint baseline correction to give accurate peak integration (Figure 42). 

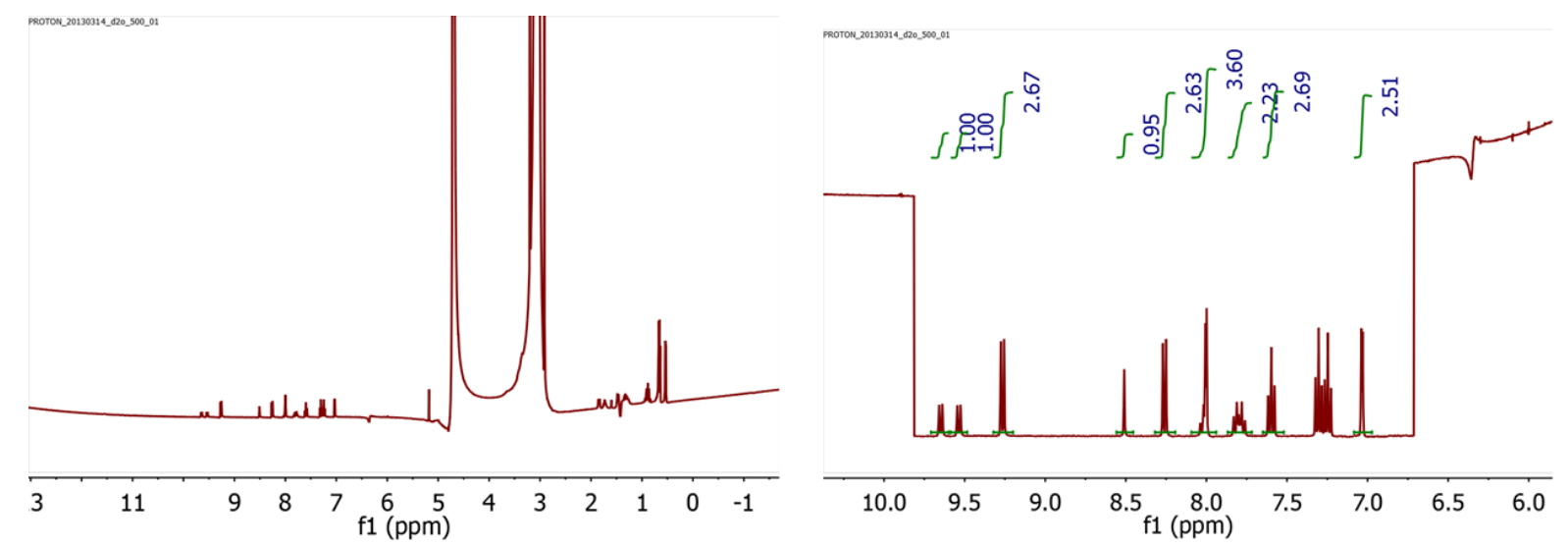

Figure 42. ${ }^{1} \mathrm{H}$ NMR spectrum of the reaction mixture of $\mathbf{1 2 2}+\mathbf{1 3 6}$ with $\mathrm{KOH}, \mathrm{MeOH}$ and $\mathrm{D}_{2} \mathrm{O}$ at $\mathrm{t}=0$. Full unaltered spectrum on left, and baseline-corrected spectrum of the relevant aromatic region on right.

Over the course of three weeks, this reaction was monitored by ${ }^{1} \mathrm{H}$ NMR spectroscopy, with at least one spectrum being obtained per day.

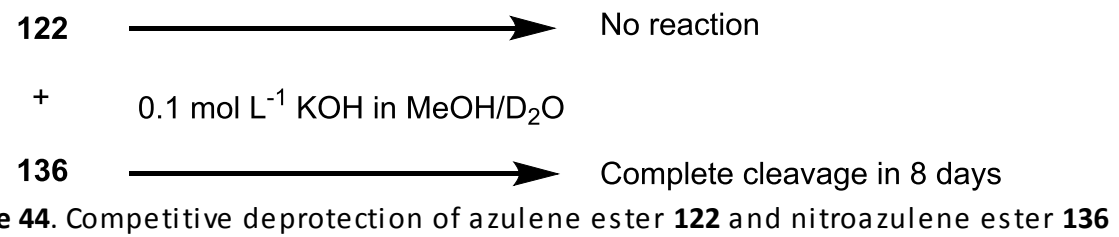

The 3-nitroazulene menthyl ester 136 underwent complete cleavage over 8 days, however no change in the concentration of the unactivated azulene ester 122 was observed over the course of the 3 week experiment (Scheme 44). This result differs somewhat from the observed 3\% deprotection of $\mathbf{1 2 2}$ at r.t. recorded in Table 5, and is possibly due to the NMR sample having a lower concentration of $\mathrm{KOH}$, or possibly because the base was slowly neutralised through reaction with the borosilicate glass surface of the NMR tube.

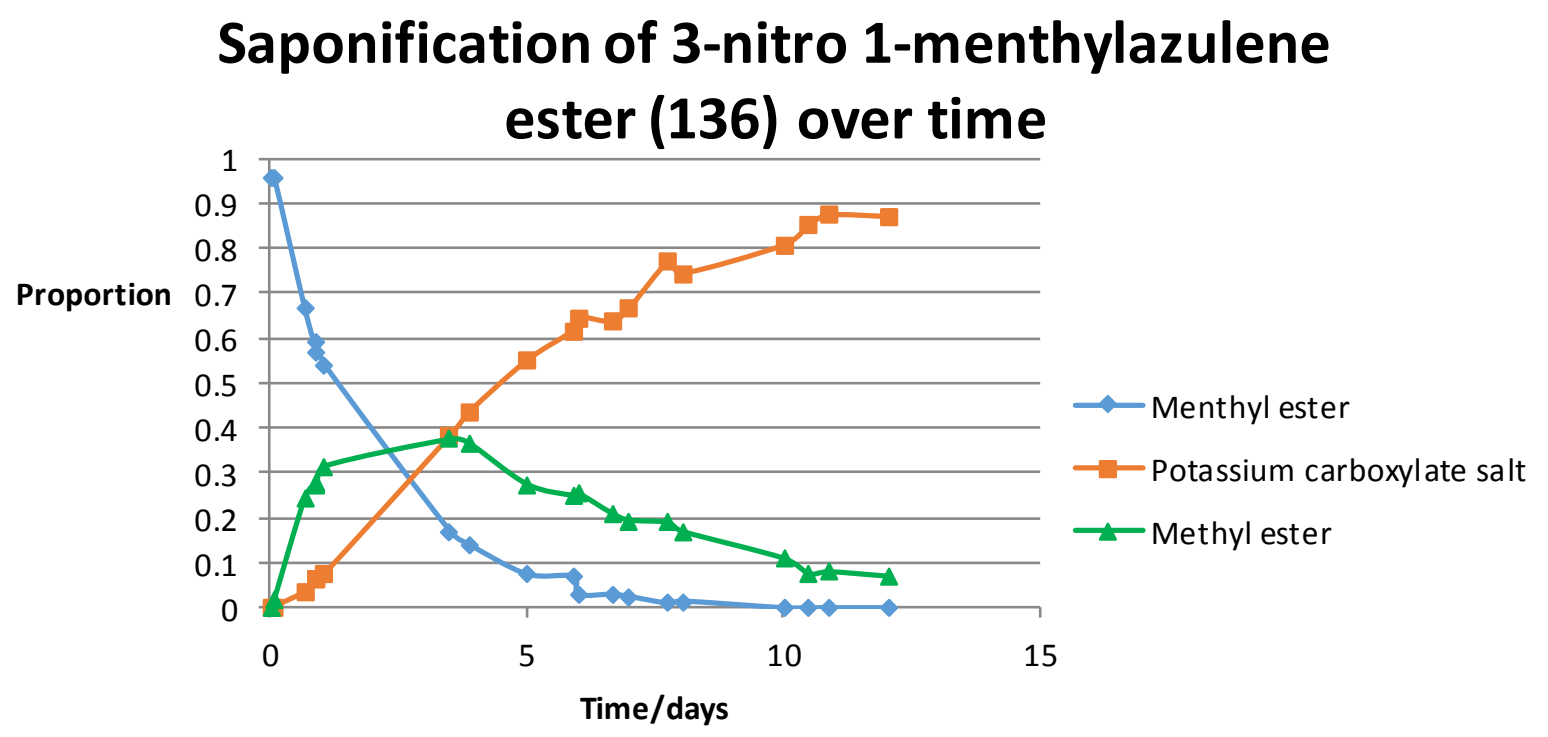

Figure 43. Graph of the saponification of the nitroazulene ester $\mathbf{1 3 6}$ over time 
Formation of an intermediate species was observed in the ${ }^{1} \mathrm{H}$ NMR spectra during the saponification of the nitroazulene menthyl ester (Figure 43). This was thought to be the methyl ester, an intermediate resulting from transesterification with methoxide that then proceeds to be saponified in the basic conditions. The rate of deprotection is consistent with a pseudo-first order reaction rate, with the log of the proportion of menthyl ester taking the form of a straight line, with a $\mathrm{R}^{2}$ value of 0.9835 (Figure 44). This gives a half-life of the nitroazulene menthyl ester as 1730 minutes, or 29 hours under these reaction conditions.

\section{Natural log of 3-nitroazulene menthyl ester (136) proportion over time Time/days}

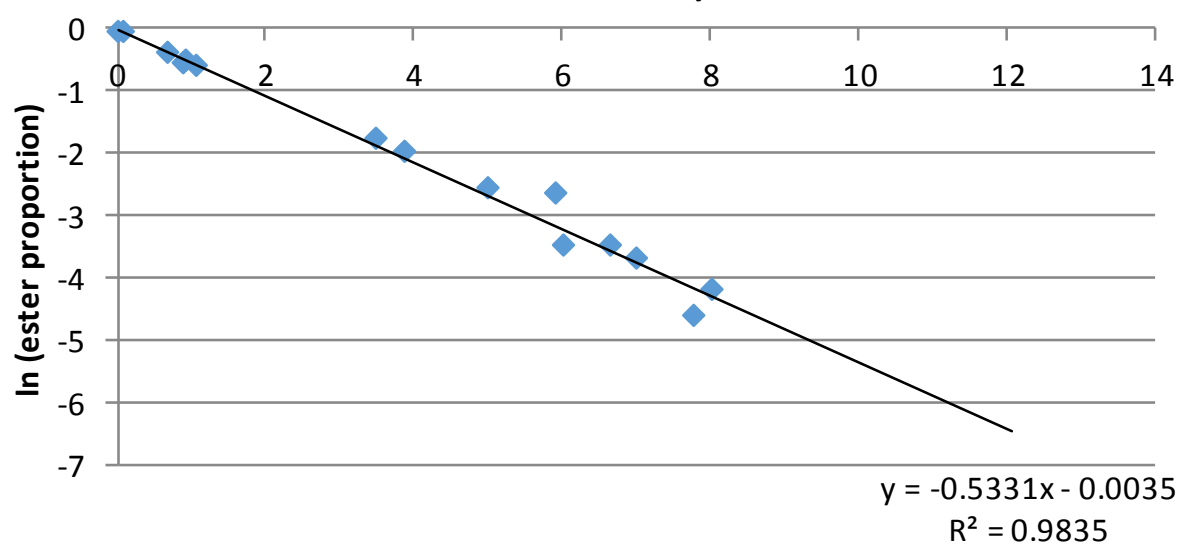

Figure 44. Logarithm of the proportion of menthyl ester $\mathbf{1 3 6}$ over time, demonstrating a linear trend

While no direct comparison between the rates of azulene and nitroazulene menthyl ester deprotection was achieved, the nitroazulene ester is estimated to be about two orders of magnitude faster. While a 100 -fold increase in reaction rate may sound impressive, it was deemed insufficient to make this into a viable protecting group. The nitroazulene ester $\mathbf{1 3 6}$ is still highly recalcitrant, and the conditions required to effect deprotection are comparable to those of the most stable esters used as protecting groups, pivaloate (0.5 M NaOH, EtOH, r.t. 12 hours) and mesitoate (2 $\mathrm{M} \mathrm{NaOH}$ in EtOH, r.t., 20 hours). ${ }^{13}$

\subsubsection{Other activation methods - $\pi$-complexation and breaking of aromaticity}

One final strategy for activation of the azulene ring towards deprotection was investigated. It was thought that by disrupting the aromaticity of the azulene ring system, the highly electron-donating properties of the azulene could be diminished. 
One approach for disrupting the aromaticity involved attack of the 7-membered ring at the electrophilic 4- and 8-positions of azulene with a nucleophilic alkyl species (Scheme 45). Addition of methyllithium to azulene without an oxidation step results in formation of the 4-methyl dihydroazulene species $\mathbf{1 3 7 . 1 4}$

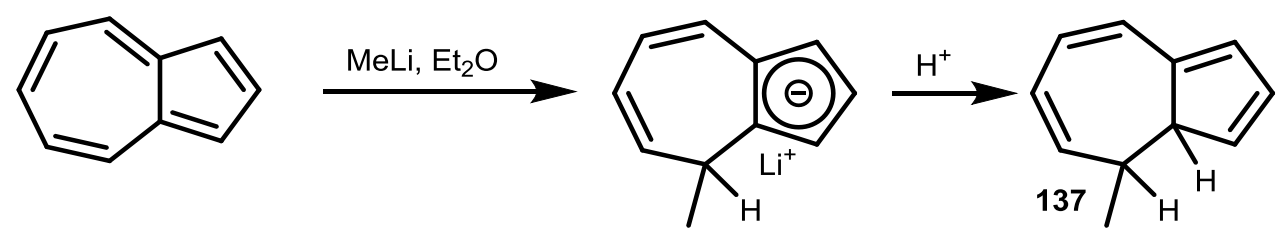

Scheme 45. Disruption of a romaticity through nucleophilic attack at the 4-position

There are three possible locations for the nucleophilic attack of methyllithium onto an azulene-1carboxylate ester: two on the azulene ring itself, at the 4- and 8-positions, and one at the ester carbonyl (Scheme 46). In any case, addition of methyllithium would either directly cleave the ester or activate it towards cleavage.
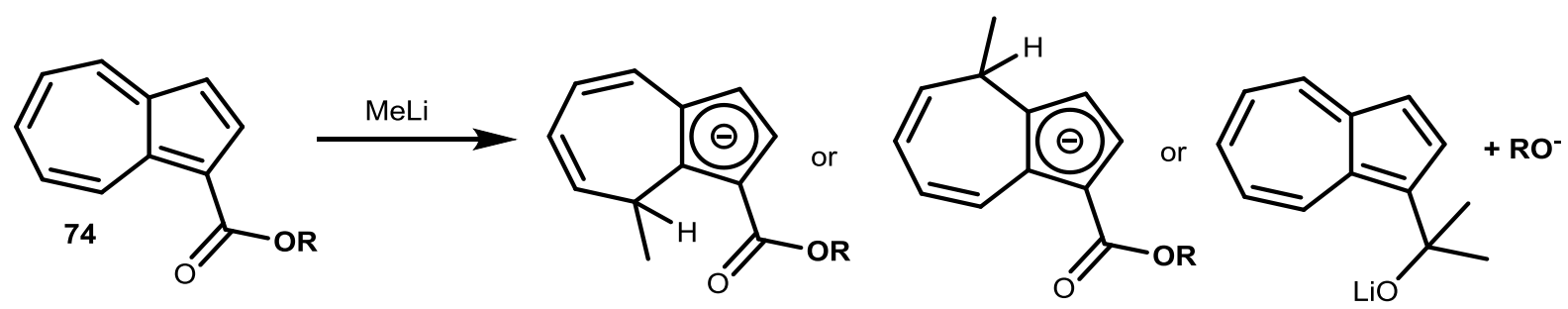

Scheme 46. Possible products of nucleophilic attack on 74 by methyllithium

To test this approach, methyllithium was added to the 1-menthyl ester $\mathbf{1 2 2}$. The purple colour of the azulene was lost, indicating attack on the ring itself. The species formed were unstable and the ${ }^{1} \mathrm{H}$ NMR spectrum of the crude reaction mixture was largely unintelligible. This was not a major issue, because recovery of the protecting group body from the deprotection reaction is not necessary. The reaction was repeated, except with the subsequent addition of KOH in ethanol 0.2 M), followed by heating at reflux overnight to effect deprotection. Menthol was present in the reaction mixture in large amounts, but the number of other products present in the reaction mixture with similar $\mathrm{R}_{\mathrm{f}}$ values made it impossible to purify. The addition of methyllithium may have had some effect on the saponification rate, but the messy nature of the reaction mixture introduced problems for purification, and the necessary deprotection conditions were nevertheless still quite harsh, so this line of investigation was abandoned.

\section{$\pi$-Complexation with tetracyanoethylene}

The previously discussed computational studies in section 3.4.4 suggested that while the expected changes in reactivity from $\pi$-complexation of electron-deficient aromatic rings such as 
trinitrobenzene were modest, complexation with a metal complex such as diironpentacarbonyl or with tetracyanoethylene would produce much greater activation.

Tetracyanoethylene reacts with 1,3-disubstituted azulenes irreversibly to form a bridged structure, such as 138 or $\mathbf{1 3 9}$, with the tetracyanoethylene moiety stretching between the 5 - and 8- positions. It is unclear whether these form through a radical or concerted pericyclic process. Takekuma etal. propose a radical mechanism. ${ }^{14}$ It is important that both the 1-and 3-positions are substituted, otherwise tetracyanoethylene will undergo nucleophilic attack by the azulene ring instead, resulting in loss of HCN and formation of a tricyanovinyl compound such as $\mathbf{1 4 0}$ (Scheme 47). ${ }^{15}$
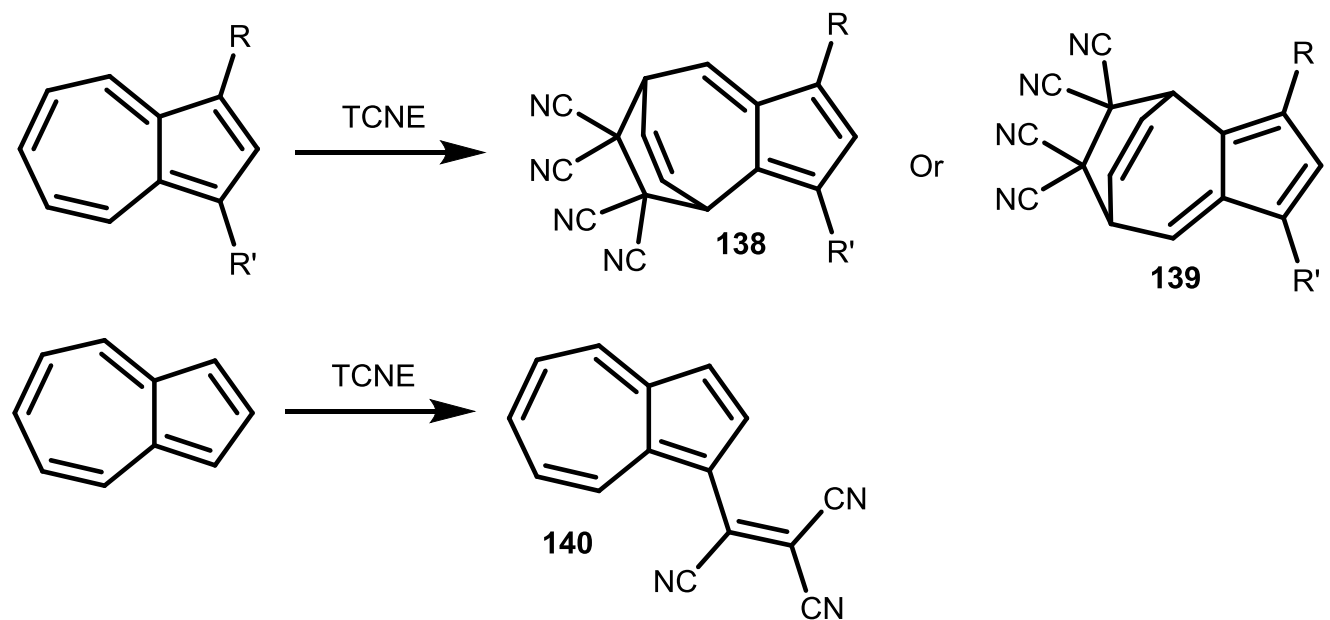

Scheme 47. Possible products from TCNE reaction with 1,3-disubstituted a zulenes (above) and with azulene itself (below)

What is not explained in the literature is the regioselectivity of the bridge formation when the 1and 3-positions are substituted unsymmetrically. The only two azulene-TCNE adducts that have been described in the literature are symmetric azulene and guaiazulene derivatives ${ }^{16}$ - which can only have one stereochemical outcome, either due to symmetry or due to substitution at the 4-and 7-positions. The outcome of this reaction is important, as the regioisomer $\mathbf{1 3 2}$ was predicted computationally (shown in Table 6) to have a much greater activation effect than the other regioisomer 133.

Ultimately, it was decided not to investigate TCNE activation, in part due to safety concerns (TCNE evolves hydrogen cyanide gas in the presence of water), and also due to the uncertainty in the amount of activation that the $\pi$-complex would allow.

\section{$\pi$-Complexation using an iron carbonyl complex}

There is precedence in the literature for the formation of azulene-metal complexes (such as iron and molybdenum) wherein the metal is bonded to the face of azulene through $\pi$-complexation. A 
diiron pentacarbonyl species in particular was looked at as a potential mode for azulene activation (Figure 45). The crystal structure of azulene diiron pentacarbonyl 141, elucidated by M. R. Churchill shows some noteworthy features. ${ }^{16}$ One of the iron atoms lies directly over the top of the 5-membered ring and has two carbonyl ligands, while the iron that lies over the 7-membered ring is positioned to one side with a hapticity of two, with one double bond on the 7membered ring not participating in iron ligation. This results in a loss of symmetry of the azulene ring system, as well as a slight bending of the 7-membered ring out of plane. The author notes that the Fe-Fe bond is also unusually long, at $2.78 \AA$, which is relatively close to the longest known FeFe bond, which is $2.88 \AA \AA^{17}$

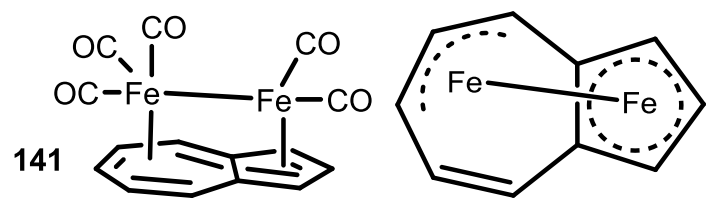

Figure 45. Azulene diironpentacarbonyl, side on (left) and view from above (right), depicting the asymmetrical arrangement of the Fe atoms over the ring system (CO ligands omitted for clarity)

There are two reported synthetic methods for making the azulene iron pentacarbonyl complex. One involves heating azulene at reflux with iron pentacarbonyl in petroleum ether (b.p. 100$120^{\circ} \mathrm{C}$ fraction) for 5 days. ${ }^{17}$ This synthesis was unacceptable as an activation protocol due to the harshness and extreme duration of these conditions and the potential toxicity of the liquid iron pentacarbonyl. The other synthesis involves heating at reflux in pentane with the addition of diiron nonacarbonyl - an orange solid that is much more easily handled and therefore safer. The reference given for this procedure by R. Burton et al.18 was a 'personal communication', and no other information regarding relative quantities or reaction time was provided about the procedure, so the relevant conditions had to be determined experimentally.

Heating azulene at reflux in $n$-hexane with diiron nonacarbonyl for 3 hours gave the desired $\pi$ complex 142 in only 10\% yield. The same conditions with 4,6,8-trimethylazulene gave a similarly low $23 \%$ yield. When the solvent were changed to toluene (also at reflux), the yield was instead quantitative (Scheme 48). All the aromatic protons in these complexes appeared in a range between 5.5 and 3.5 ppm, typical of aromatic ligands of metal complexes. 
<smiles>c1ccc2cccc-2cc1</smiles>

1

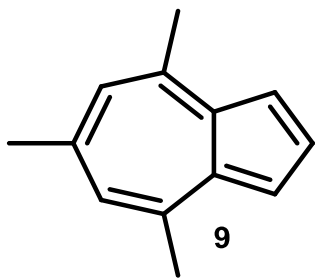

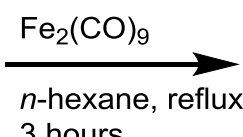

3 hours

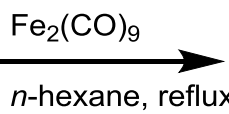

3 hours

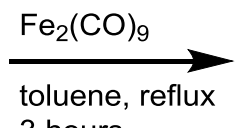

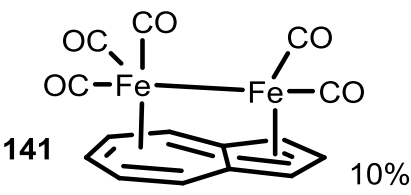

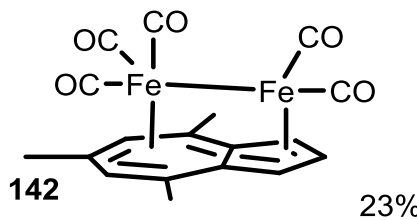

142

$99 \%$

Scheme 48. Syntheses of azulene and 4,6,8-trimethylazulene iron carbonyl complexes

The azulene menthyl ester was then subjected to these same conditions - heating at reflux in toluene with diiron nonacarbonyl. After 6 hours only partial reaction had occurred (Scheme 49). This is not surprising, as the $\pi$-system of the menthyl ester $\mathbf{1 2 2}$ will be much less electron rich than that of azulene. This reaction gave an orange-red compound 143 in $19 \%$ yield. The ${ }^{13} \mathrm{C}$ NMR spectrum revealed that all peaks were doubled, probably due to the formation of diastereomers (although positional isomers differing in the placement of the Fe over the 7-membered ring are also possible).
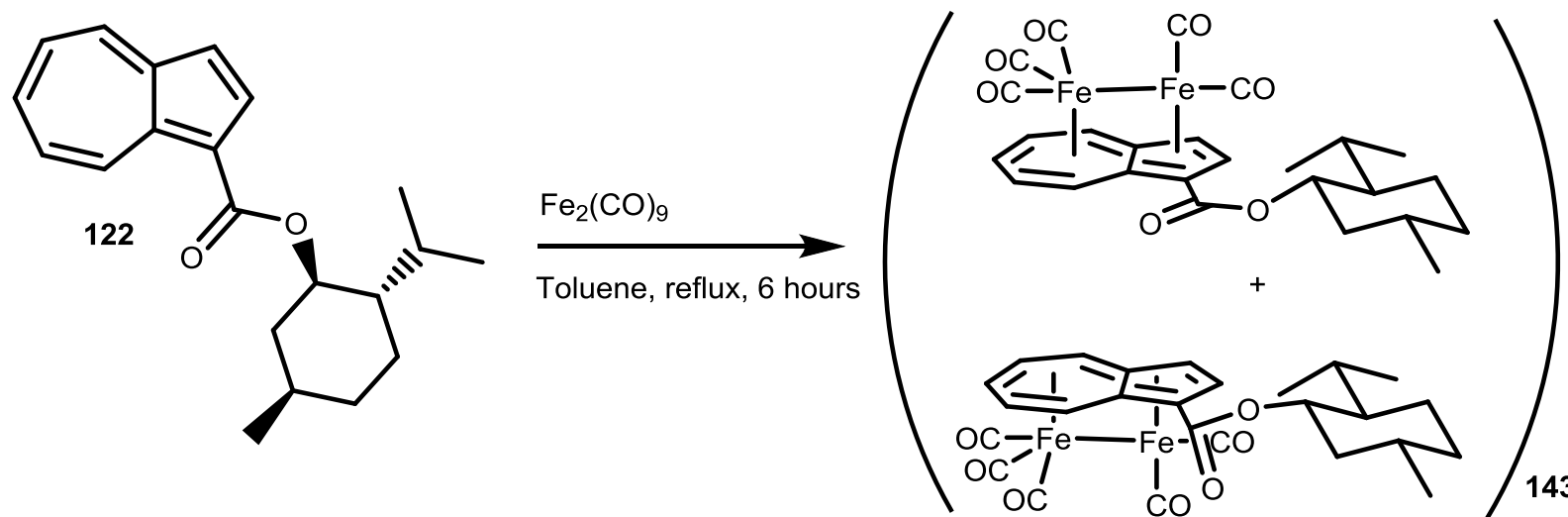

Scheme 49. Reaction of menthyl ester 122 with diiron nonacarbonyl generated the complex $\mathbf{1 4 3}$ (thought to be a mixture of diastereomers)

The azulene iron pentacarbonyl complex 143 was then subjected to saponification conditions of $\mathrm{KOH}$ in EtOH at room temperature. Unfortunately, these conditions resulted in removal of the iron carbonyl complex instead of cleavage of the ester linkage, regenerating the starting menthyl ester with no evidence of deprotection (Scheme 50). 

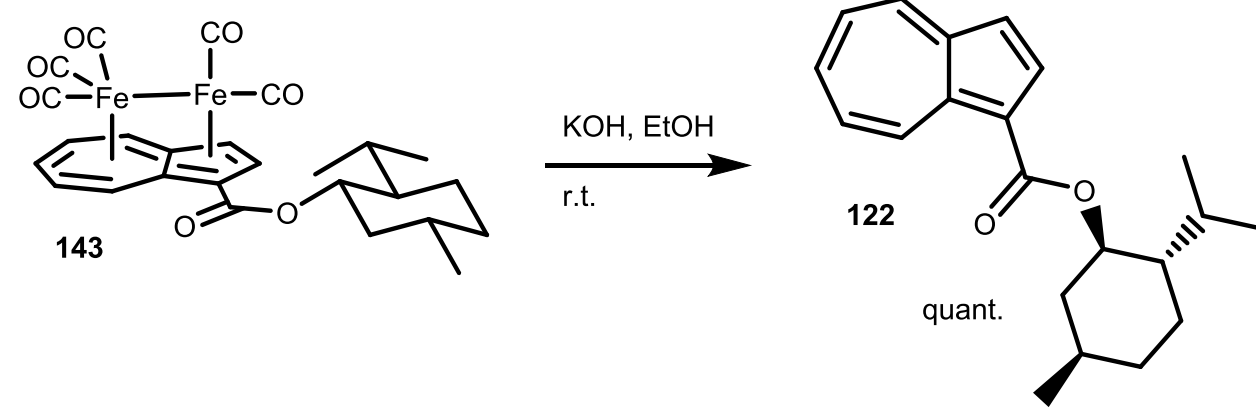

No deprotection

Scheme 50. Attempted deprotection only regenerated the ester $\mathbf{1 2 2}$

\subsubsection{Substituted azulenes and 'tethered' deprotections}

Investigation of an alternate, 'tethered' deprotection mechanism involving 4-methyl-substituted azulenes was undertaken. The basic idea was that functionalisation of the adjacent 4-methyl substituent with a methylene alkoxide would facilitate an alternate deprotection route through intramolecular attack on the ester, forming a 7-membered lactone ring and displacing the alcohol substrate (Scheme 51). For this investigation, three different azulene derivatives were explored: 4-methylazulene, guaiazulene and 4,6,8-trimethylazulene.
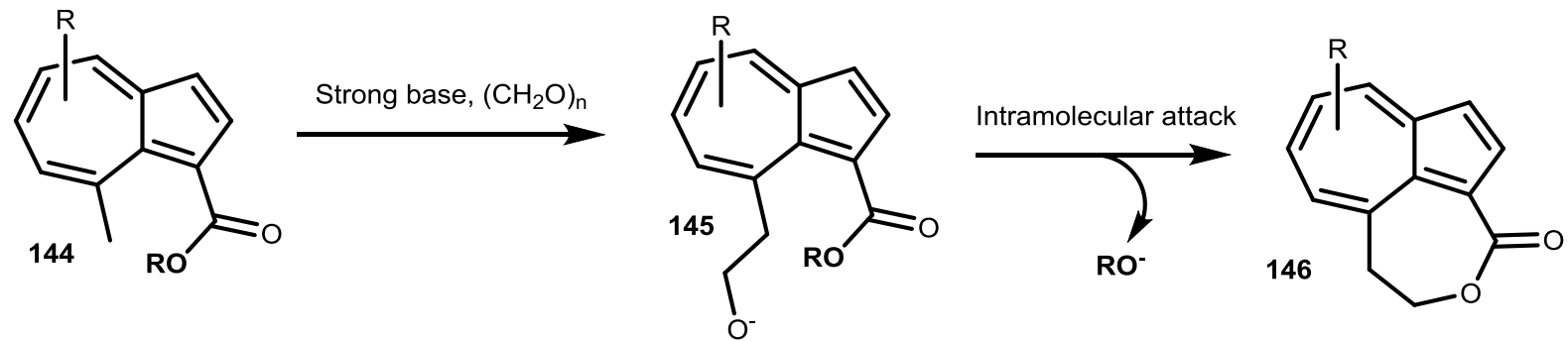

Scheme 51. Generalised depiction of te thered deprotection involving deprotonation of the 4- position of 144 followed by al koxymethylenation with paraformaldehyde to form $\mathbf{1 4 5}$ and intramolecular nucleophilic attack to release the substrate RO and form the lactone 146

4-Methylazulene was the first to be investigated for this purpose. Firstly, the regioselectivity of the reaction between 4-methylazulene and oxalyl chloride needed to be elucidated. Plausible rationalisations exist for the functionalization of either side of the 5-membered ring. By invoking electronics, the 3-position would be favoured due to electron donation from the 4-methyl group. By invoking sterics, the 1-position would be favoured due to steric crowding of the 4-methyl grouparound the 3-position. 


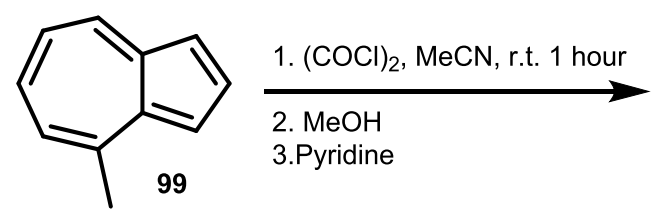

99

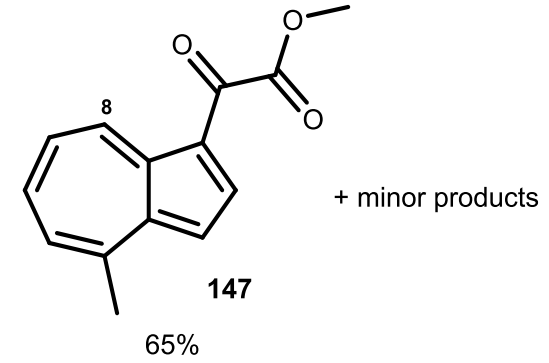

$65 \%$

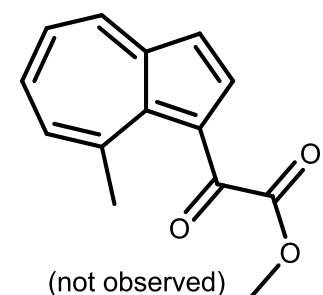

observed)

Scheme 52. Reaction of 4-methylazulene (99) gave the undesired isomer 147

Upon addition of oxalyl chloride to 4-methylazulene (99), only substitution of the 1-position was observed (Scheme 52). The major product was the ketoester 147, and several unidentified minor compounds were also present. Common to all these compounds was a doublet in the ${ }^{1} \mathrm{H}$ NMR spectrum containing at $10 \mathrm{ppm}$, a shift diagnostic for a proton at the 8-position on an azulene adjacent to a keto-ester or similar highly electron-withdrawing functional groups. This unwanted reactivity precluded use of 4-methylazulene as a precursor for a tethered deprotection-based protectinggroup.

\section{The guaiazulene ester and tethered deprotection}

Guaiazulene avoids the regioselectivity issue faced by 4-methylazulene, through the presence of the methyl on the 1-position blocking off electrophilic attack. Acylation of guaiazulene was well understood as it was a focus of my Master's research, though adaption of this chemistry into a protection reaction had not been performed previously.

Yields of the methyl ester of guaiazulene of up to $90 \%$ were obtained during my Master's work when using an excess of methanol. A protocol for protection is necessarily different, as the alcohol substrate must be the limiting reagent. Repeating the protocols previously developed with menthol as a limiting reagent gave a low $42 \%$ yield of $\mathbf{1 4 8}$, and significant formation (46\% w.r.t guaiazulene) of the diguaiazulenyl ketone by-product 149 (Scheme 53).

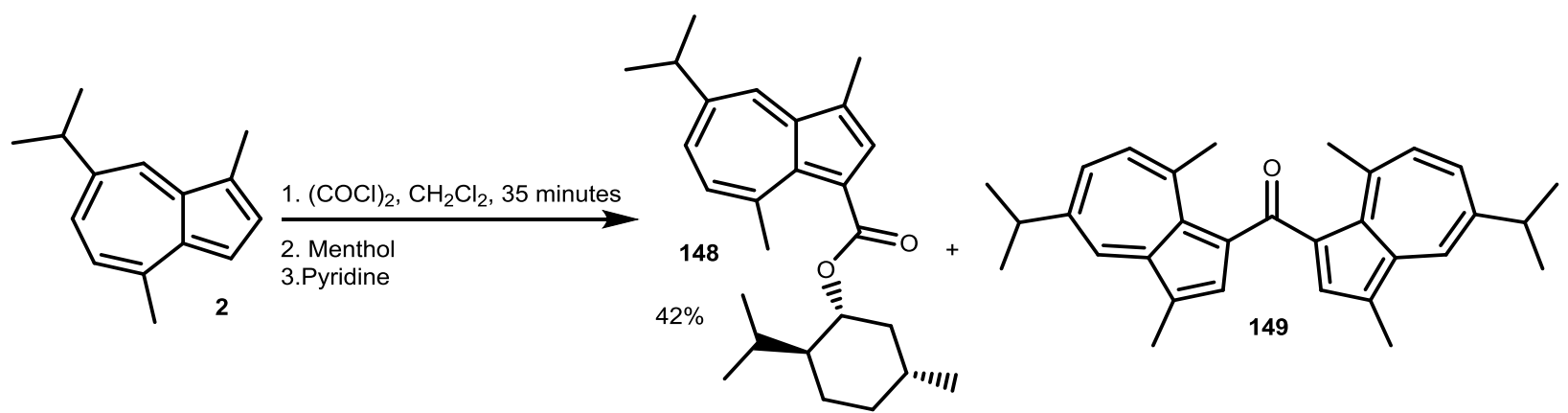

Scheme 53. Synthesis of the guaiazulene menthyl ester $\mathbf{1 4 8}$

Nevertheless, enough of the desired material 148 was obtained to allow exploration of the deprotection conditions, through both acidic ester hydrolysis and saponification, in addition to the tethered deprotection route. 
Guaiazulene esters are even more resistant to saponification than azulene esters. Heating 148 at reflux overnight in $0.7 \mathrm{M} \mathrm{KOH}$ in EtOH gave only 25\% deprotection (as measured by ${ }^{1} \mathrm{H}$ NMR spectroscopy of the crude mixture), in comparison to full deprotection of the azulene ester $\mathbf{1 2 2}$ in the same conditions. However, the guaiazulene ester 148 was much more amenable to acidic hydrolysis, giving complete deprotection when subjected to $10 \%$ aq. $\mathrm{HCl}$ in $\mathrm{EtOH}$ and heated at reflux over two hours (Scheme 54). In comparison, the azulenyl ester 122 gave only 8\% deprotection under similar conditions. This pattern of reactivity can be explained through the presence of the alkyl groups around the guaiazulene ring making it more electron rich in comparison to azulene, and thereby promoting the acidic hydrolysis pathway while impeding the basic hydrolysis pathway.

Neither of these reactions are particularly useful as deprotection protocols as they nevertheless require conditions that are much harsher than those that an organic substrate of any complexity could be expected to be compatible with.

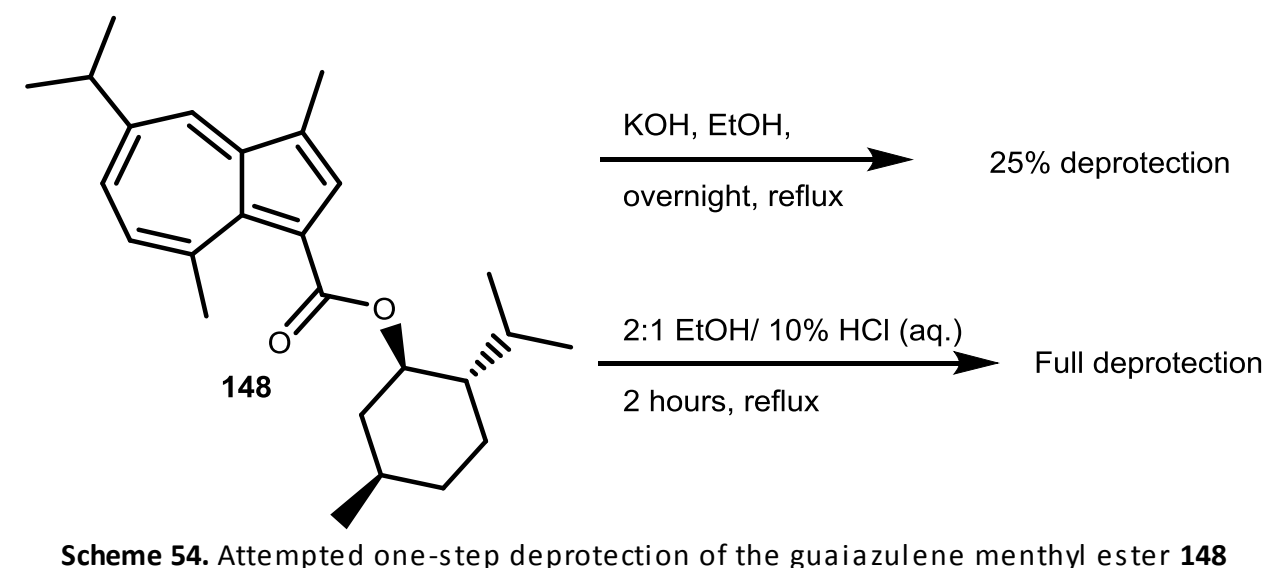

Two general strategies of tethered deprotection were envisaged. The deprotonationhydroxymethylation of a 4-methylazulene could be performed as a two-step process involving irreversible deprotonation of the 4-methyl group with a strong base followed by addition of paraformaldehyde, or in a single step by addition of a weaker base (so as to form an equilibrium with the deprotonated species) in the presence of paraformaldehyde. The first strategy was carried out by using alkali metal hexamethyldisilazide salts as strong bases (which, although insufficient to deprotonate unfunctionalised methylazulenes, are sufficiently basic to completely deprotonate methylazulenes with electron withdrawing substituents such as esters). Addition of KHMDS in $\mathrm{Et}_{2} \mathrm{O}$ resulted in a rapid deprotonation (indicated by a colour change from purple to light brown), however addition of paraformaldehyde only gave $40 \%$ reclaimed starting material and unidentifiable degradation material. The same reaction performed with LiHMDS gave $22 \%$ of the hydroxymethylated product $\mathbf{1 5 0}$ and trace amounts $(<1 \%)$ of the intended 7 -membered lactone 151, as well as trace amounts of free menthol (Scheme 55). 


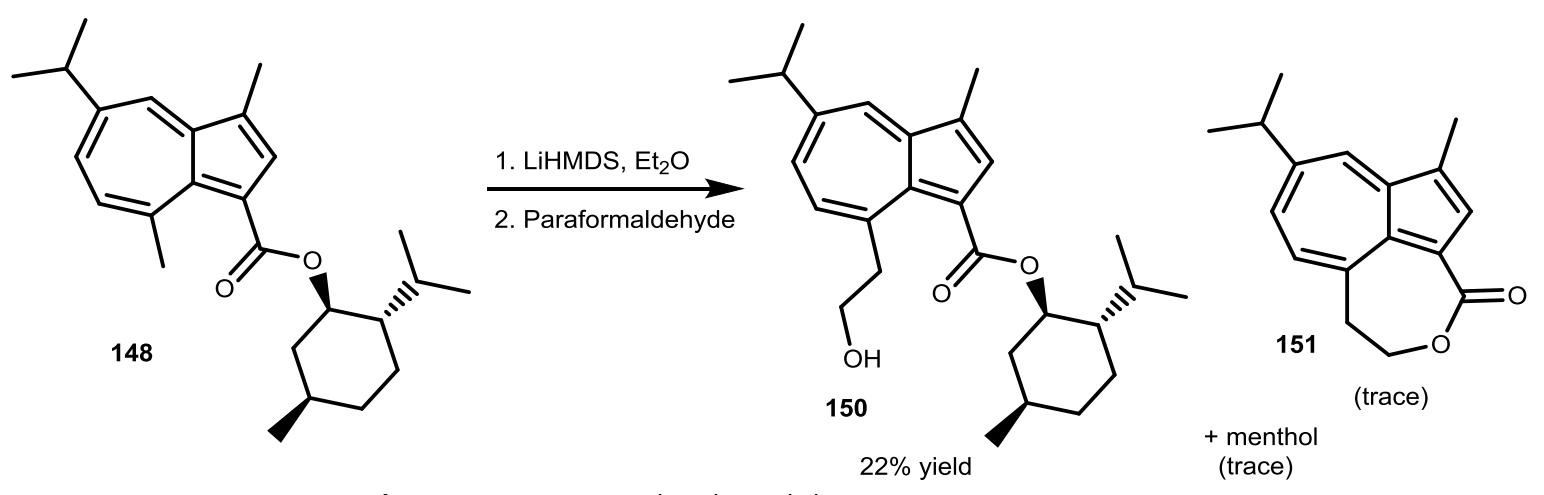

Scheme 55. Attempted tethered deprotection using LiHMDS

The second strategy involved addition of DBU in MeCN. DBU, while being much stronger than any known amine base, is weaker than alkali HMDS salts. (In THF, the conjugate acid of KHMDS has a pKa of 26, whereas that of DBU has a pKa of 16.9). ${ }^{19}$

Addition of DBU and paraformaldehyde to $\mathbf{1 4 8}$, followed by a two day reaction period at room temperature afforded the hydroxymethylated product $\mathbf{1 5 0}$ in $16 \%$ yield and the bishydroxymethylated product 152 in 38\% yield (Scheme 56). No menthol was detected.

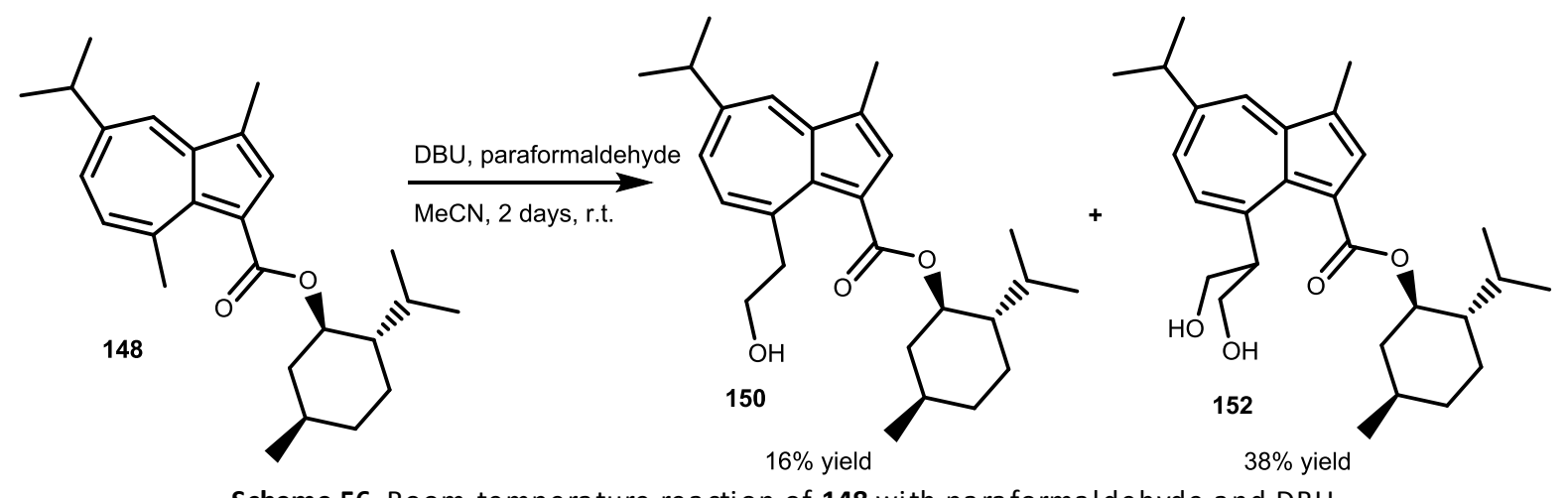

Scheme 56. Room-temperature reaction of $\mathbf{1 4 8}$ with paraformaldehyde and DBU

Repeating the reaction at elevated temperatures ( 1 day at $\sim 35^{\circ} \mathrm{C}$, followed by 1 day at reflux) gave a more complicated mixture of products. In addition to the products $\mathbf{1 5 0}$ and $\mathbf{1 5 2}$ at $16 \%$ and 10\% yields respectively, this reaction returned the purple lactone $\mathbf{1 5 1}$ in $10 \%$ yield and a hydroxymethylated lactone 153 in 3\% yield. By ${ }^{1} \mathrm{H}$ NMR integration, 17\% yield of deprotected menthol was observed for this reaction (Scheme 57).
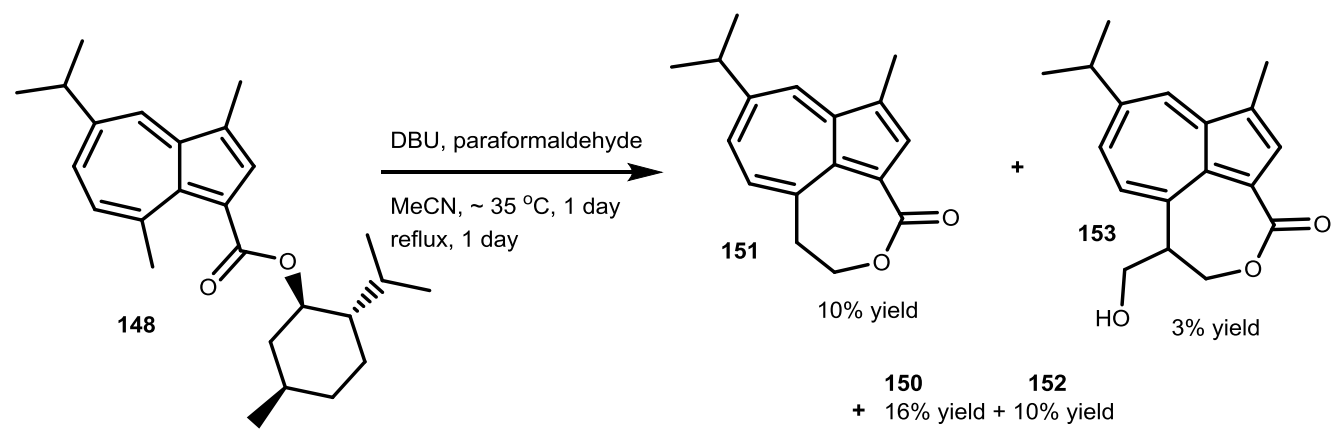

Scheme 57. Reaction of 148 a t elevated temperatures gave some deprotection a nd formation of the lactones 151 and 153 
While these reaction conditions were milder than the conditions required for saponification, they are still too harsh and low-yielding for use in an end-stage deprotection reaction.

As an aside, the lactones $\mathbf{1 5 1}$ and $\mathbf{1 5 3}$ are a different colour to the acyclic esters, being purple rather than indigo. The carbonyl of the lactone is constrained in a co-planar arrangement with the azulene ring system, and it is thought that the heightened degree of overlap between the $\pi$ systems of the two results in a greater electron withdrawing effect from the carbonyl, and consequently a hypsochromic shift in absorbance towards the purple.

\section{The 4,6,8-trimethylazulene ester and tethered deprotection}

Finally, the reactivity towards acidic, basic and tethered deprotection was investigated for 4,6,8trimethylazulene esters.

The protection step was performed using the same general technique as employed with the azulene esters, but, as is usual with this class of compounds, various idiosyncrasies in the reactivity of 4,6,8-trimethylazulene had to be overcome.

In refluxing $\mathrm{MeCN}$, the 4,6,8-trimethylazulene ketoacid chloride 154 would convert to an acid chloride 155 in approximately 7 - 8 minutes, which was accompanied by a colour change from orange to an intense red-pink. At the 20 minute mark, this red-pink changed to a deeper 'brickcoloured' red-brown, and at this point addition of the alcohol followed by pyridine saw the product obtained in only single digit yields (9\% and 3\% after 20 minutes and 30 minutes at reflux respectively). The major product was a highly-insoluble brick-red powder that is believed to be the 4,6,8-trimethyazulene ketone oligomer 156, on the basis of its colour and insolubility (Scheme 58).
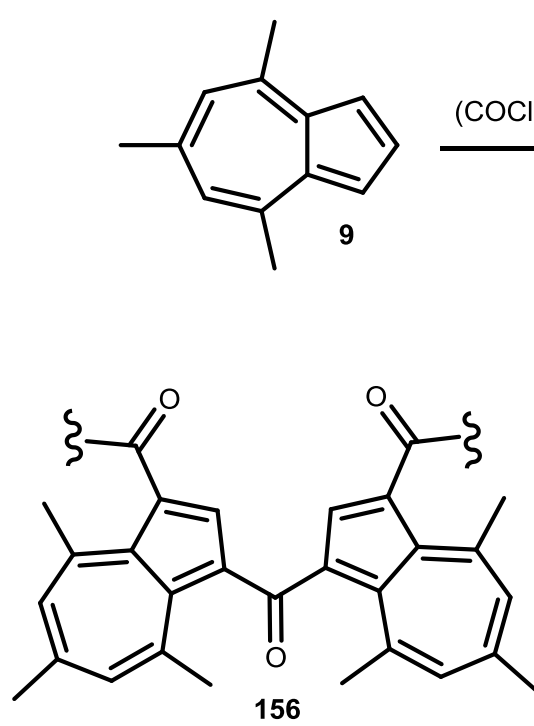

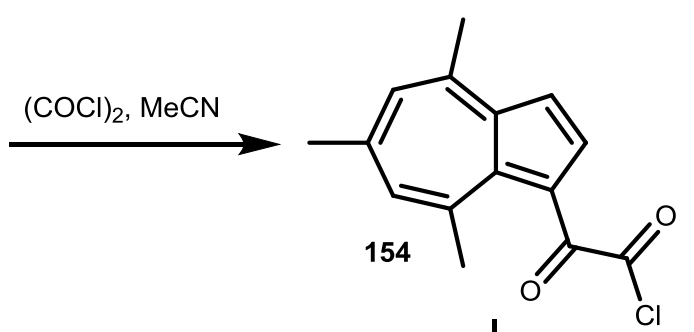

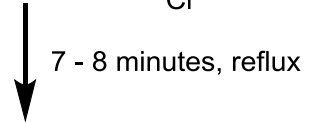<smiles>Cc1cc(C)c(C)c2c(C(=O)Cl)cc(C)c-2c1</smiles> 
The highest yields of these protection reactions were obtained by addition of menthol at the 7 minute mark, immediately after the colour change from orange to pink-red was observed. This returned a 50\% yield of the desired ester 157 (Scheme 59).

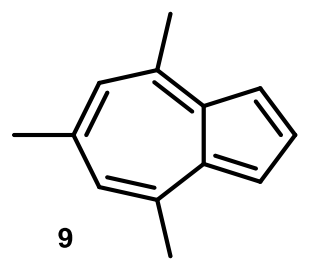

1.(COCl $)_{2}$ (1 eq.), MeCN 7 min reflux

2.Menthol

3.Pyridine

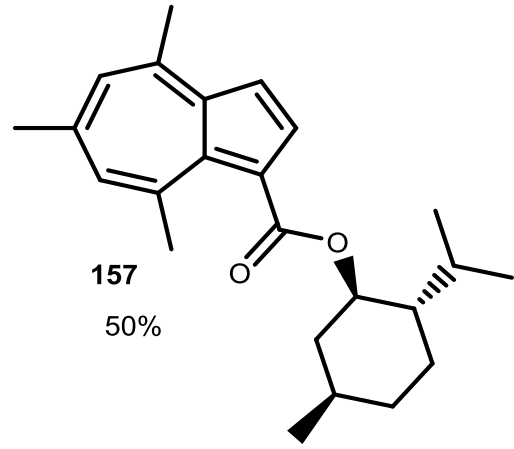

$+44 \% \mathrm{SM}$

Scheme 59. Synthesis of the 4,6,8-trimethylazulene menthyl ester 157

A slightly higher yield was obtained by adding an excess of menthol (making this method inapplicable as an example of a protection reaction) after 9 minutes. A 62\% yield of the desired product was obtained, along with 36\% yield of the bubblegum-pink menthyl diester $\mathbf{1 5 8}$ (Scheme 60).

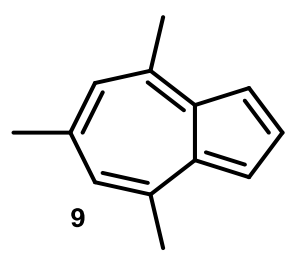

1.(COCl $)_{2}(2.3$ eq. $), \mathrm{MeCN}, 9$ min reflux

2.Menthol (excess) 3.Pyridine

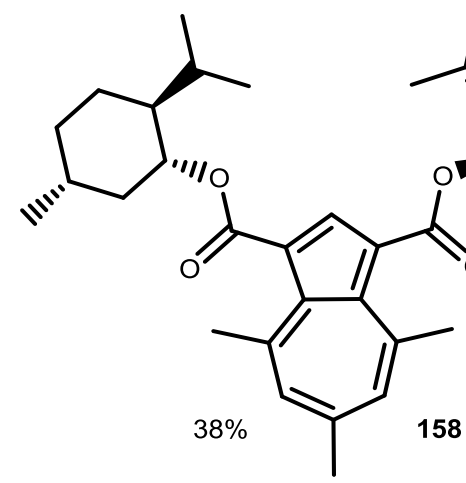

Scheme 60. Synthesis of the mono-ester 157 and di-ester 158

The observed reactivity of the 1- and 3-positions in the formation of $\mathbf{1 5 8}$ is unusual with respect to other azulenes. 1,3-Bis-addition is not normally observed in the reactions of azulene with oxalyl chloride, despite the potential for bis-addition of both azulene and oxalyl chloride. This is because the initial product of the reaction between azulene and oxalyl chloride - an azulene acyl chloride or keto-acyl chloride - is less nucleophilic than azulene and less electrophilic than oxalyl chloride. When reaction times are relatively short and there is no vast excess of one reagent over the other, such dimers are not observed (Figure 46). 


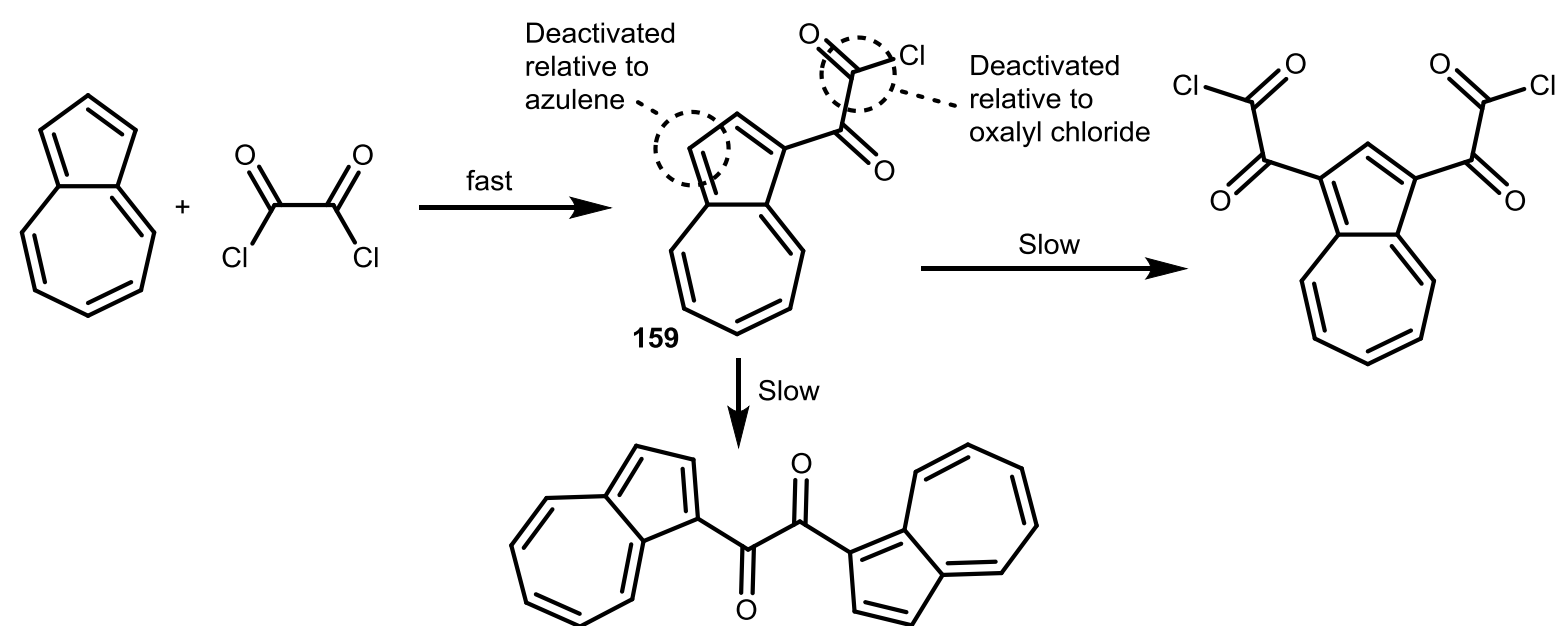

Figure 46. Azulene keto-acid chloride $\mathbf{1 5 9}$ can react with a second equivalent of azulene or oxalyl chloride, but both pathways are much slower than the initial reaction

The presence of dimers in the reactions involving 4,6,8-trimethylazulene suggests that these dimer-forming reaction steps are more favoured than they are for azulene. Since the electronic effects of the electron donating methyl groups should affect the reactivity of all azulene species equally, this effect is thought to be steric in nature - the presence of the methyl group causes the carbonyl group to be pushed out of plane with the azulene (Figure 47), thereby mitigating the electron withdrawing effect on the azulene system and allowing the 3-position to undergo nucleophilic attack on oxalyl chloride more readily. Such an effect also explains why the formation of other azulene-based analogues of the insoluble compound 156 are not observed.
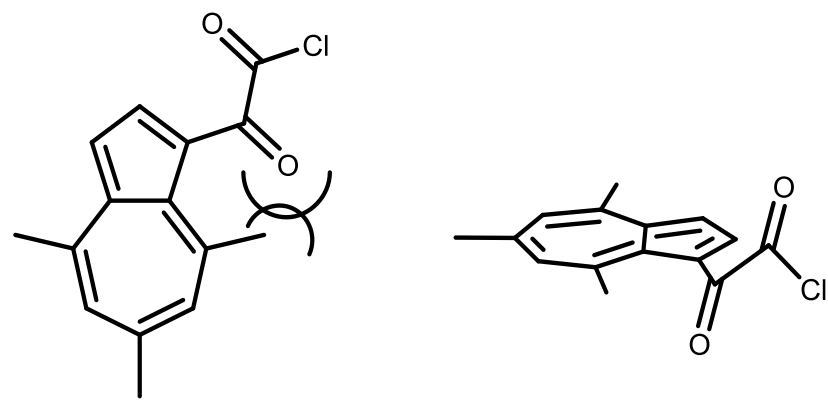

Figure 47. The presence of the methyl group at the 4-position is thought to force the keto-acid chloride out of plane, diminishing its electron-withdrawing effect over the azulene ring system

With sufficient material to proceed, the deprotection characteristics of the 4,6,8-trimethylazulene menthyl ester were subsequently investigated.

The 4,6,8-trimethylazulene menthyl ester 157 proved even more resistant to basic hydrolysis than the guaiazulene ester, providing $12 \%$ consumption of the starting material and only $6 \%$ yield of menthol from heating at reflux overnight in $0.6 \mathrm{M} \mathrm{KOH}$ in EtOH. Surprisingly, this ester also proved to be less amenable to acidic hydrolysis than the guaiazulene menthyl ester 148, with complete deprotection occurring in aqueous $\mathrm{HCl} /$ methanol at reflux after 4.5 hours (Scheme 61). 


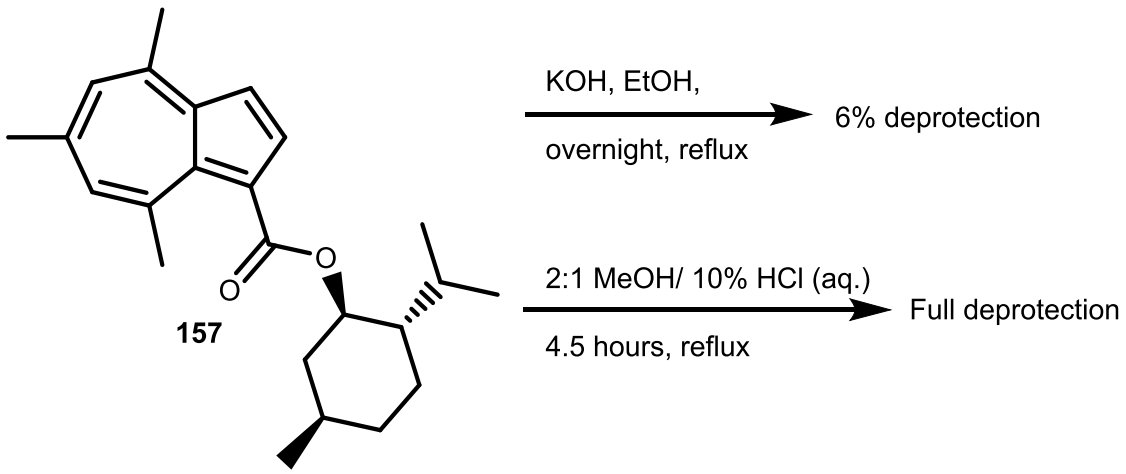

Scheme 61. One-step deprotection characteristics of the 4,6,8-trimethylazulene menthyl ester 157

Finally, the tethered deprotection chemistry was investigated. It was expected that the ester 157 would fare even more poorly than the guaiazulene ester 148, due to the additional sites at which superfluous hydroxymethylation could take place, so 157 itself was not investigated. Instead, 157 was nitrated to form the 1-nitroazulene ester 160 in 52\% yield using Menke nitration (Scheme 62). It was hoped that the 1-nitro group would not only aid in deprotonation and hydroxymethylation, but would also increase the reactivity of the ester towards nucleophilic attack.

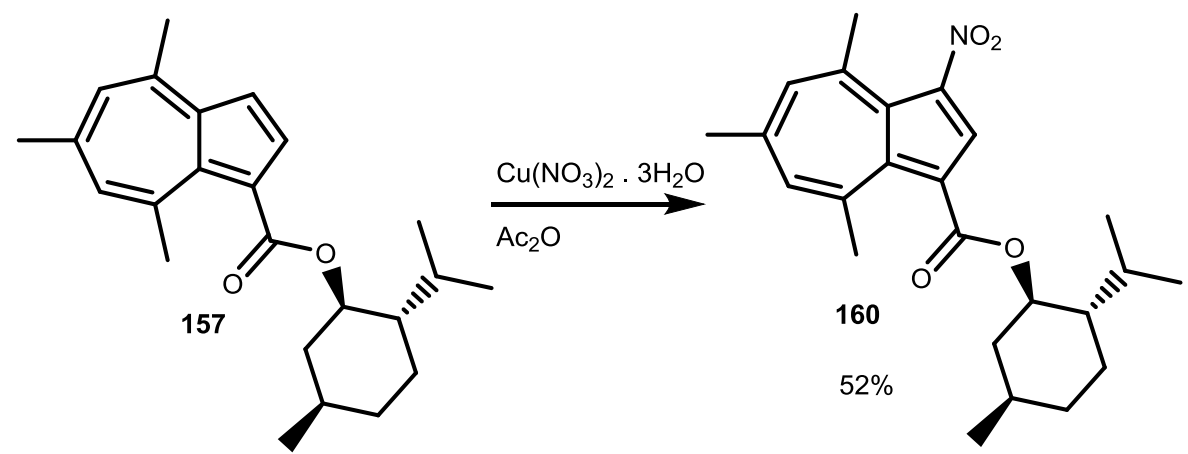

Scheme 62. Nitration of 157 to form 160

A tethered deprotection was then attempted. DBU was added to a solution of $\mathbf{1 6 0}$ and paraformaldehyde in MeCN. An immediate colour change from orange to yellow was observed, indicating deprotonation (Scheme 63). Over the course of the reaction, highly polar orange material was produced (as measured by TLC), suggesting hydroxymethylation. However, no free menthol could be observed on TLC or in the 1 H NMR spectrum of the crude sample.

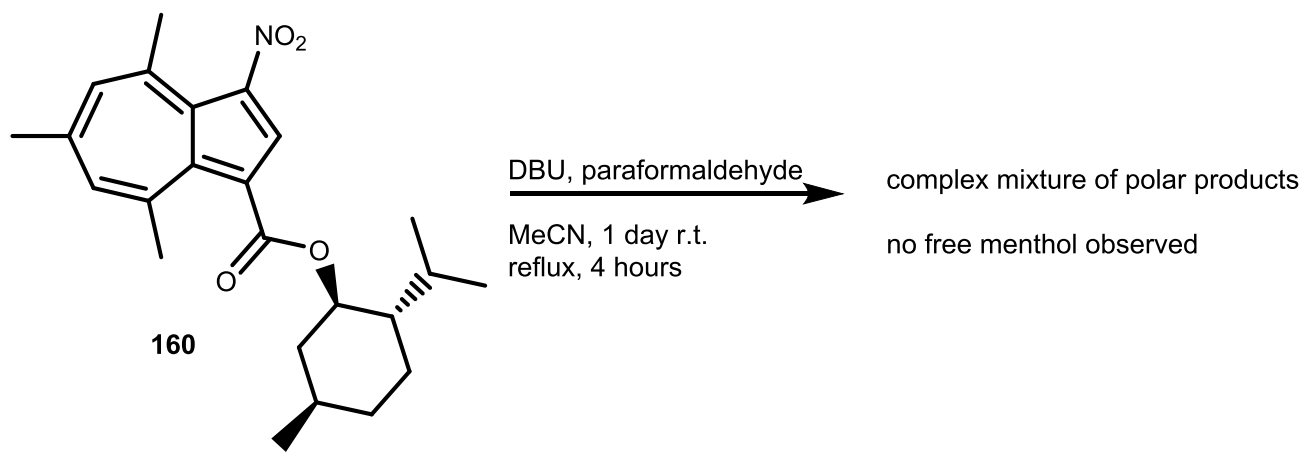


With the lack of success of the guaiazulene and 4,6,8-trimethylazulene esters towards tethered deprotection, this line of investigation was halted.

\subsection{Summary of chapter 3}

Flaws were exposed at an early stage for the protecting group candidates $\mathbf{7 9}$ and $\mathbf{8 2}$, with the 1azulenylmethylene $\mathbf{7 9}$ being too unstable in open air to be usable, and the 1-azulenesulfonamide 82 expected to be far too difficult to remove with mild conditions.

Protection and deprotection methods for alcohols with the 1-azulenyl ester $\mathbf{7 4}$ were demonstrated. Unfortunately, the successful deprotection methods found for this protecting group were far too harsh to be of practical use in most total syntheses - especially as it is intended for these protecting groups to be removed during the synthetic endgame, where the need for mild reaction conditions is paramount. One-step deprotection required harsh basic conditions of $\mathrm{KOH} / \mathrm{EtOH}$ at reflux overnight in order to proceed to completion, and the most successful two step deprotection - nitration - only succeeded in increasing the reactivity by about a hundredfold, which was deemed insufficient to allow for acceptably mild deprotection conditions for use in complex molecule synthesis.

Replacing azulene with other alkyl-substituted azulenes gave even more recalcitrant ester protecting groups. Tethered deprotection using guaiazulene esters was shown to work in principle, but in practice the reactivity was far too low and the reaction conditions far too harsh for this method to be useful as a deprotection strategy.

Due to these factors, it was decided that 1-azulenyl esters would not be viable protecting groups in organic synthesis. While they are not precluded entirely from some sort of protecting group function (i.e. in uses such as coloured tags where deprotection is not important, or in a synthesis where the final substrate is sufficiently stable to withstand the harsh deprotection conditions), these applications were deemed too far outside the scope of this project to pursue.

\subsection{References}

1. Bevan, T. Investigations of azulene derivatives as self-indicating chromophores for applications in protecting group strategies and solid-phase purification. VUW, MSc thesis, 2011.

2. Timmer, M. S. M.; Stocker, B. L.; Northcote, P. T.; Burkett, B. A., Az-a Colourful AzuleneDerived Protecting Group. Tetrahedron Lett. 2009, 50, 7199-7204. 
3. Inanga, J.; Hirata, K.; Saeki, H.; Katsuki, T.; Yamaguchi, M., A rapid esterification by means of mixed anhydride and its application to large-ring lactonisation. Bull. Chem. Soc. Jpn. 1978, 52, 1989-1993.

4. (a) Clark, J. H., Green Chemistry: Challenges and Opportunities. Green Chemistry 1999, 1, 1; (b) Schaetz, A.; Zeltner, M.; Michl, T. D.; Rossier, M.; Fuhrer, R.; Stark, W. J., Magnetic Silyl Scaffold Enables Efficient Recycling of Protecting Groups. Chem. Eur. J. 2011, 17, 1056610573.

5. Longridge, J. L.; Long, F. A., The Decarboxylation of Azulene-1-carboxylic Acid in Acidic Aqueous Solution.J. Am. Chem. Soc. 1968, 90, 3092-3098.

6. Neuvonen, H.; Neuvonen, K., Correlation Analysis of Carbonyl Carbon ${ }^{13} \mathrm{C}$ NMR Chemical Shifts, IR Absorption Frequencies and Rate Coefficients of Nucleophilic Acyl Substitutions. A Novel Explanation for the Substituent Dependence of Reactivity. J. Chem. Soc.,Perkin Trans. 1999, 2, 1497-1502.

7. Yanai, T.; Tew, D. P.; Handy, N. C., A new Hybrid Exchange-Correlation Functional using the Coulomb-attenuating Method (CAM-B3LYP). Chem. Phys. Lett. 2004, 393, 51-57.

8. Riley, K. E.; Holt, B. T. Op't. Holt.; K. M. Mertz Jr., Critical Assessment of the Performance of Density Functional Methods for Several Atomic and Molecular Properties. J. Chem. Theory Comput. 2007, 3, 407-433.

9. Hansch, C.; Taft, R. W.; Leo, A., A Survey of Hammett Substituent Constants and Resonance and Field Parameters. Chem. Rev. 1991, 91, 165-195.

10. Menke, J. B., Nitrieren mit Nitraten. Recueil des Travaux Chimiques des Pays-Bas 1925, 44, 141-149.

11. Bordwell, F. G.; Jr., E. W. G., Nitrations with Acetyl Nitrate. I. The Nature of the Nitrating Agent and the Mechanism of Reaction with Simple Alkenes. J. Am. Chem. Soc. 1960, 82, 3588-3598.

12. Olah, G. A.; Narang, S. C.; Olah, J. A.; Lammertsma, K., Recent Aspects of Nitration: New Preparative Methods and Mechanistic Studies (A Review). Proc. Natl. Acad. Sci. 1982, 79, 4487-4494.

13. Wuts, P. G. M.; Greene, T. W., Protective groups in organic synthesis. Wiley: 2007.

14. Elwahy, A. H. M.; Hafner, K., Synthesis and Characterization of Novel Oligoazulenes with Mixed Ethynyl and Butadiynyl Bridges. Eur. J. Org. Chem. 2006, 3910-3916.

15. Takekuma, S.-i.; Nagata, K.; Yoshioka, Y.; Obata, H.; Minami, T.; Tanaka, T.; Yashima, K.; Minematsu, T.; Takekuma, H., Preparation, Molecular Structures, and Characteristic properties of (E)-1-(2-furyl)- and (E)-1-(2-thienyl)-2-(3-guaiazulenyl)ethylenes and (E)-1(3-furyl)- and (E)-1- (3-thienyl)-2-(3-guaiazulenyl)ethylenes. Tetrahedron 2012, 68, 6737-6758. 
16. Shoji, T.; Ito, S.; Higashi, J.; Morita, N., Synthesis of 1-Heteroaryl- and 1,3Bis(heteroaryl)azulenes: Electrophilic Heteroarylation of Azulenes with the Triflates of NContaining Heteroarenes. Eur. J. Org. Chem. 2011, 5311-5322.

17. Churchill, M. R., The Crystal and Molecular Structure of Azulene Diiron Pentacarbonyl. Inorganic Chemistry 1967, 6, 190-196.

18. Burton, R.; Pratt, L.; Wilkinson, G., Transition-metal Complexes of Seven-membered Ring Systems. Part II . Azulenemetal Carbonyls. J. Chem. Soc. 1960, 4290-4303.

19. Leito, I.; Kütt, A.; Kaljurand, I.; Rõõm, E.-I.; Rodima, T.; Koppel, I. A., Brønsted Acidity of Neutral and Cationic Acids in Nonaqueous Solvents: Recent Developments. http://tera.chemut.ee/ ivo/HA UT/Acidity Basicity INOR 1036 ACS 2007.pdf, Ed. (last accessed 17/2/16) 


\section{Chapter 4. Base-labile 6-substituted azulene protecting groups for carboxylic acids, alcohols and amines}

\subsection{Introduction}

After exhausting the possibilities for mild deprotection of azulene 1-esters, 6-substituted azulenes were investigated for their potential as protecting groups, with 6-(2-hydroxyethyl)azulene as the precursor (Scheme 64). Despite early difficulties with synthesising sufficient material for testing, this project met with significantly more success than the 1-azulenyl ester chemistry discussed in Chapter 3, with mild and high-yielding protection and deprotection (one and two-step) methodology developed.
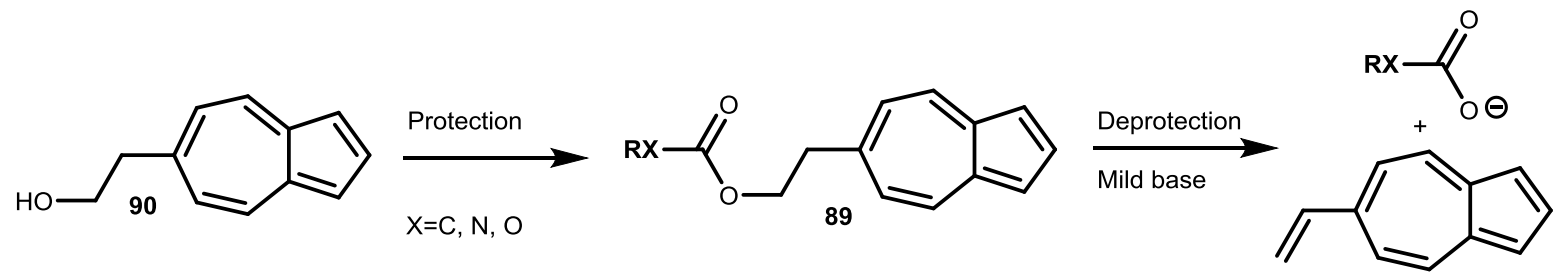

Scheme 64 . The protecting group candidate $\mathbf{8 9}$

The key to the function of 6-substituted azulene protecting groups was exploitation of the acidity of the protons adjacent to the 6-position. Upon treatment with the appropriate (typically amine) base, an equilibrium is formed between the starting material and the resonance-stabilised carbanion 161 (Scheme 65). This induces an elimination through an E1cB mechanism, regenerating the original substrate. ${ }^{1}$
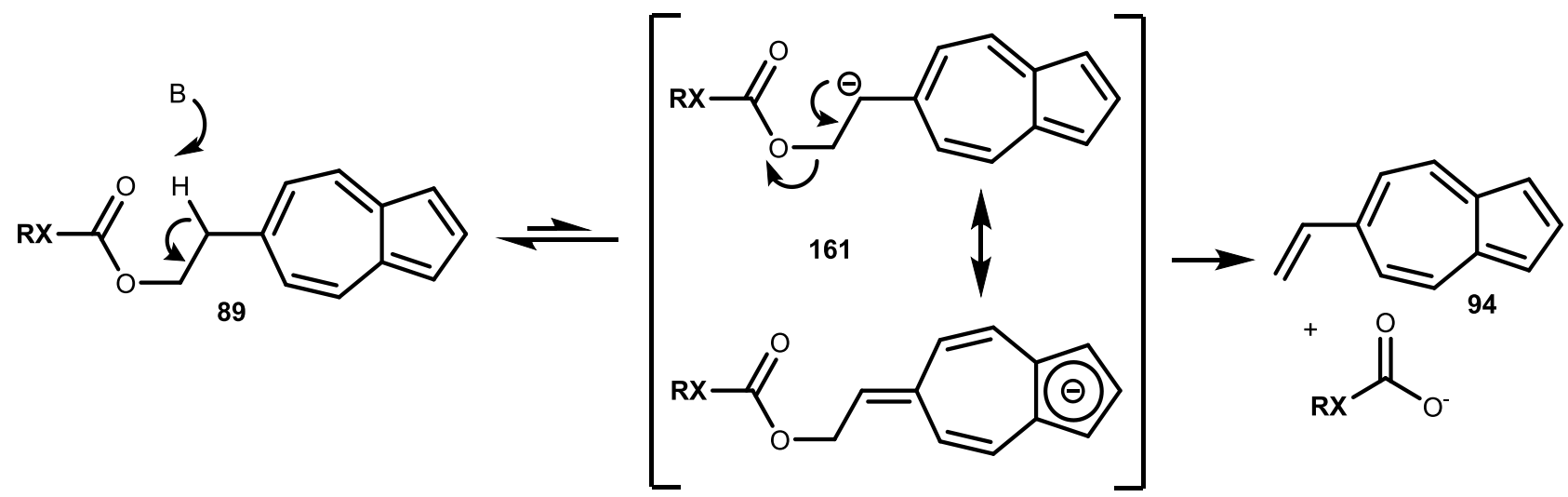

Scheme 65. General deprotection mechanism of the protecting group candidate $\mathbf{8 9}$ 


\subsection{Synthesis of 6-(2-hydroxyethyl)azulene}

From the outset, it was clear that large quantities of 6-(2-hydroxyethyl)azulene (90) would be needed for this project. For this, the synthesis of 1-methyl-4-(2-hydroxyethyl)-7-isopropylazulene 162 from guaiazulene that was developed during my Master's research was adapted (Scheme 66). ${ }^{2}$ Hydroxymethylation was achieved through deprotonation with LDA at $0{ }^{\circ} \mathrm{C}$, followed by addition of paraformaldehyde.

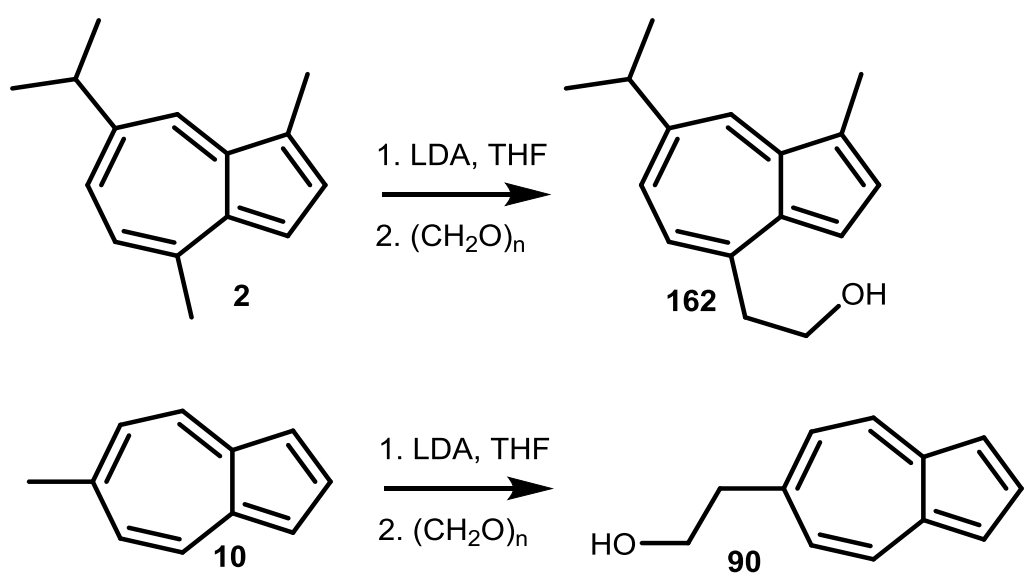

Scheme 66. Hydroxymethylation of guaiazulene (2) and 6-methylazulene (10)

It was known from this previous work on guaiazulene that deprotonation/hydroxymethylation often gave low and inconsistent yields of 162 (ranging from 13\% to 67\% with an average of $35 \%) .^{2} \mathrm{~A}$ few initial reactions with 6-methylazulene indicated that this pattern would be repeated, with yields ranging between $20 \%$ and $68 \%$ despite little variation in the reaction protocol. Considerable effort was therefore invested in finding higher-yielding and more reliable reaction conditions and determining the causes of the low and variable yields. While no definitive explanation of this variability was found, reaction protocols (described in section 4.2.2) were eventually established that gave more consistent yields of 50-60\% with a highest yield of $73 \%$.

There are many factors at play in this chemistry, some of which are controllable - such as concentration and reaction temperature - and others are inherent to the chemistry of the reagents, such as the complexity of organolithium chemistry and the quantity of water present in paraformaldehyde. Obtaining definite correlations between these variables and yield was fraught with difficulties, and often a clear correlation would appear, only to disappear after additional reactions were performed. 


\subsubsection{Investigation of the deprotonation step with methyl iodide}

The chemistry of LDA-mediated deprotonation is notoriously complicated. ${ }^{3}$ Optimisation of the deprotonation step of the synthesis, and exploration of the fidelity of the deprotonation step is essential to the optimisation of the overall reaction.

LDA was typically made by addition of $n$-BuLi (1.7-1.9 $\mathrm{M}$ in cyclohexane) to diisopropylamine in THF at $0{ }^{0} \mathrm{C}$. Deprotonation of 6-methylazulene (and likewise, guaiazulene and 4,6,8trimethylazulene) produces a colour change from indigo to a colour ranging from golden brown to grey-brown depending on the concentration. The resulting azulenide anion 163 absorbs much less strongly in the visible region, and reaction mixtures that are rendered completely opaque by the starting material often become somewhat transparent after deprotonation. As a result, even a small amount of 6-methylazulene - whether unreacted or reprotonated - will discolour the deprotonated reaction mixture quite noticeably to a khaki green. This allows the observer to tell if the deprotonation has reached completion or not with relative confidence.

After full deprotonation was visually confirmed through colour change, the reaction was quenched in order to determine the extent of reaction. Initially this was done through the addition of $\mathrm{D}_{2} \mathrm{O}$, but use of methyl iodide was found to be more amenable to analysis. Methyl iodide reacts somewhat faster with the anion than $\mathrm{D}_{2} \mathrm{O}$, and the proportions of the resulting reaction mixture of 6-methyl- and 6-ethylazulene 164 were easier to evaluate by ${ }^{1} \mathrm{H}$ NMR integration (Scheme 67).
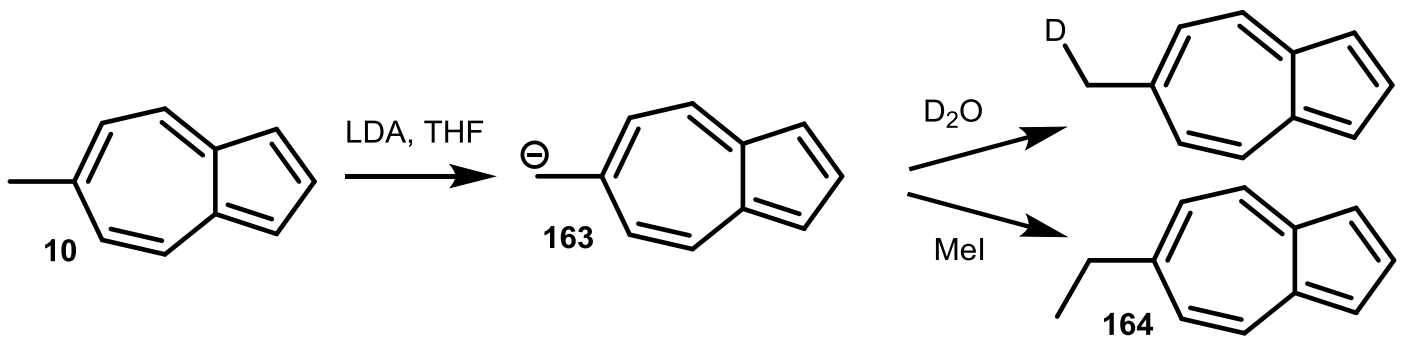

Scheme 67. The two methods of investigating the extent of deprotection

Initially, deprotonation was carried out such that LDA was added to the reaction mixture, rather than the reaction mixture to LDA. Through this method, yields were obtained ranging from $50 \%$ to $80 \%$ of 6-ethylazulene 164. This method suffered from two drawbacks. The first was determining the concentration of LDA: LDA would be titrated prior to use (see titration method in chapter 7), but the values obtained would often vary from the calculated values (the number of moles of $n$-BuLi divided by the total volume of LDA, THF and the $n$-BuLi solution) by about $30 \%$ in either direction, creating an uncertainty in the quantity needed for addition into the reaction mixture. Reasonable doubt can be placed on the accuracy of both estimations - the titration 
method gave concentrations that varied considerably between individual runs (in some cases up to $40 \%$ variation). In comparison, titration of $n$-BuLi using the same method gave a much smaller variation, of the order of 5\%. Calculation of the concentration of LDA from the volumes of the added reagents diisopropylamine, $n$-BuLi and THF, assumes that no change in density takes place upon mixing, which is not necessarily the case.

The second drawback was formation of small ( $\sim 2 \%$ yield) quantities of a dimeric by-product 165 (Figure 48). Through addition of the 6-methylazulene-containing reaction mixture to the LDA solution, a slightly better overall yield of $83 \%$ was obtained. The issues with determining the concentration of the LDA mixture were simply bypassed. Also, formation of the dimeric impurity $\mathbf{1 6 5}$ was no longer observed in these reactions. It is believed that $\mathbf{1 6 5}$ is formed through a radical mechanism when 6-methylazulene and the azulenide 163 are both present in the same mixture. When adding the reaction mixture to LDA through a cannula, LDA is always in excess so mixtures of 6-methylazulene and 163 cannot occur. While the overall yield did not see much improvement ( $83 \%$ vs $80 \%$ for best case) between these two addition methods, the reverse addition method did improve the reliability and ease of the process, preventing the formation of 165 which occasionally was observed to degrade during chromatography and foul up the column.

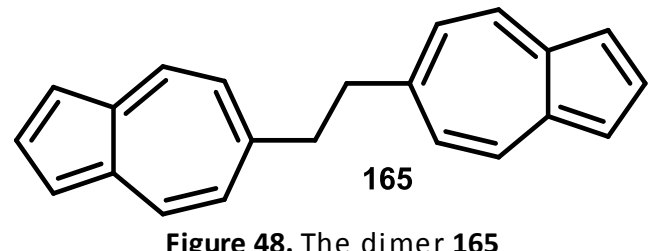

Figure 48. The dimer 165

Nevertheless, the high $83 \%$ conversion obtained indicates that the deprotonation step in the formation of 6-(2-hydroxyethyl)azulene is not the primary cause of the low yields of $\mathbf{9 0 .}$

\subsubsection{Optimisation of the formation of 90}

Following the model reactions with methyl iodide, the reaction of the azulenide 163 with paraformaldehyde was investigated in depth. Firstly, the same reverse addition method used in section 4.2.1 was adopted. Despite this, the yields of these reactions were lower than those that formed the ethylazulene 164, typically between $25-50 \%$ and never above $61 \%$, with notinsignificant quantities of starting material (10-20\%) recovered and other by-products being generated.

There are two highly polar products that were regularly observed in these reactions. The azulene derivative 166 was often seen in $\sim 10 \%$ yields, presumably from addition of paraformaldehyde to the nucleophilic 1-position (Figure 49). Smaller quantities of an even more polar compound were 
sometimes observed. This compound has never been isolated in sufficient purity to identify by NMR, but it is thought to be the triol $\mathbf{1 6 7}$ based on this polarity.
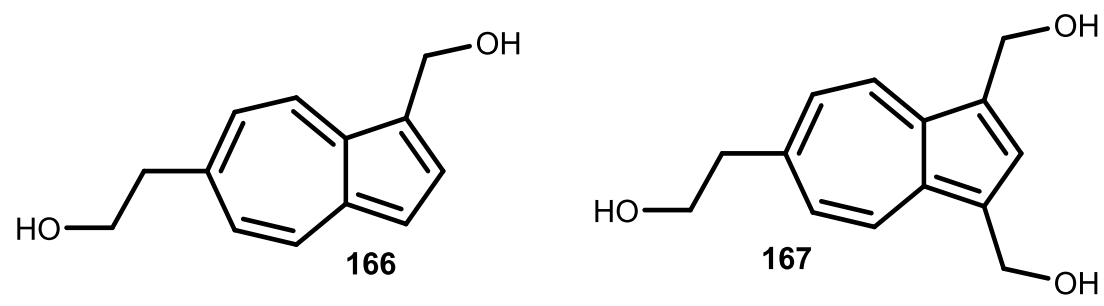

Figure 49. By-products of the formation of $\mathbf{9 0}$

Hydroxymethylation of the 1-position of azulene by paraformaldehyde is typically performed under acidic conditions. ${ }^{4}$ It is not clear how the formation of 166 and 167 takes place. When azulene was treated with paraformaldehyde in the presence of $\mathrm{KOH}$, no reaction occurred. Likewise, when the reaction is repeated with 6-ethylazulene and in the additional presence of diisopropylamine, no reaction occurred. Only when azulene was treated with LDA before addition of formaldehyde gas was the 1-hydroxymethylazulene 111 formed in 9\% yield (Scheme 68).

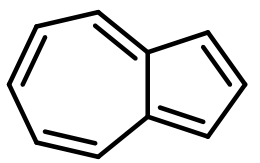

THF, $\mathrm{KOH},\left(\mathrm{CH}_{2} \mathrm{O}\right)_{\mathrm{n}}$
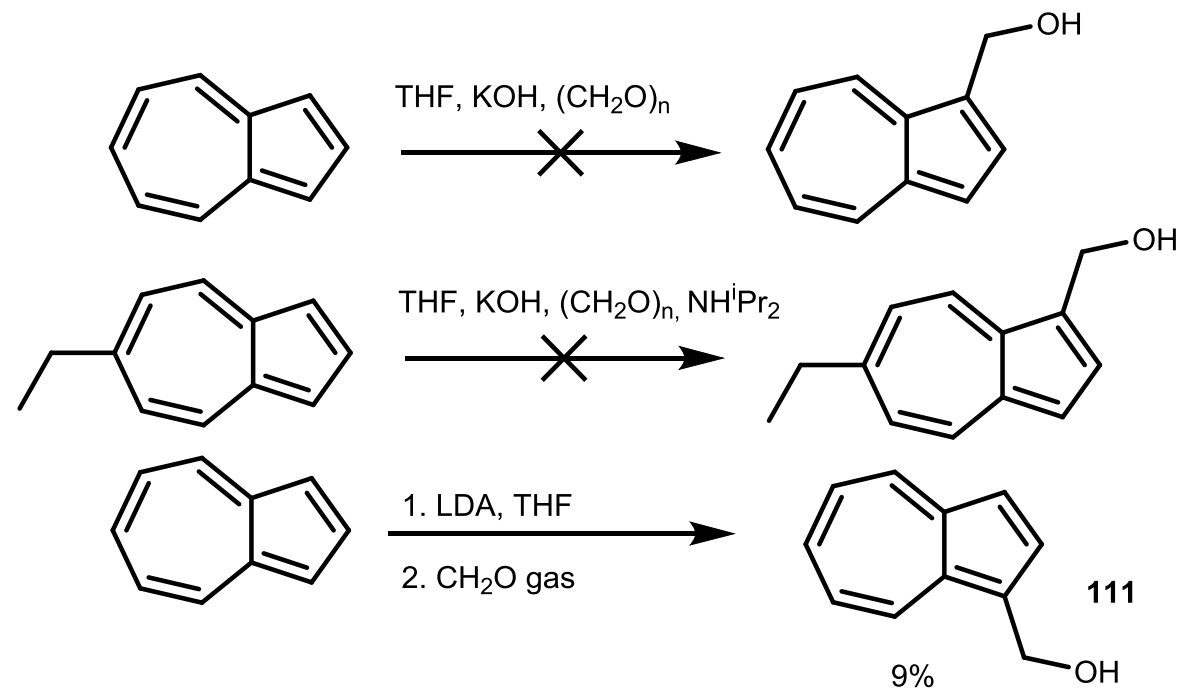

Scheme 68. Attempted replication of the conditions that form 166 and $\mathbf{1 6 7}$

When summed, the yields of the starting material 10, the product 90 and the by-products 166 and 167 did not always account for the entire mass balance - significant quantities of degradation to highly-polar green material was often observed. The lost material ranged in yield from $1 \%$ to $60 \%$, and the resultant green material would in extreme cases discolour the column during chromatography and obfuscate the eluting blue fractions. 


\section{Investigation of the paraformaldehyde addition step}

Further understanding of the nature of formaldehyde and paraformaldehyde was needed in order to optimise the reaction conditions. Formaldehyde gas itself is unstable, and readily polymerises to polyoxymethylene on cold surfaces (Figure 50). As a result, formaldehyde is almost never handled as a gas on its own.

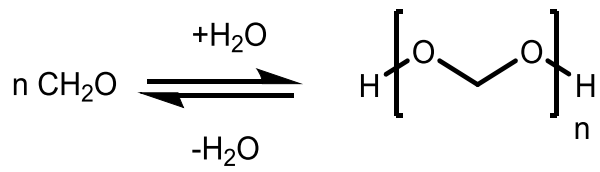

Figure 50. Equilibrium of formaldehyde with polyoxymethylene

There are three main ways of storing and using formaldehyde. The first, formalin, is a solution in water typically consisting of $37 \%$ formaldehyde by mass. The second is 1,3,5-trioxane, the cyclic trimer of formaldehyde. 1,3,5-Trioxane is a solid, and forms an equilibrium with formaldehyde in the presence of acid, and can decompose to formaldehyde at $270{ }^{\circ} \mathrm{C}$ or higher. 5 The third is paraformaldehyde, a specific type of polyoxymethylene powder, usually 8-100 monomer units long capped by $\mathrm{OH}$ groups. Paraformaldehyde can be depolymerised to formaldehyde in the presence of base, or it can be decomposed by heat to form formaldehyde gas.

Of the three forms, only paraformaldehyde was seriously considered as a formaldehyde source. 1,3,5-Trioxane was tried a handful of times as a reagent, but with no reaction observed. Formalin was rejected because the aqueous environment was incompatible with the chemistry.

Paraformaldehyde is a frustratingly ill-defined material. The main concern affecting reactivity is that every oligomeric unit contains one equivalent of water. This one equivalent cannot be removed by drying, as it makes up the end cap of the oligomer and is only released on depolymerisation. On a molar basis, an oligomer $\mathrm{n}$ units long will have a water content of $100 /(n+1) \%$ and therefore will be $100 *(n /(n+1)) \%$ pure. Little information is given on the distribution of chain length in individual batches (a range of 8-100 is normally cited), but the average degree of polymerisation can be calculated from the stated purity of the paraformaldehyde. Commercial batches usually come with a purity of $90 \%$, with the other $5 \%$ being primarily water. From this, it can be determined that one twentieth of the substance is water and therefore that the average chain length is 20 .

The water content and chain length matters, as it affects not only the amount of water present in the reaction, but also the rate of reaction. The mechanism of paraformaldehyde depolymerisation is roughly the same irrespective of the mode of decomposition: the terminal protons are removed either by base or by heat, and the polymer spontaneously depolymerises from the ends inwards. 
The rate of depolymerisation is proportional to the quantity of capping hydroxyl groups present, and therefore inversely proportional to the average chain length of the paraformaldehyde oligomer. The greater the average polymer chain length, the slower the release of formaldehyde.

There was a slight negative trend observed between the excess amount of paraformaldehyde and the reaction yield that suggests the water evolved from paraformaldehyde does play a detrimental part (Figure 51).

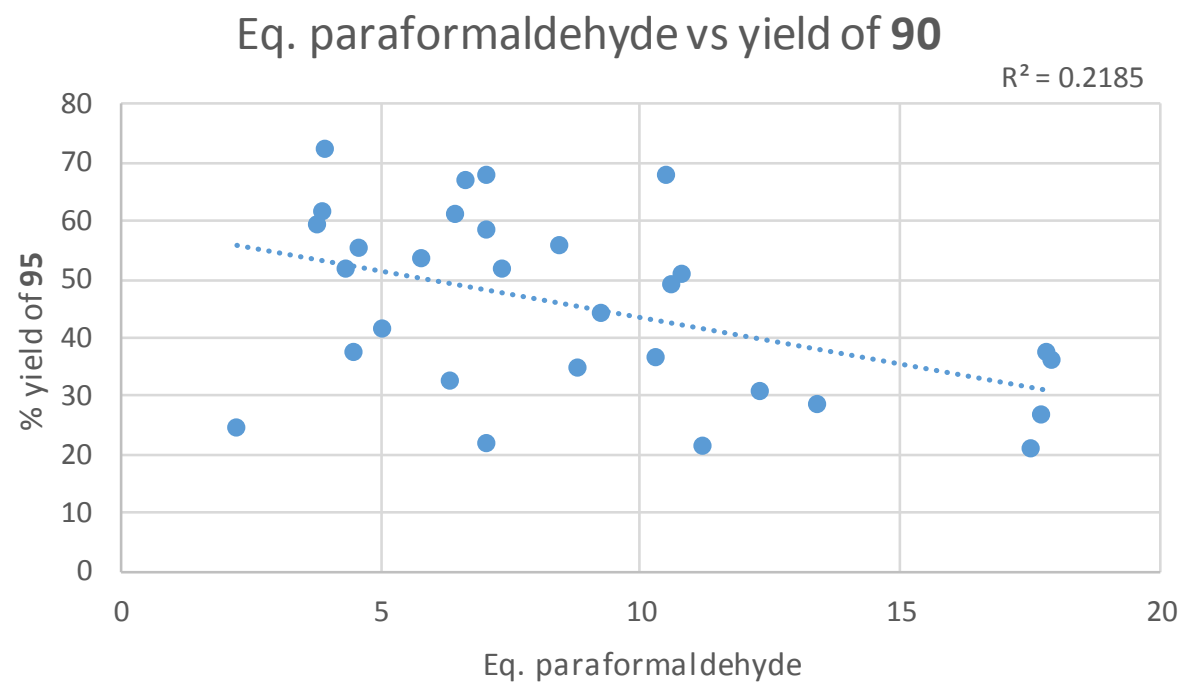

Figure 51. Relationship between the excess of paraformaldehyde and the isolated yield of $\mathbf{9 0}$

When plotting the number of equivalents of paraformaldehyde versus the yield of $\mathbf{9 0}$, a negative correlation with an $\mathrm{R}^{2}$ value of 0.21 was seen. An $\mathrm{R}^{2}$ value this small cannot be used as anything more than the barest suggestion of a correlation, though it is worth noting that a poor correlation would be observed even from a strong dependence, due to the lack of systematic control over the other variables in these reactions.

Formaldehyde gas was typically introduced to the reaction mixture by heating a separate vessel containing paraformaldehyde and connected to the reaction vessel via cannula. Typically, no other flow of gas was present. Occasionally, when blockages in the cannula occurred, the remaining paraformaldehyde powder was added directly to the reaction mixture. Both methods have their disadvantages. Direct addition required the septum be removed briefly in order to add the powder, potentially introducing oxygen and water to the reaction mixture. Paraformaldehyde powder also reacts comparatively slowly - sometimes taking half an hour at $0{ }^{\circ} \mathrm{C}$ before going to completion. Cannulation of formaldehyde gas resulted in a much faster reaction, such that the reaction vessel was occasionally observed to go from golden-brown to blue almost instantaneously. However, the newly-formed formaldehyde gas is hot, which creates the potential for unwanted side-reactions. 


\section{Distribution of reaction yields by mode of addition of paraformaldehyde}

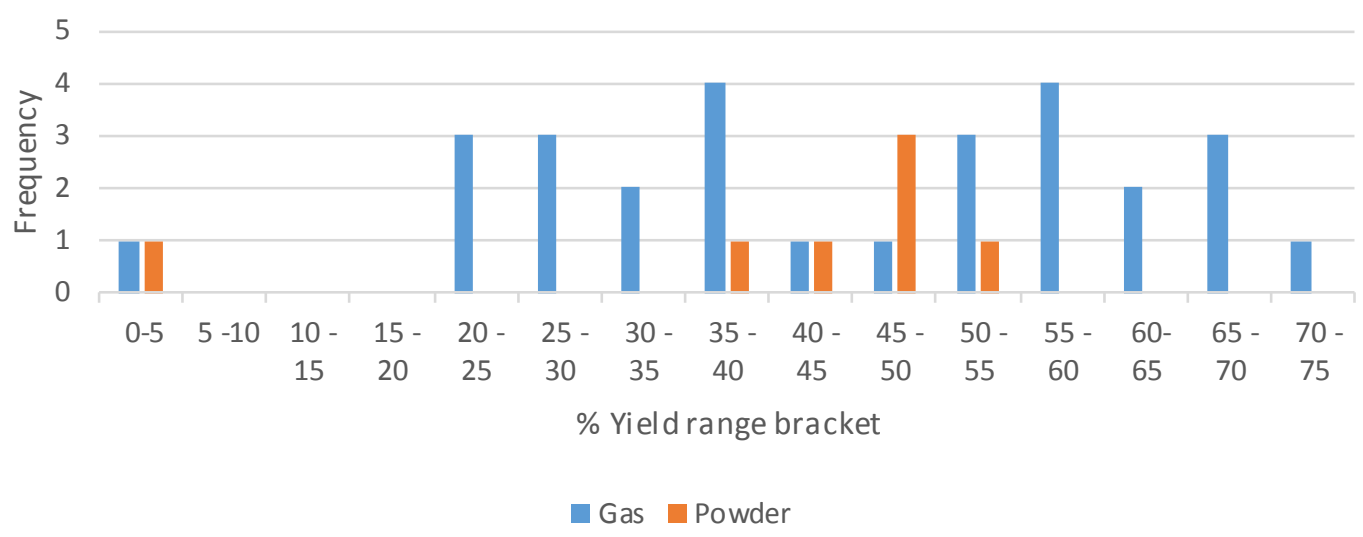

Figure 52. Distribution of yields of $\mathbf{9 0}$ for the mode of addition of formaldehyde

From Figure 52, it can be seen that the yields from formaldehyde added as a gas are much more variable then the yields from addition of paraformaldehyde powder. The average yields (when discarding the outlier at $0-5 \%$ ) are both approximately the same $-46.3 \%$ for powder addition, and $45.7 \%$ for gas addition. Addition of formaldehyde as a gas is clearly necessary if optimum yields are to be obtained, but without eliminating the source of the poor and variable reactivity, both methods would be equally efficient over the long run.

\section{Investigation of concentration effects}

Reaction concentration was another factor that was investigated, but was ultimately found to have no effect on overall yield. There was a concern over a certain aspect of lithium chemistry, in that the lithium cation coordinates readily to any compound carrying a lone pair, and the number of possible species in any given reaction mixture can be combinatorially vast. ${ }^{6}$ There have been examples before of higher-order aggregates inhibiting reaction rates in concentrated solutions, and it was thought that by increasing the dilution, the formation of such aggregates would be reduced.

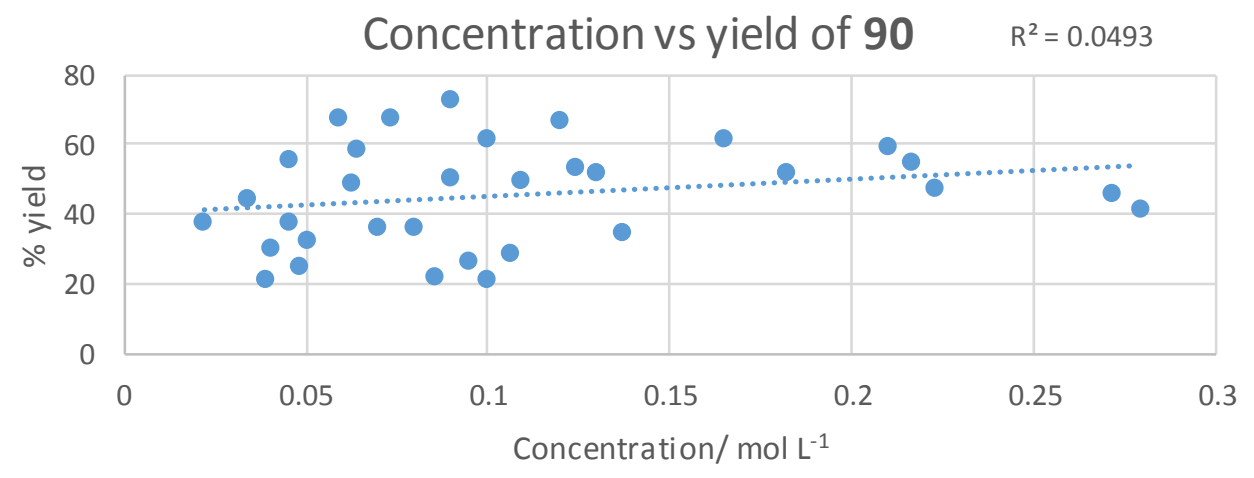

Figure 53. Relationship between concentration and the yield of $\mathbf{9 0}$ 
When plotting reaction concentration vs yield, no correlation was observed $\left(\mathrm{R}^{2}=0.05\right)$ (Figure 53). While this does not rule out aberrant behaviour resulting from lithium coordination, it does present strong evidence of a lack of aggregation effects.

\section{Effect of dry ice/ethylene glycol bath and scaling up}

The ice-water bath used to maintain the temperature of the reaction was considered to be a potential source of reprotonation. Despite all reactions being performed with a rubber septum under inert atmosphere, a slow colour change of the golden-brown solution of the azulenide $\mathbf{1 6 3}$ to green-blue upon sitting was often observed, suggesting reprotonation through penetration of water through the septum. When performing this reaction using an ethylene glycol/ $/ \mathrm{CO}_{2}$ bath $\left(-30{ }^{\circ} \mathrm{C}\right)$, the golden-brown azulenide species 163 retained its colour for much longer. The first attempt at performing this reaction with an ethylene glycol $/ \mathrm{CO}_{2}$ bath gave a record $73 \%$ yield that so far has notbeen matched.

Nevertheless, encouraged by this high yield, the reaction was gradually scaled up from the $100 \mathrm{mg}$ scale to the gram scale. The yields from these reactions typically ranged from $52 \%$ to $62 \%$, a marked increase in consistency. It is not clear whether this increased consistency is due to the larger scale or due to the use of the ethylene glycol/ $\mathrm{CO}_{2}$ bath. Unfortunately, at the gram scale, the cannula transferring formaldehyde gas into the reaction mixture would regularly get blocked with paraformaldehyde solid, necessitating direct addition of the powder and resulting in diminished yields. This would occur even in a 14-gauge (1.5 mm inner diameter) cannula, the largest bore available in the lab.

\section{Other variables and their effects}

Several other variables were considered. Glassware and reagents were subject to varying amounts of drying - including storage of all reagents in a desiccator over phosphorus pentoxide, and rigorous drying of all glassware with a heat gun under vaccuum. To our knowledge, use of rigorous drying techniques over and above the normal precautions (oven-dried glassware, dry solventsand inert atmosphere) had no measurable effect.

The formaldehyde gas is typically transferred via cannula from a sealed vial containing paraformaldehyde. In an attempt to circumvent blockage of the cannula during addition, a positive nitrogen flow through the cannula was tried, which resulted in very low ( $\sim 25 \%)$ yields. Attempting to dry the formaldehyde gas by passing it through a calcium chloride plug resulted in no gas flow beyond the plug. 
Washing the product 90 with dilute hydrochloric acid during the workup procedure was found to cause rapid degradation. Even after removal of the aqueous layer and the subsequent chromatography of the organic layer, 6-(2-hydroxyethyl)azulene would degrade to a green material in minutes.

\section{Formation of the unsymmetrical dimer 168}

Alongside 6-(2-hydroxyethyl)azulene (90), these reactions often gave small quantities of an unsymmetrical dimer 168 (Figure 54). It had been occasionally observed that the 6-(2hydroxyethyl)azulene spot on the TLC plate would over time resolve into two spots with very similar $\mathrm{R}_{\mathrm{f}}$ values; one major and one minor, but the minor would seemingly disappear during column chromatography and in the ${ }^{1} \mathrm{H}$ NMR spectrum. It was only when an NMR spectrum was obtained of 6-(2-hydroxyethyl)azulene with peaks attributable to ${ }^{13} \mathrm{C}$ satellites appearing to be in $10 \%$ abundance, that it was realised these peaks in fact belonged to the missing minor product (Figure 55).

Frustratingly, this compound was very difficult to separate from 6-(2-hydroxyethyl)azulene (90). The two compounds have very similar $\mathrm{R}_{\mathrm{f}}$ values in many solvents, with a significant degree of overlap. Purification of large quantities of the impure $\mathbf{9 0}$ was tedious, as performing column chromatography on the mixed fraction gave a separation of approximately $50 \%$ each time. Even after successful separation from 90 , the fraction containing 168 was not wholly pure.

The ${ }^{1} \mathrm{H}$ NMR spectrum of compound 168 (Figure 56) shows that the aromatic proton peaks are shifted simultaneously upfield and downfield, on account of the compound's slightly asymmetrical structure. The off-centre hydroxymethyl appears to slightly shield one azulene ring and deshield the other. When compounded with its relative abundance of $2-4 \%$ it was able to almost flawlessly masquerade as satellites of 90 (Figure 55).

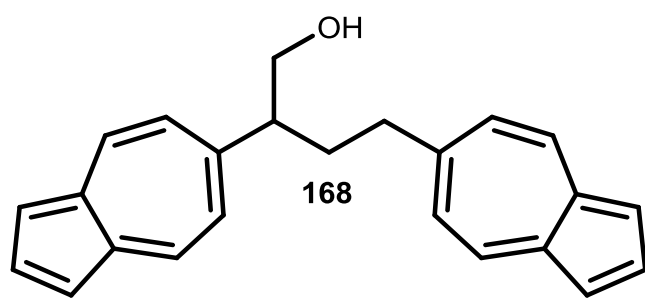

Figure 54. The unsymmetrical dimer 168 


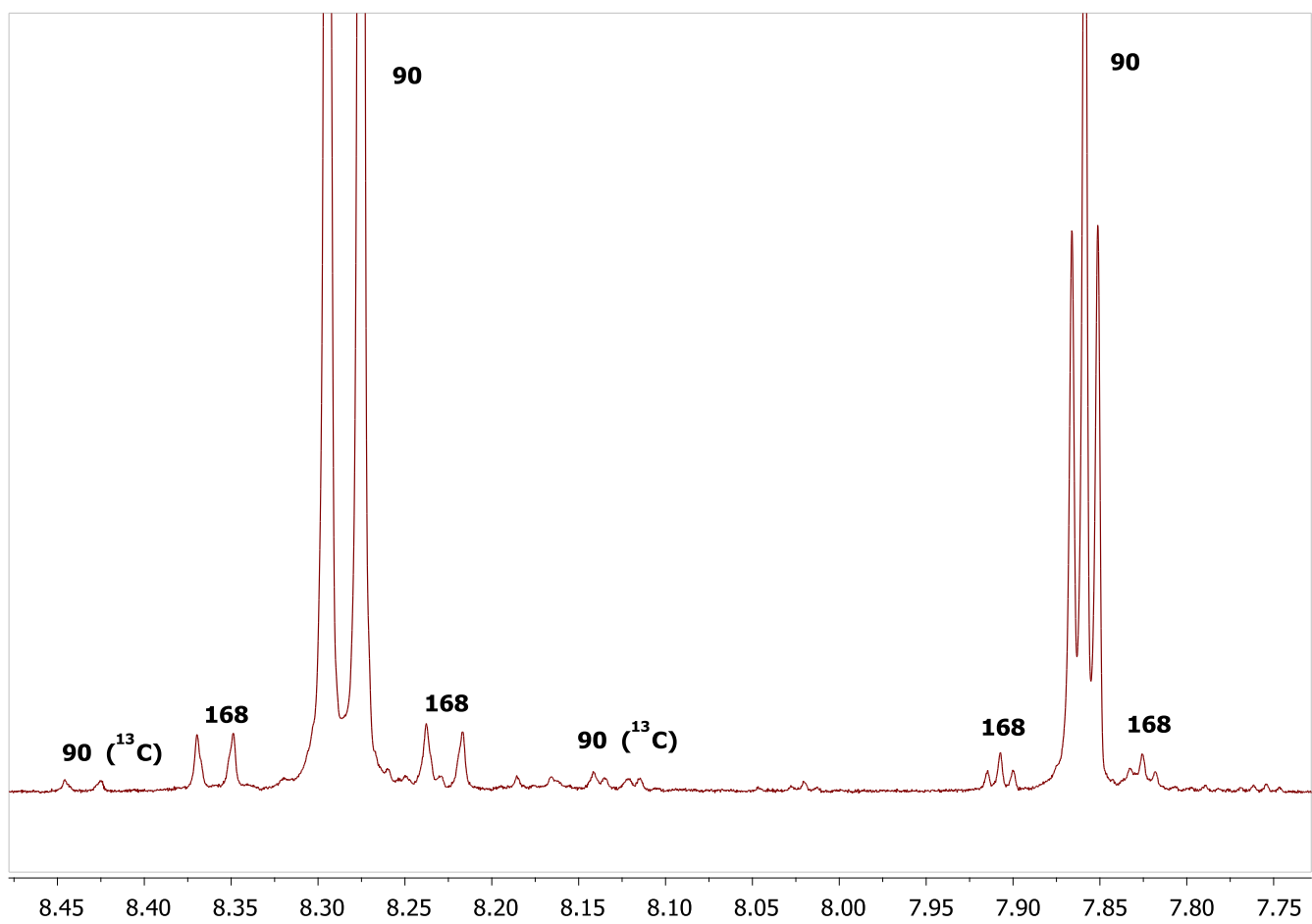

Figure 55. An expansion of the ${ }^{1} \mathrm{H}$ NMR spectrum of i mpure 90 , showing peaks belonging to the 4 - a nd 2-positions with the peaks belonging to the dimer $\mathbf{1 6 8}$ masquerading as carbon satellites, and the satellites themselves.

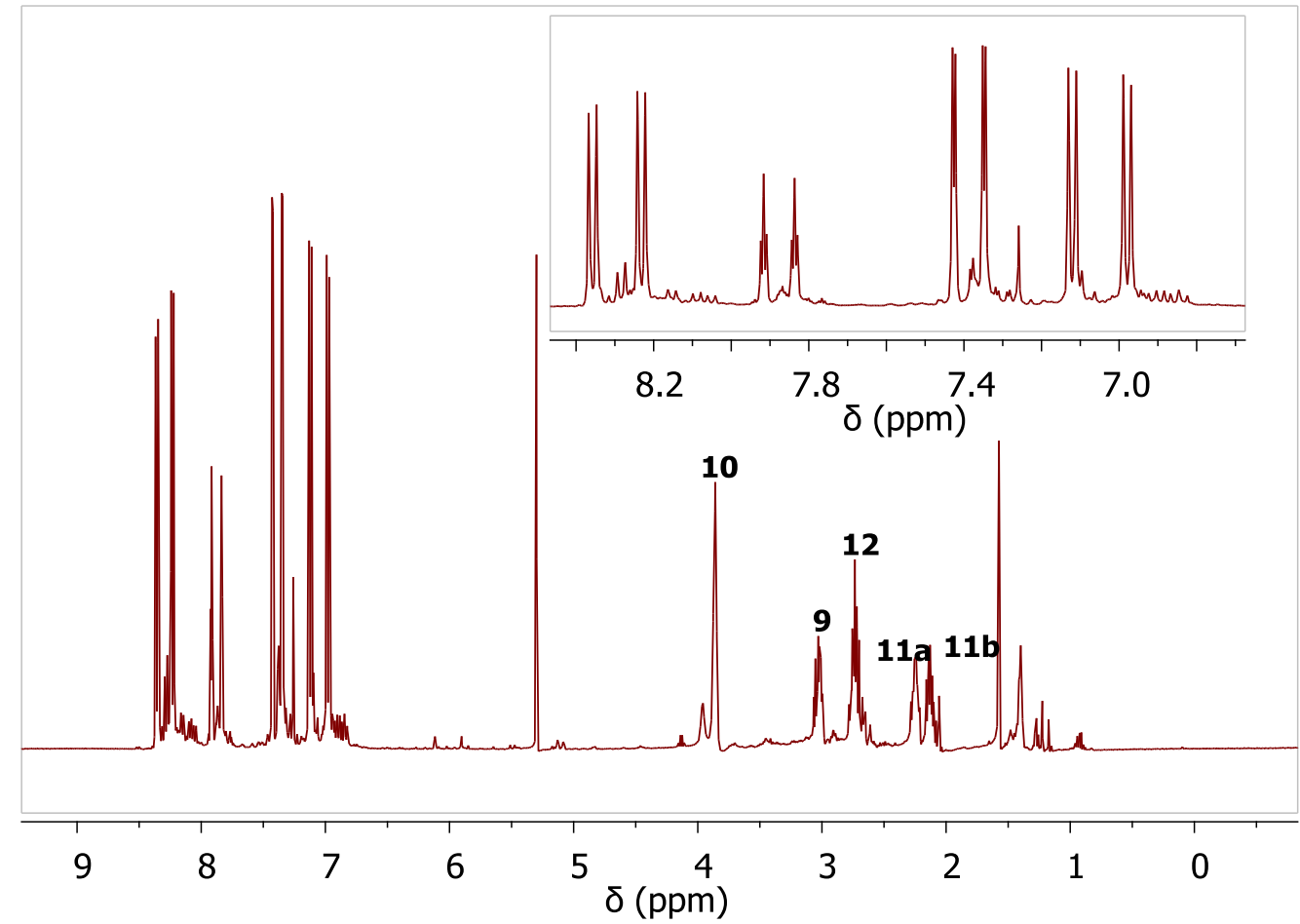

Figure 56. Spectrum of $\mathbf{1 6 8}$ with blowup of a romatic region

The assignment of this structure was based on the COSY and HSQC data from the aliphatic region, as well as the presence of two non-identical azulene rings. The HSQC spectrum shows four unique carbon environments, with three methylenes and a methine. The protons on one methylene are non-equivalent. Performing a COSY walk from $3.86\left(\mathrm{CH}_{2}\right.$, adjacent to the hydroxyl) gives the sequence $3.02(\mathrm{CH}) \rightarrow 2.13\left(\mathrm{CH}_{2}\right) \rightarrow 2.73\left(\mathrm{CH}_{2}\right)$ (Figure 57). 


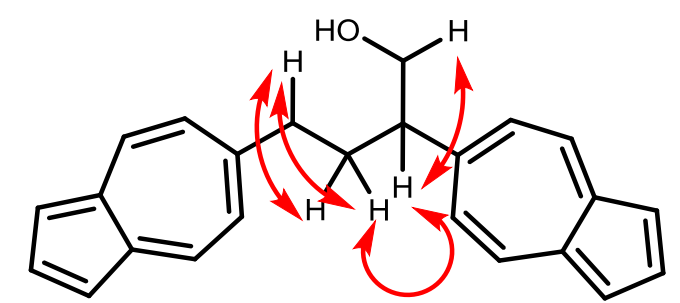

Figure 57. COSY correlations for the structural assignment of $\mathbf{1 6 8}$

It is unclear how the compound $\mathbf{1 6 8}$ forms in the synthesis of $\mathbf{9 0}$. A brief retrosynthetic treatment suggests that one equivalent of 6-(2-hydroxyethyl)azulene becomes deprotonated at the sidechain adjacent to the 6-position, which then goes on to react with positive charge beta to the 6-position on the other equivalent (Scheme 69).

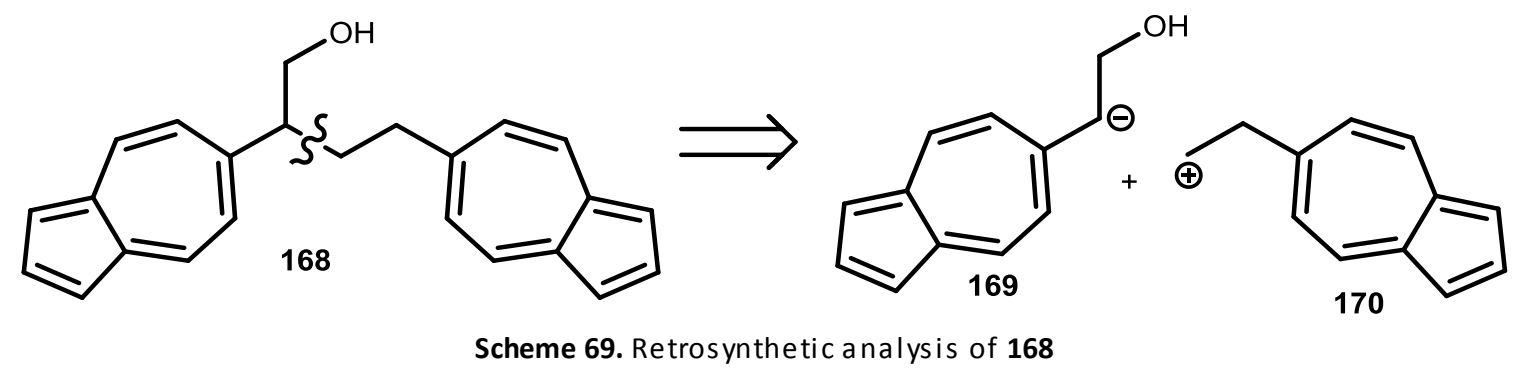

Formation of a carbanion on a compound with a free hydroxyl (whether protonated or deprotonated) is unlikely, so this suggests the hydroxyl on 169 is substituted somehow. There are two main possible identities for the carbocation synthon 170, either 6-vinylazulene or a 6-(2alkoxyethyl)azulene 171 substituted with a leaving group (Figure 58). 6-Vinylazulene (94) was judged to be unlikely, asit has never been observed as a product of these reactions.

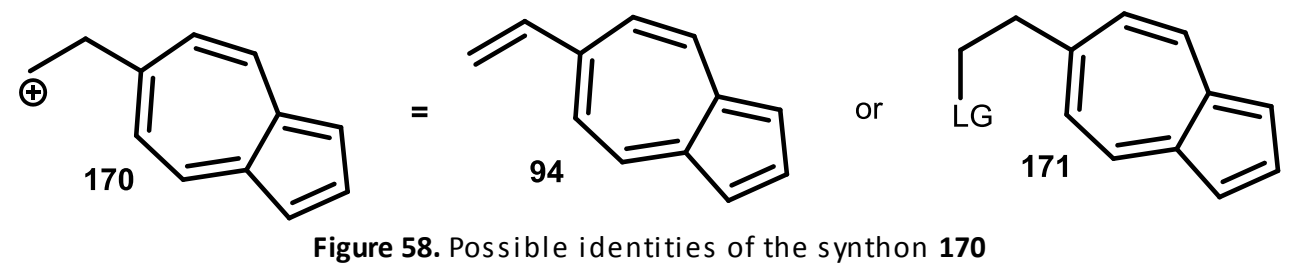

One plausible mechanism involves the formation of an oxymethylene chain on the end of 6-(2-oxyethyl)azulene $\mathbf{1 7 2}$ to form $\mathbf{1 7 3}$. Formaldehyde gas readily condenses and repolymerises in solution to form an equilibrium with paraformaldehyde, so the formation of 6-(2-ethylenyl)azulene end-capped polyoxymethylene $\mathbf{1 7 4}$ is not outside the realm of possibility (Scheme 70.) Formation of $\mathbf{1 7 4}$ circumvents the two mechanistic problems discussed earlier the acidic position adjacent to the 6-position on azulene is spatially more removed from the hydroxyl functionality at the end of the oxymethylene chain, and the chain also functions as an acceptable leaving group. 


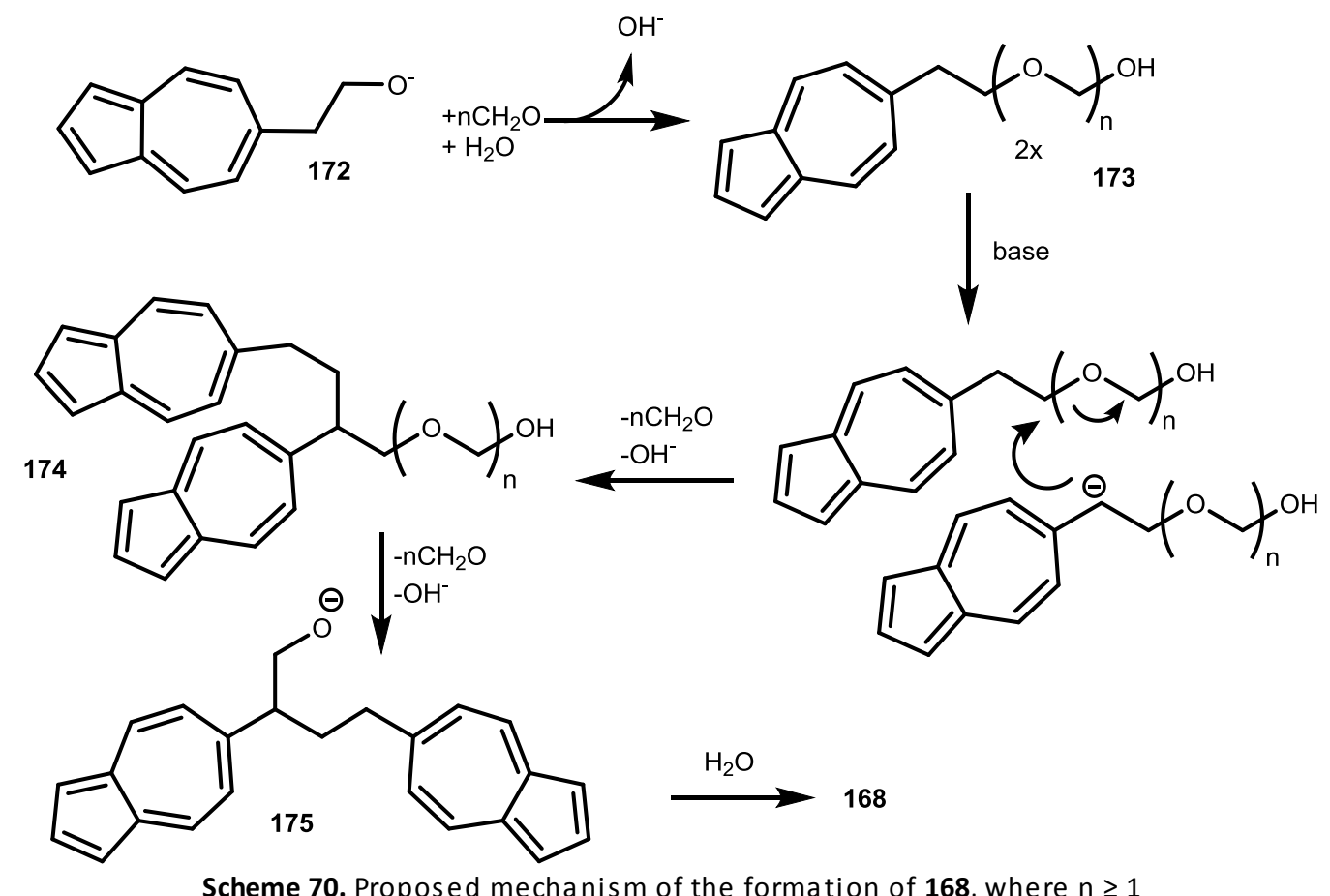

\section{Summary}

Efficient synthesis of large quantities of 6-(2-hydroxyethyl)azulene (90) was crucial to the success of the 6-substituted protecting group 89. Many variables were investigated and found to have no detectable effect on the overall yield. Use of an ethylene glycol/dry ice bath gave much more consistent results and resulted in a modest overall increase in yield. There nevertheless remain possible ways of increasing the overall yield (perhaps by further preparation or manipulation of paraformaldehyde), but nevertheless enough pure 6-(2-hydroxyethyl)azulene had been generated for subsequent protection and deprotection studies.

\subsection{Development of protection methodology}

With 6-(2-hydroxyethyl)azulene in hand, exploration of the conditions necessary for alcohol, amine and carboxylic acid protection commenced (Scheme 71).

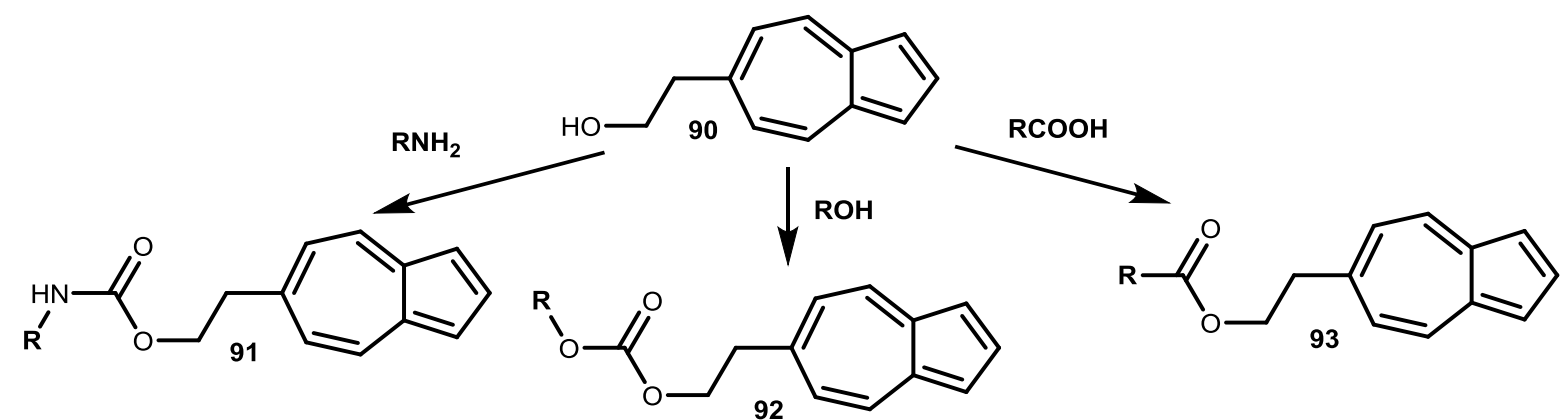

Scheme 71. Amines, alcohols and carboxylic a cids were ta rgeted as substrates for development of protection methodology 
Carboxylic acid protection was to be achieved through esterification of the acid substrate with 90. There are many easy and high-yielding esterification protocols available from which a protection reaction could be developed, such as Yamaguchi, ${ }^{7}$ Steglich, ${ }^{8}$ Mitsunobu, ${ }^{9}$ Corey-Nicolaou, ${ }^{10}$ and other protocols (Scheme 72).

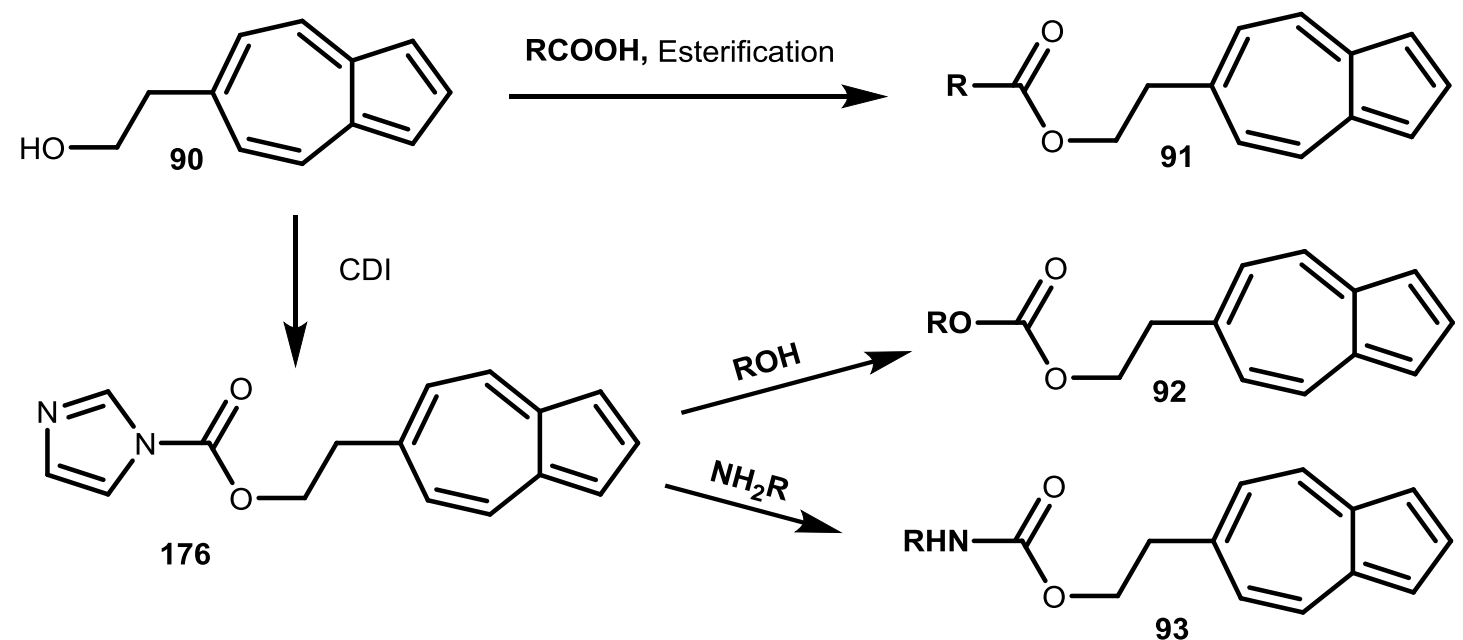

Scheme 72. General strategy of protection of alcohols, amines and carboxylic acids

Access to alcohol and amine protection was to be achieved through treatment of $\mathbf{9 0}$ with carbonyldiimidazole (CDI) to form the carbonylimidazole species $\mathbf{1 7 6}$, followed by treatment (either in one pot or after a purification step on 176) with the alcohol or amine substrate to form the corresponding carbonate 92 or carbamate $\mathbf{9 3}$, respectively.

\subsubsection{Carboxylic acid protection}

Protection of carboxylic acids was chosen as the first area of investigation. There are many effective and high-yielding procedures for ester formation, so the development of protection methodology was expected to be relatively easy in comparison to the methodology for alcohol and amine protection. This was indeed the case, and efficient protection through Steglich esterification allowed rapid progress to be made towards investigation of the deprotection conditions covered in chapter $\mathbf{5}$.

The carboxylic acid chosen for the initial investigation was cinnamic acid 177. This is because cinnamic acid is relatively non-polar for a carboxylic acid, easy to handle, easy to identify and integrate in a ${ }^{1} \mathrm{H}$ NMR spectrum and easy to visualise on TLC, whether under UV or with a permanganate stain.

The original intention was to do a survey of many established esterification reactions to find suitable protection conditions. This turned out to be unnecessary, as after trialling Yamaguchi 
esterification and thionyl chloride-based activation of cinnamic acid, Steglich esterification was settled on as a reliable, high yielding, mild protection protocol.

The yields for Yamaguchi esterification were low, typically producing the cinnamate $\mathbf{1 7 8}$ in yields of less than 50\%. An inseparable by-product was present in the purified material, and the ${ }^{1} \mathrm{H}$ NMR spectrum of the mixture suggested the presence of the trichlorobenzoate ester 179 (Scheme 73).
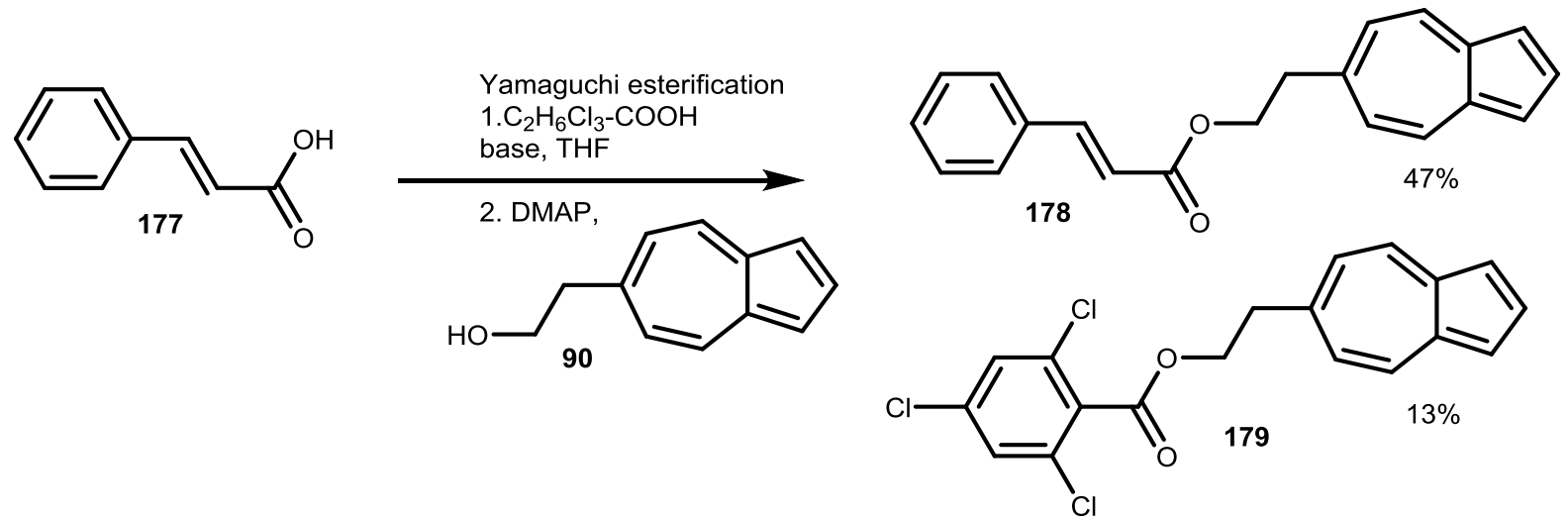

Scheme 73. Ya ma guchi esterification of cinnamic acid $\mathbf{1 7 7}$ with $\mathbf{9 0}$ gave incomplete conversion to $\mathbf{1 7 8}$ a nd small a mounts of a compound thought to be $\mathbf{1 7 9}$

Direct reaction of $\mathbf{9 0}$ with an acid chloride generated using thionyl chloride resulted in complete degradation of the azulene moiety, with no detectable azulene species present by TLC (Scheme 74). It is believed this degradation is due to the sensitivity of 6-(2-hydroxyethyl)azulene to the acidic conditions present in the reaction mixture. As mentioned in section 4.2.2, hydrochloric acid would induce rapid degradation of 6-(2-hydroxyethyl)azulene.

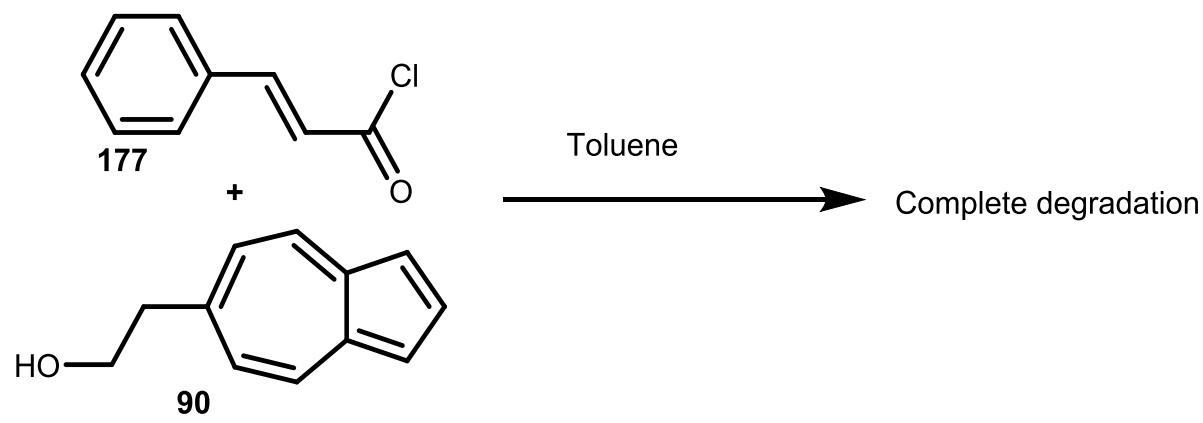

Scheme 74. Attempted coupling of $\mathbf{9 0}$ with cinnamyl chloride

\section{Steglich esterification}

Steglich esterification involves the use of stoichiometric quantities of the carbodiimide DCC (although EDCI can be employed to the same effect) and catalytic quantities of DMAP to deprotonate the acid and reprotonate the species 180 (Scheme 75). ${ }^{8}$ It is a robust and mild way of generating esters at room temperature. As a protection reaction, these features are ideal, with the only drawbacks being the inability to protect a carboxylic acid when an unprotected hydroxyl 
group is present elsewhere in the molecule (as intermolecular esterification and/or lactonisation can occur) and the occasional difficulties with removing the dicyclohexylurea by-product 181.

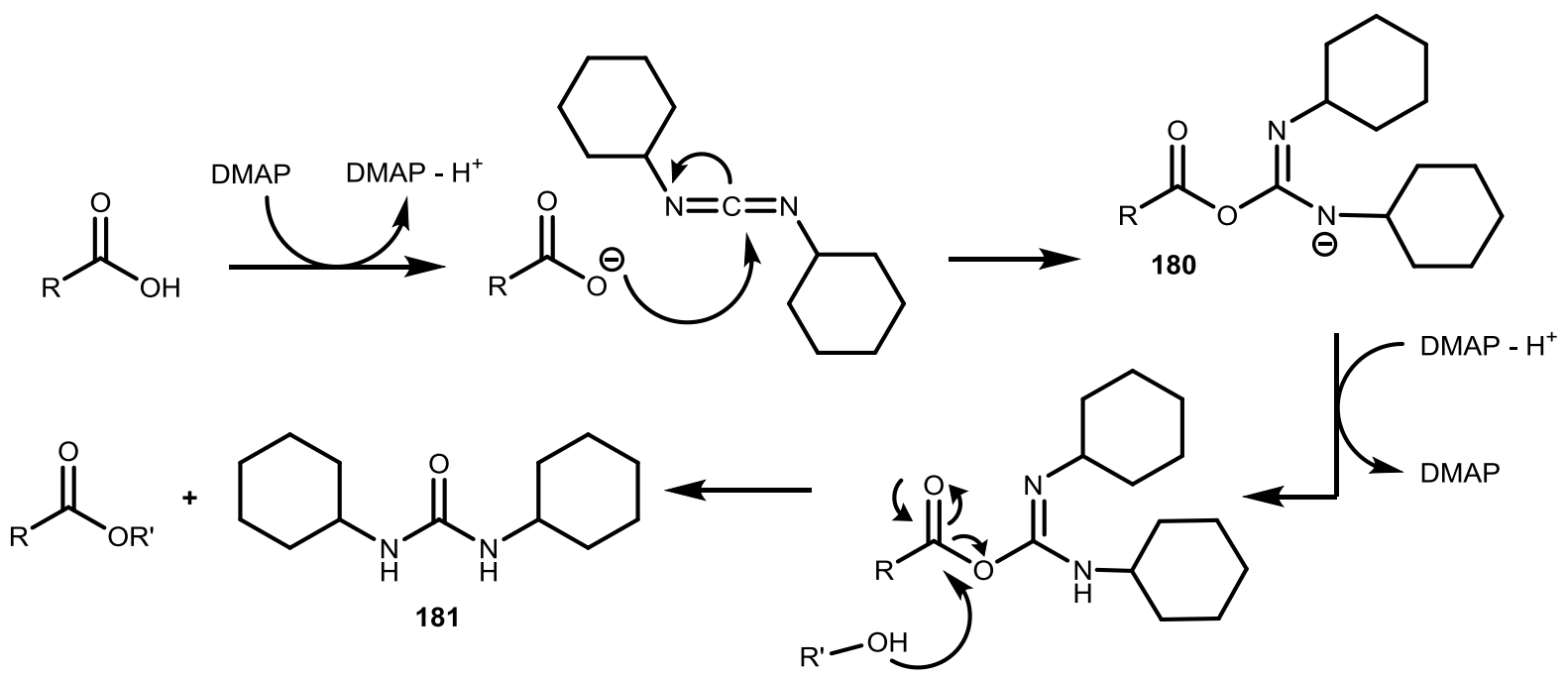

Scheme 75. Mechanism of Steglich esterification

Steglich esterification of cinnamic acid with 6-(2-hydroxyethyl)azulene using DCC as the coupling reagent gave the desired ester in yields ranging from $83 \%$ to $93 \%$ in 3 hours (Table 8). Use of EDCI resulted in more sluggish esterification, which would explain the somewhat lower yield of 79\% over the same time period. The ester coupling for 178 (and all other esters) was confirmed by HMBC correlation between the carboxylate carbon and the methylene protons on the distal end of the 6-(2-oxyethyl)azulene moiety.

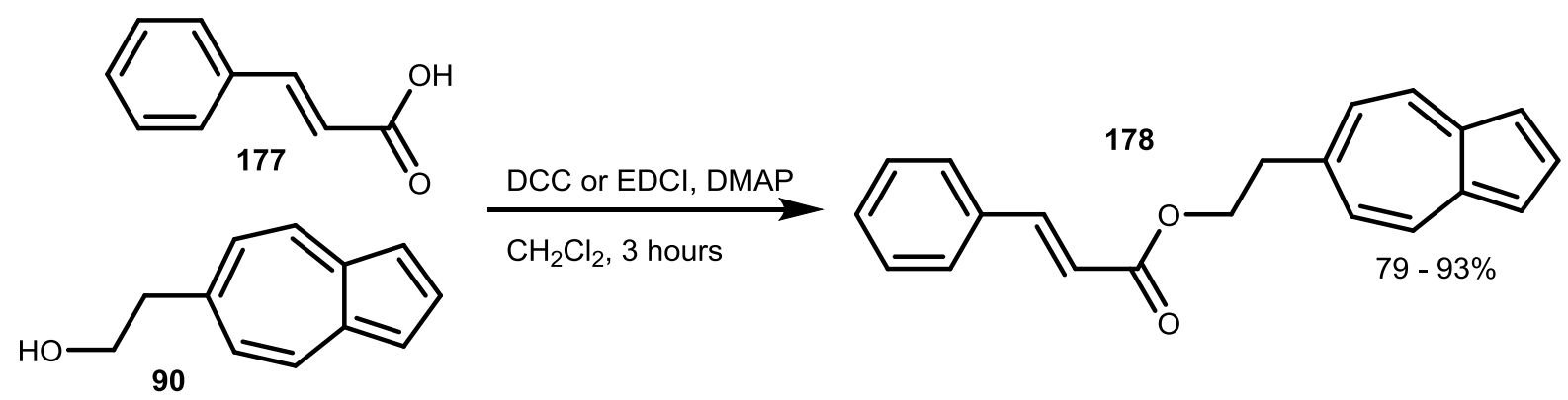

Table 8. Steglich esterification of cinnamic acid (177) with 90

\begin{tabular}{|l|l|l|l|l|}
\hline Coupling agent & Eq. coupling agent & Eq.90 & DMAP / mol\% & Yield \% \\
\hline DCC & 1.27 & 0.77 & 10 & 83 \\
\hline EDCI & 1.1 & 1.18 & 12 & 79 \\
\hline DCC & 1.1 & 1.13 & 17 & 93 \\
\hline DCC & 1.09 & 0.91 & 32 & 89 \\
\hline
\end{tabular}

With such high yielding and clean reactions, the protocol was scaled up to produce sufficient amounts of material for further deprotection studies, as well as create a selection of other protected acids with differing physical and electronic properties (Table 9). 


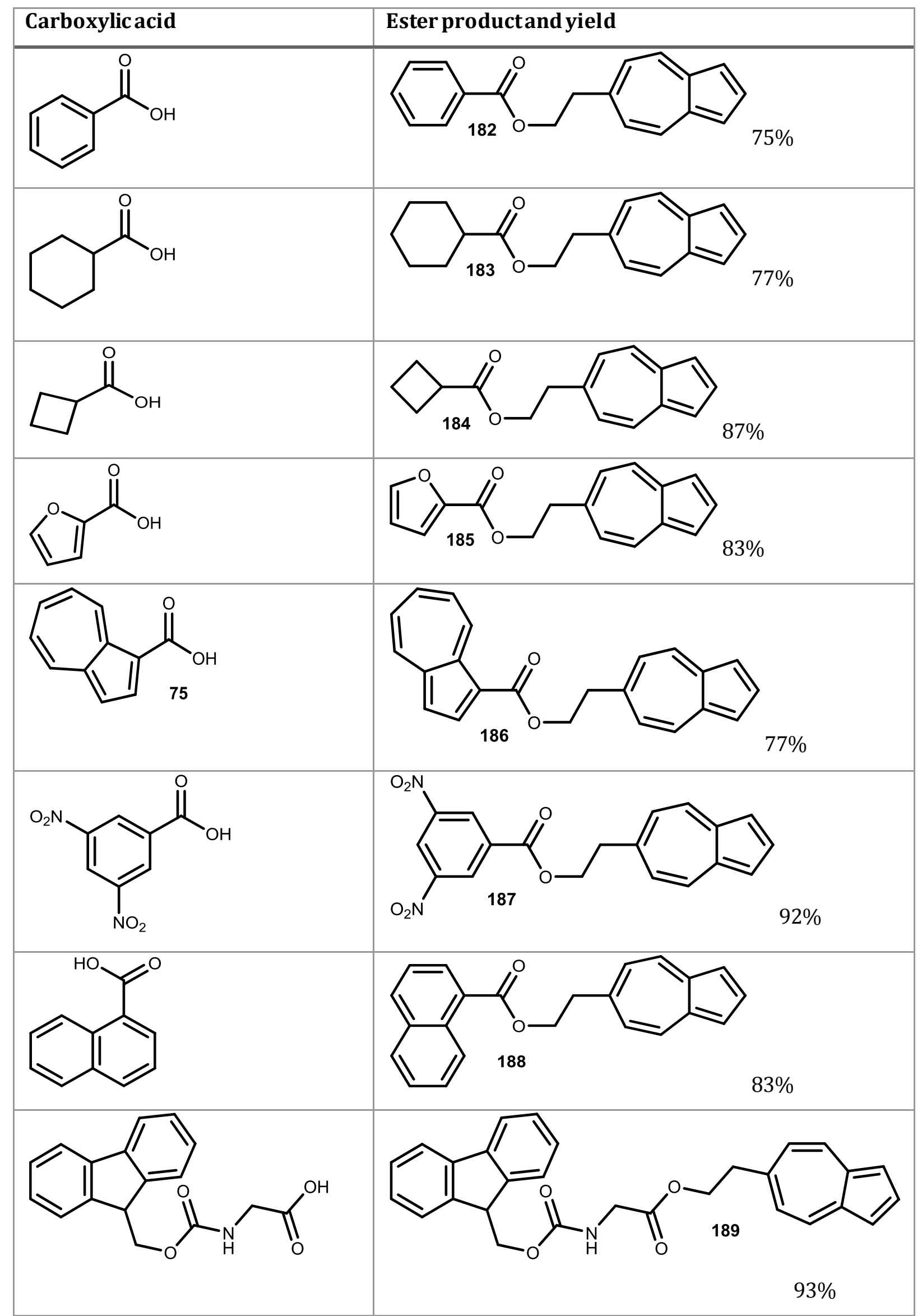

Table 9. A selection of carboxylic acids were protected through Steglich methodology 
With the exception of the esterification of azulene-1-carboxylic acid $\mathbf{7 5}$, all of these reactions were complete in $3-4$ hours. For the coupling of $\mathbf{7 5}$, the reaction required heating at reflux overnight and stoichiometric quantities of DMAP to proceed to completion. The sluggishness of this reaction was presumably due to the low acidity of the carboxylic acid group. Azulene-1-carboxylic acid has a pKa of 7 on account of the electron-donating nature of the azulene ring, whereas other carboxylic acids have pKas that typically range from 3.5 to $5 .{ }^{11}$ As a result, 75 is more resistant to deprotonation by DMAP.

\section{Solid-state protection of carboxylic acids}

Protection of carboxylic acids through Steglich esterification is by no means restricted to the solution phase.12 High-yielding solid-state esterifications have been shown to be possible by Cedric McLeland et al. ${ }^{13}$

The cinnamate 178 and benzoate 182 have been made by grinding the starting materials in a mortar \& pestle for approximately 10 minutes (Table 10). The yields for these reactions were usually slightly lower than those of solution-phase Steglich esterifications, probably due to the incomplete mixing that is typical of solid-phase reactions. Both DCC and EDCI can be used in these solid-state reactions, although as before, EDCI reacted more sluggishly and required a longer grinding period.

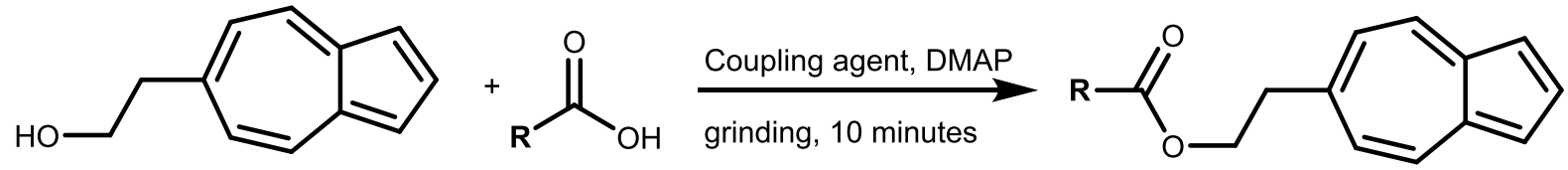

Table 10. Solid state Steglich syntheses of esters 178 and 182

\begin{tabular}{|l|l|l|l|}
\hline Carboxylic acid & Coupling agent & Eq. coupling agent & Yield \\
\hline Cinnamic acid & DCC & 1.5 & $78 \%$ \\
\hline Benzoic acid & DCC & 1.3 & $75 \%$ \\
\hline Benzoic acid & EDCI a & 1.5 & $77 \%$ \\
\hline
\end{tabular}

a 15-20 minutes of grinding.

\subsubsection{Protection of amines and primary alcohols}

Development of protocols for alcohol and amine protection proved somewhat trickier. One of the primary ways of forming carbonates and carbamates involves use of phosgene. ${ }^{14}$ Use of phosgene was not pursued due to toxicity concerns and because 6-(2-hydroxyethyl)azulene wold almost certainly react with phosgene at the 1-position, possibly faster than at the hydroxyl group. 
Carbonyldiimidazole is often employed as a milder and less toxic phosgene analogue, and has been used for carbonate and carbamate formation as well as esterifications. ${ }^{15}$ Initial difficulties were encountered with the use of CDI, but they were overcome and protocols were developed with acceptable yields for the protection of alcohols and amines.

\section{One-step protocol for alcohol protection}

The reaction protocols that were followed came originally from Davis et al. ${ }^{16}$ In these protocols, unsymmetrical carbonates are prepared by adding one alcohol to a reaction mixture containing $\mathrm{CDI}$ and catalytic quantities of $\mathrm{KOH}$ in toluene at $60{ }^{\circ} \mathrm{C}$, followed by addition of the second alcohol after several hours. The authors mention that the alkyl carbonyl imidazole intermediate 190 is much less reactive than CDI itself, (Scheme 76) which means that while CDI can react with any alcohol, the intermediate $\mathbf{1 9 0}$ will only react with sufficiently sterically un-demanding primary alcohols at anything resembling an appreciable rate, so the resulting unsymmetrical carbonate can only ever have one secondary or tertiary alcohol attached. 6-(2-Hydroxyethyl)azulene is a primary alcohol, so in principle protection should be accessible for all alcohol species.<smiles>O=C(n1ccnc1)n1ccnc1</smiles>

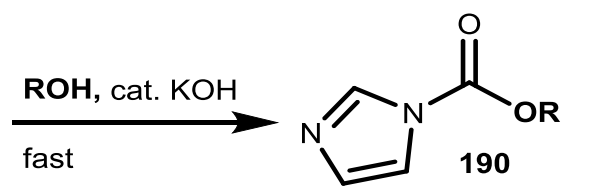<smiles>[R]O[C@H](C)[C@H](C)O</smiles><smiles>[R]OC(=O)O[R2]</smiles>

Scheme 76. Generalised depiction of reactions with CDI

A one-step procedure for carbonate formation was attempted with 6-(2-hydroxyethyl)azulene and cyclohexanemethanol as the other alcohol species. $\mathbf{9 0}$ was added first, so as to aid reaction troubleshooting (rendering all subsequent reaction intermediates visible). With 1.3 equivalents of cyclohexanemethanol, a 97\% yield of the product was achieved (Scheme 77).
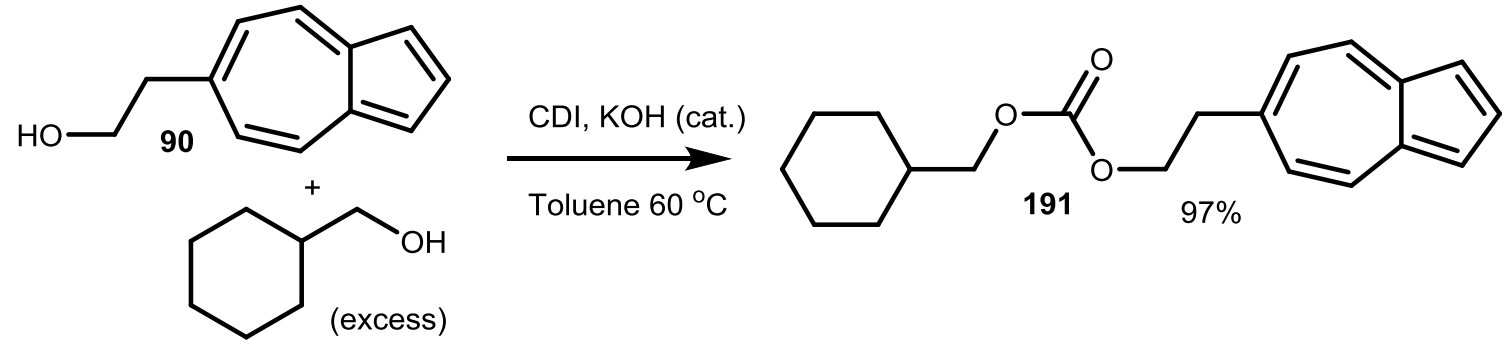

Scheme 77. Synthesis of the cyclohexanemethanol carbonate 191

An issue with one-pot generation of unsymmetrical carbonates is that the reaction stoichiometry must be precisely equimolar, otherwise an excess of one material over another leads to disproportionate amounts of symmetrical carbonate formation. To illustrate, if CDI is in $20 \%$ excess after the addition of the first alcohol (the protecting group), addition of the second alcohol substrate (which should be the limiting reagent) will result in preferential reaction with CDI over 
the monoimidazole carbamate $\mathbf{1 9 0}$, leading to a $20 \%$ loss of the substrate as an unwanted carbonyl monoimidazole. The remaining 0.8 equivalents of the second alcohol can then react with either of the carbonyl imidazole species, generating either the intended carbonate or a symmetrical carbonate by-product. A loss of material ranging from 20 - 40\% can ultimately occur, depending on the comparative rates of reaction between the carbonyl monoimidazole species.

Furthermore, if CDI is in a $20 \%$ deficit (i.e. 0.8 eq.), then after addition of the first alcohol, the remaining 0.2 equivalents of this alcohol can react to form a symmetrical carbonate, leaving only 0.6 equivalents of the mono-imidazole intermediate to react with the substrate.

These issues of stoichiometry are compounded by the fact that CDI is highly sensitive to water, and easily degrades to imidazole, losing $\mathrm{CO}_{2}$ in the process. Even unopened bottles of CDI can contain significant quantities of imidazole, making it necessary to determine the CDI content by ${ }^{1} \mathrm{H}$ NMR integration (using $\mathrm{CDCl}_{3}$ dried with molecular sieves) and adjusting quantities accordingly.

\section{Two-step protocol}

Because of these stoichiometic issues, a two-step approach towards protection was tried. Generation of the carbonyl monoimidazole of 6-(2-hydroxyethyl)azulene 176 proceeded quantitatively, even at room temperature without catalytic KOH (Scheme 78). The reaction can be performed with excess CDI, as it is removed during purification. This material is surprisingly stable to air and moisture, and can be kept for up to a week in open air before requiring repurification. In fact, its handling properties are better than 6-(2-hydroxyethyl)azulene, as it dries more easily and forms a fluffy solid instead of a solid film that is difficult to remove via spatula.

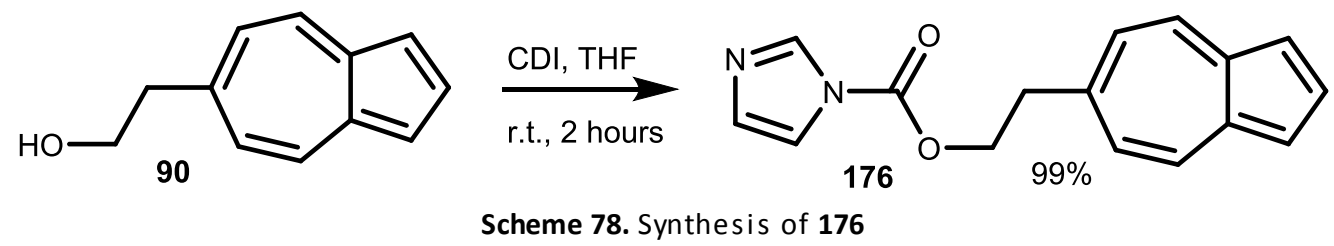

Coupling of this intermediate to cyclohexanemethanol proved more difficult than expected. When the literature protocol was followed, a poor yield of $16 \%$ was obtained even after extending the reaction time to overnight. Typically, a large quantity of starting material would remain, along with small quantities of other products such as 6-vinyl azulene (94), 6-(2-hydroxyethyl)azulene (90) and the symmetrical carbonate 192 (Table 11). 


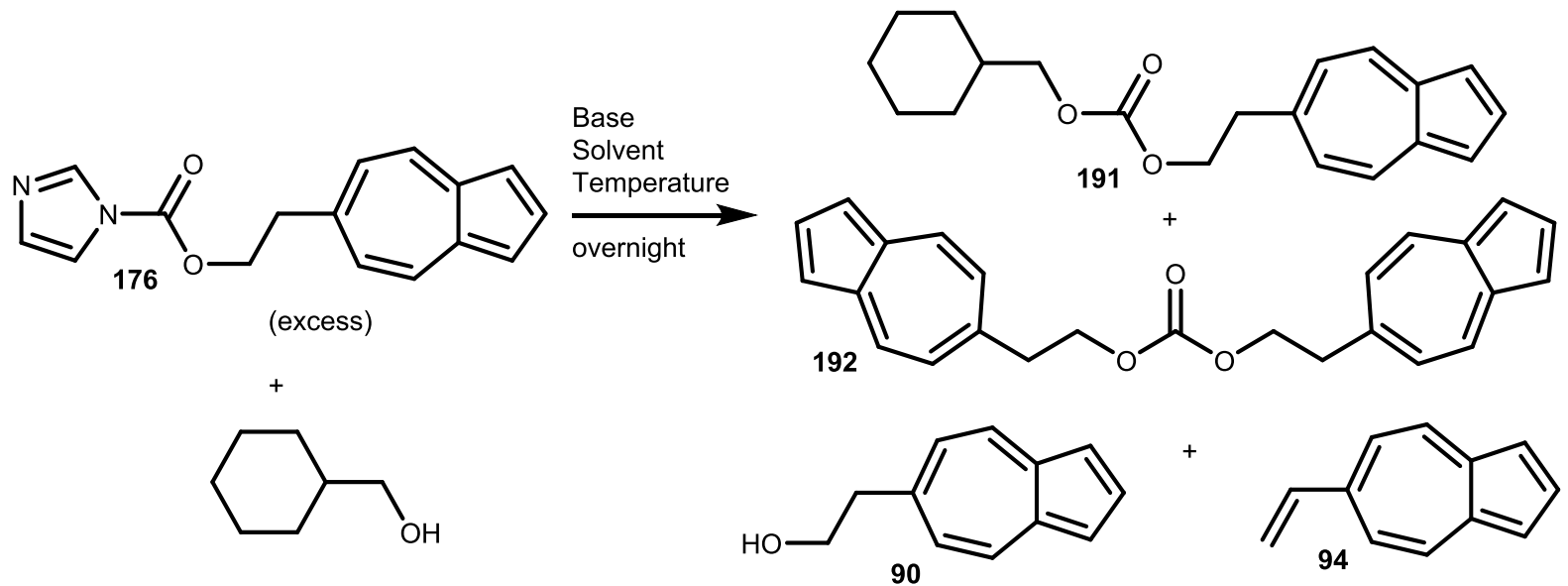

Table 11. Attempted optimisation of the formation of 191

\begin{tabular}{|c|c|c|c|c|c|c|c|c|c|}
\hline Entry & $\begin{array}{ll}\text { Eq. of } \\
176\end{array}$ & Base & Solvent & Temp. & $\begin{array}{l}\% \text { yield } \\
191^{a}\end{array}$ & $\begin{array}{l}\text { \% yield } \\
192\end{array}$ & $\begin{array}{l}\text { \% yield } \\
90\end{array}$ & $\begin{array}{l}\% \text { yield } \\
94\end{array}$ & $\begin{array}{l}\text { RSM } \\
176\end{array}$ \\
\hline 1 & $\begin{array}{l}1.01 \\
\text { eq. }\end{array}$ & $\begin{array}{l}\mathrm{KOH} \\
(12 \mathrm{~mol} \%)\end{array}$ & Toluene & $60^{\circ} \mathrm{C}$ & 16 & - & 11 & trace & 77 \\
\hline 2 & $\begin{array}{l}1.12 \\
\text { eq. }\end{array}$ & $\begin{array}{l}\mathrm{K}_{2} \mathrm{CO}_{3} \text { (1 eq.) } \\
\mathrm{KOH}(0.5 \text { eq.) }\end{array}$ & Toluene & $65^{\circ} \mathrm{C}$ & 26 & 12 & 30 & trace & 36 \\
\hline 3 & $\begin{array}{l}1.14 \\
\text { eq. }\end{array}$ & $\begin{array}{l}\mathrm{KOH} \\
(69 \mathrm{~mol} \%)\end{array}$ & THF & reflux & 25 & - & - & 3 & 74 \\
\hline 4 & $\begin{array}{l}0.87 \\
\text { eq. }\end{array}$ & - & $\mathrm{MeCN}$ & r.t. & 5 & - & - & - & 77 \\
\hline 5 & $\begin{array}{l}0.79 \\
\text { eq. }\end{array}$ & $\begin{array}{l}\mathrm{KOH} \\
(34 \mathrm{~mol} \%)\end{array}$ & $\mathrm{MeCN}$ & reflux & 60 & - & - & 27 & 5 \\
\hline $6^{b}$ & $\begin{array}{l}1.02 \\
\text { eq. }\end{array}$ & $\begin{array}{l}\mathrm{KOH} \\
(19 \mathrm{~mol} \%)\end{array}$ & Toluene & Reflux & 63 & - & 8 & 7 & 14 \\
\hline
\end{tabular}

a Calculated relative to cyclohexanemethanol. b 4 hour reaction time.

Several alterations in the reaction conditions were tried. Stoichiometric amounts of $\mathrm{NEt}_{3}$ gave only starting material, and only modest reactivity was observed when using $\mathrm{K}_{2} \mathrm{CO}_{3}$ (Table 11). The solvents THF, MeCN and toluene were tried at different temperatures, but little change in reactivity was seen between solvents at the same temperature (compare entry 1 and 3 in Table 11). Refluxing in acetonitrile $\left(\sim 80{ }^{\circ} \mathrm{C}\right)$ gave a $60 \%$ yield, which suggested that the primary variable affecting yield was temperature. Finally, heating at toluene reflux $\left(\sim 110{ }^{\circ} \mathrm{C}\right)$ gave the slightly higher $63 \%$ yield.

In an attempt to better understand the reactivity patterns, a hypothesised reaction network of the protection reaction was drawn up (Scheme 79). The active basic species were assumed to be the potassium alkoxide of the substrate and the potassium imidazolate species. The desired species 91 is susceptible to deprotection from the catalytic quantity of base present, resulting in formation of $\mathbf{9 4}$. The base is nevertheless regenerated from this elimination, so small amounts of formation of $\mathbf{9 4}$ do not impede the overall reaction. The mechanism of formation of 192 is hypothesised in Scheme 80. 


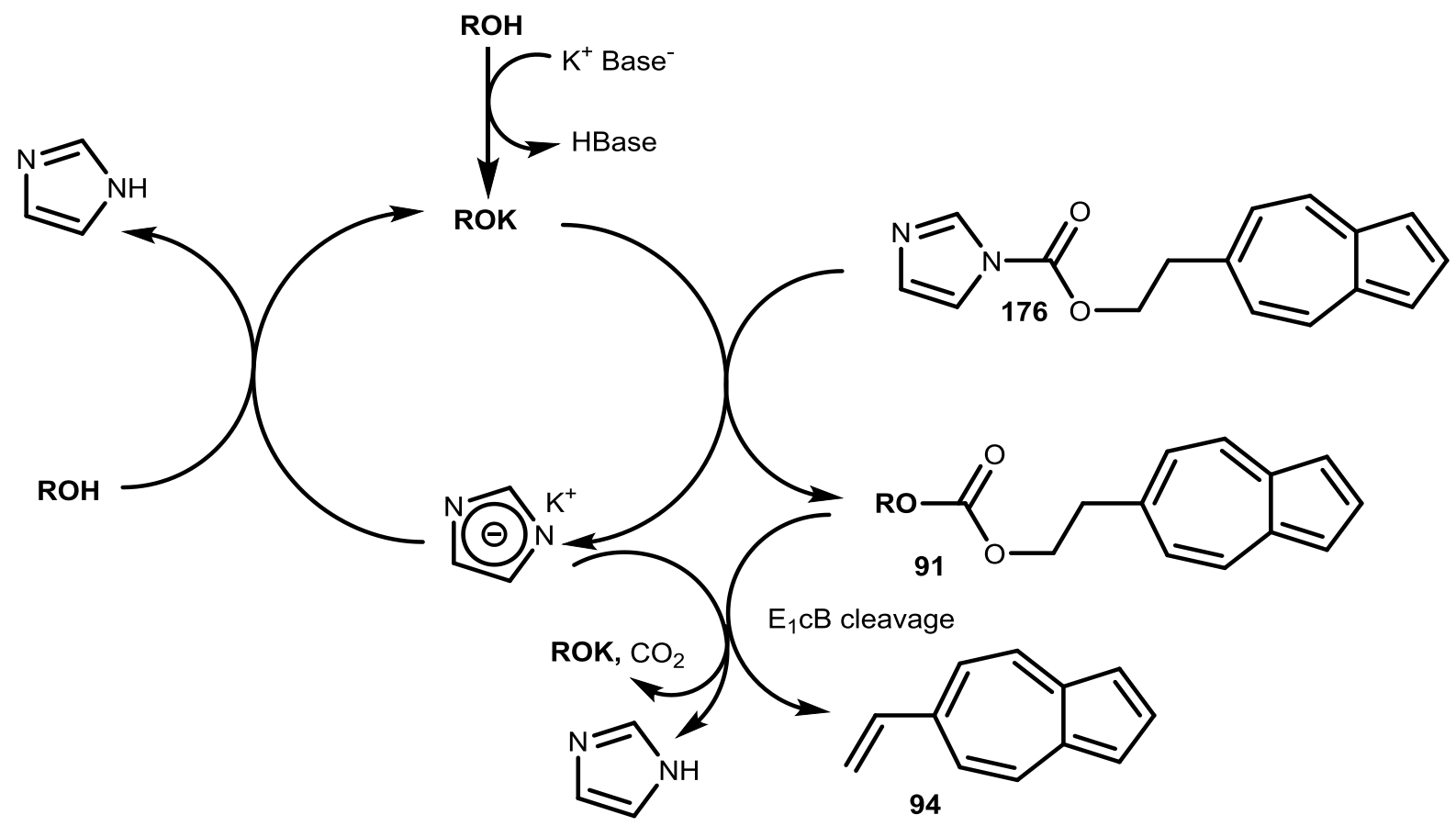

Scheme 79. Proposed reaction network for the formation of $\mathbf{9 1}$
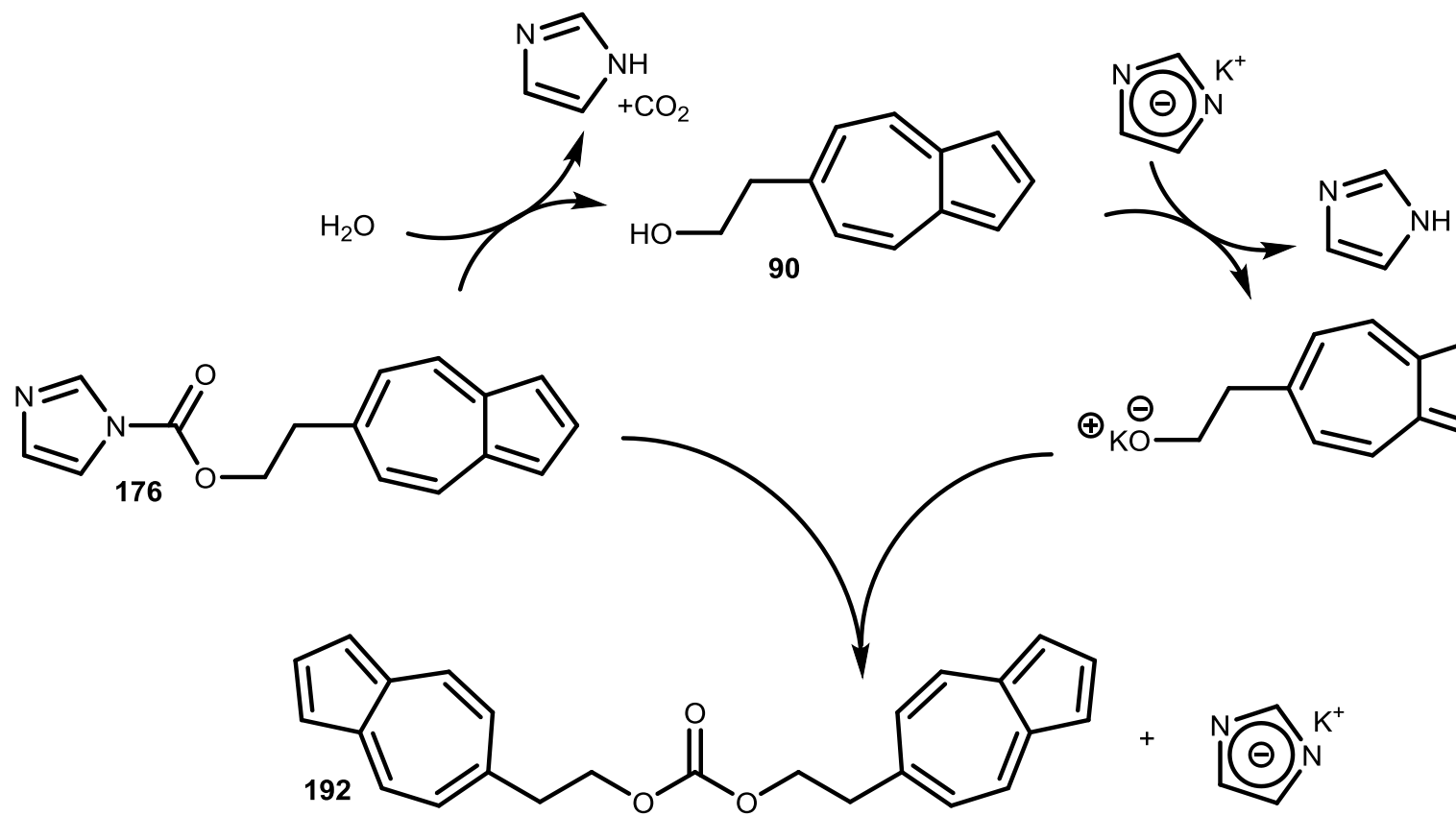

Scheme 80. Propos ed reaction network responsible for the formation of the symmetric dimer $\mathbf{1 9 2}$ when wa ter is present

There appeared to be a tendency for these reactions to stall; the reaction would initially proceed rapidly, and significant product could often be seen after 10 minutes by TLC analysis. After several hours there would reach a point where no further reaction seemed to take place, irrespective of how much longer it was left to react. This points to two possibilities: either the catalytic quantity of $\mathrm{KOH}$ is being poisoned or lost, or one of the reaction products is hindering reaction in an antiautocatalytic fashion. 
More drastic modifications to the protection protocol were later pursued. A solid-state reaction was performed with 176, KOH and cetyl alcohol using a Mortar \& Pestle (Scheme 81). (Cetyl alcohol was used instead of cyclohexanemethanol at this point because it is solid, and was in plentiful supply).

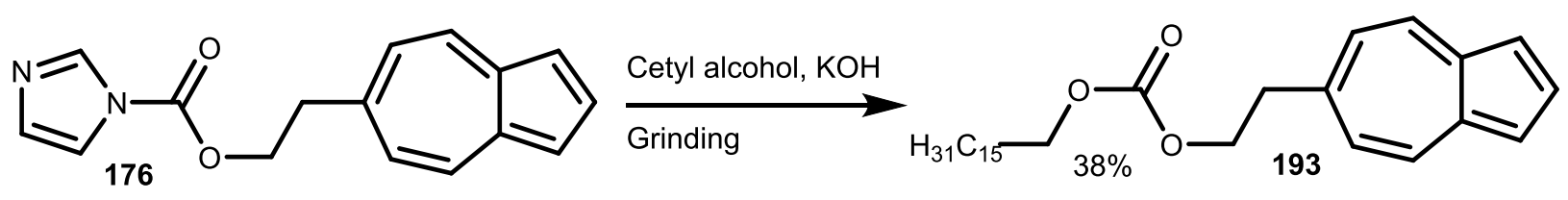

Scheme 81. Solid state formation of the cetyl carbonate 197

After approximately 15 minutes of grinding, with occasional addition of small $(<1 \mathrm{~mL})$ quantities of $\mathrm{CH}_{2} \mathrm{Cl}_{2}$ to ensure formation of a paste, a 38\% yield of 193 was obtained, along with $17 \%$ yield of the symmetrical carbonate 192, 7\% yield of 94 and 41\% RSM. The formation of the symmetrical carbonate 192 is presumably due to the lack of anhydrous conditions. While these reaction conditions are too moisture sensitive for grinding to be an effective technique, it did reveal how fast the reaction could take place given the right conditions.

Further experiments were performed in the solution phase, using KHMDS instead of KOH as the basic catalyst. KHMDS (as a $0.42 \mathrm{M}$ solution in toluene) has the advantage of being easier to add in small quantities than $\mathrm{KOH}$. When performing the reaction with KHMDS (14 mol \%) in THF at room temperature, a $62 \%$ yield was obtained after 1 hour, a yield equal to that of entry 6 of Table 11 with much less time and heat expenditure. In addition, generation of the by-products 192, 94 and $\mathbf{9 0}$ seem to be reduced through use of KHMDS.

The use of very small catalytic loadings of KHMDS failed to produce favourable results. Loadings of $0.4 \mathrm{~mol} \%$ and $1.6 \mathrm{~mol} \%$ gave only $19 \%$ and 29\% yields of 193 respectively, even after reaction overnight. As before, when monitoring the reaction by TLC, the reaction appeared to stall. This stalling was eventually circumvented through the repeated addition of small quantities of KHMDS over the course of the reaction. Addition of 8 drops in total of KHMDS solution (equating to $26 \mathrm{~mol} \%$ total) irregularly over the course of four days gave an astonishing $80 \%$ yield of 193 . Finally, the yield was increased to $83 \%$ through performing the reaction in silanised glassware (see method for glassware silanisation in Chapter 7) and adding a single drop of KHMDS solution (18 mol\% in total) every half hour over 2.5 hours.

From these results, it is believed that the acidic silanol groups present on the surface of the glassware were neutralising the active basic species in the solution. These reactions were performed at approximately one hundredth the scale of those reported by Davis et al. ${ }^{11}$ which is presumably why they did not observe the same effects. 
Nevertheless, a high-yielding (if somewhat cumbersome) protocol was developed that allowed protection of alcohols as 6-(2-oxyethyl)azulene carbonates.

\section{Protection of amines}

Reaction of the azulene carbonylimidazole species with the test substrate cyclohexylamine to produce the carbamate 194 was carried out. The protocol used is based on the protocol of Davis et al., ${ }^{17}$ similar to the protocol given for the formation of carbonates. ${ }^{13}$ In comparison to the alcohols explored previously, cyclohexylamine was more reactive, presumably owing to the higher nucleophilicity of amines over alcohols (Table 12). Even so, the same stalling of the reaction rate was observed again. Without catalytic base, the highest yield achieved was 50\%. With catalytic $\mathrm{KOH}$ and toluene at reflux, the highest yield achieved was 75\%.
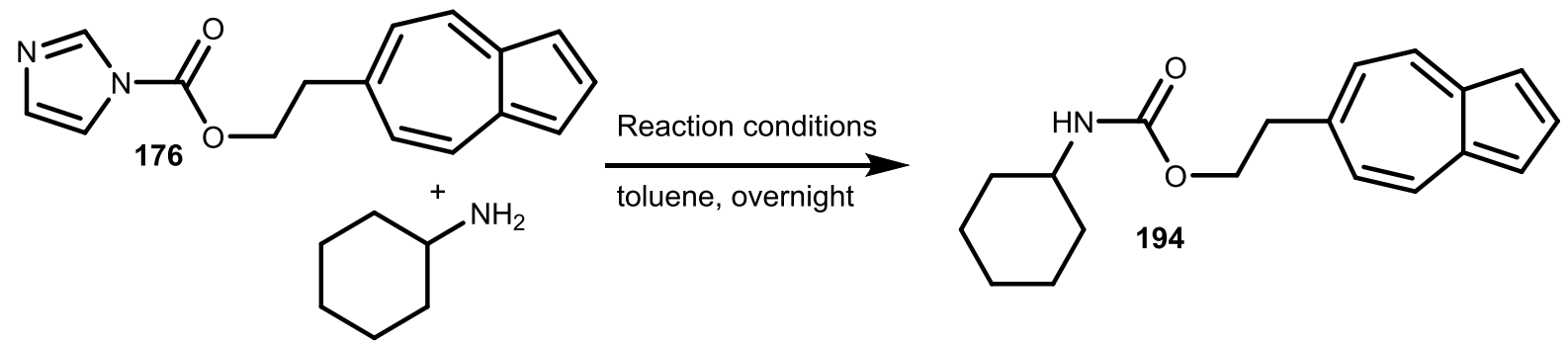

Table 12. Optimisation of the formation of 194

\begin{tabular}{|l|l|l|l|l|l|l|l|l|}
\hline Entry & Base & temperature & $\begin{array}{l}\mathbf{\%} \text { yield } \\
\mathbf{1 9 4} \text { a }\end{array}$ & $\begin{array}{l}\text { yield } \\
\mathbf{1 9 2}\end{array}$ & $\begin{array}{l}\text { yield } \\
\mathbf{9 0}\end{array}$ & $\begin{array}{l}\text { yield } \\
\mathbf{9 4}\end{array}$ & $\begin{array}{l}\text { RSM } \\
\mathbf{1 7 6}\end{array}$ \\
\hline 1 & KOH $180 \mathrm{~mol}^{\circ}{ }^{\mathrm{b}}$ & r.t. & $6 \%$ & $23 \%$ & $40 \%$ & - & $21 \%$ \\
\hline 2 & - & $65^{\circ} \mathrm{C}$ & $29 \%$ & - & - & - & $68 \%$ \\
\hline 3 & - & Reflux & $50 \%$ & & & $5 \%$ & $45 \%$ \\
\hline 4 & KOH 7 mol\% & Reflux & $76 \%$ & $3 \%$ & - & $4 \%$ & $9 \%$ \\
\hline
\end{tabular}

a Calculated relative to cyclohexylamine $\mathrm{b}$ This high base loading was accidental

\subsection{Deprotection}

Since this 6-substituted azulene protecting group shares the same mechanism of deprotection as FMOC, the first conditions tried were the traditional deprotection conditions of FMOC: piperidine in DMF18 at room temperature overnight on the azulene-protected cinnamate $\mathbf{1 7 8}$ (Scheme 82). This returned a $28 \%$ yield of deprotected cinnamic acid, along with the piperidine adduct of 6 vinylazulene 195 and $72 \%$ returned starting material. 

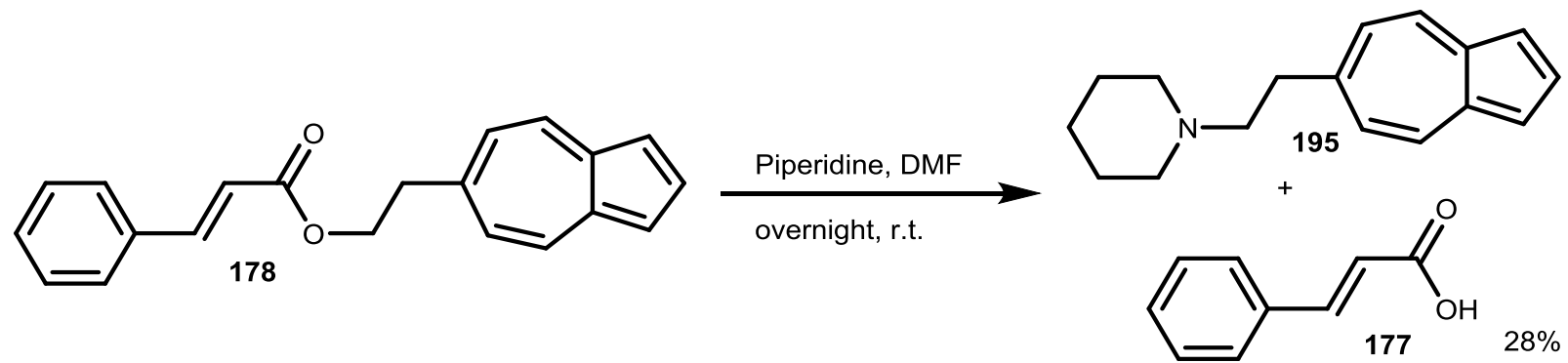

Scheme 82. Initial deprotection conditions investigated for $\mathbf{1 7 8}$

The same deprotection was tried with other solvents, such as THF, MeCN and EtOH, with no deprotection observed. These results indicated that the 6-substituted azulene is more stable to base than FMOC, and stronger bases were needed for complete deprotection.

Addition of the stronger amidine base DBU (with a pKa of 24.3 in $\mathrm{MeCN}$, as compared to piperidine, which has a pKa of 19.3 in $\mathrm{MeCN})^{19}$ to the protected cinnamate in MeCN gave full deprotection within three hours (Scheme 83). Complete consumption of the starting material and quantitative regeneration of cinnamic acid (as calculated from the integration of the ${ }^{1 \mathrm{H}} \mathrm{NMR}$ spectrum of the crude mixture) as well as generation of the blue 6-vinylazulene (94) was achieved. Interestingly, the 6-vinylazulene produced in this reaction did not appear to react further with DBU - the adduct 196 was not observed. This is presumably because DBU is much less nucleophilic than piperidine, despite being far more basic.

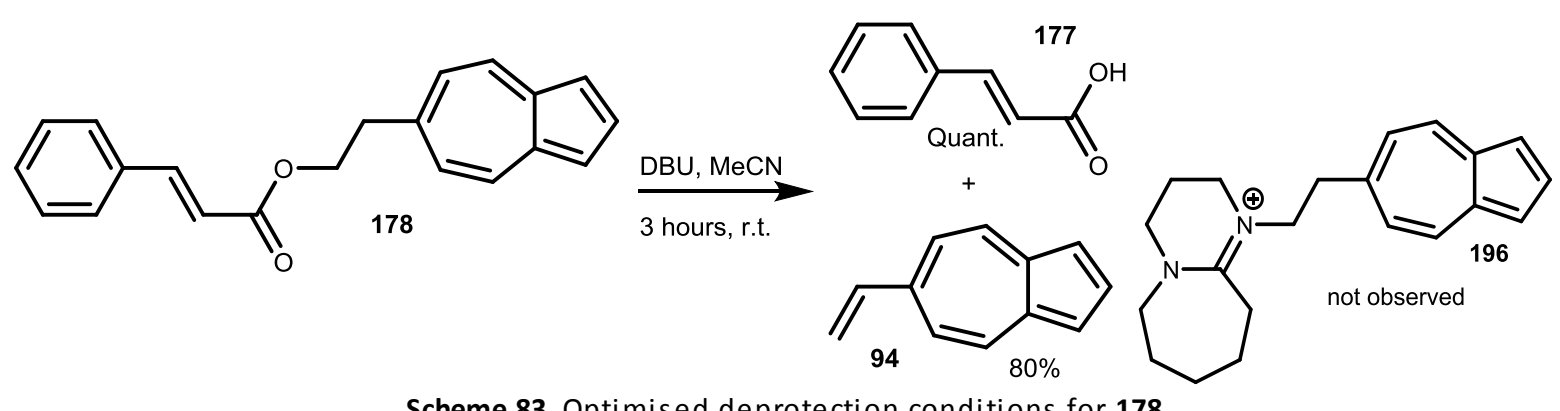

Scheme 83. Optimised deprotection conditions for $\mathbf{1 7 8}$

This deprotection has several attractive features. Firstly, the azulene-based products are easy to separate from the reaction mixture. If deprotected with a nucleophilic base such as piperidine, an amine species such as $\mathbf{1 9 5}$ will be formed that can be protonated and easily separated from the desired product during aqueous workup. If deprotected with a less nucleophilic base such as DBU, 6-vinylazulene 94 is produced, which is sufficiently non-polar to be eluted with petroleum ether during column chromatography. Secondly, 6-vinylazulene has a different colour to the 6-alkylsubstituted azulenes. It is blue, rather than indigo, and this colour change is noticeable enough to allow the extent of deprotection to be assessed visually, thus making the deprotection selfindicating (Figure 59). 


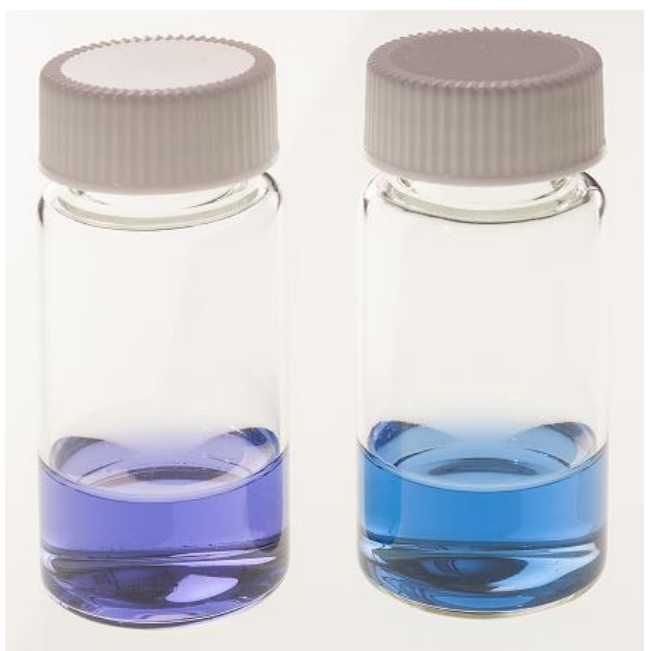

Figure 59. Cinnamate ester $\mathbf{1 7 8}$, on left, and 6-vinylazulene $\mathbf{9 4}$ on right, demonstrating colour change

\subsubsection{Two-step deprotection}

With the success of the single-step deprotection demonstrated, the feasibility of two-step deprotection was examined. Because the reaction rate is dependent on the stabilisation of the negative charge generated by the 6-position, and because the anion sees good overlap with the plane of the azulene $\pi$-system, nitration at the 1-position was once again chosen as an exploratory activating reaction (Figure 60).

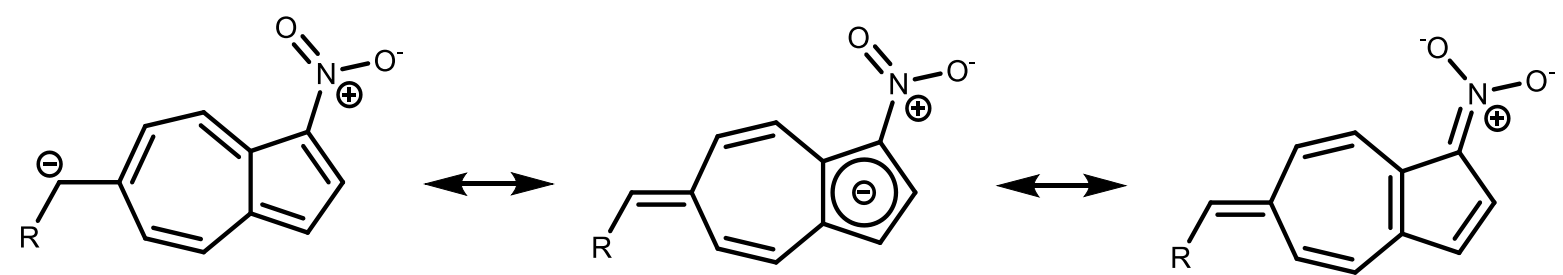

Figure 60. The nitro group confers additionals tabilisation of the negative charge by the 6-position, facilitating cleavage.

The cinnamate 178 was nitrated using the Menke nitration protocol at $10{ }^{\circ} \mathrm{C}$ to give a $20 \%$ yield of the intended product 192 (Scheme 84). As discussed before in Chapter 3, testing of the effects of nitration on deprotection was deemed to be more important than optimisation of the yield, and sufficient quantities of $\mathbf{1 9 7}$ were generated to allow for these tests.

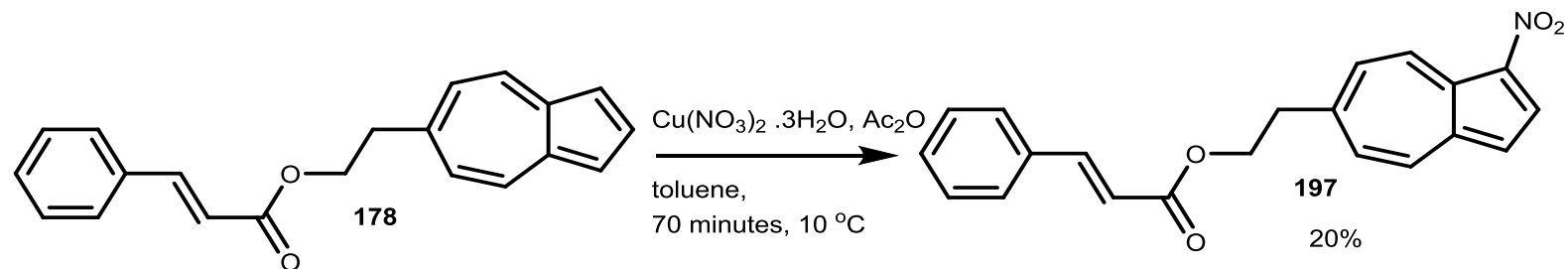

Scheme 84. Nitration of $\mathbf{1 7 8}$

Morpholine was chosen as the initial base for the deprotection studies of the activated nitroazulene protecting group, as it is relatively weak (pKa of 16 in $\mathrm{MeCN}$ ) and was expected to fail. Surprisingly, $76 \%$ deprotection (as measured by ${ }^{1} \mathrm{H}$ NMR) was attained after a 20 hour period 
(Scheme 85). This is a vast increase in reactivity over the unactivated azulene protecting group, estimated to be a ten million-fold increase in reaction rate. This figure was arrived at on the basis of DBU and morpholine having a difference in basicity of 8.3 pKa units (corresponding to DBU being $10^{8.3}=200,000,000$ times more basic) and the deprotection of 197 with morpholine taking roughly ten times as long as the DBU deprotection of $\mathbf{1 7 8 .}$
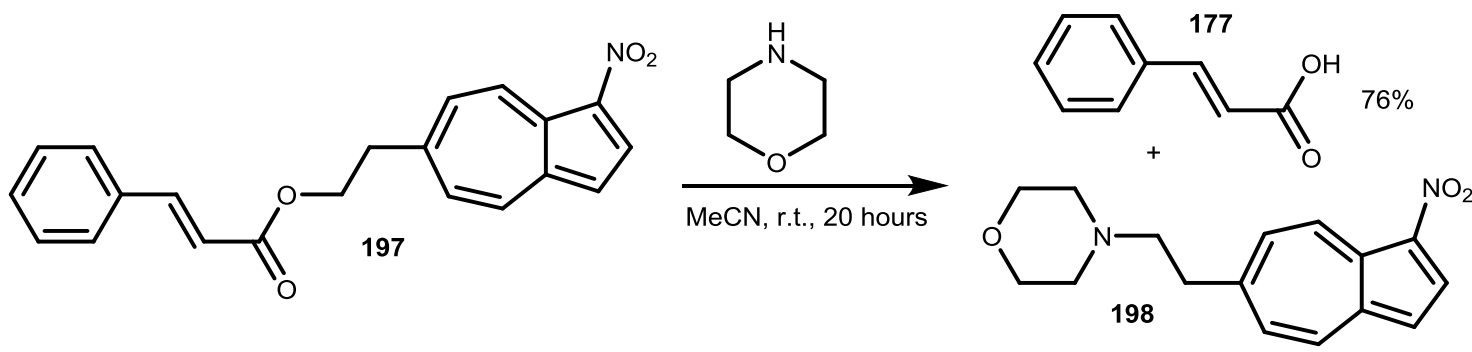

Scheme 85. Cleavage of the nitrated azulene ester 197

\subsection{Summary}

These initial results show the 6-substituted azulene $\mathbf{8 9}$ holds much promise as a protecting group for amines, alcohols and carboxylic acids. Mild and easy protocols have been found for protection of carboxylic acids. The amine and alcohol protection protocols gave good yields but were somewhat user-unfriendly and could still be improved.

The conditions required for deprotection of carboxylic acids were found to be relatively mild. Addition of DBU in MeCN at room temperature over 3 hours is sufficient to give full deprotection. Furthermore, nitration of the azulene ring revealed this chemistry is highly amenable to activation, and a two-step process could be a viable orthogonal deprotection procedure, providing a highyielding activation reaction can be found.

With these results in hand, it was decided to focus the rest of this research project on exploring the scope and limitations of the 6-substituted azulene as a viable protecting group (Chapter 5).

\subsection{References}

1. McDonald, R. N.; Wolfe, L; Petty, H. E., Nonbenzenoid Aromatic Systems. VIII."' Buffered Acetolysis of 2-(4- and 2-(6-Azulyl)ethyl Arenesulfonates and 3-(4-Azulyl)-l-propyl Nosylate. Examples of Ar3-5 and Ar3-6 Mechanisms. J. Org. Chem. 1973, 38, 1106-1113.

2. Bevan, T. Investigations of Azulene Derivatives as Self-Indicating Chromophores for Applications in Protecting Group Strategies and Solid-Phase Purification.VUW, MSc thesis, 2011. 
3. Reich, H. J., What's Going on with These Lithium Reagents? J. Org. Chem. 2012, 77, 54715491.

4. Jr, A. G. A.; Anderson, R. G.; Fujita, T. S., Displacement Reactions on 1Azulylmethyltrimethylammonium Iodide.J. Org. Chem. 1962,27, 4535-4539.

5. Aldridge, H. K.; Liu, X.; Lin, M. C.; Melius, C. F., Thermal Unimolecular Decomposition of 1,3,5-Trioxane: Comparison of Theory and Experiment. Int. J. Chem. Kinet. 1991, 23, 947956.

6. (a) Reich, H. J., Role of Organolithium Aggregates and Mixed Aggregates in Organolithium Mechanisms. Chem. Rev. 2013, 113, 7130-7178; (b) Hoepker, A. C.; Collum, D. B., Computational Studies of Lithium Diisopropylamide Deaggregation. J. Org. Chem. 2011, 76, 7985-7993.

7. Inanga, J.; Hirata, K.; Saeki, H.; Katsuki, T.; Yamaguchi, M., A rapid esterification by means of mixed anhydride and its application to large-ring lactonisation. Bull. Chem. Soc. Jpn. 1978, 52, 1989-1993.

8. Neises, B.; Steglich, W., Simple Method for the Esterification of Carboxylic Acids. Angew. Chem. Int. Ed. Engl. 1978, 17, 522-524.

9. Fletcher, S., The Mitsunobu reaction in the 21st century. Org. Chem. Front. 2015, 2, 739.

10. Corey, E. J.; Nicolaou, K. C., An Efficient and Mild Lactonization Method for the Synthesis of MacrolidesJ. Am. Chem. Soc. 1974, 5614-5616.

11. McDonald, R. N.; Reitz, R. R., Nonbenzenoid Aromatic Systems. V1.1 pKa Values of Azuloic Acids. J. Org. Chem. 1972,17, 2703-2705.

12. Fernandez-Bertran, J. F., Mechanochemistry: an Overview. Pure Appl. Chem. 1999, 71, 581586.

13. McLeland, C. W., DCC/DMAP-Promoted Esterification Reactions in Ionic Liquids and under Solvent-Free Conditions. In ICOS20, Budapest, Hungary, 2014.

14. Babad, H.; Zeiler, A. G., The Chemistry of Phosgene. Chem. Rev. 1973, 73, 75-91.

15. (a) Heller, S. T.; Sarpong, R., On the reactivity of imidazole carbamates and ureas and their use as esterification and amidation reagents. Tetrahedron 2011, 67, 8851-8859; (b) Staab, H. A., Syntheses Using Heterocyclic Amides (Azolides). Angew. Chem. Int. Ed. Engl. 1962, 1, 351-367.

16. Rannard, S. P.; Davis, N. J., Controlled Synthesis of Asymmetric Dialkyl and Cyclic Carbonates Using the Highly Selective Reactions of Imidazole Carboxylic Esters. Org. Lett. 1999, 1, 933-936. 
17. Rannard, S. P.; Davis, N. J., The Selective Reaction of Primary Amines with Carbonyl Imidazole Containing Compounds: Selective Amide and Carbamate Synthesis. Org. Lett. 2000, 2, 2117-2120.

18. Carpino, L. A; Han, G. Y., The 9-Fluorenylmethoxycarbonyl Amino-Protecting Group. J. Org. Chem. 1972,37, 3404-3409.

19. Leito, I.; Kütt, A.; Kaljurand, I.; Rõõm, E.-I.; Rodima, T.; Koppel, I. A., Brønsted Acidity of Neutral and Cationic Acids in Nonaqueous Solvents: Recent Developments. http://tera.chem.ut.ee/ ivo/HA UT/Acidity Basicity INOR 1036 ACS 2007.pdf, Ed. (Last accessed on 17/2/16) 


\section{Chapter 5. Exploration of the utility of the Azul protecting group}

\subsection{Overview}

With the 6-substituted azulene group showing promise as a protecting group, an investigation of its utility in the broader sphere of synthetic chemistry was initiated.

At this point, it was decided that the 6-(2-oxyethyl)azulene protecting group would be christened $A z u l$ when used as an ester for carboxylic acid protection and AzulOC (Azulene OxyCarbonate or OxyCarbamate) when used for hydroxyl/amine protection (Figure 61).

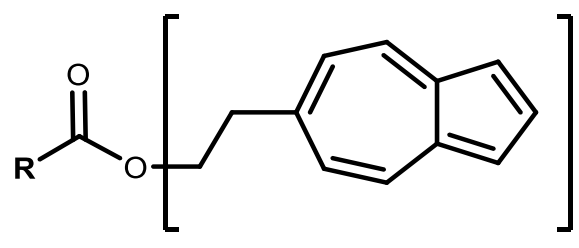

Azul

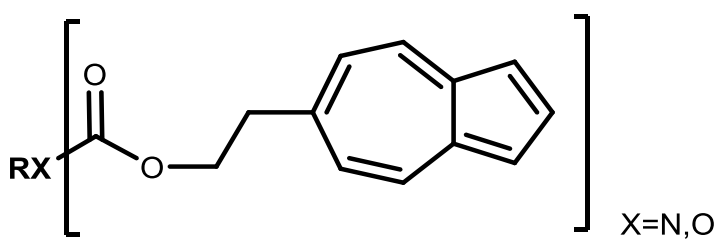

AzulOC

Figure 61. The Azul protecting group for carboxylic acids, and the AzulOC protecting group for a mines and alcohols

In order to better establish Azul as a protecting group, several aspects of its compatibility with the broader area of organic chemistry had to be investigated, such as reactivity with various bases, compatibility with other reactions and orthogonality to other protecting groups, as well as improvements to the deprotection methods.

Several additional deprotection conditions were found for the one-step deprotection method, and an efficient and high-yielding process for two-step deprotection was found. This expanded list of deprotection conditions allowed both selective and global deprotection to take place in the presence of other protecting groups such as TBS and FMOC, demonstrating a high degree of orthogonality in the process.

Investigating the compatibility with various reaction conditions was also judged to be an important part of the development of this protecting group. The synthetic chemist ideally wants to know beforehand the compatibility of the protecting group with the reactions they intend to carry out. While it is completely impractical to test the compatibility of the Azul protecting group with every known variant of every known reaction, much information can be gleaned by choosing a few representative reactions that both cover a wide area of 'reaction space' (i.e. oxidation, reduction, palladium catalysis, nucleophilic, electrophilic, acidic, basic) and are relatively commonly used in synthesis. From this body of results, the intention is that one could infer 
compatibility of a similar reaction with this Azul protecting group. The Azul protecting group was found to be incompatible with Swern oxidation and partly incompatible with Suzuki crosscoupling, but was otherwise inert to the range of reaction conditions that were tested.

Most of this work was performed with the Azul-protected acids, rather than protected alcohols or amines. The primary reason for this was that alcohol and amine protection reactions were only developed relatively late in the project, and initial experiments indicated they behaved in much the same way as the protected acids. It was judged that investigation of the wider scope of Azulprotected acids would have higher priority than development of Azul-protected alcohols and amines, because if a fundamental flaw of the protected acids were to be discovered, it would likely be shared by the protected alcohols and amines.

\section{A note on quantitative NMR spectroscopy}

For much of the work in this chapter, quantitative NMR techniques were used to determine the outcomes of reactions. Quantitative NMR involves adding a known amount of an internal standard to the sample, whose integral is compared to that of the components in the sample.

In order to increase the accuracy of NMR quantitation, the pulse sequencewas slightly modified. A 90 degree pulse (as opposed to the standard 60 degree pulse) was used, along with a long relaxation decay and acquisition time. Integration becomes much more reliable when using such a pulse sequence, as integrals belonging to different protons on the same compound can vary by $10 \%$ or more when produced by the standard ${ }^{1} \mathrm{H}$ NMR pulse sequence, while those same integrals only vary by $2 \%$ or less when recorded through these quantitative NMR techniques.

The internal standard used was nitromethane. A sharp singlet at $4.3 \mathrm{ppm}$ is present in the ${ }^{1} \mathrm{H}$ NMR spectrum, and usually did not overlap with other nearby peaks. Nitromethane boils at $100{ }^{\circ} \mathrm{C}$, making it sufficiently involatile to prevent evaporation during preparation of the NMR sample and long-term storage, but volatile enough to be removed by vacuum when needed. A standard solution of $3 \%$ nitromethane in $\mathrm{CDCl}_{3}$ was prepared and $50 \mu \mathrm{L}$ of this solution (a calculated $0.0273 \pm 0.0005 \mathrm{mmol}$ ) was added to each sample analysed.

There were several reasons why quantitative NMR was used over isolation or other quantitative analytical techniques involving HPLC, GCMS or LCMS. Firstly, a large number of small-scale reactions were planned - reactions involving testing Azul against various bases and against other protecting groups. Isolating every product of a reaction takes time, and at small scales the weighed value may be wildly inaccurate. Secondly, in the case of the reaction compatibility 
experiments, analysis of the reaction mixture as a whole rather than isolation of specific compounds was the goal.

NMR quantitation was chosen over HPLC, GCMS or LCMS quantitation primarily for practical reasons - there was no access to walkup LCMS and only restricted access to HPLC and GCMS, while NMR access was unrestricted. In terms of the overall usefulness of NMR quantitation, it holds distinct advantages over HPLC quantitation, as HPLC relies on the compound in question having a unique and well-resolved $\mathrm{R}_{\mathrm{f}}$, and does not give unambiguous identification of the compound. When compared to MS techniques, the setup and run time of the experiments is approximately the same, but NMR has a greater capacity for the identification of minor byproducts.

However, it became apparent of the course of these investigations that the above method consistently overestimated yields by a factor of 1.2 to 1.3 . This was also observed when quantifying known masses of material. As such, most of the results in this chapter are discussed on the basis of material conversion determined through ${ }^{1} \mathrm{H}$ NMR spectroscopy, although the calculated yield values are mentioned in Chapter $\mathbf{7}$, for the sake of completeness.

\subsection{Exploration of Azul deprotection conditions}

A range of reaction conditions was explored and a map of the regions of 'base space' in which the Azul protecting group was stable or labile was generated. The three primary variables were the identity of the base, the solvent, and the reaction temperature. The bases chosen were mostly amines, complemented with amidine bases, sodium hydroxide and TBAF. The conjugate acids of these bases have pKas in acetonitrile ranging from 12.5 to 24.3, covering approximately 12 orders of magnitude, and in Table 13 have been ordered in terms of their basicity in MeCN. ${ }^{1} \mathrm{~A}$ value for the pKa of DIPEA in MeCN could not be found, so its rank is based on its basicity in water.

The extent of deprotection was assessed by quantitative NMR of the crude reaction mixture. While a percentage deprotection was normally obtained, the relevant information to answer the question "Do these conditions cause deprotection?" can be summed up in a ternary "Yes", "no" or "partially". In the following tables, the cells representing the deprotection outcome are coloured with a traffic light motif: Red for no reaction, yellow for partial deprotection and green for full deprotection $(\leq 95 \%)$. 


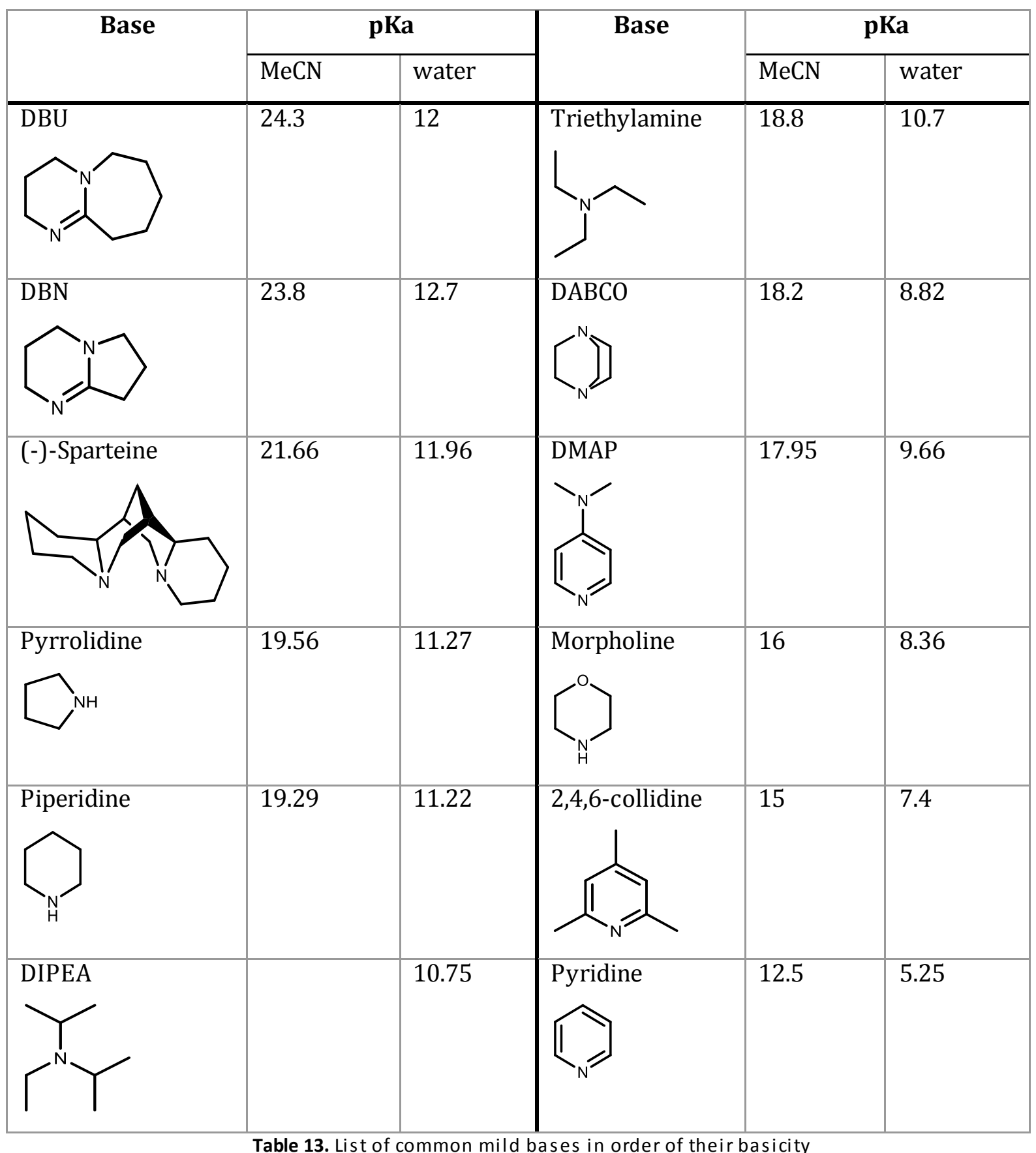

\subsubsection{Investigation of deprotection at room temperature}

An Azul-protected species was subjected to a selection of various bases in various solvents at room temperature. The cinnamate ester 178 (Azul-cinnamate) was used as the test substrate in these studies, once again due to the favourable properties of cinnamic acid (relatively non polar, identifiable using UV/permanganate stain on TLC). Four solvents were chosen for investigation: DMF, acetonitrile, ethanol and THF. Dichloromethane was excluded because of its reactivity with some of the more nucleophilic amine bases. The results are summed up in Table 14. 


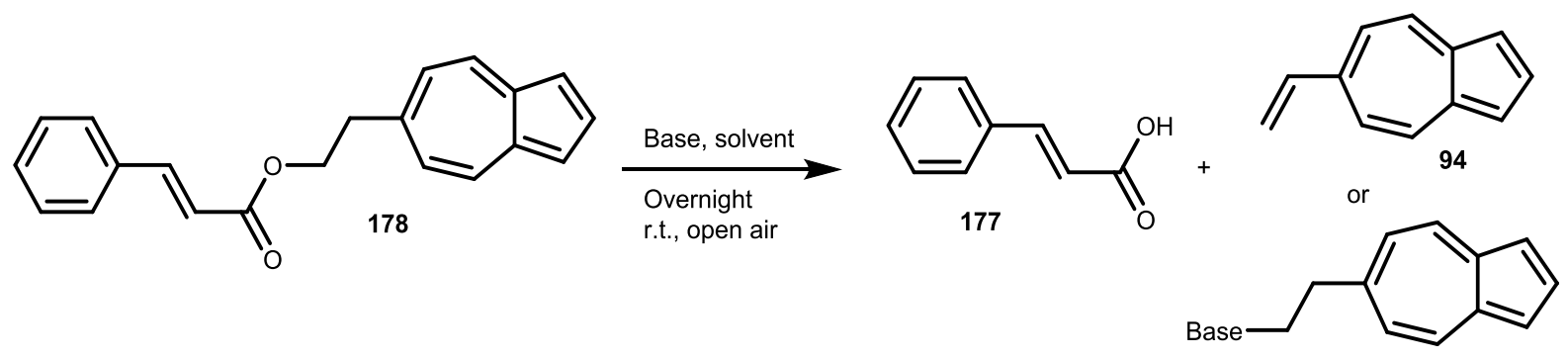

Table 14. Bases and solvents and their degree of deprotection at room temperature. All reactions were performed with overnight duration, unless otherwise stated

\begin{tabular}{|l|l|l|l|l|}
\hline & DMF & MeCN & EtOH & THF \\
\hline TBAF & & & & $100 \%, 20$ min \\
\hline DBU & & $100 \%, 3$ hours & Partiala & $100 \%, 3$ hours \\
\hline DBN & & $100 \%, 3$ hours & & \\
\hline$(-)-S p a r t e i n e$ & $\sim 2 \%$ & No reaction & & \\
\hline Pyrrolidine & $94 \%$ & No reaction & & No reaction \\
\hline Piperidine & $70 \%$ & No reaction & No reaction & $37 \%$ \\
\hline NaOH/ $\mathrm{H}_{2} \mathrm{O}$ & & & & \\
\hline DIPEA & & No reaction & & \\
\hline NEt ${ }_{3}$ & No reaction & No reaction & & \\
\hline DABCO & & $3 \%$ & & \\
\hline DMAP & & No reaction & & \\
\hline Morpholine & & No reaction & & \\
\hline
\end{tabular}

aAzul fragment appears to be Azul ethyl ether by NMR. The peaks overlap, making integration of individual species difficult. Deprotection was estimated at $90 \%$.

b 1:1 THF $/ \mathrm{H}_{2} \mathrm{O}$. Deprotection occurs primarily through saponification, with only traces of 6vinylazulene forming.

Both of the amidine bases, DBU and DBN, were able to cleave the Azul protecting group in a few hours in MeCN. The standard conditions used to cleave FMOC- piperidine in DMF- only gave partial deprotection of the Azul protecting group. Pyrrolidine in DMF gave a higher amount of cleavage, but did not quite reach completion. Sodium hydroxide in $1: 1 \mathrm{THF} / \mathrm{H}_{2} \mathrm{O}$ gave only partial deprotection. The Azul-containing fragments of piperidine and pyrrolidine deprotection were the piperidine and pyrrolidine adducts of 6-vinylazulene respectively.

TBAF was placed at the top of the list owing to its rapid deprotection of Azul. Anhydrous fluoride is known to be strongly basic, ${ }^{2}$ but no references citing the actual pKa of TBAF could be found. Addition of anhydrous TBAF to Azul-cinnamate in THF gave complete deprotection within 5 minutes. This rapid reaction rate appears to be insensitive to moisture, as this reaction can be performed with wet TBAF in open air with the same results. 


\subsubsection{Investigation of deprotection at elevated temperature}

The reaction between Azul-cinnamate and various bases at elevated temperatures was investigated (Table 15). The stronger amine bases such as pyrrolidine and piperidine can effect complete deprotection after heating at reflux overnight in MeCN. THF was less effective as a solvent, presumably because of its lower boiling point.
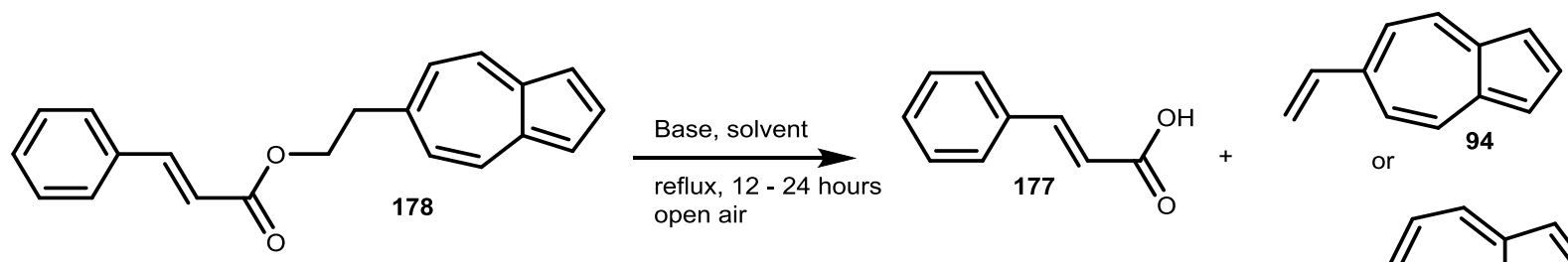

Bas

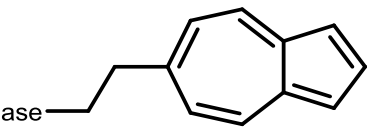

Table 15. Bases and solvents and their effect on deprotection at elevated temperatures

\begin{tabular}{|l|l|l|l|}
\hline & MeCN & EtOH & THF \\
\hline Pyrrolidine & Full deprotection & & \\
\hline Piperidine & Full deprotection & $23 \%$ deprotection & $18 \%$ deprotection \\
\hline Triethylamine & $11 \%$ deprotection & & \\
\hline DABCO & $94 \%$ deprotection & & \\
\hline
\end{tabular}

\subsubsection{Two-step activation - deprotection methodology}

As shown in the previous chapter, nitration of the 1-position of the azulene moiety gave a protecting group that was much more reactive towards $\beta$-elimination. However, the $20 \%$ yield of the nitrated derivative was entirely too low to be useful. In order for two-step deprotection to be practical, the activation step must be both relatively mild and as high yielding as possible. Other methods were sought to achieve efficient activation.

One potential advantage the Azul protecting group had over the other protecting group candidates that were explored in Chapter 3 , was that the 5-membered ring could potentially be substituted twice. A set of potential mono- and di- activated azulene targets was drawn up (Figure 62). 


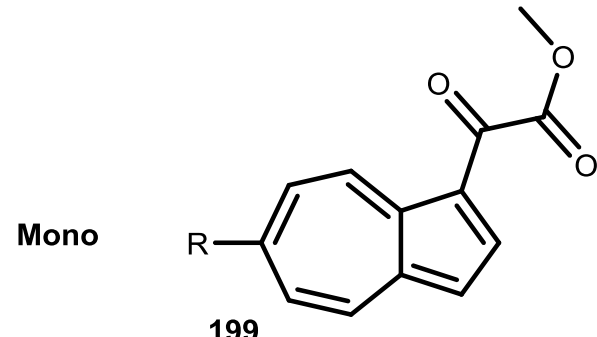

199

Di

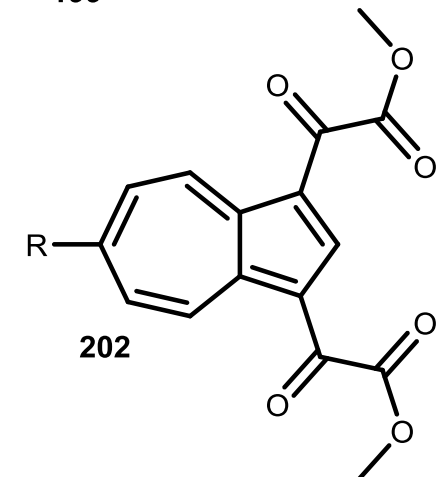

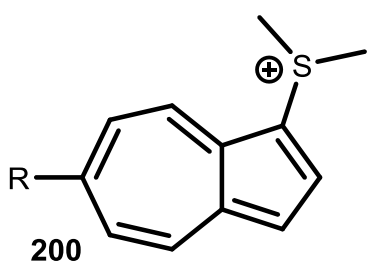
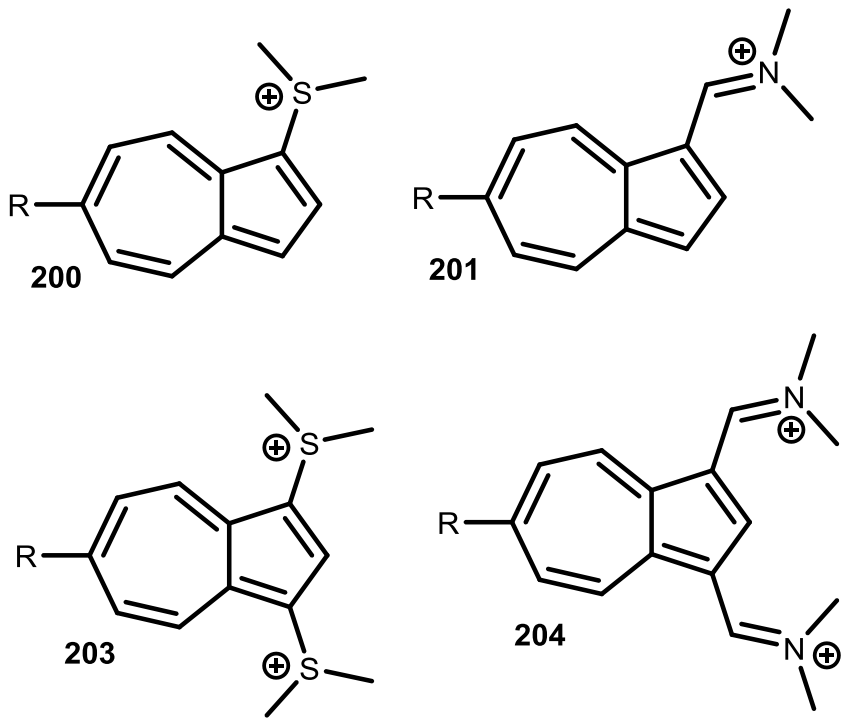

Figure 62. Possible mono- and di-EWG-substituted azulene derivatives that could act as activated Azul species

The mono-substituted targets not only represented compounds known in the literature, but were also accessible through reactions that had been performed previously during this project for other purposes. Access to the di-substituted azulenes were a lot less clear, as it was not known whether the deactivated mono-substituted azulene ring would still be sufficiently nucleophilic to react with the relevant electrophile a second time.

\section{Addition of a methyl ketoester}

Ketoesters are very easy to introduce to an azulene moiety, ${ }^{3}$ and their effect on the electronics of the azulene ring can be inferred to be similar to the nitro group from the similar orange-red colours of the compounds (compare $\lambda_{\max }$ of $499 \mathrm{~nm}$ for nitro-substituted Azul-cinnamate 197 and $505 \mathrm{~nm}$ for methyl ketoester-substituted Azul-cinnamate 205). This made the addition of the methyl ketoester a natural choice for the activation reaction. Addition of oxalyl chloride to azulene in open air, followed 20-30 seconds later by methanol and then after another 30-60 seconds by pyridine gave a quantitative yield of the ketoester 7 (Scheme 86). These brief intervals of time are necessary if this two-step methodology is to be used with acid-sensitive substrates, as anhydrous $\mathrm{HCl}$ is produced during the reaction. All the reagents were of standard quality and were not pre-dried. 

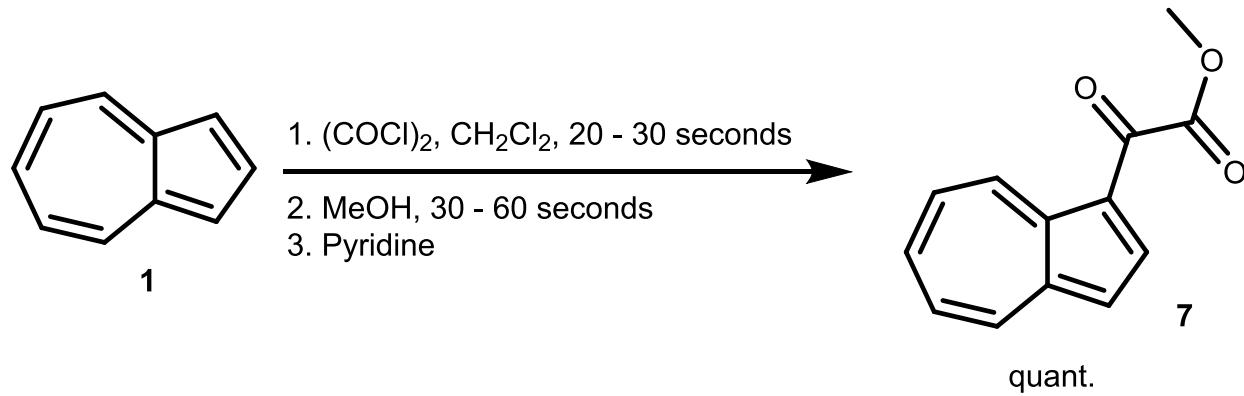

Scheme 86. Addition of a methyl ketoester to azulene

This reaction was repeated on the Azul-protected cinnamate, once again giving an almost quantitative yield and confirming that the procedure is compatible with the ester linkage (Scheme 87).

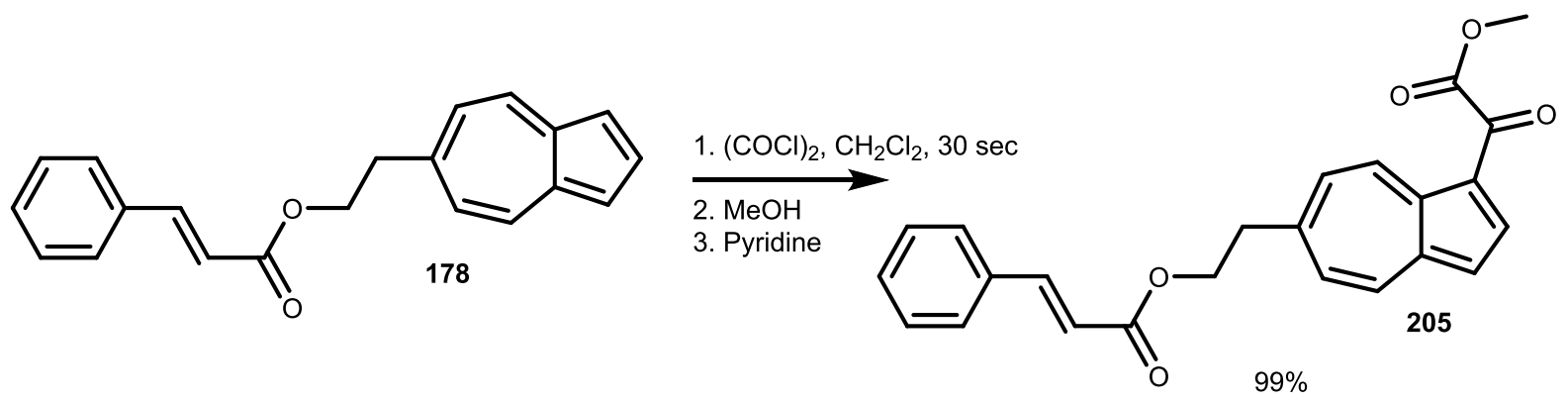

Scheme 87. Activation of Azul-cinnamate $\mathbf{1 7 8}$ to form $\mathbf{2 0 5}$ quantitatively.

An attempt was made at adding a second methyl ketoester onto 7. This substrate proved to be much less reactive than azulene itself, and after 5 minutes no product had appeared (Scheme 88). This reaction could have theoretically been pushed further in terms of time and temperature, but it was decided that it would then no longer meet the criterion of being a mild reaction (at the very least, such strongly acidic conditions might endanger the ester link between Azul and the substrate).

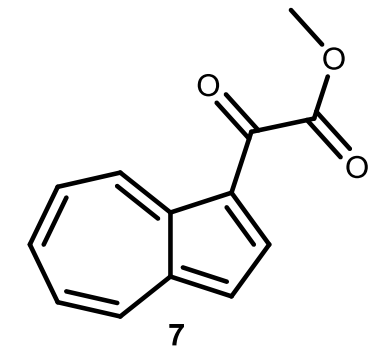

1. $(\mathrm{COCl})_{2}, \mathrm{CH}_{2} \mathrm{Cl}_{2}, 5$ minutes

3. Pyridine

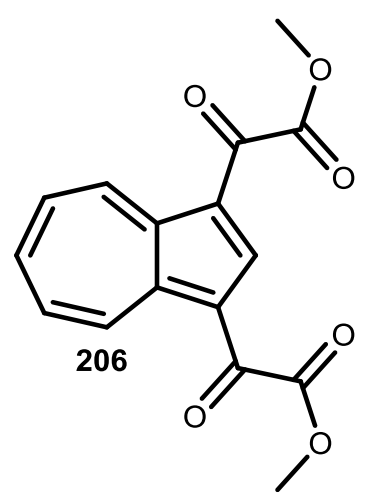

Scheme 88. Unsuccessful attempt at adding a second methyl ketoester to 7

As an activating group, the performance of the methyl ketoester was slightly better than that of the nitro group explored in Chapter 4. Essentially complete (97\%) deprotection could be achieved by treating 205 with morpholine overnight at room temperature (as opposed to 76\% 
deprotection from the nitro-activated species 197), and employment of stronger bases gave much more rapid reactions (Table 16).
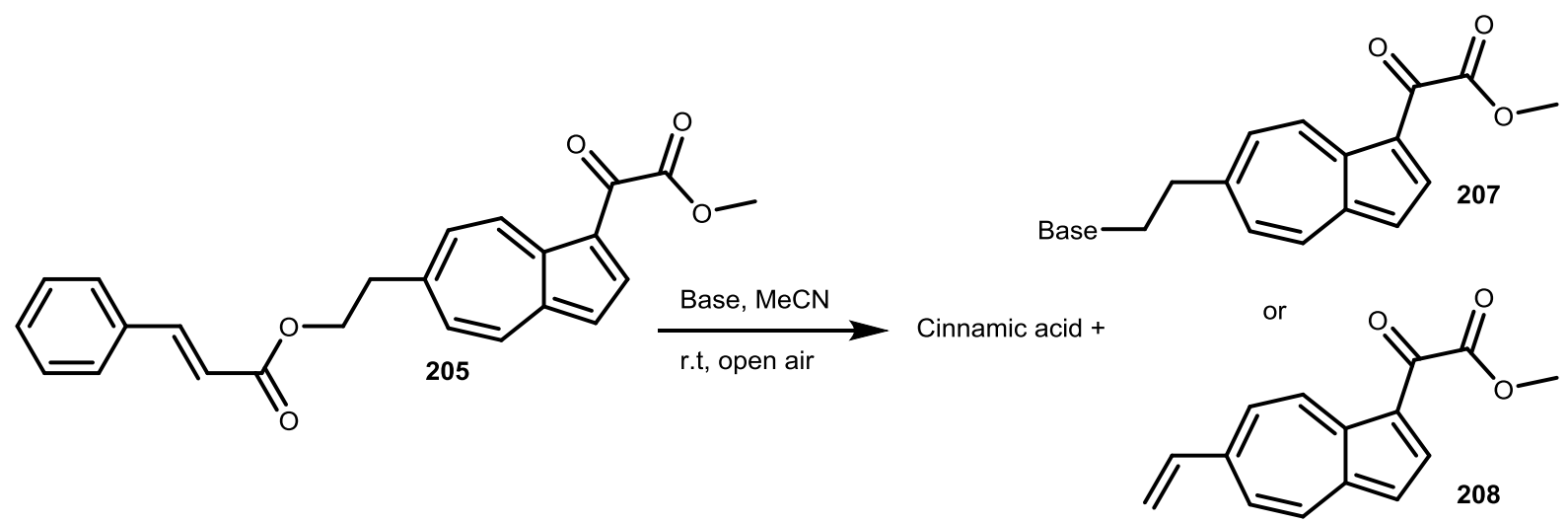

Table 16. Exploration of the sensitivity of $\mathbf{2 0 5}$ towards various bases

\begin{tabular}{|l|l|}
\hline Base & Reactivity \\
\hline DBU & Full deprotection. <10 seconds \\
\hline Piperidine & Full deprotection, <25 minutes \\
\hline Triethylamine & Full deprotection, 5 hours \\
\hline DABCO & Full deprotection, <1 minute \\
\hline Morpholine & $97 \%$ deprotection, overnight \\
\hline 2,4,6-collidine & No reaction, overnight \\
\hline Pyridine & No reaction, overnight \\
\hline
\end{tabular}

The vinylazulene deprotection product 208 appears to readily react with the bases DBU, piperidine and morpholine (forming adducts of the form 207), based on the formation of highly polar orange material seen with TLC analysis after the deprotection was complete.

\section{Addition of dimethylsulfonium chloride}

Under the Swern oxidation reaction conditions, azulenes react rapidly with the intermediate chlorodimethyl sulfonium chloride to form the corresponding 1-dimethylsulfonium chloride, rapidly changing the colour of the azulene species from indigo to magenta (Scheme 89). ${ }^{4}$

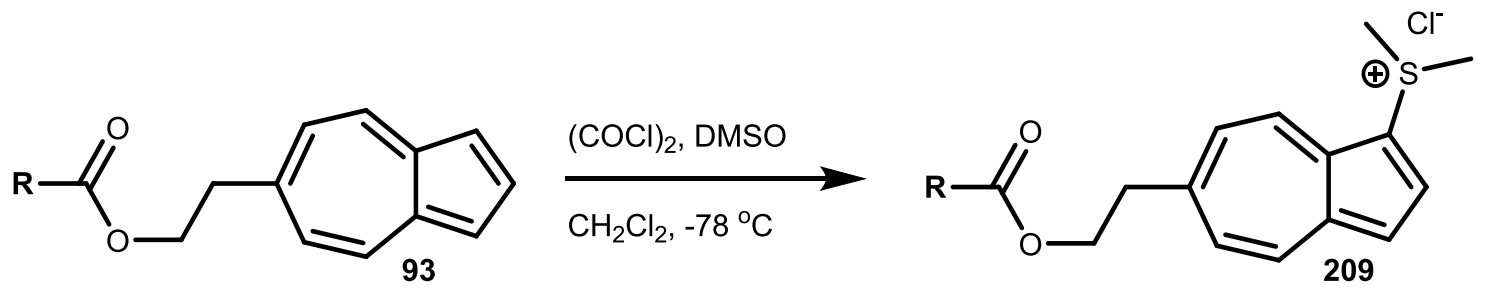

Scheme 89. Addition of dimethylsulfonium to Azul can be carried out under Swe rn oxidation conditions 
Due to the relatively minor colour change in comparison to addition of a nitro group or ketoester, and the corresponding indication that the dimethylsulfonium moiety would be less electronwithdrawing than either of the aforementioned groups, this approach was not expected to increase the reactivity of Azul towards deprotection to the same degree. Nevertheless, any increase in reaction rate may be beneficial, and so this method of activation was explored.

An attempted addition of dimethylsulfonium on 178 was carried out using Swern oxidation conditions (Scheme 90). A rapid colour change from indigo to magenta was observed. The conversion of the Azul species to the dimethylsulfonium appeared to be more or less quantitative according to TLC. However, the resulting azulene derivative stubbornly partitioned into the aqueous layer during phase separations. Even for relatively non-polar species such as Azulcinnamate 178, the resulting sulfonium ion is only sparingly soluble in organic solvents and was not amenable to purification through normal or reverse-phase chromatography. Another regrettable feature is that once the azulene dimethylsulfonium species is dissolved in organic solvents, degradation would occur. As a result, neither yields nor clean NMR spectra could be obtained of the pure compounds.

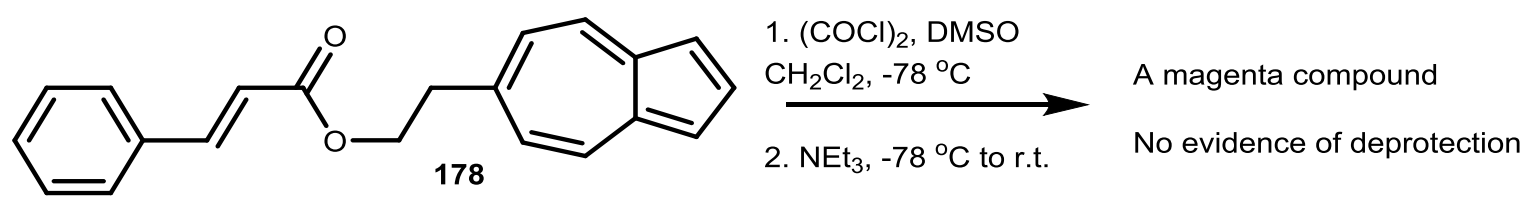

Scheme 90. Attempted dimethylsulfonium addition-deprotection of $\mathbf{1 7 8}$ using $\mathrm{NEt}_{3}$ as a base

Lack of isolability of the intermediate does not necessarily preclude its use as an activation reaction - it merely restricts the process to a one-pot activation-deprotection. In this case, however, the sulfonium ion was insufficiently electron-withdrawing to allow $\mathrm{NEt}_{3}$ to induce deprotection, and no sign of cinnamic acid was found in the ${ }^{1} \mathrm{H}$ NMR spectrum. On this basis, the dimethylsulfonium mode of activation was not pursued further.

\section{Vilsm eier-Haack formylation}

Using the Vilsmeier-Haack reagent to introduce an activating dimethyliminium moiety was also considered. Hydrolysis of the resulting iminium to the corresponding aldehyde was rejected, not only because the aldehyde functionality was deemed too weakly electron withdrawing to impart sufficient activation on the azulene ring, but also because the conditions required to generate the aldehyde - aqueous base - would likely induce the deprotection of the activated Azul protecting group regardless. 


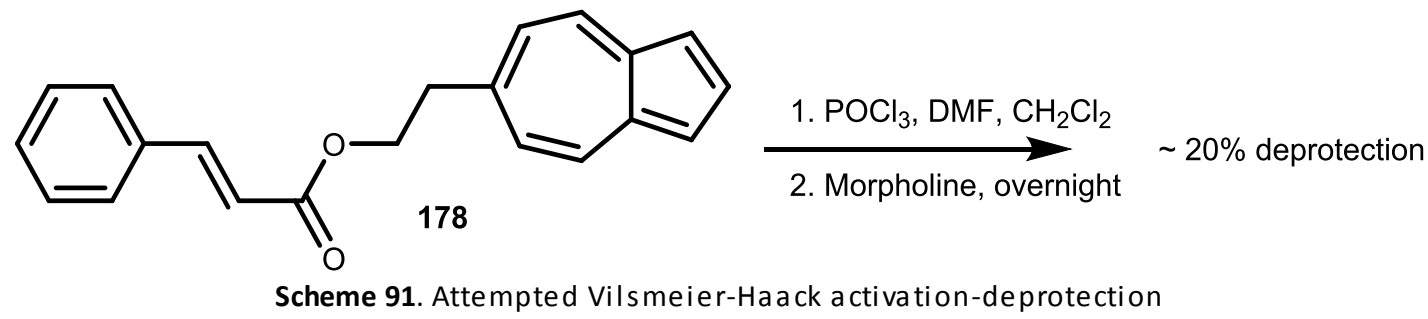

A one pot Vilsmeier-Haack activation-deprotection on Azul-cinnamate was tried (Scheme 91), using morpholine as a base. This gave approximately $20 \%$ deprotection based on ${ }^{1} \mathrm{H}$ NMR integration. While this initially seemed promising, multiple species were produced, giving a complex ${ }^{1} \mathrm{H}$ NMR spectrum and obscuring the relevant peaks. Furthermore, some of the byproducts were of roughly the same polarity as cinnamic acid and were prohibitively difficult to separate by column chromatography. While this reaction could conceivably be developed into an effective activation-deprotection method, it was judged to be inferior to the ketoester-based activation method described earlier.

\subsubsection{Deprotection of other substrates}

Various carboxylic acids and the cetyl Azul carbonate 193 (cetyl AzulOC) were subjected to the standard deprotection conditions consisting of DBU in MeCN (Table 17).

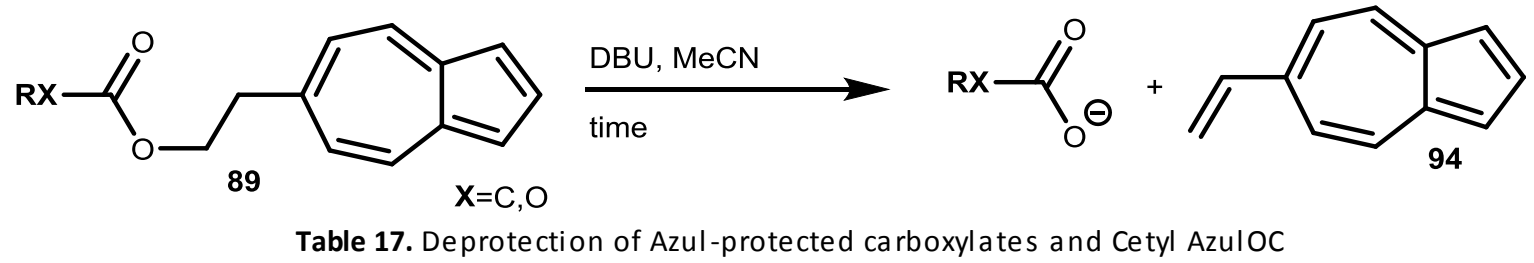

\begin{tabular}{|l|l|l|l|}
\hline Protected substrate & Deprotected substrate & Reaction duration & Yielda \\
\hline Azul-cinnamate 178 & cinnamic acid & 8 hours & $94 \%$ \\
\hline Azul-benzoate $\mathbf{1 8 2}$ & benzoic acid & 3 hours & $99 \%$ \\
\hline Azul-2-furoate $\mathbf{1 8 5}$ & 2-furoic acid & 4 hours & $97 \%$ \\
\hline Azul-1-naphthoate $\mathbf{1 8 8}$ & 1-naphthoic acid & 3 hours & $97 \%$ \\
\hline Azul-azulene-1-carboxylate $\mathbf{1 8 6}^{\text {b }}$ & azulene-1-carboxylic acid & overnight & $80 \%$ \\
\hline Cetyl AzulOC $\mathbf{1 9 3}^{\text {b }}$ & cetyl alcohol & 6 hours & $97 \%$ \\
\hline
\end{tabular}

a Isolated yield

b THF added as a co-solvent to aid dissolution

Most of the deprotections took place within 3 - 6 hours and returned close to quantitative yields. In the case of Azul cinnamate 182, the deprotection rate was lowered due to the sparing solubility of the material in MeCN. The previous deprotections of $\mathbf{1 8 2}$ presented in section 5.2.1 proceeded 
more quickly due to the reaction being performed at smaller scales with the same quantity of solvent, and were therefore at higher dilution. The sluggishness of the deprotection of $\mathbf{1 8 6}$ is explored in the following section.

\subsection{Deprotection kinetics}

It was noticed with the deprotection of Azul-azulene-1-carboxylate 186 that the rate of deprotection was much slower than for other Azul-protected substrates. This was thought to be due to the poor leaving ability of the azulenecarboxylate anion. To test the influence of the leaving group ability on the deprotection rate, several Azul-protected carboxylic esters were subjected to the identical deprotection conditions of 3.5\% DBU in MeCN while being continuously monitored with a UV-Vis spectrophotometer. The molar excess of DBU was sufficiently high for pseudo-first order kinetics to be assumed. The change in colour between the Azul-protected substrate and 6vinylazulene allowed the reaction rate to be monitored at visible wavelengths (Figure 63).

\section{Visible absorbance spectra of protected and deprotected Azul-cyclohexanecarboxylate 183}

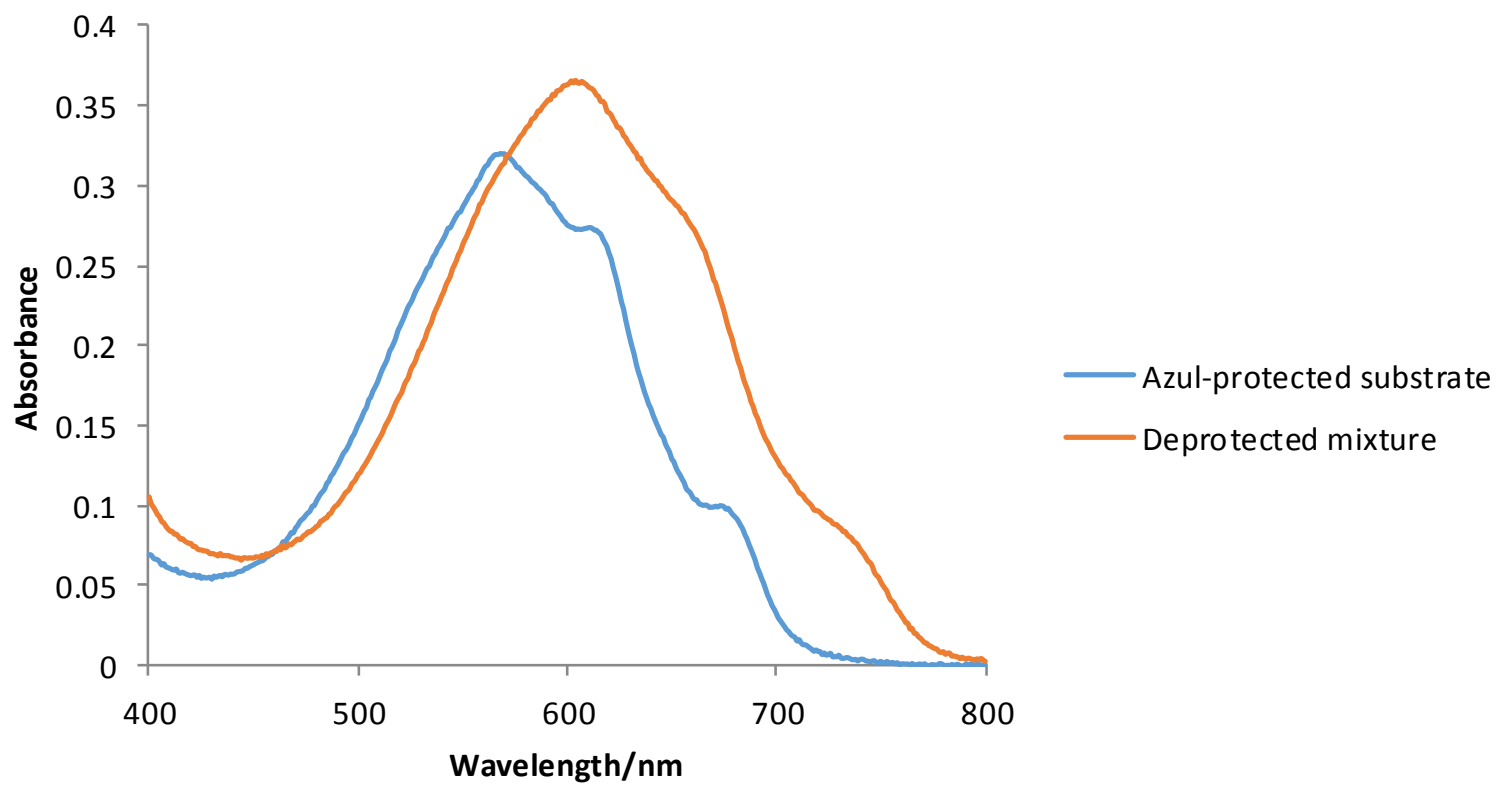

Figure 63. Absorbance spectra of protected and deprotected Azul-cycl ohexanecarboxylate 183, describing the observed change in colour

Eight Azul-protected carboxylic acids were investigated in this fashion, but only five of them gave useful data (Table 18). Of the successful experiments, the range of pKas was $6.99-3.12$, which was sufficiently broad to give a noticeable trend. 
Each substrate was observed to have a first-order decay profile, consistent with the expectation of pseudo-first order kinetics (183 serving as an example in Figure 64). The rate constant was extracted through finding an exponential of best fit, as discussed in chapter 7 .

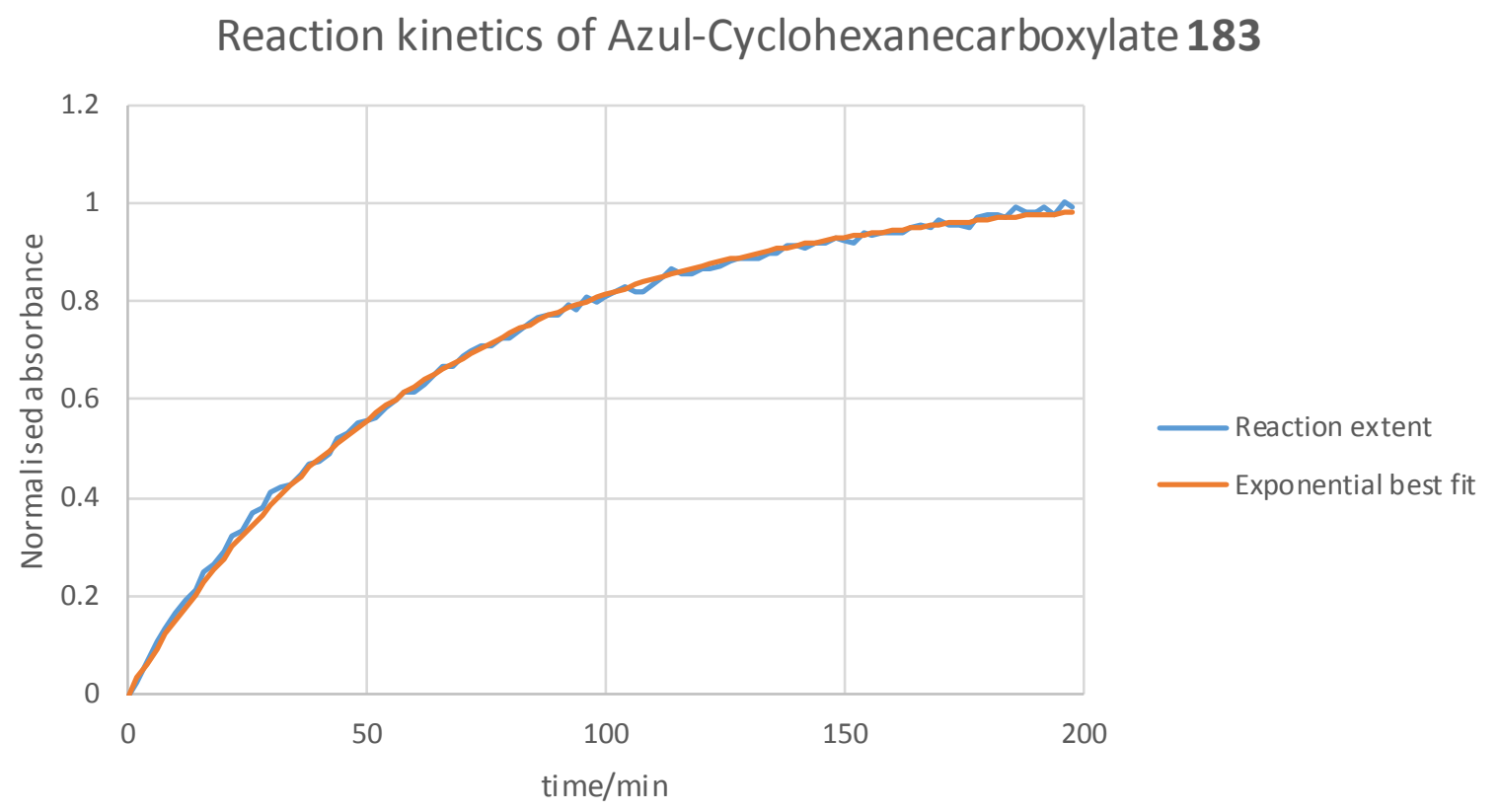

Figure $\mathbf{6 4}$. Reaction rate of $\mathbf{1 8 3}$, showing a reaction rate consistent with first order kinetics

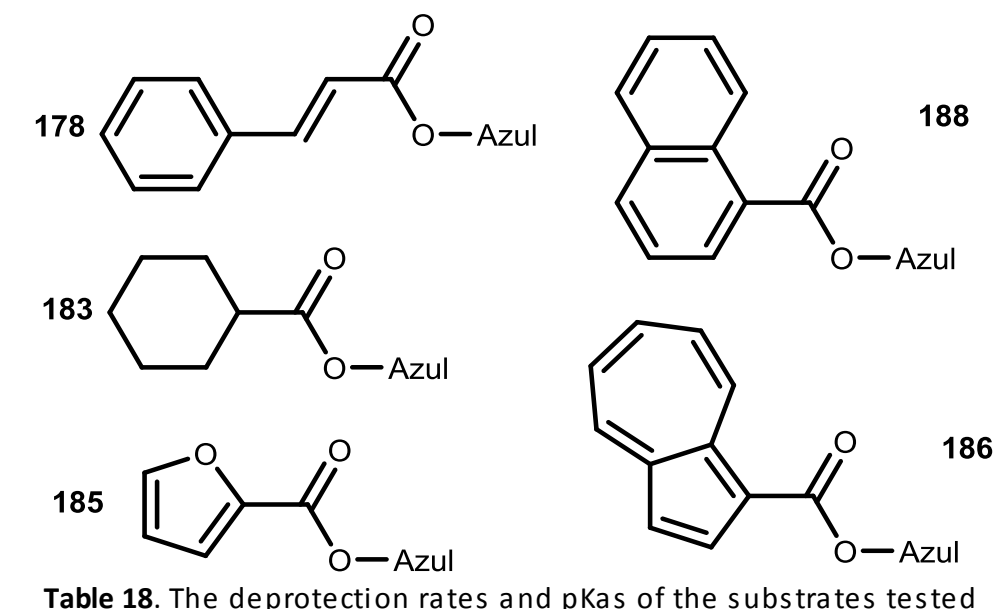

\begin{tabular}{|l|l|l|l|}
\hline Compound & $\begin{array}{l}\text { Deprotection } \\
\text { rate*1000/L } \\
\text { mol-1 }^{-1}\end{array}$ & $\begin{array}{l}\text { Half } \\
\text { life/min }\end{array}$ & $\begin{array}{l}\text { pKa of corresponding } \\
\text { carboxylic acid }\end{array}$ \\
\hline Azul-Furoate 185 & $2.20 \pm 0.64$ & 25 & 3.12 \\
\hline Azul-Naphthoate 188 & $1.48 \pm 0.34$ & 37 & 3.69 \\
\hline Azul-Cinnamate 178 & $1.12 \pm 0.43$ & 47 & 4.44 \\
\hline Azul-Cyclohexanecarboxylate 183 & $1.15 \pm 0.26$ & 44 & 4.9 \\
\hline Azul-Azulene-1-carboxylate 186 & $0.54 \pm 0.15$ & 103 & 6.99 \\
\hline
\end{tabular}




\section{Ln(k) vs leaving group pKa of Azul-carboxylate deprotection}

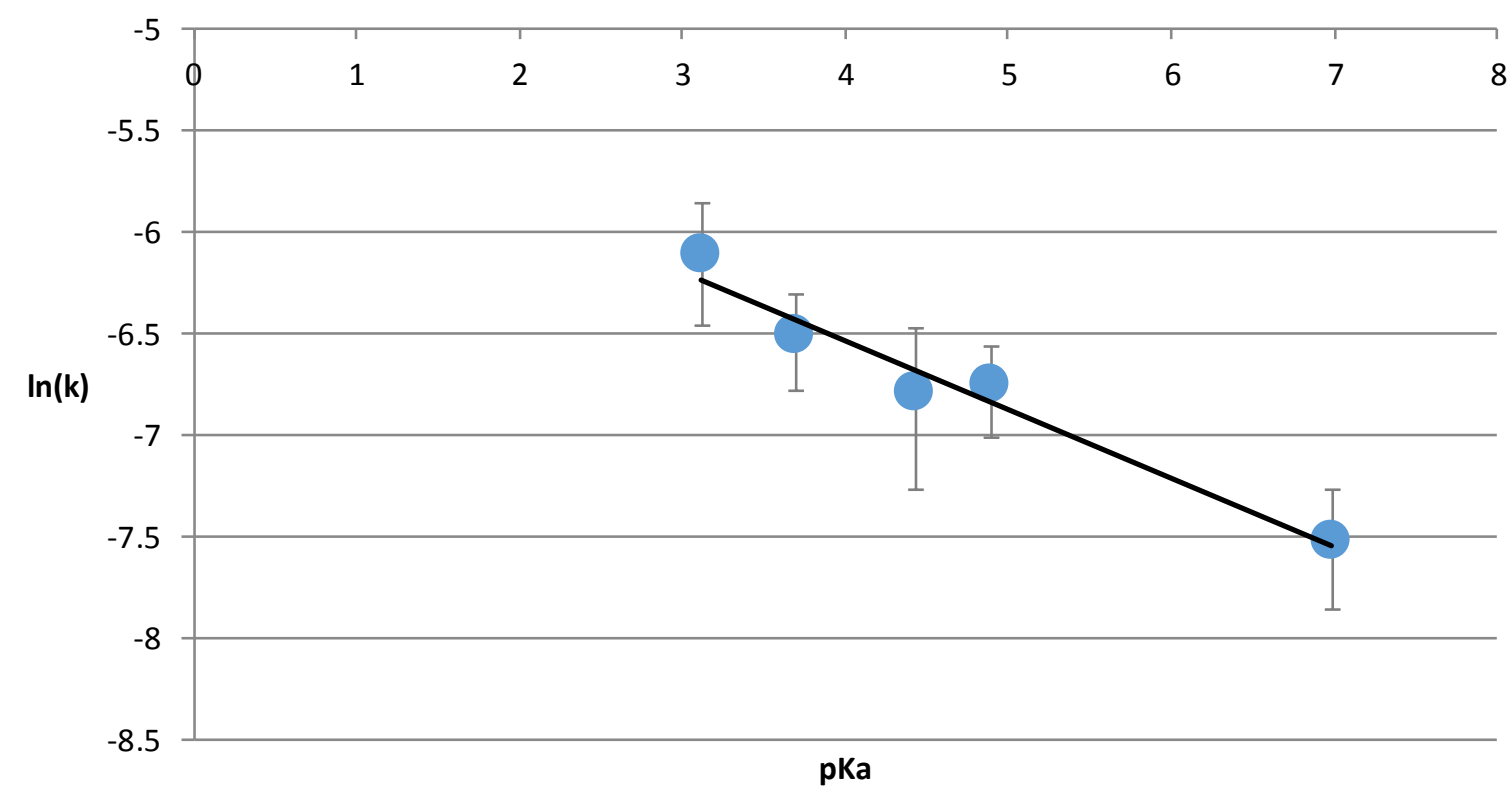

Figure 65. Correlation between the logarithm of the reaction rate and pKa of the leaving group

An obvious negative correlation can be seen between the pKa of the leaving group and the logarithm of the reaction rate $\left(\mathrm{R}^{2}=0.96\right)$ (Table 18, Figure 65). This indicates the negativelycharged transition state of the elimination mechanism is stabilised by the electron-withdrawing tendencies of the adjacent leaving group. It also suggests that the rate limiting step is the elimination step, rather than the deprotonation step (Figure 66). A flattening of the observed reaction rates at lower pKa ranges would indicate the switching of rate limiting steps from the elimination step to the deprotonation step, and the left hand side of Figure 65 would appear to level out.<smiles></smiles>

Figure 66. The elimination step - believed to be the rate-limiting step

The two esters with the most acidic corresponding carboxylic acids - the trichloroacetate $\mathbf{2 1 0}$ and dichloroacetate $\mathbf{2 1 1}$ did not exhibit an appreciable change in spectrum or colour upon addition of DBU. By NMR of the products, it appears the ester is hydrolysed directly with adventitious water and 6-(2-hydroxyethyl)azulene is produced, rather than 6-vinylazulene (Scheme 92). This indicates that the mechanism for ester cleavage changes from $\mathrm{E} 1 \mathrm{cB}$ to direct hydrolysis for sufficiently electron-poor esters. 

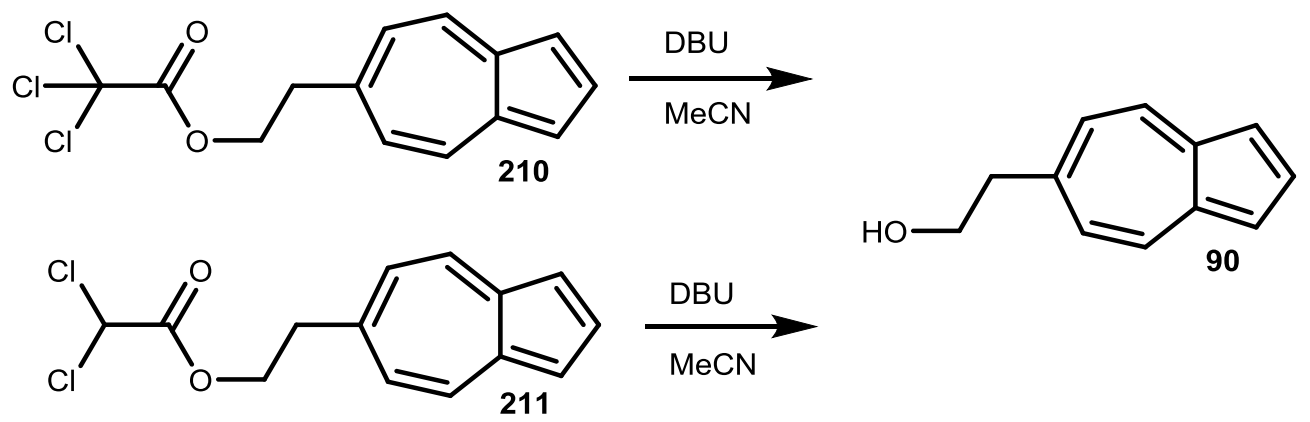

Scheme 92. Ester cl eavage of trichloroacetate $\mathbf{2 1 0}$ a nd dichloroacetate $\mathbf{2 1 1}$ proceeds through saponification, rather than $\beta$ elimination

The Azul-3,5-dinitrobenzoate ester 187 behaved in a markedly different and more surprising way. Upon addition of DBU to the reaction mixture, an immediate colour change from indigo to a much more intense crimson colour was observed (Figure 67). A change in the azulene ring system was tentatively ruled out, as there were no species present in the reaction mixture that could conceivably create the crimson colour by either substituting on to an odd position and being sufficiently electron poor, or substituting onto an even position and being sufficiently electron rich. The intensity of the crimson colour was also a red flag - azulene containing compounds with much higher extinction coefficients are usually heavily conjugated molecules with donor/acceptor functionalities.

\section{Azul 3,5-dinitrobenzoate, before and after addition of DBU}

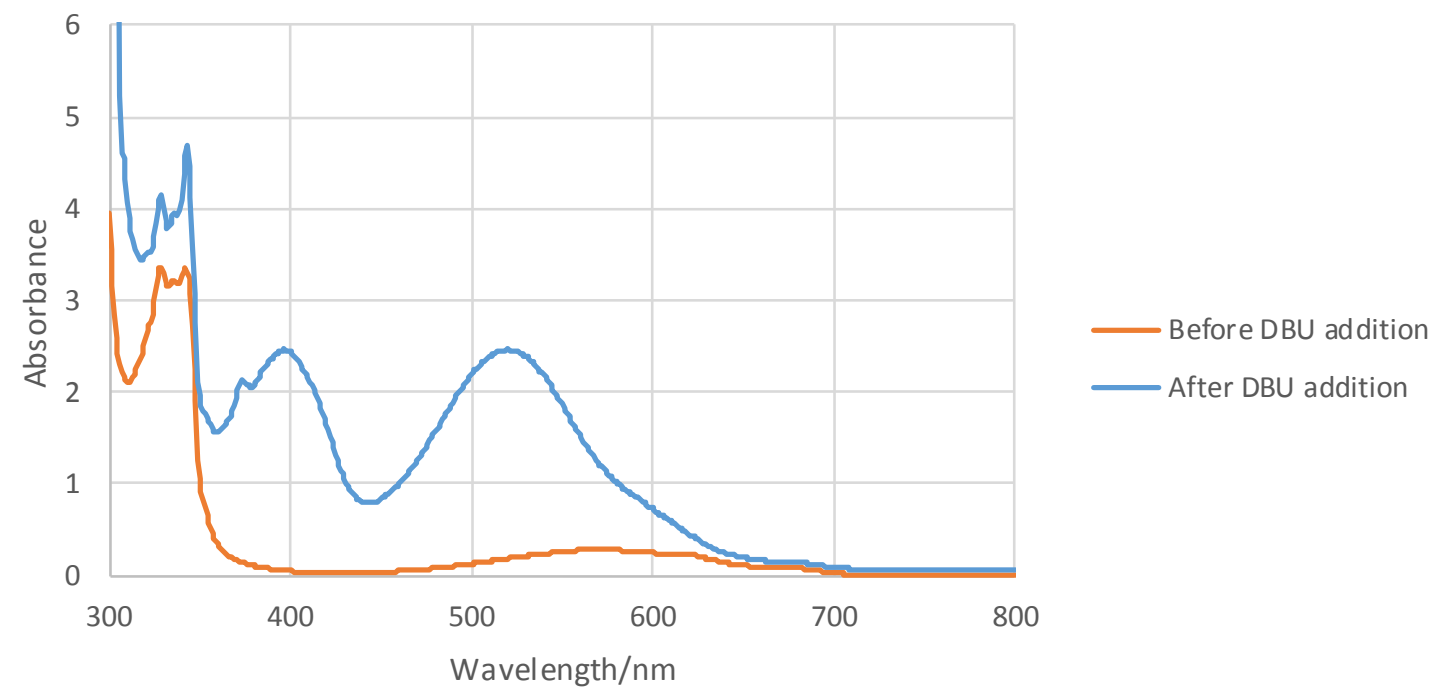

Figure 67. Addition of DBU ca uses formation of a Meisenheimer complex that is more strongly absorbing than the Azul species

The colour change was ultimately explained by formation of a Meisenheimer complex, which is a relatively stable negatively charged species resulting from nucleophilic attack of an electron deficient aromatic ring, in this case attack of DBU onto the 4-position of the dinitrobenzoate moiety (Scheme 93). 5 The intense crimson completely overwhelmed the UV-Vis spectrum and 
obscured the colour change associated with deprotection. Some spectral info on the reaction rate could be gleaned from the shoulder at $669 \mathrm{~nm}$ that gave a tentative deprotection rate of $0.005 \mathrm{~s}^{-1}$ or a half-life of 10 minutes, but this result can only be tentative, as the chosen wavelength is different from the wavelengths of the other substrates studied.<smiles>C1CCN=C2CCCCN2CC1</smiles><smiles>CCCCc1cc(C(=O)O[Al])cc([N+](=O)[O-])c1</smiles>

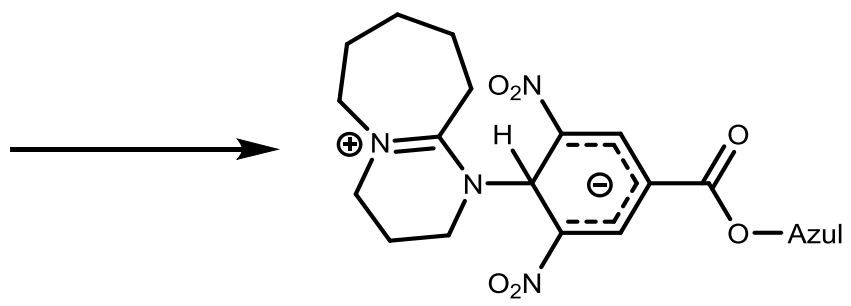

Scheme 93. Formation of a coloured Meisenheimer complex from DBU and $\mathbf{1 8 7}$

Interestingly, Azul 3,5-dinitrobenzoate appears to form a Wheland-Meisenheimer complex with itself in the solid phase (Figure 68). A Wheland-Meisenheimer complex occurs when the attacking nucleophile is an aromatic species capable of forming a positively charged conjugated system alongside the negatively charged Meisenheimer complex. ${ }^{6}$ While the compound appears indigo in solution, once it is allowed to dry it becomes a dirty green-brown colour typical of oxidised azulene species, indicating addition at the 1-position of the azulene. This aggregation is reversible, and upon addition of solvent this oligomeric material slowly dissolves and monomerises to give an indigo solution once again.

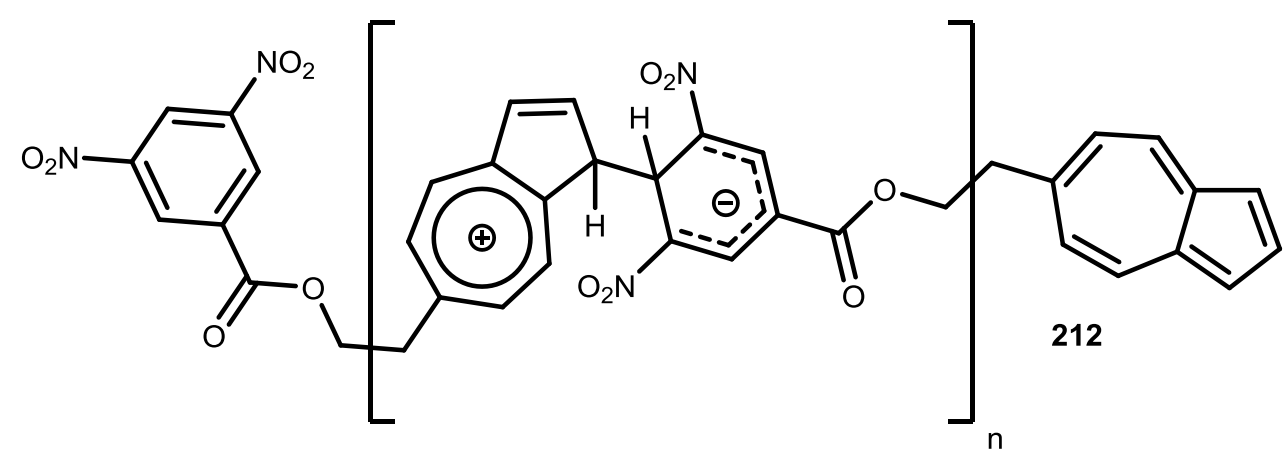

Figure 68. The postulated structure of the oligomeric Wheland-Meisenheimer complex generated from 187

\subsection{Regeneration of 6-(2-hydroxyethyl)azulene (90)}

As discussed previously in section 4.3, the prospect of being able to recycle a protecting group after cleavage is an attractive one, especially when the protecting group employed is expensive or scarce. A large quantity of 6-vinylazulene was accumulated over the course of this project as a result of deprotection reactions, and would otherwise be of no value if it could not be regenerated into starting material. 


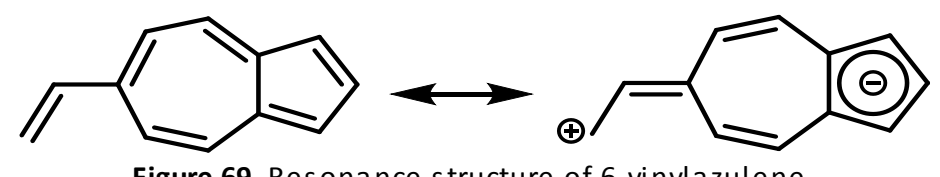

Figure 69. Resonance structure of 6-vinylazulene

Addition of a hydroxyl group to the end of the double bond was expected to be relatively easy, as 6-vinylazulene is electrophilic at that position on account of the stabilisation of the negative charge on the zwitterionic resonance structure (Figure 69). This stabilisation overwhelms the Markovnikov effect typically responsible for substitution at the more substituted position.

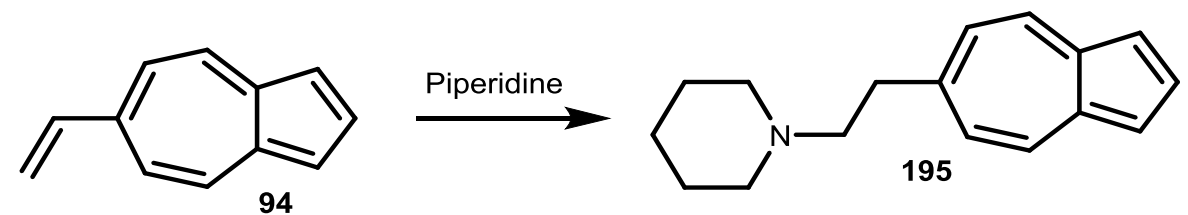

Scheme 94. The piperidine adduct 195 is generated in Azul deprotections involving piperidine

Nucleophilic attack at the less-substituted position had already been observed with piperidine in the deprotection of Azul-cinnamate 178, as mentioned earlier in Chapter 4 (Scheme 94). The nucleophile in question would either be water or another species that could be converted to a hydroxyl group in a subsequent step (Scheme 95). The choice of nucleophile was informed by Herbert Mayr's excellent tables of nucleophilicities. ${ }^{7}$

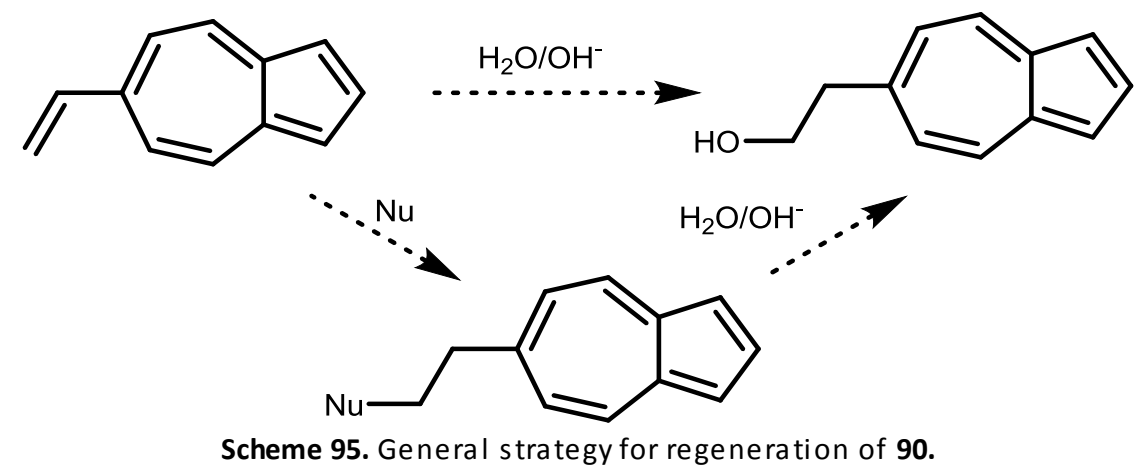

Many attempts were made to regenerate 6-(2-hydroxyethyl)azulene from 6-vinylazulene over the course of this project. Initial attempts involved either acid-catalysed alkene hydration or direct nucleophilic attack of $\mathrm{H}_{2} \mathrm{O} / \mathrm{OH}^{-}$(Schemes 96 and 97).

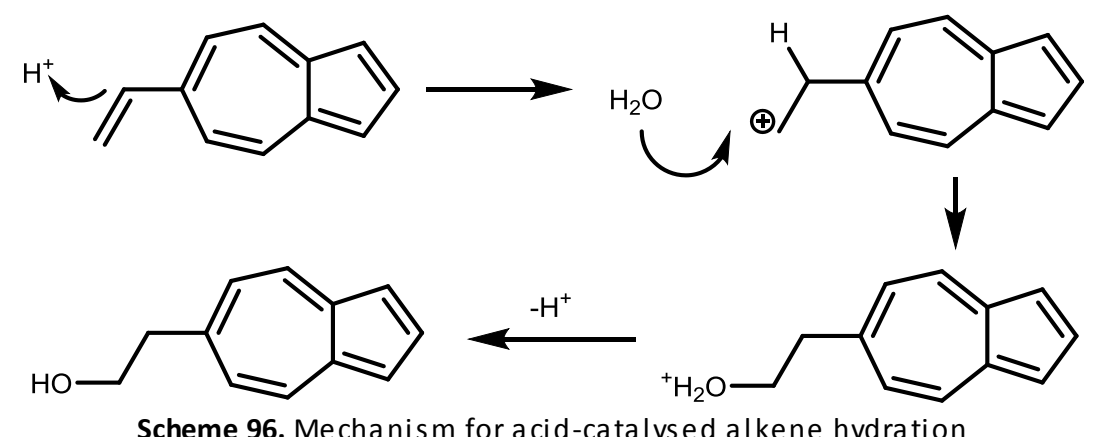




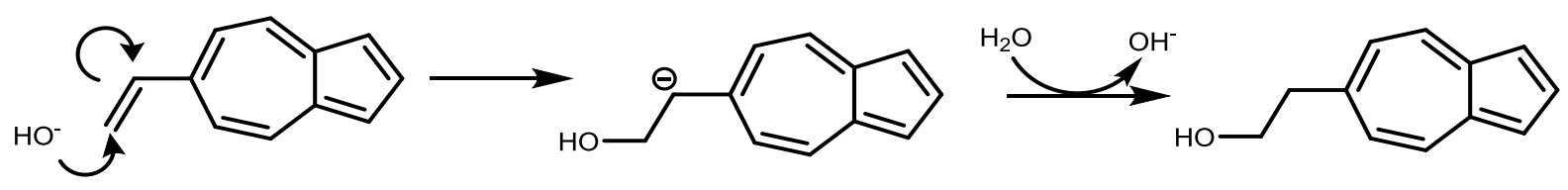

Scheme 97. Mechanism for basic alkene hydration

Neither water nor hydroxide were sufficiently nucleophilic to result in reaction and these reactions would invariably either return SM or result in degradation (Table 19). Mayr's tables of nucleophilicity give values of 5.5 for $\mathrm{H}_{2} \mathrm{O}, 10$ for $\mathrm{OH}$, and 19 for piperidine..$^{7-8}$ The scale is logarithmic.

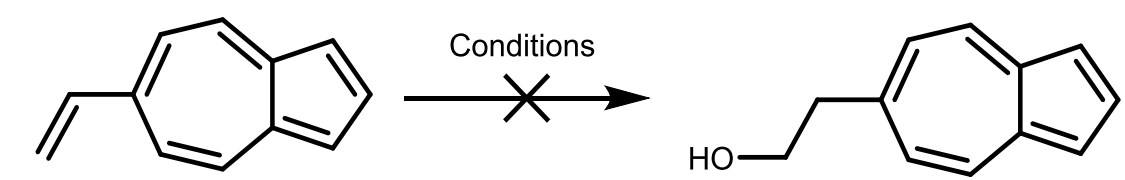

Table 19. Attempts at synthesising 90 from 94 through direct addition of $\mathrm{H}_{2} \mathrm{O}$ or $\mathrm{OH}^{-}$

\begin{tabular}{|l|l|l|}
\hline Reaction conditions & Temperature & Outcome \\
\hline $\mathrm{NaOH}, 1: 1 \mathrm{H}_{2} \mathrm{O} / \mathrm{THF}$, overnight & r.t. & No reaction \\
\hline $10 \% \mathrm{HCl}$ (aq.), THF, overnight & r.t. & Degradation \\
\hline $\mathrm{KOH}, 1: 1 \mathrm{THF} / \mathrm{H}_{2} \mathrm{O}$, overnight & Reflux & Degradation \\
\hline $\mathrm{NEt}_{3} / \mathrm{H}_{2} \mathrm{O} 2: 1$, overnight & r.t. & No reaction \\
\hline $\mathrm{MeCN} / \mathrm{H}_{2} \mathrm{O} 2: 1, \mathrm{NEt}_{3}, p-\mathrm{TsOH}$ & $\sim 30^{\circ} \mathrm{C}$ & No reaction \\
\hline$p-\mathrm{TsOH}, \mathrm{H}_{2} \mathrm{O}, \mathrm{THF}$, overnight & r.t. & SM + degradation \\
\hline $\mathrm{KOH}, \mathrm{H}_{2} \mathrm{O}, \mathrm{DME}, 1$ week & r.t. & $\mathrm{SM}+$ degradation \\
\hline
\end{tabular}

$\mathrm{THF} / \mathrm{H}_{2} \mathrm{O}$ mixtures will separate into different phases upon addition of aqueous base, diminishing the capacity for reaction. It was found that $\mathrm{DME} / \mathrm{H}_{2} \mathrm{O}$ mixtures remained monophasic when treated with aqueous base. Nevertheless, no reaction was observed to take place in $\mathrm{DME} / \mathrm{H}_{2} \mathrm{O}$ with addition of $\mathrm{KOH}$.

From the results of the compatibility studies with low-temperature Suzuki cross-coupling (discussed in more detail in section 5.7.2), 8\% conversion of Azul-benzoate 182 to 6-(2-hydroxyethyl)azulene (90) was observed. It was postulated that deprotection was taking place, generating and hydrating 6-vinylazulene (94) in situ through the action of the palladium catalyst (Scheme 98). 


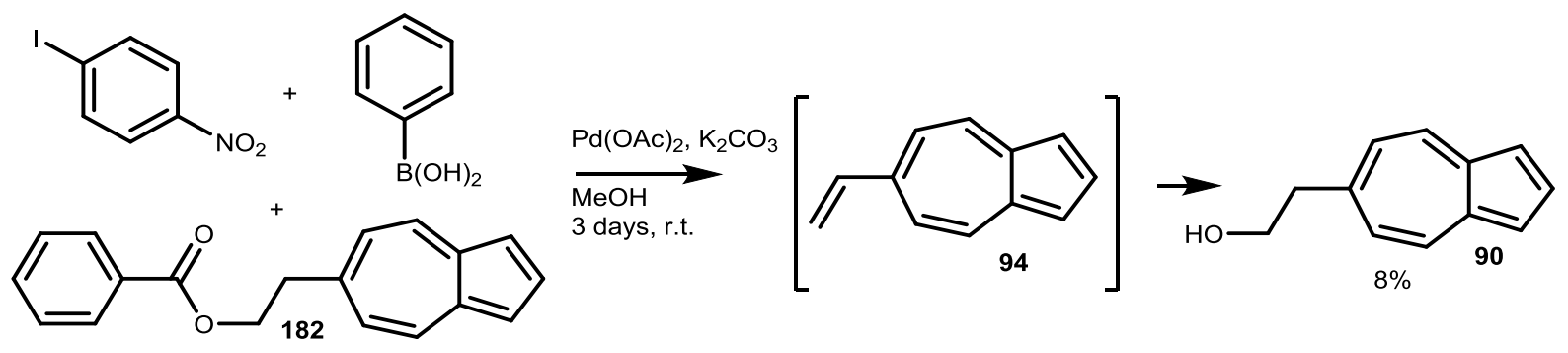

Scheme 98. Low-temperature Suzuki cross coupling in the presence of $\mathbf{1 8 2}$ generated a small amount of $\mathbf{9 0}$

To explore the possibility of this mechanism taking place, 6-vinylazulene was subjected to conditions similar to the original reaction: $\mathrm{Pd}(\mathrm{OAc})_{2}, \mathrm{~K}_{2} \mathrm{CO}_{3}$ in a $3: 1 \mathrm{THF} / \mathrm{H}_{2} \mathrm{O}$ mixture $(\mathrm{MeOH}$ was not used due to the possibility of (Scheme 99). After two days at r.t. no reaction had been observed, so the mixture was heated at reflux for one further day, after which still no reaction took place. These results suggest that generation of 6-(2-hydroxyethyl)azulene in the lowtemperature Suzuki reaction was likely to occur through ester saponification rather than a deprotection-hydration sequence.
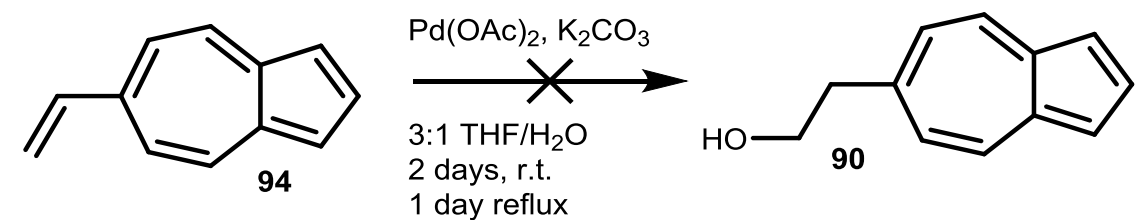

Scheme 99. Attempted synthesis of $\mathbf{9 0}$ by repeating the conditions found in Scheme 96

With the failure of these single-step methods for the regeneration of $\mathbf{9 0}$, two-step procedures were considered. It had been observed previously that in deprotections involving DBU that after approximately one week, the blue colour of 6-vinylazulene would revert to indigo. This suggested the formation of a DBU adduct to 6-vinylazulene. If the DBU moiety could be hydrolysed by base, a route to regeneration of 6-(2-hydroxyethyl)azulene might be achieved (Scheme 100).

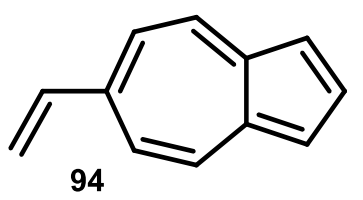

94

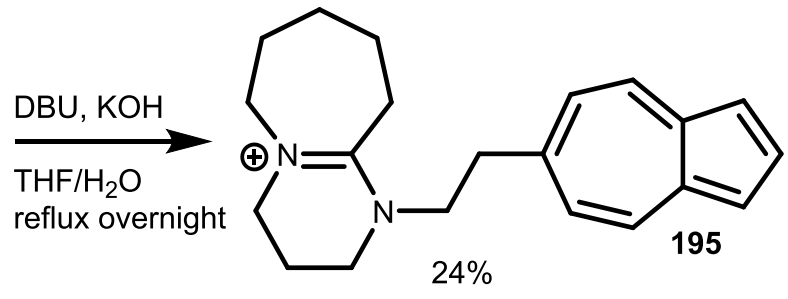

Scheme 100. Synthesis of the DBU adduct 195

Heating 6-vinylazulene at reflux in the presence of DBU gave the adduct 195 in $24 \%$ yield. Subsequent treatment of $\mathbf{1 9 5}$ with $\mathrm{KOH}$ in $\mathrm{H}_{2} \mathrm{O}$ /THF at room temperature gave no reaction, even after a week, while heating the same mixture to reflux resulted in degradation with traces of 6vinylazulene present. Postulating that the DBU moiety was too poor a leaving group on account of the pKa of DBU, activation with DMAP (the conjugate acid of which has a pKa of 17.95 in MeCN, 
compared to 24.3 for $\mathrm{DBU}-\mathrm{H}^{+}$, making it a better leaving group) was investigated. No reaction occurred between 6-vinylazulene and DMAP, even at reflux in THF (Scheme 101).

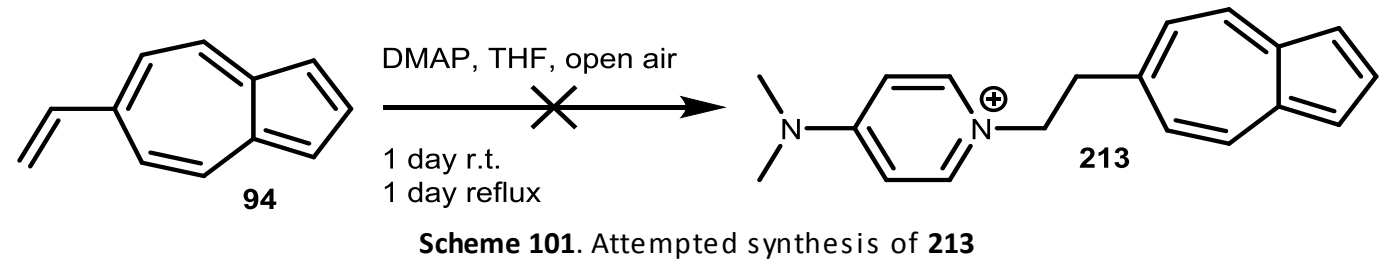

The next nucleophile considered was sodium nitrite. According to Mayr's tables, the nitrite anion has a relatively high nucleophilicity - around 17. Nitrite can react both at the nitrogen and the oxygen position, depending seemingly arbitrarily on the specific reaction conditions. ${ }^{9}$ If the reaction occurs on the oxygen, the resulting compound is a nitrite ester, which can subsequently be hydrolysed. However, after subjecting 6-vinylazulene to sodium nitrite in refluxing acetonitrile, no reaction was observed (Scheme 102).
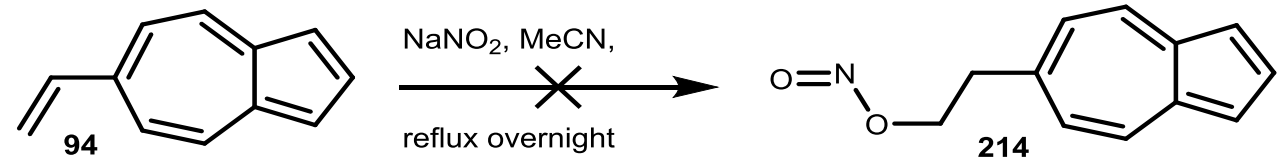

Scheme 102. Attempted synthesis of $\mathbf{2 1 4}$

The nucleophilic hydroperoxide anion (HOO-) was also considered. The nucleophilicity of HOO- is approximately 5 orders of magnitude higher than hydroxide, ${ }^{7}$ and nucleophilic attack on 6vinylazulene was expected to produce 6-(2-hydroperoxyethyl)azulene 215 which could then plausibly be reduced to obtain the desired compound 90 (Scheme 103).

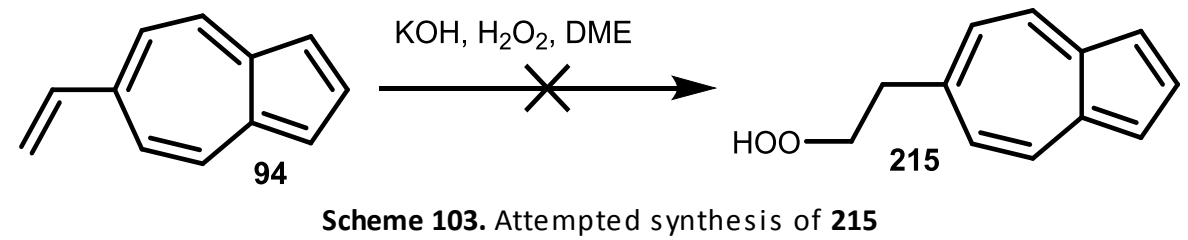

Hydrogen peroxide disproportionates to water and oxygen rapidly in the presence of base, and vigorous bubbling would occur until all the hydrogen peroxide had been consumed, without appearing to react with 6-vinylazulene to any detectable level. No reaction was observed when using DME as a solvent. When $\mathrm{MeOH}$ was used a highly non-polar indigo compound was returned in $27 \%$ yield (Scheme 104). The ${ }^{1} \mathrm{H}$ NMR spectrum showed evidence of an Azul moiety and a terminal methyl, and HRMS confirmed the structure as Azul-methyl ether 216.

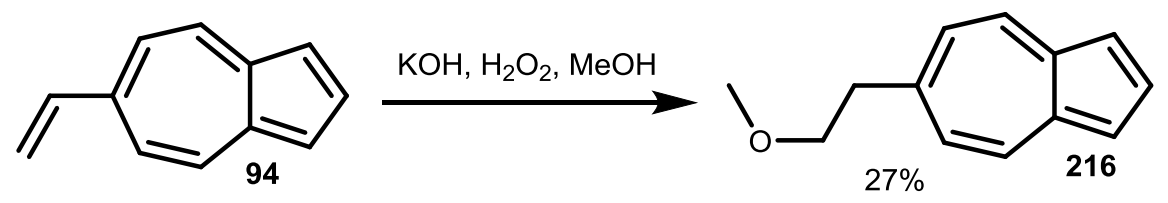

Scheme 104. The methyl ether $\mathbf{2 1 6}$ was generated from the methanolic KOH present in the reaction 
The surprising reactivity of 6-vinylazulene to methoxide suggested an alternative route to 6-(2-hydroxyethyl)azulene: addition of an alcoholic protecting group precursor followed by deprotection (Scheme 105).<smiles>C=Cc1ccc2cc(C=C)cc-2cc1</smiles>

Scheme 105. Generalised two-step route to regeneration of $\mathbf{9 0}$

3,4-Dimethoxybenzyl alcohol was chosen as the protecting group precursor, as deprotection of DMB using DDQ is relatively easy to perform. Addition of 6-vinylazulene to a 1:1 solution of 3,4dimethoxybenzyl alcohol in DME in the presence of $\mathrm{KOH}$ gave the desired product $\mathbf{2 1 7}$ in $49 \%$ yield after 4 hours (Scheme 106). Further reaction times resulted in increasing amounts of degradation.
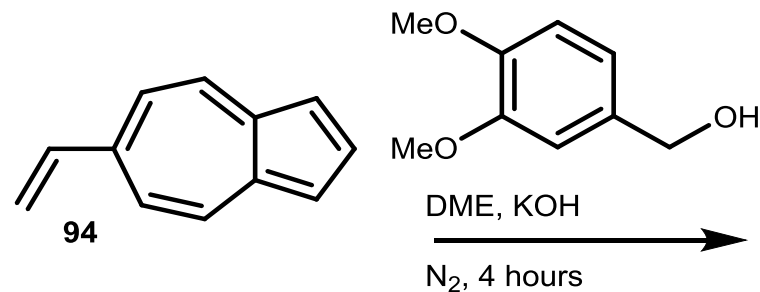

$\mathrm{N}_{2}, 4$ hours

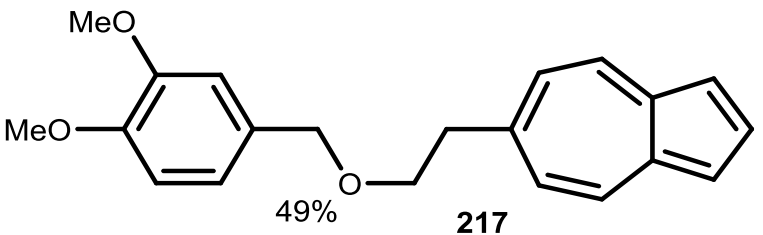

217

Scheme 106. Synthesis of the Azul 3,4-dimethoxybenzyl ether 217

Unfortunately, azulenes degrade rapidly and completely in the presence of DDQ (Scheme 107). A sticky green substance resulted from the addition of DDQ to $\mathbf{2 1 7}$, and repeating the reaction with azulene resulted in a similar degradation, which indicates this tendency towards degradation is inherent to the azulene moiety and not just a feature of $\mathbf{2 1 7}$.

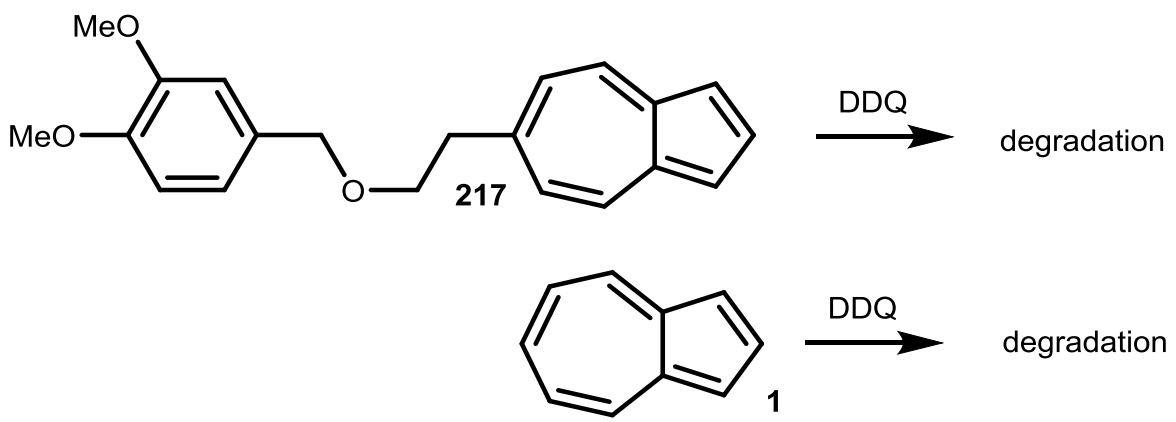

Scheme 107. Addition of DDQ resulted in rapid degradation of the azulene moiety

An alternative set of DMB deprotection conditions was tried - cerium chloride and sodium iodide. ${ }^{10}$ Several products were present in very small quantities, but were difficult to separate and had indistinct ${ }^{1} \mathrm{H}$ NMR spectra. None of the observed spectra corresponded to 6-(2-hydroxyethyl)azulene. There is circumstantial evidence of substitution at the 1-position for 
some of the products. A polar blue (as opposed to indigo) product was present, with a ${ }^{1} \mathrm{H}$ NMR spectrum lacking triplet multiplicity for $\mathrm{H}$-2. It is possible that the deprotection by-product of this reaction, 3,4-dimethyoxybenzyl iodide, reacted with the 1-position of azulene through a cerium chloride-catalysed Friedel-Crafts alkylation to form a compound such as $\mathbf{2 1 8}$ (Figure 70). Such a result would be unusual, as azulenes are notoriously difficult to functionalise through FriedelCrafts alkylation. ${ }^{11}$ However, this reaction was not investigated further, owing to time constraints.

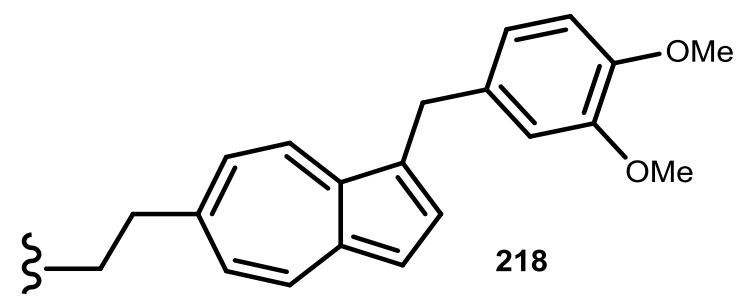

Figure 70. Possible structure of one of the products resulting from $\mathrm{CeCl}_{3}$-mediated deprotection of DMB

Regeneration of 6-(2-hydroxyethyl)azulene from 6-vinylazulene remains elusive. It is believed that reintroduction of the hydroxyl group should be possible under the right conditions, and that an efficient method for regenerating $\mathbf{9 0}$ would be an attractive feature of the Azul protecting group.

\subsection{Deprotection summary}

Removal of the Azul protecting group is very easily accomplished. One-step deprotection can be performed with amidine bases such as DBU or DBN or with TBAF at room temperature, and do not require dry or inert-atmosphere conditions to proceed. At elevated temperatures, piperidine and pyrrolidine in acetonitrile can be used for deprotection. The majority of amine bases do not react at all with the Azul protecting group, which is an advantageous feature considering how commonly certain amine bases (such as DABCO, Hünig's base, DMAP and $\mathrm{NEt}_{3}$ ) are employed as reagents. The colour change from indigo to blue that is typically observed upon deprotection allows self-indicating reaction reaction to self-indicate.

Two-step deprotection can be accomplished through the introduction of a methyl ketoester, followed by the addition of a very mild base such as morpholine. The activation reaction is quantitative and gives a colour change from indigo to an orange-red, and increased the reactivity towards deprotonation by an estimated seven orders of magnitude. Other activation methods were explored, but were deemed to be insufficiently effective or mild. 


\subsection{Comparisons with other protecting groups}

\subsubsection{Overview}

Crucial to the development of a new protecting group is the assessment of its compatibility with other commonly used protecting groups. Ideally, there should exist conditions where either the new or pre-existing protecting group be cleaved in the presence of the other. The ability to perform a global deprotection isn't a requirement, but is a nice bonus. Any incompatibilities between protecting groups need to be fully understood if the protecting group is to be useful for the wider synthetic organic community.

Originally, the comparison was to be performed on a single substrate with both protecting groups attached. Through initial tests on FMOC-Gly-OAzul 189, it was found that large numbers of similar compounds could be formed through these experiments, confounding ${ }^{1} \mathrm{H}$ NMR analysis. Also, the syntheses of these compounds proved challenging. For instance, the comparison with TBS ethers was originally intended to be performed on the substituted glycolate $\mathbf{2 2 0}$, but isolation of the polar intermediate $\mathbf{2 1 9}$ from the contents of the previous reaction proved to be prohibitively difficult (Scheme 108).

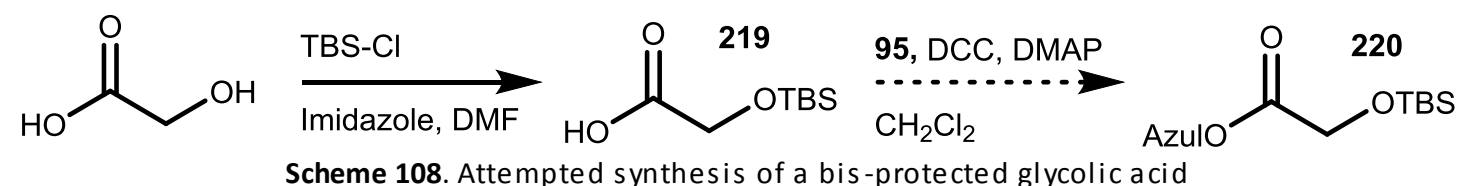

Instead, the orthogonality comparisons were typically performed on two separate species - a protected subject of the protecting group in question, and an Azul-protected carboxylic acid.

Conditions were found that allowed the Azul protecting group to be fully compatible with the TBS and MOM protecting groups, whereas for FMOC only partial orthogonality could be achieved.

\subsubsection{Orthogonality to TBS}

The TBS ether protecting group is one of the most important and widely used protecting group for hydroxyls. TBS ethers are typically cleaved through treatment with acid or fluoride, but are relatively stable to base. This combination immediately suggests some selective cleavage methods, as the Azul protecting group is stable to acid, but labile to base and fluoride. TBS-cyclohexanemethanol $\mathbf{2 2 1}$ was used as the TBS-protected substrate. Mixtures of 221 and Azul cinnamate were made up, typically in around 1:1 ratios in preparation for the selective deprotection reactions (Scheme 109). 


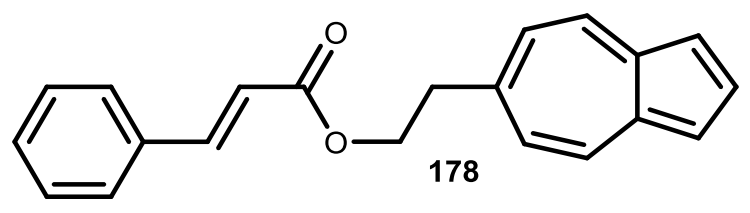

$\overbrace{221}^{T B S}$

Deprotection conditions

Scheme 109. Various conditions for deprotection were explored on the mixture of $\mathbf{1 7 8}$ and $\mathbf{2 2 1}$

Selective cleavage of the TBS ether and global deprotection were found relatively easily (Table 20). Treatment of the mixture with $4: 1 \mathrm{MeOH} / 10 \%$ aq. $\mathrm{HCl}$ solution overnight at room temperature gave complete cleavage of the TBS group, but also a small amount of oxidative degradation of the azulene moiety. To circumvent this problem, the weak acid PPTS was used instead. Addition of PPTS followed by heating at reflux overnight in MeCN gave complete TBS cleavage with no cleavage of the Azul group.

Deprotection of both the TBS-ether and Azul-cinnamate (i.e. global deprotection) was achieved by reaction with TBAF in THF for 3.5 hours. Shorter reaction times resulted in only partial cleavage of both protecting groups.

Finding a workup protocol for selective Azul deprotection was somewhat trickier on account of having to remove DBU under non-acidic conditions. Fortunately, DBU is sufficiently basic to reversibly form a complex with water (DBU- $\mathrm{H}^{+} \mathrm{OH}^{-}$) and could be removed through repeated washes of water during phase separations. ${ }^{12}$ The cinnamate salt was then extracted from the aqueous wash using aqueous hydrochloric acid and copious extraction with $\mathrm{CH}_{2} \mathrm{Cl}_{2}$.

\begin{tabular}{|l|l|l|}
\hline & No TBS deprotection & Full TBS deprotection \\
\hline $\begin{array}{l}\text { No Azul } \\
\text { Deprotection }\end{array}$ & & $\begin{array}{l}1: 410 \% \mathrm{HCl}_{(\mathrm{aq})} / \mathrm{MeOH} \text { r.t. overnight. } \\
\text { Or PPTS, MeCN reflux overnight }\end{array}$ \\
\hline $\begin{array}{l}\text { Full Azul } \\
\text { deprotection }\end{array}$ & DBU, THF, 7 hours r.t. & TBAF, THF, 3.5 hours, r.t. \\
\hline
\end{tabular}

Table 20. Selective and global deprotection conditions for Azul and TBS mixtures

From these results, it was concluded that full orthogonality between Azul and TBS can be achieved. 


\subsubsection{Orthogonality to FMOC}

The FMOC protecting group was considered to serve as a useful comparison to Azul, owing to their shared mode of deprotection through base-induced $\beta$-elimination. As FMOC-glycine was readily available, the Azul group was introduced via the method discussed in Chapter 4 to obtain the compound 189 (Figure 71).

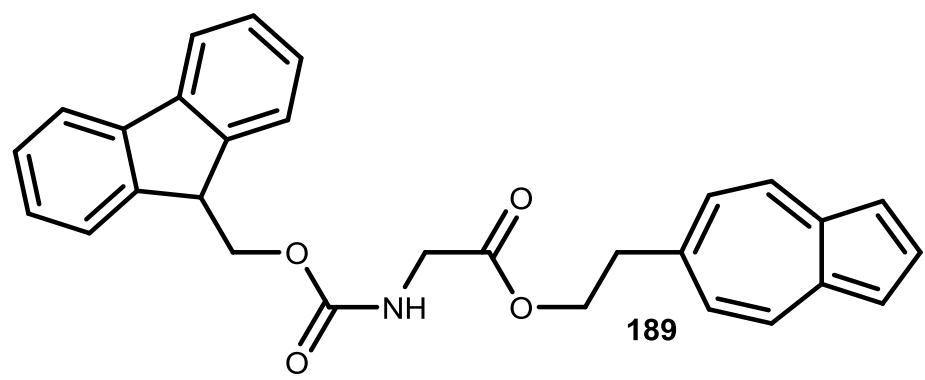

Figure 71. FMOC-Gly-OAzul

FMOC was found to be much more labile to basic conditions than the Azul group, and full selective FMOC deprotection was easily achieved by reacting with piperidine in MeCN for 30 minutes at r.t., while global deprotection could be achieved by treatment with typical conditions for Azul deprotection - namely DBU in MeCN for 8 hours, or TBAF in THF for 30 minutes (Table 21).

The rate of FMOC deprotection precludes the development of a one-step selective Azul deprotection protocol, so the use of a two-step protocol was investigated.

189

1. $(\mathrm{COCl})_{2}, \mathrm{CH}_{2} \mathrm{Cl}_{2}$, 20 seconds, r.t.

2. $\mathrm{MeOH}, 30$ seconds

3. Pyridine

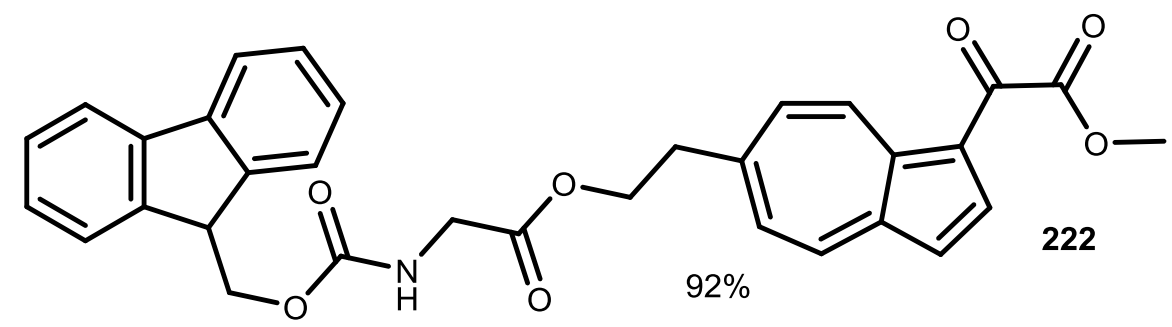

Scheme 110. Activation of FMOC-Gly-OAzul in preparation for selective Azul deprotection

The Azul protecting group was activated using the methodology discussed in section 5.2.3 to give the compound 222 in high yields (Scheme 110). Despite the activation, selective Azul deprotection was difficult to achieve, as FMOC was only slightly less reactive than the activated Azul protecting group. The most successful result involved using $\mathrm{NEt}_{3}$ in $\mathrm{MeCN}$ at $0{ }^{0} \mathrm{C}$ to give full Azul deprotection and 25\% FMOC deprotection. 


\begin{tabular}{|l|l|l|}
\hline & No FMOC deprotection & Full FMOC deprotection \\
\hline No Azul Deprotection & & Piperidine, MeCN, 30 minutes r.t. \\
\hline Full Azul deprotection & $\begin{array}{l}\text { 1. }(\mathrm{COCl})_{2}, \mathrm{CH}_{2} \mathrm{Cl}_{2} \\
2 . \mathrm{NEt}_{3}, \mathrm{MeCN}, 0{ }^{\circ} \mathrm{C} \text { a }\end{array}$ & $\begin{array}{l}\text { TBAF, THF, 30 minutes r.t. } \\
\text { or DBU, MeCN, 8 hours r.t. }\end{array}$ \\
\hline
\end{tabular}

Table 21. Selective and global deprotection conditions for FMOC-Gly-OAzul

a25\% deprotection of FMOC was observed

These results show that partial orthogonality between Azul and FMOC can be achieved. Through the right choice of solvent and temperature, it is anticipated that selective Azul deprotection in the presence of FMOC is attainable.

\subsubsection{Orthogonality to MOM}

The MOM-protected substrate used in this study was the MOM-cetyl ether 223. MOM is typically only deprotected through acidic or Lewis acidic conditions, and this left little room for the design of a global deprotection method. Azul cinnamate was used as the Azul protected substrate in this study (Scheme 111).

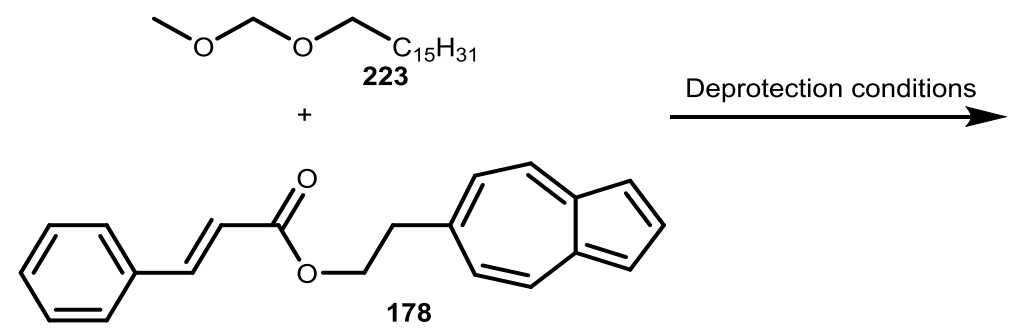

Scheme 111. Various conditions for deprotection were explored on the mixture of $\mathbf{1 7 8}$ and $\mathbf{2 2 3}$

Azul-cinnamate was deprotected selectively in the presence of MOM through addition of DBU to MeCN (Table 22). Selective MOM deprotection was attempted though heating the mixture at reflux in a 1:30 v/v solution of $10 \% \mathrm{HCl}$ (aq.) in $\mathrm{MeOH}$, which gave $\mathrm{MOM}$ deprotection concurrently with Azul degradation. Use of the mild acid PPTS while heating at reflux in a 3:1 mixture of EtOH/MeCN (MeCN present to aid solubility) gave full selective MOM deprotection and no observable degradation of Azul-cinnamate. These results show that full orthogonality of Azul with MOM can be achieved

\begin{tabular}{|l|l|ll|}
\hline & No MOM deprotection & Full MOM deprotection & \\
\hline No Azul Deprotection & & $\begin{array}{l}\text { PPTS, 3:1 EtOH/MeCN reflux } \\
\text { overnight }\end{array}$ \\
\hline Full Azul deprotection & DBU, MeCN, r.t. overnight & \\
\hline
\end{tabular}

Table 22. Selective deprotection conditions for Azul and MOM mixtures 


\subsection{Reaction compatibility}

\subsubsection{Overview}

Knowledge of a protecting group's compatibility with various reaction conditions is crucial when designing a synthetic scheme. If the effects of a common protocol are unknown on a new protecting group, chemists will be reluctant to use the protecting group in their syntheses.

Several commonplace reactions were investigated to this end, to explore not only the effect of the reaction on the protecting group, but also the effect of the protecting group on the reaction. A set of representative reactions was sought, based on both diverse chemical phenomena and common synthetic requirements. These reactions included: Suzuki cross coupling, sodium borohydride reduction, Masamune-Roush, and Swern oxidation

The azulene-protected species chosen for this work was the benzoate ester $\mathbf{1 8 2}$. This ester was chosen in particular due to the expected inertness of the benzoate functionality to the reaction conditions explored. (Figure 72).

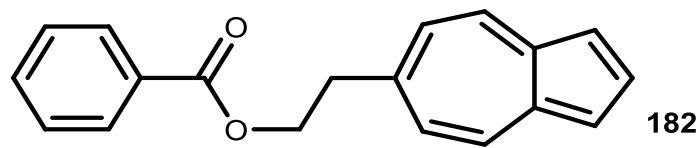

Figure 72. Azul-benzoate $\mathbf{1 8 2}$ was used as the Azul-protected substrate

Compatibility was tested by running the reactions in duplicate, with one flask charged with Azulbenzoate and one control without any azulene-based contamination. These reactions were run side-by-side and the reaction conditions (relative quantities of reagents, timing, temperature) were kept as similar to each other as possible. Afterwards, each reaction was subjected to some form of simple workup designed to allow an intelligible ${ }^{1} \mathrm{H}$ NMR spectrum of the crude product mixture to be obtained, from which the extent of reaction could be assessed through integration of the peaks corresponding to the relevant chemical species.

Incompatibilities were found in only two reactions - high-temperature Suzuki cross-coupling and Swern oxidation. The azulene moiety was found to be compatible to all the other reaction conditions explored.

\subsubsection{Suzuki cross-coupling}

The Suzuki cross-coupling is one of the most popular and widely used reactions for carbon-carbon bond formation, making it ideal as the subject of the first reaction compatibility investigation. ${ }^{13}$ 
There were two potential points of failure for this reaction, one being the possibility of deprotection through the presence of base, and the other being the potential for palladiumcatalysed activation and substitution on the nucleophilic 1-position of the azulene moiety.

A traditional (i.e. high temperature) Suzuki cross-coupling between phenylboronic acid (224) and 2-bromoanisole (225) catalysed by palladium tetrakis(triphenylphosphine) was performed (Scheme 112). After 2 hours, the control reaction gave 70\% conversion of the starting material $\mathbf{2 2 5}$, while the azulene-doped reaction gave $45 \%$ conversion to $\mathbf{2 2 6}$, in addition to approximately $17 \%$ conversion of the Azul-benzoate to other species, including 6-vinylazulene (94) and various highly polar materials that degraded during workup.

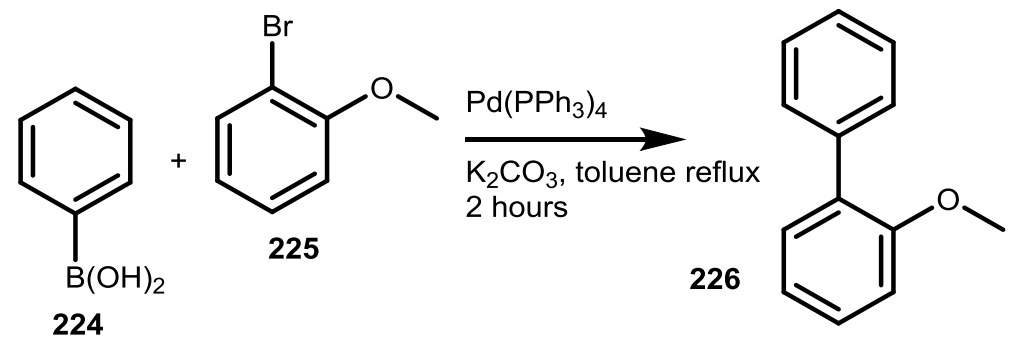

Scheme 112. The reaction used to assess the compatibility of Suzuki cross coupling with Azul

It is not clear what the cause of the Azul-benzoate deprotection is. A follow-up reaction involving Azul-benzoate in the presence of palladium tetrakis(triphenylphosphine) and potassium carbonate in refluxing toluene (without the cross-coupling reactants) gave only $8 \%$ conversion of 186 to 6-vinylazulene after heating at reflux overnight - much less than the $17 \%$ conversion over two hours observed previously. A similar reaction without the palladium catalyst (using potassium carbonate and phenylboronic acid only) gave only trace quantities of 6-vinylazulene. This suggests an active intermediate in the cross-coupling mechanism may be responsible for the observed deprotection.

Of course, the Suzuki cross-coupling has seen much development since the original publication, ${ }^{14}$ and considerably milder methods exist that allow for coupling at ambient temperatures. Coupling of phenylboronic acid to the electron-deficient $p$-nitroiodobenzene (227) can be performed using palladium acetate at room temperature (Scheme 113).

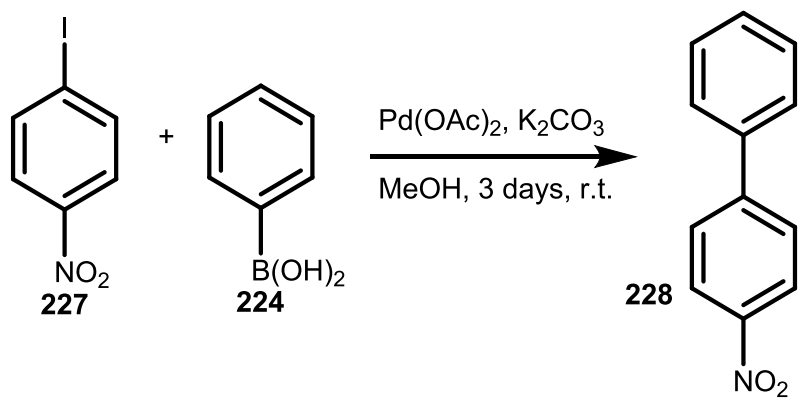

Scheme 113. The reaction used to assess Azul compatibility with low-temperature Suzuki cross coupling 
The low-temperature Suzuki reactions were allowed to proceed for 3 days, despite being essentially complete after one day. Yields of $\mathbf{2 2 8}$ were quantitative for both the Azul-benzoate doped reaction and the control, although $8 \%$ conversion of Azul-benzoate to 6-(2-hydroxyethyl)azulene was observed. Considering the extended length of this reaction, it is reasonable to assume that reaction time can be shortened to give a lower amount of deprotection.

Overall, Suzuki cross-coupling was judged to be partially compatible with the Azul protecting group. Compatibility is achievable, providing the appropriate choice of alkyl halide (or possibly triflate) is made.

\subsubsection{Reduction using $\mathrm{NaBH}_{4}$}

Reduction of carbonyl-containing functionalities to alcohols is commonly performed using reagents such as sodium borohydride and lithium aluminium hydride. 15

The reduction of benzophenone (229) to diphenylmethanol (230) with sodium borohydride was chosen as the test reaction (Scheme 114). Once again this reaction was allowed to proceed for much longer than the time it took to reach completion, for the purposes of over-engineering. The reaction was left overnight, despite being complete after approximately half an hour. In both cases, the conversion of benzophenone to diphenylmethanol was complete, with only trace evidence of Azul benzoate being consumed. From this, it was concluded that the Azul protecting group is compatible with $\mathrm{NaBH}_{4}$ reduction.
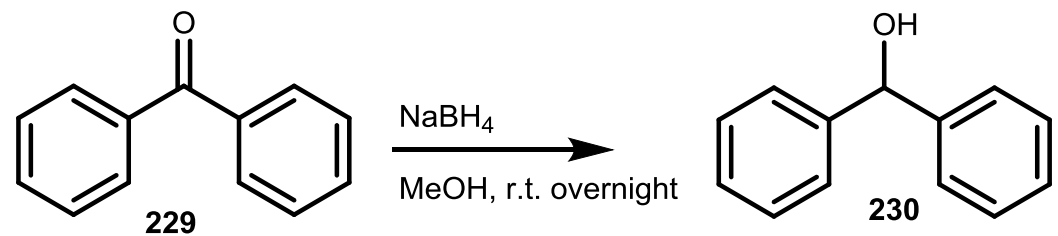

Scheme 114. The reaction used to assess Azul compatibility with $\mathrm{NaBH}_{4}$ reduction

\subsubsection{Masamune-Roush olefination}

One of the concerns over the Azul protecting group was that it would be incompatible with the strongly basic conditions of the Wittig-type reactions (i.e. LDA, NaH and KHMDS). These reactions are synthetic workhorses and are regularly applied to stitch together two molecular fragments or introduce a conjugated alkyl-protected ester, especially in the early stages of a synthesis, so the ability to perform these reactions in the presence of the Azul protecting group is highly desirable. 


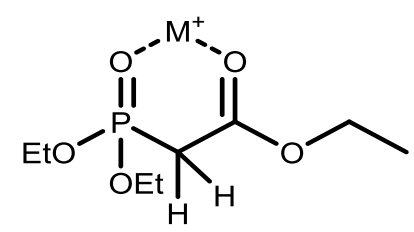

Figure 73. The a cidity of the $\alpha$-proton is increased through the complexation of a metal to the phosphonoacetate in the Masamune Roush olefination

One variant, the Masamune-Roush modification, ${ }^{16}$ can be performed using triethylamine as a base, which the Azul protecting group is stable to (based on results in section 5.2). The acidic $\alpha$-proton of the phosphonoacetate can be made much more acidic through the bidentate complexation of the carbonyl and the $\mathrm{P}=0$ to a Lewis acid; typically $\mathrm{LiBr}$ or $\mathrm{MgBr}_{2}$ is used for this reaction (Figure 73). ${ }^{17}$

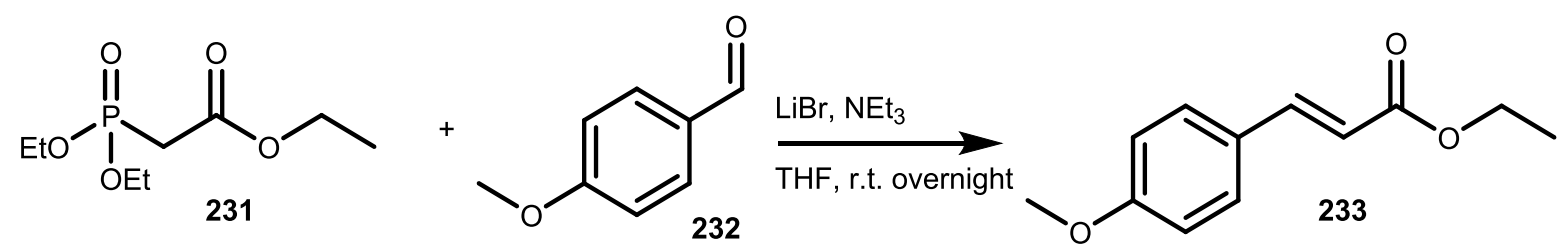

Scheme 115. The reaction used to assess Azul compatibility with Masamune-Roush olefination

Anisaldehyde was coupled to triethylphosphonoacetate using lithium bromide and triethylamine (Scheme 115). Complete conversion was achieved in both cases with only trace evidence of conversion of the Azul-benzoate. From this, it was concluded that the Azul group is compatible with the Masamune-Roush olefination

\subsubsection{Swern oxidation}

Oxidation of alcohols to aldehydes and ketones is an integral part of synthesis, owing to the synthetic utility of the resulting carbonyl. From the investigations into activation-deprotection reactions in section 5.2.3, it was already known that the Swern reaction conditions ${ }^{18}$ would unfavourably react with the Azul protecting group. Nevertheless, it is still important to test the compatibility of Azul-benzoate with this reaction, for two reasons. First, it was not known how close the competition is between the alcohol and Azul-benzoate towards nucleophilic attack on chlorodimethylsulfonium chloride. There is the possibility of one reaction being selected for over the other. Second, it was not known if the Azul protecting group would have an adverse effect on the oxidation of the alcohol substrate beyond using up equivalents of chlorodimethylsulfonium chloride.

Cetyl alcohol was chosen as the substrate for oxidation, mainly because it was a primary alcohol (the resulting aldehyde peak would be easy quantify by ${ }^{1} \mathrm{H}$ NMR integration), readily available, and both it and the oxidised product hexadecanal are non-volatile (Scheme 116). This choice of 
substrate led to some problems, though. Cetyl alcohol is barely soluble in $\mathrm{CH}_{2} \mathrm{Cl}_{2}$ at room temperature - at $-78{ }^{\circ} \mathrm{C}$ the compound is not noticeably soluble at all. By using THF as a co-solvent, this problem was avoided.

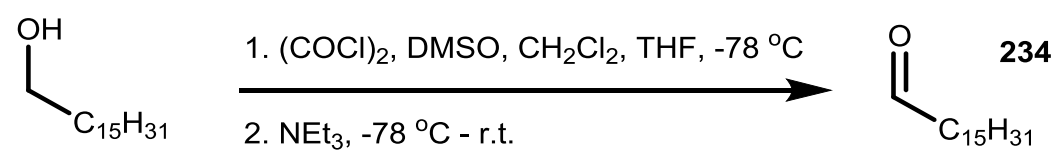

Scheme 116. The reaction used to assess Azul compatibility with Swern oxidation

Attack of the dimethylsulfonium moiety on Azul-benzoate was rapid and complete, with a colour change from indigo to magenta observed over the course of twenty seconds, suggesting formation of the compound 235. In comparison, addition to the alcohol occurs much more slowly, over the course of 2 hours - as evidenced by previous attempts at this reaction whereby triethylamine was added early (after 30 minutes), and returning large quantities of unreacted cetyl alcohol. (Scheme 117).

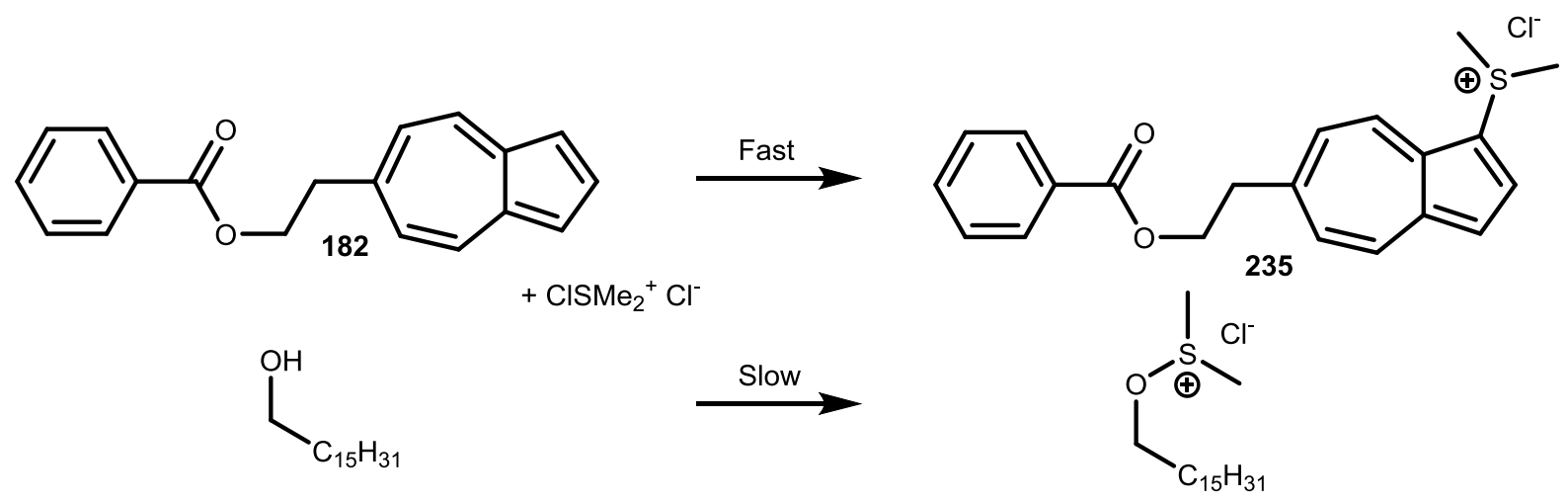

Scheme 117. Compa rison of the reaction ra tes between a ddition of the dimethylsulfonium chloride to an al cohol and to Azul

The control reaction gave a conversion of $86 \%$, while the Azul-benzoate reaction gave a slightly lower conversion of 75\%. Approximately 5 equivalents of chlorodimethylsulfonium chloride were present in both reactions. From this, it can be concluded that Swern oxidation is not compatible with the Azul protecting group, although oxidation of alcohols can still proceed, providing an excess of chlorodimethylsulfonium chloride is present.

\subsection{Summary}

Azul shows promise as a protecting group. It has been tested against a wide variety of bases, and these tests show the Azul protecting group inhabits a 'Goldilocks Zone' where it is labile to some of the stronger bases such as amidines and TBAF, but is stable to the vast majority of amine bases including the more commonly used organocatalysts. Finding a quantitative and easy-to-perform 
activation method for two-step deprotection was fortuitous, as it allows for so much more flexibility and orthogonality.

Orthogonality between Azul and other common protecting groups was demonstrated. Full orthogonality with TBS-ethers and MOM-ethers was observed, and partial orthogonality with FMOC was observed. These experiments served as a good demonstration of the flexibility of the Azul protecting group towards deprotection.

Regeneration of the deprotection by-product 6-vinylazulene (94) was investigated, but no methods were found to achieve this transformation.

The compatibility of the Azul protecting group with a selection of common reactions such as Suzuki cross-coupling, $\mathrm{NaBH}_{4}$ reduction, Masamune-Roush and Swern oxidation was assessed. Some incompatibilities were found with high-temperature Suzuki reactions and Swern oxidations, but otherwise the Azul group performed well.

\subsection{References}

1. Leito, I.; Kütt, A.; Kaljurand, I.; Rõõm, E.-I.; Rodima, T.; Koppel, I. A., Brønsted Acidity of Neutral and Cationic Acids in Nonaqueous Solvents: Recent Developments. http://tera.chemut.ee/ ivo/HA UT/Acidity Basicity INOR 1036 ACS 2007.pdf, Ed. (Last accessed on $17 / 2 / 16)$

2. Clark, J. H., Fluoride Ion as a Base in Organic Synthesis. Chem. Rev. 1980, 80, 429-452.

3. Timmer, M. S. M.; Stocker, B. L.; Northcote, P. T.; Burkett, B. A., Az-a Colourful AzuleneDerived Protecting Group. Tetrahedron Lett. 2009, 50, 7199-7204.

4. Shoji, T.; Higashi, J.; Ito, S.; Toyota, K.; Asao, T.; Yasunami, M.; Fujimori, K.; Morita, N., Synthesis and Redox Behavior of 1-Azulenyl Sulfides and Efficient Synthesis of 1,1Biazulenes. Eur.J. Org. Chem. 2008, 1242-1252.

5. Sutherland, J. K., Reaction of 1,8-diazabicyclo[5.4.0]undec-8-ene with Methyl 3,5Dinitrobenzoate and 1,3,5-Trinitrobenzene. Chem. Commun 1997,325.

6. Boga, C.; Vecchio, E. D.; Forlani, L.; Mazzanti, A.; Todesco, P. E., Evidence for CarbonCarbon Meisenheimer-Wheland Complexes between Superelectrophilic and Supernucleophilic Carbon Reagents. Angew. Chem. Int. Ed. Engl 2005, 117, 3349-3353.

7. Mayr, H. http://www.cup.lmu.de/oc/mayr/reaktionsdatenbank/. (Last acessed on $17 / 2 / 16)$

8. Kanzian, T.; Nigst, T. A.; Maier, A.; Pichl, S.; Mayr, H., Nucleophilic Reactivities of Primary and Secondary Amines in Acetonitrile. Eur.J. Org. Chem. 2009, 6379-6385. 
9. Tishkov, A. A.; Schmidhammer, U.; Roth, S.; Riedle, E.; Mayr, H., Ambident Reactivity of the Nitrite Ion Revisited. Angew. Chem. Int. Ed. Engl. 2005, 44, 4623-4626.

10. Cappa, A.; Marcantoni, E.; Torregiani, E., A Simple Method for the Selective Deprotection of p-Methoxybenzyl Ethers by Cerium(III) Chloride Heptahydrate and Sodium Iodide. J. Org. Chem. 1999, 64, 5696-5699.

11. Jr, A. G. A.; Cowles, E. J.; Tazuma, J. J.; Nelson, J. A., Azulene. V. Alkylation Experiments. Chloromercuration. J. Am. Chem. Soc. 1955, 77, 6321-6323.

12. Cota, I.; Chimentao, R; Sueiras, J.; Medina, F., The DBU- $\mathrm{H}_{2} \mathrm{O}$ Complex as a New Catalyst for Aldol Condensation Reactions. Catalysis Communications 2008, 9, 2090-2094.

13. Suzuki, A., Recent Advances in the Cross-coupling Reactions of Organoboron Derivatives with Organic Electrophiles, 1995-1998.J. Organomet. Chem. 1999, 576, 147-168.

14. Littke, A. F.; Dai, C.; Fu, G. C., Versatile Catalysts for the Suzuki Cross-Coupling of Arylboronic Acids with Aryl and Vinyl Halides and Triflates under Mild Conditions. J. Am. Chem. Soc. 2000,122, 4020-4028.

15. Chaikin, S. W.; Brown, W. G., Reduction of Aldehydes, Ketones and Acid Chlorides by Sodium Borohydride. J. Am. Chem. Soc. 1949, 71, 122-125.

16. Blanchette, M. A.; Choy, W.; Jeffery T. Davis; Essenfeld, A. P.; Masamune, S.; Roush, W. R.; Sakai, T., Horner-Wadsworth-Emmons Reaction: Use of Lithium Chloride and an Amine for Base-Sensitive Compounds. Tetrahedron Lett. 1984,25, 2183-2186.

17. Rathke, M. W.; Nowak, M., The Horner-Wadsworth-Emmons Modification of the Wittig Reaction Using Triethylamine and Lithium or Magnesium Salts. J. Org. Chem. 1985, 50, 2624-2626.

18. (a) Kanji Omura; Swern, D., Oxidation of Alcohols by "Activated" Dimethyl Sulfoxide. a Preparative, Steric and Mechanistic Study. Tetrahedron 1978, 34, 1651-1660; (b) Tidwell, T. T., Oxidation of Alcohols by Activated Dimethyl Sulfoxide and Related Reactions: An Update. Synthesis 1990,10, 857-870. 


\section{Chapter 6: Future work and conclusions}

\subsection{Future work}

As with any open-ended project, there is no definable endpoint to research. Termination of the project seems arbitrary, and leaves a dizzying array of possibilities left unexplored and paths not taken.

\subsubsection{Further development of the Azul protecting group for carboxylic acids}

While the Azul protecting group for carboxylic acids has been well investigated at this point, there still remain numerous additional tests for orthogonality and reaction compatibility. The orthogonality of the PMB protecting group to Azul was to be investigated, but time restraints did not permit adequate study. Incompatibility with DDQ was demonstrated through the attempts at regenerating 6-(2-hydroxyethyl)azulene from the DMB ether $\mathbf{2 1 7}$, but a more formal investigation with PMB-protected species is nevertheless needed. Hydrogenation is an alternate method for cleaving PMB ethers, and may provide an interesting test of compatibility with the Azul moiety.

There were several other salient reactions whose compatibility with Azul were to be tested. Additional reductions with stronger reducing agents such as $\mathrm{LiBH}_{4}$ and $\mathrm{LiAlH}_{4}$, or with alkyl substituted reducing agents such as $K$-selectride or DIBAL-H are needed. Finding a compatible alcohol-to-aldehyde oxidation method is also a high priority. Parikh-Doering and Dess-Martin oxidations were investigated but suitable procedures for generating crude mixtures with sensible ${ }^{1} \mathrm{H}$ NMR spectra could not be found. Anecdotal evidence exists towards the compatibility of Azul with Parikh-Doering in that no azulenedimethylsulfonium species was produced, a somewhat surprising result given the similarity of Parikh-Doering to the Swern oxidation. Other important reactions considered for evaluation were alkene metathesis, Yamaguchi esterification, Huisgen cycloaddition and reactions involving radical chemistry.

Alternative modes of protection with the Azul protecting group and multi-step one-pot reactions warrant investigation. For instance, the compatibility of the Masamune-Roush reaction with Azul opens up the possibility of introducing the Azul protecting group through a Masamune-Roush olefination. Reaction of the Azul-phosphonoacetate 236 with an aldehyde or ketone-containing 
substrate under Masamune-Roush conditions would provide a convenient Azul protection and two-carbon homologation in a single step (Scheme 118).

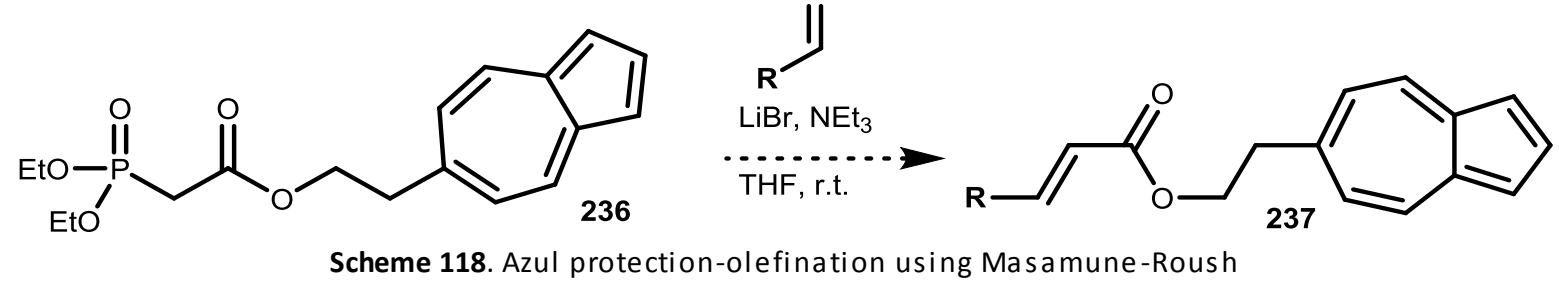

Alternatively, the same process might be achieved through the use of the Bestmann ylide (Scheme 119). ${ }^{1}$ Reaction of 90 with an aldehyde or ketone in the presence of Bestmann ylide would give the same product 237.

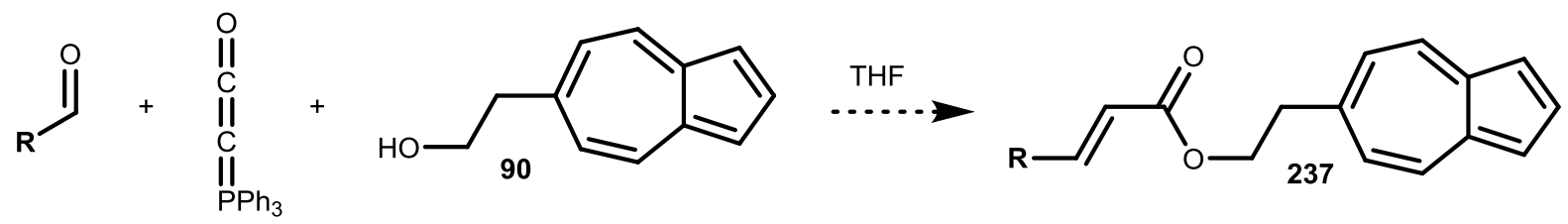

Scheme 119. Azul protection-olefination using Bestmann ylide

Such procedures are synthetically useful not only due to their step efficiency, but also because they avoid generation of free carboxylic acids that may be difficult to handle and purify.

Tandem deprotection-esterification or deprotection-lactonisation reactions are also expected to be highly useful (Scheme 120). The typical eventual fate of a carboxylic acid produced as a synthetic intermediate is as part of an ester or lactone. Azul deprotection results in a carboxylate salt and 6-vinylazulene. There is no hydroxyl functionality that can possibly re-esterify the carboxylate salt. Introduction of an alcohol under esterification conditions (such as Mitsunobu or Yamaguchi) could provide the desired ester efficiently, while lactonisation could conceivably be achieved through global deprotection of a substrate such as $\mathbf{2 3 8}$ to generate the conjugate base of a seco-acid such as $\mathbf{2 3 9}$ which subsequently lactonises under the appropriate conditions.

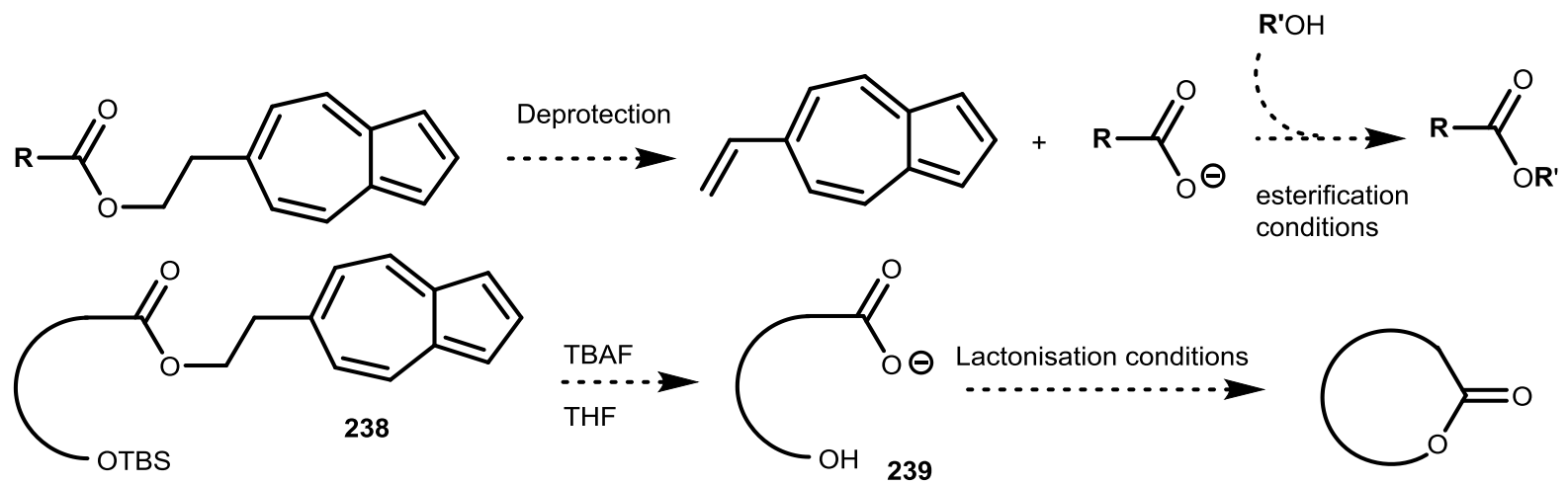

Scheme 120. Possible methods of tandem deprotection-esterification or deprotection-lactonisation 


\subsubsection{Development of the AzulOC protecting group for alcohols and amines}

While methods were found to introduce the AzulOC protecting group onto amines and alcohols in relatively high yields ( $75 \%$ and $83 \%$ respectively), the reaction conditions are not especially userfriendly and result in a large number of azulene-based by-products. It is possible that protection may be improved by using the more reactive (and more expensive) $N, N^{\prime}$-disuccinimidyl carbonate in place of CDI.

At present, only two primary alcohols - cetyl alcohol and cyclohexanemethanol - have been protected with AzulOC. The methodology needs to be extended to secondary and tertiary alcohols, as well as phenols. Selective protection methodology for differentiating between hydroxyl functionalities is also highly desirable.

Much work needs to be done before AzulOC is a viable protecting group for amines. Along with protocols that cover a wide variety of nitrogen-containing compounds (such as primary and secondary amines, amino acids and heterocyclic compounds such as indoles), protocols for deprotection and the subsequent separation of the amine substrate from DBU need to be developed.

The array of orthogonality and compatibility reactions that were performed on the Azul carboxylic acid protecting group must also be performed on the AzulOC amine and alcohol protecting groups. While it is expected that AzulOC will behave very similarly to the Azul protecting group, it is nevertheless necessary to investigate this area fully.

The final test for the Azul protecting group is its incorporation into a complex molecule synthesis. While many of the scenarios encountered during a synthesis can be anticipated and investigated separately, there will always be some combination of factors that cannot be anticipated and must be experienced and overcome at the time. The proofof the pudding, as they say, is in the eating.

\subsubsection{Other azulene based protecting groups}

There is room for making additional variants of the Azul protecting group. The difficulty of scaleup of the 6-(2-hydroxyethyl)azulene (90) preparation remains a bottleneck in the process, on account of the handling properties of paraformaldehyde. Large quantities of the protecting group precursor must be cheaply and readily available if other synthetic chemists are to adopt its use. Other protecting groups with similar function could conceivably be developed. Paraformaldehyde could be replaced with other aldehydes or ketones in the synthesis step of the protecting group to 
give compounds with additional steric bulk. For instance, reaction of 6-methylazulene with LDA followed by benzophenone would give the bulky tertiary alcohol 240. As benzophenone is much more easily handled than paraformaldehyde, higher yields and larger scales are expected to be achievable for the synthesis of this protecting group precursor (Scheme 121).

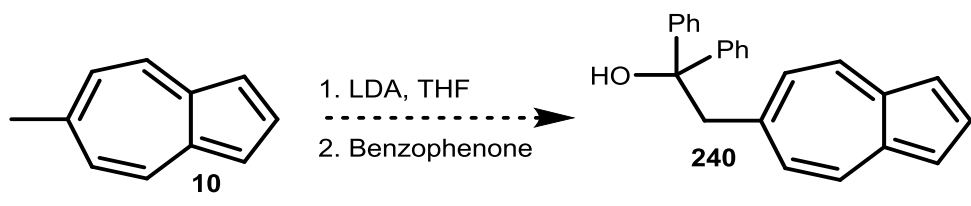

Scheme 121. Synthesis of the protecting group precursor $\mathbf{2 4 0}$

Protection with a hindered species such as $\mathbf{2 4 0}$ would likely be more difficult than protection with the Azul protecting group, and protection of alcohols would likely be limited only to primary alcohols. ${ }^{2}$ Deprotection may be expedited through the generation of additional conjugation in the compound 242, and may give access to a slightly different regime of lability in 'base space' (Scheme 122). The increased conjugation of 242 could also result in a more noticeable colour change upon deprotection.

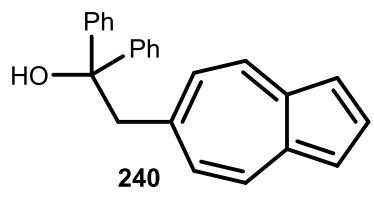

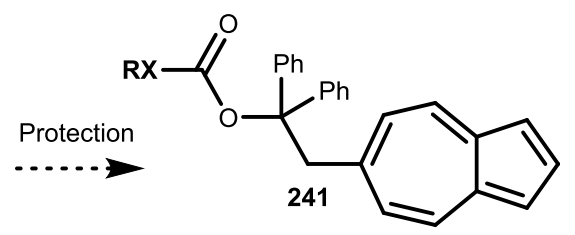

Scheme 122. Protection of $\mathbf{2 4 0}$ as $\mathbf{2 4 1}$, and its deprotection

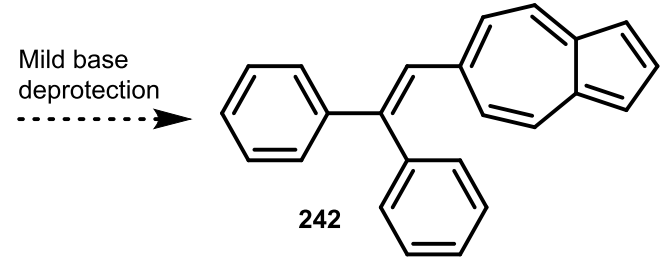

A 1-(2-hydroxyethyl)azulene-based protecting group is also feasible. The progenitor alcohol 243 has been synthesised from azulene in one step using oxalyl chloride and lithium borohydride. Unlike the Azul protecting group, the azulene ring is not expected to have much influence over the deprotection conditions - it is merely anticipated to be a coloured tag. Such a protecting group would deprotect through the usual ester or carbonate cleavage conditions (i.e. acidic or basic hydrolysis) rather than through $\beta$-elimination and would be expected to be stable to the nonaqueous bases that deprotect Azul (Scheme 123).
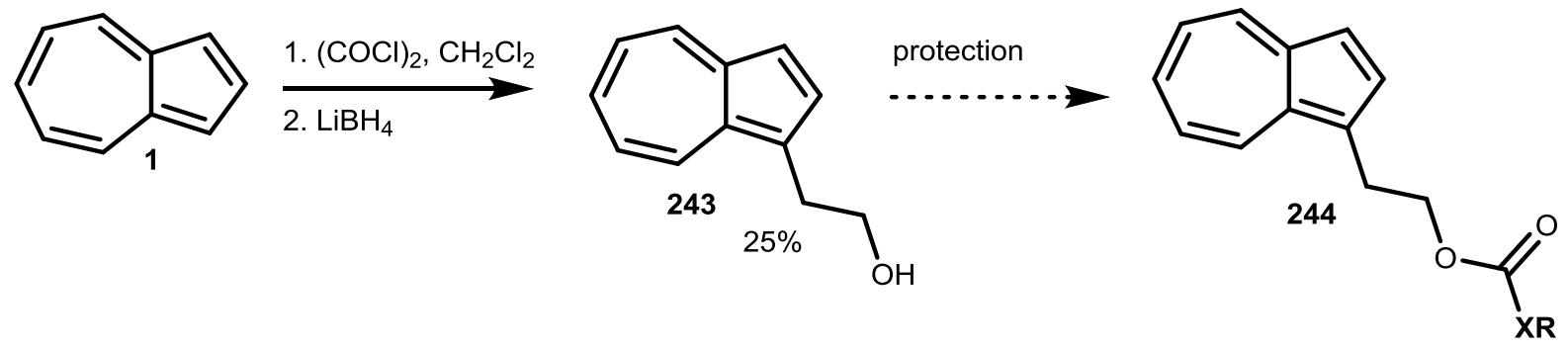

Scheme 123. Generation of $\mathbf{2 4 3}$ and protection of a substrate as $\mathbf{2 4 4}$ 


\subsection{Concluding remarks}

The use of coloured protecting groups greatly expedites synthetic chemistry by allowing purification to be performed more easily and by allowing the chemist to track and account for material more easily. This allow the chemist to be more productive over a given timescale, and may also benefit non-specialists, researchers who are not career organic chemists who nevertheless need to use synthetic techniques to carry out research.

The physical and chemical properties of azulenes make them ideally suited for use as protecting groups. The intensity of visible light absorption is high but not too high, and so negligible quantities of material do not remain highly coloured. The azulene moiety is both non-polar and structurally compact, a combination of traits highly unusual amongst other organic chromophoric species. Stabilisation of both positive and negative charges allows for some unusual and potentially orthogonal modes of deprotection.

Several azulene-based protecting group candidates were explored. Two of these candidates, the 1azulenylmethyl group $\mathbf{7 9}$ and the azulenesulfonamide 82 were swiftly abandoned. 79 was dropped due to a tendency to degrade, and $\mathbf{8 2}$ was dropped due to difficulties in synthesis and the realisation that the predicted deprotection conditions required would likely be too harsh for use in complex molecule synthesis. The azulene-1-carboxylate ester $\mathbf{7 4}$ and guaiazulene and 4,6,8trimethylazulene variants were heavily investigated as protecting groups. Despite finding favourable protection conditions, the basic deprotection conditions required were judged to be unacceptably harsh for use in complex molecule synthesis. Various two-step approaches were investigated, including nitration, $\pi$-complex activation and tethered deprotection from the neighbouring 4-methyl position. None of these approaches gave sufficiently improved protocols for deprotection.

The 6-(2-oxyethyl)azulene-based protecting group, nicknamed Azul for carboxylic acids and AzulOC for alcohols and amines has met with much greater success. It has demonstrated excellent flexibility when it comes to deprotection conditions and orthogonality, and compatibility with several common reaction conditions has also been demonstrated. As a protecting group for carboxylic acids, protection is easily carried out through Steglich chemistry. Protection reactions for alcohols and amines still shows room for improvement and more research is needed before they can be used as a general protocol. The Azul protecting group fulfils most of the requirements stipulated in Chapter 1 for a useful azulene-based protecting group, and it can tentatively be said to be ready for inclusion in a total synthesis. 


\subsection{References}

1. Bartlett, M., (Triphenylphosphoranylidene)ketene: The Bestmann Ylide. Synlett. 2013, 24, 773-774.

2. Rannard, S. P.; Davis, N. J., Controlled Synthesis of Asymmetric Dialkyl and Cyclic Carbonates Using the Highly Selective Reactions of Imidazole Carboxylic Esters. Org. Lett. 1999, $1(6), 933-936$. 


\section{Experimental}

\section{General procedures}

All reactions were performed under a nitrogen atmosphere in glassware dried using a heat gun under vacuum, unless otherwise specified. All reaction solvents were obtained dry, either from a Innovative Technology PureSolv solvent dispenser (THF, DCM, toluene, diethyl ether, hexane) or from distillation over sodium metal (methanol, ethanol, isopropanol, DME) or calcium hydride (pyridine, acetonitrile, diethylamine, diisopropylamine, DMSO), unless otherwise specified.

Phase separations of mixtures involving coloured substrates were typically performed using $\mathrm{CH}_{2} \mathrm{Cl}_{2} / \mathrm{H}_{2} \mathrm{O}$, and repeated as necessary until the colour had been removed from the aqueous layer. No drying procedures are performed prior to concentration by rotary evaporation.

All column chromatography was performed using silica gel (40-63 micron from PureScience). Material collection was typically performed by collecting single fractions for each coloured band into round-bottomed flasks, unless for desired colourless compounds or particularly difficult separations, in which case multiple fractions were collected using test tubes.

All NMR data were acquired using a Varian Unity Inova 500 (operating at $500 \mathrm{MHz}$ for ${ }^{1} \mathrm{H}$ and 125 $\mathrm{MHz}$ for ${ }^{13} \mathrm{C}$ ). All structural assignments were made on the basis of 2D NMR data (COSY, HSQCAD, HMBCAD). J coupling values are rounded to the nearest $0.5 \mathrm{~Hz}$. All IR data were obtained from a Bruker Alpha Platinum with ATR fitting. All mass spectrometry data were acquired from an Agilent Technologies 6530 Accurate Mass Q-TOF LC/MS instrument. All UV/Vis data were obtained from a Varian Cary 100scan UV-Visible spectrophotometer, using quartz cuvettes with a $10 \mathrm{~mm}$ path length, and spectra were recorded of the regions between 400 and $800 \mathrm{~nm}$.

Thin layer chromatography was performed using Machery-Nagel SilG/UV $\mathrm{U}_{254}$ polyester-backed silica TLC sheets. When performing TLC on mixtures consisting only of coloured compounds, it is not necessary to use a TLC stain. In cases where azulene-based compounds were insufficiently concentrated to be visible to the naked eye, a $254 \mathrm{~nm}$ UV source effectively visualised the spots (due to azulene absorbing approximately five times more strongly in the UV region than in the visible), or alternatively an anisaldehyde stain was used (prepared from $15 \mathrm{~mL} \mathrm{AcOH,} 3.5 \mathrm{~mL} p$ anisaldehyde, $350 \mathrm{~mL}$ EtOH and $50 \mathrm{~mL} \mathrm{H}_{2} \mathrm{SO}_{4}$ ), under which azulene moieties char to a deep red colour and become much more intense. 
Specific optical rotation measurements were attempted on chiral molecules, but the concentrations required for transparency of the solution at $589 \mathrm{~nm}$ were too dilute to allow measurements above background noise to be made.

\section{A note on descriptions of colour}

Many of the names for colours that fall between blue and red on the colour wheel are used interchangeably (such as the colours violet, purple and magenta) or with ambiguity (indigo the classical colour is a slightly purple blue, whereas indigo dye is a slightly green blue). It is necessary to provide a visual description of these colours, so that phrases such as 'colour change from purple to violet' still confer meaning (Figure 74).
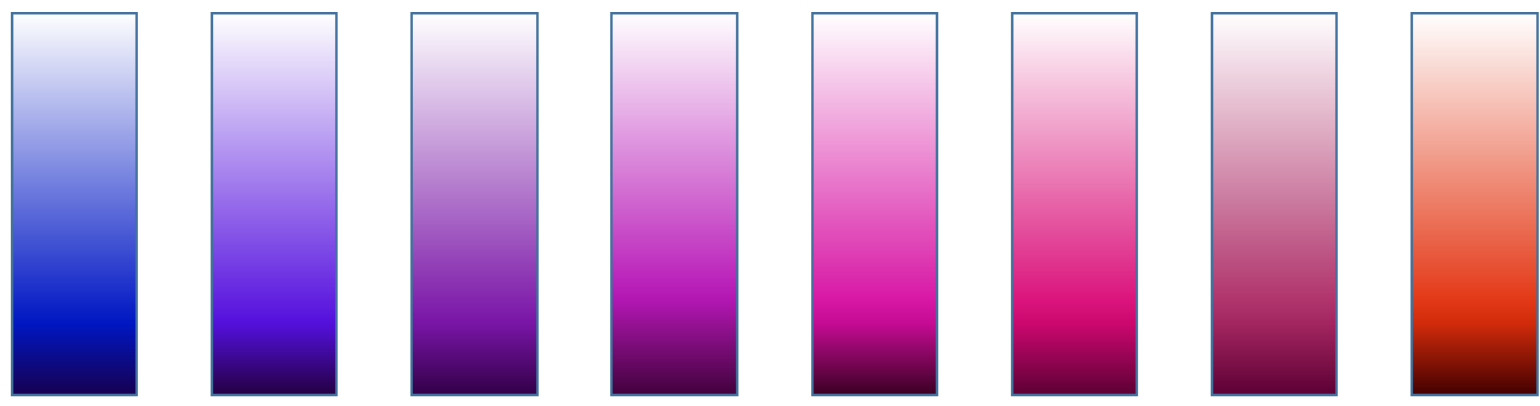

Blue Indigo Violet

Purple

Magenta Crimson

Maroon Orange-red

Figure 74. Depiction of the colours encountered throughout this project

\section{Azulene (1)}<smiles>C1=CC2=NC=PSC2=PS1</smiles>

1-Chloro-2,4-dinitrobenzene ( $6.88 \mathrm{~g}, 34 \mathrm{mmol}, 1$ eq.) was added to a RBF, followed by pyridine (70 mL, $870 \mathrm{mmol}, 25.5$ eq.), upon which a red-orange colour appeared. This solution was heated at reflux for 70 minutes, during which time a precipitate had formed throughout the entire liquid volume. The reaction was left to cool to r.t. over 20 minutes, then cooled further in an ice bath for 10 minutes. Diethylamine (7.5 mL, $72.5 \mathrm{mmol}, 2.1$ eq.) was added and the mixture stirred for 20 minutes, then left to warm to r.t. and left to stir until all the precipitate had dissolved (ca. 1 hour). During this time, a solution of $\mathrm{NaOMe}$ was prepared by adding $\mathrm{MeOH}$ (18 $\mathrm{mL}$ ) slowly to sodium metal (2.12 g, $92.6 \mathrm{mmol}, 2.7$ eq.). Freshly cracked cyclopentadiene ( $7.5 \mathrm{~mL}, 89 \mathrm{mmol}, 2.6$ eq.) was slowly added to the reaction mixture, followed immediately by the NaOMe solution. The reaction mixture was then heated at reflux for 30 minutes, after which a distillation head was attached in 
order to remove some of the more volatile components of the reaction (approximately $40 \mathrm{~mL}$ of the mixture, mostly methanol and diethylamine). The distillation head was then swapped back to the reflux condenser and reflux was resumed. After a few hours, a dirty green-blue colour was visible in the reaction mixture.

After heating at reflux overnight, the distillation head was re-fitted and the reaction mixture was distilled to dryness. A portion of azulene co-distilled with the pyridine. Pet. ether was periodically added and distilled off to recover additional azulene through azeotrope formation. This distillate was reduced via rotary evaporation to remove pyridine and pet. ether. The solid remaining in the reaction vessel was broken up manually and repeatedly washed with dichloromethane to extract the remaining azulene. The resulting solution was then passed through a silica plug with pet. ether as the eluent to remove the most polar impurities. The two azulene-containing mixtures were then combined and purified with column chromatography using pet. ether. The pure solution was then repeatedly reduced via rotary evaporation and the azulene-containing distillate that collected in the splash-head was returned to the solution. Finally, a small amount of diethyl ether was added in order to remove remaining hexane through azeotrope formation, and the solution reduced to dryness through rotary evaporation with a wad of cotton wool wrapped in aluminium foil fitted into the lower arm of a splash-head, to obtain azulene 1 (2.2 g, 51\%) as a flaky blueindigo powder. ${ }^{1} \mathrm{H}$ NMR (500 MHz, $\mathrm{CDCl}_{3}$ ) $\delta 8.44(\mathrm{~d}, J=10.0 \mathrm{~Hz}, 2 \mathrm{H}, \mathrm{H}-4,8), 8.02(\mathrm{t}, J=3.5 \mathrm{~Hz}, 1 \mathrm{H}, \mathrm{H}-$ 2), 7.66 (t, J=10.0 Hz, 1H, H-6), 7.51 (d, J=10.0 Hz, 2H, H-1,3), 7.24 (t, J=9.5 Hz, 2H, H-5,7). ${ }^{13} \mathrm{C}$ NMR (125 MHz, $\mathrm{CDCl}_{3}$ ) $\delta 140.2$ (C, C-3a,8a), 137.4 (CH, C-6), 137.1 (CH, C-2), 136.7 (CH, C-4,8), 122.8 (CH, C-5,7), $118.1(\mathrm{CH}, \mathrm{C}-1,3), \lambda_{\text {max: }} 575 \mathrm{~nm}$. Spectral data matched those reported previously. ${ }^{1}$

\section{2,4,6-Trimethylpyrylium tetrafluoroborate (100)}<smiles>CC1=CC(C)=C(Br)P(C)S1</smiles>

Acetic anhydride (50.0 mL, $530 \mathrm{mmol}, 9.8$ eq.) and $t$-butanol ( $4.0 \mathrm{~mL}, 42 \mathrm{mmol}, 1$ eq.) was added to a conical flask with stirrer under open air. Tetrafluoroboric acid $(7.0 \mathrm{~mL}, 48 \% \mathrm{v} / \mathrm{v}$ in water) was added dropwise at a rate such that the temperature of the solution in the flask was maintained around $100{ }^{\circ} \mathrm{C}$. During this time the solution slowly turned a dark brown. The reaction mixture was left to cool to $80{ }^{\circ} \mathrm{C}$ before being placed in an ice bath, upon which a beige precipitate formed. $\mathrm{Et}_{2} \mathrm{O}(30 \mathrm{~mL})$ was added to aid further precipitation. The reaction mixture was filtered and washed with $\mathrm{Et}_{2} \mathrm{O}$, and a further filtration was performed on the filtrate to yield a second crop. 
The solids were combined to yield 2,4,6-trimethylpyrylium tetrafluoroborate 101 (4.568 g, 51\%) as a beige powder. This material was used in the next reaction without undue delay.

\section{4,6,8-Trimethylazulene (9)}<smiles></smiles>

Isopropanol (60 mL) was added to sodium metal (1.73g, $75 \mathrm{mmol}, 3.3 \mathrm{eq}$.) in a RBF fitted with a condenser and was heated until all sodium had reacted. Freshly distilled cyclopentadiene $(6.5 \mathrm{~mL}$, $77 \mathrm{mmol}, 3.5$ eq.) was then added to the still-warm reaction mixture and a slight pink colour formed. 2,4,6-Trimethylpyrylium tetrafluoroborate $\mathbf{1 0 0}$ (4.704 g, $22.3 \mathrm{mmol}$ ) was added and the reaction was left to reflux for 2 hours. The reaction mixture briefly went purple, followed by orange.

The still-warm reaction mixture was then filtered through a silica plug and washed with pet. ether. The crude mixture was concentrated via rotary evaporation to remove the remaining isopropanol, and was then subjected to another silica plug in pet. ether followed by column chromatography in pet. ether to afford 4,6,8-trimethylazulene 9 (667 mg, 17.5\% yield) as purple waxy crystals. ${ }^{1} \mathrm{H}$ NMR (500 MHz, CDCl $) \delta 7.66$ (t, J=4.0 Hz, 1H, H-2), 7.26 (d, J= 4.0 Hz, 2H, H-1,3), 7.06 (s, 2H, H5,7), 2.88 (s, 6H, 4-Me,8-Me), 2.65 (s, 3H, 6-Me). $\left.{ }^{13} \mathrm{C} \mathrm{NMR} \mathrm{(125} \mathrm{MHz,} \mathrm{CDCl}\right) \delta 146.4$ (C, C-6), 145.1 (C, C-4,8), 136.64 (C, C-3a,8a), 132.62 (CH, C-2), 127.3 (CH, C-5,7), 116.2 (CH, C-1,3), 28.9 ( $\mathrm{CH}_{3}, 6-$ $\mathrm{Me}), 25.3\left(\mathrm{CH}_{3}, 4-\mathrm{Me}, 8-\mathrm{Me}\right), \lambda_{\max }: 540 \mathrm{~nm}$. Spectroscopic data matched those reported previously. ${ }^{2}$

\section{1,4-Dimethylpyridinium iodide (106)}<smiles>CC1=C=[N+](C)OP=C1</smiles>

4-Methylpyridine (15 mL, $152 \mathrm{mmol}, 1$ eq.) was added to a RBF fitted with a Vigreaux column open to the air in an ice bath. Methyl iodide (9.5 mL, $153 \mathrm{mmol}, 1.004$ eq.) was added slowly in 1 $\mathrm{mL}$ portions. A beige precipitate gradually formed, followed by a rapid exotherm during which the reaction mixture completely changed to a salmon-coloured solid. A recrystallisation was then 
performed in the same vessel, using a minimum (ca. $20 \mathrm{~mL}$ ) of hot ethanol, and the solid was washed with diethyl ether to afford 1,4-dimethylpyridinium iodide 106 (33.877 g, 95\%) as salmon-coloured deliquescent crystals possessing a sweet-sickly amine-like odour. This compound was used in the next reaction without undue delay.

\section{6-Methylazulene (10)}<smiles>CP1SC=C2C=CN=C2C=[SH]1</smiles>

Isopropanol ( $145 \mathrm{~mL})$ was added slowly to potassium metal ( $8.23 \mathrm{~g}, 210 \mathrm{mmol}, 2.33 \mathrm{eq}$.) at $0{ }^{0} \mathrm{C}$ in a RBF fitted with a condenser. Freshly distilled cyclopentadiene (18 mL, $214 \mathrm{mmol}, 2.37 \mathrm{eq}$.) was added to this reaction mixture and a cream-coloured precipitate with hints of pink developed. 1,4Dimethylpyridinium iodide 106 (21.241 g, $90.3 \mathrm{mmol}, 1$ eq.) was added and the mixture was heated to $40{ }^{\circ} \mathrm{C}$ for 30 minutes followed by heating to $70{ }^{\circ} \mathrm{C}$ for 2 hours. After this period, the reaction mixture appeared blue-green. The reaction mixture was allowed to cool.

The reaction mixture was added directly to a silica plug filled with silica and topped with celite. During this process the reaction mixture became much more brown, but this is presumably due to the atmospheric oxidation of the unreacted reaction components, not loss of 6-methylazulene. The column was flushed sequentially with pet. ether and $\mathrm{CH}_{2} \mathrm{Cl}_{2}$, and concentrated by rotary evaporation, taking care not to evaporate to dryness. This crude mixture was then subjected to another two silica plugs in pet. ether to remove the most polar by-products, after which the crude mixture had regained its blue colour. A final column in pet. ether was performed to remove the remaining impurities, followed by repeated rotary evaporation to separate volatile 6methylazulene from pet. ether. The material was re-dissolved in $\mathrm{Et}_{2} \mathrm{O}$ and concentrated by rotary evaporation to afford 6-methylazulene 10 (4.493 g, 35\%) as indigo flakes. ${ }^{1} \mathrm{H}$ NMR (500 MHz, $\mathrm{CDCl}_{3}$ ) $\delta 8.28$ (d, J=10.5 Hz, 2H, H-4,8), 7.89 (t, J=4.0 Hz, 1H, H-2), 7.42 (d, J=4.0 Hz, 2H, H-1,3), 7.14 (d, J=10.0 Hz, 2H, H-5,7), 2.70 (s, 3H, 6-Me). $\left.{ }^{13} \mathrm{C} \mathrm{NMR} \mathrm{(125} \mathrm{MHz,} \mathrm{CDCl}\right) \delta 148.8$ (C, C-6), 138.8 (C, C-3a,8a), 135.8 (CH, C-4,8), 135.6 (CH, C-2), 124.3 (CH, C-5,7), 118.0 (CH, C-1,3), 21.1 ( $\mathrm{CH}_{3}, 6-\mathrm{Me}$ ). $\lambda_{\text {max: }} 561 \mathrm{~nm}$. Spectroscopic data matched those reported previously. ${ }^{3}$

\section{6-p-Tolyl azulene (107)}

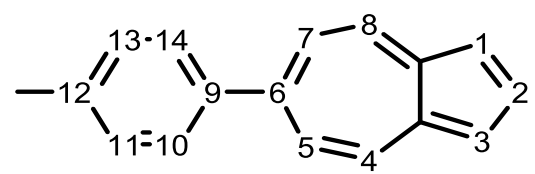


This compound formed in tiny $(\sim 0.01 \%$ yield $)$ amounts in syntheses of 6-methylazulene that contain an excess of sodium or potassium to cyclopentadiene. Indigo needles. ${ }^{1 H} \mathrm{NMR}(500 \mathrm{MHz}$, $\mathrm{CDCl}_{3}$ ) $\delta 8.41$ (d, J=10.0 Hz, 2H, H-4,8), 7.90 (t, J=3.5 Hz, 1H, H-2), 7.57 (d, J=7.5 Hz, 2H, H-10,14), 7.42 (d, J=4.0 Hz, 2H, H-1,3), 7.41 (d, J=9.0 Hz, 2H, H-5,7), 7.31 (d, J=8.0 Hz, 2H, H-11,13), 2.46 (s, 3H, 12-Me). ${ }^{13} \mathrm{C}$ NMR (125 MHz, CDCl $) \delta 151.0$ (C, C-6), 142.5 (C, C-9), 138.8 (C, C-3a,8a), 137.9 (C, C-12), 136.6 (CH, C-2), 135.9 (CH, C-4,8), 129.5 (CH, C-11), 128.4 (CH, C-10), 123.4 (CH, C-5,7), 118.2 (CH, C-1,3). Spectroscopic data matched those reported previously. ${ }^{4}$

\section{5-Methylazulene (11)}

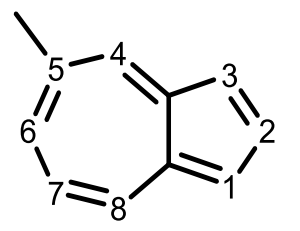

3-Methylpyridine (30 mL, $303 \mathrm{mmol}$, obtained from bottle without purification) was added to 1-chloro-2,4-dinitrobenzene (1.878 g, $9.3 \mathrm{mmol}, 1$ eq.) in a RBF fitted with condenser. The reaction mixture went red-brown. After 40 minutes at reflux, reaction mixture went black and viscous. This was then cooled to $0{ }^{\circ} \mathrm{C}$ and diethylamine $(2.0 \mathrm{~mL}, 19.3 \mathrm{mmol}, 2.1 \mathrm{eq}$.) was added and the reaction changed colour to maroon. Concurrently, NaOMe solution was prepared by adding $\mathrm{MeOH}$ ( $10 \mathrm{~mL}$ ) to sodium metal (556 mg, $24 \mathrm{mmol}, 2.6 \mathrm{eq}$.). This solution was added to the reaction mixture followed by freshly distilled cyclopentadiene $(2.0 \mathrm{~mL}, 23.7 \mathrm{mmol}, 2.56 \mathrm{eq}$.$) and$ the reaction mixture was heated to reflux.

After overnight reaction, the reaction mixture had become thick and black. This mixture was transferred directly to a silica plug and eluted with pet. ether to obtain a blue solution. A separation was performed with pet. ether/ $10 \%$ aq. $\mathrm{HCl}$ to remove remaining 3-methylpyridine, and the organic layer was purified by column chromatography in pet. ether to afford 5methylazulene 11 (93 mg, 7\% yield) as a blue solid. ${ }^{1} \mathrm{H}$ NMR (500 MHz, $\left.\mathrm{CDCl}_{3}\right) \delta 8.30(\mathrm{~s}, 1 \mathrm{H}, \mathrm{H}-4)$, 8.25 (d, J=10.0 Hz, 1H, H-8), 7.88 (broad s, 1H, H-2), 7.52 (d, J=10.5 Hz, 1H, H-6), 7.29 (m, 2H, H1,3), 7.07 (t, J=10.0 Hz, 1H, H-7), 2.65 (s, 3H, 5-Me). ${ }^{13} \mathrm{C} \mathrm{NMR} \mathrm{(125} \mathrm{MHz,} \mathrm{CDCl} 3$ ) $\delta 139.8$ (C, C-3a), 139.5 (C, C-8a), 138.4 (CH, C-4), 138.2 (CH, C-6), 137.0 (CH, C-2), 134.9 (CH, C-8), 131.9 (C, C-5), 121.9 (CH, C-7), 116.65 (CH, C-1 or C-3), 116.61 (CH, C-3 or C-1), $26.5\left(\mathrm{CH}_{3}, 5-\mathrm{Me}\right)$. Spectroscopic data matched those reported previously. ${ }^{5}$ 


\section{4-Methylazulene (99)}<smiles>Cc1spcpc2npc1-2</smiles>

Azulene 1 (178.9 mg, $1.395 \mathrm{mmol}$ ) was added to a RBF and dissolved with $\mathrm{Et}_{2} \mathrm{O}(10 \mathrm{~mL}) .1 \mathrm{~mL}$ of MeLi soln (3 M in diethoxymethane, $3 \mathrm{mmol}, 2.15$ eq.) was added in two $0.5 \mathrm{~mL}$ portions. After 15 minutes, the blue colour had been lost and a light pink colour was observed. The reaction mixture was cooled to $-78{ }^{\circ} \mathrm{C}$ and $\mathrm{MeOH}(1.1 \mathrm{~mL}, 25 \mathrm{mmol}, 18 \mathrm{eq}$.) was added, followed by $p$-chloranil (461 $\mathrm{mg}, 1.87 \mathrm{mmol}, 1.3 \mathrm{eq}$.) and the reaction was left to return to room temperature. After 45 minutes, the reaction mixture appeared brown-green.

A separation was performed using pet. ether/aq. $\mathrm{KOH}$, and column chromatography was performed on the organic layer using pet. ether, to afford 4-methylazulene $\mathbf{9 9}$ ( $69.6 \mathrm{mg}, 35 \%$ yield) as an indigo viscous oil. ${ }^{1} \mathrm{H}$ NMR (500 MHz, $\left.\mathrm{CDCl}_{3}\right) \delta 8.37(\mathrm{~d}, J=9.5 \mathrm{~Hz}, 1 \mathrm{H}, \mathrm{H}-8), 7.86(\mathrm{t}, J=3.5 \mathrm{~Hz}$, 1H, H-2), 7.55 (t, J=9.5 Hz, 1H, H-6), 7.45 (d, J=3.5 Hz, 1H, H-1), 7.40 (d, J=3.5 Hz, 1H, H-3), 7.20 (d, $J=10.0 \mathrm{~Hz}, 1 \mathrm{H}, \mathrm{H}-5), 7.14$ (t, J=9.5 Hz, 1H, H-7), 2.95 (s, 3H, 4-Me). ${ }^{13} \mathrm{C}$ NMR (125 MHz, CDCl 3 ) $\delta$ 146.8 (C, C-4), 140.0 (C, C-3a), 137.6 (C, C-8a), 136.9 (CH, C-8), 136.3 (CH, C-6), 135.3 (CH, C-2), 126.3 (CH, C-5), 121.8 (CH, C-7), 118.7 (CH, C-3), 115.7 (CH, C-1), $24.5\left(\mathrm{CH}_{3}, 4-\mathrm{Me}\right)$. Spectroscopic data matched those reported previously. ${ }^{3}$

\section{1-Formylazulene (78)}

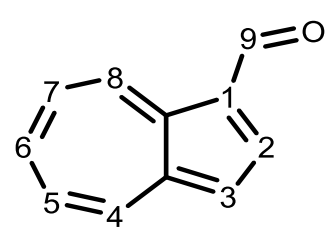

Vilsmeier reagent solution $\left(0.25 \mathrm{~mL} \mathrm{POCl}_{3}\right.$ in $\left.1 \mathrm{~mL} \mathrm{DMF}\right)$ was made at $0{ }^{0} \mathrm{C}$ and $0.6 \mathrm{~mL}$ of this solution was added to a solution of azulene 1 (121.7 mg, $0.95 \mathrm{mmol}$ ) in $\mathrm{CH}_{2} \mathrm{Cl}_{2}$ (4 mL). The reaction mixture rapidly changed colour from blue to orange. A solution of $\mathrm{KOH}$ (84 mg, $1.5 \mathrm{mmol}$, 1.5 eq.) in $10 \mathrm{~mL} \mathrm{H}_{2} \mathrm{O}$ was added and the reaction mixture was stirred for 2 hours, over which time the reaction mixture became purple.

A separation was performed using $\mathrm{CH}_{2} \mathrm{Cl}_{2} / \mathrm{H}_{2} \mathrm{O}$, to which further $\mathrm{NaOH}$ (aq.) was added until the orange aqueous layer became colourless. The organic fraction was concentrated by rotary evaporation and subjected to a second phase separation using pet. ether/sat. $\mathrm{CuSO}_{4}$ (aq.), and the organic fraction was concentrated by rotary evaporation. Column chromatography in $\mathrm{CH}_{2} \mathrm{Cl}_{2}$ was 
performed on the crude mixture to obtain the title compound $\mathbf{7 8}$ (138.6 $\mathrm{mg}$, 93\% yield) as a purple oil. ${ }^{1} \mathrm{H}$ NMR (500 MHz, $\left.\mathrm{CDCl}_{3}\right) \delta 10.4(\mathrm{~s}, 1 \mathrm{H}, \mathrm{H}-9), 9.59$ (d, J=9.5 Hz, 1H, H-8), 8.51 (d, J=10.0 Hz, 1H, H-4), 8.28 (d, J=4.5 Hz, 1H, H-2), 7.86 (t, J=10.0 Hz, 1H, H-6), 7.63 (t, J=9.5 Hz, 1H, H-7), 7.54 ( $\mathrm{t}, J=10.0 \mathrm{~Hz}, 1 \mathrm{H}, \mathrm{H}-5), 7.34$ (d, J=4.0 Hz, 1H, H-3). ${ }^{13} \mathrm{C}$ NMR (125 MHz, CDCl 3 ) $\delta 186.7$ (CH, C-9), 146.2 (C, 8a), 142.0 (CH, C-2), 140.3 (C, C-3a), 139.8 (CH, C-6), 139.0 (CH, C-4), 137.6 (CH, C-8), 129.6 (CH, C-7), 128.3 (CH, C-5), 126.0 (C, C-1), 119.1 (CH, C-3). Spectroscopic data matched those reported previously. ${ }^{6}$

\section{1-Methylazulene (101)}<smiles>Cp1pcsc2cpbpcpc1-2</smiles>

1-Formylazulene 78 (30 mg, $0.19 \mathrm{mmol}$ ) was dissolved in a RBF with THF ( $5 \mathrm{~mL}$ ) followed by addition of $0.4 \mathrm{~mL}$ of a $\mathrm{LiBH}_{4}$ solution (2M in THF, $0.8 \mathrm{mmol}, 4.2$ eq.). The reaction mixture changed colour from purple to blue. Two drops of $\mathrm{BF}_{3} \cdot \mathrm{Et}_{2} \mathrm{O}$ were added (undistilled), and after 5 minutes, diethyl ether was added to dilute the reaction mixture, and the reaction was quenched with saturated aq. $\mathrm{NaHCO}_{3}$.

A separation was performed with $\mathrm{Et}_{2} \mathrm{O} /$ sat. aq. $\mathrm{NaHCO}_{3}$ and the organic fraction was concentrated by rotary evaporation, and purified by a gradient column starting with pet. ether and ending with 3:1 pet. ether $/ \mathrm{Et}_{2} \mathrm{O}$ to elute 1-methylazulene 101 (11.6 mg, 43\% yield) as a vivid blue oil, and a blue substance that appeared by ${ }^{1} \mathrm{H}$ NMR spectroscopy to be various 1 -azulylmethylene oligomers. 1H NMR (500 MHz, CDCl $) \delta 8.26$ (d, J=9.5 Hz, 2H, H-4,8), 7.77 (d, J=3.5 Hz, 1H, H-2), 7.55 (t, J=3.5 Hz, 1H, H-6), 7.34 (d, J=4.0 Hz, 1H, H-3), 7.10 (t, J=9.5 Hz, 1H, H-7) 7.07 (t, J=9.5 Hz, 1H, H-5), 2.71 (s, 3H, 1-Me). Spectroscopic data matched those reported previously. ${ }^{7}$

\section{1-(N-Pyrrolidinylmethyl)azulene (110) and 1-hydroxymethylazulene (111)}

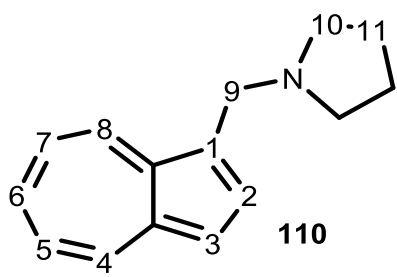

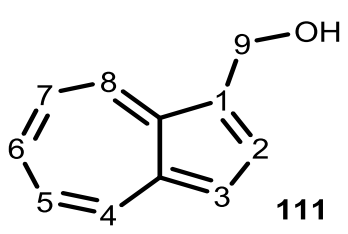


1-Formylazulene 78 (75 mg, $0.48 \mathrm{mmol}, 1$ eq.) was added to a RBF and dissolved in THF (7 mL). $p$ TsOH. $\mathrm{H}_{2} \mathrm{O}$ (138 mg, $1.25 \mathrm{mmol}, 2.6$ eq.) was added and a slight colour change towards crimson was observed. After 30 minutes, pyrrolidine $(0.15 \mathrm{~mL}, 1.8 \mathrm{mmol}, 3.8$ eq.) was added, and after a further 10 minutes, $\mathrm{NaBH}_{4}$ (580 mg, $15 \mathrm{mmol}, 32$ eq.) was added, at which point the reaction mixture effervesced and changed colour to blue.

A phase separation was performed using $\mathrm{CH}_{2} \mathrm{Cl}_{2} / \mathrm{H}_{2} \mathrm{O}$ and the organic fraction was concentrated by rotary evaporation and purified by column chromatography, eluting fraction $\mathrm{A}$ in $\mathrm{CH}_{2} \mathrm{Cl}_{2}$ and fraction B in 3:1 pet. ether/EtOAc. Fraction A was concentrated by rotary evaporation to give 110 (16.5 mg, 16\% yield) as a blue oil, and fraction B was concentrated by rotary evaporation to give 111 (30.6 $\mathrm{mg}, 40 \%$ yield) as a blue oil.

110: ${ }^{1} \mathrm{H}$ NMR (500 MHz, CDCl $) \delta 8.43$ (d, J=9.5 Hz, 1H, H-8), 8.40 (d, J=9.5 Hz, 1H, H-4), 7.92 (d, $J=4.0 \mathrm{~Hz}, 1 \mathrm{H}, \mathrm{H}-2), 7.70(\mathrm{t}, J=10.0 \mathrm{~Hz}, 1 \mathrm{H}, \mathrm{H}-6), 7.41$ (d, J=4.0 Hz, 1H, H-3), 7.33 (t, J=10.0 Hz, 1H, H7), 7.29 (t, J=9.5 Hz, 1H, H-5), 4.63 (s, 2H, H-9), 3.16-3.11 (m, 2H, H-10a), 2.92-2.85 (m, 2H, H-10b), 2.20-2.14 (m, 2H, H-11a), 1.66-1.59 (m, 2H, H-11b). ${ }^{13} \mathrm{C}$ NMR (125 MHz, CDCl 3 ) $\delta 141.6$ (C, C-8a), 140.9 (CH, C-2), 139.4 (C, C-3a), 138.2 (CH, C-6), 137.3 (CH, C-4), 134.1 (CH, C-8), 124.3 (CH, C-5), 123.7 (CH, C-7), 119.2 (C, C-1), 117.2 (CH, C-3), $57.7\left(\mathrm{CH}_{2}, \mathrm{C}-10\right), 56.7\left(\mathrm{CH}_{2}, \mathrm{C}-9\right), 27.9\left(\mathrm{CH}_{2}, \mathrm{C}-11\right)$. HRMS: $\mathrm{m} / \mathrm{z} \mathrm{C}_{15} \mathrm{H}_{18} \mathrm{~N}^{+}[\mathrm{M}+\mathrm{H}]+$ Calculated 212.1434, found 212.1427. $\lambda_{\max }: 577 \mathrm{~nm}$.

111: ${ }^{1} \mathrm{H}$ NMR (500 MHz, CDCl $) \delta 8.51$ (d, J=10 Hz, 1H, H-8), 8.34 (d, J=9.5 Hz, 1H, H-4), 7.93 (d, $J=3.5 \mathrm{~Hz}, 1 \mathrm{H}, \mathrm{H}-2), 7.63(\mathrm{t}, J=9.5 \mathrm{~Hz}, 1 \mathrm{H}, \mathrm{H}-6), 7.34$ (d, J=3.5 Hz, 1H, H-3), 7.23 (t, J=10.0 Hz, 1H, H7), 7.19 (t, J=9.5 Hz, 1H, H-5), 5.16 (s, 2H, H-9). 141.3 (C, C-8a), 137.9 (CH, C-6), 137.3 (CH, C-2), 137.2 (CH, C-4), 136.5 (C, C-3a), 133.9 (CH, C-8), 128.7 (C, C-1), 123.3 (CH, C-5), 123.0 (CH, C-7), 117.8 (CH, C-3), $58.5\left(\mathrm{CH}_{2}, \mathrm{C}-9\right)$.

\section{Methyl 1-azulenecarboxylate (8) and methyl oxo-1-azulenecarboxylate}

(7)
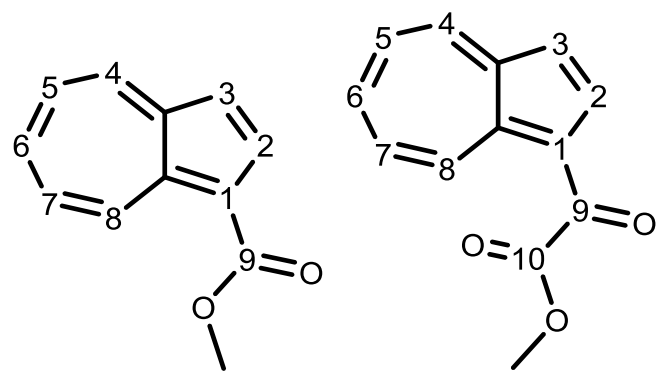

Azulene (49.2 mg, $0.38 \mathrm{mmol}, 1$ eq.) was added to a RBF fitted with condenser and dissolved with MeCN (6 mL). A solution of oxalyl chloride was prepared ( $0.14 \mathrm{~mL}$ in $1 \mathrm{~mL}$ MeCN.) and an aliquot 
( $0.25 \mathrm{~mL}, 1.06$ eq.) was added to the reaction mixture, which rapidly changed colour to orange. The reaction was heated at reflux for 80 minutes, during which time the colour changed from orange to crimson. $\mathrm{MeOH}(0.5 \mathrm{~mL}, 12.4 \mathrm{mmol}, 32 \mathrm{eq}$.) was added and the reaction was left to cool to r.t. for 30 minutes, after which pyridine $(0.7 \mathrm{~mL}, 8.7 \mathrm{mmol}, 23 \mathrm{eq}$.) was added.

A separation was immediately performed with $\mathrm{CH}_{2} \mathrm{Cl}_{2} / \mathrm{H}_{2} \mathrm{O}$, and the organic fraction was passed through a silica plug in $\mathrm{CH}_{2} \mathrm{Cl}_{2}$ to remove baseline matter, to obtain $73.4 \mathrm{mg}$ of a crude mixture of 25:1 methyl 1-azulenecarboxylate 8 and methyl oxo-1-azulenecarboxylate 7, equivalent to a 95\% yield of 8 and a $4 \%$ yield of 7.

8: ${ }^{1} \mathrm{H}$ NMR (500 MHz, CDCl $) \delta 9.66$ (d, J=10.0 Hz, 1H, H-8), 8.48 (d, J=9.5 Hz, 1H, H-4), 8.38 (d, $J=4.0 \mathrm{~Hz}, 1 \mathrm{H}, \mathrm{H}-2), 7.82(\mathrm{t}, J=10.0 \mathrm{~Hz}, 1 \mathrm{H}, \mathrm{H}-6), 7.57$ (t, J=10.0 Hz, 1H, H-7), 7.47 (t, J=10.0 Hz, 1H, H5), 7.31 (d, J=4.5 Hz, 1H, H-3), 3.97 (s, 3H, O-Me). ${ }^{13} \mathrm{C}$ NMR (125 MHz, CDCl 3 ) $\delta 165.9$ (C, C-9), 144.7 (C, C-8a), 140.7 (C, C-3a), 140.2 (CH, C-2), 139.0 (CH, C-6), 138.2 (CH, C-4), 137.7 (CH, C-8), 127.66 (CH, C-7), 126.7 (CH, C-5), 117.6 (CH, C-3), 116.7 (C, C-1), $51.2\left(\mathrm{CH}_{3}, \mathrm{O}-\mathrm{Me}\right)$. Proton NMR data corresponds to data reported previously. ${ }^{8}$

7: ${ }^{1} \mathrm{H}$ NMR (500 MHz, CDCl $) \delta 9.89$ (d, J=10.0 Hz, 1H, H-8), 8.54 (d, J=9.5 Hz, 1H, H-4), 8.47 (d, $J=4.5 \mathrm{~Hz}, 1 \mathrm{H}, \mathrm{H}-2$ ) , 7.94 (t, J=10.5 Hz, 1H, H-6), 7.76 (t, J=9.5 Hz, 1H, H-7), 7.64 (t, J=10.0 Hz, 1H, H5), 7.32 (d, J=4.5 Hz, 1H, H-3), 3.99 (s, 3H, O-Me). ${ }^{13} \mathrm{C} \mathrm{NMR} \mathrm{(125} \mathrm{MHz,} \mathrm{CDCl} 3$ ) $\delta 180.7$ (C, C-9), 164.9 (C, C-10), 147.3 (C, C-8a), 142.91 (C, C-3a), 142.89 (CH, C-2), 140.2 (CH, C-6), 139.7 (CH, C-8), 139.0 (CH, C-4), 131.2 (CH, C-7), 129.6 (CH, C-5), 120.5 (C, C-1), 119.3 (CH, C-3), $52.6\left(\mathrm{CH}_{3}, \mathrm{O}-\mathrm{Me}\right)$. Spectroscopic data matched those reported previously. ${ }^{9}$

\section{Methyl oxo-(3-methylazulene-1-carboxylate)(119)}

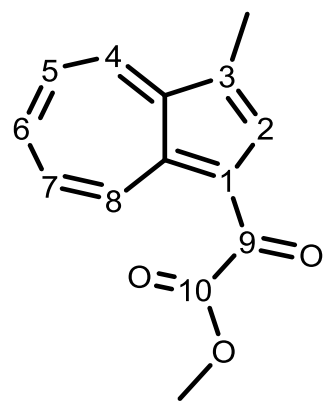

1-Methylazulene (11.6 mg, $0.08 \mathrm{mmol}, 1$ eq.) was dissolved in $3 \mathrm{~mL} \mathrm{CH}_{2} \mathrm{Cl}_{2}$ in a RBF. A solution of oxalyl chloride was prepared $\left(1: 9 \mathrm{v} / \mathrm{v}\right.$ oxalyl chloride in $\left.\mathrm{CH}_{2} \mathrm{Cl}_{2}\right)$ and an aliquot of this solution $(0.08 \mathrm{~mL}, 0.093 \mathrm{mmol}, 1.1 \mathrm{eq})$ was added to the reaction mixture. The reaction changed colour from blue to orange-red. After 80 minutes, $\mathrm{MeOH},(0.4 \mathrm{~mL}, 9.8 \mathrm{mmol}, 120$ eq.) was added and a 
colour change was observed to brown. Pyridine ( $0.1 \mathrm{~mL}, 1.2 \mathrm{mmol}, 15$ eq.) was added after 1 minute.

A separation was performed with $\mathrm{Et}_{2} \mathrm{O} / 10 \%$ aq. $\mathrm{HCl}$, and the organic layer was concentrated by rotary evaporation and purified by column chromatography in 3:2 pet. ether/Et $\mathrm{t}_{2} \mathrm{O}$ to afford methyl oxo-(3-methylazulene-1-carboxylate) 119 (13.5 mg, 72.5\% yield) as a brown solid, and trace amounts of a pink material that was assumed to be methyl (3-methylazulene-1-carboxylate). ${ }_{1} \mathrm{H} \mathrm{NMR} \mathrm{(500} \mathrm{MHz,} \mathrm{CDCl}_{3}$ ) $\delta 9.79$ (d, J=9.5 Hz, 1H, H-8), 8.40 (d, J=9.5 Hz, 1H, H-4), 8.27 (s, 1H, H-2), 7.87 (t, J=10.0 Hz, 1H, H-6), 7.66 (t, J=10.5 Hz, 1H, H-7), 7.58 (t, J=10.0 Hz, 1H, H-5), 3.99 (s, 3H, 0Me), 2.60 (s, 3H, 1-Me). ${ }^{13} \mathrm{C}$ NMR (125 MHz, CDCl $) \delta 180.2$ (C, C-9), 165.7 (C, C-10), 144.3 (C, C-3a), 143.4 (C, C-8a), 143.1 (CH, C-2), 140.0 (CH, C-6), 139.0 (CH, C-8), 135.9 (CH, C-4), 130.75 (CH, C-7), 128.5 (CH, C-5), 127.4 (C, C-3), 118.3 (C, C-1), $52.5\left(\mathrm{CH}_{3}, \mathrm{O}-\mathrm{Me}\right), 12.5\left(\mathrm{CH}_{3}, 3-\mathrm{Me}\right)$.

\section{Methyl 1-(4,6,8-trimethylazulenecarboxylate) (120) and methyl oxo-1- (4,6,8-trimethylazulenecarboxylate) (121)}
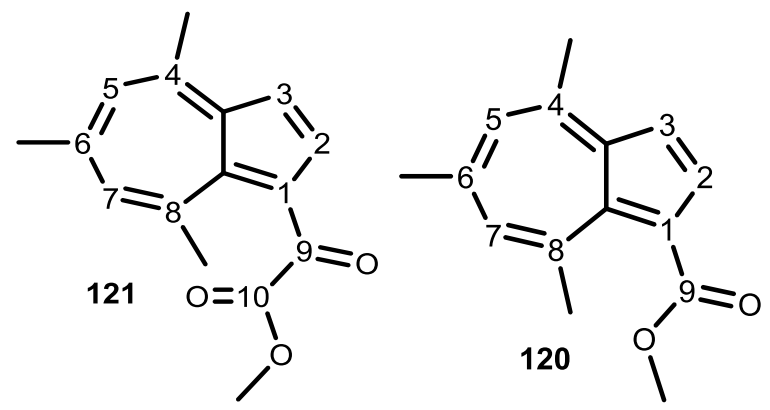

4,6,8-Trimethylazulene (27.4 mg,0.16 mmol, 1 eq.) was dissolved in $\mathrm{CH}_{2} \mathrm{Cl}_{2}(3 \mathrm{~mL}$ ) in a RBF. A solution of oxalyl chloride was prepared $\left(1: 9 \mathrm{v} / \mathrm{v}\right.$ in $\left.\mathrm{CH}_{2} \mathrm{Cl}_{2}\right)$ and an aliquot of this solution $(0.16$ $\mathrm{mL}, 0.18 \mathrm{mmol}, 1.16$ eq.) was added, and a colour change was observed from purple to orange. After 80 minutes, $\mathrm{MeOH}$ (0.4 mL, $9.8 \mathrm{mmol}, 61$ eq.) was added and a slight colour change towards red was observed. Pyridine $(0.1 \mathrm{~mL}, 1.2 \mathrm{mmol}, 7.7$ eq. $)$ was added after one minute.

A separation was performed using $\mathrm{Et}_{2} \mathrm{O} / 10 \%$ aq. $\mathrm{HCl}$, and column chromatography was performed on the organic layer, eluting with 3:2 pet. ether/Et $2 \mathrm{O}$ to afford 120 (25.1 mg, 24\% yield) as a magenta oil and $\mathbf{1 2 1}$ (25.4 $\mathrm{mg}, 62 \%$ yield) as a peach-coloured solid.

120: ${ }^{1} \mathrm{H}$ NMR (500 MHz, $\mathrm{CDCl}_{3}$ ) $\delta 7.96$ (d, J=4.5 Hz, 1H, H-2), 7.27 (s, 1H, H-7), 7.23 (s, 1H, H-5), 7.21 (d, J=4.0 Hz, 1H, H-3), 3.93 (s, 3H, 0-Me), 2.97 (s, 3H, 8-Me), 2.89 (s, 3H, 4-Me), 2.66 (s, 3H, 6Me). ${ }^{13} \mathrm{C}$ NMR (125 MHz, CDCl 3 ) $\delta 168.3$ (C, C-9), 148.4 (C, C-8), 147.5 (C, C-6), 146.9 (C, C-4), 139.8 (C, C-3a), 137.0 (CH, C-2), 134.6 (C, C-8a), 131.6 (CH, C-7), 130.1 (CH, C-5), 120.1 (C, C-1), 114.6 
(CH, C-3), $51.8\left(\mathrm{CH}_{3}, \mathrm{O}-\mathrm{Me}\right), 28.46\left(\mathrm{CH}_{3}, 8-\mathrm{Me}\right), 28.44\left(\mathrm{CH}_{3}, 4-\mathrm{Me}\right), 25.8\left(\mathrm{CH}_{3}, 6-\mathrm{Me}\right)$. IR (ATR): $v_{\max }$ $2952,2922,2852,1731,1618,1583,1500,1388,1330,1244,1201,1079 \mathrm{~cm}^{-1}$.

121: ${ }^{1} \mathrm{H}$ NMR (500 MHz, CDCl $) \delta 8.05$ (d, J=4.5 Hz, 1H, H-2), 7.44 (s, 1H, H-7), 7.39 (s, 1H, H-5), 7.18 (d, J=4.5 Hz, 1H, H-3), 3.99 (s, 3H, 0-Me), 3.00 (s, 3H, 8-Me), 2.90 (s, 3H, 6-Me), 2.68 (s, 3H, 4Me). ${ }^{13} \mathrm{C}$ NMR (125 MHz, CDCl $) \delta 180.4$ (C, C-9), 165.5 (C, C-10), 151.2 (C, C-8), 148.7 (C, C-6), 147.7 (C, C-4), 143.8 (C, C-3a), 140.3 (CH, C-2), 137.6 (C, C-8a), 134.4 (CH, C-7), 132.8 (CH, C-5), 124.4 (C, C-1), 115.9 (CH, C-3), $52.6\left(\mathrm{CH}_{3}, \mathrm{O}-\mathrm{Me}\right), 29.6\left(\mathrm{CH}_{3}, 8-\mathrm{Me}\right), 28.5\left(\mathrm{CH}_{3}, 4-\mathrm{Me}\right), 26.1\left(\mathrm{CH}_{3}, 6-\right.$ Me). HRMS: $m / z \mathrm{C}_{16} \mathrm{H}_{17} \mathrm{O}_{3^{+}}[\mathrm{M}+\mathrm{H}]^{+}$Calculated 257.1172, found 257.1164. IR (ATR): $v_{\max } 2951$, $2923,2852,1731,1618,1499,1388,1243,1201,1078,731 \mathrm{~cm}^{-1} . \lambda_{\max }: 487 \mathrm{~nm}$.

\section{$(1 R, 2 S, 5 R)-M e n t h y l$ azulene-1-carboxylate (122), azulene-1-carboxylic acid (75) and azulene-1-carboxylic anhydride (123)}

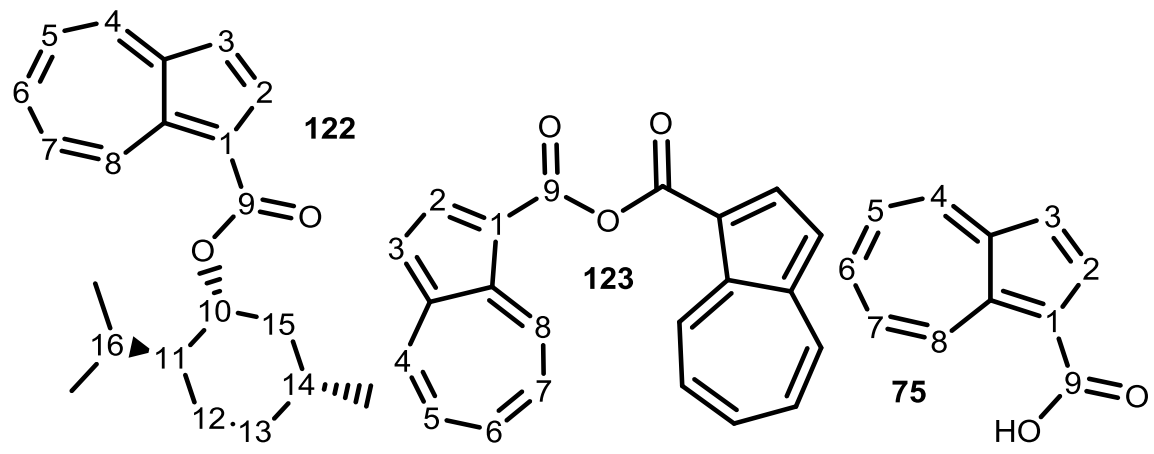

Azulene 1 (154 mg, $1.2 \mathrm{mmol}, 1.18$ eq.) was dissolved in MeCN (10 mL) in a RBF fitted with condenser. An oxalyl chloride solution was prepared ( $0.4 \mathrm{~mL}$ in $1 \mathrm{~mL} \mathrm{MeCN}$ ) and an aliquot (0.35 $\mathrm{mL}, 1.16 \mathrm{mmol}, 1.14$ eq.) was added to the reaction mixture, which changed from blue to orange. The reaction mixture was heated at reflux for 30 minutes, then a (-)-menthol solution (158.8 mg in $2 \mathrm{~mL} \mathrm{MeCN}, 1.02 \mathrm{mmol}, 1$ eq.) was added. The reaction mixture was removed from the oil bath, and after 45 seconds $\mathrm{NEt}_{3}(0.3 \mathrm{~mL}, 2.2 \mathrm{mmol}, 2.1$ eq.) was added, producing a white vapour upon addition.

A separation was performed with $\mathrm{CH}_{2} \mathrm{Cl}_{2} / \mathrm{H}_{2} \mathrm{O}$, with both phases being coloured. The aqueous layer was indigo and was assumed to contain the triethylammonium azulene 1-carboxylate salt. The purple organic layer was concentrated by rotary evaporation and purified by column chromatography, eluting fraction $\mathrm{A}$ in $1: 1$ pet. ether $/ \mathrm{CH}_{2} \mathrm{Cl}_{2}$ and fractions $\mathrm{B}$ and $\mathrm{C}$ in $\mathrm{Et}_{2} \mathrm{O}$. Fraction A was concentrated by rotary evaporation to afford 122 (285.9 mg, 90.6\% yield) as a purple oil. Fraction B was concentrated by rotary evaporation to afford 123 (20.3 mg, 5\% yield w.r.t. oxalyl 
chloride) as a crimson solid and fraction $\mathrm{C}$ was concentrated by rotary evaporation to afford $\mathbf{7 5}$ (7.1 mg, 3.5\% yield w.r.t. oxalyl chloride) as a maroon solid.

122: ${ }^{1} \mathrm{H}$ NMR (500 MHz, CDCl $) \delta 9.69$ (d, J=10.0 Hz, 1H, H-8), 8.46 (d, J=9.5 Hz, 1H, H-4), 8.39 (d, $J=4.5 \mathrm{~Hz}, 1 \mathrm{H}, \mathrm{H}-2), 7.80$ (t, J=10.0 Hz, 1H, H-6), 7.55 (t, J=10.5 Hz, 1H, H-7), 7.44 (t, J=9.5 Hz, 1H, H5), 7.30 (d, J=4.5 Hz, 1H, H-3), 5.01 (td, J=10.5, 4.5 Hz, 1H, H-10), 2.22 (dm, J=12.0 Hz, 1H, H-15a), 2.08 (septd, J=7.0, $2.5 \mathrm{~Hz}, 1 \mathrm{H}, \mathrm{H}-16$ ) , 1.78-173 (m, 2H, H-13a, H-12a), 1.65-1.60 (m, 1H, H-11), 1.60-1.58 (m, 1H, H-14), 1.22-1.14 (m, 1H, H-12b), 1.15 (m, 1H, H-15b), 0.96 (m, 1H, H-13b), 0.95 (d, J=5.0 Hz, 3H, 16-Me), 0.94 (d, J=4.5 Hz, 3H, 14-Me), 0.84 (d, J=7.0 Hz, 3H, 16-Me). ${ }^{13} \mathrm{C}$ NMR (125 $\mathrm{MHz}, \mathrm{CDCl}_{3}$ ) $\delta 165.0$ (C, C-9), 144.7 (C, C-8a), 140.7 (C, C-3a), 140.2 (CH, C-2), 138.9 (CH, C-6), 138.0 (CH, C-4), 137.8 (CH, C-8), 127.5 (CH, C-7), 126.5 (CH, C-5), 117.44 (C, C-1), 117.42 (CH, C-3), 73.4 (CH, C-10), 47.4 (CH, C-11), $41.4\left(\mathrm{CH}_{2}, \mathrm{C}-15\right), 34.4\left(\mathrm{CH}_{2}, \mathrm{C}-13\right), 31.6$ (CH, C-14), 26.6 (CH, C16), $23.7\left(\mathrm{CH}_{2}, \mathrm{C}-12\right), 22.1\left(\mathrm{CH}_{3}, 16-\mathrm{Me}\right), 20.8\left(\mathrm{CH}_{3}, 14-\mathrm{Me}\right), 16.6\left(\mathrm{CH}_{3}, 16-\mathrm{Me}\right)$. HRMS: $\mathrm{m} / \mathrm{z}$ $\mathrm{C}_{21} \mathrm{H}_{27} \mathrm{O}_{2}+[\mathrm{M}+\mathrm{H}]+$ Calculated 311.2006, found 311.2008. IR (ATR): $v_{\max } 2951,2924,2867,1681$, $1418,1211,1143,991,769 \mathrm{~cm}^{-1}$.

123: ${ }^{1} \mathrm{H}$ NMR ( $500 \mathrm{MHz}, \mathrm{CDCl}_{3}$ ) $\delta 9.79$ (d, J=10.0 Hz, 2H, H-8), 8.54 (d, J=9.5 Hz, 2H, H-4), 8.50 (d, $J=4.5 \mathrm{~Hz}, 2 \mathrm{H}, \mathrm{H}-2), 7.90(\mathrm{t}, J=9.5 \mathrm{~Hz}, 2 \mathrm{H}, \mathrm{H}-6), 7.69(\mathrm{t}, J=10.0 \mathrm{~Hz}, 2 \mathrm{H}, \mathrm{H}-7), 7.58(\mathrm{t}, J=9.5 \mathrm{~Hz}, 2 \mathrm{H}, \mathrm{H}-5)$, 7.35 (d, J=4.0 Hz, 2H, H-3). ${ }^{13} \mathrm{C}$ NMR (125 MHz, CDCl $) \delta 161.1$ (C, C-9), 146.2 (C, C-8a), 142.6 (C, C3a), 141 (CH, C-2), 139.7 (CH, C-6), 138.7 (CH, C-4), 138.4 (CH, C-8), 129.0 (CH, C-7), 128.0 (CH, C5), 118.1 (CH, C-3), 115.6 (C, C-1). HRMS: $m / z_{22} \mathrm{C}_{22} \mathrm{H}_{14} \mathrm{O}_{3} \mathrm{Na}^{+}$[M+Na]+Calculated 349.0835, found 349.0839. IR (ATR): $v_{\max } 3052,2954,2922,2853,1727,1674,1397,1201,1026,936,742,563 \mathrm{~cm}^{-}$ 1. $\lambda_{\max }: 522 \mathrm{~nm}$.

75: ${ }^{1} \mathrm{H}$ NMR (500 MHz, $\mathrm{CDCl}_{3}$ ) $\delta 9.72$ (d, J=10 Hz, 1H, H-8), 8.51 (obscured d, 1H, H-4), 8.50 (obscured d, 1H, H-2), 7.86 (t, J=10.0 Hz, 1H, H-6), 7.62 (t, J=10.0 Hz, 1H, H-7), 7.51 (t, J=9.5 Hz, 1H, H-5), 7.34 (d, J=4.0 Hz, 1H, H-3). ${ }^{13} \mathrm{C}$ NMR (125 MHz, CDCl $) \delta 170.6$ (C, C-9), 145.5 (C, C-8a). 141.5 (C, C-3a), 141.2 (CH, C-2), 139.2 (CH, C-6), 138.4 (CH, C-4), 138.0 (CH, C-8), 128.2 (CH, C-7), 127.4 (CH, C-5), 118.0 (CH, C-3), 116.0 (CH, C-1). HRMS: $m / z_{11} \mathrm{C}_{9} \mathrm{O}_{2}{ }^{+}[\mathrm{M}+\mathrm{H}]+$ Calculated 173.0597, found 173.0599. Spectra match those reported in literature. ${ }^{7}$

\section{Deprotection of menthyl azulene-1-carboxylate}

Menthyl azulene-1-carboxylate 122 ( $41 \mathrm{mg}, 0.13 \mathrm{mmol}, 1$ eq.) was dissolved in $10 \mathrm{~mL}$ wet EtOH in a RBF fitted with condenser under open air. $\mathrm{KOH}$ (346 mg, $6.1 \mathrm{mmol}, 46 \mathrm{eq}$.) was added and reaction was heated to reflux and stirred overnight. Colour had changed from purple to violet. The reaction was removed from the oil bath and allowed to cool. 
A separation was performed using $\mathrm{CH}_{2} \mathrm{Cl}_{2} / \mathrm{H}_{2} \mathrm{O}$, with the violet colour inhabiting the aqueous layer. Saturated aq. $\mathrm{NH}_{4} \mathrm{Cl}$ was added and the aqueous layer was washed two times with $\mathrm{CH}_{2} \mathrm{Cl}_{2}$, and concentrated by rotary evaporation to obtain a crude mass of $22.7 \mathrm{mg}$, which was shown by ${ }^{1} \mathrm{H}$ NMR integration to be $86 \%$ menthol. This translates to a $94 \%$ yield of menthol as determined by ${ }^{1}$ H NMR spectroscopy.

This crude mixture was then subjected to column chromatography (gradient elution running from pure pet. ether to $\mathrm{CH}_{2} \mathrm{Cl}_{2}$ ) to obtain menthol ( $8.7 \mathrm{mg}$, $42 \%$ yield) as white needles, along with traces of starting material and other by-products.

\section{Regeneration of azulene from azulene-1-carboxylic acid}

Azulene-1-carboxylic acid (59.5 mg, $0.34 \mathrm{mmol}$ ) was added to a RBF fitted with distillation head. Methanol $(10 \mathrm{~mL})$ and $10 \%$ aq. $\mathrm{HCl}(4.5 \mathrm{~mL})$ were added to the reaction vessel under open air and the reaction vessel was heated to boiling. A solution of azulene in methanol was collected while the reaction mixture slowly changed to green. The blue distillate was concentrated via rotary evaporation to remove methanol, and a separation was performed using $\mathrm{CH}_{2} \mathrm{Cl}_{2} / \mathrm{H}_{2} \mathrm{O}$ to remove water. The organic fraction was then concentrated by rotary evaporation and the resulting material was filtered to a silica plug in pet. ether to obtain azulene ( $35.1 \mathrm{mg}$, $79 \%$ yield).

\section{1-Nitroazulene (135)}

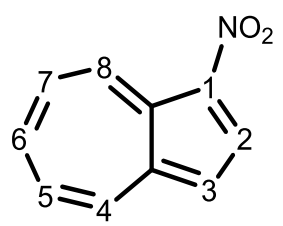

Azulene (36.8 mg, 0.29 mmol, 1 eq.) was added to a RBF and dissolved in $\mathrm{CH}_{2} \mathrm{Cl}_{2}(6 \mathrm{~mL}) .3 .5 \mathrm{~mL} \mathrm{~A}$ copper nitrate solution was prepared ( $432 \mathrm{mg} \mathrm{Cu}\left(\mathrm{NO}_{3}\right)_{2} .3 \mathrm{H}_{2} \mathrm{O}$ in $10 \mathrm{~mL} \mathrm{Ac} 2 \mathrm{O}$ ) and an aliquot (3.5 $\mathrm{mL}, 0.62 \mathrm{mmol}, 2.2 \mathrm{eq}$.) was added to the reaction mixture, which after a few seconds had changed colour to a dirty brown-green.

After 5 minutes, the reaction was quenched with saturated aq. $\mathrm{NaHCO}_{3}$, and a phase separation was performed using $\mathrm{NaHCO}_{3}$ (aq.) and $\mathrm{CH}_{2} \mathrm{Cl}_{2}$. The organic fraction was concentrated by rotary evaporation and column chromatography was performed on the resulting material in a gradient of pet. ether to $\mathrm{CH}_{2} \mathrm{Cl}_{2}$, to afford 1-nitroazulene 135 (18.2 $\mathrm{mg}, 37 \%$ yield) as an orange-red solid. 1H NMR (500 MHz, CDCl $) \delta 9.79$ (d, J=10.5 Hz, H, H-8), 8.56 (d, J=10.0 Hz, 1H, H-4), 8.48 (d, J=4.5 $\mathrm{Hz}, 1 \mathrm{H}, \mathrm{H}-2$ ) , 8.00 (t, J=9.5 Hz, 1H, H-6), 7.80 (t, J=10.0 Hz, 1H, H-7), 7.67 (t, J=10.0 Hz, 1H, H-5), 
7.24 (d, J=4.5 Hz, 1H, H-3). ${ }^{13} \mathrm{C}$ NMR (125 MHz, $\mathrm{CDCl}_{3}$ ) $\delta 143.9$ (C, C-3a), 141.0 (CH, C-6), 140.6 (CH, C-4), 138.2 (CH, C-8), 134.8 (CH, C-2), 134.0 (C, C-8a), 131.1 (CH, C-7), 129.6 (CH, C-5), 116.87 (CH, C-3). C-1 not observed. HRMS: $m / z \mathrm{C}_{10} \mathrm{H}_{8} \mathrm{NO}_{2}{ }^{+}[\mathrm{M}+\mathrm{H}]^{+}$calculated 174.0550, found 174.0547. IR (ATR): $v_{\max } 2917,1540,1476,1321,1259,1217,1044,758$.

\section{1,3-Dinitroazulene (6)}

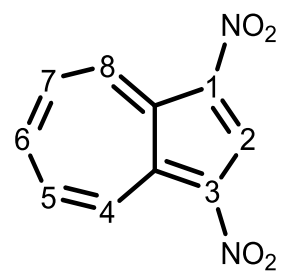

Azulene (66.6 mg, $0.52 \mathrm{mmol}, 1$ eq.) was added to a RBF with copper nitrate trihydrate (556 mg, $2.3 \mathrm{mmol}, 4.4$ eq.) and acetic anhydride ( $6 \mathrm{~mL}, 64 \mathrm{mmol}, 120$ eq.). A colour change to green was observed after 5 minutes, followed by evolution of a brown gas after a further 5 minutes. At this point, the reaction was quenched with water.

A separation was performed with $\mathrm{CH}_{2} \mathrm{Cl}_{2}$ /aq. $\mathrm{NaHCO}_{3}$, and the organic fraction was concentrated by rotary evaporation. The crude product was purified by column chromatography (gradient elution of pet. ether to $\mathrm{CH}_{2} \mathrm{Cl}_{2}$ ) to afford 1,3-dinitroazulene 6 (30.6 mg, 27\% yield) as vivid orange needles. ${ }^{1} \mathrm{H}$ NMR (500 MHz, $\left.\mathrm{CDCl}_{3}\right) \delta 10.05$ (d, J=10.0 Hz, 2H, H-4,8), 9.14 (s, 1H, H-2), 8.35 (t, $J=10.0 \mathrm{~Hz}, 1 \mathrm{H}, \mathrm{H}-6), 8.17$ (t, J=10.0 Hz, 2H, H-5,7). ${ }^{13} \mathrm{C}$ NMR (125 MHz, CDCl 3 ) $\delta 144.6$ (CH, C-6), 141.0 (CH, C-4,8), 135.2 (CH, C-5,7), 134.8 (C, C-3a,8a), 132.5 (CH, C-2). C-1,3 not observed. HRMS: $m / z \mathrm{C}_{10} \mathrm{H}_{7} \mathrm{~N}_{2} \mathrm{O}_{4}{ }^{+}[\mathrm{M}+\mathrm{H}]+$ Calculated 219.0400, found 219.0409. $\lambda_{\max }: 475 \mathrm{~nm}$. 


\section{$(1 R, 2 S, 5 R)-M e n t h y l$ (3-nitroazulene-1-carboxylate) (136)}

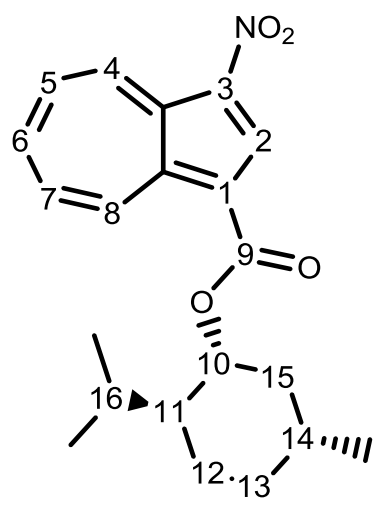

Menthyl azulene-1-carboxylate 122 (53.5 mg, $0.17 \mathrm{mmol}, 1$ eq.) was added to a RBF with copper nitrate trihydrate (154 mg, $0.63 \mathrm{mmol}, 3.7 \mathrm{eq}$.). The reaction vessel was cooled to $0{ }^{0} \mathrm{C}$ and acetic anhydride $(5 \mathrm{~mL})$ was added. The reaction was stirred for an hour during which time the reaction vessel warmed up to room temperature and a colour change had occurred from purple to brown.

A separation was performed in sat. aq. $\mathrm{NaHCO}_{3} / \mathrm{CH}_{2} \mathrm{Cl}_{2}$ and the organic layer was concentrated by rotary evaporation and purified by column chromatography (gradient elution of pet. ether to $\mathrm{CH}_{2} \mathrm{Cl}_{2}$ ) to afford 136 (31.6 mg, 52\% yield) as an orange oil. ${ }^{1} \mathrm{H}$ NMR (500 MHz, $\left.\mathrm{CDCl}_{3}\right) \delta 10.03(\mathrm{~d}$, $J=10.0 \mathrm{~Hz}, 1 \mathrm{H}, \mathrm{H}-8$ ), 9.91 (d, J=10.0 Hz, 1H, H-4), 8.97 (s, 1H, H-2), 8.17 (t, J=10.0 Hz, 1H, H-6), 7.99 (t, J=10.0 Hz, 1H, H-5), 7.96 (t, J=10.0 Hz, 1H, H-7), 5.01 (td, J=11.0, 4.4 Hz, 1H, H-10), 2.16 (dm, $J=12.0 \mathrm{~Hz}, 1 \mathrm{H}, \mathrm{H}-15 \mathrm{a}$ ), 2.02 (septd, J=7.0, 3.0 Hz, 1H, H-16), 1.80-1.73 (m, 2H, H-12a, H-13a), 1.641.56 (m, 2H, H-11, H-14), 1.20-1.13 (m, 2H, H-12b, H-15b), 0.99 (obscured m, 1H, H-13b), 0.96 (d, $J=6.5 \mathrm{~Hz}, 3 \mathrm{H}, 16-\mathrm{Me}), 0.95$ (d, J=7.0 Hz, 3H, 14-Me), 0.83 (d, J=7.0 Hz, 3H, 16-Me). ${ }^{13} \mathrm{C}$ NMR (125 MHz, $\mathrm{CDCl}_{3}$ ) $\delta 163.8$ (C, C-9), 142.8 (C, C-3a), 142.6 (CH, C-6), 141.6 (CH, C-8), 139.1 (CH, C-4), 137.9 (CH, C-2), 136.6 (C, C-8a), 133.4 (CH, C-5), 133.0 (CH, C-7), 115.6 (C, C-1), 74.5 (CH, C-10), 47.2 (CH, C-11), 41.1 ( $\left.\mathrm{CH}_{2}, \mathrm{C}-15\right), 34.3$ ( $\left.\mathrm{CH}_{2}, \mathrm{C}-13\right), 31.6$ (CH, C-14), 26.3 (CH, C-16), $23.6\left(\mathrm{CH}_{2}, \mathrm{C}-\right.$ 12), $22.1\left(\mathrm{CH}_{3}, 17-\mathrm{Me}\right), 20.8\left(\mathrm{CH}_{3}, 14-\mathrm{Me}\right), 16.5\left(\mathrm{CH}_{3}, 16-\mathrm{Me}\right)$. $\mathrm{C}-3$ was not observed. HRMS: $m / z$ $\mathrm{C}_{21} \mathrm{H}_{26} \mathrm{NO}_{4}+[\mathrm{M}+\mathrm{H}]+$ Calculated 356.1856, found 356.1865. IR (ATR): $v_{\max } 3126,2957,2911,2871$, $1688,1500,1419,1280,1211,1178,1018,757 \mathrm{~cm}^{-1} . \lambda_{\max }: 478 \mathrm{~nm}$. 


\section{(1R,2S,5R)-Menthyl 3-(1,4-dimethyl, 7-isopropylazulenecarboxylate)}

(148)

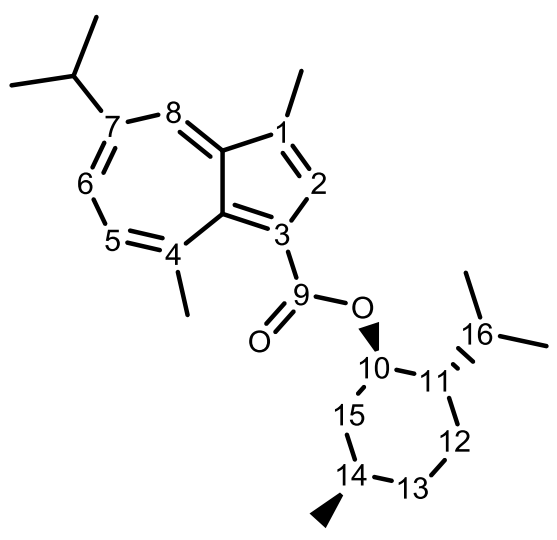

Guaiazulene (320 mg, $1.6 \mathrm{mmol}, 1.08$ eq.) was added to a $\mathrm{RBF}$ in $\mathrm{CH}_{2} \mathrm{Cl}_{2}$ (20 mL) and oxalyl chloride ( $0.13 \mathrm{~mL}, 1.5 \mathrm{mmol}, 1.02 \mathrm{eq}$.) was added, causing a colour change from blue to orangebrown. After 35 minutes, the reaction mixture had become purple. Added a solution of (-)menthol (232.5 mg in $5 \mathrm{~mL} \mathrm{CH} \mathrm{Cl}_{2}, 1.47 \mathrm{mmol}, 1$ eq.), was added, followed by $\mathrm{NEt}_{3}$ (0.6 mL, 4.3 mmol, 2.9 eq.) and the reaction changed colour to green.

A separation was performed with $\mathrm{CH}_{2} \mathrm{Cl}_{2} / \mathrm{H}_{2} \mathrm{O}$ and the organic fraction was concentrated by rotary evaporation and the remaining material was further purified by column chromatography in $\mathrm{CH}_{2} \mathrm{Cl}_{2}$ to give 148 (259.2 mg, 42\% yield) as an indigo oil. ${ }^{1 \mathrm{H}} \mathrm{NMR}\left(500 \mathrm{MHz}, \mathrm{CDCl}_{3}\right) \delta 8.24(\mathrm{~d}$, $J=2.0 \mathrm{~Hz}, 1 \mathrm{H}, \mathrm{H}-8$ ), 7.90 (s, 1H, H-2), 7.52 (dd, J=11.0, $2.0 \mathrm{~Hz}, 1 \mathrm{H}, \mathrm{H}-6), 7.25$ (d, J=10.5 Hz, 1H, H-5), 4.96 (td, J=11.0, 4.5 Hz, 1H, H-10), 3.11 (sep, J=7.5 Hz, 1H, 7-iPr CH), 2.97 (s, 3H, 4-Me), 2.60 (s, 3H, 1-Me), 2.21 (dm, J=11.0 Hz, 1H, H-15a), 2.11 (septd, J=7.0, 3.0 Hz, 1H, H-16), 1.74 (m, 2H, H-12a, H13a), 1.58 (m, 1H, H-14), 1.56 (m, 1H, H-11), 1.37 (d, J=7.0 Hz, 6H, 7-iPr CH $\mathrm{CH}_{3}, 1.17$ (m, 1H, H-15b), 1.14 (m, 1H, H-12b), 0.98 (m, 1H, H-13b), 0.96 (d, J=7.0 Hz, 3H, 16-Me), 0.94 (d, J=7.0 Hz, 3H, 14Me), 0.85 (d, J=7.0 Hz, 3H, 17-Me). ${ }^{13}$ C NMR (125 MHz, CDCl 3 ) $\delta 166.8$ (C, C-9), 147.2 (C, C-4), 143.3 (C, C-7), 140.2 (C, C-3a), 139.7 (CH, C-2), 135.76 (C, C-8a), 135.73 (CH, C-6), 134.3 (CH, C-8), 130.3 (CH, C-5), 123.9 (C, C-3), 117.7 (C, C-1), 73.9 (CH, C-10), 47.5 (CH, C-11), $41.1\left(\mathrm{CH}_{2}, \mathrm{C}-15\right), 37.9$ (CH, 7-iPr), 34.6 ( $\left.\mathrm{CH}_{2}, \mathrm{C}-13\right), 31.5$ (CH, C-14), $27.7\left(\mathrm{CH}_{3}, 4-\mathrm{Me}\right), 26.3(\mathrm{CH}, \mathrm{C}-16), 24.5\left(\mathrm{CH}_{3}, 7-\mathrm{Pr} \mathrm{CH}\right)$, $23.4\left(\mathrm{CH}_{2}, \mathrm{C}-12\right), 22.2\left(\mathrm{CH}_{3}, 17-\mathrm{Me}\right), 21.0\left(\mathrm{CH}_{3}, 14-\mathrm{Me}\right), 16.2\left(\mathrm{CH}_{3}, 16-\mathrm{Me}\right), 12.9\left(\mathrm{CH}_{3}, 7-\mathrm{i} \mathrm{Pr} \mathrm{CH}_{3}\right)$. HRMS: $m / z \mathrm{C}_{26} \mathrm{H}_{37} \mathrm{O}_{2}+[\mathrm{M}+\mathrm{H}]+$ Calculated 381.2788, found 381.2805. 
(1R,2S,5R)-Menthyl 4-(2-Hydroxyethyl)-7-isopropyl-1-methylazulene-3carboxylate (150) and 1H-7-isopropyl-9-methylazuleno[3,4-cd]3,4dihydrooxepin-1-one (151) and (1R,2S,5R)-menthyl 4-((1hydroxymethyl)2-hydroxyethyl)-7-isopropyl-1-methylazulene-3carboxylate (152) and 1H-7-isopropyl-9-methylazuleno[3,4-cd]4hydroxymethyl-3,4-dihydrooxepin-1-one (153)

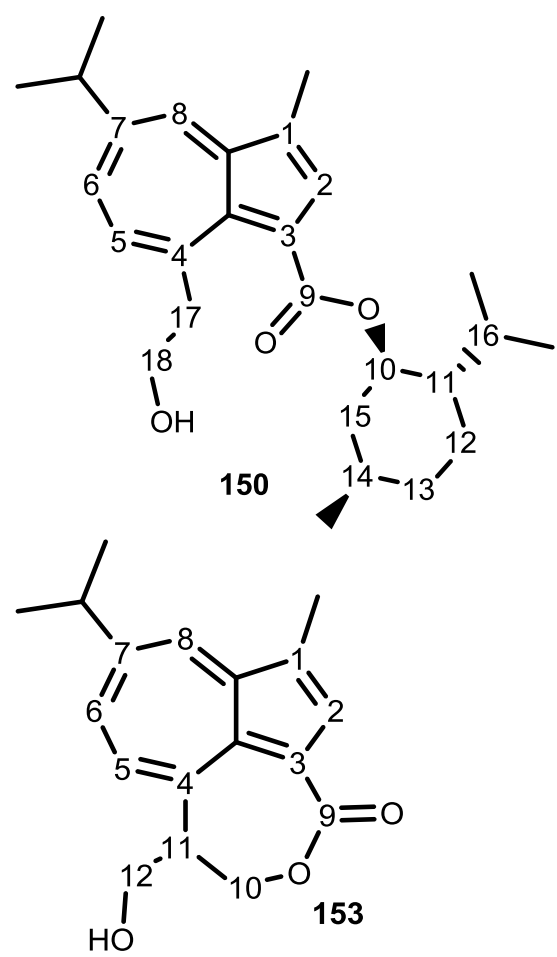

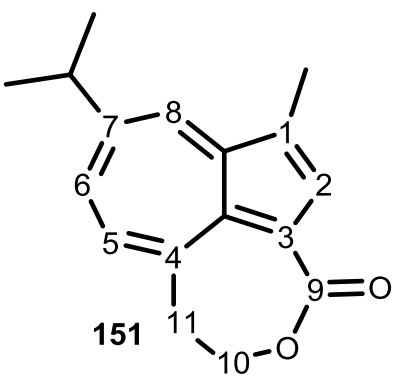

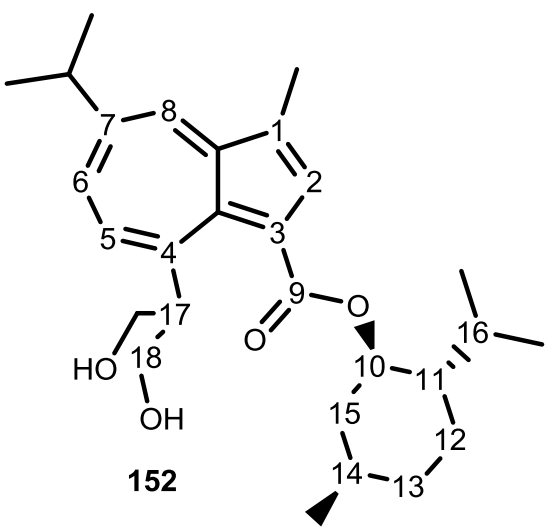

148 (40.8 mg, 0.1 mmol, 1 eq.) was added to an RBF fitted with condenser and dissolved in MeCN (12 mL). Paraformaldehyde (118 mg, $3.9 \mathrm{mmol}, 37 \mathrm{eq}$.) was added to the reaction mixture, followed by DBU $\left(0.05 \mathrm{~mL}, 0.3 \mathrm{mmol}, 3\right.$ eq.). The reaction was heated at $65{ }^{\circ} \mathrm{C}$ for 1 hour then cooled down to $37{ }^{\circ} \mathrm{C}$ after paraformaldehyde was observed condensing on the glassware. The reaction was left at this temperature overnight, and then the reaction was heated to reflux and left for another night.

The reaction was cooled down to r.t. and a separation was performed with $\mathrm{CH}_{2} \mathrm{Cl}_{2} / \mathrm{H}_{2} \mathrm{O}$, the organic fraction was washed with $10 \%$ aq. $\mathrm{HCl}$, and was concentrated by rotary evaporation. The resulting residue was purified by column chromatography (gradient elution from pet. ether to $\mathrm{CH}_{2} \mathrm{Cl}_{2}$ to $\mathrm{Et}_{2} \mathrm{O}$ to $10 \% \mathrm{MeOH}$ in $\mathrm{CH}_{2} \mathrm{Cl}_{2}$ ), to provide recovered starting material and four products, 
$\mathbf{1 5 0}$ (9.9 mg, 22.5\% yield) as an indigo oil, $\mathbf{1 5 1}$ (2.6 mg, 9.5\% yield) as a violet oil, $\mathbf{1 5 2}$ (4.9 mg, 10\% yield) as an indigo oil and $\mathbf{1 5 3}$ (1 mg, 3\% yield) as a violet oil.

150: ${ }^{1} \mathrm{H}$ NMR (500 MHz, CDCl 3 ) $\delta 8.24$ (s, 1H, H-8), 8.02 (s, 1H, H-2), 7.60 (d, J=11.0 Hz, 1H, H-6), 7.36 (d, J=11.0 Hz, 1H, H-5), 4.97 (td, J=11.0, 4.5 Hz, 1H, H-10), 4.18 (q, J=6.5 Hz, 2H, H-18), 3.53 (t, $J=6.0 \mathrm{~Hz}, 2 \mathrm{H}, \mathrm{H}-17$ ), 3.12 (sept, J=7.0 Hz, 1H, 7-iPr CH), 2.59 (s, 3H, 1-Me), 2.18 (m, J=11.5 Hz, 1H, H-15a), 2.09 (septd, J=7.0, 2.5 Hz, 1H, H-16), 1.78-1.72 (m, 2H, H-12a, H-13a), 1.63-1.55 (m, 2H, H11, H-14), 1.37 (d, J=7.0 Hz, 6H, 7-iPr $\mathrm{CH}_{3}$ ), 1.23-1.12 (m, 1H, H-15b), 1.15 (m, 1H, H-12b), 0.98 (obscured m, 1H, H-13a), 0.97 (d, J=6.0 Hz, 3H, 16-Me), 0.95 (d, J=6.5 Hz, 3H, 14-Me), 0.85 (d, J=7.0 Hz, 3H, 16-Me). ${ }^{13} \mathrm{C}$ NMR (125 MHz, $\mathrm{CDCl}_{3}$ ) $\delta 167.8$ (C, C-9), 149.1 (C, C-4), 143.9 (C, C-7), 141.5 (CH, C-2), 140.6 (C, C-3a), 138.0 (C, C-8a), 136.5 (CH, C-6), 134.5 (CH, C-8), 130.0 (CH, C-5), 124.5 (C, C-1), 115.8 (C, C-3), 74.3 (CH, C-10), 63.7 ( $\left.\mathrm{CH}_{2}, \mathrm{C}-18\right), 47.5$ (CH, C-11), $41.7\left(\mathrm{CH}_{2}, \mathrm{C}-17\right), 41.1$ $\left(\mathrm{CH}_{2}, \mathrm{C}-15\right), 37.9$ (CH, 7-iPr CH), $34.4\left(\mathrm{CH}_{2}, \mathrm{C}-13\right), 31.5$ (CH, C-14), 26.3 (CH, C-16), $24.5\left(\mathrm{CH}_{3}, 7-\mathrm{i} \mathrm{Pr}\right.$ $\left.\mathrm{CH}_{3}\right)$, $23.4\left(\mathrm{CH}_{2}, \mathrm{C}-12\right), 22.1\left(\mathrm{CH}_{3}, 16-\mathrm{Me}\right), 21.0\left(\mathrm{CH}_{3}, 14-\mathrm{Me}\right), 16.3\left(\mathrm{CH}_{3}, 16-\mathrm{Me}\right), 12.9\left(\mathrm{CH}_{3}, 1-\mathrm{Me}\right)$. HRMS: $m / z \mathrm{C}_{27} \mathrm{H}_{39} \mathrm{O}_{3^{+}}[\mathrm{M}+\mathrm{H}]+{ }^{+}$Calculated 411.2894, found 411.2893. IR (ATR): $v_{\max } 3419,2954$, 2926, 2868, 1691, 1664, 1523, 1455, 1406, 1369, 1194, 1021, $753 \mathrm{~cm}^{-1} . \lambda_{\max }: 564 \mathrm{~nm}$.

151: ${ }^{1} \mathrm{H}$ NMR (500 MHz, $\mathrm{CDCl}_{3}$ ) $\delta 8.32(\mathrm{~s}, 2 \mathrm{H}, \mathrm{H}-2,8), 7.60(\mathrm{dd}, J=11.0,2 \mathrm{~Hz}, 1 \mathrm{H}, \mathrm{H}-6), 7.24(\mathrm{~d}$, $J=10.5 \mathrm{~Hz}, 1 \mathrm{H}, \mathrm{H}-5), 4.63$ (t, J=4.5 Hz, 2H, H-10), 3.61 (broad m, 2H, H-11), 3.17 (sept, J=7.0 Hz, 1H,

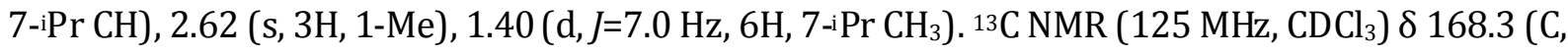
C-9), 147.8 (C, C-4), 145.8 (C, C-7), 144.1 (CH, C-2), 141.7 (C, C-3a), 136.2 (CH, C-6), 135.5 (CH, C-8), 135.49 (C, C-8a), 128.9 (CH, C-5), 126.9 (C, C-1), 114.4 (C, C-3), $66.0\left(\mathrm{CH}_{2}, \mathrm{C}-10\right), 41.4\left(\mathrm{CH}_{2}, \mathrm{C}-11\right)$, 38.4 (CH, 7-iPr CH), $24.7\left(\mathrm{CH}_{3}, 7-\mathrm{iPr} \mathrm{CH}_{3}\right), 12.7\left(\mathrm{CH}_{3}, 1-\mathrm{Me}\right)$. HRMS: $m / z \mathrm{C}_{17} \mathrm{H}_{19} \mathrm{O}_{2^{+}}[\mathrm{M}+\mathrm{H}]^{+}$, Calculated 255.1385, found 255.1386.

152: ${ }^{1} \mathrm{H}$ NMR (500 MHz, CDCl 3 ) $\delta 8.26$ (s, $\left.1 \mathrm{H}, \mathrm{H}-8\right), 8.04$ (s, 1H, H-2), 7.66 (d, J=11.5 Hz, 1H, H-6), 7.38 (d, J=12.0 Hz, 1H, H-5), 4.98 (td, J=11.0, 4.5 Hz, 1H, H-10), 4.35 (quin, J=7.0 Hz, 1H, H-17), 4.08 (dd, J=11.0, 6.5 Hz, 2H, H-18a), 3.95 (m, J=7.0 Hz, 2H, H-18b), 3.13 (sept, J=7.0 Hz, 1H, 7-iPr CH), 2.60 (s, 3H, 1-Me), 2.17 (m, J=11.0 Hz, 1H, H-15a), 2.08 (septd, J=7.0, 2.0 Hz, 1H, H-16), 1.76 (m, 2H, H-12a, H-13a), 1.58 (t, J=9.0 Hz, 2H, H-14, H-11), 1.38 (d, J=7.0 Hz, 1H, 7-iPr CH3), 1.22 (q, J=11.0 Hz, 1H, H-15b), 1.15 (m, 1H, H-12b), 0.97 (obscured m, 1H, H-13b), 0.97 (d, J=6.0 Hz, 3H, 16-Me), 0.96 (d, J=6.5 Hz, 3H, 14-Me), 0.85 (d, J=7.0 Hz, 3H, 16-Me). ${ }^{13} \mathrm{C}$ NMR (125 MHz, CDCl $) \delta 167.7$ (C, C-9), 149.7 (C, C-4), 144.8 (C, C-7), 141.62 (C, C-3a), 141.59 (CH, C-2), 139.4 (C, C-8a), 136.9 (CH, C6), 134.6 (CH, C-8), 124.9 (CH, C-5), 124.3 (C, C-1), 115.6 (C, C-3), 74.4 (CH, C-10), 65.9 ( $\left.\mathrm{CH}_{2}, \mathrm{C}-18\right)$, 51.2 (CH, C-17), 47.5 (CH, C-11), 41.2 ( $\left.\mathrm{CH}_{2}, \mathrm{C}-15\right), 37.9$ (CH, 7-iPr), $34.4\left(\mathrm{CH}_{2}, \mathrm{C}-13\right), 31.5$ (CH, C14), 26.4 (CH, C-16), $24.5\left(\mathrm{CH}_{3}, 7-\mathrm{P} P \mathrm{CH}_{3}\right), 23.4(\mathrm{CH}, \mathrm{C}-12), 22.1\left(\mathrm{CH}_{3}, 16-\mathrm{Me}\right), 21.0\left(\mathrm{CH}_{3}, 14-\mathrm{Me}\right)$, 
$16.3\left(\mathrm{CH}_{3}, 16-\mathrm{Me}\right), 13.0\left(\mathrm{CH}_{3}, 1-\mathrm{Me}\right)$. HRMS: $\mathrm{m} / \mathrm{z} \mathrm{C}_{28} \mathrm{H}_{41} \mathrm{O}_{4^{+}}[\mathrm{M}+\mathrm{H}]^{+}$, Calculated 441.2999, found 441.2996. IR (ATR): $\nu_{\max } 3340,2954,2921,2863,1664,1557,1409,1201,1032 \mathrm{~cm}^{-1} . \lambda_{\max }: 567 \mathrm{~nm}$.

153: ${ }^{1} \mathrm{H}$ NMR (500 MHz, CDCl 3 ) $\delta 8.33$ (s, 2H, H-8, H-2), 7.65 (d, J=11.0 Hz, 1H, H-6), 7.33 (d, J=10.5 Hz, 1H, H-5), 4.77 (apparent s, 2H, H-12), 4.22 (td, J=9.5, 4.5 Hz, 1H, H-10a), 3.99 (dt, J=11.0, 5.5 Hz, 1H, H-10b), 3.63 (m, 1H, H-11), 3.17 (sept, J=7.0 Hz, 1H, 7-iPr CH), 2.62 (s, 3H, 1-Me), 1.40 (d, J=6.5 $\mathrm{Hz}, 6 \mathrm{H}, 7-\mathrm{i} \mathrm{Pr} \mathrm{CH}_{3}$ ). ${ }^{13} \mathrm{C}$ NMR (150 MHz, CDCl 3 ) $\delta 168.2$ (C, C-9), 148.0 (C, C-4), 146.6 (C, C-7), 144.6 (CH, C-8), 142.0 (C, C-3a), 136.3 (CH, C-6), 135.6 (CH, C-2), 135.2 (C, C-8a), 130.1 (CH, C-5), 127.4 (C, C-1), 113.3 (C, C-3), $66.2\left(\mathrm{CH}_{2}, \mathrm{C}-12\right), 62.8\left(\mathrm{CH}_{2}, \mathrm{C}-10\right), 52.8$ (CH, C-11), 38.4 (CH, 7-i.Pr CH), 24.6 $\left(\mathrm{CH}_{3}, 7-\mathrm{i} P r \mathrm{CH}_{3}\right), 12.9\left(\mathrm{CH}_{3}, 1-\mathrm{Me}\right)$. HRMS: $\mathrm{m} / \mathrm{z} \mathrm{C}_{18} \mathrm{H}_{21} \mathrm{O}_{3}{ }^{+}[\mathrm{M}+\mathrm{H}]+$ Calculated 285.1485, found 285.1488.

\section{(1R,2S,5R)-Menthyl 4,6,8-trimethylazulene-1-carboxylate (157) and (1R,2S,5R)-Menthyl4,6,8-trimethylazulene-1,3-dicarboxylate (158)}

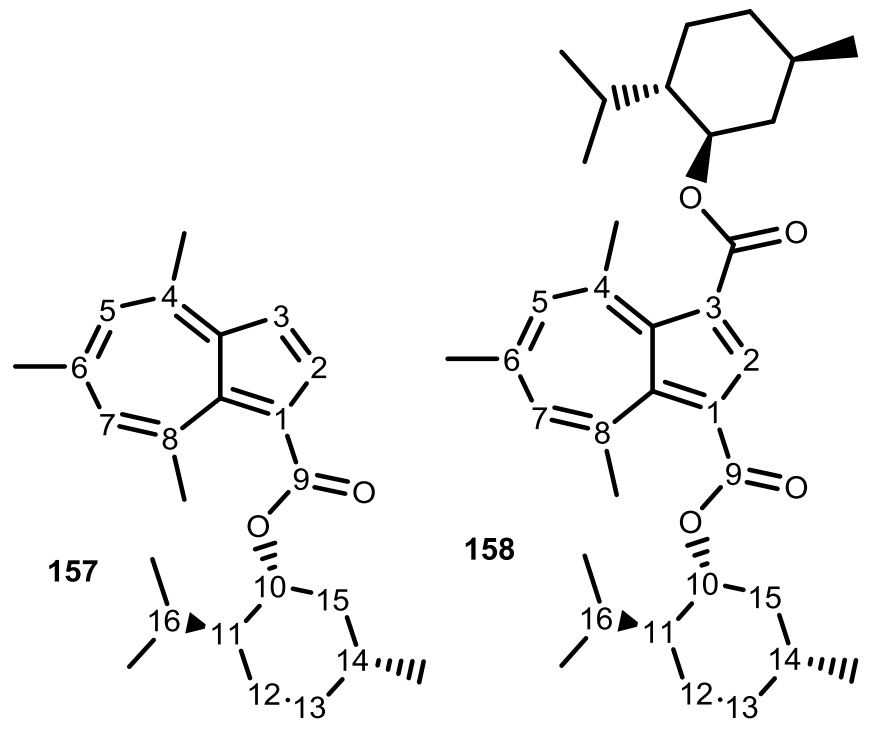

4,6,8-Trimethylazulene ( $85.8 \mathrm{mg}, 0.50 \mathrm{mmol}, 1$ eq.) was added to a RBF fitted with condenser and dissolved in MeCN ( $9 \mathrm{~mL}$ ). Oxalyl chloride ( $0.1 \mathrm{~mL}, 1.2 \mathrm{mmol}, 2.3 \mathrm{eq}$.) was added to the reaction mixture, which changed colour from purple to orange. The reaction was heated at reflux, and after about 7 minutes, the reaction mixture appeared an intense red-pink. A solution of $(-)$-menthol (223.4 mg in $2 \mathrm{~mL} \mathrm{MeCN}, 1.42 \mathrm{mmol}, 2.8$ eq.) was added at this point (a longer delay results in the reaction mixture turning brick red and forming oligomeric material) and the reaction changed colour to crimson. Pyridine $(0.2 \mathrm{~mL}, 2.5 \mathrm{mmol}, 5 \mathrm{eq}$.) was added and the reaction was allowed to cool to r.t.

A phase separation was performed in $\mathrm{CH}_{2} \mathrm{Cl}_{2} / \mathrm{H}_{2} \mathrm{O}$ and the organic fraction was concentrated by rotary evaporation. The resulting residue was purified by column chromatography (gradient 
elution from pet. ether to $\mathrm{CH}_{2} \mathrm{Cl}_{2}$ to $\mathrm{Et}_{2} \mathrm{O}$ ), to obtain 157 (114 mg, 62\% yield) as a magenta oil, and 158 (101.3 $\mathrm{mg}, 36 \%$ yield) as a pink-red crystalline film.

157: ${ }^{1} \mathrm{H}$ NMR (500 MHz, $\mathrm{CDCl}_{3}$ ) $\delta 7.91$ (d, J=4.5 Hz, 1H, H-2), 7.24 (s, 1H, H-7), 7.20 (s, 1H, H-5), 7.19 (obscured d, 1H- H-3), 4.95 (td, J=10.5, 4.5 Hz, 1H, H-10), 2.98 (s, 3H, 8-Me), 2.88 (s, 3H, 4Me), 2.65 (s, 3H, 6-Me), 2.22 (m, J=12.0 Hz, 1H, H-15a), 2.12 (septd, J=7.0, 2.5 Hz, 1H, H-16), 1.74 (m, 2H, H-12a, H-13a), 1.57 (m, 2H, H-14, H-11), 1.16 (m, 2H, H-12b, H-15b), 0.96 (d, J=7.0 Hz, 3H, 16-Me), 0.93 (d, J=7.5 Hz, 3H, 14-Me), 0.85 (d, J=7.0 Hz, 3H, 16-Me). ${ }^{13} \mathrm{C}$ NMR (125 MHz, CDCl $) \delta$ 167.51 (C, C-9), 148.3 (C, C-8), 147.3 (C, C-6), 146.7 (C, C-4), 139.7 (C, C-3a), 136.5 (CH, C-2), 134.2 (C, C-8a), 131.4 (CH, C-7), 129.5 (CH, C-5), 121.6 (C, C-1), 114.4 (CH, C-3), 74.1 (CH, C-10), 47.3 (CH, C-11), 41.0 ( $\left.\mathrm{CH}_{2}, \mathrm{C}-15\right), 34.4$ ( $\left.\mathrm{CH}_{2}, \mathrm{C}-13\right), 31.5$ ( $\left.\mathrm{CH}, \mathrm{C}-14\right), 28.5$ ( $\left.\mathrm{CH}_{3}, 8-\mathrm{Me}, 6-\mathrm{Me}\right), 26.3$ (CH, C-16), $25.9\left(\mathrm{CH}_{3}, 4-\mathrm{Me}\right), 23.4\left(\mathrm{CH}_{2}, \mathrm{C}-12\right), 22.1\left(\mathrm{CH}_{3}, 16-\mathrm{Me}\right), 20.9\left(\mathrm{CH}_{3}, 14-\mathrm{Me}\right), 16.2\left(\mathrm{CH}_{3}, 16-\mathrm{Me}\right)$. HRMS: $m / z \mathrm{C}_{24} \mathrm{H}_{33} \mathrm{O}_{2}+[\mathrm{M}+\mathrm{H}]+$ Calculated 353.2475, found 353.2476. IR (ATR): $v_{\max } 2949,2923,2863$, $1687,1580,1389,1370,1216,1143,1102,987 \mathrm{~cm}^{-1}, \lambda_{\max }: 517 \mathrm{~nm}$.

158: ${ }^{1} \mathrm{H}$ NMR (500 MHz, CDCl 3 ) $\delta 8.14(\mathrm{~s}, 1 \mathrm{H}, \mathrm{H}-2), 7.32$ (s, 2H, H-5,7), $4.94(\mathrm{td}, J=11.0,4.5 \mathrm{~Hz}, 2 \mathrm{H}$, H-10), 2.94 (s, 6H, 4-Me, 8-Me), 2.63 (s, 3H, 6-Me), 2.22 (m, J=11.5 Hz, 2H, H-15a), 2.11 (septd, $J=7.0,2.5 \mathrm{~Hz}, 2 \mathrm{H}, \mathrm{H}-16), 1.74$ (m, 4H, H-12a, H-13a), 1.58 (m, 4H, H-14, H-11), 1.17 (m, 4H, H-12a, H-15a), 0.97 (d, J=7.0 Hz, 6H, 16-Me), 0.95 (d, J=7.5 Hz, 6H, 14-Me), 0.85 (d, J=7.0 Hz, 6H, 16-Me). ${ }^{13} \mathrm{C}$ NMR (125 MHz, CDCl 3 ) $\delta 166.7$ (C, C-9), 148.8 (C, C-4,8), 148.3 (C, C-6), 139.8 (CH, C-2), 137.2 (CH, C-5,7), 133.1 (C, C-3a,8a), 119.8 (C, C-1,3), 74.5 (CH, C-10), 47.3 (CH, C-11), $40.1\left(\mathrm{CH}_{2}, \mathrm{C}-15\right)$, $34.4\left(\mathrm{CH}_{2}, \mathrm{C}-13\right), 31.4$ ( $\left.\mathrm{CH}, \mathrm{C}-14\right), 28.8\left(\mathrm{CH}_{3}, \mathrm{C}-4\right), 28.0\left(\mathrm{CH}_{3}, \mathrm{C}-6\right), 26.4(\mathrm{CH}, \mathrm{C}-16), 23.3\left(\mathrm{CH}_{2}, \mathrm{C}-12\right)$, $22.2\left(\mathrm{CH}_{3}, 16-\mathrm{Me}\right), 21.0\left(\mathrm{CH}_{3}, 14-\mathrm{Me}\right), 16.2\left(\mathrm{CH}_{3}, 16-\mathrm{Me}\right)$. HRMS: $m / z \mathrm{C}_{35} \mathrm{H}_{51} \mathrm{O}_{4}{ }^{+}[\mathrm{M}+\mathrm{H}]+$ Calculated 535.3782, found 535.3778. IR (ATR): $v_{\max } 2953,2920,2867,1698,1584,1440,1400,137,114$, 1106, 1011, $989 \mathrm{~cm}^{-1} . \lambda_{\max }: 504 \mathrm{~nm}$.

\section{(1R,2S,5R)-Menthyl3-nitro-4,6,8-trimethylazulene-1-carboxylate (160)}

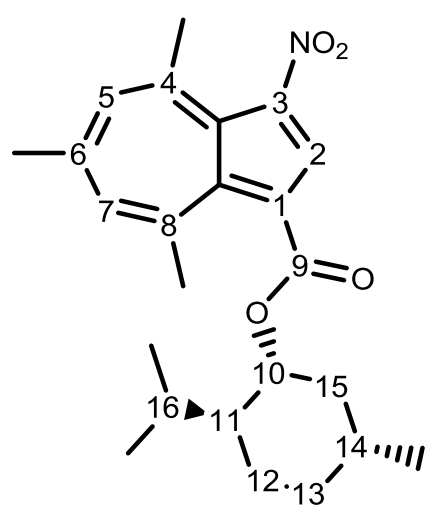


157 (34.7 mg, $0.098 \mathrm{mmol}, 1$ eq.) and acetic anhydride ( $6 \mathrm{~mL}, 63.5 \mathrm{mmol}$ ) were added to a RBF maintained at $10{ }^{\circ} \mathrm{C}$. Copper nitrate trihydrate $(259.5 \mathrm{mg}, 1.07 \mathrm{mmol}, 10.9 \mathrm{eq}$.) was added and the reaction was left for 30 minutes, during which a blue-green colouration developed.

A separation was performed using $\mathrm{CH}_{2} \mathrm{Cl}_{2}$ /sat. aq. $\mathrm{NaHCO}_{3}$, and the organic layer was purified by column chromatography in $1: 1$ pet. ether $/ \mathrm{CH}_{2} \mathrm{Cl}_{2}$ to give the title compound ( $20.5 \mathrm{mg}, 52 \%$ yield) as a peach-coloured solid.

$\left.{ }_{1}^{1} \mathrm{H} \mathrm{NMR} \mathrm{(500} \mathrm{MHz,} \mathrm{CDCl}\right) \delta 8.31$ (s 1H, H-2), 7.51 (s, 1H, H-5 or H-7), 7.50 (s, 1H, H-5 or H-7), 4.95 (td, J=11.0, 4.0 Hz, 1H, H-10), 2.98 (s, 3H, 4-Me or 6-Me or 8-Me), 2.85 (s, 3H, 4-Me or 6-Me or 8Me), 2.72 (s, 3H, 4-Me or 6-Me or 8-Me), 2.16 (m, H-15a), 2.05 (obscured septd, J=7.0, 3.0 Hz, 1H, H-16), 1.78-1.72 (m, 2H, H-12a, H-13a) 1.61-1.53 (obscured m, 2H, H-14, H-11), 1.18-1.11 (m, 2H, H-12b, H-15b), 0.96 (d, J=6.5 Hz, 3H, 16-Me), 0.95 (d, J=7.0 Hz, 3H, 14-Me), 0.93 (obscured m, 1H, H-13b), 0.84 (d, J=7.0 Hz, 3H, 16-Me). ${ }^{13} \mathrm{C}$ NMR (125 MHz, CDCl 3 ) $\delta 165.8$ (C, C-9), 151.3 (C, C-4 or C-6 or C-8), 150.5 (C, C-4 or C-6 or C-8), 149.4 (C, C-4 or C-6 or C-8), 135.8 (CH, C-5 or C-7), 135.7 (C, C-3a or C-8a), 135.3 (CH, C-5 or C-7), 135.1 (CH, C-2), 131.2 (C, C-3a or C-8a), 118.5 (C, C-1), 75.1 (CH, C-10), 47.2 (CH, C-11), $40.9\left(\mathrm{CH}_{2}, \mathrm{C}-15\right), 34.3\left(\mathrm{CH}_{2}, \mathrm{C}-13\right), 31.5(\mathrm{CH}, \mathrm{C}-14), 28.9\left(\mathrm{CH}_{3}, 4-\right.$ Me or 6-Me or 8-Me), $28.2\left(\mathrm{CH}_{3}, 4-\mathrm{Me}\right.$ or 6-Me or 8-Me), $28.0\left(\mathrm{CH}_{3}, 4-\mathrm{Me}\right.$ or 6-Me or 8-Me), 26.4 (CH, C-16), $23.3\left(\mathrm{CH}_{2}, \mathrm{C}-12\right), 22.1\left(\mathrm{CH}_{3}, 16-\mathrm{Me}\right), 20.9\left(\mathrm{CH}_{3}, 14-\mathrm{Me}\right), 16.1\left(\mathrm{CH}_{3}, 16-\mathrm{Me}\right) . \mathrm{C}-3$ was not observed. HRMS: $m / z \mathrm{C}_{24} \mathrm{H}_{32} \mathrm{NO}_{4}{ }^{+}[\mathrm{M}+\mathrm{H}]+{ }^{+}$Calculated 398.2326, found 398.2338. $\lambda_{\max }$ : Shoulder 473 nm.

\section{Azulene diiron pentacarbonyl (143)}

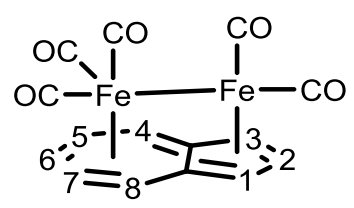

Azulene (47.8 mg, $0.37 \mathrm{mmol}, 1 \mathrm{eq}$ ) and diiron nonacarbonyl (168 mg, $0.46 \mathrm{mmol}, 1.2 \mathrm{eq}$.) were added to a RBF fitted with a condenser. Hexane $(8 \mathrm{~mL})$ was added and the reaction was heated at reflux for three hours.

A separation was performed with $\mathrm{CH}_{2} \mathrm{Cl}_{2} / \mathrm{H}_{2} \mathrm{O}$, and the organic layer was purified by column chromatography in pet. ether followed by $1: 1$ pet. ether $/ \mathrm{CH}_{2} \mathrm{Cl}_{2}$ to give azulene $(32.8 \mathrm{mg}, 69 \%)$ and 143 (15.2 $\mathrm{mg}, 10 \%$ yield) as an orange red substance.

${ }^{1} \mathrm{H} \mathrm{NMR}\left(500 \mathrm{MHz}, \mathrm{CDCl}_{3}\right) \delta 5.47(\mathrm{t}, J=9.0 \mathrm{~Hz}, 1 \mathrm{H}, \mathrm{H}-5), 5.28(\mathrm{~m}, 1 \mathrm{H}, \mathrm{H}-4), 5.25(\mathrm{~m}, 1 \mathrm{H}, \mathrm{H}-7), 5.10(\mathrm{~s}$, 1H, H-1), 5.05 (s, 1H, H-3), 4.78 (d, J=5.0 Hz, 1H, H-8), 3.69 (s, 1H, H-2), 3.48 (t, J=7.5 Hz, 1H, H-6). 
${ }^{13} \mathrm{C}$ NMR (125 MHz, CDCl $) \delta 216.3$ (C, C三0), 130.5 (CH, C-5), 118.5 (CH, C-4), 85.7 (CH, C-1), 84.5 (C, C-3a or C-8a), 81.5 (CH, C-3), 80.4 (CH, C-7), 78.1 (C, C-8a or C-3a) 77.3 (CH, C-2), 58.0 (CH, C-6), $57.8(\mathrm{CH}, \mathrm{C}-8) .{ }^{1} \mathrm{H}$ NMR data corresponds with those reported previously. ${ }^{10}$

\section{4,6,8-Trimethylazulene diiron pentacarbonyl (144)}

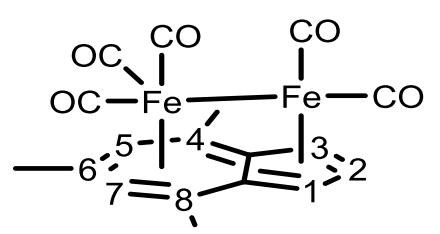

4,6,8-Trimethylazulene (17.4 mg, $0.1 \mathrm{mmol}, 1$ eq.) and diiron nonacarbonyl (161.9 mg, 0.445 mmol, 4.5 eq.) were added to a RBF fitted with condenser, followed by $5 \mathrm{~mL}$ toluene. The reaction mixture was heated at reflux for 3 hours, after which no trace of starting material could be seen.

Column chromatography was performed on the crude material with a mixture of pet. ether and $\mathrm{CH}_{2} \mathrm{Cl}_{2}$, to afford 144 (46.2 mg, 99\% yield) as an orange solid. $\left.{ }^{1} \mathrm{H} \mathrm{NMR} \mathrm{(500} \mathrm{MHz,} \mathrm{CDCl}_{3}\right) \delta 5.18$ (s, 1H, H-1), 5.07 (s, 1H, H-3), 3.87 (s, 1H, H-2), 2.88 (s, 1H, H-5 or 7), 2.62 (s, 1H, H-7 or 5), 2.10 (s, 3H, 6-Me), 1.76 (s, 3H, 4-Me or 8-Me), 1.62 (s, 3H, 8-Me or 4-Me). ${ }^{1} \mathrm{H}$ NMR data corresponds with those reported previously. 10

\section{$(1 R, 2 S, 5 R)-M e n t h y l$ azulene-1-carboxylate diiron pentacarbonyl (145)}
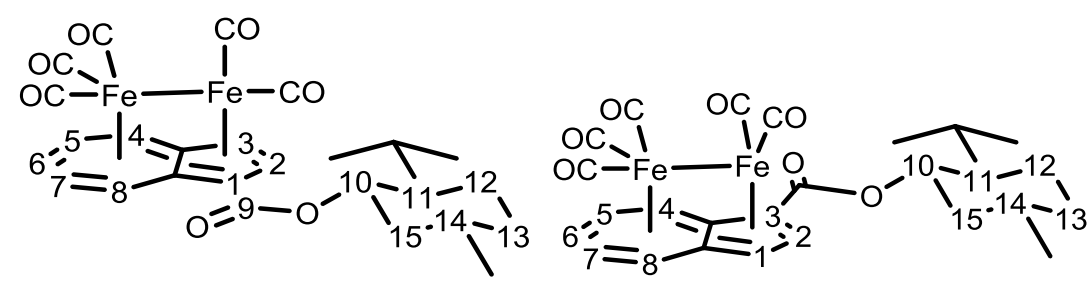

123 (47.3 mg, $0.15 \mathrm{mmol}, 1$ eq.) and diiron nonacarbonyl (120.9 mg, $0.33 \mathrm{mmol}, 2.2$ eq.) were added to a RBF fitted with condenser. Toluene $(6 \mathrm{~mL})$ was added and reaction was heated at reflux for 6 hours. After this period, the reaction was allowed to cool, and column chromatography was performed on the crude mixture using $1: 1$ pet. ether $/ \mathrm{CH}_{2} \mathrm{Cl}_{2}$ to obtain 145 (17.5 mg, 19\% yield) as orange-red solid diastereomers.

${ }^{1} \mathrm{H} \mathrm{NMR} \mathrm{(500} \mathrm{MHz,} \mathrm{CDCl}_{3}$ ) $\delta 5.88$ and 5.83 (d, J= $6.0 \mathrm{~Hz}, \mathrm{H}-4$ or H-8), 5.62 and 5.60 (s, H-5 or H-7), 5.55 (t, J=9.0 Hz, H-2 or H-3), 5.40 (broad t, H-5 or H-7), 5.23 and 5.22 (s, H-2 or H-3), 5.16 (s, H-4 or H-8), 4.65 (d, J=9.0 Hz, H-10), 3.46 (broad t, H-6), 2.27 (m), 1.97 (m), 1.84 (m), 1.66 (m), 1.25 (m), $0.88(\mathrm{~m}) .{ }^{13} \mathrm{C}$ NMR (125 MHz, $\mathrm{CDCl}_{3}$ ) $\delta 214.7$ (C, C $\left.\equiv 0\right), 165.7$ and $165.3(\mathrm{C}, \mathrm{C}-9$ ), 132.02, 132.01, 118.43, 118.4, 86.7, 86.68, 85.5, 85.3, 83.4, 83.2, 81.14, 81.12, 75.3, 75.1, 60.3, 60.0, 57.3, 
57.2, 46.90, 46.88, 40.7, 40.2, 34.1, 31.92, 31.87, 31.4, 31.3, 29.7, 26.5, 25.4, 23.5, 22.8, 22.7, 22.1, $21.9,20.8,20.7,16.5,15.4,14.1$.

\section{Reaction kinetics of menthyl azulene-1-carboxylate (122) and menthyl 3-nitroazulene-1-carboxylate (136) deprotection}

122 ( $8.5 \mathrm{mg}, 0.027 \mathrm{mmol}, 1$ eq.) and 136 (10.8 mg, $0.0303 \mathrm{mmol}, 1.1$ eq.) were each dissolved in $\mathrm{MeOH}(0.5 \mathrm{~mL})$ and the solutions combined. A basic solution was prepared $(200.8 \mathrm{mg} \mathrm{KOH}, 10 \mathrm{~mL}$ $\mathrm{MeOH}, 1 \mathrm{~mL} \mathrm{D}_{2} \mathrm{O}$ and one drop of $\mathrm{SiMe}_{4}$ ) and $0.5 \mathrm{~mL}$ was added to this mixture, which was subjected periodically to ${ }^{1} \mathrm{H}$ NMR analysis. A baseline correction was made on the aromatic region of the NMR spectrum, allowing the integrals of the azulene peaks to be obtained despite the overwhelming oxymethyl and HOD peaks present elsewhere in the spectrum. ${ }^{1} \mathrm{H}$ NMR spectra were obtained approximately once per day over the course of a month.

\section{6-(2-Hydroxyethyl)azulene (90) and 6-(2-hydroxyethyl)-1- hydroxymethylazulene (166)}

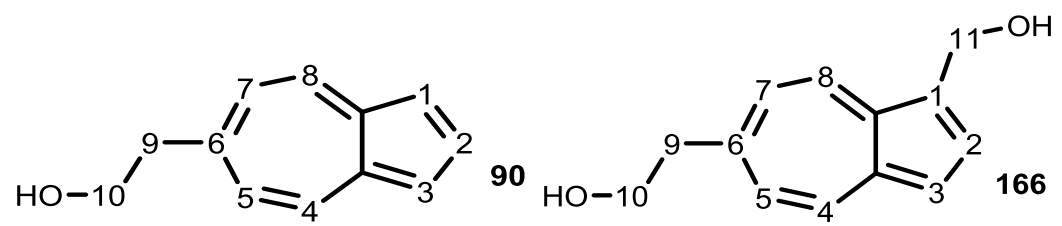

An LDA solution was prepared by mixing THF ( $6 \mathrm{~mL}$ ) and diisopropylamine, $(0.18 \mathrm{~mL}, 1.27 \mathrm{mmol}$, 1,15 eq.), followed by $n$-BuLi ( $0.71 \mathrm{~mL}$ of a $1.77 \mathrm{M}$ in cyclohexane solution, $1.25 \mathrm{mmol}, 1.13 \mathrm{eq}$.) at $-30{ }^{0} \mathrm{C}$ (dry ice/ethylene glycol bath), and to this reaction mixture, a chilled $\left(-30{ }^{\circ} \mathrm{C}\right)$ solution of 6-methylazulene (156.9 mg, $1.104 \mathrm{mmol}, 1$ eq.) in THF (3 mL) was added via cannula. The reaction mixture rapidly changed colour from indigo to golden brown. A vial containing paraformaldehyde (129.5 mg, $4.3 \mathrm{mmol}, 3.9 \mathrm{eq}$.) was heated to the point of sublimation and formaldehyde gas was transferred from this vessel into the reaction mixture via cannula, which caused the reaction mixture to change colour back to blue. Water ( $3 \mathrm{~mL}$ ) was added and the reaction was stirred for one minute before workup.

A separation was performed using $\mathrm{CH}_{2} \mathrm{Cl}_{2} / \mathrm{H}_{2} \mathrm{O}$ and the organic layer was washed with saturated aqueous brine and concentrated by rotary evaporation. Column chromatography was performed on the residue using silica gel prepared with $2 \% \mathrm{NEt}_{3}$ in pet. ether, obtaining returned starting material (22.2 mg, $14 \%$ ) eluting in 1:1 pet. ether $/ \mathrm{CH}_{2} \mathrm{Cl}_{2}, 90$ (138.6 mg, 73\% yield) as an indigo 
solid eluting in 2:3 EtOAc/ $\mathrm{CH}_{2} \mathrm{Cl}_{2}$, and 166 (26.3 mg, $12 \%$ yield) as a teal-blue solid eluting in $\mathrm{Et}_{2} \mathrm{O} / \mathrm{MeOH}$.

90: ${ }^{1} \mathrm{H}$ NMR (500 MHz, CDCl $) \delta 8.29$ (d, J=9.5 Hz, 2H, H-4,8), 7.87 (t, J=3.5 Hz, 1H, H-2), 7.39 (d, $J=3.5 \mathrm{~Hz}, 2 \mathrm{H}, \mathrm{H}-1,3$ ) , 7.11 (d, J=9.5 Hz, 2H, H-5,7), 3.96 (t, J=7.0 Hz, 2H, H-10), 3.05 (t, J=6.5 Hz, 2H, H-9). ${ }^{13} \mathrm{C}$ NMR (125 MHz, CDCl 3 ) $\delta 148.9$ (C, C-6), 140.0 (C, C-3a,8a), 136.3 (CH, C-2), 135.9 (CH, C4,8), 124.3 (CH, C-5,7), 118.2 (CH, C-1,3), $64.2\left(\mathrm{CH}_{2}, \mathrm{C}-10\right), 45.3\left(\mathrm{CH}_{2}, \mathrm{C}-9\right)$. HRMS: $m / z \mathrm{C}_{12} \mathrm{H}_{13} \mathrm{O}^{+}$ $[\mathrm{M}+\mathrm{H}]+$ Calculated 173.0961, found 173.0964. IR (ATR): $v_{\max } 3218,2944,2895,1577,1392,1039$, 822, 751, $744 \mathrm{~cm}^{-1}$. m.p. $82.3-83.8^{\circ} \mathrm{C}$.

166: ${ }^{1} \mathrm{H}$ NMR (500 MHz, CDCl 3 ) $\delta 8.43$ (d, J=9.5 Hz, 1H, H-8), 8.26 (d, J=10.0 Hz, 1H, H-4), 7.87 (d, $J=3.5 \mathrm{~Hz}, 1 \mathrm{H}, \mathrm{H}-2$ ), 7.31 (d, J=3.5 Hz, 1H, H-3), 7.17 (d, J=9.5 Hz, 1H, H-7), 7.13 (d, J=10.0 Hz, 1H, H5), 5.15 (s, 2H, H-11), 3.98 (t, J=6.5 Hz, 2H, H-10), 3.09 (t, J=6.5 Hz, 2H, H-9). ${ }^{13 C}$ NMR (125 MHz, $\mathrm{CDCl}_{3}$ ) $\delta 149.7$ (C, C-6), 140.2 (C, C-3a or C-8a), 136.6 (CH, C-2), 136.5 (CH, C-4), 135.5 (C, C-3a or C-8a), 133.2 (CH, C-8), 1.29.0 (C, C-1), 124.8 (CH, C-5), 124.4 (CH, C-7), 117.1 (CH, C-3), $64.2\left(\mathrm{CH}_{2}\right.$, C-10), $58.5\left(\mathrm{CH}_{2}, \mathrm{C}-11\right), 45.2\left(\mathrm{CH}_{2}, \mathrm{C}-9\right)$. HRMS: $m / z \mathrm{C}_{13} \mathrm{H}_{15} \mathrm{O}_{2}+[\mathrm{M}+\mathrm{H}]+$ Calculated 203.1067, found 203.1059. IR (ATR): $\nu_{\max } 3297,3017,2926,2868,1704,1577,1441,1396,1034,977,833,750 \mathrm{~cm}^{-}$ 1.

\section{1,2-Bis(azulen-6-yl)ethane (165)}

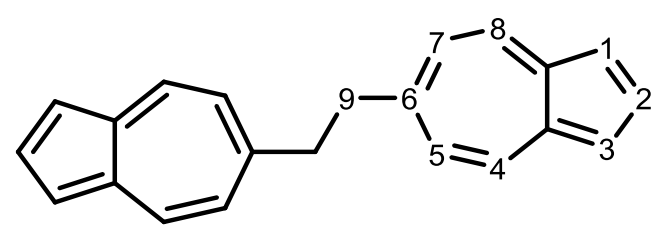

This teal solid was produced in small quantities (1-3\% yield) from LDA deprotonation of 6methylazulene.

${ }^{1} \mathrm{H}$ NMR (500 MHz, CDCl $) \delta 8.25$ (d, J=9.0 Hz, 4H, H-4,8), 7.85 (t, J=3.5 Hz, 2H, H-2), 7.36 (d , J=3.5 Hz, 4H, H-1,3), 7.07 (d, J=10.0 Hz, 4H, H-5,7), 3.22 (s, 4H, H-9). $\lambda_{\text {max: }} 564$ nm. Spectra matched those reported previously. ${ }^{11}$

\section{2,4-Bis(azulen-6-yl) butan-1-ol (168)}

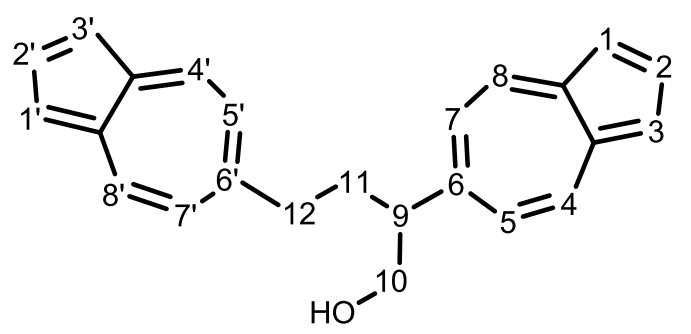


This compound is an indigo oil that was formed in small quantities (approx. 1-6\% yield) in reactions that produce 6-(2-hydroxyethyl)azulene (90). ${ }^{1} \mathrm{H} \mathrm{NMR}\left(500 \mathrm{MHz}, \mathrm{CDCl}_{3}\right) \delta 8.36(\mathrm{~d}$, $J=10.5 \mathrm{~Hz}, 2 \mathrm{H}, \mathrm{H}-4,8), 8.23$ (d, J=10.5 Hz, 2H, H-4', $8^{\prime}$ ), 7.92 (t, J=3.5 Hz, 1H, H-2), 7.84 (t, J=4.0 Hz, 1H, H-2') 7.43 (d, J=4.0 Hz, 2H, H-1,3), 7.35 (d, J=3.5 Hz, 2H, H-1', 3'), 7.12 (d, J=10.0 Hz, 2H, H-5,7), 6.98 (d, J=10.5 Hz, 2H, H-5', $7^{\prime}$ ), 3.86 (s, 2H, H-10), 3.02 (m, 1H, H-9), 2.73 (m, 2H, H-12), 2.24 (m, 1H, H-11a), 2.13 (m, 1H, H-11b). ${ }^{13}$ C NMR (125 MHz, CDCl 3 ) $\delta 152.5$ (C, C-6'), 151.9 (C, C-6), 139.2 (C, C-3a,8a), 138.8 (C, C-3a',8a'), 136.8 (CH, C-2), 136.1 (CH, C-4,8), 136.0 (CH, C-4', 8'), 135.9 (CH, C-2') $123.8\left(\mathrm{CH}, \mathrm{C}-5^{\prime}, 7^{\prime}\right), 123.1(\mathrm{CH}, \mathrm{C}-5,7), 118.4(\mathrm{CH}, \mathrm{C}-1,3), 118.0\left(\mathrm{CH}, \mathrm{C}-1^{\prime}, 3^{\prime}\right), 67.7\left(\mathrm{CH}_{2}, \mathrm{C}-10\right)$, $53.2(\mathrm{CH}, \mathrm{C}-9), 39.8\left(\mathrm{CH}_{2}, \mathrm{C}-12\right), 35.2\left(\mathrm{CH}_{2}, \mathrm{C}-11\right)$. HRMS: $m / z \mathrm{C}_{24} \mathrm{H}_{23} \mathrm{O}^{+}[\mathrm{M}+\mathrm{H}]+$ Calculated 327.1743 , found 327.1745 .

\section{2-(Azulen-6-yl)ethyl (E)-cinnamate (Azul-cinnamate) (178)}

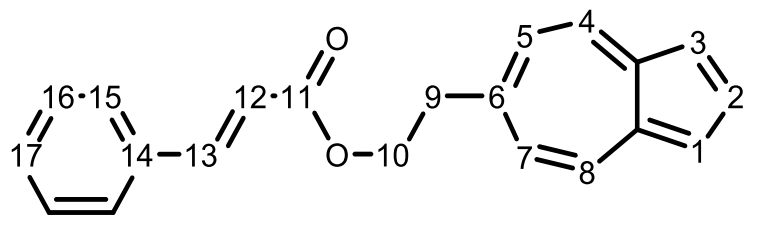

Cinnamic acid (160.7 mg, 1.08 mmol, 1 eq.), DMAP (22.5 mg, 0.18 mmol, 0.16 eq.), DCC (255 mg, $1.23 \mathrm{mmol}, 1.14$ eq.) and 6-(2-hydroxyethyl)azulene (207.1 mg, $1.20 \mathrm{mmol}, 1.1 \mathrm{eq}$.) were added to a $\mathrm{RBF}$ and dissolved in $\mathrm{CH}_{2} \mathrm{Cl}_{2}(10 \mathrm{~mL})$.

After 3 hours, a phase separation was performed with $\mathrm{H}_{2} \mathrm{O} / \mathrm{CH}_{2} \mathrm{Cl}_{2}$ and the organic fraction was concentrated by rotary evaporation. Column chromatography using $\mathrm{CH}_{2} \mathrm{Cl}_{2}$ wasperformed on the resulting product, eluting 178 (304.6 mg, 93\% yield) as an indigo powder. A small amount of 6-(2-hydroxyethyl)azulene starting material was obtained, but was heavily contaminated with dicyclohexylurea. ${ }^{1} \mathrm{H}$ NMR $\left(500 \mathrm{MHz}, \mathrm{CDCl}_{3}\right) \delta 8.30(\mathrm{~d}, J=10.5 \mathrm{~Hz}, 2 \mathrm{H}, \mathrm{H}-4,8), 7.87(\mathrm{t}, J=4.0 \mathrm{~Hz}, 1 \mathrm{H}$, H-2), 7.68 (d, J=16.0 Hz, 1H, H-13), 7.52 (m, 2H, H-15), 7.40-7.38 (complex m, 5H, H-1, H-3, H-16, H-17), 7.15 (d, J=10.5 Hz, 2H, H-5,7), 6.42 (d, J=16.0 Hz, 1H, H-12), 4.54 (t, J=7.0 Hz, 2H, H-10), 3.21 $(\mathrm{t}, J=7.0 \mathrm{~Hz}, 2 \mathrm{H}, \mathrm{H}-9) .{ }^{13} \mathrm{C} \mathrm{NMR}\left(125 \mathrm{MHz}, \mathrm{CDCl}_{3}\right) \delta 166.8$ (C, C-11), 148.0 (C, C-6), 145.1 (CH, C-13), 139.1 (C, C-3a,8a), 136.4 (CH, C-2), 135.9 (CH, C-4,8), 134.3 (C, C-14), 130.4 (CH, C-17), 128.9 (CH, C-16), 128.1 (CH, C-15), 124.2 (CH, C-5,7), 118.3 (CH, C-1,3), 117.8 (CH, C-12), 65.3 ( $\left.\mathrm{CH}_{2}, \mathrm{C}-10\right)$, $41.2\left(\mathrm{CH}_{2}, \mathrm{C}-9\right)$. HRMS: $m / z \mathrm{C}_{21} \mathrm{H}_{19} \mathrm{O}_{2}{ }^{+}[\mathrm{M}+\mathrm{H}]+$ Calculated 303.1380, found 303.1388. IR (ATR): $v_{\max }$ $3079,2944,1710,1633,1562,1307,1168,979,837,761,740$ cm$^{-1}$. m.p. $144.6-146.0^{\circ}$ C. $\lambda_{\text {max }} 567$ $\mathrm{nm}$. 


\section{Solid phase method:}

Cinnamic acid (13.2 mg, $0.09 \mathrm{mmol}, 1$ eq.), 6-(2-hydroxyethyl)azulene (19.6 mg, $0.11 \mathrm{mmol}, 1.3$ eq.), DCC (27.7 mg, $0.13 \mathrm{mmol}, 1.5$ eq.) and DMAP (5.7 mg, $0.04 \mathrm{mmol}, 0.5 \mathrm{eq}$ ) were added together in a mortar. The solid mixture was ground for approximately 9 minutes over three 3 minute sessions. Approximately one minute after grinding commenced, the mixture turns into a thick paste. This paste returned to a solid after a few further minutes of grinding.

This mixture was dissolved in $\mathrm{CH}_{2} \mathrm{Cl}_{2}$, and purified by column directly, with the product 178 eluting in $\mathrm{CH}_{2} \mathrm{Cl}_{2}$ in $78 \%$ yield

\section{2-(Azulen-6-yl)ethyl benzoate (Azul-benzoate) (182)}

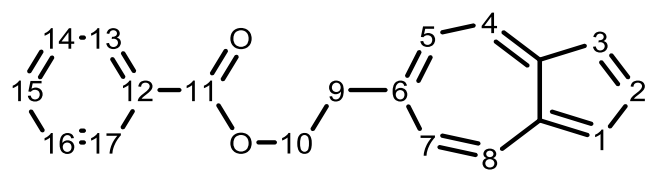

Benzoic acid (39.6 mg, $0.32 \mathrm{mmol}, 1.01$ eq.), 90 (55.2 mg, $0.32 \mathrm{mmol}, 1$ eq.), DCC (76.7 mg, 0.37 mmol, 1.16 eq.) and DMAP (10.1 mg, $0.082 \mathrm{mmol}, 0.26$ eq.) were added together and dissolved in $\mathrm{CH}_{2} \mathrm{Cl}_{2}(6 \mathrm{~mL})$.

After overnight reaction, a separation was performed with $\mathrm{CH}_{2} \mathrm{Cl}_{2} / \mathrm{H}_{2} \mathrm{O}$ and the organic fraction was concentrated by rotary evaporation. Column chromatography was performed on the crude material using $\mathrm{CH}_{2} \mathrm{Cl}_{2}$ to obtain 182 (66.8 mg, 75\% yield) as an indigo semi-crystalline solid. ${ }^{1} \mathrm{H}$ NMR (500 MHz, CDCl $)_{3} \delta 8.30$ (d, J=10.0 Hz, 2H, H-4,8), 8.01 (d, J=7.0 Hz, 2H, H-13), 7.87 (t, J=3.5 $\mathrm{Hz}, 1 \mathrm{H}, \mathrm{H}-2$ ) , 7.56 (t, J=7.0 Hz, 1H, H-15), 7.43 (t, J=7.5 Hz, 2H, H-14), 7.38 (d, J=4.0 Hz, 2H, H-1,3), 7.18 (d, J=10.0 Hz, 2H, H-5,7), 4.65 (t, J=7.0 Hz, 2H, H-10), 3.27 (t, J=7.0 Hz, 2H, H-9). ${ }^{13} \mathrm{C}$ NMR (125 $\mathrm{MHz}, \mathrm{CDCl}_{3}$ ) $\delta 166.5$ (C, C-11), 148.0 (C, C-6), 139.1 (C, C-3a,8a), 136.4 (CH, C-2), 135.8 (CH, C-4,8), 133.1 (CH, C-15), 130.1 (C, C-12), 129.5 (CH, C-13), 128.4 (CH, C-14), 124.2 (CH, C-5,7), 118.3 (CH, $\mathrm{C}-1,3), 65.7\left(\mathrm{CH}_{2}, \mathrm{C}-10\right), 41.3\left(\mathrm{CH}_{2}, \mathrm{C}-9\right)$. HRMS: $m / z \mathrm{C}_{19} \mathrm{H}_{17} \mathrm{O}_{2}+[\mathrm{M}+\mathrm{H}]+$ Calculated 277.1223, found 277.1224. IR (ATR): $v_{\max } 3067,2952,1711,1580,1261,1105,835,743,701 \mathrm{~cm}^{-1}$, m.p. 119.6$120.0{ }^{\circ}$ C. $\lambda_{\text {max }}: 566 \mathrm{~nm}$.

\section{2-(Azulen-6-yl)ethyl 2-furoate (Azul-2-furoate) (185)}

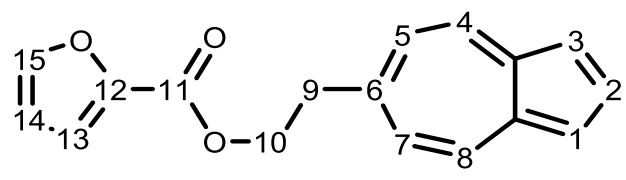


2-Furoic acid (66.9 mg, $0.59 \mathrm{mmol}, 1$ eq.), 90 (106 mg, $0.61 \mathrm{mmol}, 1.03$ eq.), EDCI (147.7 mg, $0.0 .95 \mathrm{mmol}, 1.6 \mathrm{eq}$.) and DMAP (25.3 mg, $0.2 \mathrm{mmol}, 0.35$ eq.) were added to a RBF and dissolved in $\mathrm{CH}_{2} \mathrm{Cl}_{2}(4 \mathrm{~mL})$.

After 4 hours, a separation was performed in $\mathrm{CH}_{2} \mathrm{Cl}_{2} / \mathrm{H}_{2} \mathrm{O}$ and the organic fraction was concentrated by rotary evaporation. The crude mixture was subjected to column chromatography using $\mathrm{CH}_{2} \mathrm{Cl}_{2}$ to obtain 185 (132.5 mg, 83\% yield) as an indigo solid. ${ }^{1} \mathrm{H}$ NMR (500 MHz, $\mathrm{CDCl}_{3}$ ) $\delta$ 8.29 (d, J=10.0 Hz, 2H, H-4,8), 7.87 (t, J=4.5 Hz, 1H, H-2), 7.58 (s, 1H, H-15), 7.38 (d, J=4.0 Hz, 2H, H-1,3), 7.15 (m, 3H, H-5,7, H-13), 6.50 (td, J=1.5, 0.5 Hz, 1H, H-14), 4.62 (t, J=7.0 Hz, 2H, H-10), 3.24 (t, J=7.0 Hz, 2H, H-9). ${ }^{13} \mathrm{C}$ NMR (125 MHz, CDCl $) \delta 158.5$ (C, C-11), 147.6 (C, C-6), 146.5 (CH, C-15), 144.5 (C, C-12), 139.1 (C, C-3a,8a), 136.5 (CH, C-2), 135.8 (CH, C-4,8), 124.2 (CH, C-5,7), 118.3 (CH, C-1,3), 118.2 (CH, C-13), 111.9 (CH, C-14), $65.6\left(\mathrm{CH}_{2}, \mathrm{C}-10\right)$, $41.2\left(\mathrm{CH}_{2}, \mathrm{C}-9\right)$. HRMS: $m / z \mathrm{C}_{17} \mathrm{H}_{15} \mathrm{O}_{3}{ }^{+}$ [M+H]+ Calculated 267.1016, found 267.1016. IR (ATR): $v_{\max } 3131,3117,2984,2946,1703,1571$, $1472,1398,1302,1279,1111,792,750 \mathrm{~cm}^{-1}$. m.p. $92.8-93.7^{0}$ C. $\lambda_{\max }: 569 \mathrm{~nm}$.

\section{2-(Azulen-6-yl)ethyl 1-naphthoate (Azul-1-naphthoate) (188)}

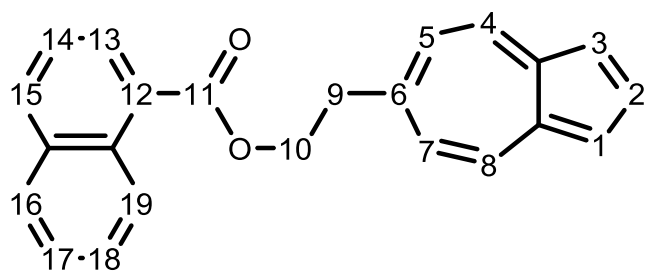

1-Naphthoic acid ( $95.7 \mathrm{mg}, 0.55 \mathrm{mmol}, 1$ eq.), 90 (120.1 mg, $0.70 \mathrm{mmol}, 1.25$ eq.), EDCI (187.8 mg, $1.2 \mathrm{mmol}, 2.2$ eq.) and DMAP (11.6 mg, $0.094 \mathrm{mmol}, 0.17$ eq.) were added together in a RBF and dissolved in $\mathrm{CH}_{2} \mathrm{Cl}_{2}(4 \mathrm{~mL})$.

After 4 hours, a separation was performed using $\mathrm{CH}_{2} \mathrm{Cl}_{2} / \mathrm{H}_{2} \mathrm{O}$ and the organic fraction was concentrated by rotary evaporation and purified by column chromatography in $\mathrm{CH}_{2} \mathrm{Cl}_{2}$ to afford $188\left(167.1 \mathrm{mg}, 82 \%\right.$ yield) as an indigo solid. ${ }^{1} \mathrm{H}$ NMR (500 MHz, $\left.\mathrm{CDCl}_{3}\right) \delta 8.78(\mathrm{~d}, J=8.5 \mathrm{~Hz}, 1 \mathrm{H}, \mathrm{H}-$ 19), 8.31 (d, J=10.5 Hz, 2H, H-4,8), 8.12 (dd, J=7.5, $1.5 \mathrm{~Hz}, 1 \mathrm{H}, \mathrm{H}-13$ ), 8.01 (d, J=8.0 Hz, 1H, H-15), 7.88 (t, J=3.5 Hz, 1H, H-2), 7.87 (d, J=6.5 Hz, 1H, H-16), 7.51 (m, 2H, H-17, H-18), 7.47 (m, 1H, H14), 7.40 (d, J=3.5 Hz, 2H, H-1,3), 7.22 (d, J=10.5 Hz, 2H, H-5,7). 4.75 (t, J=7.0 Hz, 2H, H-10), 3.33 (t, $J=7.0 \mathrm{~Hz}, 2 \mathrm{H}, \mathrm{H}-9) .{ }^{13} \mathrm{C}$ NMR (125 MHz, CDCl $) \delta 167.5$ (C, C-11), 148.2 (C, C-6), 139.2 (C, C-3a,8a), 136.5 (CH, C-2), 135.9 (CH, C-4,8), 133.8 (C, C-15a), 133.4 (CH, C-15), 131.3 (C, C-19a), 130.2 (CH, C-13), 128.5 (CH, C-16), 127.7 (CH, C-18), 127.1 (C, C-12), 126.2 (CH, C-17), 125.8 (CH, C-19), 124.5 (CH, C-14), 124.3 (CH, C-5,7), 118.4 (CH, C-1,3), $65.9\left(\mathrm{CH}_{2}, \mathrm{C}-10\right), 41.3\left(\mathrm{CH}_{2}, \mathrm{C}-9\right)$. HRMS: $m / z$ $\mathrm{C}_{23} \mathrm{H}_{19} \mathrm{O}_{2}+[\mathrm{M}+\mathrm{H}]+$ Calculated 327.1380, found 327.1388. IR (ATR): $v_{\max } 3052,2949,2890,1701$, $1575,1393,1234,1194,1130,1013,778,760 \mathrm{~cm}^{-1}$. m.p. 87.4-88. ${ }^{\circ} \mathrm{C} . \lambda_{\max }: 568 \mathrm{~nm}$. 


\section{2-(Azulen-6-yl)ethyl cyclohexanecarboxylate}

\section{(Azul-cyclohexanecarboxylate) (183)}

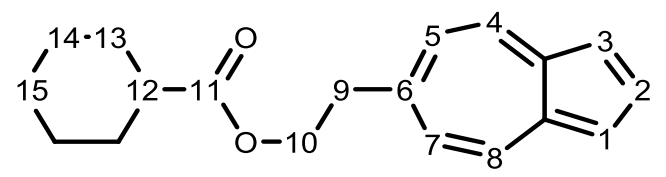

Cyclohexanecarboxylic acid (41.3 mg, $0.32 \mathrm{mmol}, 1$ eq., heated to its m.p. $\left(32{ }^{0} \mathrm{C}\right)$ and added as liquid.), 90 (56.7 mg, $0.33 \mathrm{mmol}, 1.02$ eq.), DCC (73.2 mg, $0.35 \mathrm{mmol}, 1.1$ eq.) and DMAP (12.1 mg, $0.1 \mathrm{mmol}, 0.3$ eq.) were added together in a RBF and dissolved in $\mathrm{CH}_{2} \mathrm{Cl}_{2}(6 \mathrm{~mL})$. The reaction was left overnight.

A separation was performed in $\mathrm{CH}_{2} \mathrm{Cl}_{2} / \mathrm{H}_{2} \mathrm{O}$ and the organic fraction was concentrated by rotary evaporation. The crude mixture was subjected to column chromatography using $\mathrm{CH}_{2} \mathrm{Cl}_{2}$ to afford 183 (70.1 mg, 77\% yield) as an indigo solid with dendritic crystals. ${ }^{1} \mathrm{H}$ NMR (500 $\left.\mathrm{MHz}, \mathrm{CDCl}_{3}\right) \delta$ 8.28 (d, J=10.5 Hz, 2H, H-4,8), 7.87 (t, J=3.5 Hz, 1H, H-2), 7.38 (d, J=3.5 Hz, 2H, H-1,3), 7.11 (d, $J=10.0 \mathrm{~Hz}, 2 \mathrm{H}, \mathrm{H}-5,7), 4.39$ (t, J=7.0 Hz, 2H, H-10), 3.12 (t, J=7.0 Hz, 2H, H-9), 2.28 (tt, J=11.5, $3.5 \mathrm{~Hz}$, 1H, H-12), 1.87 (dd, J=13.5, 2.5 Hz, 4H, H-13a), 1.74 (m, 4H, H-14a), 1.63 (m, 2H, H-15a), 1.42 (qd, $J=12.0,3.5 \mathrm{~Hz}, 1 \mathrm{H}, \mathrm{H}-13 \mathrm{~b}), 1.25$ (m, 3H, H-14b, 15b). ${ }^{13} \mathrm{C}$ NMR (125 MHz, $\mathrm{CDCl}_{3}$ ) $\delta 175.9$ (C, C-11), 148.2 (C, C-6), 139.0 (C, C-3a,8a), 136.4 (CH, C-2), 135.8 (CH, C-4,8), 124.2 (CH, C-5,7), 118.1 (CH, $\mathrm{C}-1,3), 64.9\left(\mathrm{CH}_{2}, \mathrm{C}-10\right), 43.2(\mathrm{CH}, \mathrm{C}-12), 41.1\left(\mathrm{CH}_{2}, \mathrm{C}-9\right), 28.9\left(\mathrm{CH}_{2}, \mathrm{C}-13\right), 25.7\left(\mathrm{CH}_{2}, \mathrm{C}-15\right), 25.4$ $\left(\mathrm{CH}_{2}, \mathrm{C}-14\right)$. HRMS: $m / z \mathrm{C}_{19} \mathrm{H}_{23} \mathrm{O}_{2}{ }^{+}[\mathrm{M}+\mathrm{H}]+$ Calculated 283.1693, found 283.1700. IR (ATR): $v_{\max }$ $3079,2930,2849,1723,1578,1395,1167,1130,839,743$ cm$^{-1}$. m.p. $85.4-85.9^{0}$ C. $\lambda_{\max }: 571 \mathrm{~nm}$.

\section{2-(Azulen-6-yl)ethyl}

cyclobutanecarboxylate

(Azul-

\section{cyclobutanecarboxylate) (184)}

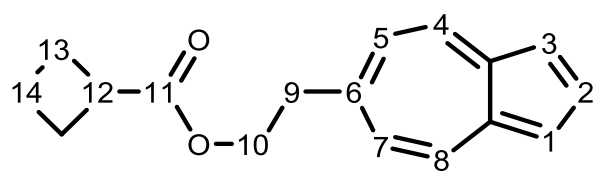

Cyclobutanecarboxylic acid (31.4 mg, 0.31 mmol, 1 eq.), 90 (56.1 mg, 0.32 mmol, 1.04 eq.), DCC ( $85.9 \mathrm{mg}, 0.42 \mathrm{mmol}, 1.32$ eq.) and DMAP (14.8 mg, $0.12 \mathrm{mmol}, 0.38$ eq.) were added to an RBF and dissolved in $\mathrm{CH}_{2} \mathrm{Cl}_{2}(6 \mathrm{~mL})$.

After 5 hours, a separation was performed in $\mathrm{CH}_{2} \mathrm{Cl}_{2} / \mathrm{H}_{2} \mathrm{O}$ and the organic fraction was concentrated by rotary evaporation and subjected to column chromatography in $\mathrm{CH}_{2} \mathrm{Cl}_{2}$ to afford 184 (69.3 mg, 87\% yield) as an indigo viscous oil. $\left.{ }^{1} \mathrm{H} \mathrm{NMR} \mathrm{(500} \mathrm{MHz,} \mathrm{CDCl}_{3}\right) \delta 8.28$ (d, J=10.0 Hz, 
2H, H-4,8), 7.87 (t, J=3.5 Hz, 1H, H-2), 7.38 (d, J=4.0 Hz, 2H, H-1,3), 7.10 (d, J=10.0 Hz, 2H, H-5,7), 4.40 (t, J=6.5 Hz, 2H, H-10), 3.13 (t, J=6.5 Hz, 2H, H-9), 3.11 (obscured m, 1H, H-12), 2.25 (quin, $J=9.0 \mathrm{~Hz}, 2 \mathrm{H}, \mathrm{H}-13 \mathrm{a}), 2.18$ (m, 2H, H-13b), 1.95 (m, J=9.5 Hz, 1H, H-14a), 1.87 (m, 1H, H-14b). ${ }^{13} \mathrm{C}$ NMR (125 MHz, CDCl 3 ) $\delta 175.5$ (C, C-11), 147.8 (C, C-6), 139.05 (C, C-3a,8a), 136.4 (CH, C-2), 134.8 (CH, C-4,8), 124.2 (CH, C-5,7), 118.2 (CH, C-1,3), $65.0\left(\mathrm{CH}_{2}, \mathrm{C}-10\right), 41.2\left(\mathrm{CH}_{2}, \mathrm{C}-9\right), 38.0$ (CH, C-12), $25.3\left(\mathrm{CH}_{2}, \mathrm{C}-13\right), 18.4$ ( $\left.\mathrm{CH}_{2}, \mathrm{C}-14\right)$. IR (ATR): $v_{\max } 3065,2931,2116,1723,1580,1398,1344,1248$, 1173, 971, 837, $744 \mathrm{~cm}^{-1}$. HRMS: $m / z \mathrm{C}_{17} \mathrm{H}_{19} \mathrm{O}_{2}{ }^{+}[\mathrm{M}+\mathrm{H}]^{+}$Calculated 255.1380, found 255.1376. m.p. 85.7-86. ${ }^{\circ}$ C. $\lambda_{\max }: 569 \mathrm{~nm}$.

\section{2-(Azulen-6-yl)ethyl azulene-1-carboxylate (Azul-azulenecarboxylate)}

\section{(186)}

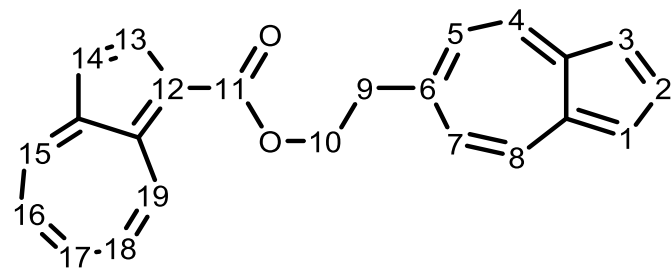

Azulene-1-carboxylic acid 75 (43.2 mg, 0.25 mmol, 1.007 eq.), 90 (42.9 mg, 0.25 mmol, 1 eq.), DCC (76.2 mg, $0.37 \mathrm{mmol}, 1.5$ eq.) and DMAP ( $9.3 \mathrm{mg}, 0.08 \mathrm{mmol}, 0.3$ eq.) were added to a RBF and dissolved in $\mathrm{CH}_{2} \mathrm{Cl}_{2}$ (6 mL). After a few hours a further $41 \mathrm{mg}$ DMAP (1.3 eq.) was added and the reaction was heated at reflux overnight.

A separation was performed in $\mathrm{CH}_{2} \mathrm{Cl}_{2} / \mathrm{H}_{2} \mathrm{O}$ and the organic fraction was concentrated by rotary evaporation. The crude mixture was subjected to column chromatography using $\mathrm{CH}_{2} \mathrm{Cl}_{2}$ to afford 186 (63.5 mg, 78\% yield) as a purple solid. ${ }^{1} \mathrm{H}$ NMR (500 MHz, $\left.\mathrm{CDCl}_{3}\right) \delta 9.56$ (d, J=10.0 Hz, 1H, H19), 8.45 (d, J=10.0 Hz, 1H, H-15), 8.35 (d, J=4.0 Hz, 1H, H-13), 8.32 (d, J=10.0 Hz, 2H, H-4,8), 7.87 ( $\mathrm{t}, J=4.0 \mathrm{~Hz}, 1 \mathrm{H}, \mathrm{H}-2), 7.70$ (t, J=10.0 Hz, 1H, H-17), 7.47 (t, J=10.0 Hz, 1H, H-18), 7.44 (t, J=10.0 Hz, 1H, H-16), 7.39 (d, J=3.5 Hz, 2H, H-1,3), 7.29 (d, J=4.0 Hz, 1H, H-14), 7.24 (d, J=10.0 Hz, 2H, H-5,7), $4.71(\mathrm{t}, J=6.5 \mathrm{~Hz}, 2 \mathrm{H}, \mathrm{H}-10), 3.32$ (t, J=7.0 Hz, 2H, H-9). ${ }^{13} \mathrm{C}$ NMR (125 MHz, CDCl 3 ) $\delta 165.2$ (C, C-11), 148.7 (C, C-6), 144.8 (C, C-14a), 140.8 (C, C-19a), 140.2 (CH, C-13), 139.1 (C, C-3a,8a), 139.0 (CH, C-17), 138.2 (CH, C-15), 137.8 (CH, C-19), 136.3 (CH, C-2), 135.9 (CH, C-4,8), 127.7 (CH, C-18), 126.8 (CH, C-16), 124.4 (CH, C-5,7), 118.2 (CH, C-1,3), 117.7 (CH, C-14), 116.6 (C, C-12), 64.7 ( $\mathrm{CH}_{2}$, C-10), $41.6\left(\mathrm{CH}_{2}, \mathrm{C}-9\right)$. HRMS: $m / z \mathrm{C}_{23} \mathrm{H}_{19} \mathrm{O}_{2}{ }^{+}[\mathrm{M}+\mathrm{H}]^{+}$Calculated 327.1380, found 327.1387. IR (ATR): $v_{\max } 3068,2956,2928,2850,1660,1574,1392,1224,1136,1006,120,754 \mathrm{~cm}^{-1}$. m.p. $116.5-117.4{ }^{0}$ C. $\lambda_{\max }: 514,530 \mathrm{~nm}$. 


\section{2-(Azulen-6-yl)ethyl 3,5-dinitrobenzoate (Azul-3,5-dinitrobenzoate) (187)}

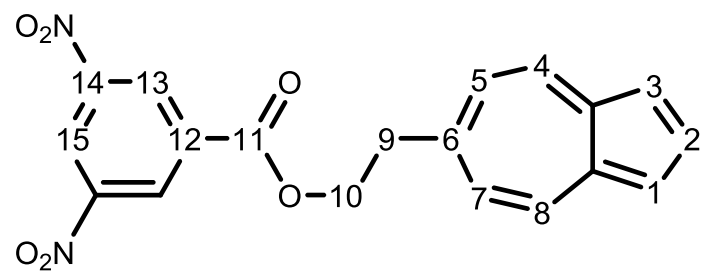

3,5-Dinitrobenzoic acid (71.8 mg, $0.33 \mathrm{mmol}, 1.06$ eq.), 90 (54.9 mg, $0.32 \mathrm{mmol}, 1$ eq.), DCC (79 $\mathrm{mg}, 0.38 \mathrm{mmol}, 1.2$ eq.) and DMAP (10.4 mg, $0.09 \mathrm{mmol}, 0.27$ eq.) were added together and dissolved in $\mathrm{CH}_{2} \mathrm{Cl}_{2}(5 \mathrm{~mL})$.

After 3 hours, a separation was performed with $\mathrm{CH}_{2} \mathrm{Cl}_{2} / \mathrm{H}_{2} \mathrm{O}$ and the organic fraction was concentrated by rotary evaporation. The crude mixture was subjected to column chromatography using $\mathrm{CH}_{2} \mathrm{Cl}_{2}$ to afford 187 (108.2 mg, 93\% yield) as a brown-green solid that appears indigo in solution. ${ }^{1} \mathrm{H}$ NMR (500 MHz, CDCl 3 ) $\delta 9.20$ (t, J=2.0 Hz, 1H, H-15), 9.11 (d, J=2.0 Hz, 2H, H-13), 8.32 (d, J=10.0 Hz, 2H, H-4,8), 7.88 (t, J=3.5 Hz, 1H, H-2), 7.39 (d, J=4.0 Hz, 2H, H-1,3), 7.17 (d, J=10.0 Hz, 2H, H-5,7), 4.75 (t, J=7.0 Hz, 2H, H-10), 3.32 (t, J=7.0 Hz, 2H, H-9). ${ }^{13} \mathrm{C}$ NMR (125 MHz, CDCl $\left.{ }_{3}\right) \delta$ 162.2 (C, C-11), 148.6 (C, C-12), 146.8 (C, C-6), 139.1 (C, C-3a,8a), 136.9 (CH, C-2), 135.9 (CH, C4,8), 129.4 (CH, C-13), 124.0 (CH, C-5,7), 122.4 (CH, C-15), 118.7 (CH, C-1,3), $67.4\left(\mathrm{CH}_{2}, \mathrm{C}-10\right)$ ), 41.0 $\left(\mathrm{CH}_{2}, \mathrm{C}-9\right)$. C-14 not observed. HRMS: $m / z \mathrm{C}_{19} \mathrm{H}_{14} \mathrm{~N}_{2} \mathrm{O}_{6}{ }^{+}[\mathrm{M}+\mathrm{H}]^{+}$Calculated 367.0925, found 367.0943. IR (ATR): $v_{\max } 310,2954,2929,2871,1724,1536,1338,1274,1163,981,846$, $715 \mathrm{~cm}^{-1}$. m.p. $217.1-218.1^{\circ}$ C. Colour change to deep blue observed upon melting. $\lambda_{\max }: 570 \mathrm{~nm}$.

\section{$N$-fluorenylmethyloxycarbonyl glycine 2-(azulen-6-yl)ethyl ester (FMOC-Gly-OAzul) (189)}

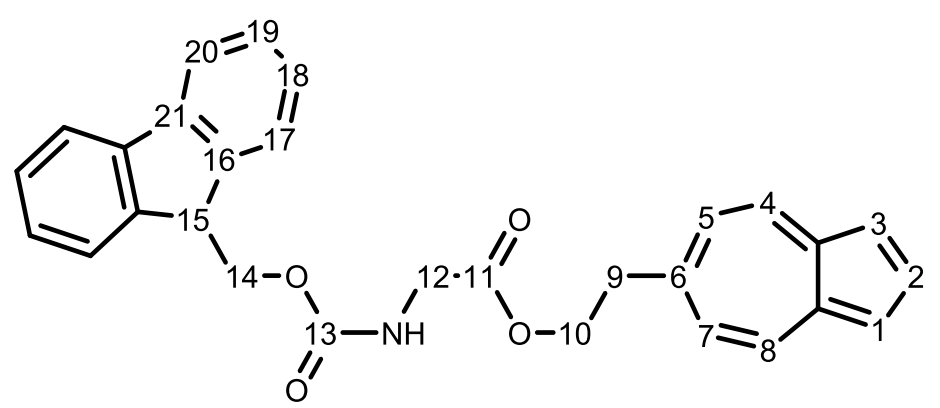

FMOC-Gly-OH (159 mg, 0.5 mmol, 1 eq.), 90 (98.7 mg, 0.57 mmol, 1.07 eq.), DCC (149.4 mg, 0.72 mmol, 1.35 eq.) and DMAP (25.8 mg, $0.21 \mathrm{mmol}, 0.39$ eq.) were added to a RBF and dissolved in $\mathrm{CH}_{2} \mathrm{Cl}_{2}(10 \mathrm{~mL})$. 
After 3 hours, a separation was performed with $\mathrm{CH}_{2} \mathrm{Cl}_{2} / \mathrm{H}_{2} \mathrm{O}$, and the organic fraction was purified by column chromatography using 9:1 $\mathrm{CH}_{2} \mathrm{Cl}_{2} / \mathrm{Et}_{2} \mathrm{O}$ to obtain 189 (224.9 mg, 93\% yield) as an indigo gummy oil. ${ }^{1} \mathrm{H}$ NMR (500 MHz, $\left.\mathrm{CDCl}_{3}\right) \delta 8.29$ (d, J=10.0 Hz, 2H, H-4,8), $7.89(\mathrm{t}, J=3.5 \mathrm{~Hz}, 1 \mathrm{H}$, H-2), 7.79 (d, J=7.5 Hz, 2H, H-20), 7.62 (d, J=8.0 Hz, 2H, H-17), 7.47 (t, J=8.0 Hz, 2H, H-19), 7.39 (d, $J=4.0 \mathrm{~Hz}, 2 \mathrm{H}, \mathrm{H}-1,3), 7.34$ (t, J=7.0 Hz, 2H, H-18), 7.07 (d, J=10.0 Hz, 2H, H-5,7), 5.30 (s, 1H, N-H), 4.48 (t, J=7.0 Hz, 2H, H-10), 4.43 (d, J=7.0 Hz, 2H, H-14), 4.25 (t, J=7.0 Hz, 1H, H-15), 3.98 (d, J=5.5 $\mathrm{Hz}, 2 \mathrm{H}, \mathrm{H}-12$ ), 3.14 (t, J=7.0 Hz, 2H, H-9). ${ }^{13} \mathrm{C}$ NMR (125 MHz, CDCl $) \delta 170.0$ (C, C-11), 156.2 (C, C13), 147.3 (C, C-6), 143.8 (C, C-16), 141.3 (C, C-21), 139.1 (C, C-3a,8a), 136.6 (CH, C-2), 135.9 (CH, C-4,8), 127.7 (CH, C-19), 127.1 (CH, C-18), 125.0 (CH, C-17), 124.0 (CH, C-5,7), 120.0 (CH, C-20), $118.3(\mathrm{CH}, \mathrm{C}-1,3), 67.2\left(\mathrm{CH}_{2}, \mathrm{C}-14\right), 66.1\left(\mathrm{CH}_{2}, \mathrm{C}-10\right), 47.1(\mathrm{CH}, \mathrm{C}-15), 42.7\left(\mathrm{CH}_{2}, \mathrm{C}-12\right), 41.0\left(\mathrm{CH}_{2}, \mathrm{C}-\right.$ 9). HRMS: $m / z \mathrm{C}_{29} \mathrm{H}_{26} \mathrm{NO}_{4}{ }^{+}[\mathrm{M}+\mathrm{H}]+$ Calculated 452.1856, found 452.1875. IR (ATR): $v_{\max } 3336$, $3065,3013,2950,1703,1578,1515,1447,1395,1181,1048,1001,737 \mathrm{~cm}^{-1} . \lambda_{\max }: 568 \mathrm{~nm}$.

\section{2-(Azulen-6-yl)ethyl trichloroacetate (Azul-trichloroacetate) (210)}

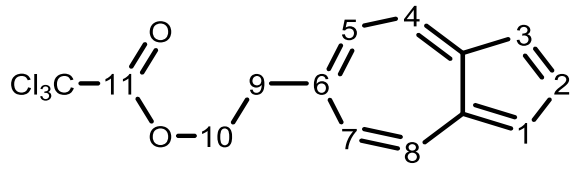

90 (21.8 mg, $0.12 \mathrm{mmol}, 1$ eq.) was dissolved in $\mathrm{CH}_{2} \mathrm{Cl}_{2}(5 \mathrm{~mL})$ and trichloroacetyl chloride (0.07 $\mathrm{mL}, 0.62 \mathrm{mmol}, 5$ eq.) was added, followed by pyridine ( $0.2 \mathrm{~mL}, 2.5 \mathrm{mmol}, 20$ eq.).

After 3 hours a colour change towards maroon was observed. A separation was performed with $\mathrm{CH}_{2} \mathrm{Cl}_{2} / \mathrm{H}_{2} \mathrm{O}$ and the organic fraction was concentrated by rotary evaporation. The crude mixture was purified by column chromatography with $1: 1$ pet. ether $/ \mathrm{CH}_{2} \mathrm{Cl}_{2}$ to afford $\mathbf{2 1 0}(21.5 \mathrm{mg}, 53 \%$ yield) as an indigo oil. ${ }^{1} \mathrm{H}$ NMR (500 MHz, $\left.\mathrm{CDCl}_{3}\right) \delta 8.28$ (d, J=10.5 Hz, 2H, H-4,8), 7.88 (t, J=3.5 Hz, 1H, H-2), 7.39 (d, J=3.5 Hz, 2H, H-1,3), 7.12 (d, J=10.5 Hz, 2H, H-5,7), 4.66 (t, J=7.0 Hz, 2H, H-10), 3.25 (t, J=7.0 Hz, 2H, H-9). ${ }^{13} \mathrm{C}$ NMR (125 M'Hz, CDCl $) \delta 161.9$ (C, C-11), 146.2 (C, C-6), 139.2 (C, C3a,8a), 136.8 (CH, C-2), 135.9 (CH, C-4,8), 124.1 (CH, C-5,7), 118.5 (CH, C-1,3), 89.7 (C, $\mathrm{CCl}_{3}$ ), 69.8 $\left(\mathrm{CH}_{2}, \mathrm{C}-10\right), 40.7\left(\mathrm{CH}_{2}, \mathrm{C}-9\right)$. HRMS: $m / z \mathrm{C}_{14} \mathrm{H}_{12} \mathrm{Cl}_{3} \mathrm{O}_{2}{ }^{+}[\mathrm{M}+\mathrm{H}]+$ Calculated 316.9897, found 316.9885. $\lambda_{\text {max }}: 568 \mathrm{~nm}$.

\section{2-(Azulen-6-yl)ethyl dichloroacetate (Azul-dichloroacetate) (211)}

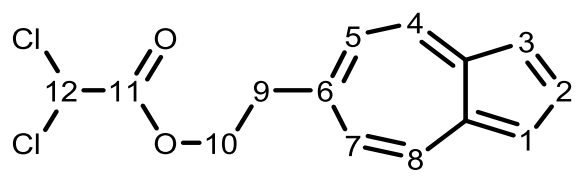


90 (27.3 mg, 016 mmol, 1 eq.) was dissolved in $\mathrm{CH}_{2} \mathrm{Cl}_{2}$ (5 mL), and dichloroacetyl chloride (0.05 $\mathrm{mL}, 0.52 \mathrm{mmol}, 3.3 \mathrm{eq}$.) was added, followed by pyridine $(0.1 \mathrm{~mL}, 1.2 \mathrm{mmol}, 7.8$ eq.).

After 3 hours, a separation was performed in $\mathrm{CH}_{2} \mathrm{Cl}_{2} / \mathrm{H}_{2} \mathrm{O}$ and the crude mixture was purified by column chromatography using $1: 1 \mathrm{CH}_{2} \mathrm{Cl}_{2}$ /pet. ether to afford 211 (31.5 mg, 70\%) as an indigo oil. ${ }^{1} \mathrm{H} \mathrm{NMR}\left(500 \mathrm{MHz}, \mathrm{CDCl}_{3}\right.$ ) $\delta 8.28$ (d, J=10.5 Hz, 2H, H-4,8), 7.88 (t, J=3.5 Hz, 1H, H-2), 7.39 (d, J=3.5 Hz, 2H, H-1,3), 7.1 (d, J=10.0 Hz, 2H, H-5,7), 5.92 (s, 1H, H-12), 4.58 (t, J=7.0 Hz, 2H, H-10), 3.2 (t, $J=7.0 \mathrm{~Hz}, 2 \mathrm{H}, \mathrm{H}-9) .{ }^{13} \mathrm{C}$ NMR (125 MHz, $\mathrm{CDCl}_{3}$ ) $\delta 164.4$ (C, C-11), 146.6 (C, C-6), 139.1 (C, C-3a,8a), 136.7 (CH, C-2), 136.0 (CH, C-4,8), 124.1 (CH, C-5,7), 118.5 (CH, C-1,3), $68.0\left(\mathrm{CH}_{2}, \mathrm{C}-10\right), 64.1$ (CH, C-12), $40.6\left(\mathrm{CH}_{2}, \mathrm{C}-9\right)$. HRMS: $m / z \mathrm{C}_{14} \mathrm{H}_{13} \mathrm{Cl}_{2} \mathrm{O}_{2}{ }^{+}[\mathrm{M}+\mathrm{H}]+{ }^{+}$Calculated 283.0287, found 283.0284. $\lambda_{\max }$ : $566 \mathrm{~nm}$.

\section{General procedure for deprotection of Azul-esters and Azul-carbonates}

Azul-ester or -carbonate ( $0.5 \mathrm{mmol}$ ) was dissolved in MeCN (3 mL) under open air, and DBU (0.2 $\mathrm{mL}$ ) was added. After 3-5 hours, a colour change from indigo to blue was observed. $\mathrm{HCl}$ ( $2 \mathrm{~mL}, 10 \%$ in $\mathrm{H}_{2} \mathrm{O}$ ) was added, and a phase separation was performed using $\mathrm{CH}_{2} \mathrm{Cl}_{2}$ and $\mathrm{H}_{2} \mathrm{O}$. The aqueous layer was washed three times with $\mathrm{CH}_{2} \mathrm{Cl}_{2}$ then twice with EtOAc. The organic phase was then concentrated by rotary evaporation. The crude mixture was purified by column chromatography, eluting 6-vinylazulene with pet. ether and the deprotected alcohol or carboxylic acid with a mixture of pet. ether/ethyl acetate (typically 2:1 or lower). The deprotected alcohol or carboxylic acid was returned in excellent yield. Occasionally a green tint remained in the product from degraded azulene material. This was a very minor impurity and could be removed by filtering through a silica plug.

\section{6-Vinylazulene (94)}

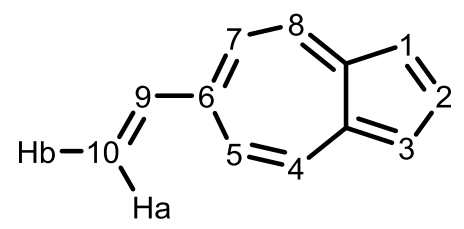

A blue crystalline solid was obtained as a by-product of Azul deprotection, typically in yields corresponding to the extent of deprotection. ${ }^{1} \mathrm{H}$ NMR $\left(500 \mathrm{MHz}, \mathrm{CDCl}_{3}\right) \delta 8.31(\mathrm{~d}, J=10.5 \mathrm{~Hz}, 2 \mathrm{H}, \mathrm{H}-$ 4,8), 7.85 (t, J=3.5 Hz, 1H, H-2), 7.36 (d, J=3.5 Hz, 2H, H-1,3), 7.31 (d, J=10.0 Hz, 2H, H-5,7), 6.92 (dd, $J=17.5,10.8 \mathrm{~Hz}, 1 \mathrm{H}, \mathrm{H}-9$ ), 5.97 (d, J=17.5 Hz, 1H, H-10b), 5.47 (d, J=10.5 Hz, 1H, H-10a). ${ }^{13 C}$ NMR (125 MHz, CDCl ${ }_{3}$ ) $\delta 145.7$ (C, C-6), 141.3 (CH, C-9), 139.5 (C, C-3a,8a), 136.9 (CH, C-2), $135.6(\mathrm{CH}$, C-4,8), $121.4(\mathrm{CH}, \mathrm{C}-5,7), 118.3(\mathrm{CH}, \mathrm{C}-1,3), 117.6\left(\mathrm{CH}_{2}, \mathrm{C}-10\right)$. HRMS: $m / z \mathrm{C}_{12} \mathrm{H}_{11^{+}}[\mathrm{M}+\mathrm{H}]^{+}$ 
Calculated 155.0855, found 155.0857. $\lambda_{\max }$ : $608 \mathrm{~nm}$. ${ }^{1} \mathrm{H}$ NMR spectrum corresponds with that reported previously. ${ }^{12}$

\section{2-(1-Nitroazulen-6-yl)ethyl (E)-cinnamate (197)}

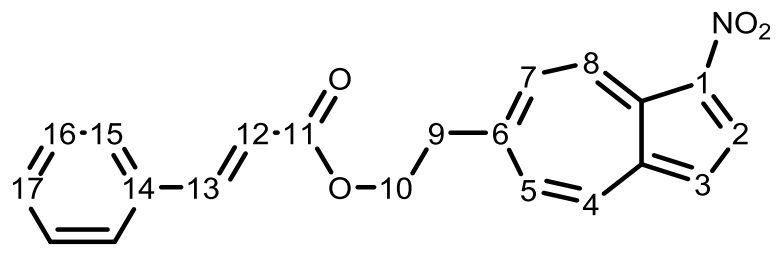

Copper nitrate trihydrate (199.1 mg) and acetic anhydride (5 mL) were added together and the mixture was left to sit until the colour changed from blue to turquoise. An aliquot of this mixture ( $3 \mathrm{~mL}$, corresponding to 16 eq. nitrate) was added to a vessel containing 178 (18.6 mg, $0.06 \mathrm{mmol}$, 1 eq.) in toluene $(5 \mathrm{~mL})$ at $0{ }^{\circ} \mathrm{C}$. The reaction was allowed to warm up to r.t. and after 70 minutes, the reaction mixture had becomegreen.

A separation was performed with $\mathrm{CH}_{2} \mathrm{Cl}_{2}$ /sat. aq. $\mathrm{Na}_{2} \mathrm{CO}_{3}$ and the organic fraction was concentrated by rotary evaporation. The dark orange residue was purified by column chromatography in $\mathrm{CH}_{2} \mathrm{Cl}_{2}$ to give 197 (6.6 mg, 31\% yield) as an orange solid. ${ }^{1} \mathrm{H}$ NMR (500 MHz, $\mathrm{CDCl}_{3}$ ) $\delta 9.70$ (d, J=10.5 Hz, 1H, H-8), 8.48 (d, J=11.0 Hz, 1H, H-4), 8.42 (d, J=4.5 Hz, 1H, H-2), 7.76 (d, J=10.5 Hz, 1H, H-7), 7.66 (d, J=16.0 Hz, 1H, H-13), 7.62 (d, J=10.0 Hz, 1H, H-5), 7.51 (m, 2H, H15), 7.40-7.38 (complex m, 3H, H-16, H-17), 7.19 (d, J=4.5 Hz, 1H, H-3), 6.39 (d, J=16.0 Hz, 1H, H12), 4.58 (t, J=7.0 Hz, 2H, H-10), 3.35 (d, J=6.5 Hz, 2H, H-9). ${ }^{13} \mathrm{C}$ NMR (125 MHz, CDCl $) \delta 166.7$ (C, C-11), 153.3 (C, C-6), 145.6 (CH, C-13), 142.8 (C, C-8a), 139.8 (CH, C-4), 137.3 (CH, C-8), 134.2 (CH, C-2), 134.1 (C, C-14), 132.9 (C, C-3a), 132.7 (CH, C-7), 131.0 (CH, C-5), 130.5 (CH, C-17), 129.0 (CH, C-16), 128.1 (CH, C-15), 117.2 (CH, C-12), 117.0 (CH, C-3), $64.6\left(\mathrm{CH}_{2}, \mathrm{C}-10\right), 41.2\left(\mathrm{CH}_{2}, \mathrm{C}-9\right) . \mathrm{C}-1$ was not observed. HRMS: $m / z \mathrm{C}_{21} \mathrm{H}_{18} \mathrm{NO}_{4}{ }^{+}[\mathrm{M}+\mathrm{H}]^{+}$Calculated 348.1230, found 348.1228. IR (ATR): $\nu_{\max } 2952,2921,1708,1637,1577,1483,1392,1276,1168,1152,977,852,769 \mathrm{~cm}^{-1} . \lambda_{\max } 499$ $\mathrm{nm}$.

\section{2-(1-(2-Methoxy-2-oxoacetyl)azulen-6-yl)ethyl (E)-cinnamate (205)}

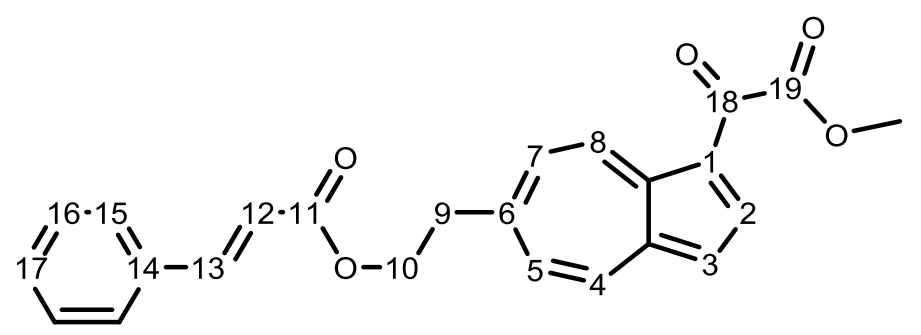


178 (78.7 mg, $0.26 \mathrm{mmol}, 1$ eq.) was dissolved in $\mathrm{CH}_{2} \mathrm{Cl}_{2}$ (3 mL). Oxalyl chloride (0.1 mL, $1.16 \mathrm{~mol}$, 4.5 eq.) was added, followed 20 seconds later by methanol ( $0.3 \mathrm{~mL}, 7.4 \mathrm{mmol}, 28 \mathrm{eq}$.) and after another 40 seconds by pyridine $(0.4 \mathrm{~mL}, 5 \mathrm{mmol}, 19 \mathrm{eq}$.$) .$

A separation was performed immediately, using $\mathrm{CH}_{2} \mathrm{Cl}_{2} / \mathrm{H}_{2} \mathrm{O}$ and the organic fraction was concentrated by rotary evaporation and purified by column chromatography in 2:1 pet. ether/ethyl acetate to give 205 (98.9 mg, 97.8\% yield) as an orange-red solid. ${ }^{1} \mathrm{H}$ NMR (500 MHz, $\mathrm{CDCl}_{3}$ ) $\delta 9.79$ (d, J=10.5 Hz, 1H, H-8), 8.45 (d, J=10.0 Hz, 1H, H-4), 8.40 (d, J=4.0 Hz, 1H, H-2), 7.70 (d, J=10.0 Hz, 1H, H-7), 7.66 (d, J=16.0 Hz, 1H, H-13), 7.58 (d, J=10.5 Hz, 1H, H-5), 7.50 (m, 2H, H15), 7.39-7.37 (m, 3H, H-16, H-17), 7.26 (d, J=4.0 Hz, 1H, H-3), 6.39 (d, J=16.0 Hz, 1H, H-12), 4.56 (t, $J=7.0 \mathrm{~Hz}, 2 \mathrm{H}, \mathrm{H}-10), 3.99$ (s, 3H, O-Me), 3.31 (t, J=6.5 Hz, 2H, H-9). ${ }^{13} \mathrm{C}$ NMR (125 MHz, $\left.\mathrm{CDCl}_{3}\right) \delta$ 180.7 (C, C-18), 167.8 (C, C-19), 166.7 (C, C-11), 152.2 (C, C-6), 146.1 (C, C-3a), 145.5 (CH, C-13), 142.3 (CH, C-2), 141.8 (C, C-8a), 138.9 (CH, C-8), 138.1 (CH, C-4), 134.1 (C, C-14), 132.5 (CH, C-7), 131.0 (CH, C-5), 130.5 (CH, C-17), 128.9 (CH, C-16), 128.2 (CH, C-15), 120.9 (C, C-1), 119.4 (CH, C3), 117.4 (CH, C-12), 64.7 ( $\left.\mathrm{CH}_{2}, \mathrm{C}-10\right), 52.6\left(\mathrm{CH}_{3}, \mathrm{O}-\mathrm{Me}\right), 41.1\left(\mathrm{CH}_{2}, \mathrm{C}-9\right)$. HRMS: $m / z \mathrm{C}_{24} \mathrm{H}_{21} \mathrm{O}_{5}{ }^{+}$ $[\mathrm{M}+\mathrm{H}]+$ Calculated 389.1384, found 389.1386. IR (ATR): $v_{\max } 3002,2950,1732,1710,1623,1409$, $1305,1174,850,769 \mathrm{~cm}^{-1} . \lambda_{\max }: 505 \mathrm{~nm}$.

\section{$N$-fluorenylmethyloxycarbonyl glycine 2-(1-(2-methoxy-2- oxoacetyl)azulen-6-yl)ethyl ester (222)}

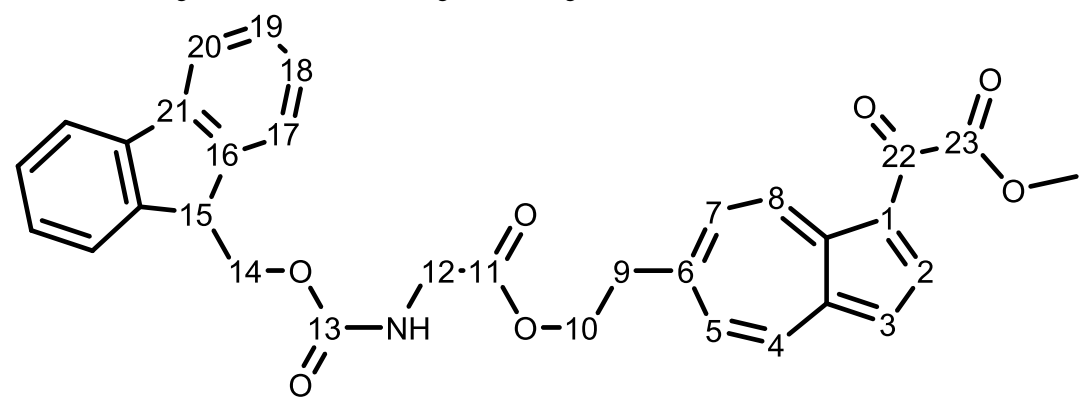

189 (189.4 mg, $0.42 \mathrm{mmol}, 1$ eq.) was dissolved in $\mathrm{CH}_{2} \mathrm{Cl}_{2}$ (4 mL) and oxalyl chloride (0.2 mL, 2.3 mmol, 5.5 eq.) was added at $0{ }^{\circ} \mathrm{C}$, followed 20 seconds later by $\mathrm{MeOH}(0.4 \mathrm{~mL}, 9.9 \mathrm{mmol}, 23 \mathrm{eq}$.) and another 30 seconds later by pyridine $(0.5 \mathrm{~mL}, 6.2 \mathrm{mmol}, 15$ eq.).

A phase separation was performed using $\mathrm{CH}_{2} \mathrm{Cl}_{2} / \mathrm{H}_{2} \mathrm{O}$, and the organic fraction was concentrated by rotary evaporation. The crude material was purified by column chromatography in ethyl acetate to afford 222 as an orange-red solid (208.5 mg, 92\%). $\left.{ }^{1} \mathrm{H} \mathrm{NMR} \mathrm{(500} \mathrm{MHz,} \mathrm{CDCl}_{3}\right) \delta 9.77$ (d, $J=10.0 \mathrm{~Hz}, 1 \mathrm{H}, \mathrm{H}-8$ ), 8.42 (d, J=10.5 Hz, 1H, H-4), 8.40 (d, J=4.5 Hz, 1H, H-2), 7.76 (d, J=7.5 Hz, 2H, H-20), 7.63 (d, J=10.0 Hz, 1H, H-7), 7.58 (d, J=7.5 Hz, 2H, H-17), 7.51 (d, J=10.0 Hz, 1H, H-5), 7.40 (t, $J=7.5 \mathrm{~Hz}, 2 \mathrm{H} \mathrm{H}-19), 7.31$ (t, J=7.5 Hz, 2H, H-18), 7.25 (d, J=4.5 Hz, 1H, H-3), 5.22 (t, J=5.5 Hz, 1H, NH), 4.51 (d, J=7.0 Hz, 2H, H-10), 4.40 (d, J=7.0 Hz, 2H, H-14), 4.22 (t, J=7.0 Hz, 1H, H-15), 3.99 (s, 3H, 
O-Me), 3.96 (d, J=5.5 Hz, 2H, H-12), 3.26 (t, J=7.0 Hz, H, H-9). ${ }^{13} \mathrm{C}$ NMR (125 MHz, CDCl $) \delta 180.6(\mathrm{C}$, C-22), 169.8 (C, C-11), 164.6 (C, C-23), 156.2 (C, C-13), 151.3 (C, C-6), 146.0 (C, C-3a or 8a), 143.7 (C, C-16), 142.4 (CH, C-2), 141.8 (C, C-3a or 8a), 141.3 (C, C-21), 138.8 (CH, C-8), 138.1 (CH, C-4), 132.2 (CH, C-7), 130.9 (CH, C-5), 127.7 (CH, C-19), 127.1 (CH, C-18), 125.0 (CH, C-17), 120.9 (C, C1), 120.0 (CH, C-20), 119.6 (CH, C-3), $67.2\left(\mathrm{CH}_{2}, \mathrm{C}-14\right), 15.6\left(\mathrm{CH}_{2}, \mathrm{C}-10\right), 52.6\left(\mathrm{CH}_{3}, \mathrm{O}-\mathrm{Me}\right), 47.1(\mathrm{CH}$, C-15), 42.7 ( $\left.\mathrm{CH}_{2}, \mathrm{C}-12\right), 40.8\left(\mathrm{CH}_{2}, \mathrm{C}-9\right)$. HRMS: $m / z \mathrm{C}_{32} \mathrm{H}_{28} \mathrm{NO}_{7^{+}}[\mathrm{M}+\mathrm{H}]^{+}$Calculated 538.1860, found 538.1876. IR (ATR): $v_{\max } 3355,3040,2951,1720,1621,1395,1255,1182,1048,783 \mathrm{~cm}^{-1}$. m.p. $63.6-65.7^{\circ}$ C. $\lambda_{\max }: 507 \mathrm{~nm}$.

\section{2-(Azulen-6-yl)ethyl 1H-imidazole-1-carboxylate (176)}

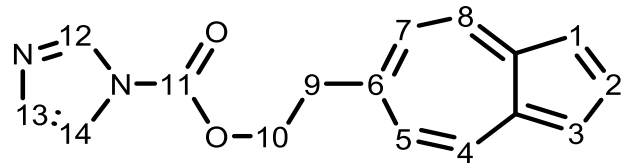

90 (164.3 mg, $0.95 \mathrm{mmol}, 1$ eq.) and CDI (259 mg, 66\% pure by ${ }^{1} \mathrm{H}$ NMR integration, equivalent to 1.1 eq. pure CDI) were dissolved in THF ( $5 \mathrm{~mL}$ ) and left to react for 1.5 hours. The reaction mixture was concentrated by rotary evaporation and purified by column chromatography with ethyl acetate to afford the title compound $\mathbf{1 7 6}$ as an indigo crystalline solid ( $251 \mathrm{mg}$, 99\% yield). ${ }_{1} \mathrm{H}$ NMR (500 MHz, CDCl $) \delta 8.29$ (d, J=10.5 Hz, 2H, H-4,8), 8.09 (s, 1H, H-12), 7.89 (t, J=3.5 Hz, 1H, H-2), 7.40 (d, J=3.5 Hz, 2H, H-1,3), 7.37 (s, 1H, H-14), 7.11 (d, J=10.0 Hz, 2H, H-5,7), 7.06 (s, 1H, H13), 4.71 (t, J=7.0 Hz, 2H, H-10), 3.27 (t, J=4.0 Hz, 2H, H-9). ${ }^{13} \mathrm{C}$ NMR (125 MHz, CDCl $) \delta 148.4(\mathrm{C}$, C-11), 146.4 (C, C-6), 139.1 (C, C-3a,8a), 137.03 (CH, C-12), 136.96 (CH, C-2), 135.9 (CH, C-4,8), 130.8 (CH, C-14), 123.8 (CH, C-5,7), 118.7 (CH, C-1,3), 117.0 (CH, C-13), $68.8\left(\mathrm{CH}_{2}, \mathrm{C}-10\right), 40.9\left(\mathrm{CH}_{2}\right.$, C-9). HRMS: $m / z \mathrm{C}_{16} \mathrm{H}_{15} \mathrm{~N}_{2} \mathrm{O}_{2}+[\mathrm{M}+\mathrm{H}]+{ }^{+}$Calculated 267.1128, found 267.1133. IR (ATR): $v_{\max } 3129$, $1752,1581,1470,1396,1288,1241,998,826,749,647 \mathrm{~cm}^{-1}$. m.p. 89.1-90.4 ${ }^{0} \mathrm{C} . \lambda_{\max }: 566 \mathrm{~nm}$.

\section{6-(2-Oxyethyl)azulene cyclohexanemethyl carbonate (AzulOC- cyclohexanemethanol) (191)}

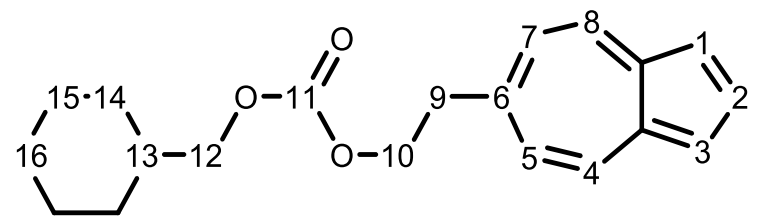

Method A. 90 (74.9 mg, 0.43 mmol, 1 eq.) and CDI (130.9 mg, 75\% pure by ${ }^{1} \mathrm{H}$ NMR integration, equivalent to 1.4 eq. pure CDI) were dissolved in toluene ( $4 \mathrm{~mL}$ ), and $\mathrm{KOH}(11.4 \mathrm{mg}, 0.2 \mathrm{mmol}$, 0.46 eq.) was added. The reaction mixture was heated at $70{ }^{\circ} \mathrm{C}$ for four hours. Cyclohexylmethanol $(0.07 \mathrm{~mL}, 0.56 \mathrm{mmol}, 1.3 \mathrm{eq}$.) was added and the reaction was left overnight. 
A separation was performed using $\mathrm{CH}_{2} \mathrm{Cl}_{2} / \mathrm{H}_{2} \mathrm{O}$ and the organic layer was purified by column chromatography in $\mathrm{CH}_{2} \mathrm{Cl}_{2}$, giving 191 (132.9 mg, 97\% yield) as an indigo solid.

Method B. 176 (48.9 mg, $0.18 \mathrm{mmol}, 1.01$ eq.) and cyclohexylmethanol (20.6 mg, $0.18 \mathrm{mmol}, 1 \mathrm{eq}$.) were dissolved in toluene (3.5 mL) and $\mathrm{KOH}(1.9 \mathrm{mg}, 0.03 \mathrm{mmol}, 0.19$ eq.) was added. The reaction was heated at reflux for 4 hours.

A separation was performed using $\mathrm{CH}_{2} \mathrm{Cl}_{2} / \mathrm{H}_{2} \mathrm{O}$ and the organic fraction was concentrated by rotary evaporation. The crude mixture was purified by column chromatography, eluting fractions $\mathrm{A}$ and $\mathrm{B}$ in $\mathrm{CH}_{2} \mathrm{Cl}_{2}$, fraction $\mathrm{C}$ in 1:1 pet. ether/ethyl acetate and fraction $\mathrm{D}$ in ethyl acetate. These fractions were concentrated by rotary evaporation, giving A as 6-vinylazulene ( $2 \mathrm{mg}$, 7\% yield), B as the title compound 191 (35.7 mg, 63.3\% yield), C as 90 (2.4 mg, 7.7\% yield), and D as the starting material 176 (6.9 mg, 14\% yield). ${ }^{1} \mathrm{H}$ NMR (500 MHz, CDCl $) \delta 8.29$ (d, J=10.0 Hz, 2H, H4,8), 7.88 (t, J=3.5 Hz, 1H, H-2), 7.39 (d, J=4.0 Hz, 2H, H-1,3), 7.11 (d, J=10.9 Hz, 2H, H-5,7), 4.45 (t, $J=7.5 \mathrm{~Hz}, 2 \mathrm{H}, \mathrm{H}-10$ ), 3.95 (d, J=7.0 Hz, 2H, H-12), 3.18 (t, J=7.5 Hz, 2H, H-9), 1.74 (m, 4H, H-14a, H15a), 1.68 (m, 2H, H-13, H-16a), 1.25 (m, 3H, H-15b, H-16b), 0.97 (m, 2H, H-14b). ${ }^{13} \mathrm{C}$ NMR (125 $\mathrm{MHz}, \mathrm{CDCl}_{3}$ ) $\delta 155.4$ (C, C-11), 147.2 (C, C-6), 139.2 (C, C-3a,8a), 136.5 (CH, C-2), 135.8 (CH, C-4,8), 124.3 (CH, C-5,7), $118.4(\mathrm{CH}, \mathrm{C}-1,3), 73.3\left(\mathrm{CH}_{2}, \mathrm{C}-12\right), 68.3\left(\mathrm{CH}_{2}, \mathrm{C}-10\right), 41.1\left(\mathrm{CH}_{2}, \mathrm{C}-9\right), 37.1$ (CH, C13), $29.5\left(\mathrm{CH}_{2}, \mathrm{C}-14\right), 26.4\left(\mathrm{CH}_{2}, \mathrm{C}-16\right), 25.6(\mathrm{CH}, \mathrm{C}-15)$. HRMS: $\left.m / z_{20} \mathrm{C}_{25} \mathrm{H}_{3^{+}} \mathrm{H} \mathrm{M}+\mathrm{H}\right]+$ Calculated 313.1798, found 313.1809. IR (ATR): $v_{\max } 2933,2858,1736,1582,1471,1400,1252,972,952$, $838,745 \mathrm{~cm}^{-1}$. m.p. $82.2-83 .{ }^{0}$ C. $\lambda_{\max }: 570 \mathrm{~nm}$.

\section{2-(Azulen-6-yl)ethyl cyclohexylcarbamate (194)}

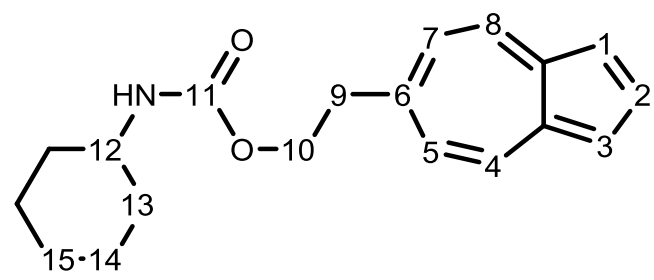

176 (249.2 mg, $0.93 \mathrm{mmol}, 1$ eq.) and cyclohexylamine (153.1 mg, $1.54 \mathrm{mmol}, 1.6$ eq.) were dissolved in toluene ( $5 \mathrm{~mL}$ ), and $\mathrm{KOH}$ ( $3.8 \mathrm{mg}, 0.067 \mathrm{mmol}, 0.072 \mathrm{eq}$.) was added to the reaction mixture. The mixture was heated at reflux overnight.

A separation was performed with $\mathrm{CH}_{2} \mathrm{Cl}_{2} / \mathrm{H}_{2} \mathrm{O}$ and the organic fraction was concentrated by rotary evaporation. The crude mixture was subjected to column chromatography. Fraction A was eluted with pet. ether, fraction $B$ eluted with $\mathrm{CH}_{2} \mathrm{Cl}_{2}$, fraction $\mathrm{C}$ eluted with 1:1 pet. ether/ethyl acetate and fraction $\mathrm{D}$ eluted with ethyl acetate. The fractions were concentrated by rotary evaporation, giving A as 6-vinylazulene (6.4 mg, 4.4\% yield), B as the symmetrical carbonate 192 (10.8 mg, 3.1\% 
yield), $\mathrm{C}$ as the title compound 194 (210.3 $\mathrm{mg}, 75.6 \%$ yield) as an indigo solid, and D as the starting material 176 (22.6 mg, 9.1\% yield). ${ }^{1} \mathrm{H}$ NMR (500 MHz, CDCl $) \delta 8.27$ (d, J=10.0 Hz, 2H, H4,8), 7.85 (t, J=4.0 Hz, 1H, H-2), 7.36 (d, J=3.5 Hz, 2H, H-1,3), 7.11 (d, J=10.0 Hz, H, H-5,7), 4.53 (broad s, 1H, N-H), 4.36 (t, J=7.0 Hz, 2H, H-10), 3.49-3.44 (m, 1H, H-12), 3.11 (t, J=7.0 Hz, 2H, H-9), 1.91 (d, J=10.0 Hz, 2H, H-13a), 1.68 (m, 2H, H-14a), 1.58 (m, 1H, H-15a), 1.39-1.25 (m, 2H, H-14b), 1.17-1.07 (m, 3H, H-13b, H-15b). ${ }^{13}$ C NMR (125 MHz, CDCl 3 ) $\delta 155.5$ (C, C-11), 148.4 (C, C-6), 139.1 (C, C-3a,8a), 136.3 (CH, C-2), 135.9 (CH, C-4,8), 124.2 (CH, C-5,7), 118.2 (CH, C-1,3), 65.4 ( $\mathrm{CH}_{2}, \mathrm{C}-$ 10), 49.8 (CH, C-12), 41.7 ( $\left.\mathrm{CH}_{2}, \mathrm{C}-9\right), 33.4\left(\mathrm{CH}_{2}, \mathrm{C}-13\right), 25.6\left(\mathrm{CH}_{2}, \mathrm{C}-15\right), 24.9\left(\mathrm{CH}_{2}, \mathrm{C}-14\right)$. HRMS: $m / z \mathrm{C}_{19} \mathrm{H}_{24} \mathrm{NO}_{2}+[\mathrm{M}+\mathrm{H}]+$ Calculated 298.1802, found 298.1800. IR (ATR): $v_{\max }$ 3311, 2931, 1851, $1683,1530,1312,1234,1065,1043,831,748,670 \mathrm{~cm}^{-1}$. m.p. $140.9-141.5^{0}$ C. $\lambda_{\max }: 568 \mathrm{~nm}$.

\section{Bis[6-(2-oxyethyl)azulene] carbonate (192)}

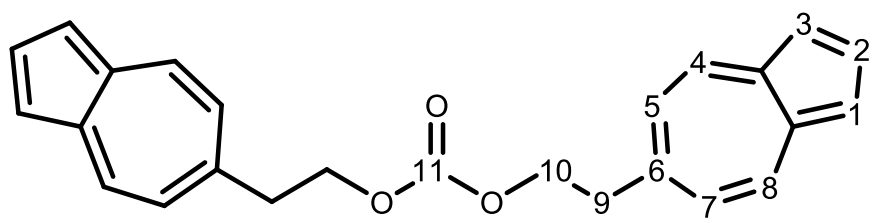

This indigo solid was formed in small quantities (yields typically between $0-10 \%$ ) in most reactions involving the use of 177. ${ }^{1} \mathrm{H}$ NMR $\left(500 \mathrm{MHz}, \mathrm{CDCl}_{3}\right) \delta 8.25(\mathrm{~d}, J=10.0 \mathrm{~Hz}, 4 \mathrm{H}, \mathrm{H}-4,8), 7.87$ (t, J=3.5 Hz, 2H, H-2), 7.37 (d, J=4.0 Hz, 4H, H-1,3), 7.07 (d, J=10.5 Hz, 4H, H-5,7), 4.42 (t, J=7.0 Hz, 4H, H-10), 3.14 (t, J=7.0 Hz, 4H, H-9). ${ }^{13} \mathrm{C}$ NMR (125 MHz, CDCl $) \delta 154.9$ (C, C-11), 147.1 (C, C-6), 139.1 (C, C-3a,8a), 136.6 (CH, C-2), 135.9 (CH, C-4,8), 124.1 (CH, C-5,7), 118.4 (CH, C-1,3), 68.5 $\left(\mathrm{CH}_{2}, \mathrm{C}-10\right), 41.1\left(\mathrm{CH}_{2}, \mathrm{C}-9\right)$. HRMS: $m / z \mathrm{C}_{25} \mathrm{H}_{23} \mathrm{O}_{3^{+}}[\mathrm{M}+\mathrm{H}]+$ Calculated 371.1642, found 371.1648.IR (ATR): $v_{\max } 2955,2916,2849,1726,1578,1493,1389,1257,953,837,825,753 \mathrm{~cm}^{-1} . \lambda_{\max }: 572 \mathrm{~nm}$.

\section{6-(2-Oxyethyl)azulene cetyl carbonate (193)}

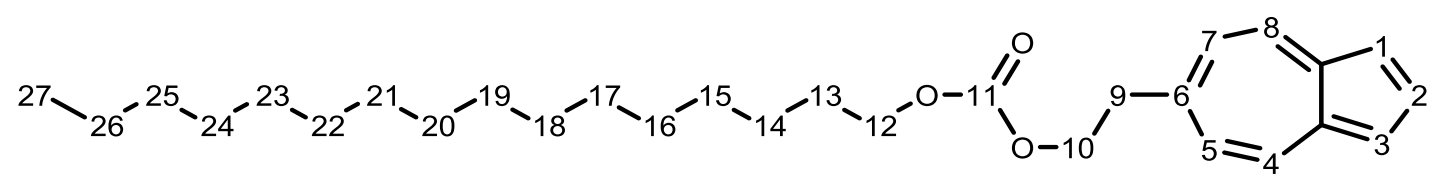

176 (46.4 mg, $0.17 \mathrm{mmol}, 1.5 \mathrm{eq}$.) and cetyl alcohol (27.9 mg, $0.12 \mathrm{mmol}, 1 \mathrm{eq}$.) were added to a silanised RBF and dissolved in THF (1 mL). KHMDS solution (0.42 M in toluene, $\sim 50 \mu \mathrm{L})$ was added at a rate of one drop every 30 minutes for a total of five drops over 2.5 hours $(0.021 \mathrm{mmol}$, 0.18 eq.) After 3 hours, the reaction mixture was concentrated by rotary evaporation and purified by column chromatography, eluting fraction A with pet. ether, fraction B with 20:1 pet. ether/ethyl acetate, fraction $\mathrm{C}$ with $\mathrm{CH}_{2} \mathrm{Cl}_{2}$, and recovered starting material 176 (26\%) with ethyl acetate. Fraction A was concentrated to give 6-vinylazulene (1.3 mg, 5\% yield w.r.t 176) Fraction 
B was concentrated to give the title compound 193 as an indigo powder (42.1 $\mathrm{mg}, 83 \%$ yield). Fraction $\mathrm{C}$ was concentrated to give the symmetrical carbonate 192 ( $5.2 \mathrm{mg}$, 8\% yield w.r.t. 176).

193: ${ }^{1} \mathrm{H}$ NMR (500 MHz, $\mathrm{CDCl}_{3}$ ) $\delta 8.28(\mathrm{~d}, J=10.0 \mathrm{~Hz}, 2 \mathrm{H}, \mathrm{H}-4,8), 7.86(\mathrm{t}, J=4.0 \mathrm{~Hz}, 1 \mathrm{H}, \mathrm{H}-2), 7.37$ (d, $J=3.5 \mathrm{~Hz}, 2 \mathrm{H}, \mathrm{H}-1,3$ ), 7.11 (d, J=10.0 Hz, 2H, H-5,7), 4.43 (t, J=7.0 Hz, H, H-10), 4.11 (t, J=7.0 Hz, 2H, $\mathrm{H}-12$ ), 3.17 (t, J=7.0 Hz, 2H, H-9), 1.64 (quin, J=7.5 Hz, 2H, H-13), 1.35-1.25 (complex m, 26H, H-14 to $\mathrm{H}-26), 0.88$ (t, J=8.0 Hz, 3H, H-27). ${ }^{13} \mathrm{C}$ NMR (125 MHz, CDCl $) \delta 155.1$ (C, C-11), 147.3 (C, C-6) 139.1 (C, C-3a,8a), 136.5 (CH, C-2), 135.9 (CH, C-4,8), 124.2 (CH, C-5,7), 118.3 (CH, C-1,3), 68.4 $\left(\mathrm{CH}_{2}, \mathrm{C}-10\right), 68.3\left(\mathrm{CH}_{2}, \mathrm{C}-12\right), 41.2\left(\mathrm{CH}_{2}, \mathrm{C}-9\right), 31.9\left(\mathrm{CH}_{2}, \mathrm{C}-26\right), 29.7-29.2\left(\mathrm{CH}_{2}, \mathrm{C}-14\right.$ to $\left.\mathrm{C}-25\right), 28.6$ ( $\left.\mathrm{CH}_{2}, \mathrm{C}-13\right), 25.6\left(\mathrm{CH}_{2}\right.$, alkyl), $22.7\left(\mathrm{CH}_{2}\right.$, alkyl), $14.1\left(\mathrm{CH}_{3}, \mathrm{C}-27\right)$. HRMS: $m / z \mathrm{C}_{29} \mathrm{H}_{45} \mathrm{O}_{3}{ }^{+}[\mathrm{M}+\mathrm{H}]^{+}$ Calculated 441.3363, found 441.3365. IR (ATR): $v_{\max } 2959,2915,2849,1737,1584,1471,1403$, $1253,948,839,746 \mathrm{~cm}^{-1}$.m.p. $74.1-75.3^{\circ} \mathrm{C}$. $\lambda_{\max }: 568 \mathrm{~nm}$.

\section{Pyrimido[1,2-a]azepinium, yl)ethyl] (196) \\ 2,3,4,6,7,8,9,10-octahydro-1-[2-(azulen-6-}

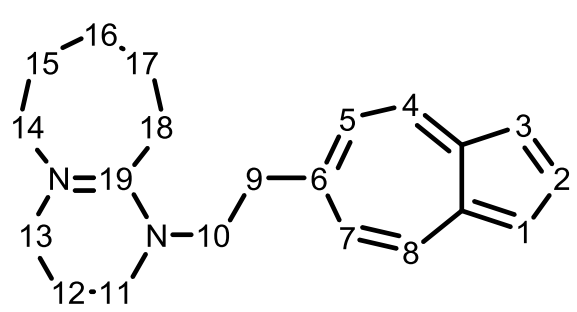

6-Vinylazulene (94) (111.1 mg, $0.72 \mathrm{mmol}, 1$ eq.) and KOH (110.2 mg, $1.9 \mathrm{mmol}, 2.7$ eq.) were dissolved in a 1:1 mixture of THF: $\mathrm{H}_{2} \mathrm{O}(6 \mathrm{~mL})$, and $\mathrm{DBU}(0.2 \mathrm{~mL}, 1.3 \mathrm{mmol}, 1.9 \mathrm{eq}$.) was added. The reaction mixture was heated at reflux overnight, over which time the colour changed from blue to indigo.

A phase separation was performed using $\mathrm{CH}_{2} \mathrm{Cl}_{2} / \mathrm{H}_{2} \mathrm{O}$, and the organic fraction was washed with sat. $\mathrm{NaHCO}_{3}$, followed by $10 \%$ aqueous $\mathrm{HCl}$, followed again by sat. $\mathrm{NaHCO}_{3}$ before being concentrated by rotary evaporation. The crude mixture was purified by column chromatography (gradient elution from $\mathrm{CH}_{2} \mathrm{Cl}_{2}$ to $\mathrm{MeOH}$ ) to give 196 (56.7 mg, 24.2\% yield), presumably as the hydroxide salt, as an indigo oil. ${ }^{1} \mathrm{H}$ NMR $\left(500 \mathrm{MHz}, \mathrm{CDCl}_{3}\right) \delta 8.28(\mathrm{~d}, J=10.0 \mathrm{~Hz}, 2 \mathrm{H}, \mathrm{H}-4,8), 7.86(\mathrm{t}$, $J=3.5 \mathrm{~Hz}, 1 \mathrm{H}, \mathrm{H}-2$ ), 7.37 (d, J=3.5 Hz, 2H, H-1,3), 7.13 (d, J=9.0 Hz, H, H-5,7), 3.42 (t, J=8.0 Hz, 2H, H13), 3.29 (m, 2H, H-14), 3.03 (t, J=6.5 Hz, 2H, H-10), 2.98 (t, J=6.5 Hz, 2H, H-9), 2.65 (t, J=7.0 Hz, 2H, $\mathrm{H}-11$ ), 2.51 (m, J=5.5 Hz, 2H, H-18), 1.71 (m, 6H, H-12,16,17), 1.61 (m, 2H, H-15). ${ }^{13} \mathrm{C}$ NMR (125 $\mathrm{MHz}, \mathrm{CDCl}_{3}$ ) $\delta 176.1$ (C, C-19), 150.6 (C, C-6), 138.9 (C, C-3a,8a), 135.98 (CH, C-2), 135.95 (CH, C4,8), 124.1 (CH, C-5,7), 118.0 (CH, C-1,3), $52.0\left(\mathrm{CH}_{2}, \mathrm{C}-10\right), 49.5\left(\mathrm{CH}_{2}, \mathrm{C}-14\right), 46.7\left(\mathrm{CH}_{2}, \mathrm{C}-11\right), 45.8$ $\left(\mathrm{CH}_{2}, \mathrm{C}-13\right), 42.5\left(\mathrm{CH}_{2}, \mathrm{C}-9\right), 37.1\left(\mathrm{CH}_{2}, \mathrm{C}-18\right), 29.9\left(\mathrm{CH}_{2}, \mathrm{C}-16\right), 28.5\left(\mathrm{CH}_{2}, \mathrm{C}-15\right), 28.1\left(\mathrm{CH}_{2}, \mathrm{C}-12\right)$, $23.5\left(\mathrm{CH}_{2}, \mathrm{C}-17\right)$. 


\section{6-(2-Oxyethyl)azulene m ethyl ether (216)}<smiles></smiles>

6-Vinylazulene (94) (13.1 mg, 0.085 mmol, 1 eq.) and KOH (104.1 mg, 1.85 mmol, 22 eq.) were added together and dissolved in $\mathrm{MeOH}(3 \mathrm{~mL}) . \mathrm{H}_{2} \mathrm{O}_{2}\left(1 \mathrm{~mL}, 30 \% \mathrm{v} / \mathrm{v}\right.$ solution in $\left.\mathrm{H}_{2} \mathrm{O}\right)$ was added and the reaction was heated at $45^{\circ} \mathrm{C}$ and vigorous bubbling was observed. A further $96.7 \mathrm{mg} \mathrm{KOH}$ was added after 3 hours and the reaction wasleft overnight.

A phase separation was performed using $\mathrm{CH}_{2} \mathrm{Cl}_{2} / \mathrm{H}_{2} \mathrm{O}$ and the organic fraction was concentrated by rotary evaporation and purified by column chromatography in $\mathrm{CH}_{2} \mathrm{Cl}_{2}$ to afford the title compound 216 (4.2 mg, 27\% yield) as an indigo oil. ${ }^{1} \mathrm{H}$ NMR (500 MHz, $\left.\mathrm{CDCl}_{3}\right) \delta 8.27$ (d, J=11.0 $\mathrm{Hz}, 2 \mathrm{H}, \mathrm{H}-4,8), 7.84$ (t, J=4.0 Hz, 1H, H-2), 7.35 (d, J=3.5 Hz, 2H, H-1,3), 7.11 (d, J=9.5 Hz, 2H, H-5,7), 3.70 (t, J=7.5 Hz, 2H, H-10), 3.36 (s, 3H, H-11), 3.08 (t, J=8.0 Hz, 2H, H-9). ${ }^{13} \mathrm{C}$ NMR (125 MHz, CDCl $)_{3}$ $\delta 149.4$ (C, C-6), 139.0 (C, C-3a,8a), 136.1 (CH, C-2), 135.9 (CH, C-4,8), 124.3 (CH, C-5,7), 118.0 (CH, C-1,3), $74.14\left(\mathrm{CH}_{2}, \mathrm{C}-10\right), 58.9\left(\mathrm{CH}_{3}, \mathrm{C}-11\right), 42.4\left(\mathrm{CH}_{2}, \mathrm{C}-9\right)$. HRMS: $m / z \mathrm{C}_{13} \mathrm{H}_{14} \mathrm{O}[\mathrm{M}+\mathrm{H}]+$ Calculated 187.1117, found 187.1115

\section{6-(2-Oxyethyl)azulene 3,4-dimethoxybenzyl ether (217)}

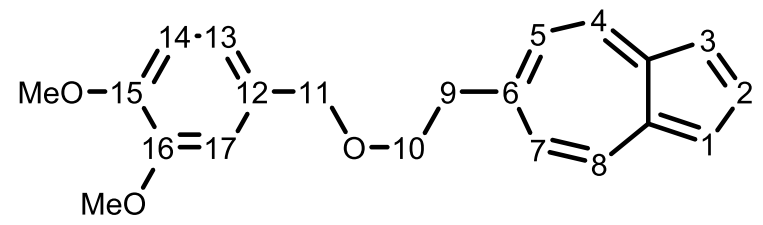

6-Vinylazulene (13.7 mg, $0.89 \mathrm{mmol}, 1$ eq.) and 3,4-dimethoxybenzyl alcohol (0.45 mL, $3.1 \mathrm{mmol}$, 34.8 eq.) were added to a RBF, followed by a single pellet of $\mathrm{KOH}$ ( $86.5 \mathrm{mg}, 1.54 \mathrm{mmol}, 17.3 \mathrm{eq}$.) and dry dimethoxyethane $(0.5 \mathrm{~mL})$. The reaction vessel was purged with $\mathrm{N}_{2}$ and sealed, and after 4 hours of reaction at $\sim 30^{\circ} \mathrm{C}$, a noticeable colour change from blue to indigo had occurred.

$\mathrm{CH}_{2} \mathrm{Cl}_{2}$ was added and the reaction mixture was purified by column chromatography, eluting 6vinylazulene ( $4.6 \mathrm{mg}, 33 \%$ yield) with pet. ether, and eluted the title compound in $\mathrm{CH}_{2} \mathrm{Cl}_{2}, 217$ (13.6 mg, 47\% yield), as an indigo oil. ${ }^{1} \mathrm{H}$ NMR (500 MHz, $\left.\mathrm{CDCl}_{3}\right) \delta 8.26$ (d, J=10.5 Hz, 2H, H-4,8), 7.84 (t, J=4.0 Hz, 1H, H-2), 7.35 (d, J=4.0 Hz, 2H, H-1,3), 7.12 (d, J=10.0 Hz, 2H, H-5,7), 6.81-6.77 (complex m, 3H, H-13, H-14, H-17), 4.47 (s, 2H, H-11), 3.86 (s, 3H, OMe), 3.78 (t, J=7.5 Hz, 2H, H10), 3.73 (s, 3H, OMe), 3.11 (t, J=7.0 Hz, 2H, H-9). ${ }^{13} \mathrm{C}$ NMR (125 MHz, CDCl 3 ) $\delta 149.6$ (C, C-6), 149.0 (C, C-15 or C-16), 148.5 (C, C-15 or C-16), 139.0 (C, C-3a,8a), 136.0 (CH, C-2), 135.8 (CH, C-4,8), 130.7 (C, C-12), 124.4 (CH, C-5,7), 120.1 (CH, C-13, 14 or C-17), 118.0 (CH, C-1,3), 110.78 (CH, C-13, 
14 or C-17), 110.76 (CH, C-13, 14 or C-17), $73.0\left(\mathrm{CH}_{2}, \mathrm{C}-11\right), 71.2(\mathrm{CH}, \mathrm{C}-10), 55.9\left(\mathrm{CH}_{3}, \mathrm{OMe}\right), 55.7$ $\left(\mathrm{CH}_{3}, \mathrm{OMe}\right), 42.6\left(\mathrm{CH}_{2}, \mathrm{C}-9\right)$. HRMS: $m / z \mathrm{C}_{21} \mathrm{H}_{23} \mathrm{O}_{3}+[\mathrm{M}+\mathrm{H}]+$ Calculated 323.1642, found 323.1662. $m / z \mathrm{C}_{19}{ }^{13} \mathrm{C}_{2} \mathrm{H}_{23} \mathrm{O}_{3}+[\mathrm{M}+\mathrm{H}]+$ Calculated 325.1709, found 325.1719.

\section{N-2-(Azulen-6-yl)ethyl piperidine (195)}

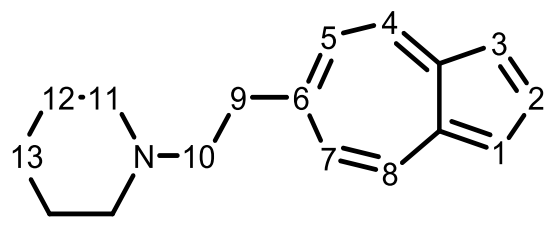

An indigo solid, formed in Azul deprotections involving the use of piperidine, obtained in quantities concordant with the extent of deprotection. ${ }^{1} \mathrm{H}$ NMR $\left(500 \mathrm{MHz}, \mathrm{CDCl}_{3}\right) \delta 8.26(\mathrm{~d}, J=10.5$ Hz, H, H-4,8), 7.83 (t, J=4.0 Hz, 1H, H-2), 7.35 (d, J=4.0 Hz, 2H, H-1,3), 7.09 (d, J=10.0 Hz, 2H, H-5,7), 3.00 (m, 2H, H-9), 2.67 (m, 2H, H-10), 2.50 (broad s, 4H, H-11), 1.64 (quin, J=6.0 Hz, 4H, H-12), 1.51.45 (m, 2H, H-13). ${ }^{13} \mathrm{C}$ NMR (125 MHz, CDCl $) \delta 151.4$ (C, C-6), 138.9 (C, C,3a,8a), 136.0 (CH, C4,8), 135.9 (CH, C-2), 124.2 (CH, C-5,7), 118.0 (CH, C-1,3), $62.1\left(\mathrm{CH}_{2}, \mathrm{C}-10\right), 54.5\left(\mathrm{CH}_{2}, \mathrm{C}-11\right), 40.0$ $\left(\mathrm{CH}_{2}, \mathrm{C}-9\right), 26.0\left(\mathrm{CH}_{2}, \mathrm{C}-12\right), 24.4\left(\mathrm{CH}_{2}, \mathrm{C}-13\right)$. HRMS: $m / z \mathrm{C}_{17} \mathrm{H}_{22} \mathrm{~N}^{+}[\mathrm{M}+\mathrm{H}]+$ Calculated 240.1747, found 240.1751. $\lambda_{\max }: 562 \mathrm{~nm}$.

\section{Methoxymethyl cetyl ether (MOM cetyl ether) (223)}

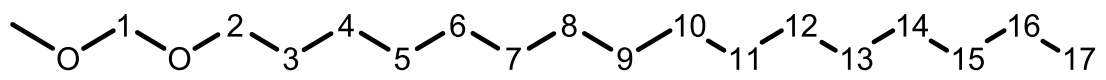

Cetyl alcohol (804.7 mg, $3.32 \mathrm{mmol}, 1$ eq.), and DMAP (11.5 mg, $0.09 \mathrm{mmol}, 0.02$ eq.) were added together in a $\mathrm{RBF}$ and dissolved in $\mathrm{CH}_{2} \mathrm{Cl}_{2}(15 \mathrm{~mL})$ and cooled to $-30{ }^{\circ} \mathrm{C}$ in an ethylene glycol/dry ice bath. Chloromethyl methyl ether $(0.5 \mathrm{~mL}, 6.6 \mathrm{mmol}, 2.0$ eq.) was added, followed by DIPEA (1 $\mathrm{mL}, 5.7 \mathrm{mmol}, 1.7 \mathrm{eq}$.$) . The reaction was left to stir overnight.$

A phase separation was performed using $\mathrm{CH}_{2} \mathrm{Cl}_{2}$ /sat. aq. $\mathrm{NaHCO}_{3}$ and the organic fraction was concentrated by rotary evaporation. Column chromatography (14:1 pet. ether/ethyl acetate with $1 \% \mathrm{NEt}_{3}$ ) was performed on the crude mixture to give 223 as a colourless oil. ${ }^{1} \mathrm{H}$ NMR (500 MHz, $\left.\mathrm{CDCl}_{3}\right) \delta 4.62(\mathrm{~s}, 2 \mathrm{H}, \mathrm{H}-1), 3.52(\mathrm{t}, J=7.0 \mathrm{~Hz}, 2 \mathrm{H}, \mathrm{H}-2$ ), 3.36 (s, 3H, O-Me), 1.59 (quin, J=6.5 Hz, 2H, H3), 1,38-1.23 (complex m, 26H, H-4 to H-16), 0.88 (t, $J=7.0 \mathrm{~Hz}, 3 \mathrm{H}, \mathrm{H}-17$ ). HRMS: $m / z \mathrm{C}_{18} \mathrm{H}_{39} \mathrm{O}_{2}{ }^{+}$ [M+H]+ Calculated 287.2945, found 287.2944. 
<smiles>CC(C)(C)[Si](C)(C)OI1CCSCS1</smiles>

Cyclohexanemethanol (409.6 mg, $3.6 \mathrm{mmol}, 1$ eq.), tert-butyldimethylsilyl chloride (1.14 g, 7.56 mmol, 2.1 eq.) and imidazole (603 mg, $8.86 \mathrm{mmol}, 2.47$ eq.) were added to a RBF and dissolved in $\operatorname{DMF}(7 \mathrm{~mL})$.

After overnight reaction, a phase separation was performed with pet. ether/sat. aq. $\mathrm{NaHCO}_{3}$, and the organic layer was concentrated by rotary evaporation. The residue was purified by column chromatography in $\mathrm{CH}_{2} \mathrm{Cl}_{2}$ to give 221 (852 mg, quant.) as a colourless oil. ${ }^{1} \mathrm{H}$ NMR (500 $\mathrm{MHz}$, $\mathrm{CDCl}_{3}$ ) $\delta 3.38$ (d, J=6.5 Hz, 2H, H-1), 1.73 (m, 5H) 1.42 (m, 1H, H-2) 1,26-1.12 (complex m, 3H), 0.91 (obscured m, 2H) 0.90 (s, 9H, Si-tBu), 0.04 (6H, Si-Me). Spectroscopic data matched those reported previously. ${ }^{13}$

\section{1-(2-Hydroxyethyl)azulene (243)}

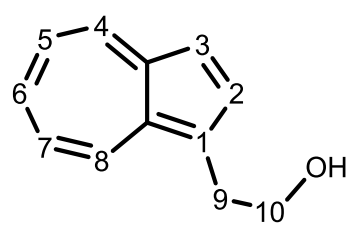

Azulene (101.9 mg, 0.795 mmol, 1 eq.) was dissolved in $\mathrm{CH}_{2} \mathrm{Cl}_{2}$ (5 mL) in a RBF. Oxalyl chloride $(0.07 \mathrm{~mL}, 0.8 \mathrm{mmol}, 1.03 \mathrm{eq}$.) was added and the reaction changed colour to orange. The reaction was cooled down to $0{ }^{\circ} \mathrm{C}$ over 9 minutes and $0.4 \mathrm{~mL} \mathrm{LiBH}_{4}$ (2M in THF, $0.8 \mathrm{mmol}, 1$ eq.) was added rapidly, causing the reaction mixture to bubble. The reaction mixture changed colour to bluegreen.

The reaction was quenched with $0.2 \mathrm{~mL} \mathrm{MeOH}$, and separated with $\mathrm{Et}_{2} \mathrm{O} /$ brine. The organic fraction was concentrated by rotary evaporation, and column chromatography was performed on the resulting material (gradient of pet. ether to $\mathrm{Et}_{2} \mathrm{O}$ ) to afford 243 (34.4 $\mathrm{mg}$, 25\% yield) as a blue oil. ${ }^{1} \mathrm{H}$ NMR (500 MHz, CDCl $) \delta 8.34$ (d, J=9.5 Hz, 1H, H-8), 8.29 (d, J=9.0 Hz, 1H, H-4), 7.84 (d, $J=4.0 \mathrm{~Hz}, 1 \mathrm{H}, \mathrm{H}-2$ ), 7.58 (t, J=9.5 Hz, 1H, H-6), 7.38 (d, J=4.0 Hz, 1H, H-3), 7.15 (t, J=9.5 Hz, 1H, H-7), 7.12 (t, J=9.5 Hz, 1H, H-5), 3.98 (t, J=6.5 Hz, 2H, H-10), 3.37 (t, J=6.5 Hz, 2H, H-9). ${ }^{13} \mathrm{C}$ NMR (125 $\mathrm{MHz}, \mathrm{CDCl}_{3}$ ) $\delta 140.8$ (C, C-3a), 137.7 (CH, C-6), 137.4 (CH, C-2), 136.8 (C, C-8a), 136.6 (CH, C-4), 
133.6 (CH, C-8), 126.4 (C, C-1), 122.4 (CH, C-5), 122.0 (CH, C-7), 117.1 (CH, C-3), $64.0\left(\mathrm{CH}_{2}, \mathrm{C}-10\right)$, $30.9\left(\mathrm{CH}_{2}, \mathrm{C}-9\right)$.

\section{Azul-ester deprotection kinetics}

A solution of the Azul-containing species in MeCN ( $0.001 \mathrm{~mol} \mathrm{~L}^{-1}$ in $\left.2.8 \mathrm{~mL}\right)$ was added to a quartz cuvette and DBU $(0.1 \mathrm{~mL})$ was added, resulting in a DBU concentration of $0.23 \mathrm{~mol} \mathrm{~L}^{-1}$. DBU was in 230 -fold excess, ensuring pseudo-first order kinetics. The cuvette was immediately placed into the UV-Vis spectrophotometer and a full scan from 800 to $400 \mathrm{~nm}$ was taken regularly, typically every two minutes, for a typical total of 100 scans.
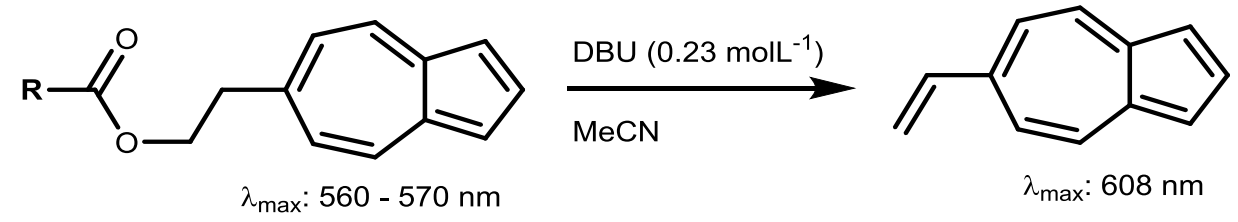

Several wavelengths around the $\lambda_{\max }$ value of 6-vinylazulene $(608 \mathrm{~nm}$ ) were chosen for analysis, typically between 600 and $620 \mathrm{~nm}$. The $\lambda_{\max }$ of the Azul-protected starting materials (around 570 $\mathrm{nm}$ ) was not used, as there is very little change in absorbance at that wavelength over the course of the deprotection. The trace from a single representative wavelength is shown for each experiment.

The raw data for the absorbance were then normalised (from 0 to 1 ) and linear regression was performed on the logarithm of the time series using Excel's LINEST feature to provide an estimate of the slope. The returned value is vulnerable to noise from the tail end of the time series (where noise dominates the signal) so an exponential of best fit was generated and its parameters altered manually until the square of the residual (the difference between the time series and the best fit) was minimised. From the best fit, the pseudo-first order rate constant was obtained, from which the actual rate constant is found by dividing the pseudo-first order rate constant by the concentration of DBU. The rate constants for each wavelength were averaged to give the final value for the rate constant and the uncertainty was calculated by dividing the range (highest value - lowest value) by two. For each compound, the UV-Vis spectrum of the start and end point are displayed with the studied wavelengths marked. The kinetics at a single representative wavelength (raw data in blue, and exponential of best fit in orange) are displayed, as well as the corresponding residual. 


\section{Azul-cinnamate (178) deprotection kinetics}

\section{Change in the visible absorbance spectrum of Azul-cinnamate 178 during deprotection}

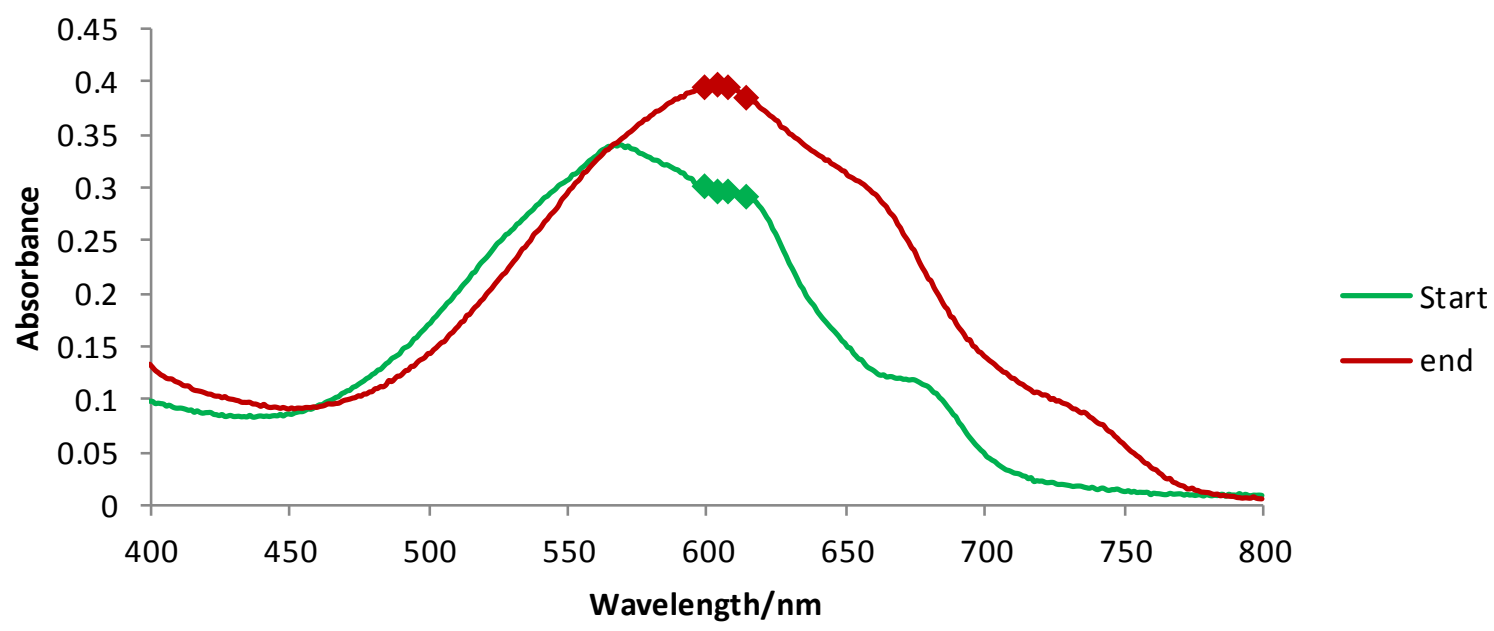

Azul-cinnamate deprotection kinetics at $600 \mathrm{~nm}$

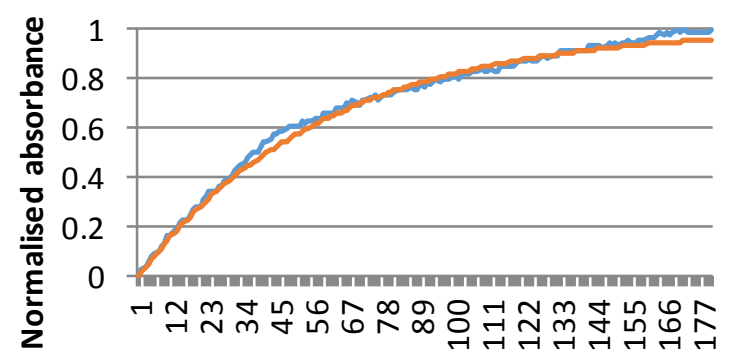
Scan
Residual at $600 \mathrm{~nm}$

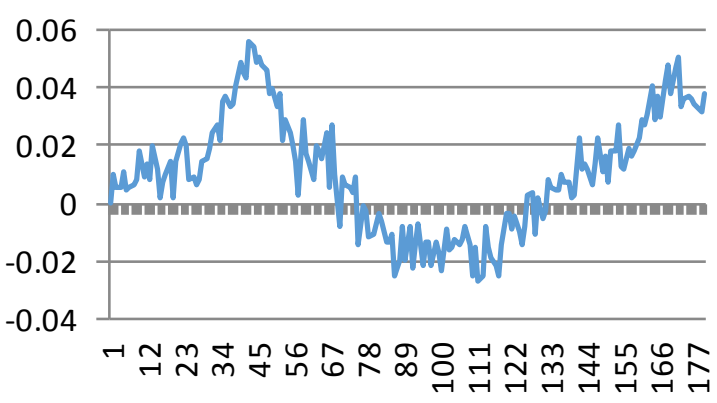

Scan

$182(13.9 \mathrm{mg}$ ) was dissolved in $1 \mathrm{~mL} \mathrm{MeCN}$, diluted 1 in 10 followed by 1 in 4.6, to give a 0.001 mol L-1 solution. One scan was run every 71 seconds for 180 scans total.

\begin{tabular}{|l|l|l|}
\hline Measured wavelength & Reaction rate $/$ Lmol $^{-\mathbf{1}} \mathbf{s}^{\mathbf{1}}$ & Sum of Residuals $^{\mathbf{2}}$ \\
\hline $600 \mathrm{~nm}$ & 0.00108 & 0.05 \\
\hline $604 \mathrm{~nm}$ & 0.00111 & 0.045 \\
\hline $608 \mathrm{~nm}$ & 0.00112 & 0.035 \\
\hline $610 \mathrm{~nm}$ & 0.00111 & 0.05 \\
\hline $615 \mathrm{~nm}$ & 0.00110 & 0.045 \\
\hline
\end{tabular}

Averaging these values for the reaction rate returns $0.0011 \pm 0.0004 \mathrm{~L} \mathrm{~mol}^{-1} \mathrm{~s}^{-1}$. The origin of the hump around scans 34-56 is not known. 


\section{Azul-cyclohexanecarboxylate (183) deprotection kinetics}

\section{Change in the visible absorbance spectrum of Azul-cyclohexanecarboxylate 183 during deprotection}

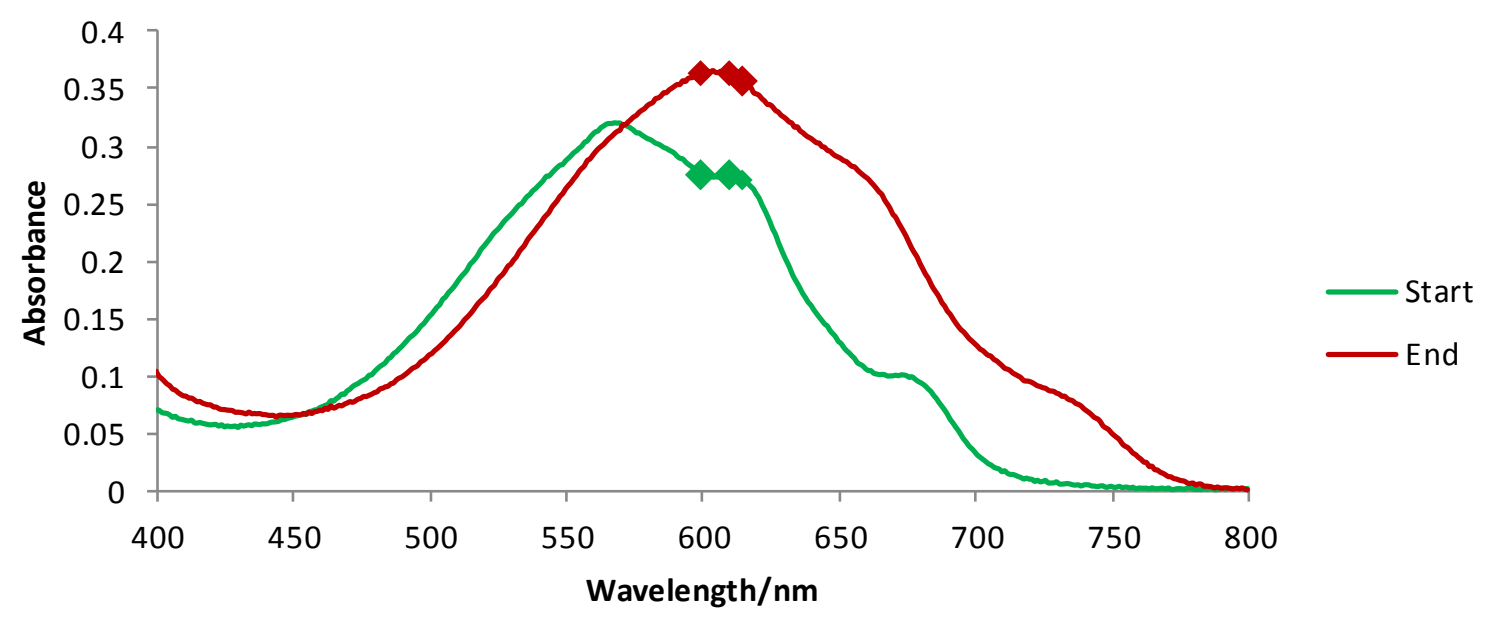

Azul-cyclohexanecarboxylate deprotection kinetics at $600 \mathrm{~nm}$

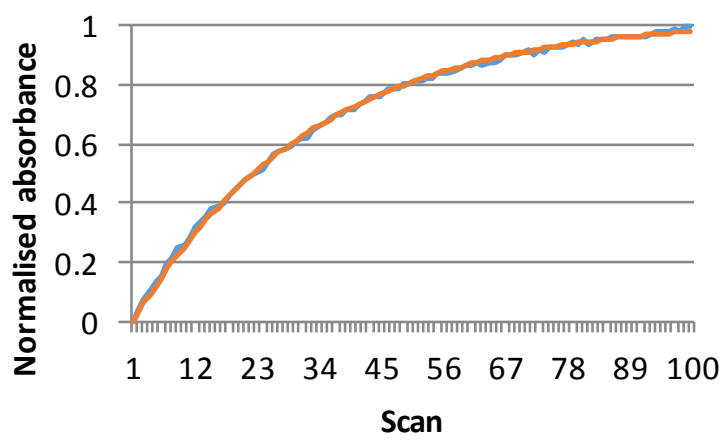

Residual at $600 \mathrm{~nm}$

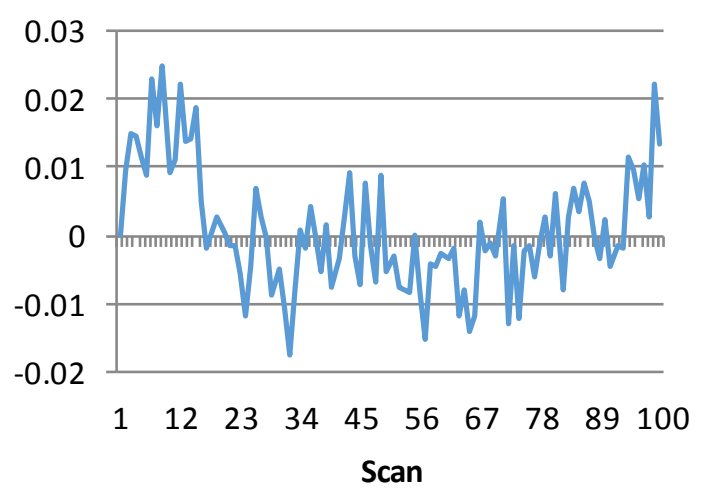

183 (6.5 mg) was dissolved in $23 \mathrm{~mL}$ MeCN to give a $0.001 \mathrm{~mol} \mathrm{~L}^{-1}$ solution. One scan was run every two minutes for 100 scans total.

\begin{tabular}{|l|l|l|}
\hline Measured wavelength & Reaction rate/ Lmol-1 $^{-1}{ }^{-1}$ & Sum of Residuals $^{2}$ \\
\hline $600 \mathrm{~nm}$ & 0.00114 & 0.0075 \\
\hline $610 \mathrm{~nm}$ & 0.00113 & 0.0085 \\
\hline $615 \mathrm{~nm}$ & 0.00110 & 0.0085 \\
\hline
\end{tabular}

Averaging these values for the reaction rate returns $0.00112 \pm 0.0002 \mathrm{~L} \mathrm{~mol}^{-1} \mathrm{~s}^{-1}$. 


\section{Azul-azulene-1-carboxylate (186) deprotection kinetics}

\section{Change in the visible absorbance spectrum of Azul-azulenecarboxylate 186 during deprotection}
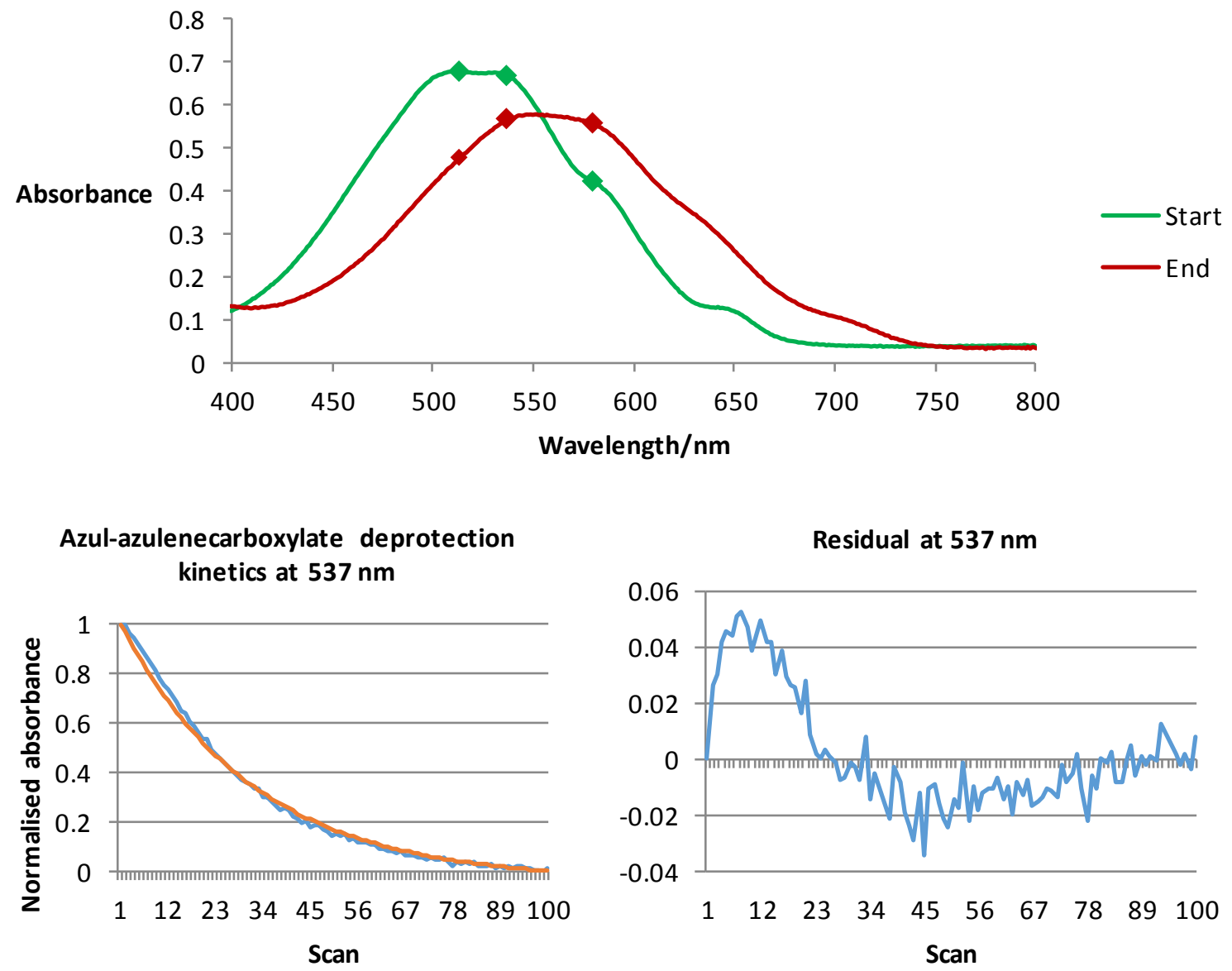

186 (7.4 mg) was dissolved in $22.6 \mathrm{~mL} \mathrm{MeCN}$ to give a $0.001 \mathrm{molL}^{-1}$ solution. One scan was run every five minutes for 100 scans total. Due to the presence of the azulenecarboxylate moiety, different wavelengths from the usual were studied for the kinetics measurements.

\begin{tabular}{|l|l|l|}
\hline Measured wavelength & Reaction rate/ Lmol-1 $\mathbf{S}^{-\mathbf{1}}$ & Sum of Residuals \\
\hline $514 \mathrm{~nm}$ & 0.00053 & 0.03 \\
\hline $537 \mathrm{~nm}$ & 0.00050 & 0.04 \\
\hline $580 \mathrm{~nm}$ & 0.00050 & 0.0075 \\
\hline
\end{tabular}

Averaging these values for the reaction rate returns $0.00051 \pm 0.0002 \mathrm{~L} \mathrm{~mol}^{-1} \mathrm{~s}^{-1}$. The mismatch between the kinetics data and the best fit at the start of the experiment (seen more clearly as the hump in the residual) appears in the 514 and $537 \mathrm{~nm}$ time series only. The cause of this is unknown. 


\section{Azul-2-furoate (185) deprotection kinetics}

\section{Change in the visible absorbance spectrum of Azul-furoate 185 during deprotection}

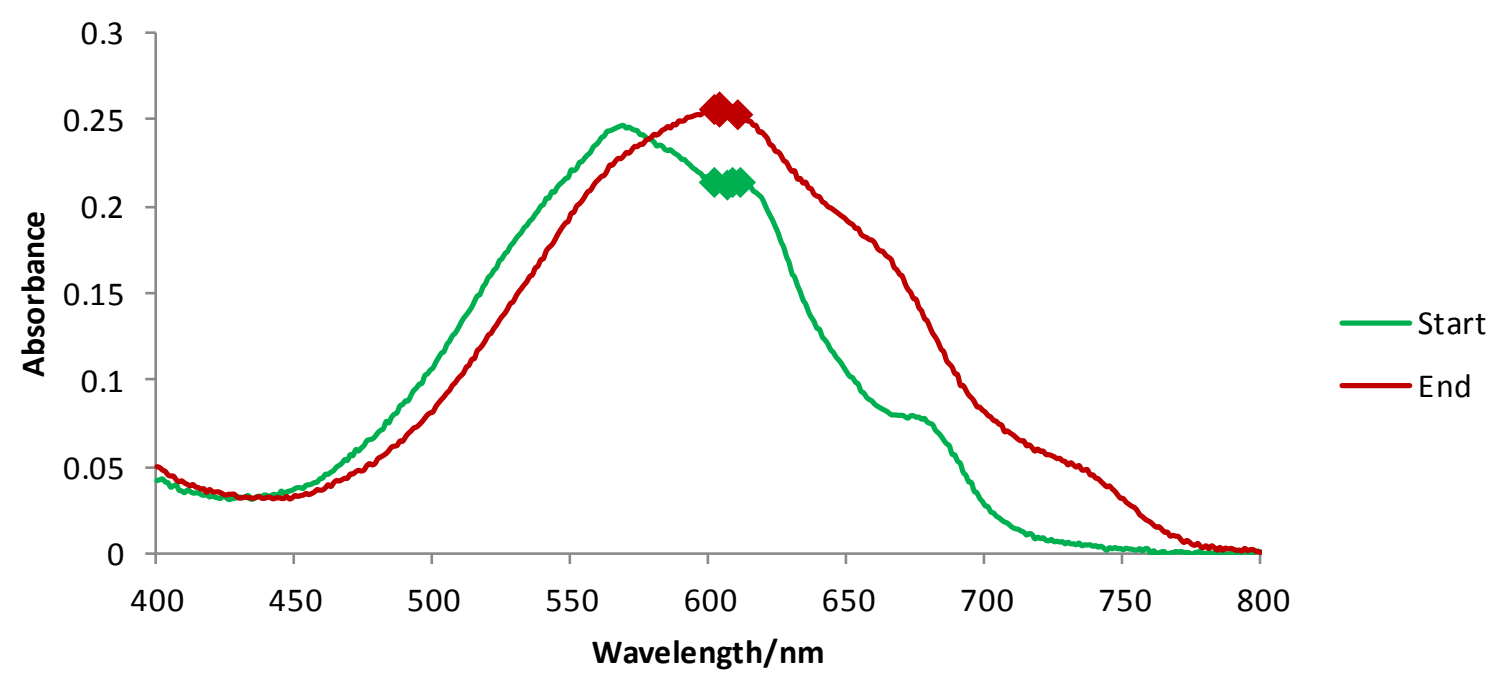

Azul-2-furoate deprotection kinetics at $610 \mathrm{~nm}$

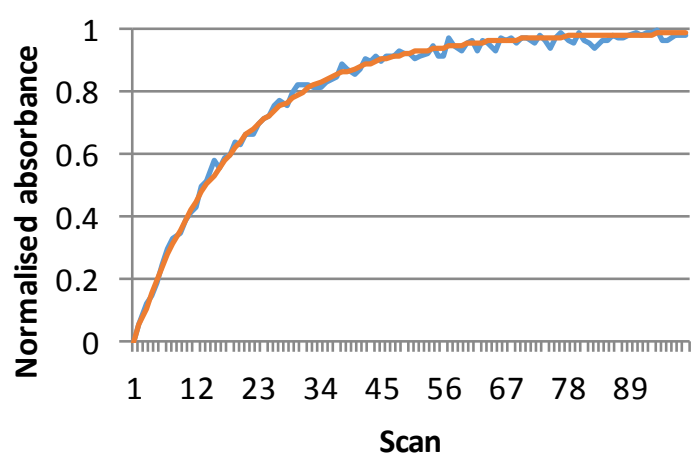

Residual at $610 \mathrm{~nm}$

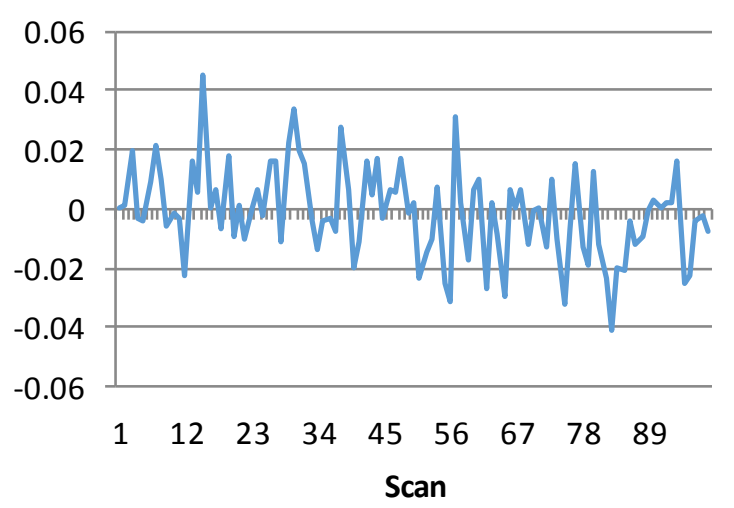

185 (2.1 mg) was dissolved in $7.9 \mathrm{~mL} \mathrm{MeCN}$ to give a $0.001 \mathrm{~mol} \mathrm{~L}^{-1}$ solution. One scan was run every two minutes for 99 scans total.

\begin{tabular}{|l|l|l|}
\hline Measured wavelength & Reaction rate/ Lmol $^{-1} \mathbf{s}^{-1}$ & Sum of Residuals \\
\hline
\end{tabular}

Averaging these values for the reaction rate returns $0.0022 \pm 0.0006 \mathrm{~L} \mathrm{~mol}^{-1} \mathrm{~s}^{-1}$. 


\section{Azul-1-naphthoate (188) deprotection kinetics}

\section{Change in the visible absorbance spectrum of Azul-1-naphthoate 188 during deprotection}

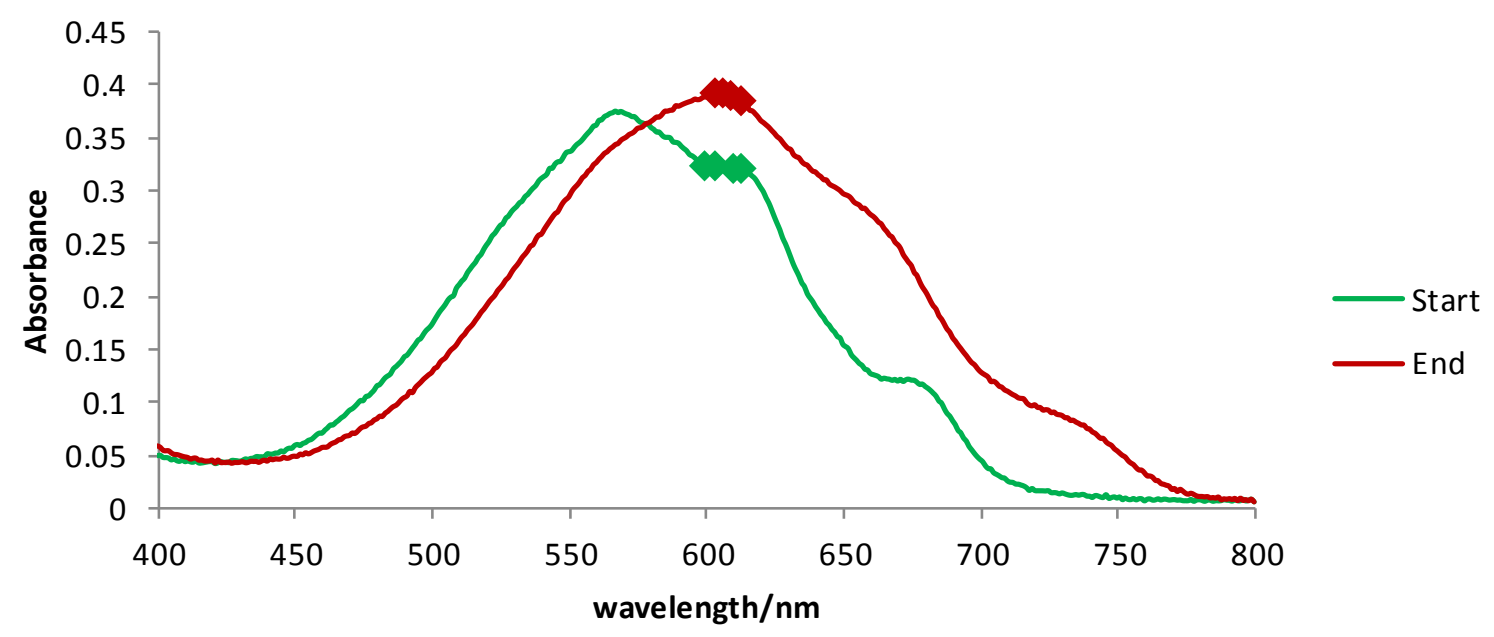

Azul-naphthoate deprotection kinetics at $610 \mathrm{~nm}$

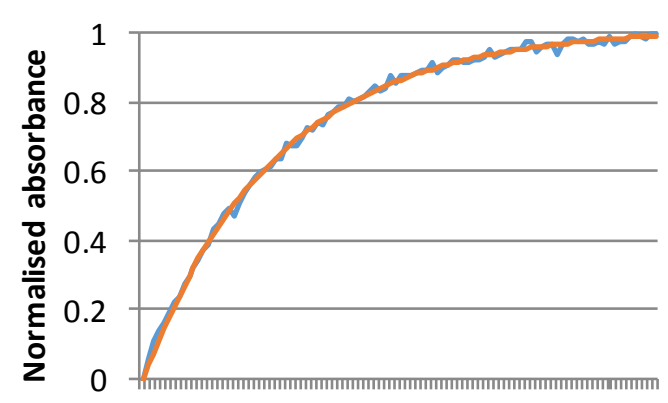

$\begin{array}{llllllllll}1 & 12 & 23 & 34 & 45 & 56 & 67 & 78 & 89 & 100\end{array}$ Scan
Residual at $610 \mathrm{~nm}$

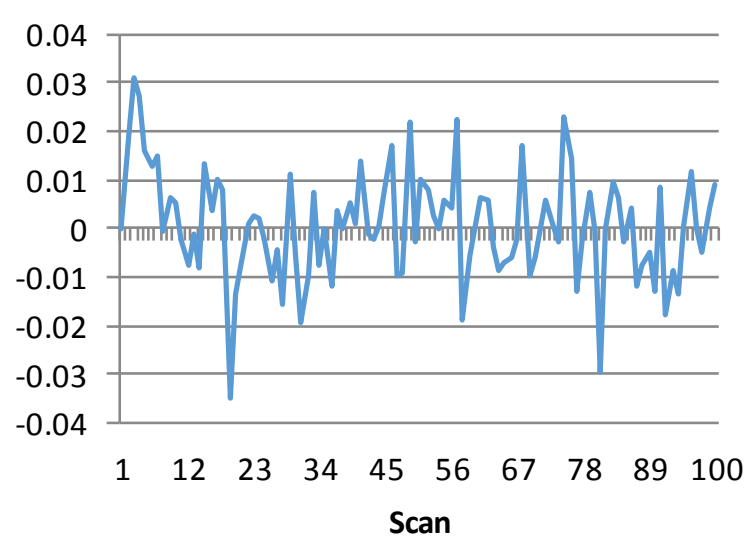

A $0.001 \mathrm{~mol} \mathrm{~L}^{-1}$ solution of $\mathbf{1 8 8}$ in MeCN was made up. One scan was run every two minutes for 100 scans total.

\begin{tabular}{|l|l|l|}
\hline Measured wavelength & Reaction rate/ Lmol-1 $^{-1} \mathbf{s}^{-1}$ & Sum of Residuals \\
\hline $603 \mathrm{~nm}$ & 0.00140 & 0.01 \\
\hline $605 \mathrm{~nm}$ & 0.00143 & 0.008 \\
\hline $610 \mathrm{~nm}$ & 0.00148 & 0.01 \\
\hline $612 \mathrm{~nm}$ & 0.00144 & 0.01 \\
\hline
\end{tabular}

Averaging these values for the reaction rate returns $0.0014 \pm 0.0003 \mathrm{~L} \mathrm{~mol}^{-1} \mathrm{~s}^{-1}$. 


\section{Quantitative NMR - general procedure}

Nitromethane $(0.3 \mathrm{~mL}, 5.60 \mathrm{mmol})$ was added to a volumetric flask and filled to $10 \mathrm{~mL}$ with dry $\mathrm{CDCl}_{3}$ (dried over $4 \AA$ molecular sieves) to make up a dry standard solution of $\mathrm{MeNO}_{2}$ in $\mathrm{CDCl}_{3}(3 \%$ or $0.560 \mathrm{~mol} \mathrm{~L}^{-1}$ ). Molecular sieves were added to the flask. A standard quantity (typically $50 \mu \mathrm{L}$ ) was measured out using a microsyringe and introduced to the mixture to be quantified, followed immediately by regular $\mathrm{CDCl}_{3}$ and mixed thoroughly to ensure complete dissolution of the mixture contents. This solution was then submitted for NMR analysis.

An uncalibrated 90 degree pulse-width was used, with a 15 second relaxation decay and 8 second acquisition time. (The defaults are a 60 degree pulse width, a 0.01 second relaxation decay and a 4.096 second acquisition time)

The raw data were processed in the following order:

Raw integral value $\rightarrow$ Corrected for proton multiplicity $\rightarrow$ Divided by corrected integral of nitromethane $\rightarrow$ Multiplied by no. moles of nitromethane $(0.0279 \mathrm{mmol}$ for a $50 \mu \mathrm{L}$ aliquot $) \rightarrow$ Divided by no. moles of limiting reagent to give the $\%$ yield value. The quantities of conversion were found by dividing the yield value of one compound with that of its progenitor.

The term 'yield value' is used, because these values often exceeded 100\% - and as such did not represent an actual estimate of the yield.

\section{Azul - TBS competitive deprotection studies}

A standardised mixture of cyclohexanemethyl tert-butyldimethylsilyl ether (1 eq.) and Azulcinnamate (1.4 eq.), called 'TBS deprotection mix', was made for use in the following experiments. The extent of reaction was measured through comparison of ${ }^{1} \mathrm{H}$ NMR integration of the starting materials (Azul-cinnamate 178 and TBS-cyclohexanemethanol 221) and the products (6vinylazulene 94, cinnamic acid 177 and cyclohexanemethanol). The salient peaks typically used for NMR integration were:

Azul-cinnamate 178: 8.29 (d, J=10.0 Hz, 2H, H-4,8), 6.41 (d, J=16.0 Hz, 1H, H-12), 4.52 (t, J=7.0 Hz, $2 \mathrm{H}, \mathrm{H}-10), 3.20$ (t, J=7.0 Hz, 2H, H-9).

TBS-cyclohexanemethanol 221: $3.38(\mathrm{~d}, J=6.5 \mathrm{~Hz}, 2 \mathrm{H}, \mathrm{H}-1)$

6-Vinylazulene 94: 8.30 (d, J=10.0 Hz, 2H, H-4), 5.97 (d, J=17.5 Hz, 1H, H-10a), 5.46 (d, J=11.0 Hz, $1 \mathrm{H}, \mathrm{H}-10 \mathrm{~b})$

Cinnamic acid 177: 6.47 (d, J=16.0 Hz, 1H, H-2) 
Cyclohexanemethanol: 3.44 (d, J=6.5 Hz, 2H, H-1)<smiles>CC(C)(C)[Si](C)(C)O[Te]1CCSCC1</smiles>

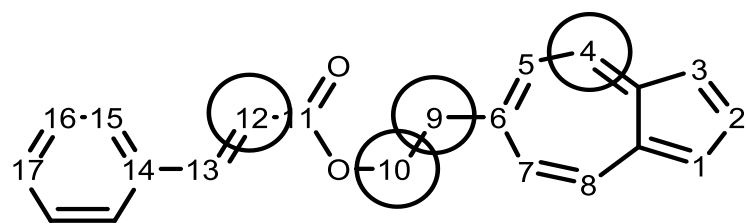

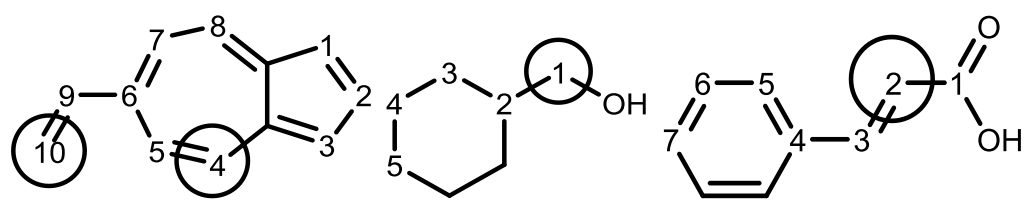

\section{Selective TBS deprotection}

TBS deprotection mix (11.2 mg, equivalent to 0.029 mmol, 1.4 eq. Azul-cinnamate 178 and 0.021 mmol, 1 eq. 221) and PPTS (84.8 mg, 16 eq.) were dissolved in MeCN ( $3 \mathrm{~mL}$ ) and heated at reflux overnight under open air.

A separation was performed with $\mathrm{CH}_{2} \mathrm{Cl}_{2} / \mathrm{H}_{2} \mathrm{O}$ and the organic phase was concentrated by rotary evaporation (taking care not to subject the mixture to high vacuum to avoid loss of cyclohexanemethanol) and this mixture was subjected to NMR analysis. ${ }^{1} \mathrm{H}$ NMR shows the presence of Azul-cinnamate (8.29, 4.52, $3.20 \mathrm{ppm}$ ) and cyclohexanemethanol (3.44 ppm), and the absence of TBS-cyclohexanemethanol and cinnamic acid.

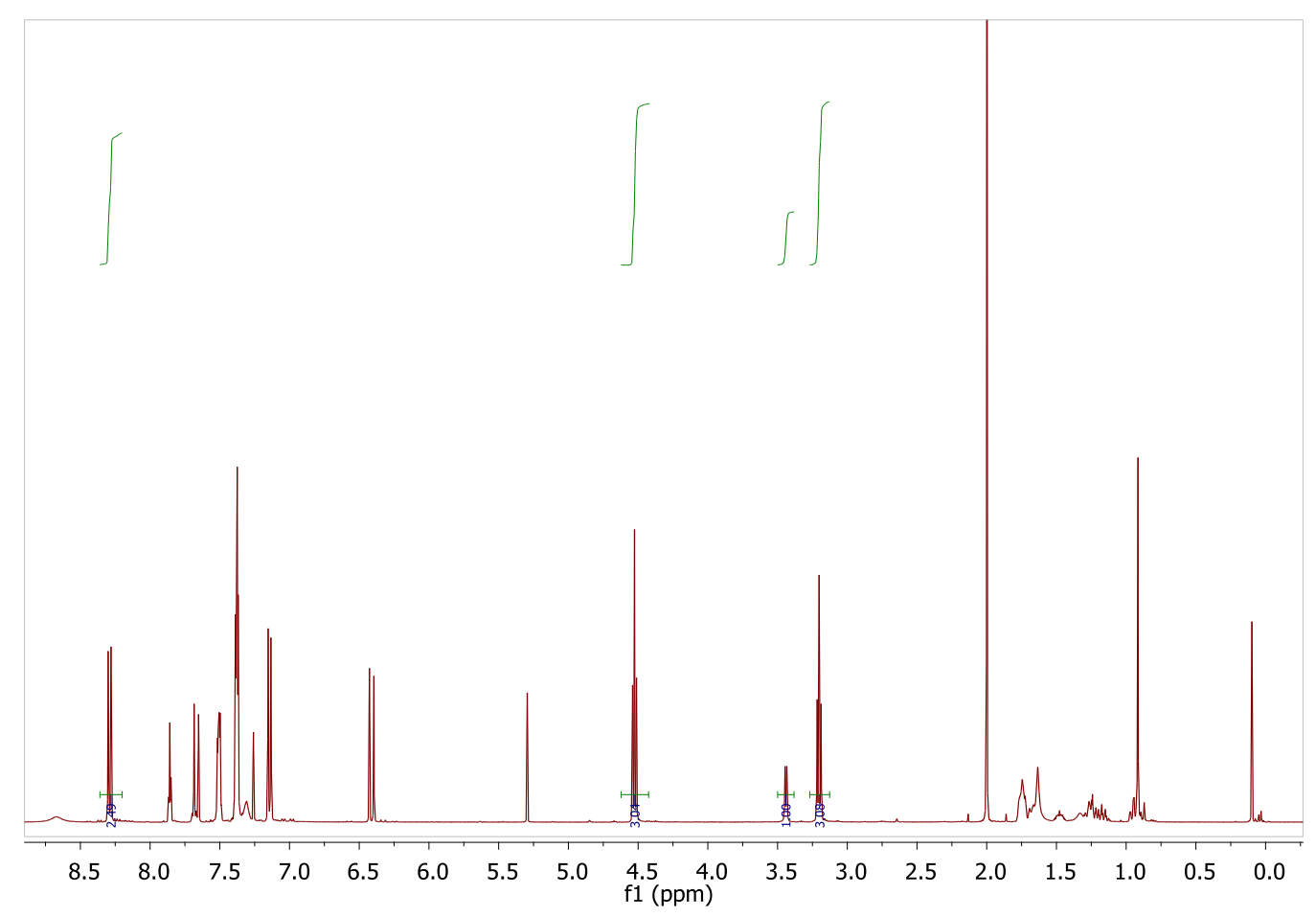

1H NMR spectrum of crude mixture after selective TBS deprotection 


\section{Selective Azul deprotection}

Azul-cinnamate (10.1 mg, $0.033 \mathrm{mmol}, 1$ eq.) and TBS-cyclohexanemethanol ( $8.4 \mathrm{mg}, 0.037 \mathrm{mmol}$, 1.07 eq.) were dissolved in THF ( $3 \mathrm{~mL}$ ), and DBU ( $0.1 \mathrm{~mL}, 0.67 \mathrm{mmol}, 18.5$ eq.) was added. After overnight reaction, a phase separation was performed using $\mathrm{H}_{2} \mathrm{O} / \mathrm{Et}_{2} \mathrm{O}$ with the organic phase being washed 4 times with $\mathrm{H}_{2} \mathrm{O}$. The organic phase was reduced via rotary evaporation under low vacuum (Fraction A). This crude mixture was submitted for quantitative NMR analysis. The ${ }^{1} \mathrm{H}$ NMR spectrum showed the presence of 6-vinylazulene $(5.95,5.45 \mathrm{ppm})$ and TBScyclohexanemethanol (3.39 ppm) and the absence of cyclohexanemethanol and Azul-cinnamate. The aqueous phase was treated with $3 \mathrm{~mL} \mathrm{HCl} \mathrm{(10 \%} \mathrm{aq.)} \mathrm{and} \mathrm{washed} \mathrm{twice} \mathrm{with} \mathrm{CH}_{2} \mathrm{Cl}_{2}$ and the organic fraction was concentrated by rotary evaporation and the crude mixture (Fraction $\mathrm{B}$ ) submitted for quantitative NMR analysis. The $1 \mathrm{H}$ NMR spectrum showed the presence of cinnamic acid (6.46 ppm).

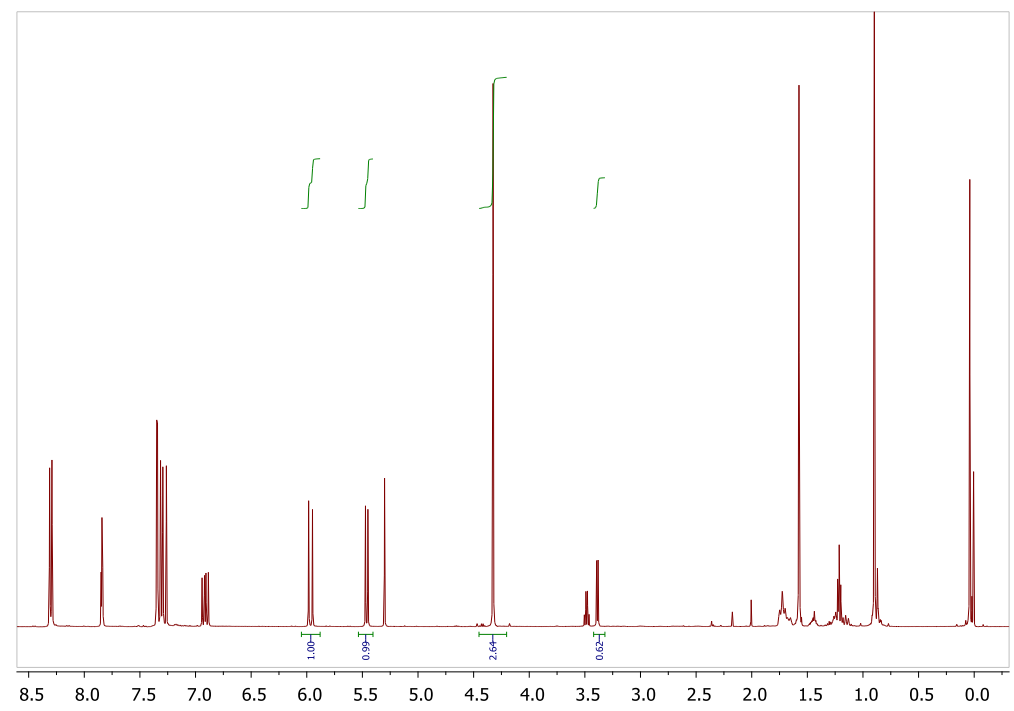

1H NMR spectrum of Fraction $A$

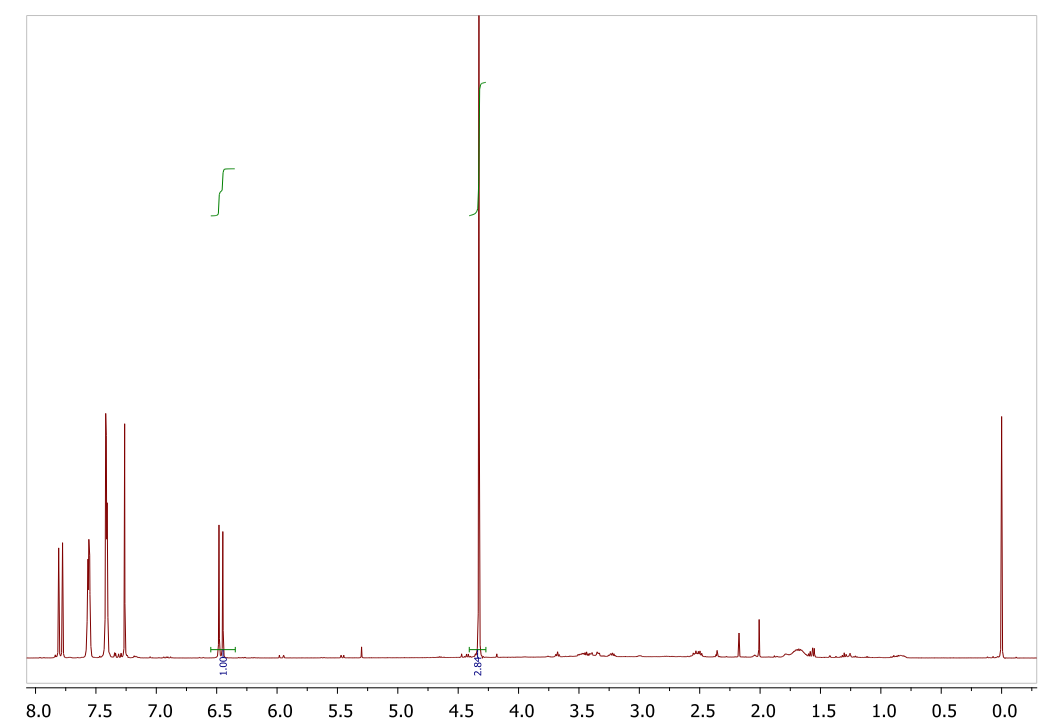

1H NMR spectrum of Fraction $B$ 


\section{Global deprotection}

Azul-cinnamate ( $8.3 \mathrm{mg}, 0.027 \mathrm{mmol}, 1$ eq.) and TBS-cyclohexanemethanol ( $6.2 \mathrm{mg}, 0.027 \mathrm{mmol}, 1$ eq.) were dissolved in THF ( $3 \mathrm{~mL}$ ), and TBAF ( $0.4 \mathrm{~mL}$ of a $1 \mathrm{M}$ solution in THF, $0.4 \mathrm{mmol}, 15$ eq.) was added. A colour change was visible after 10 minutes. After 70 minutes, $1 \mathrm{~mL}$ of $10 \%$ aq. $\mathrm{HCl}$ was added and a phase separation was performed with $\mathrm{CH}_{2} \mathrm{Cl}_{2} / \mathrm{H}_{2} \mathrm{O}$. The organic fraction was concentrated by rotary evaporation and the crude mixture submitted for quantitative NMR analysis. The ${ }^{1} \mathrm{H}$ NMR spectrum shows the presence of 6-vinylazulene $(8.27,5.94,5.43 \mathrm{ppm})$ and TBS-cyclohexanemethanol (3.44 ppm). Neither cinnamic acid nor Azul-cinnamate are observed in the mixture. The doublet at $6.66 \mathrm{ppm}$ is thought to belong to the tetrabutylammonium cinnamate salt.

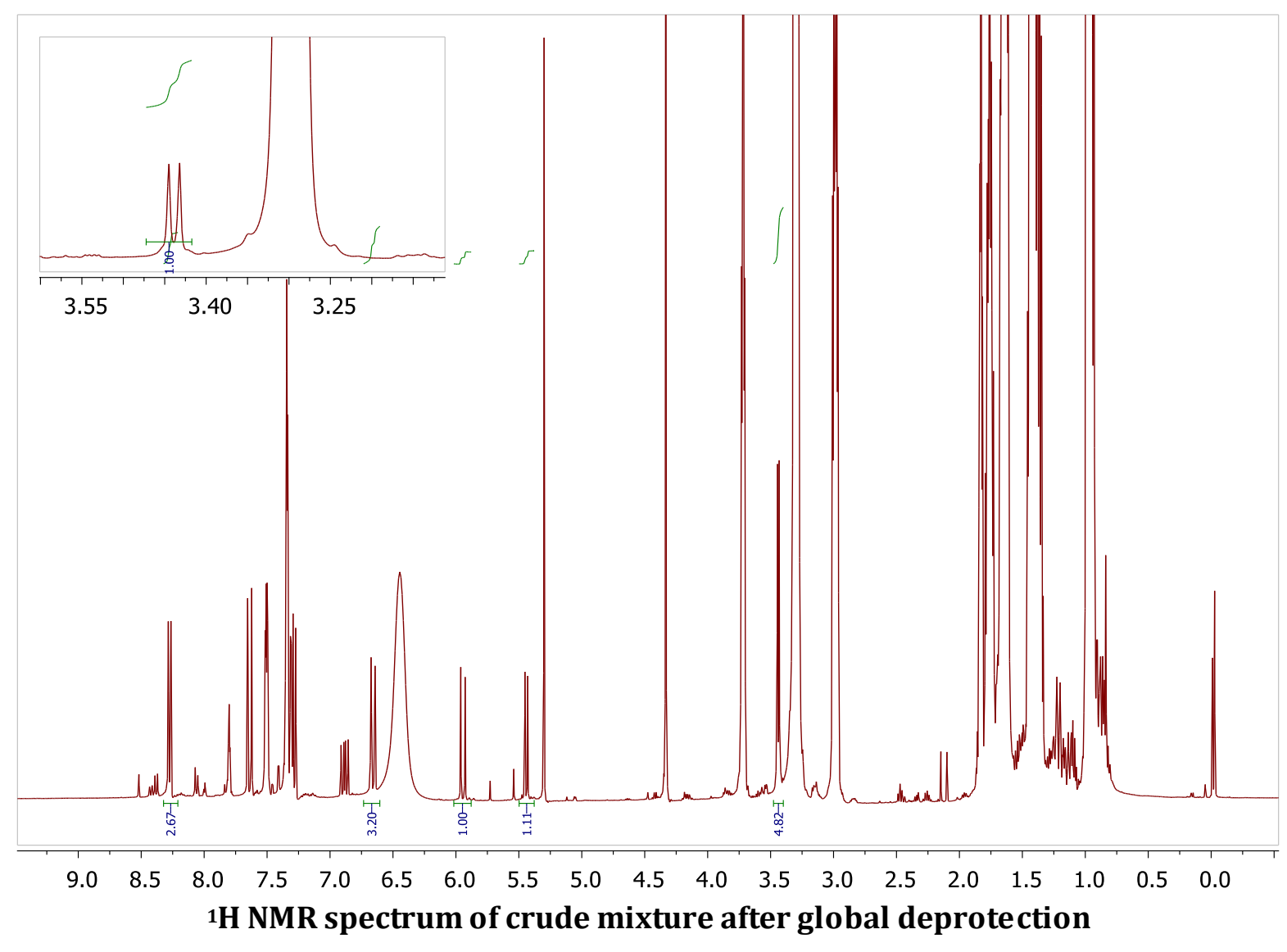




\section{Azul - FMOC competitive deprotection studies}

Extent of reaction was measured through comparison of $1 \mathrm{H}$ NMR integration of the starting material (FMOC-Gly-OAzul 189) and the products (6-vinylazulene, H-Gly-OAzul, dibenzofulvene 1-(fluoren-9-ylmethyl) piperidine). Glycine is almost completely insoluble in $\mathrm{CDCl}_{3}$, but two peaks can be seen at 2.36 and $2.18 \mathrm{ppm}$, possibly corresponding to neutral and protonated glycine. The salient peaks typically used for NMR integration were:

FMOC-Gly-OAzul: 4.25 (t,J=7.0 Hz, 1H, H-15), 3.98 (d, J=5.5 Hz, 2H, H-12).

Dibenzofulvene: 6.09 (s, $2 \mathrm{H}, \mathrm{H}-10)$.

6-Vinylazulene: 5.97 (d, J=17.5 Hz, 1H, H-10a), 5.46 (d, J=11.0 Hz, 1H, H-10b).

H-Gly-OAzul: 4.45 (t, J=7.0 Hz, 2H, H-10), 3.41 (s, 2H, H-12).

1-(fluoren-9-ylmethyl)piperidine: $4.06(\mathrm{t}, \mathrm{J}=8.0 \mathrm{~Hz}, 1 \mathrm{H}, \mathrm{H}-9)$

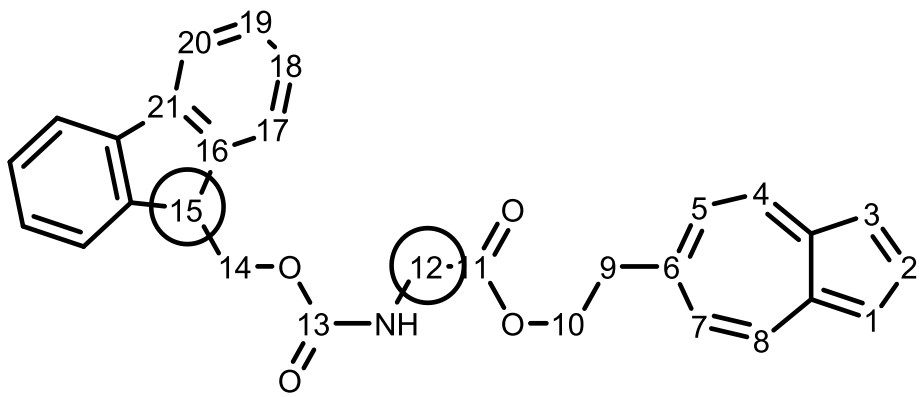

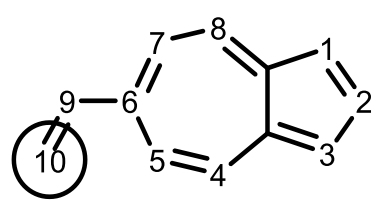<smiles>c1ccc2c(c1)-c1cpsc1-2</smiles>

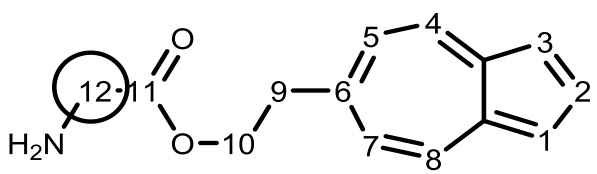

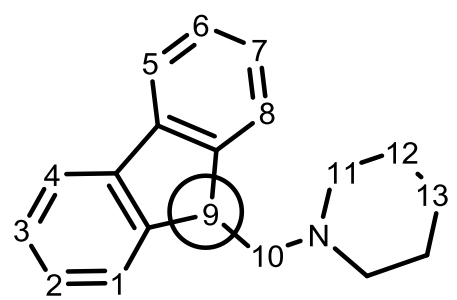

\section{Selective FMOC deprotection}

Fmoc-Gly-OAzul 189 (35.9 mg, 0.079 mmol, 1 eq.) was dissolved in MeCN (3 mL) and piperidine ( $0.1 \mathrm{~mL}, 1 \mathrm{mmol}, 13 \mathrm{eq}$.) was added to this mixture. After 30 minutes, the reaction was complete by TLC. The reaction mixture was concentrated by rotary evaporation and the crude mixture submitted for quantitative NMRanalysis.

The ${ }^{1} \mathrm{H}$ NMR spectrum shows the presence of dibenzylfulvene (6.09 ppm) and 1-(9H-fluoren ylmethyl) piperidine (4.06 ppm) in a 1:5 ratio, as well as the presence of H-Gly-OAzul. No evidence of FMOC-Gly-OAzul 189 was observed. 


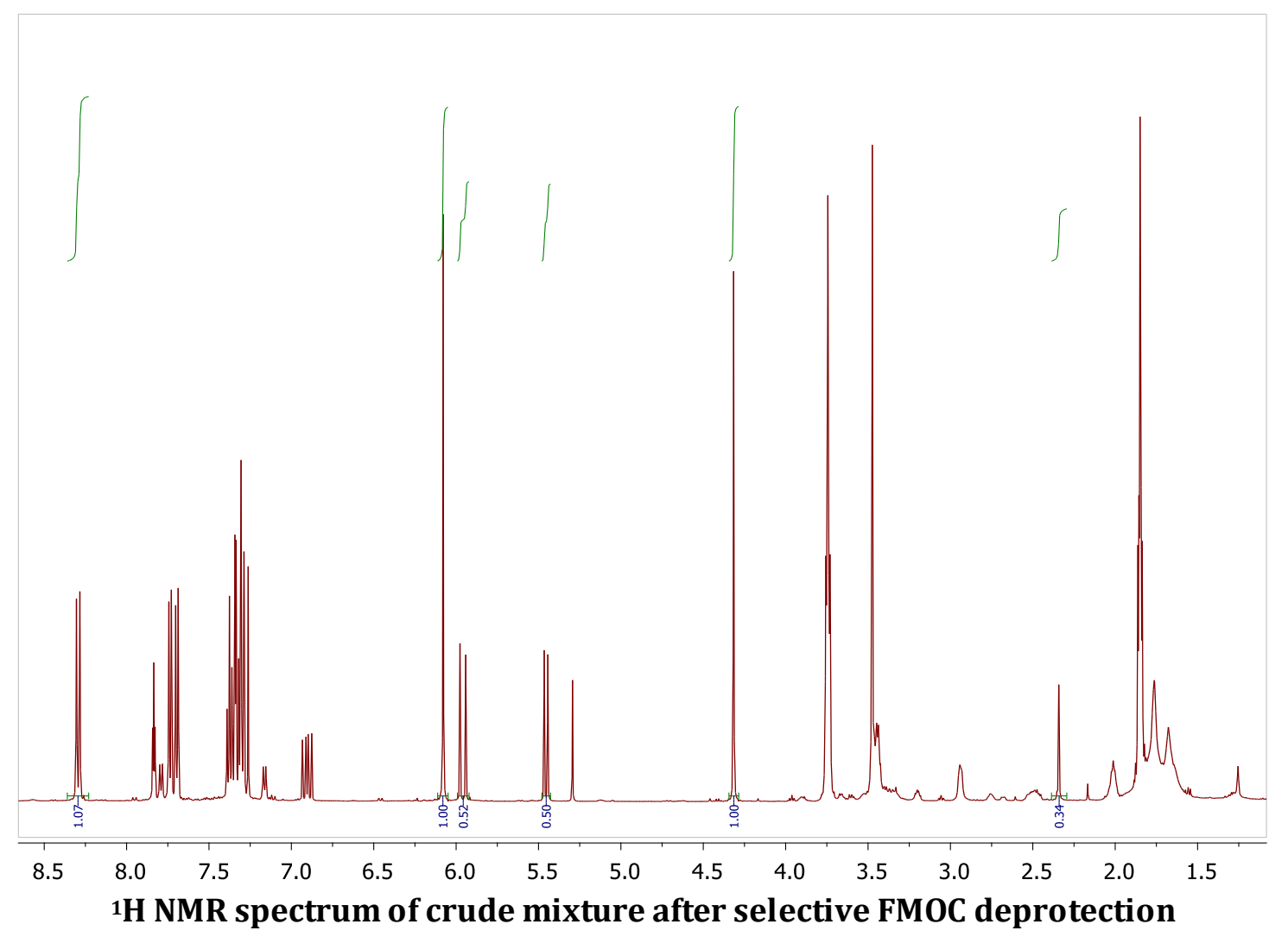

\section{Selective Azul deprotection}

FMOC-Gly-OAzul 189 was activated with oxalyl chloride to form 222 in the method described previously. 222 (37.8 mg, $0.07 \mathrm{mmol}, 1$ eq.) was dissolved in MeCN (3 mL) at $0{ }^{0} \mathrm{C}_{\text {. }} \mathrm{NEt}_{3}(0.1 \mathrm{~mL}$, $0.7 \mathrm{mmol}, 10$ eq.) was added and left to stir for 3 hours at $0{ }^{0} \mathrm{C}$. The reaction mixture was concentrated by rotary evaporation at r.t. and the crude mixture was submitted for quantitative NMR analysis.

The ${ }^{1} \mathrm{H}$ NMR spectrum shows the presence of a singlet at $6.08 \mathrm{ppm}$ corresponding to dibenzylfulvene overlapped with a doublet at 6.07 belonging to the vinylazulene species. Integration of these two peaks gives a 1:4 ratio. Complete loss of the methylenes at 4.50 and 3.25 ppm indicate complete Azul deprotection, so the 1:4 ratio indicates 25\% FMOC deprotection. 


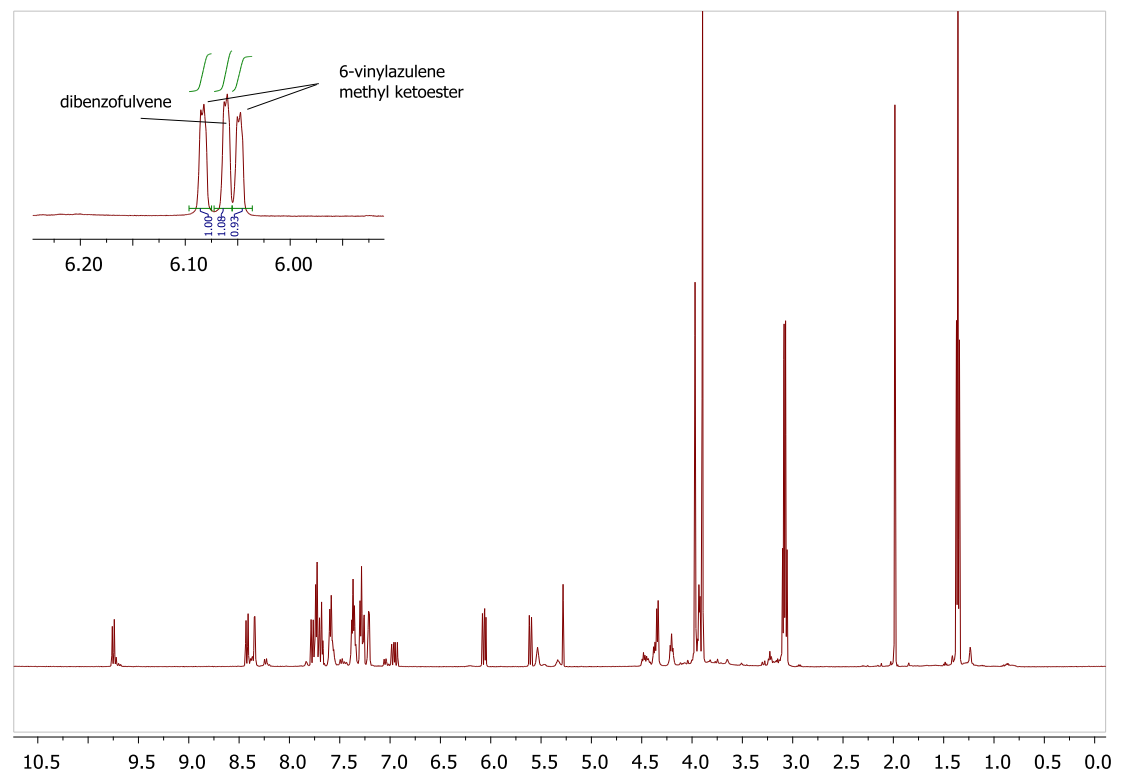

1H NMR spectrum of crude mixture after selective Azul deprotection

\section{Global deprotection}

FMOC-Gly-OAzul (23.1 mg, $0.05 \mathrm{mmol}, 1$ eq.) was dissolved in THF ( $3 \mathrm{~mL}$ ) and DBU (0.1 mL, 0.66 mmol, 13 eq.) was added. After 9 hours, the reaction mixture had changed colour from indigo to blue. $\mathrm{HCl}$ ( $1 \mathrm{~mL}, 10 \%$ aqueous solution) was added and a phase separation was performed with $\mathrm{CH}_{2} \mathrm{Cl}_{2} / \mathrm{H}_{2} \mathrm{O}$, followed by rotary evaporation of the organic fraction. The crude mixture was submitted for quantitative NMR analysis.

The $1 \mathrm{H}$ NMR spectrum shows the presence of dibenzylfulvene $(6.08 \mathrm{ppm})$ and 6 -vinylazulene $(5.97,5.45 \mathrm{ppm})$ in a 1:1 ratio. A peak at 2.34 possibly corresponding to glycine was also observed. No trace of FMOC-Gly-OAzul or any other partial deprotection products was observed.

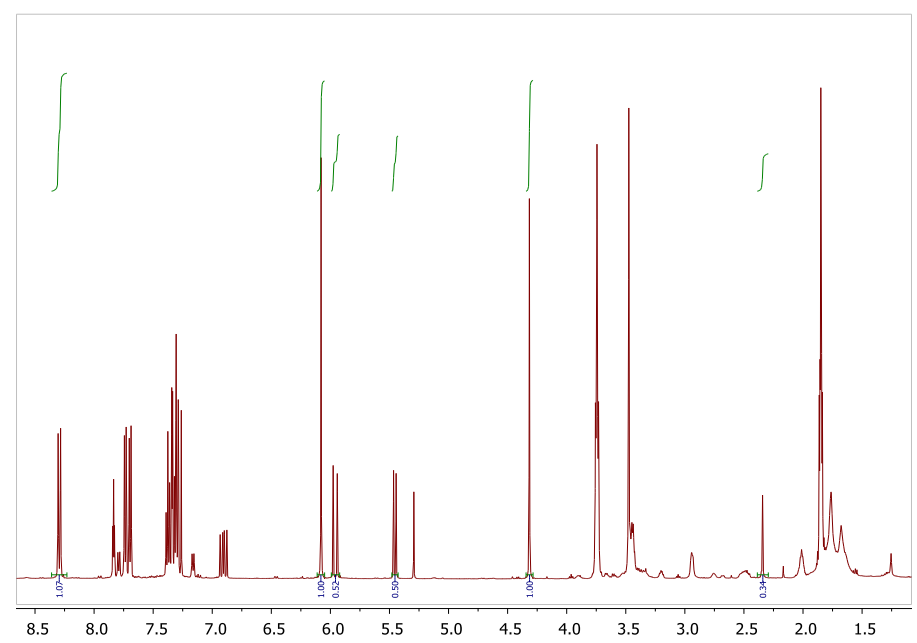

1H NMR spectrum of crude mixture after global deprotection 


\section{Azul - MOM competitive deprotection studies}

A standardised mixture of Azul-cinnamate 178 (69.7 mg, 1 eq.) and MOM-protected cetyl alcohol 223 (71.2 mg, 1.07 eq.), named 'MOM deprotection mix' was made for use in the following experiments. Extent of reaction was measured through comparison of ${ }^{1} \mathrm{H}$ NMR integration of the starting materials (Azul-cinnamate 178 and cetyl MOM ether 223) and the products (6vinylazulene 94, cinnamic acid and cetyl alcohol). The salient peaks typically used for NMR integration were:

Azul-cinnamate 178: 8.29 (d, J=10.0 Hz, 2H, H-4), 6.41 (d, J=16.0 Hz, 1H, H-12), 4.52 (t, J=7.0 Hz, $2 \mathrm{H}, \mathrm{H}-10), 3.20$ (t, J=7.0 Hz, 2H, H-9).

6-vinylazulene 94: 8.30 (d, J=10.0 Hz, 2H, H-4), 5.97 (d, J=17.5 Hz, 1H, H-10a), 5.46 (d, J=11.0 Hz, $1 \mathrm{H}, \mathrm{H}-10 \mathrm{~b})$.

Cinnamic acid 177: 6.47 (d, J=16.0 Hz, 1H, H-2)

Cetyl MOM ether 223: $4.62(\mathrm{~s}, 2 \mathrm{H}$, acetal), $3.52(\mathrm{t}, J=6.5 \mathrm{~Hz}, 2 \mathrm{H}, \mathrm{H}-1), 3.36(\mathrm{~s}, 3 \mathrm{H}$, methoxy)

Cetyl alcohol: 3.64 (q, J=6.5 Hz, 2H, H-1)
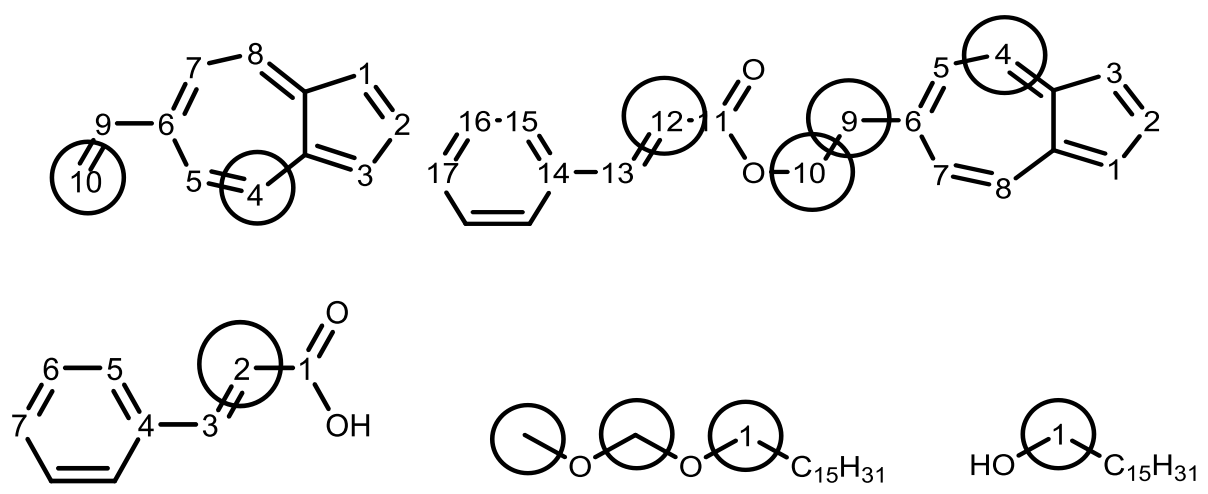

\section{Selective MOM deprotection}

MOM-deprotection mix ( $43.1 \mathrm{mg}$, equivalent to $0.070 \mathrm{mmol}$ Azul cinnamate $\mathbf{1 7 8}$ and $0.076 \mathrm{mmol}$ cetyl MOM ether 223) and PPTS (72.9 mg, $0.29 \mathrm{mmol}, 4.1 \mathrm{eq}$.) were added to a RBF fitted with condenser and dissolved in ethanol ( $3 \mathrm{~mL})$ and MeCN $(1 \mathrm{~mL})$. The reaction was heated at reflux overnight. A separation was performed using $\mathrm{CH}_{2} \mathrm{Cl}_{2}$ /sat. aq. $\mathrm{NaHCO}_{3}$ and the organic layer was submitted for quantitative NMR.

${ }^{1} \mathrm{H}$ NMR integration shows the presence of cetyl alcohol (3.64 ppm) and the absence of cetyl MOM ether 223. Azul-cinnamate $178(6.42,4.53,3.21 \mathrm{ppm})$ is present, and no evidence for other azulene species can be seen. 


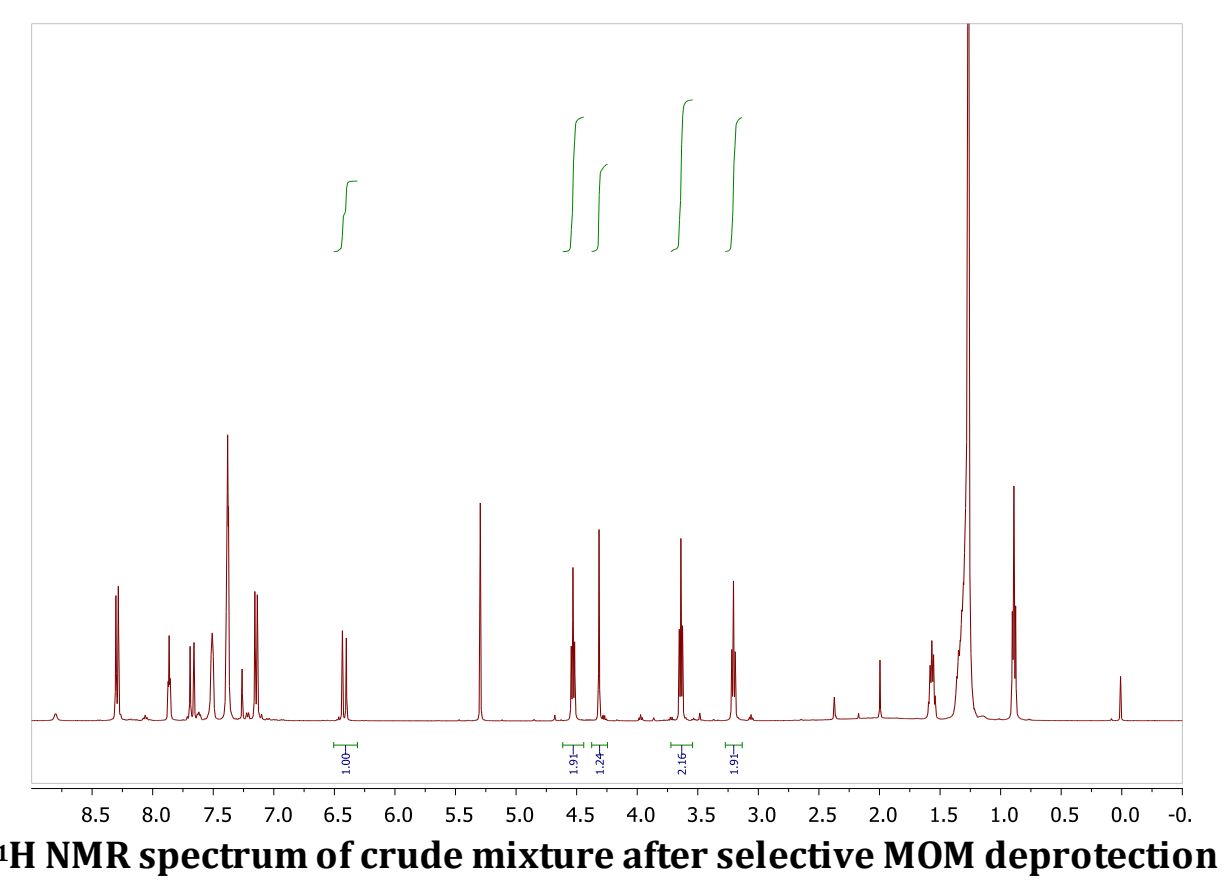

\section{Selective Azul deprotection}

MOM deprotection mix (15.6 mg, equivalent to $0.025 \mathrm{mmol}$ Azul-cinnamate $\mathbf{1 7 8}$ and $0.027 \mathrm{mmol}$ cetyl MOM ether 223) was dissolved in THF ( $3 \mathrm{~mL}$ ), and DBU ( $0.1 \mathrm{~mL}, 0.66 \mathrm{mmol})$ was added under open air. The reaction was stirred for 19 hours. 10 drops of $10 \% \mathrm{HCl}$ (aq.) were added to the reaction mixture, and a separation was performed in $\mathrm{CH}_{2} \mathrm{Cl}_{2} / \mathrm{H}_{2} \mathrm{O}$. The organic layer was concentrated by rotary evaporation and submitted for quantitative NMR.

${ }^{1} \mathrm{H}$ NMR revealed the presence of 6-vinylazulene 94 (8.30, 5.97, $\left.5.46 \mathrm{ppm}\right)$, cinnamic acid (6.47 ppm) and cetyl MOM ether (3.52 ppm) and the absence of cetyl alcohol and Azul-cinnamate.

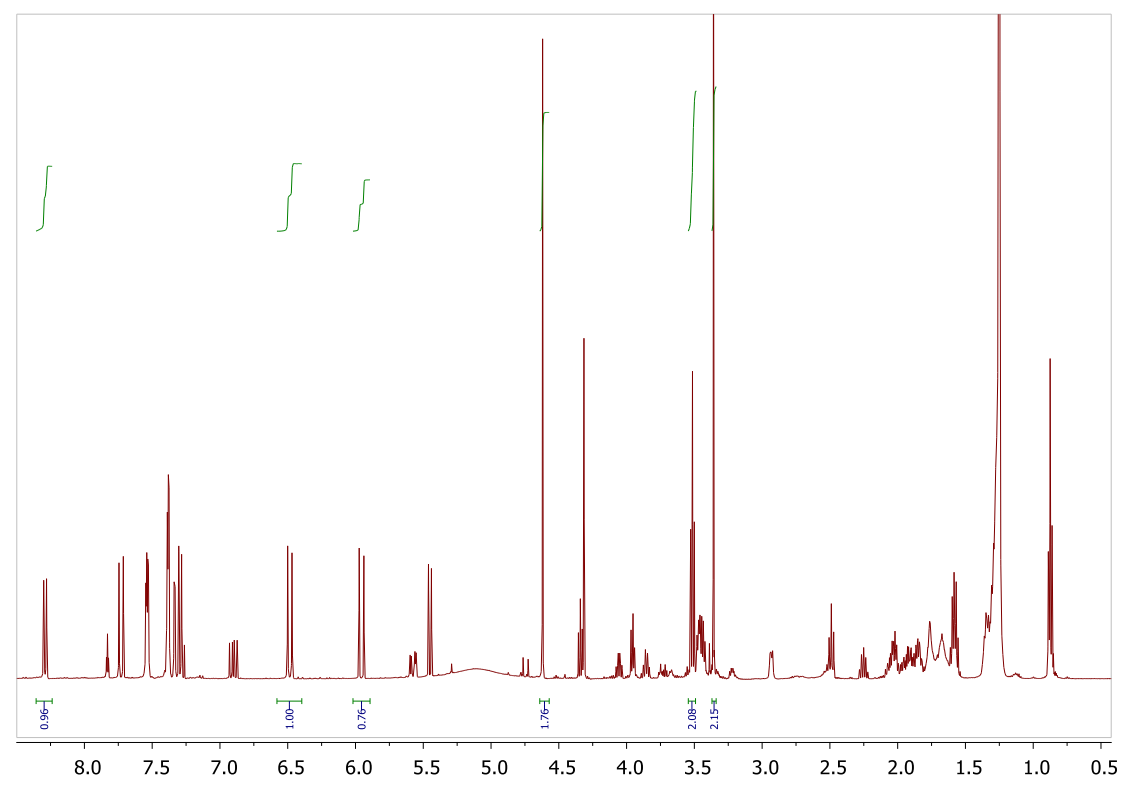

1H NMR spectrum of crude mixture after selective Azul deprotection 


\section{Azul com patibility with high-temperature Suzuki cross-coupling}

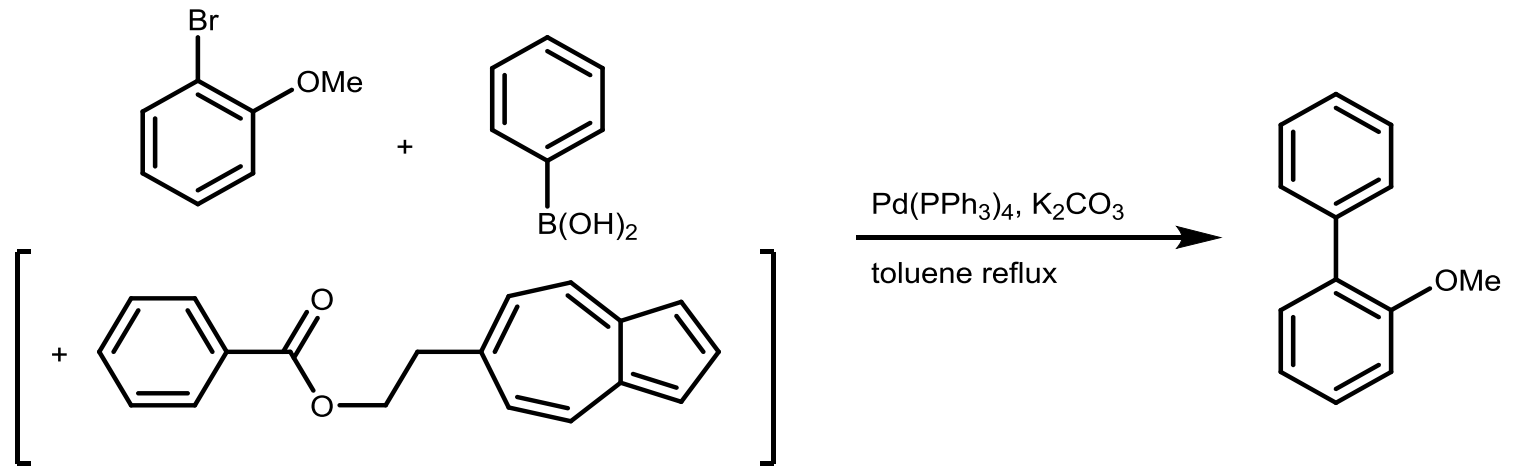

Phenylboronic acid 224 (69.2 mg), palladium tetrakis[triphenylphosphine] (64.5 mg), and potassium carbonate (130.8 $\mathrm{mg}$ ) were added together and the powders mixed thoroughly with a spatula. This mixture was partitioned into two portions - the control (135 mg) and the Azulbenzoate-containing sample (128.5 mg).

To the control sample (containing a calculated 1.2 eq. phenylboronic acid, 0.11 eq. $\mathrm{Pd}\left(\mathrm{PPh}_{3}\right)_{4}$ and 2 eq. $\left.\mathrm{K}_{2} \mathrm{CO}_{3}\right)$, toluene (3.2 mL) and 2-bromoanisole 225 ( $0.03 \mathrm{~mL}, 0.24 \mathrm{mmol}, 1$ eq. $)$ were added, and the reaction vessel was fitted with a reflux condenser and heated at reflux for 2 hours, during which time the reaction mixture changed colour from yellow to red to brown. A phase separation was performed with $\mathrm{CH}_{2} \mathrm{Cl}_{2} / \mathrm{H}_{2} \mathrm{O}$ and the organic fraction was concentrated by rotary evaporation and subjected to quantitative NMR.

To a RBF containing Azul-benzoate 182 (70.5 mg, $0.25 \mathrm{mmol}, 1.06$ eq.) and the powdered mixture described above (containing a calculated 1.15 eq. phenylboronic acid, 0.11 eq. $\mathrm{Pd}\left(\mathrm{PPh}_{3}\right)_{4}$, and 1.9 eq. $\left.\mathrm{K}_{2} \mathrm{CO}_{3}\right)$, toluene $(3.2 \mathrm{~mL})$ and 2-bromoanisole $(0.03 \mathrm{~mL}, 1$ eq.) were added, and the reaction vessel was fitted with a reflux condenser and heated at reflux for 2 hours, during which time a colour change from indigo to purple and back to indigo was observed. A phase separation was performed with $\mathrm{CH}_{2} \mathrm{Cl}_{2} / \mathrm{H}_{2} \mathrm{O}$, and the organic fraction was concentrated by rotary evaporation and subjected to quantitative NMR.

Integration of the relevant peaks in the ${ }^{1} \mathrm{H}$ NMR spectrum of the control reaction returned a $71 \%$ yield value of 2-phenylanisole (3.84 ppm) and a 30\% yield value for 2-bromoanisole (3.91 ppm), which when normalised give a conversion of $70 \%$.

Integration of the relevant peaks in the ${ }^{1} \mathrm{H}$ NMR of the Azul-benzoate-spiked reaction returned a $68 \%$ yield value of 2-phenylanisole and an $81 \%$ yield value for 2 -bromoanisole. When normalised, this gives a conversion of $45 \%$ from 2-bromoanisole to 2-phenylanisole. A yield value of $134 \%$ of Azul-benzoate $(8.34,4.65,3.30 \mathrm{ppm})$ was obtained, along with $27 \%$ 6-vinylazulene $(5.97,5.45$ ppm). When normalised, this indicates a 17\% conversion of Azul-benzoate to 6-vinylazulene. 


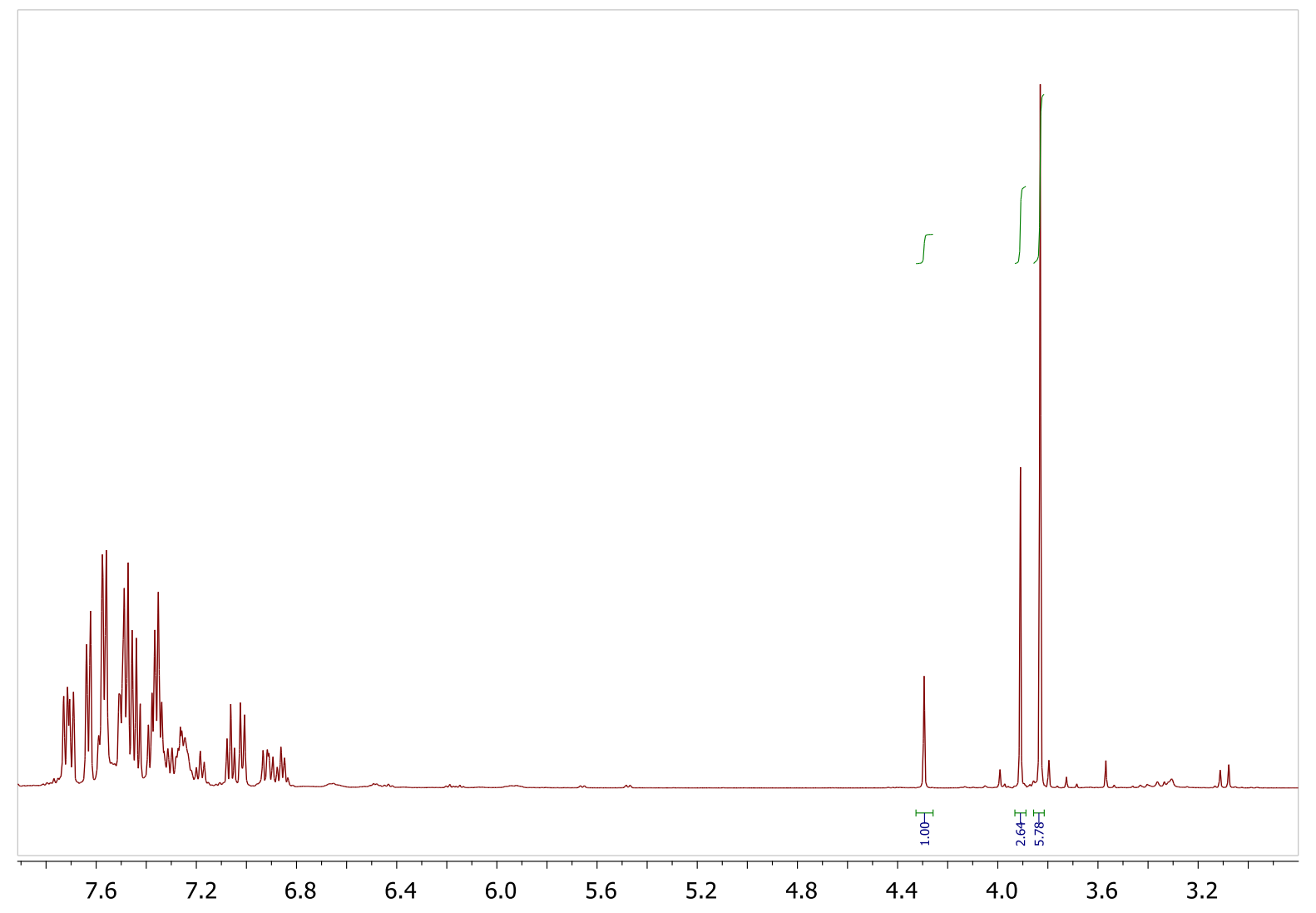

Quantitative 1H NMR spectrum of reaction spiked with Azul-benzoate

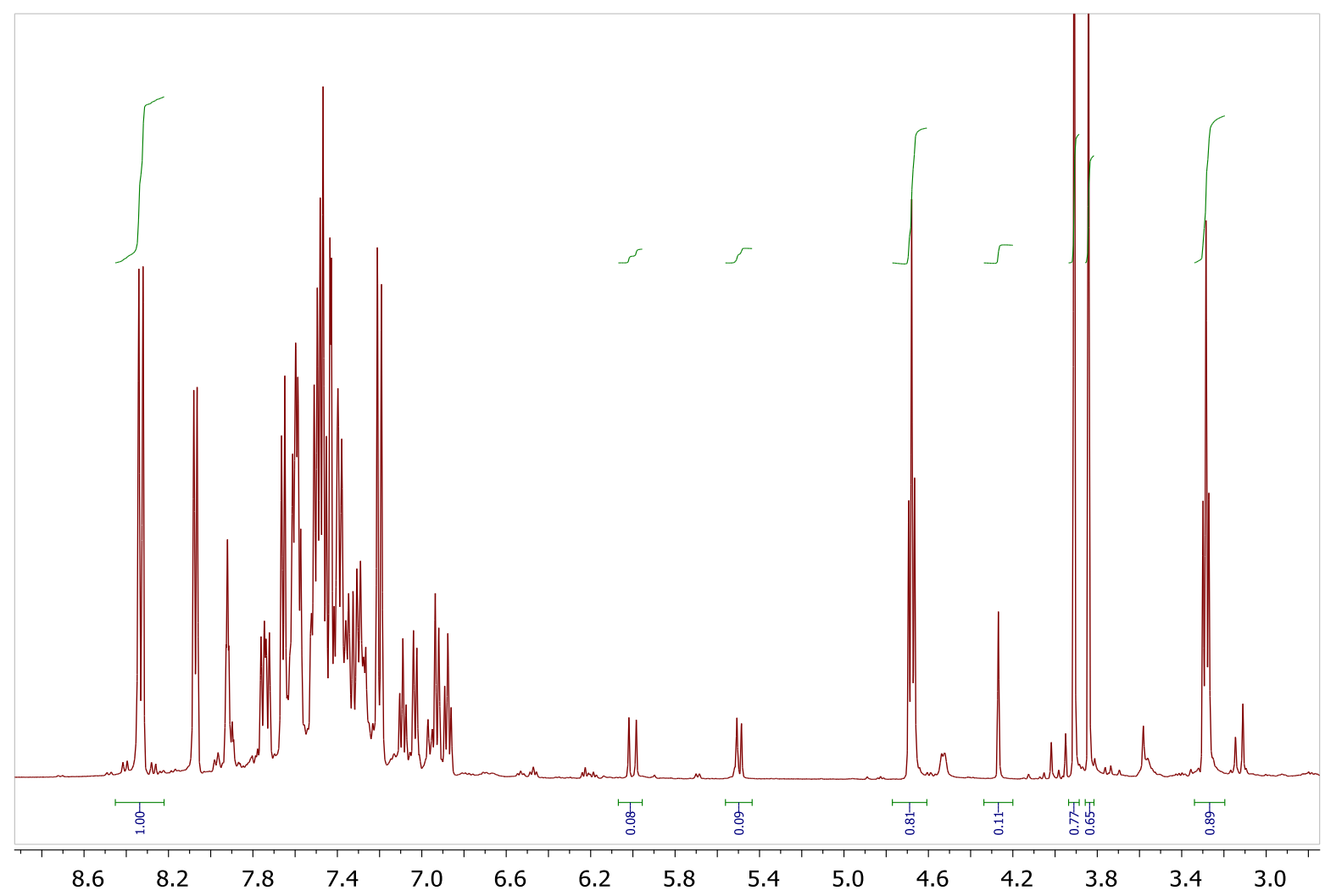




\section{Azul com patibility with low-temperature Suzuki cross-coupling}<smiles>O=C(OCCc1ccc2cccc-2cc1)c1ccccc1</smiles>

Phenylboronic acid 224 (71.5 mg), palladium acetate (15.5 mg), $p$-iodonitrobenzene 227 (108.6 $\mathrm{mg}$ ) and potassium carbonate (157.7 $\mathrm{mg}$ ) were added together and mixed as powders with a spatula. This mixture was partitioned into two portions, the control (185.4 mg) and the Azulbenzoate-spiked sample (167.9 mg).

To the control sample (containing a calculated 1.34 eq. phenylboronic acid, 0.16 eq. palladium acetate, 1 eq. $p$-iodonitrobenzene, and 2.6 eq. potassium carbonate), methanol ( $3 \mathrm{~mL}$ ) was added and the reaction was left to stir at r.t. under open air for three days. A separation was performed using $\mathrm{CH}_{2} \mathrm{Cl}_{2} / \mathrm{H}_{2} \mathrm{O}$, and the organic layer was concentrated by rotary evaporation and submitted for quantitative NMR.

To a RBF containing Azul-benzoate 182 (75.3 mg, $0.272 \mathrm{mmol}, 1.3 \mathrm{eq}$.) and the powdered mixture described above (containing a calculated 1.34 eq. phenylboronic acid, 0.16 eq. palladium acetate, 1 eq. $p$-iodonitrobenzene and 2.61 eq. potassium carbonate), methanol ( $3 \mathrm{~mL}$ ) was added and the reaction was left to stir at r.t. under open air for three days. A separation was performed using $\mathrm{CH}_{2} \mathrm{Cl}_{2} / \mathrm{H}_{2} \mathrm{O}$, and the organic layer was submitted for quantitative NMR.

Integration of the relevant peaks in the ${ }^{1} \mathrm{H}$ NMR of the control reaction returned a yield value of $123 \%$ of $p$-nitrobiphenyl $(8.30,7.75 \mathrm{ppm})$, with no detectable amount of $p$-iodonitrobenzene (7.93 ppm).

Integration of the relevant peaks in the ${ }^{1} \mathrm{H}$ NMR of the Azul-benzoate-spiked reaction returned a yield value of $125 \%$ of $p$-nitrobiphenyl $(7.75 \mathrm{ppm})$, with no detectable amount of $p$ iodonitrobenzene (7.93 ppm). A yield value of $98 \%$ for Azul-benzoate $(8.05,7.90,7.20,4.65,3.25$ ppm) was returned, with a yield value of 9\% for 6-(2-hydroxyethyl)azulene (90), which when normalised givesa 92\% retention of Azul-benzoate. 


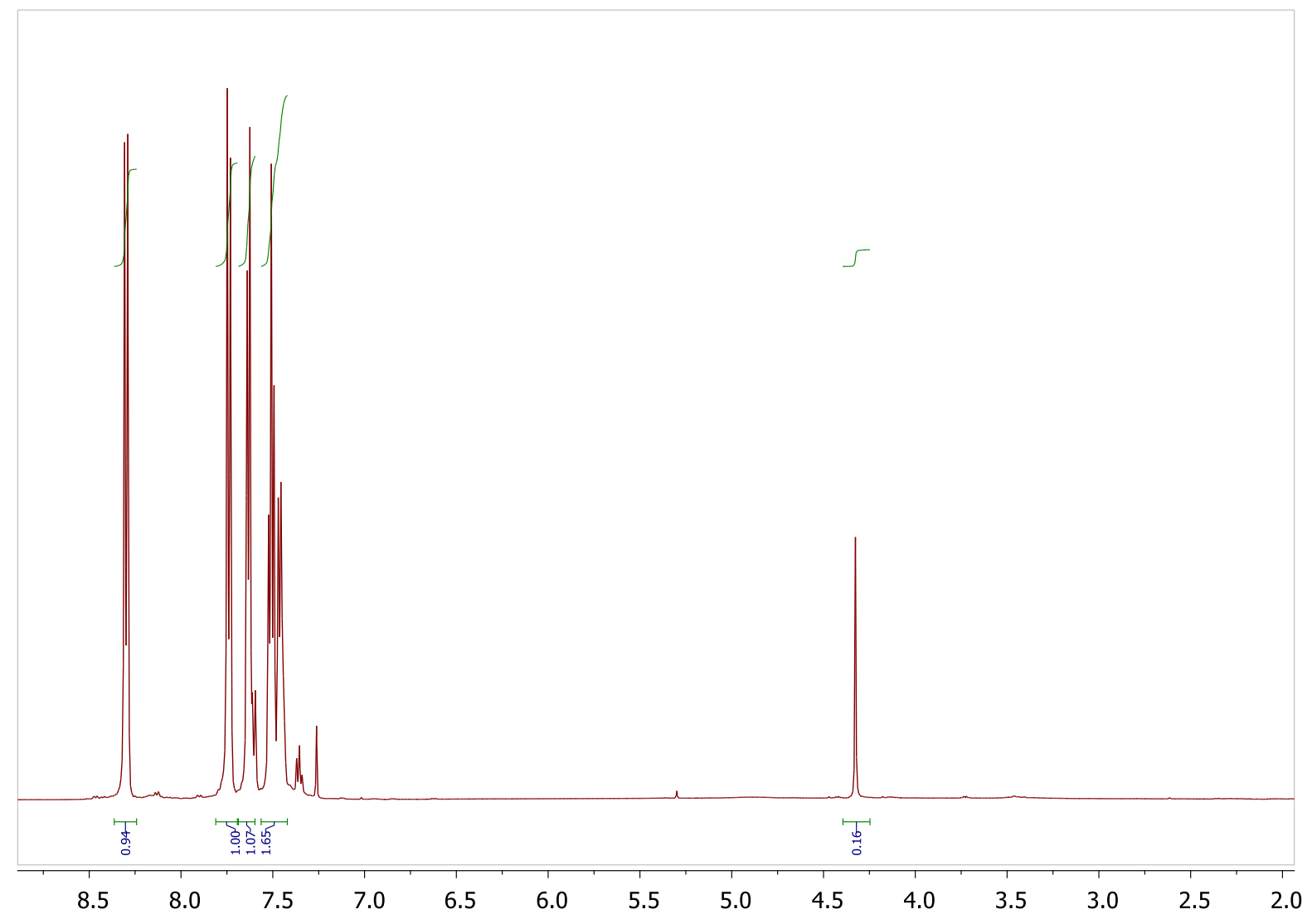

Quantitative 1 H NMR spectrum of reaction spiked with Azul-benzoate

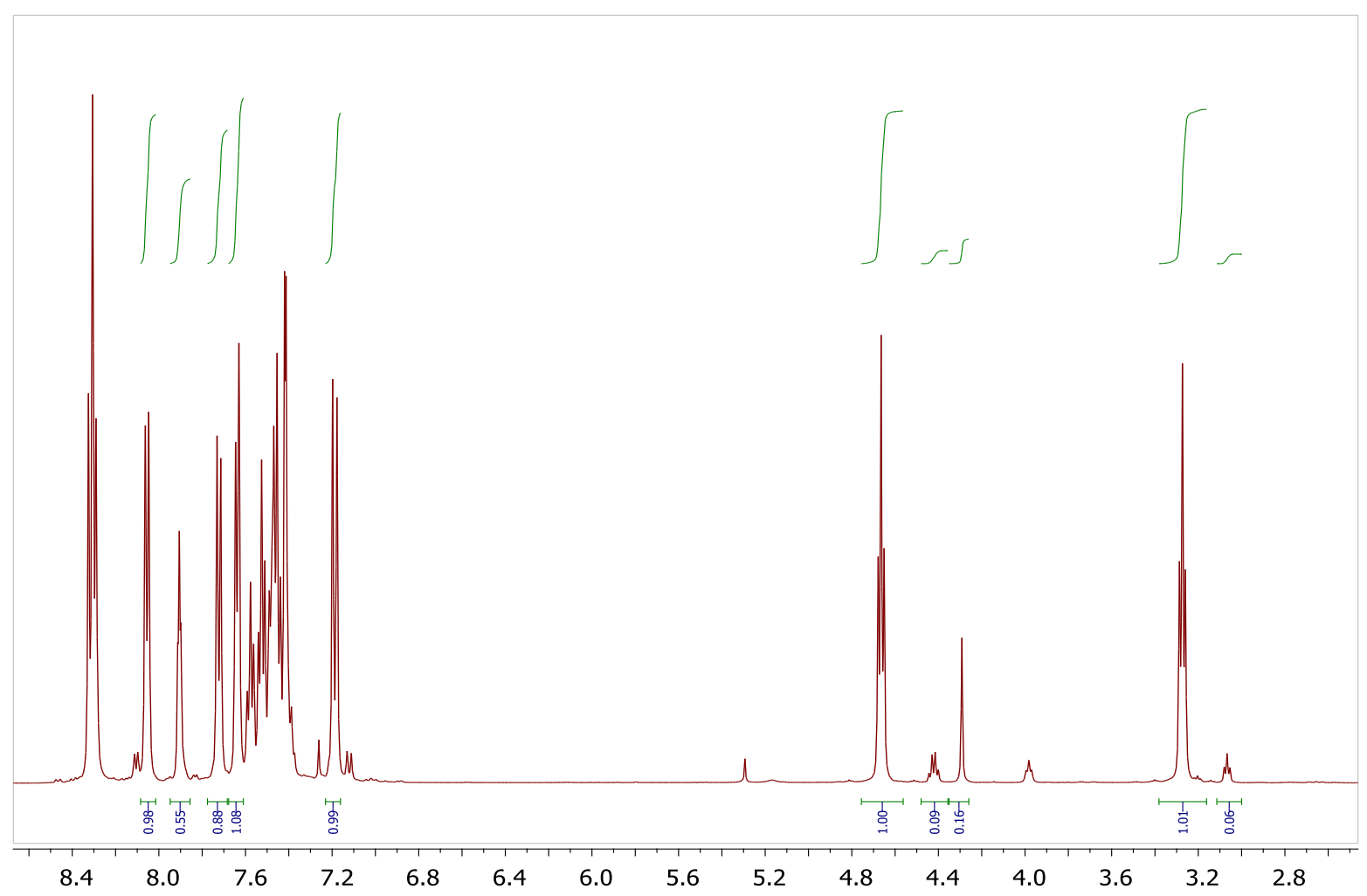




\section{Azul com patibility with $\mathrm{NaBH}_{4}$ reduction}<smiles>O=C(OCCc1ccc2cccc-2cc1)c1ccccc1</smiles>

Benzophenone 229 (146.2 mg) and $\mathrm{NaBH}_{4}(85.7 \mathrm{mg}$ ) were added together and mixed as solids. This mixture was partitioned into two portions, the control $(112.3 \mathrm{mg})$ and the Azul-benzoatespiked sample (119.6 mg).

To the control sample (containing a calculated 1 eq. benzophenone and 2.8 eq. $\mathrm{NaBH}_{4}$ ), methanol $(2 \mathrm{~mL})$ was added and the reaction was allowed to stir for 5 hours. A separation was performed using $\mathrm{CH}_{2} \mathrm{Cl}_{2} / \mathrm{H}_{2} \mathrm{O}$, and the organic layer was submitted for quantitative NMR.

To a sample containing Azul-benzoate 182 (111.8 mg, 1.04 eq.) and the mixture described above (containing a calculated 1 eq. benzophenone and 2.8 eq. $\mathrm{NaBH}_{4}$ ), methanol $(2 \mathrm{~mL}$ ) was added and the reaction was allowed to stir for 5 hours. A separation was performed using $\mathrm{CH}_{2} \mathrm{Cl}_{2} / \mathrm{H}_{2} \mathrm{O}$, and the organic layer was submitted for quantitative NMR.

Integration of the relevant peaks in the $1 \mathrm{H}$ NMR of the control reaction returned a yield value of $113 \%$ of diphenylmethanol $(7.75,7.60,5.85 \mathrm{ppm})$, with no observable benzophenone (7.79 ppm).

Integration of the relevant peaks in the ${ }^{1} \mathrm{H}$ NMR of the Azul-benzoate-spiked reaction returned a yield value of $113 \%$ of diphenylmethanol ( $5.85 \mathrm{ppm}$ ) with no observable benzophenone. A yield value of $116 \%$ of Azul-benzoate (8.32, 8.05, 4.68, $3.27 \mathrm{ppm}$ ) was obtained, with no other azulenebased species visible. 


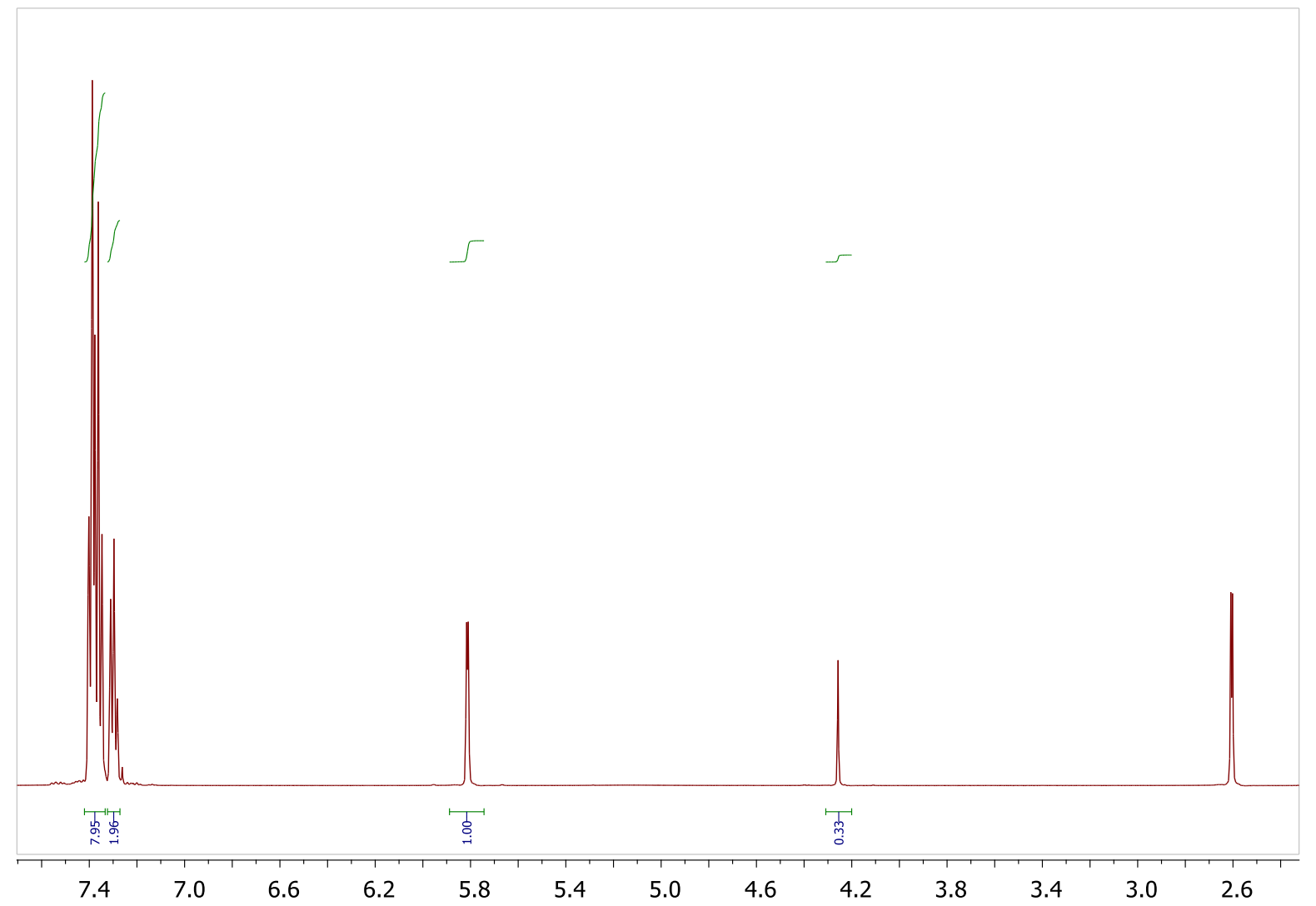

Quantitative 1H NMR spectrum of reaction spiked with Azul-benzoate

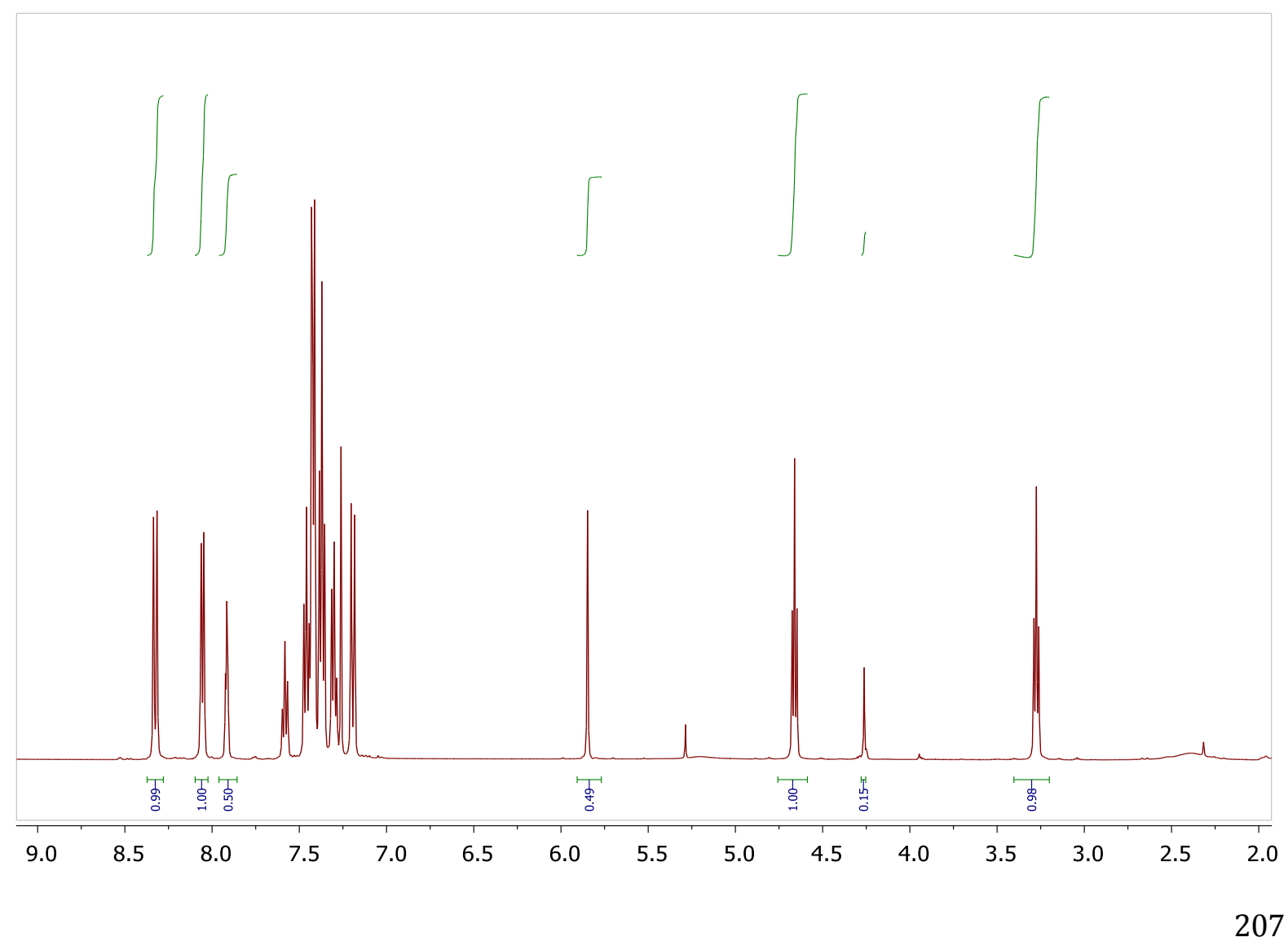




\section{Azul compatibility with Masamune-Roush}<smiles>CCOC(=O)/C=C/c1ccc(OC)cc1</smiles>

A solution of triethylphosphonoacetate $231(0.2 \mathrm{~mL})$, triethylamine $(0.28 \mathrm{~mL})$ in THF $(4 \mathrm{~mL})$ was made up.

For the control reaction, $2 \mathrm{~mL}$ of the above solution (containing a calculated 1 eq. triethylphosphonoacetate and 2 eq. $\mathrm{NEt}_{3}$ ) was added to a RBF containing $\mathrm{LiBr}$ (107.7 mg, 1.24 mmol, $2.5 \mathrm{eq})$ and left to stir for 30 minutes. Anisaldehyde $232(0.24 \mathrm{~mL}, 0.98 \mathrm{mmol}, 2 \mathrm{eq}$.) was then added and the reaction was left to proceed overnight. A separation was performed using $\mathrm{CH}_{2} \mathrm{Cl}_{2} / \mathrm{H}_{2} \mathrm{O}$, and the organic layer was submitted for quantitative NMR.

For the Azul-benzoate-spiked reaction, $2 \mathrm{~mL}$ of the above solution (containing a calculated 1 eq. triethylphosphonoacetate and 2 eq. $\mathrm{NEt}_{3}$ ) was added to a RBF containing $\mathrm{LiBr}$ (102.4 mg, 1.18 mmol, 2.4 eq.) and Azul-benzoate (105.1 mg, $0.38 \mathrm{mmol}, 0.32$ eq.) and left to stir for 30 minutes. Anisaldehyde ( $0.24 \mathrm{~mL}, 0.98 \mathrm{mmol}, 2$ eq.) was then added and the reaction was left to proceed overnight. A separation was performed using $\mathrm{CH}_{2} \mathrm{Cl}_{2} / \mathrm{H}_{2} \mathrm{O}$, and the organic layer was submitted for quantitative NMR.

Quantitative analysis of the ${ }^{1} \mathrm{H}$ NMR spectra was not possible due to deprotonation of nitromethane by remaining triethylamine. For the control reaction, the product $p$-ethylcoumarate 233 (7.60, 7.40, 6.85, 6.25, $3.85 \mathrm{ppm})$ was present in the ${ }^{1} \mathrm{H}$ NMR spectrum, while the limiting reagent triethylphosphonoacetate (2.97 ppm) was absent. For the Azul-spiked reaction, the same was observed, in addition to the presence of Azul-benzoate (8.25, 8.00, 7.50, 7.15, 4.60, $3.20 \mathrm{ppm}$ ) and absence of other azulene-based species. In both cases, $p$-anisaldehyde (9.80 ppm) was still present in the reaction mixture. 


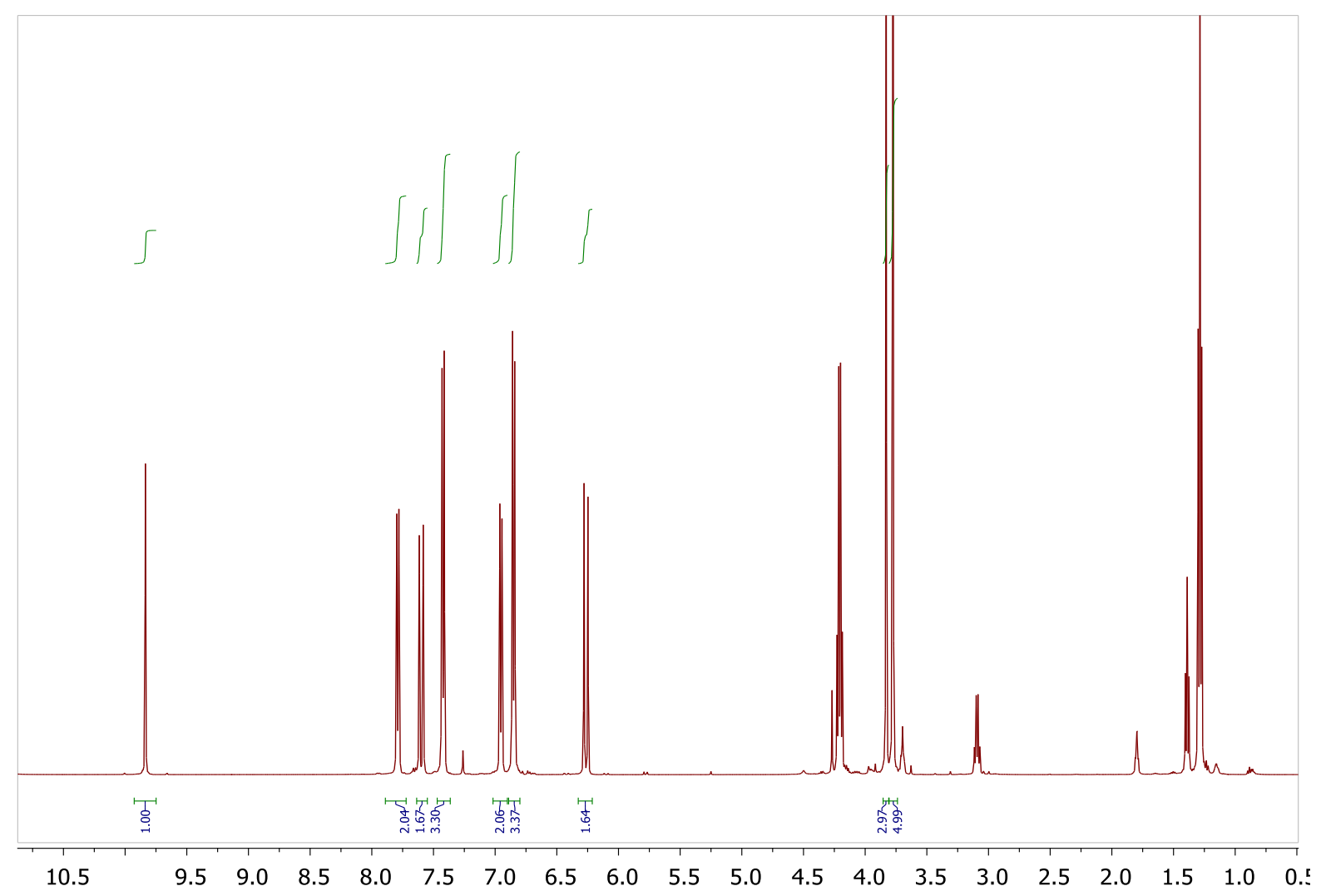

Quantitative 1H NMR spectrum of reaction spiked with Azul-benzoate

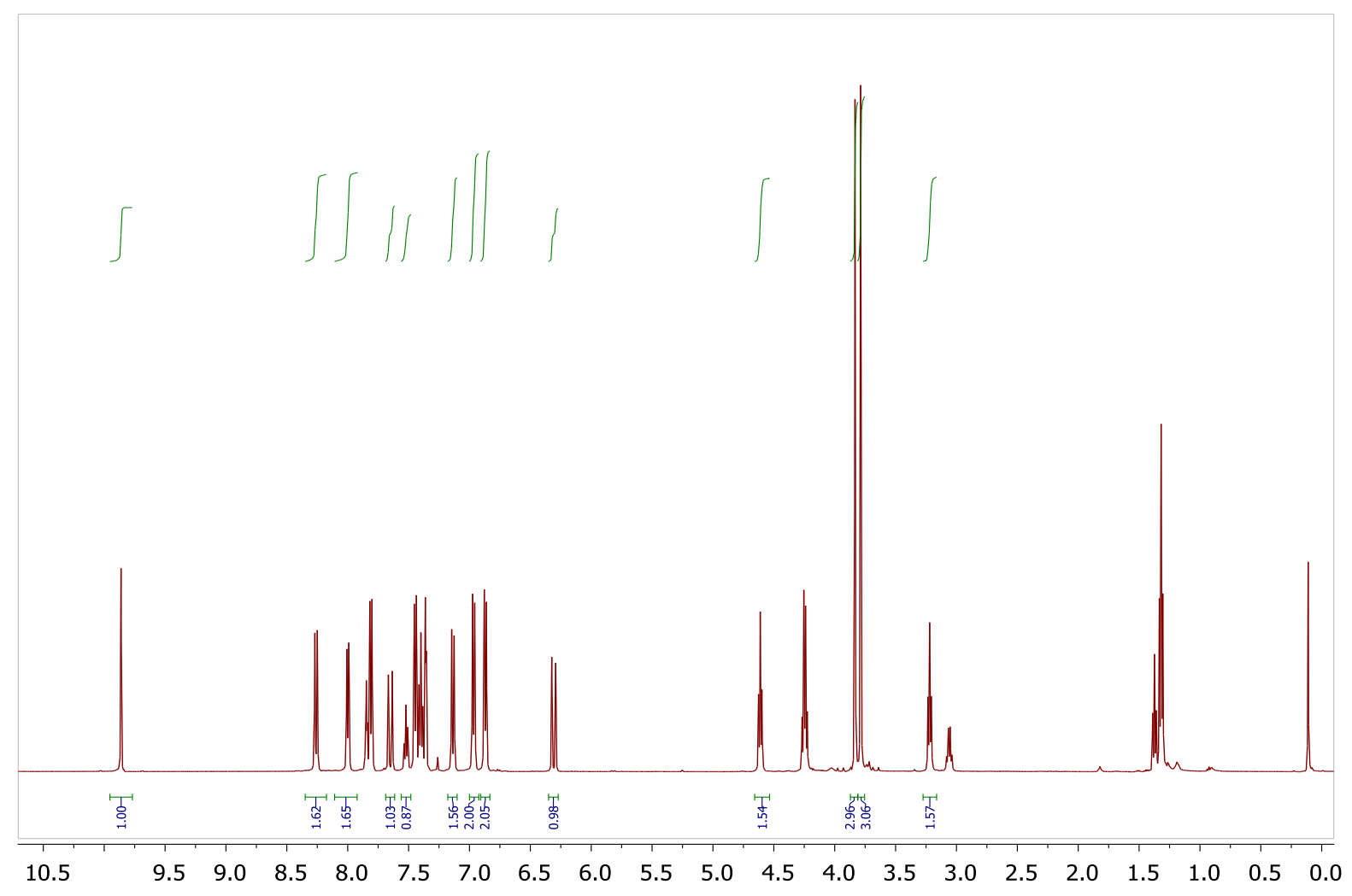




\section{Azul com patibility with Swern oxidation}

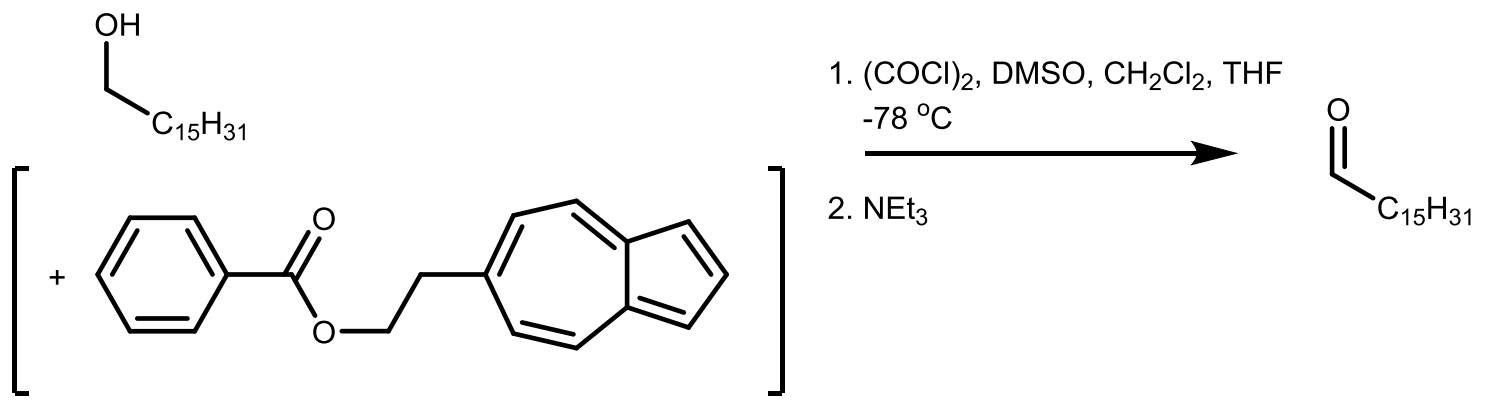

DMSO $(0.5 \mathrm{~mL}),(\mathrm{COCl})_{2}(0.2 \mathrm{~mL})$ and $\mathrm{CH}_{2} \mathrm{Cl}_{2}(5 \mathrm{~mL})$ was added together at $-78{ }^{\circ} \mathrm{C}$ and left for 30 minutes.

To the control reaction containing cetyl alcohol (53.6 mg, $0.22 \mathrm{mmol}, 1$ eq.) in THF ( $4 \mathrm{~mL}$ ) at -78 ${ }^{0} \mathrm{C}, 2 \mathrm{~mL}$ of the above mixture (containing a calculated 4.2 eq. chlorodimethylsulfonium chloride) was added. The solution became cloudy. After two hours, $\mathrm{NEt}_{3}(0.3 \mathrm{~mL}, 2.15 \mathrm{mmol}$, 9.7 eq.) was added and the reaction was maintained at $-78{ }^{\circ} \mathrm{C}$ for a further 30 minutes before allowing it to warm up to r.t. over an hour. $1 \mathrm{~mL} \mathrm{10 \%} \mathrm{HCl}$ (aq.) was added to the reaction mixture, followed by water and a separation was performed using $\mathrm{CH}_{2} \mathrm{Cl}_{2} / \mathrm{H}_{2} \mathrm{O}$, and the organic layer was submitted for quantitative NMR.

To the Azul-benzoate-spiked reaction, containing Azul-benzoate 182 (52 mg, 0.19 mmol, 0.94 eq.), cetyl alcohol (48.5 mg, $0.2 \mathrm{mmol}$, 1 eq.) in THF ( $4 \mathrm{~mL}$ ) at $-78{ }^{\circ} \mathrm{C}, 2 \mathrm{~mL}$ of the above mixture (containing a calculated 4.6 eq. chlorodimethylsulfonium chloride) was added. The indigo reaction mixture rapidly became magenta. Triethylamine $(0.3 \mathrm{~mL}, 2.15 \mathrm{mmol}, 10.8 \mathrm{eq}$.) was added after 130 minutes, and the reaction was kept at $-78{ }^{\circ} \mathrm{C}$ for a further 30 minutes before being allowed to warm up to r.t. over an hour. $1 \mathrm{~mL} \mathrm{10 \%} \mathrm{HCl}$ (aq.) was added, followed by water and a separation was performed using $\mathrm{CH}_{2} \mathrm{Cl}_{2} / \mathrm{H}_{2} \mathrm{O}$, and the organic layer was submitted for quantitative NMR. Most of the magenta material did not partition into the organic layer, despite several washes with $\mathrm{CH}_{2} \mathrm{Cl}_{2}$.

Integration of the relevant peaks in the ${ }^{1} \mathrm{H}$ NMR of the control reaction returned a yield value of $60 \%$ for hexadecanal ( $9.66 \mathrm{ppm}$ ) and 10\% for cetyl alcohol (3.65 ppm), an 86\% conversion.

Integration of the relevant peaks in the ${ }^{1} \mathrm{H}$ NMR of the Azul-benzoate-spiked reaction returned a yield value of $92 \%$ for hexadecanal (9.66 ppm) and a yield value of 30\% for cetyl alcohol (3.65 ppm), a $75 \%$ conversion. The Azul species formed had a recorded yield value of $5 \%$, though this is not representative of the quantity present, due to most of the material remaining behind in the aqueous layer during phase separation. 
Quantitative 1 H NMR spectrum of control reaction

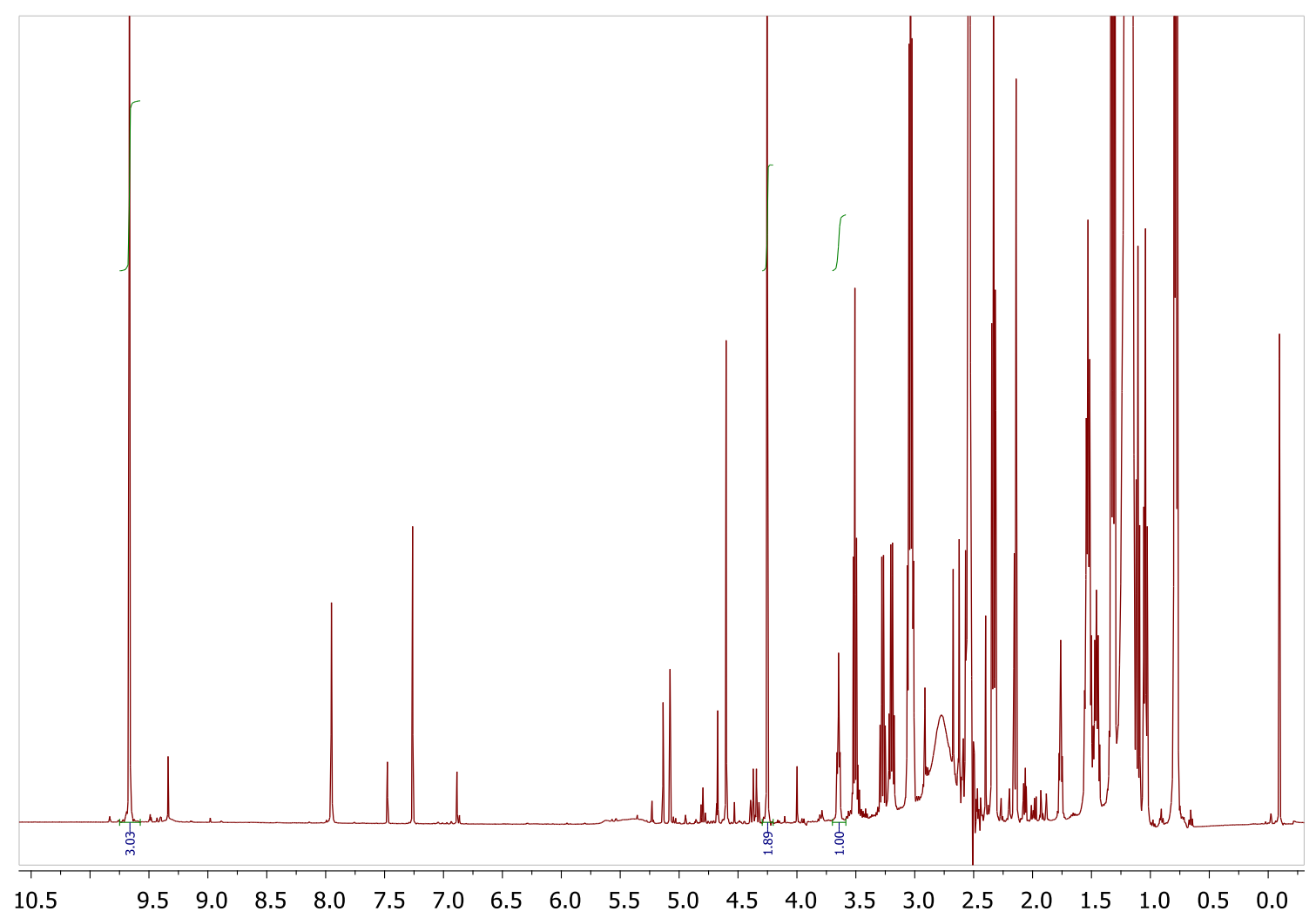

Quantitative 1 H NMR spectrum of reaction spiked with Azul-benzoate

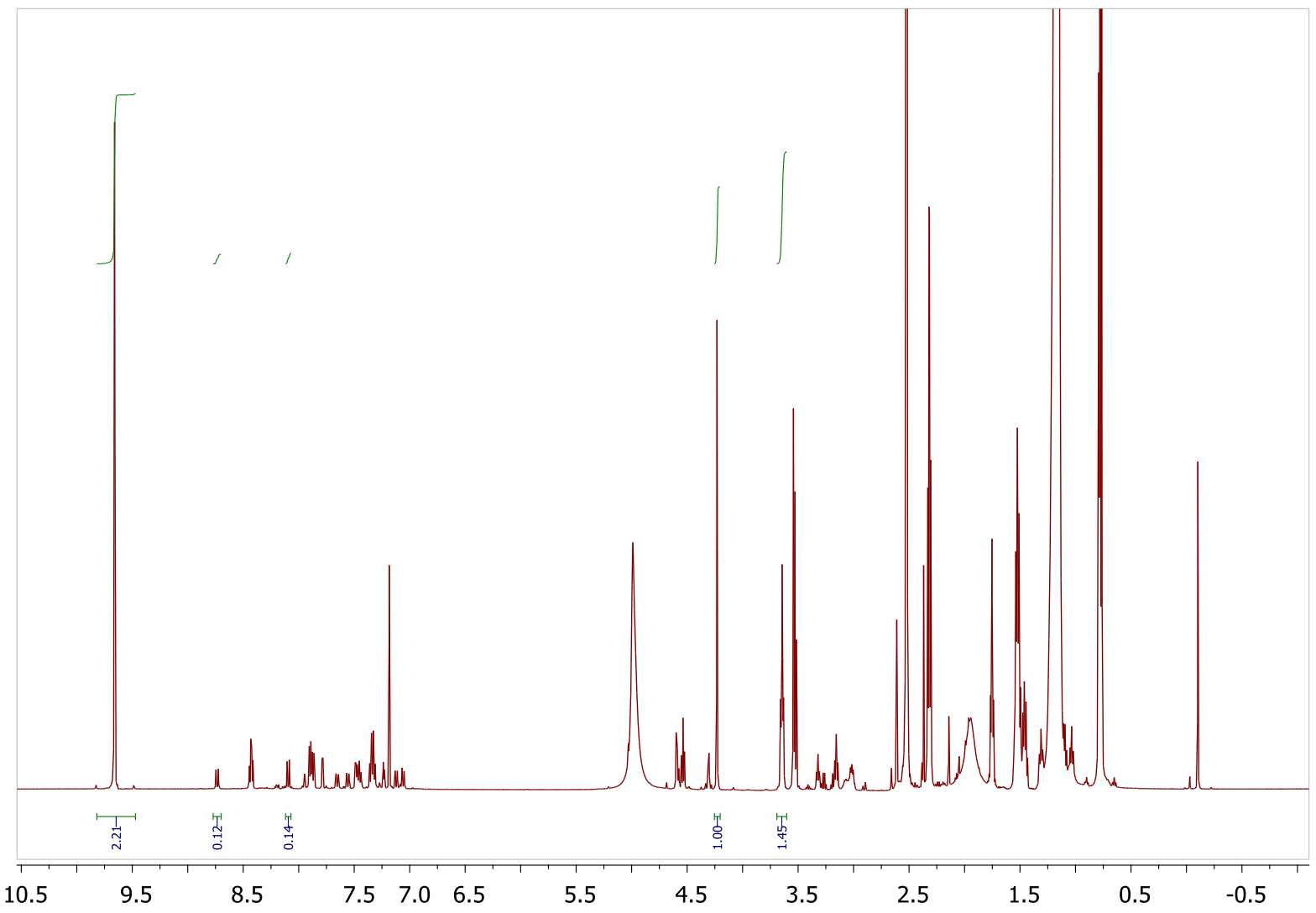




\section{Titration of $n$-BuLi, LDA and KHMDS}

Method adapted from Robert E. Ireland etal. ${ }^{14}$

A standard solution of menthol was made up (typically $0.5-1 \mathrm{M}$ in toluene or THF). A small amount of 2,2'-bipyridyl ( $\sim 2 \mathrm{mg}$ ) was dissolved in $3 \mathrm{~mL}$ THF and $n$-BuLi was added dropwise until a deep red colour formed. The standard solution of menthol was then added dropwise until the orange colour of the midpoint is attained. Known volumes of the bases in question were then added to the reaction mixture, and the menthol solution would be added dropwise until the midpoint was reached. This was repeated as needed until consistent measurements were obtained.

\section{Silanisation of glassware}

A $50 \mathrm{~mL}$ RBF was subjected to a base-bath for 2 hours (300 mg KOH in $50 \mathrm{~mL} \mathrm{1:1} \mathrm{H}_{2} \mathrm{O} / \mathrm{PrOH}$ ) before being washed with distilled $\mathrm{H}_{2} \mathrm{O}$ and dried. A solution of $1 \mathrm{~mL} \mathrm{Cl}_{2} \mathrm{SiMe}_{2}$ and $0.2 \mathrm{~mL} \mathrm{ClSiMe}$ in $40 \mathrm{~mL} \mathrm{CH} \mathrm{CH}_{2}$ was added and left to sit for 1 hour. The solution was then poured out and the remaining residue removed by evaporation under vacuum. The RBF is then washed with distilled $\mathrm{H}_{2} \mathrm{O}$ and oven dried before use.

\section{A note on degradation}

Some azulene species are known to degrade in the presence of oxygen.2,7 Guaiazulene species in particular were prone to degradation and were kept in sealed vials at $-20{ }^{\circ} \mathrm{C}$. 6-Vinylazulene (94) was also prone to degradation and was kept in sealed vials at $-20{ }^{\circ} \mathrm{C}$ and purified by column chromatography (eluting with pet. ether) immediately before use. 6-(2-Hydroxyethyl)azulene (90) was also observed to slowly degrade in open air. When stored in a RBF flushed with $\mathrm{N}_{2}$ and capped with a septum, $\mathbf{9 0}$ was much less prone to degradation and could be stored for months in this fashion.

Azul-protected species appeared to be stable to air, provided they were not stored in acidic media. Samples exposed to air for up to 2.5 years did not exhibit more than trace amounts of degradation. Azulene species with electron-withdrawing substituents (including esters but particularly ketoesters and nitro functionalities) appear to be stable to air indefinitely. 


\section{Calculation of $\mathrm{C}=0$ stretch frequencies of azulene-1- carboxylate esters}

All calculations were performed using Gaussian 09 (EM64L-G09RevD.01), with the B3LYP/6$31 \mathrm{~g}(\mathrm{~d}, \mathrm{p})$ density functional and basis set. Geometry optimisation followed by a frequency calculation was used to find and confirm an energy minimum. Charge $=0$, multiplicity $=1$ was used for all calculations.

\section{Methyl azulene-1-carboxylate (8)}

$\begin{array}{lccc}\text { C } & -3.4226 & -0.95788 & 0.29408 \\ \text { C } & -2.28602 & -1.76561 & 0.24524 \\ \text { C } & -0.94571 & -1.40017 & 0.0763 \\ \text { C } & -3.53085 & 0.43283 & 0.18342 \\ \text { C } & -0.40261 & -0.12749 & -0.08489 \\ \text { C } & -2.5151 & 1.36781 & -0.00738 \\ \text { C } & -1.14009 & 1.16297 & -0.13108 \\ \text { H } & -4.36318 & -1.48638 & 0.43643 \\ \text { H } & -2.46511 & -2.83273 & 0.35067 \\ \text { H } & -0.22392 & -2.21088 & 0.04811 \\ \text { H } & -4.53818 & 0.83579 & 0.25155 \\ \text { H } & -2.83453 & 2.40714 & -0.07181 \\ \text { C } & 0.97824 & 0.18726 & -0.23263 \\ \text { C } & 1.08018 & 1.59097 & -0.38919 \\ \text { H } & 2.00158 & 2.13194 & -0.56266 \\ \text { C } & -0.18309 & 2.17747 & -0.33253 \\ \text { H } & -0.40387 & 3.23255 & -0.43236 \\ \text { C } & 2.05763 & -0.8146 & -0.30843 \\ \text { O } & 3.33368 & -0.45243 & 0.02884 \\ \text { O } & 1.88828 & -1.95631 & -0.69309 \\ \text { C } & 3.62444 & 0.58798 & 0.96411 \\ \text { H } & 3.94567 & 1.49877 & 0.44789 \\ \text { H } & 2.76832 & 0.81783 & 1.60293 \\ \text { H } & 4.45095 & 0.22662 & 1.58122\end{array}$

\section{Methyl3-methylazulene-1-carboxylate (118)}

\begin{tabular}{|c|c|c|c|}
\hline C & -3.42259 & -0.9579 & 0.29395 \\
\hline C & -2.286 & -1.76561 & 0.24521 \\
\hline C & -0.94569 & -1.40014 & 0.07634 \\
\hline C & -3.53088 & 0.4328 & 0.18325 \\
\hline C & -0.40265 & -0.12744 & -0.08483 \\
\hline C & -2.51515 & 1.36782 & -0.00747 \\
\hline C & -1.14012 & 1.16301 & -0.13106 \\
\hline $\mathrm{H}$ & -4.36318 & -1.48642 & 0.43624 \\
\hline $\mathrm{H}$ & -2.46508 & -2.83274 & 0.35067 \\
\hline $\mathrm{H}$ & -0.22387 & -2.21083 & 0.0482 \\
\hline $\mathrm{H}$ & -4.53823 & 0.83572 & 0.2513 \\
\hline $\mathrm{H}$ & -2.83461 & 2.40713 & -0.07193 \\
\hline C & 0.9782 & 0.18732 & -0.23246 \\
\hline C & 1.08018 & 1.59103 & -0.38896 \\
\hline $\mathrm{H}$ & 2.00162 & 2.13201 & -0.56224 \\
\hline C & -0.1831 & 2.17752 & -0.33241 \\
\hline C & 2.05754 & -0.81459 & -0.30833 \\
\hline 0 & 3.33355 & -0.45267 & 0.02902 \\
\hline 0 & 1.88811 & -1.95622 & -0.69328 \\
\hline C & 3.62483 & 0.58793 & 0.96392 \\
\hline
\end{tabular}




$\begin{array}{rlr}3.94683 & 1.49823 & 0.4473 \\ 2.76872 & 0.81869 & 1.60241 \\ 4.45094 & 0.22631 & 1.58141 \\ -0.49718 & 3.67845 & -0.47443 \\ -1.16599 & 3.82667 & -1.29639 \\ -0.95384 & 4.034 & 0.42554 \\ 0.41006 & 4.21753 & -0.65111\end{array}$

\section{Methyl 3-hydroxymethylazulene-1-carboxylate (125)}

$-3.42259-0.9579$

$-2.286-1.76561$

$-0.94569-1.40014$

$-3.53088 \quad 0.4328$

$-0.40265-0.12744$

$-2.51515 \quad 1.36782$

$-1.14012 \quad 1.16301$

$-4.36318-1.48642$

$-2.46508 \quad-2.83274$

$-2.83461 \quad 2.40713$

$0.9782 \quad 0.18732$

$1.08018 \quad 1.59103$

$2.00162 \quad 2.13201$

$-0.1831 \quad 2.17752$

$2.05754-0.81459$

$3.33355-0.45267$

$1.88811-1.95622$

3.624830 .58793

$3.94683 \quad 1.49823$

$2.76872 \quad 0.81869$

$4.45094 \quad 0.22631$

$-4.5224 \quad 0.82939$

$-0.2344-2.19901$

$-0.49718 \quad 3.67845$

$-0.958394 .07787$

$-1.11596$

0.40381

0.00441
0.29395

0.24521

0.07634

0.18325

$-0.08483$

$-0.00747$

$-0.13106$

0.43624

0.35067

$-0.07193$

$-0.23246$

$-0.38896$

$-0.56224$

$-0.33241$

$-0.30833$

0.02902

$-0.69328$

0.96392

0.4473

1.60241

1. 58141

0.25023

0.04861

$-0.47443$

0.40457

$-1.32414$

$4.53857-0.65321$

$5.40669-0.74514$

\section{Dimethyl azulene-1,3-dicarboxylate (126)}

C
C
C
C
C
C
C
H
H
H
C
C
H
C
C
O
O
C
H
H
H
H
H

$\begin{array}{lc}-3.42259 & -0.9579 \\ -2.286 & -1.76561 \\ -0.94569 & -1.40014 \\ -3.53088 & 0.4328 \\ -0.40265 & -0.12744 \\ -2.51515 & 1.36782 \\ -1.14012 & 1.16301 \\ -4.36318 & -1.48642 \\ -2.46508 & -2.83274 \\ -2.83461 & 2.40713 \\ 0.9782 & 0.18732 \\ 1.08018 & 1.59103 \\ 2.00162 & 2.13201 \\ -0.1831 & 2.17752 \\ 2.05754 & -0.81459 \\ 3.33355 & -0.45267 \\ 1.88811 & -1.95622 \\ 3.62483 & 0.58793 \\ 3.94683 & 1.49823 \\ 2.76872 & 0.81869 \\ 4.45094 & 0.22631 \\ -4.5224 & 0.82939 \\ -0.2344 & -2.19901\end{array}$

0.29395

0.24521

0.07634

0.18325

$-0.08483$

$-0.00747$

$-0.13106$

0.43624

0.35067

$-0.07193$

$-0.23246$

$-0.38896$

$-0.56224$

$-0.33241$

$-0.30833$

0.02902

$-0.69328$

0.96392

0.4473

1.60241

1.58141

0.25023

0.04861 


$\begin{array}{lrlr}C & -0.49718 & 3.67845 & -0.47443 \\ 0 & 0.43364 & 4.50641 & -0.65225 \\ \text { O } & -1.85123 & 4.13286 & -0.40399 \\ \text { C } & -1.88955 & 5.54995 & -0.59183 \\ H & -2.90381 & 5.8881 & -0.54916 \\ H & -1.32166 & 6.02742 & 0.17917 \\ H & -1.47183 & 5.79469 & -1.54604\end{array}$

\section{Methyl 3-formylazulene-1-carboxylate (127)}

$-3.42259-0.9579$

$-2.286 \quad-1.76561$

$-0.94569-1.40014$

$-3.53088 \quad 0.4328$

$-0.40265-0.12744$

$-2.51515 \quad 1.36782$

$-1.14012 \quad 1.16301$

$-4.36318-1.48642$

$-2.46508 \quad-2.83274$

$-2.83461 \quad 2.40713$

$0.9782 \quad 0.18732$

$1.08018 \quad 1.59103$

$2.00162 \quad 2.13201$

$-0.1831 \quad 2.17752$

$2.05754-0.81459$

$3.33355-0.45267$

$1.88811-1.95622$

$3.62483 \quad 0.58793$

$3.94683 \quad 1.49823$

2.768720 .81869

$4.45094 \quad 0.22631$

$-4.5224 \quad 0.82939$

$-0.2344 \quad-2.19901$

$-0.49718 \quad 3.67845$

$0.38156 \quad 4.51733$

$-1.57159$
0.29395

0.24521

0.07634

0.18325

$-0.08483$

$-0.00747$

$-0.13106$

0.43624

0.35067

$-0.07193$

$-0.23246$

$-0.38896$

$-0.56224$

$-0.33241$

$-0.30833$

0.02902

$-0.69328$

0.96392

0.4473

1.60241

1. 58141

0.25023

0.04861

$-0.47443$

$-0.6488$

$3.95125-0.40862$

\section{Methyl 3-tricy anovinylazulene-1-carboxylate (128)}

$\mathrm{C}$
$\mathrm{C}$
$\mathrm{C}$
$\mathrm{C}$
$\mathrm{C}$
$\mathrm{C}$
$\mathrm{C}$
$\mathrm{H}$
$\mathrm{H}$
$\mathrm{H}$
$\mathrm{C}$
$\mathrm{C}$
$\mathrm{H}$
$\mathrm{C}$
$\mathrm{C}$
$\mathrm{O}$
$\mathrm{O}$
$\mathrm{C}$
$\mathrm{H}$
$\mathrm{H}$
$\mathrm{H}$
$\mathrm{H}$
$\mathrm{H}$
$\mathrm{C}$
$\mathrm{C}$
$-3.5250$

$-2.48672$

$-1.19889$

$-3.55889$

$-0.62397$

$-2.54822$

$-1.2552$

$-4.44222$

$-2.70643$

$-2.7969$

0.6908

0.8667

1.78

$-0.29208$

1. 68666

2. 69965

1. 68473

2. 57507

3. 09807

1.53086

3.05293

$-4.47883$

$-0.55379$

0.47312

1.63548
$-0.68703$

$-1.22322$

$-0.72184$

0.49235

0.44961

1.43544

1.45139

$-1.27255$

$-2.16741$

2. 28098

0.92164

2.13214

2.7127

2.4506

0.19025

0.88339

$-1.01902$

2. 24887

2. 91087

2. 56051

2. 32735

0.6945

$-1.31875$

4. 60474

4. 71847
1.08086

1.84311

2.06361

0. 32856

1.57595

0.15121

0.67636

1.06677

2. 33522

$-0.4888$

1.85195

1. 13847

1. 11457

0.43217

2. 65684

3. 26215

2. 79054

3. 66426

2. 96609

3.74396

4.64407

$-0.17912$

2. 67389

$-0.66697$

0. 33682 


$\begin{array}{llll}\mathrm{N} & 2.51103 & 4.80413 & 1.09292 \\ \mathrm{C} & 0.41736 & 5.544 & -1.8861 \\ \mathrm{~N} & 0.37537 & 6.25149 & -2.8044 \\ \mathrm{C} & -0.49718 & 3.67845 & -0.47443 \\ \mathrm{C} & -1.74023 & 4.08035 & -1.28984 \\ \mathrm{~N} & -2.67655 & 4.38309 & -1.90405\end{array}$

\section{Methyl3-nitroazulene-1-carboxylate (129)}

$\begin{array}{lccc}\text { C } & -3.42259 & -0.9579 & 0.29395 \\ \mathrm{C} & -2.286 & -1.76561 & 0.24521 \\ \mathrm{C} & -0.94569 & -1.40014 & 0.07634 \\ \mathrm{C} & -3.53088 & 0.4328 & 0.18325 \\ \mathrm{C} & -0.40265 & -0.12744 & -0.08483 \\ \mathrm{C} & -2.51515 & 1.36782 & -0.00747 \\ \mathrm{C} & -1.14012 & 1.16301 & -0.13106 \\ \mathrm{H} & -4.36318 & -1.48642 & 0.43624 \\ \mathrm{H} & -2.46508 & -2.83274 & 0.35067 \\ \mathrm{H} & -2.83461 & 2.40713 & -0.07193 \\ \mathrm{C} & 0.9782 & 0.18732 & -0.23246 \\ \mathrm{C} & 1.08018 & 1.59103 & -0.38896 \\ \mathrm{H} & 2.00162 & 2.13201 & -0.56224 \\ \mathrm{C} & -0.1831 & 2.17752 & -0.33241 \\ \mathrm{C} & 2.05754 & -0.81459 & -0.30833 \\ \mathrm{O} & 3.33355 & -0.45267 & 0.02902 \\ \mathrm{O} & 1.88811 & -1.95622 & -0.69328 \\ \mathrm{C} & 3.62483 & 0.58793 & 0.96392 \\ \mathrm{H} & 3.94683 & 1.49823 & 0.4473 \\ \mathrm{H} & 2.76872 & 0.81869 & 1.60241 \\ \mathrm{H} & 4.45094 & 0.22631 & 1.58141 \\ \mathrm{H} & -4.5224 & 0.82939 & 0.25023 \\ \mathrm{H} & -0.2344 & -2.19901 & 0.04861 \\ \mathrm{~N} & -0.49718 & 3.67845 & -0.47443 \\ \mathrm{O} & 0.38808 & 4.46588 & -0.64355 \\ \mathrm{O} & -1.78495 & 4.11062 & -0.40744 \\ & & & \end{array}$

\section{Methyl3-chloroazulene-1-carboxylate (130)}

$\begin{array}{lccc}\text { C } & -3.42259 & -0.9579 & 0.29395 \\ \text { C } & -2.286 & -1.76561 & 0.24521 \\ \text { C } & -0.94569 & -1.40014 & 0.07634 \\ \text { C } & -3.53088 & 0.4328 & 0.18325 \\ \text { C } & -0.40265 & -0.12744 & -0.08483 \\ \text { C } & -2.51515 & 1.36782 & -0.00747 \\ \text { C } & -1.14012 & 1.16301 & -0.13106 \\ \text { H } & -4.36318 & -1.48642 & 0.43624 \\ \text { H } & -2.46508 & -2.83274 & 0.35067 \\ \text { H } & -2.83461 & 2.40713 & -0.07193 \\ \text { C } & 0.9782 & 0.18732 & -0.23246 \\ \text { C } & 1.08018 & 1.59103 & -0.38896 \\ \text { H } & 2.00162 & 2.13201 & -0.56224 \\ \text { C } & -0.1831 & 2.17752 & -0.33241 \\ \text { C } & 2.05754 & -0.81459 & -0.30833 \\ \text { O } & 3.33355 & -0.45267 & 0.02902 \\ \text { O } & 1.88811 & -1.95622 & -0.69328 \\ \text { C } & 3.62483 & 0.58793 & 0.96392 \\ \text { H } & 3.94683 & 1.49823 & 0.4473 \\ \text { H } & 2.76872 & 0.81869 & 1.60241 \\ \text { H } & 4.45094 & 0.22631 & 1.58141 \\ \text { H } & -4.5224 & 0.82939 & 0.25023 \\ \text { H } & -0.2344 & -2.19901 & 0.04861 \\ \text { Cl } & -0.49718 & 3.67845 & -0.47443\end{array}$


Methyl azulene-1-carboxylate 5,8-tetracyanoethylene bridging complex (132)

\begin{tabular}{|c|c|c|c|}
\hline C & 1.18017 & 0.55243 & -2.23715 \\
\hline C & -0.18804 & 1.95306 & -0.62958 \\
\hline C & 0.63878 & -0.66044 & -2.14091 \\
\hline C & -1.24285 & 1.14703 & -0.40274 \\
\hline C & -1.19469 & -0.32583 & -0.46561 \\
\hline $\mathrm{H}$ & 1.62477 & 0.92585 & -3.15324 \\
\hline $\mathrm{H}$ & -0.29909 & 3.03013 & -0.53898 \\
\hline $\mathrm{H}$ & 0.60546 & -1.35446 & -2.9738 \\
\hline C & -2.62582 & 1.52742 & -0.04062 \\
\hline C & -3.32678 & 0.37663 & 0.09078 \\
\hline $\mathrm{H}$ & -4.37313 & 0.27321 & 0.3469 \\
\hline C & -2.44916 & -0.7805 & -0.1627 \\
\hline C & 0.05207 & -1.0879 & -0.80756 \\
\hline $\mathrm{H}$ & -0.15618 & -2.15878 & -0.80834 \\
\hline C & 1.18912 & 1.44019 & -1.00616 \\
\hline $\mathrm{H}$ & 1.84927 & 2.29506 & -1.16195 \\
\hline C & 1.19534 & -0.87755 & 0.30341 \\
\hline C & 1.849 & 0.60824 & 0.19415 \\
\hline C & 1.69072 & 1.37043 & 1.4464 \\
\hline $\mathrm{N}$ & 1.58041 & 2.02772 & 2.39564 \\
\hline C & 3. 29612 & 0.53006 & -0.08449 \\
\hline $\mathrm{N}$ & 4.42904 & 0.54289 & -0.33306 \\
\hline C & 2.21307 & -1.91807 & 0.06184 \\
\hline $\mathrm{N}$ & 2.95373 & -2.78551 & -0.14939 \\
\hline C & 0.62681 & -1.12743 & 1.63856 \\
\hline $\mathrm{N}$ & 0.15068 & -1.37286 & 2.66731 \\
\hline C & -3.10222 & 2.93437 & 0.13916 \\
\hline $\mathrm{H}$ & -2.54312 & 3.45072 & 0.9287 \\
\hline $\mathrm{H}$ & -2.98551 & 3.52297 & -0.77895 \\
\hline $\mathrm{H}$ & -4.15938 & 2.95092 & 0.41394 \\
\hline C & -2.913 & -2.1715 & -0.09688 \\
\hline 0 & -4.06782 & -2.47619 & 0.14314 \\
\hline 0 & -1.9293 & -3.18155 & -0.33571 \\
\hline C & -2.5745 & -4.44738 & -0.49788 \\
\hline $\mathrm{H}$ & -1.84507 & -5.19059 & -0.74378 \\
\hline $\mathrm{H}$ & -3.06508 & -4.71707 & 0.41399 \\
\hline $\mathrm{H}$ & -3.29612 & -4.38163 & -1.28518 \\
\hline
\end{tabular}

Methyl azulene-1-carboxylate 4,7-tetracyanoethylene bridging complex (133)

$\begin{array}{lrrl}\text { C } & -0.93214 & -0.70767 & -2.22795 \\ \text { C } & 0.82167 & -1.54445 & -0.61121 \\ \text { C } & -0.82484 & 0.61817 & -2.15681 \\ \text { C } & 1.53368 & -0.41999 & -0.40168 \\ \text { C } & 1.00341 & 0.94859 & -0.46636 \\ \text { H } & -1.21941 & -1.22523 & -3.1368 \\ \text { H } & 1.31391 & -2.50587 & -0.50775 \\ \text { H } & -1.02073 & 1.26607 & -3.00443 \\ \text { C } & 2.95108 & -0.3008 & -0.0212 \\ \text { C } & 3.20843 & 1.03125 & 0.13638 \\ \text { H } & 4.16456 & 1.46061 & 0.41738 \\ \text { C } & 2.00438 & 1.82126 & -0.13193 \\ \text { C } & 1.96195 & 3.31305 & -0.02001 \\ \text { H } & 1.01802 & 3.73455 & -0.37322 \\ \text { H } & 2.09289 & 3.62448 & 1.02301 \\ \text { H } & 2.77228 & 3.77197 & -0.5975 \\ \text { C } & -0.42235 & 1.24262 & -0.83367 \\ \text { H } & -0.57915 & 2.32251 & -0.85703 \\ \text { C } & -0.64919 & -1.5245 & -0.98053\end{array}$




$\begin{array}{llr}-0.98861 & -2.55278 & -1.11566 \\ -1.43899 & 0.68819 & 0.27943 \\ -1.5473 & -0.93415 & 0.20955 \\ 3.91063 & -1.38435 & 0.15787 \\ 3.66651 & -2.56941 & -0.01348 \\ -1.14089 & -1.56038 & 1.48093 \\ -0.82413 & -2.10355 & 2.45552 \\ -2.93657 & -1.35638 & -0.05486 \\ -4.00046 & -1.75374 & -0.29099 \\ -2.74647 & 1.31751 & 0.01176 \\ -3.73301 & 1.882 & -0.22023 \\ -0.9983 & 1.15523 & 1.60525 \\ -0.63655 & 1.58855 & 2.61853 \\ 5.21498 & -0.95778 & 0.55985 \\ 6.14083 & -2.0354 & 0.39728 \\ 7.12996 & -1.69219 & 0.61802 \\ 5.88238 & -2.83134 & 1.06406 \\ 6.10292 & -2.38903 & -0.61189\end{array}$

\section{Methyl azulene-1-carboxylate diiron pentacarbonyl (134)}

\begin{tabular}{lccl} 
C & -1.93284 & -1.71824 & -1.15798 \\
C & -0.88836 & -2.75178 & -1.15275 \\
C & 0.44647 & -2.57492 & -1.187 \\
C & -1.90282 & -0.54832 & -1.99904 \\
C & 1.10089 & -1.275 & -1.15833 \\
C & -0.80093 & 0.31907 & -2.01954 \\
C & 0.52669 & 0.00776 & -1.52851 \\
H & -2.93006 & -2.14891 & -1.0759 \\
H & -1.26042 & -3.77223 & -1.09969 \\
H & 1.0911 & -3.44624 & -1.17752 \\
H & -2.79595 & -0.24338 & -2.54178 \\
H & -0.91099 & 1.2512 & -2.56507 \\
C & 2.45262 & -1.04737 & -0.71345 \\
C & 2.69725 & 0.345 & -0.73587 \\
H & 3.62192 & 0.84142 & -0.47443 \\
C & 1.53442 & 1.00875 & -1.24599 \\
C & 1.48043 & 2.45681 & -1.5059 \\
O & 2.42231 & 3.20245 & -1.30788 \\
Fe & 1.06406 & 0.00572 & 0.52411 \\
C & 1.22609 & -1.18822 & 1.80586 \\
O & 1.477 & -1.98566 & 2.60429 \\
C & 1.23554 & 1.36307 & 1.64368 \\
O & 1.41853 & 2.26742 & 2.33835 \\
Fe & -1.7029 & 0.12866 & -0.07329 \\
C & -3.44768 & 0.31238 & 0.06968 \\
O & -4.58972 & 0.41396 & 0.20692 \\
C & -1.43686 & 1.845 & 0.34065 \\
O & -1.29336 & 2.9535 & 0.62113 \\
C & -1.46889 & -0.63755 & 1.51559 \\
O & -1.61711 & -1.16484 & 2.53783 \\
H & 3.15839 & -1.8039 & -0.44061 \\
O & 0.25362 & 2.96179 & -2.0396 \\
C & 0.35962 & 4.37584 & -2.22431 \\
H & 1.15092 & 4.58728 & -2.91281 \\
H & -0.56233 & 4.75483 & -2.61324 \\
H & 0.56958 & 4.84347 & -1.28509 \\
& & & \\
\hline
\end{tabular}




\section{References}

1. $\quad$ http://sdbs.db.aist.go.jp/sdbs/cgi-bin/direct_frame_top.cgi (Last accessed 11/1/16),

2. Matsubara, Y.; Takekuma, S.-i.; Yokoi, K.; Yamamoto, H.; Nozoe, T., Autoxidation of Guaiazulene and 4,6,8-trimethylazulene in Polar Aprotic Solvent: Structural Proof of Products. Bull. Chem. Soc.Jpn. 1987, 60, 1415-1428.

3. Collins, M. J.; Sternhell, S.; Tansey, C. W., N.M.R. Studies of Bond Order in Azulene, Biphenylene and 1,6-Methano[10]annulene. Aust.J. Chem. 1990,43, 1541-1546.

4. Balschukat, D.; Dehmlow, E. V., Neuartige 2,6-disubstitutierte Azulene. Chemische Berichte 1986, 119, 2272-2288.

5. Berger, S.; Zeller, K.-P., A ${ }^{13} \mathrm{C}^{13} \mathrm{C}$ Spin-Spin Coupling Matrix for Azulene. J. Org. Chem. 1984, 49,3725-3728.

6. Löber, S.; Tschammer, N.; Hübner, H.; Melis, M. R.; Argiolas, A.; Gmeiner, P., The Azulene Framework as a Novel Arene Bioisostere: Design of Potent Dopamine D4 Receptor Ligands Inducing Penile Erection. ChemMedChem 2009, 4, 325-328.

7. Takekuma, S.-i.; Matsubara, Y.; Yamamoto, H.; Nozoe, T., Autoxidation of 1-Methyl- and 1,3-Dimethylazulenes in Polar Aprotic Solvents: Structural Proof for Products and Reaction Pathways. Bull. Chem. Soc.Jpn. 1987, 10, 3721.

8. Muehle, E., Kernresonanzspektren einiger Azulenderivate. Annalen der Physik 1966, 18, 130-137.

9. Timmer, M. S. M.; Stocker, B. L.; Northcote, P. T.; Burkett, B. A., Az-a Colourful AzuleneDerived Protecting Group. Tetrahedron Lett. 2009, 50, 7199-7204.

10. Burton, R.; Pratt, L.; Wilkinson, G., Transition-metal Complexes of Seven-membered Ring Systems. Part II . Azulenemetal Carbonyls. J. Chem. Soc. 1960, 4290-4303.

11. Chen, S.-L.; Klein, R.; Hafner, K., Synthesis and Properties of syn-[2.2](1,6)- and $(4,6)$ Azulenophanes and Macrocyclic Azulenophanes. Eur. J. Org. Chem. 1998, 423-433.

12. McDonald, R. N.; Wolfe, L.; Petty, H. E., Nonbenzenoid Aromatic Systems. VIII."' Buffered Acetolysis of 2-(4- and 2-(6-Azulyl)ethyl Arenesulfonates and 3-(4-Azulyl)-l-propyl Nosylate. Examples of Ar3-5 and Ar3-6 Mechanisms. J. Org. Chem. 1973,38, 1106-1113.

13. Hattori, K.; Sajikip, H.; Hirota, K., Undesirable Deprotection of O-TBDMS groups by Pd/CCatalyzed Hydrogenation and Chemoselective Hydrogenation using a $\mathrm{Pd} / \mathrm{C}(\mathrm{en})$ Catalyst. Tetrahedron 2001,57, 2109-2114.

14. Ireland, R. E.; Meissner, R. S., Convenient Method for the Titration of Amide Base Solutions. J. Org. Chem. 1991, 56, 4566-4568. 


\section{Appendix $1 .{ }^{1} \mathrm{H}$ and ${ }^{13} \mathrm{C}$ NMR spectra of selected compounds}

Azulene (1)<smiles></smiles>
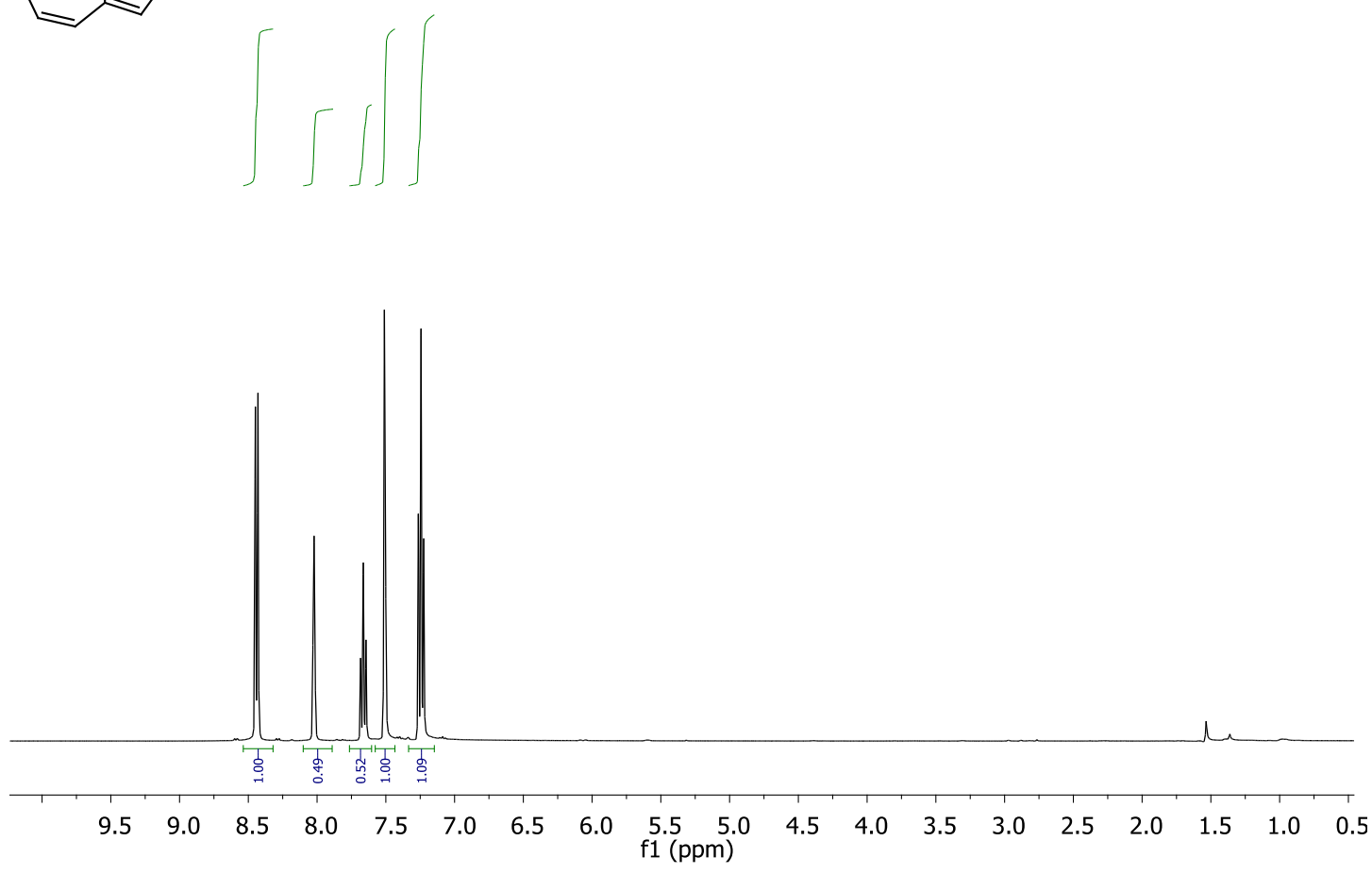

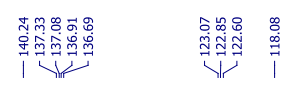

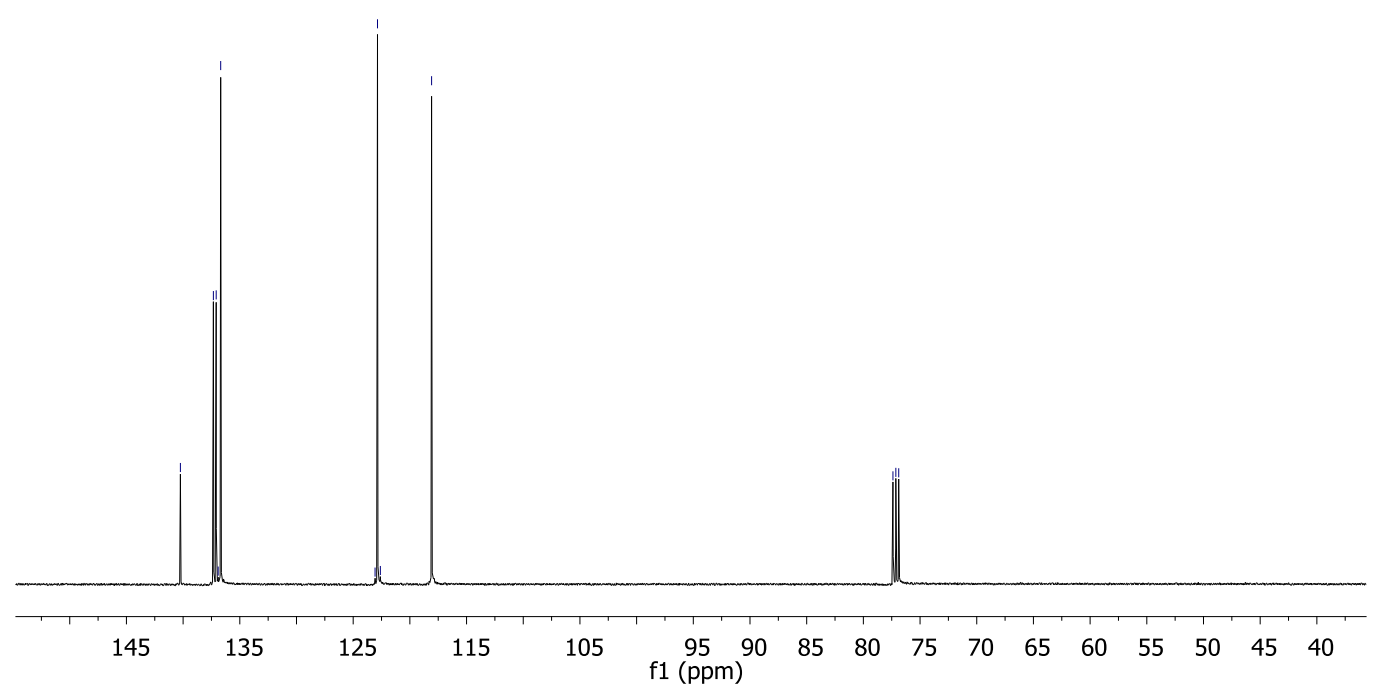


4,6,8-trimethylazulene (9)
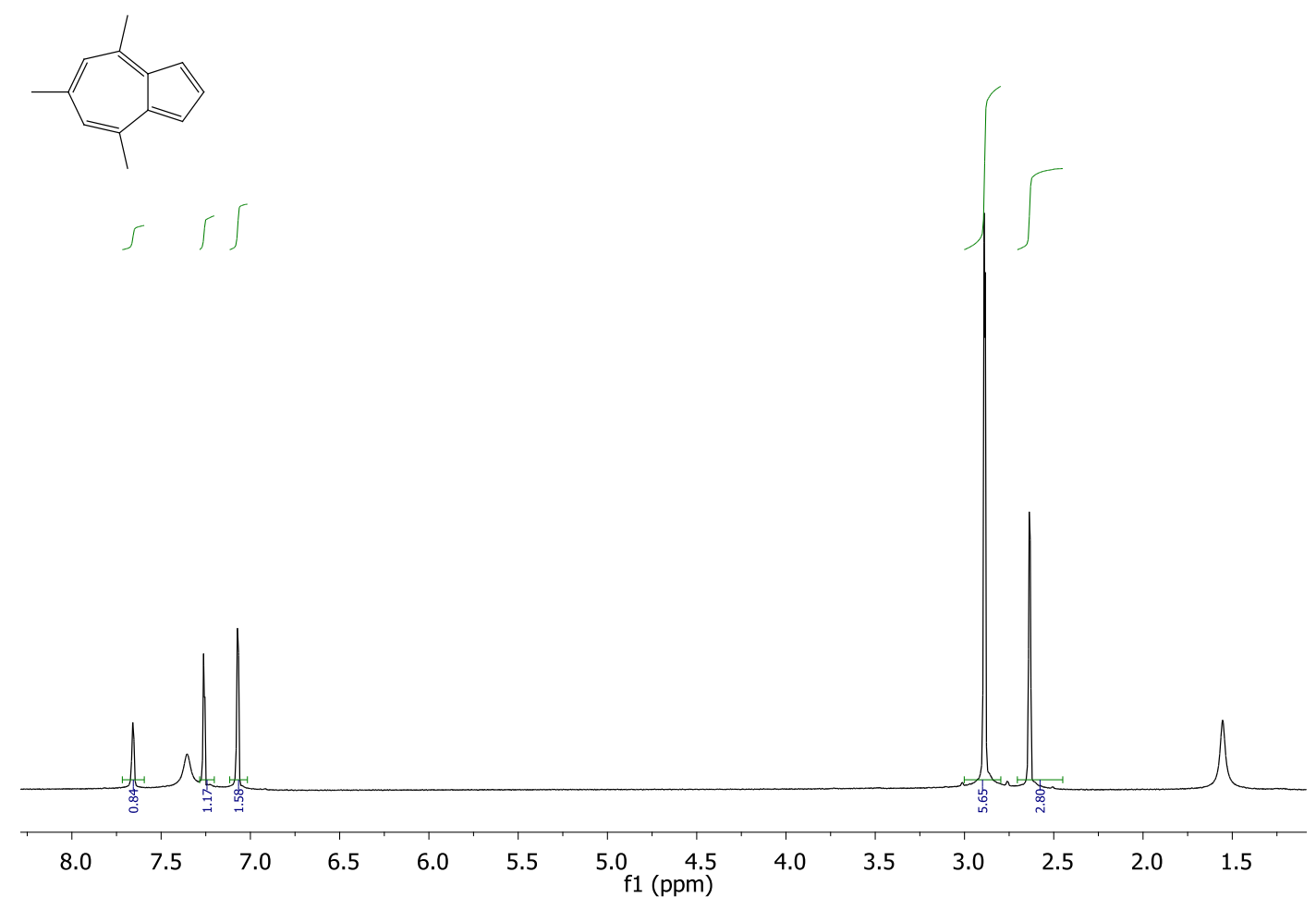

恝11

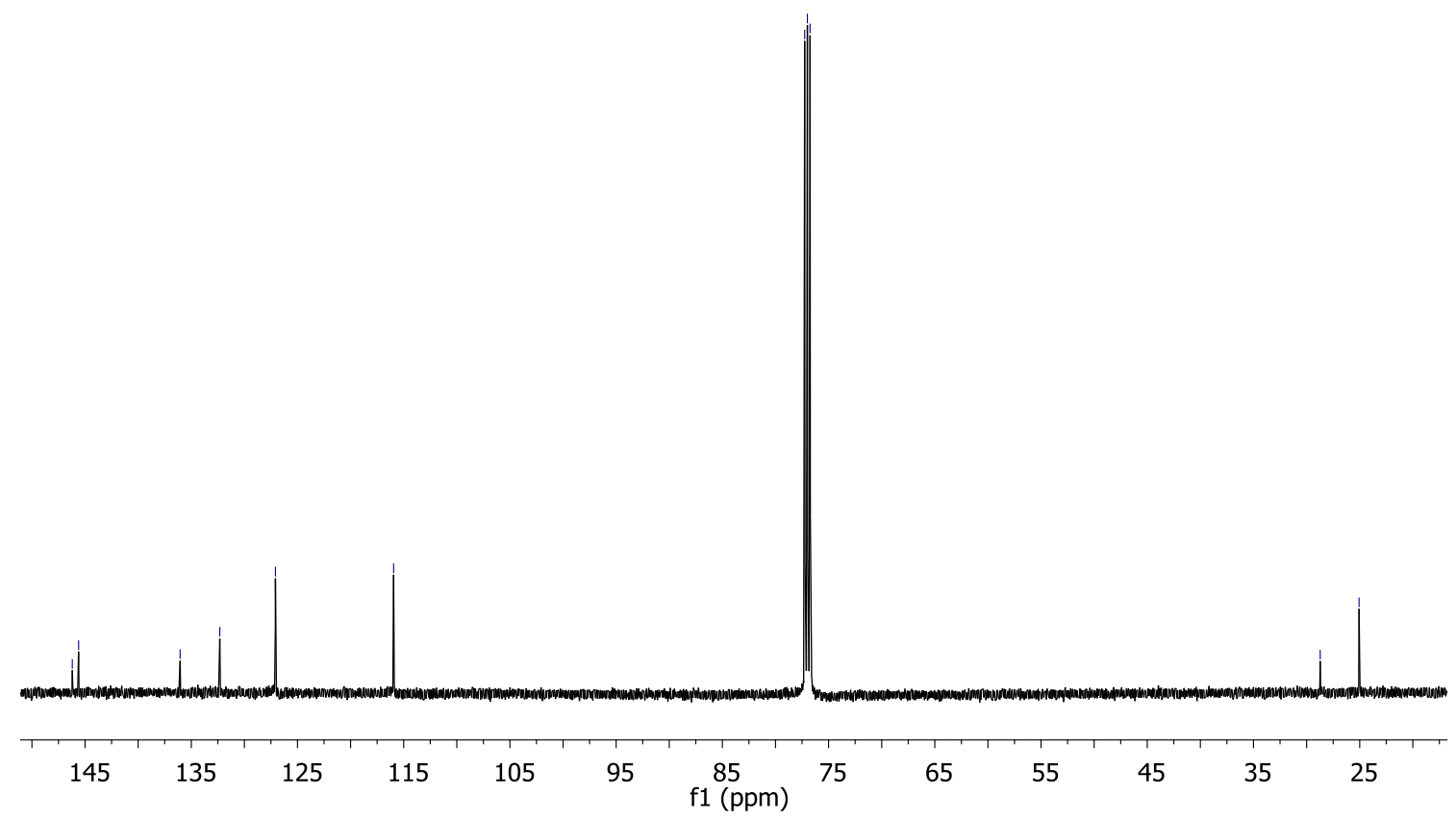




\section{6-Methylazulene (10)}
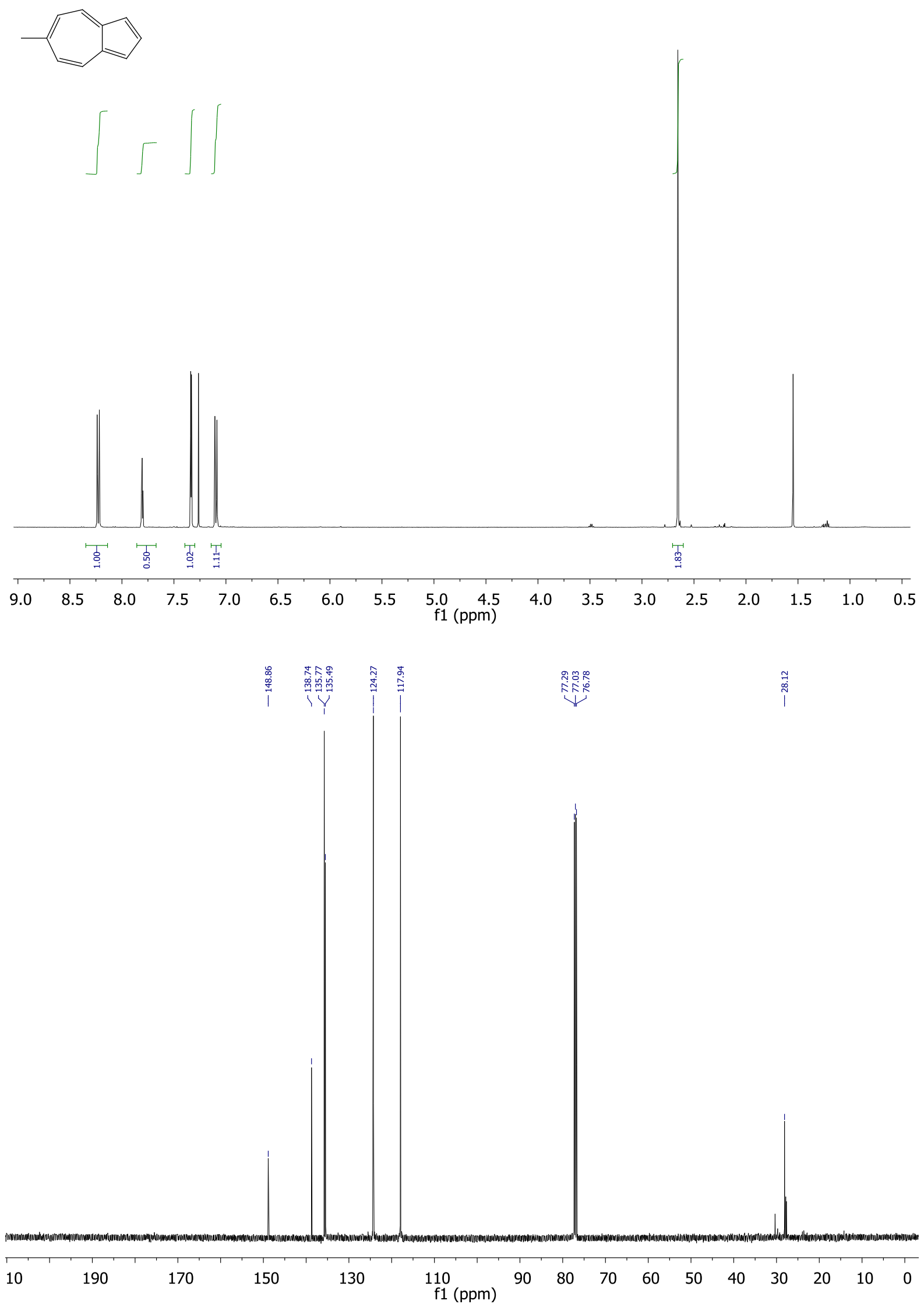
1-(N-Pyrrolidinylmethyl)azulene (110)

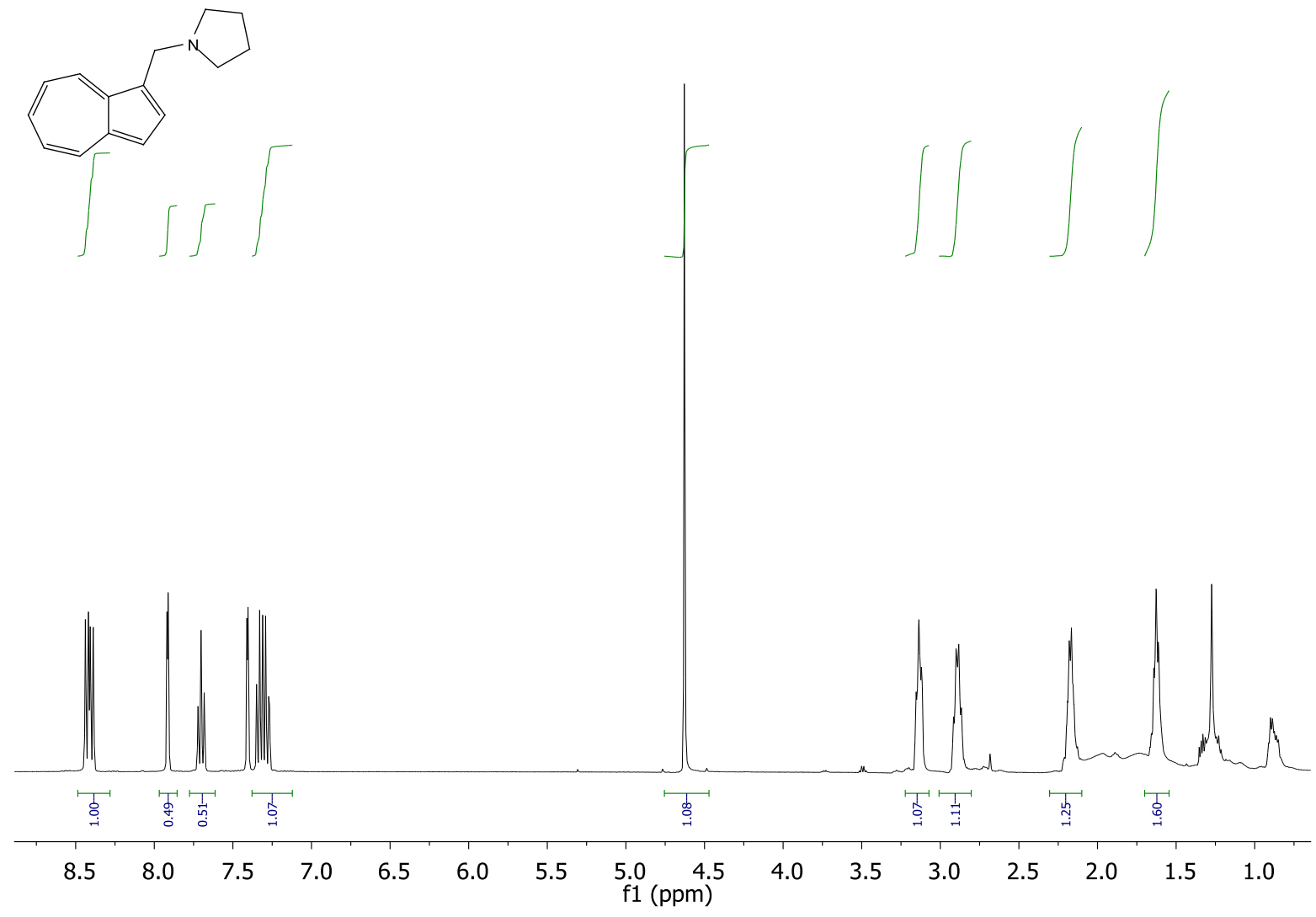

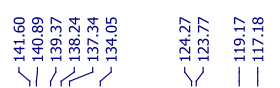

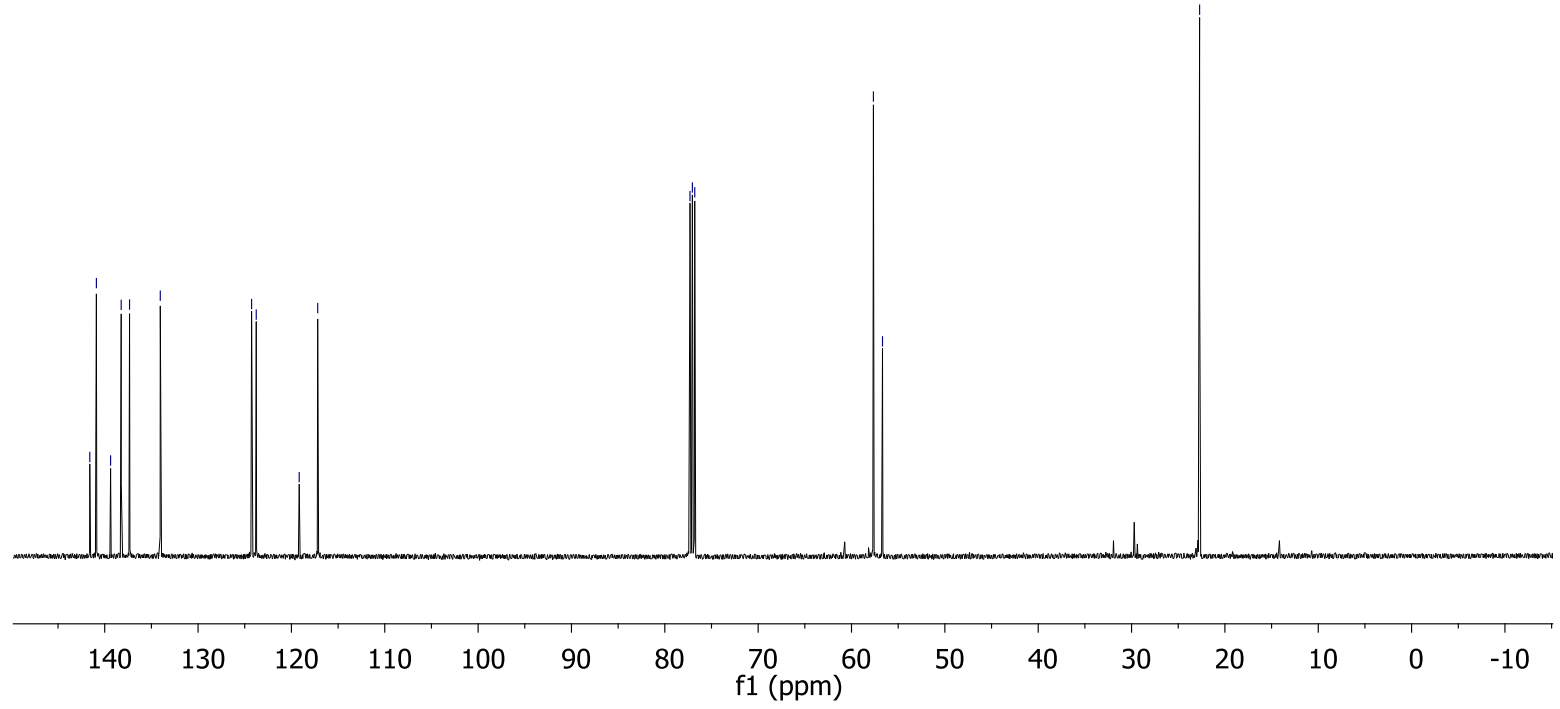


Menthyl azulene-1-carboxylate (122)
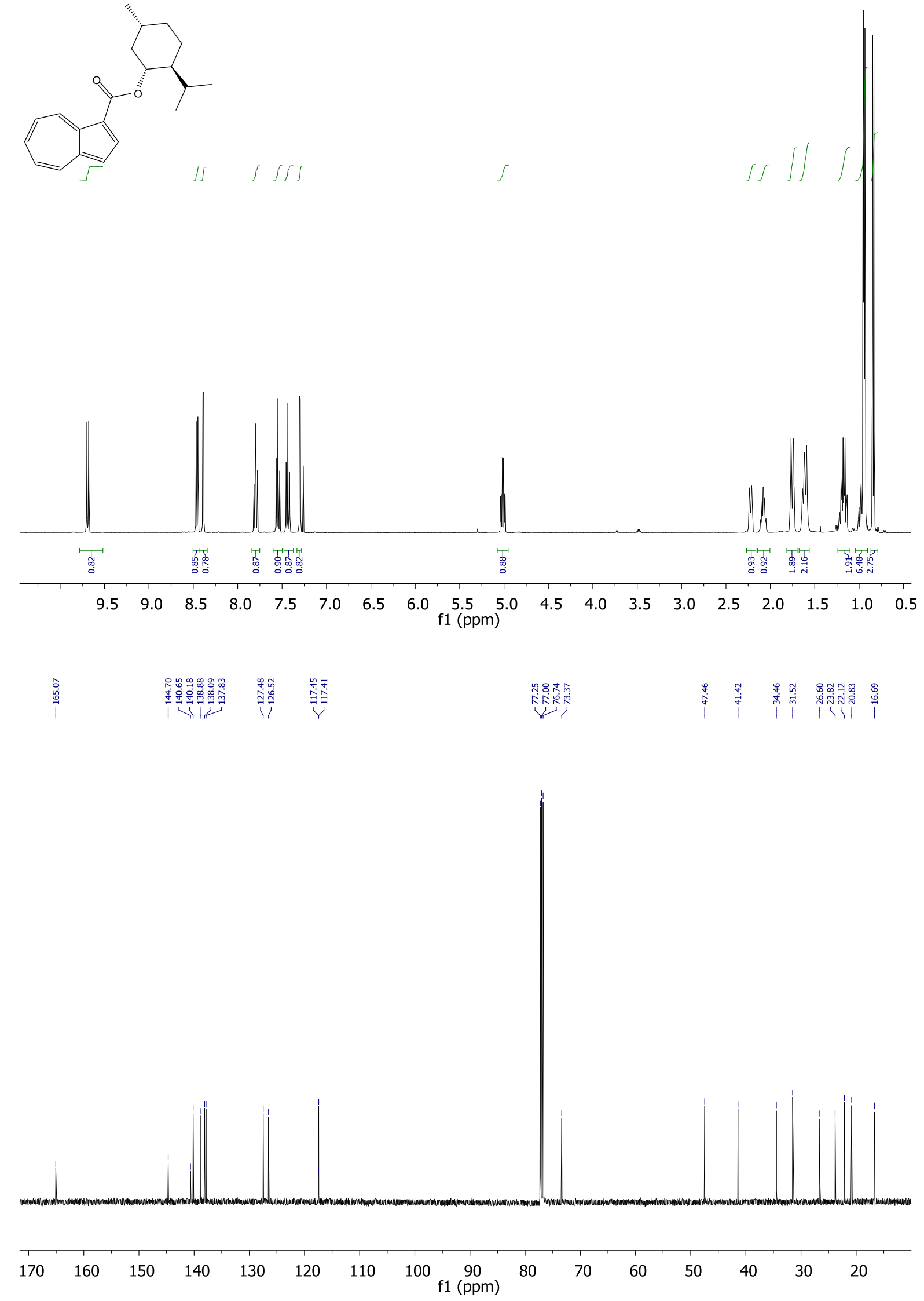


\section{Menthyl3-nitroazulene-1-carboxylate (136)}

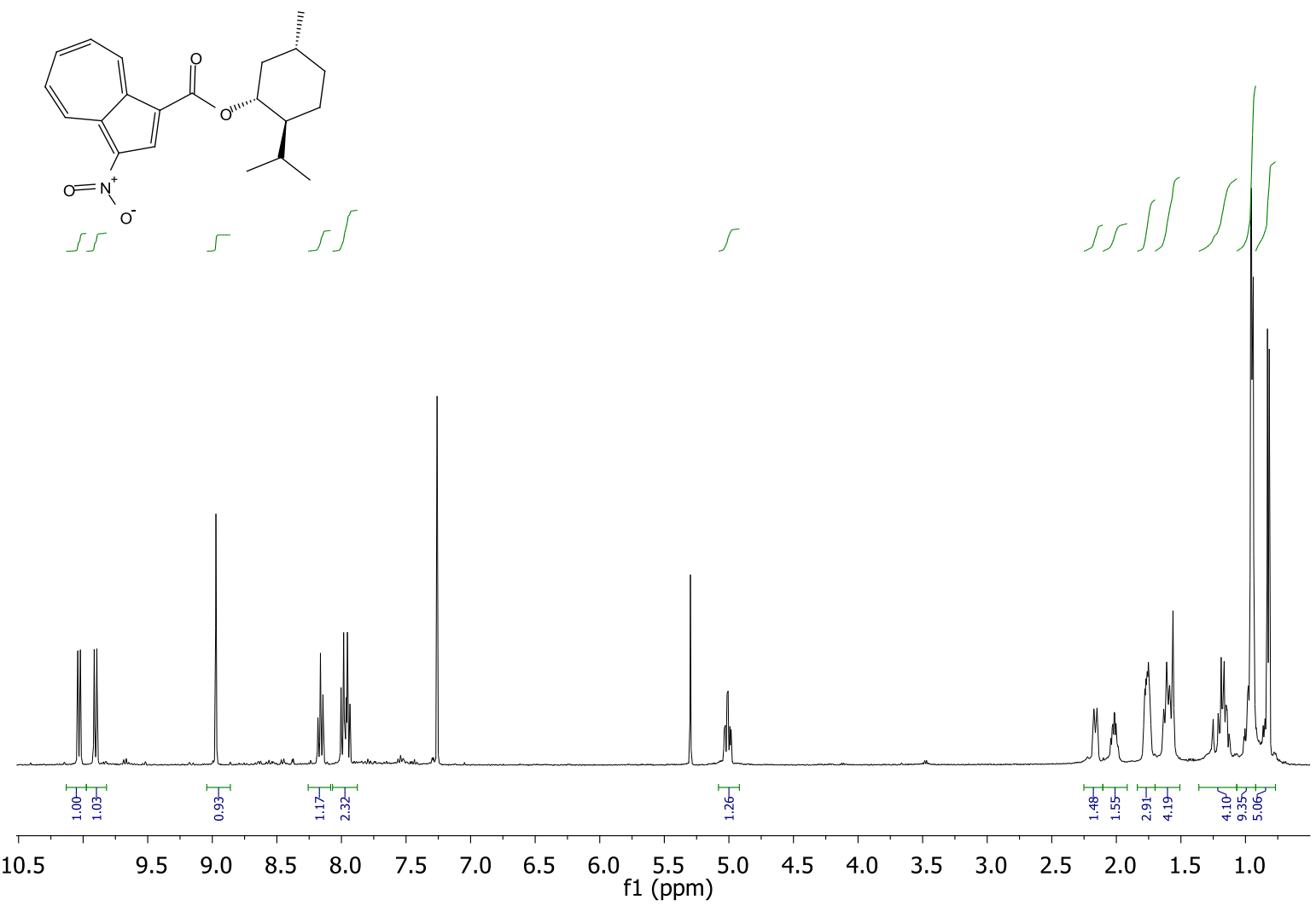

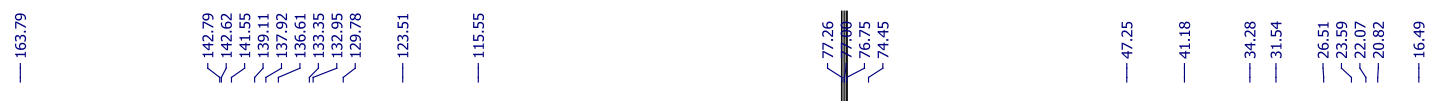

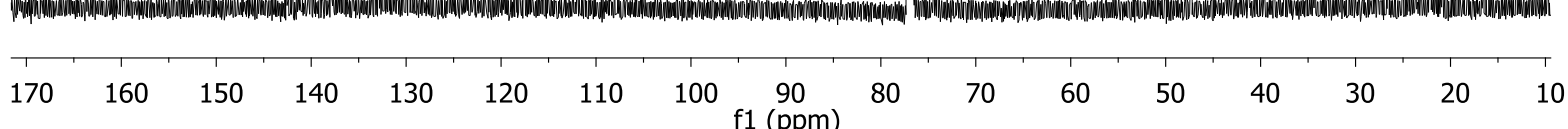


Menthyl guaiazulene-3-carboxylate (148)
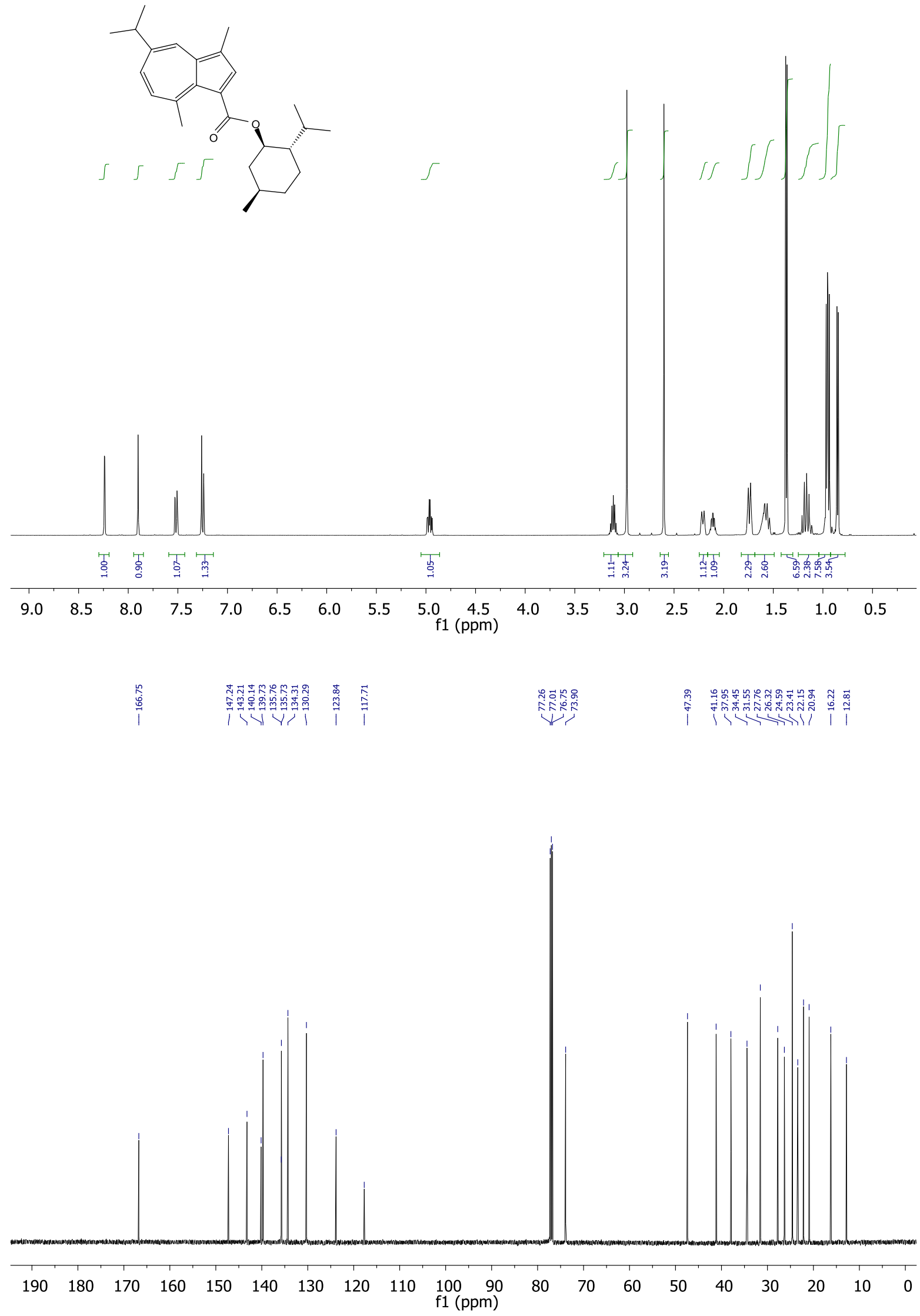
1H-7-isopropyl-9-methylazuleno[3,4-cd]3,4-dihydrooxepin-1-one (151)

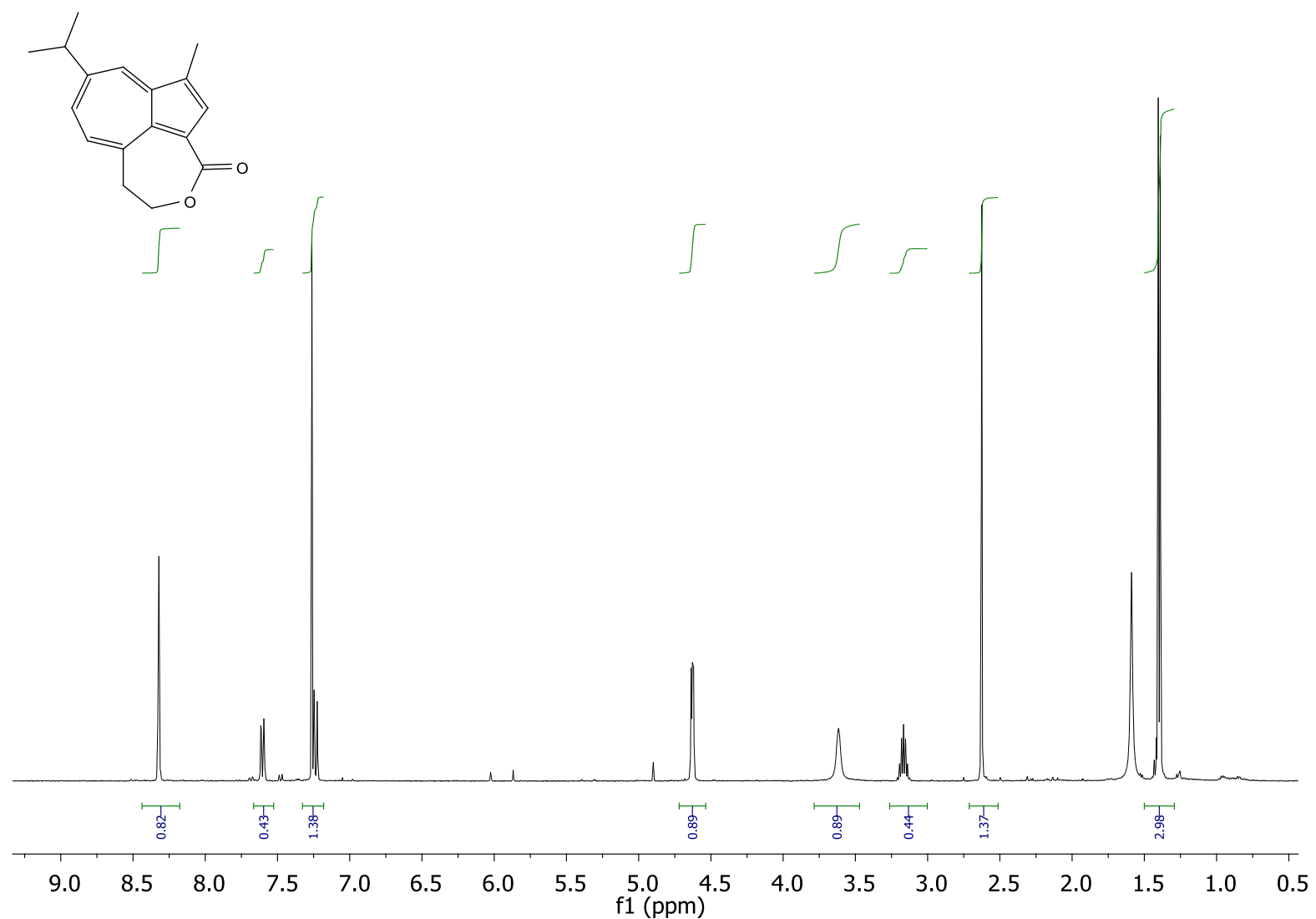

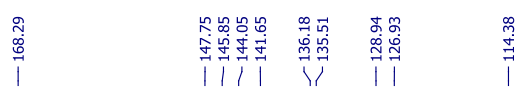

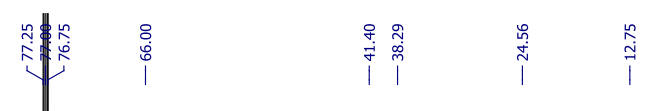

$\begin{array}{lllllllllllllllll}170 & 160 & 150 & 140 & 130 & 120 & 110 & 100 \underset{f 1(\mathrm{ppm})}{90} & 80 & 70 & 60 & 50 & 40 & 30 & 20 & 10\end{array}$ 
Menthy14,6,8-trimethylazulene-1-carboxylate (157)
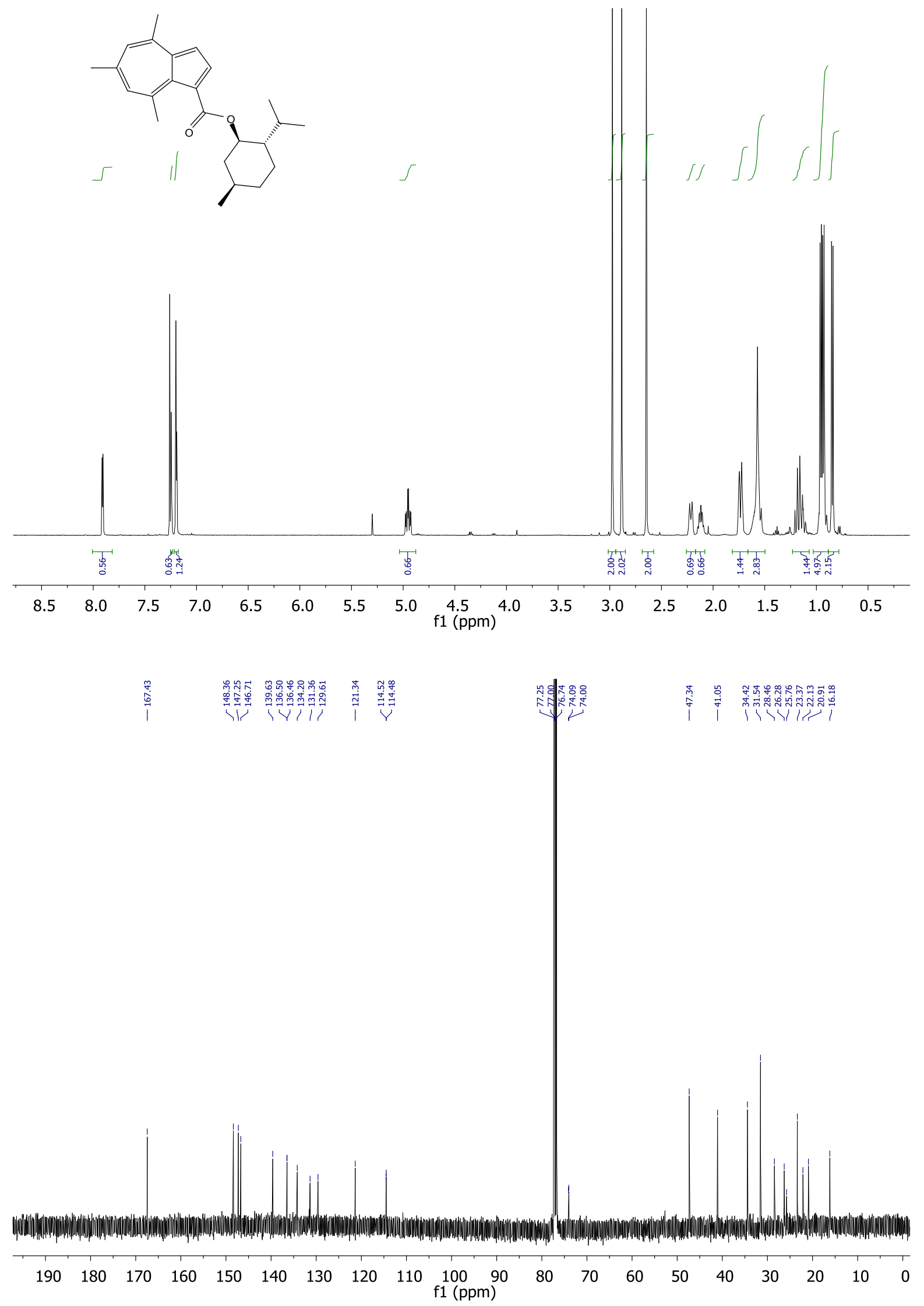


\section{Azulene diiron pentacarbonyl (143)}
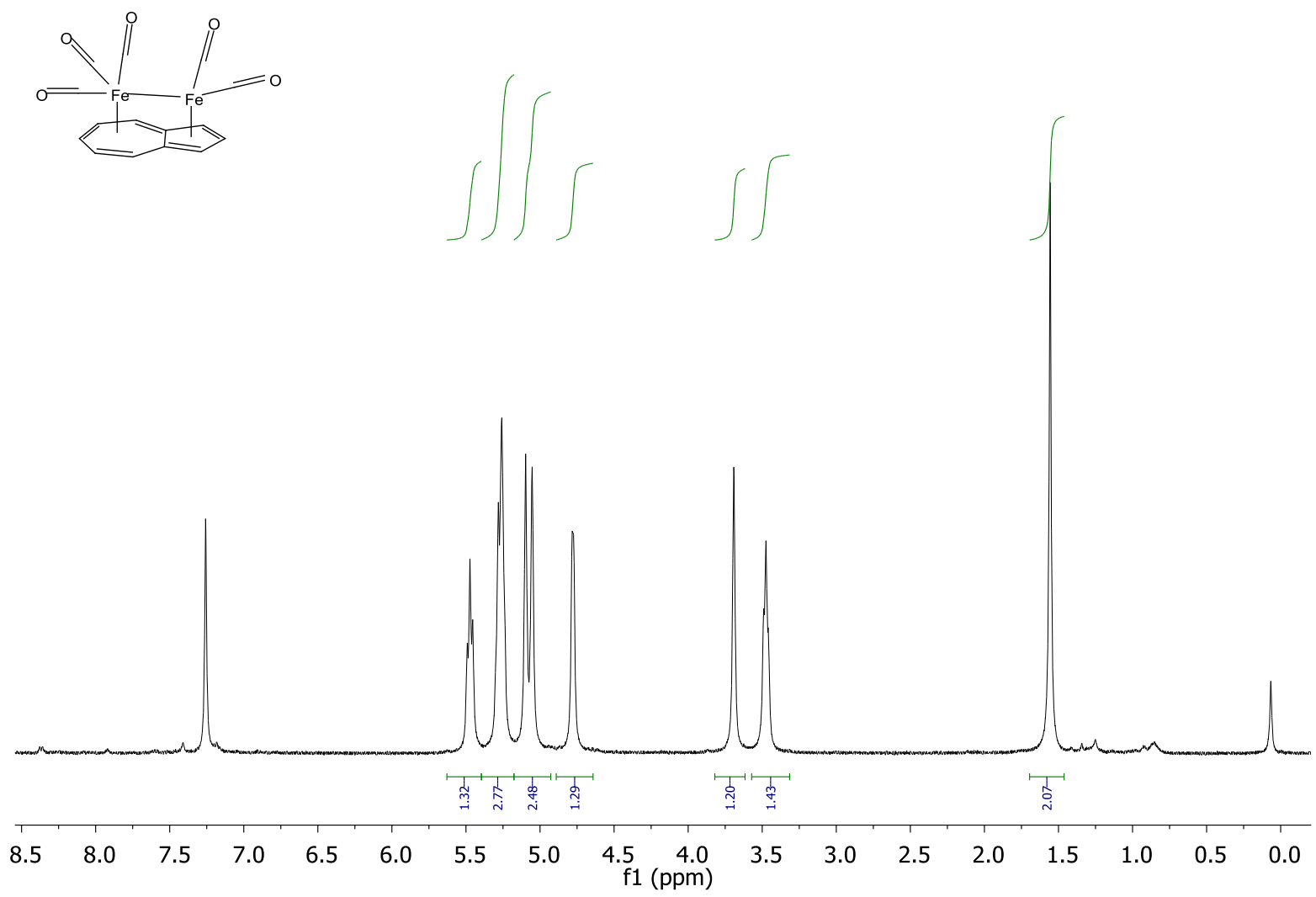

$\vec{m}$
$\stackrel{0}{0}$
$i$
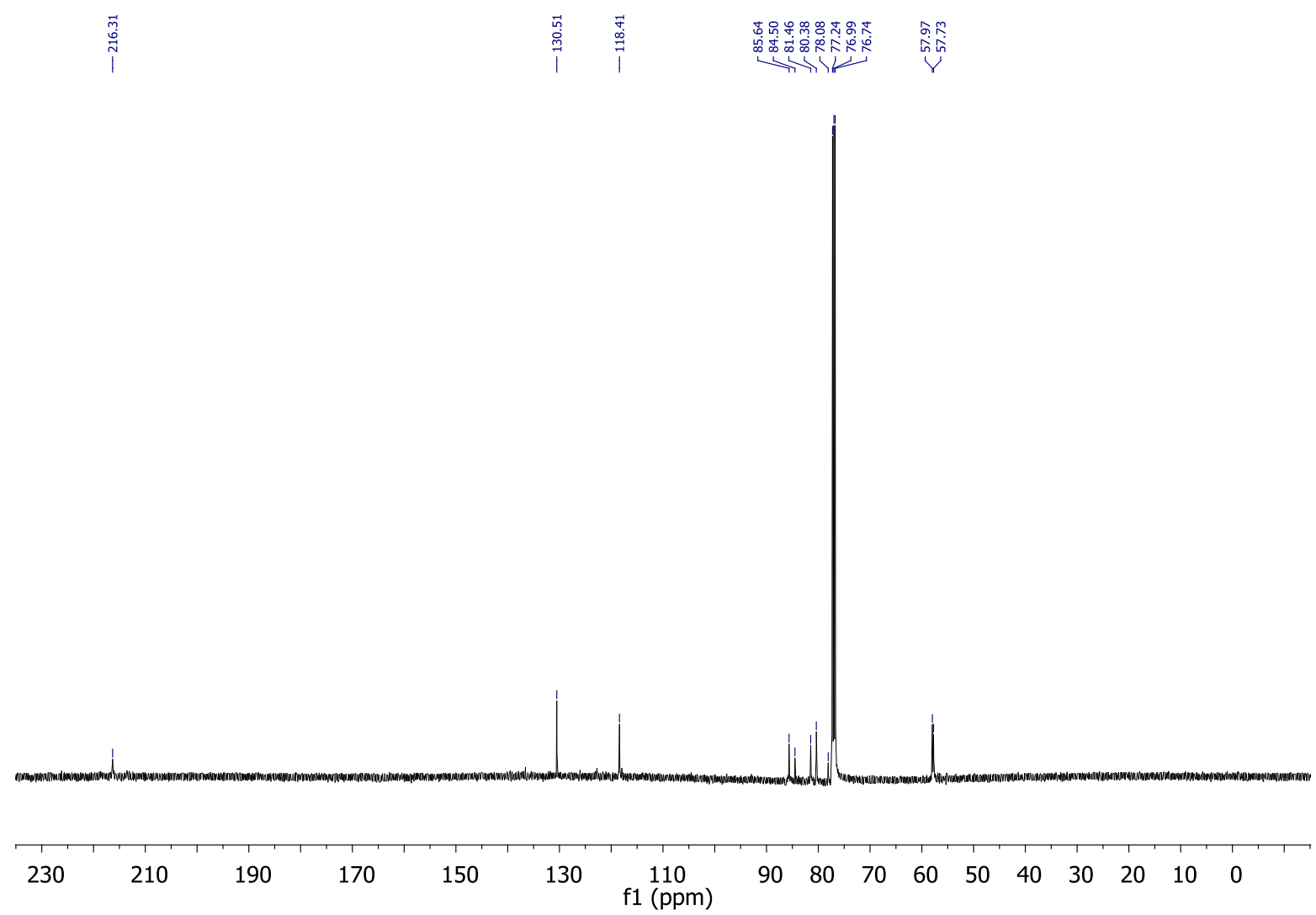
Menthyl azulene-1-carboxylate diiron pentacarbonyl (145)
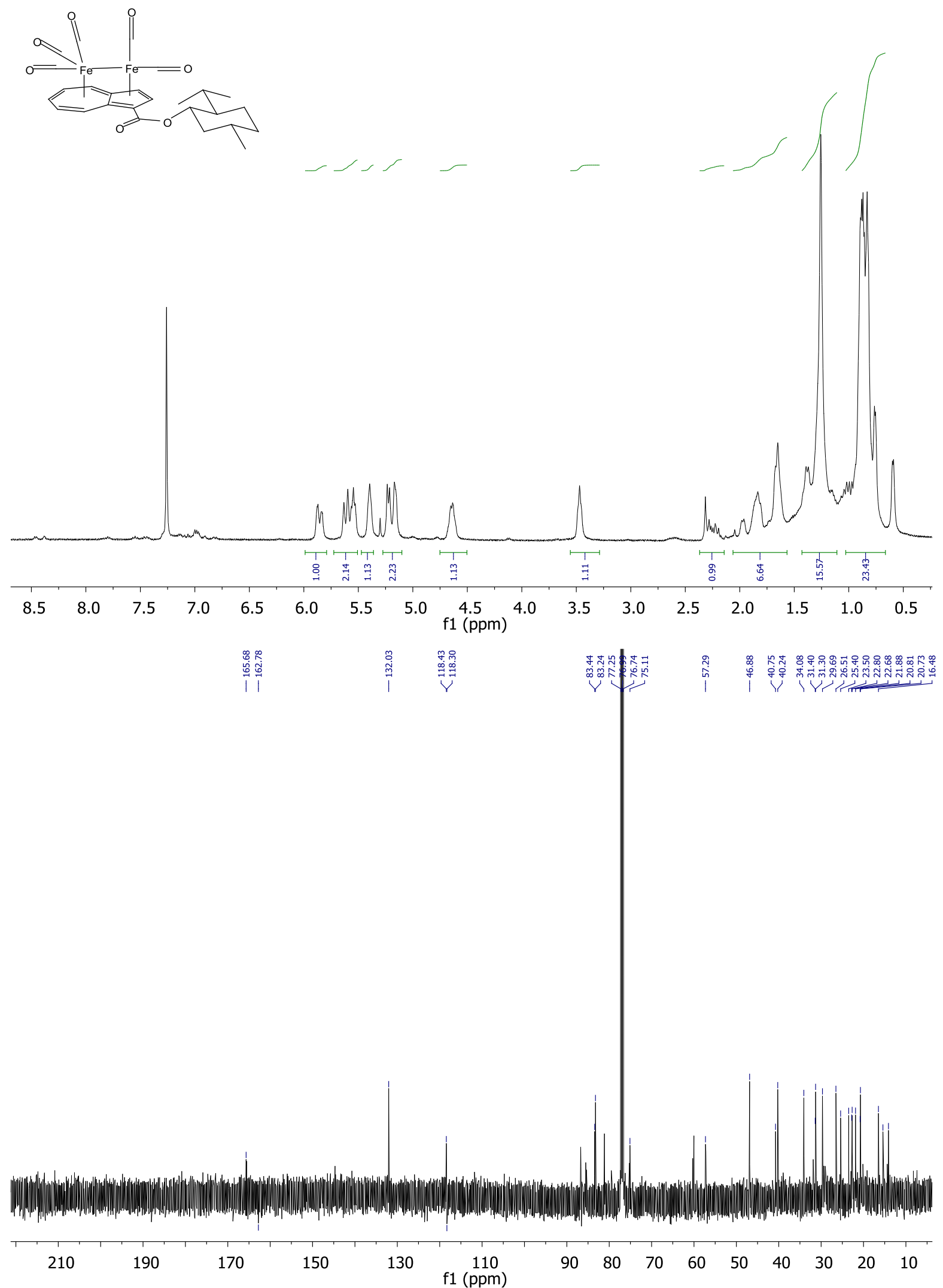


\section{6-(2-hydroxyethyl)azulene (90)}

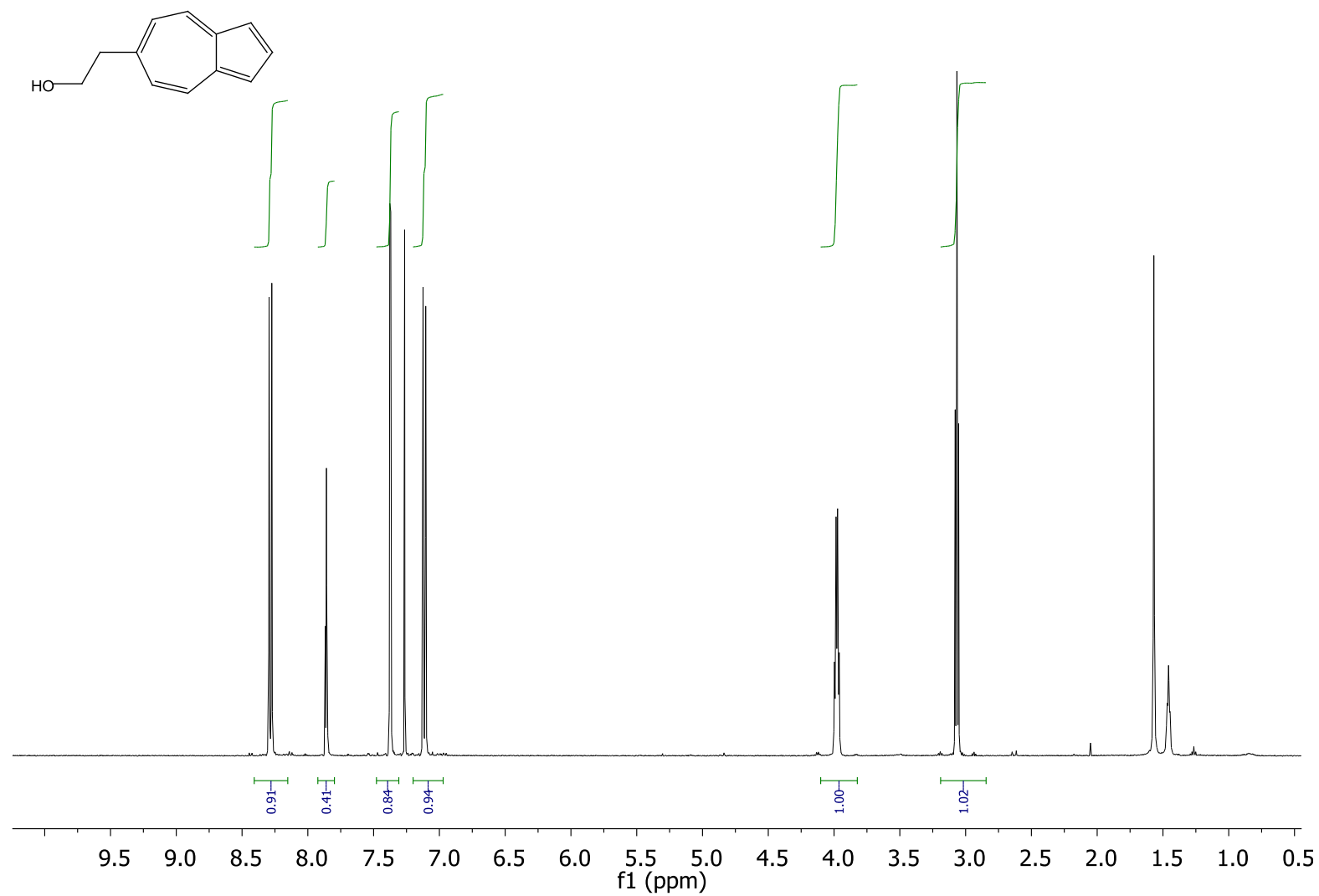

|

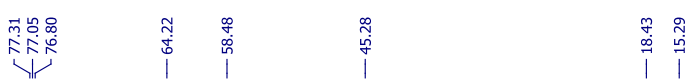

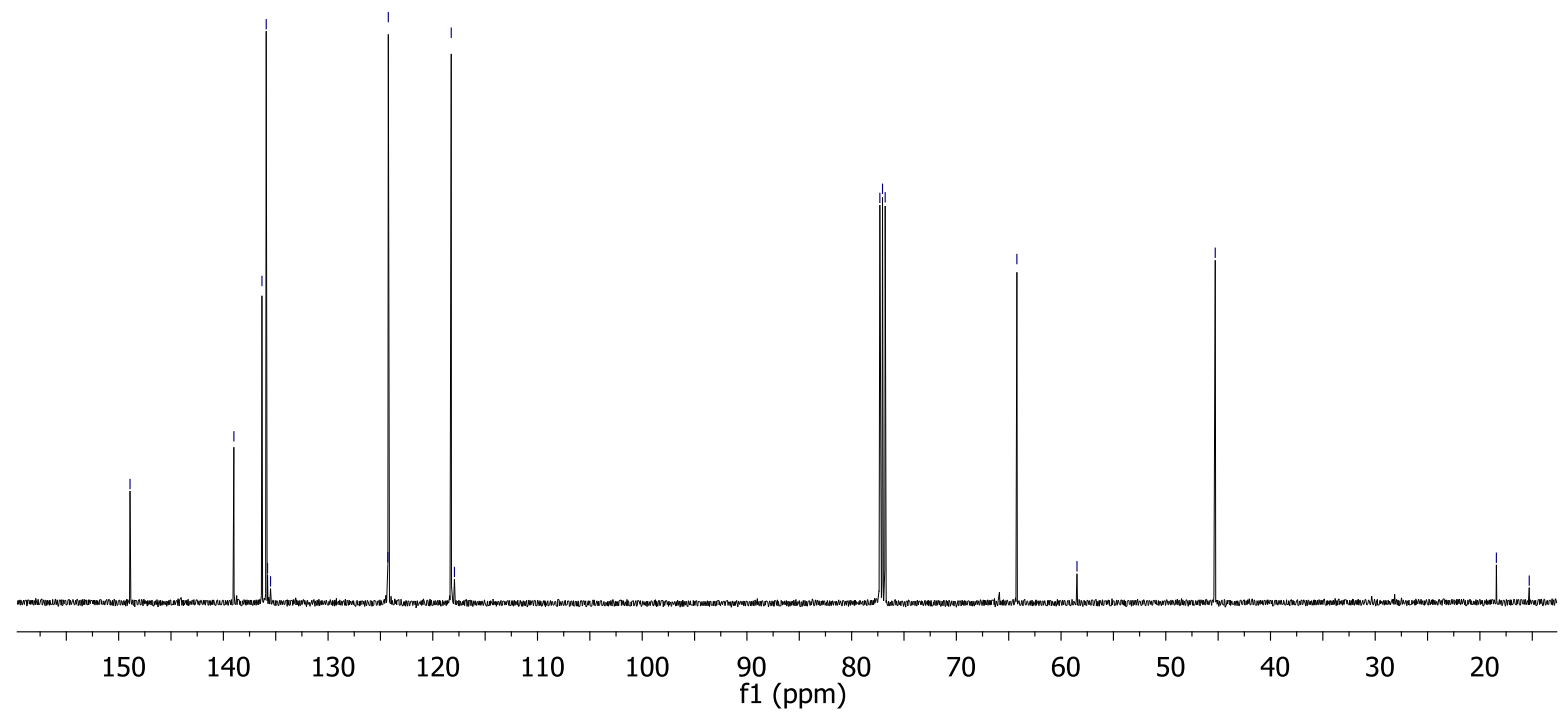


2-(Azulen-6-yl)ethyl (E)-cinnamate (Azul-cinnamate) (178)

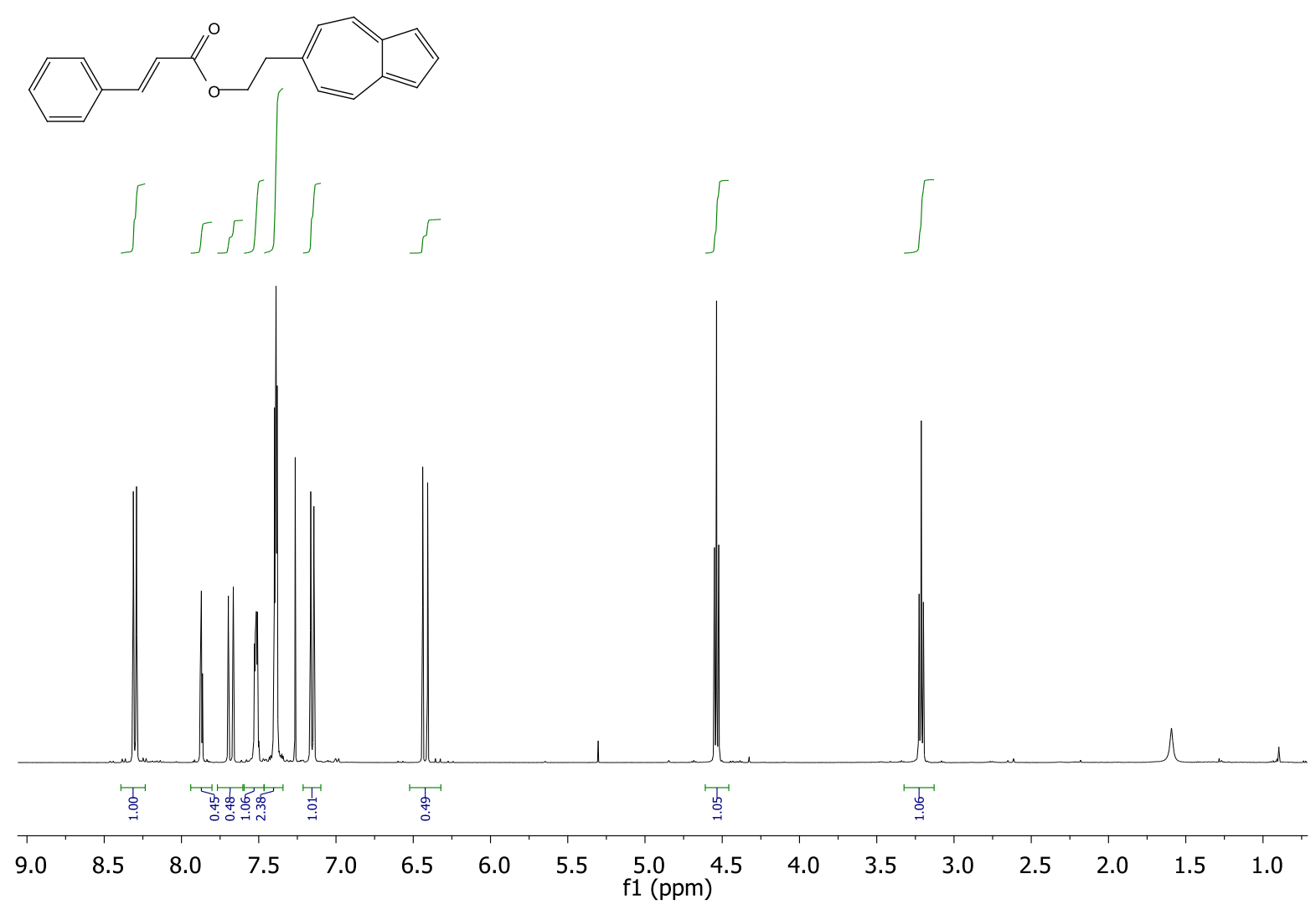

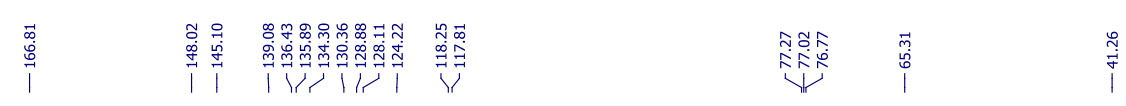

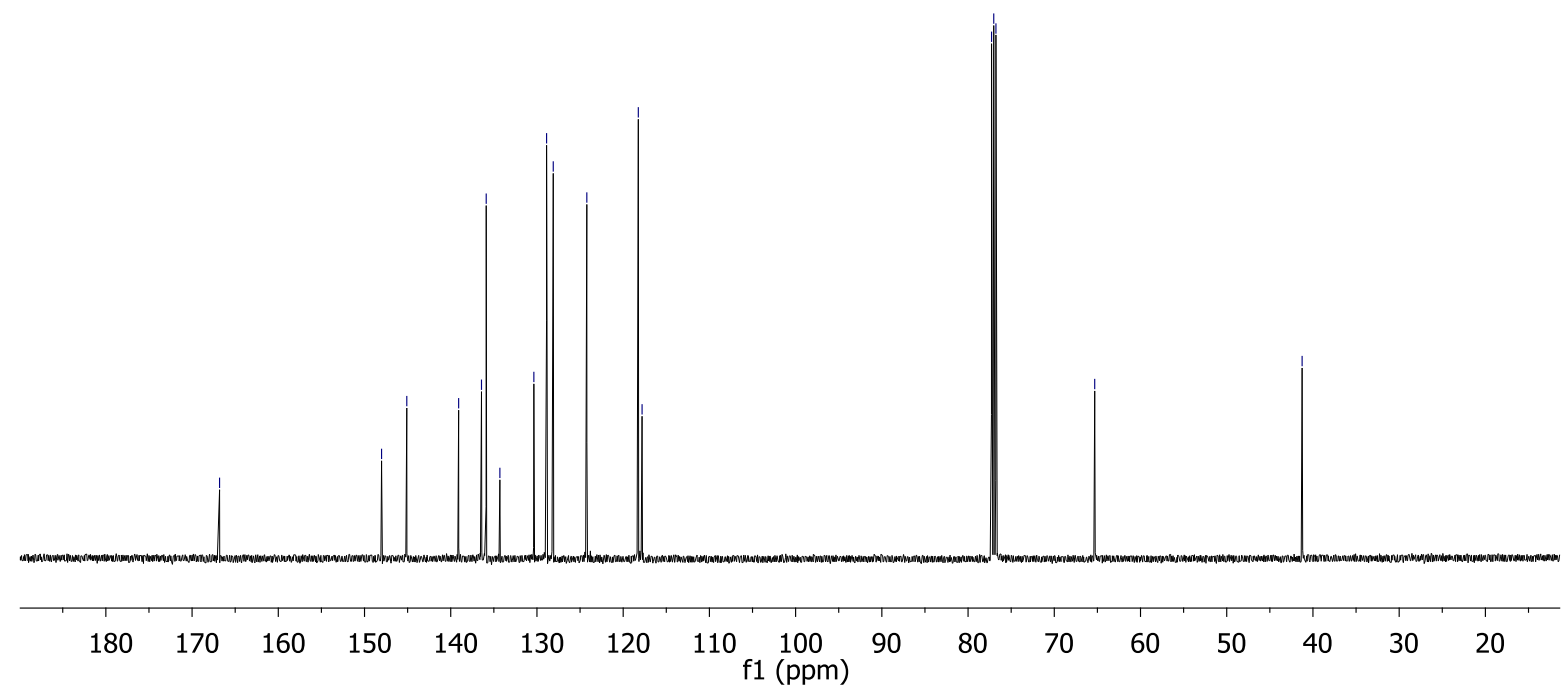


2-(Azulen-6-yl)ethyl benzoate (Azul-benzoate) (182)
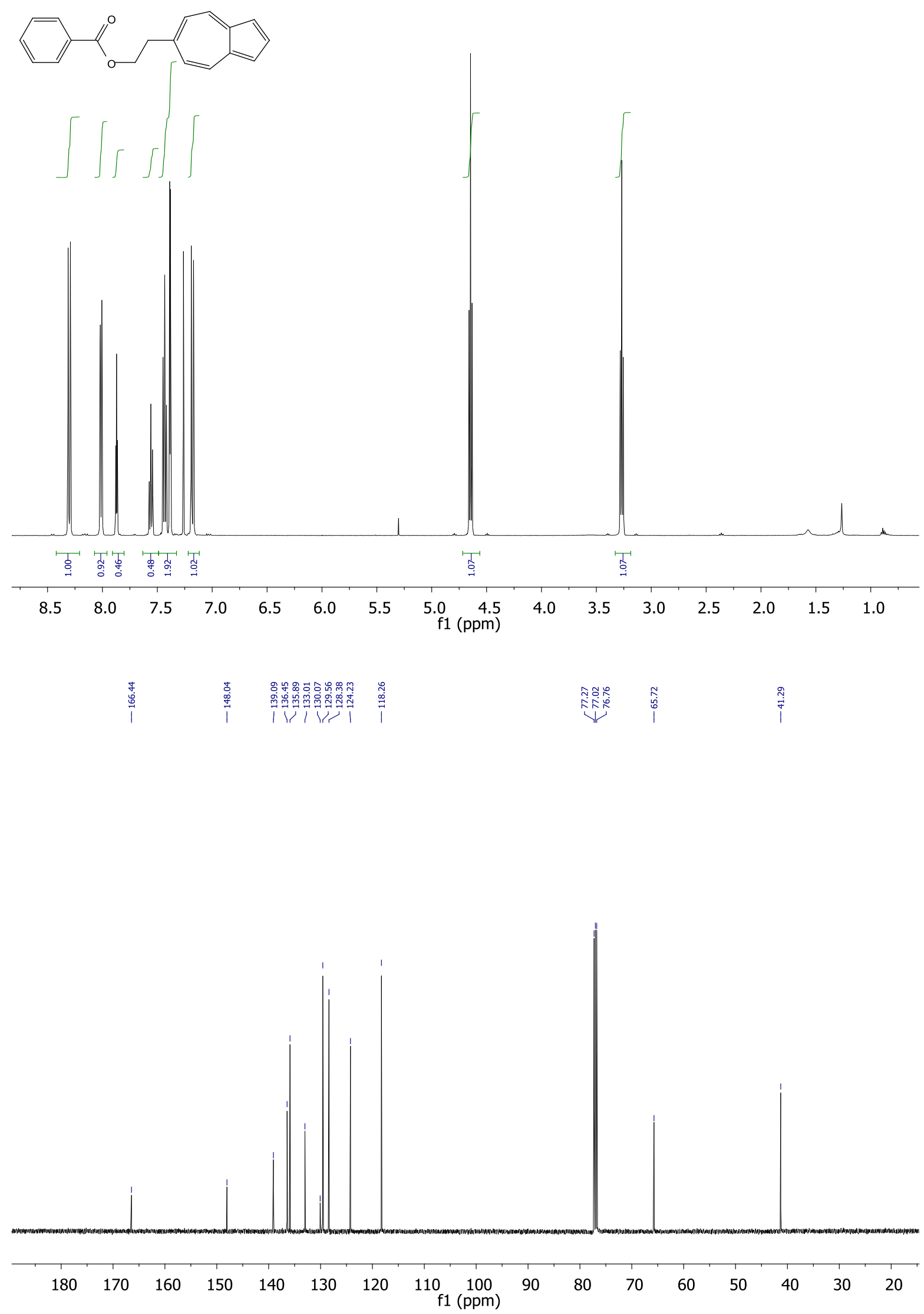
2-(Azulen-6-yl)ethyl 2-furoate (Azul-2-furoate) (185)

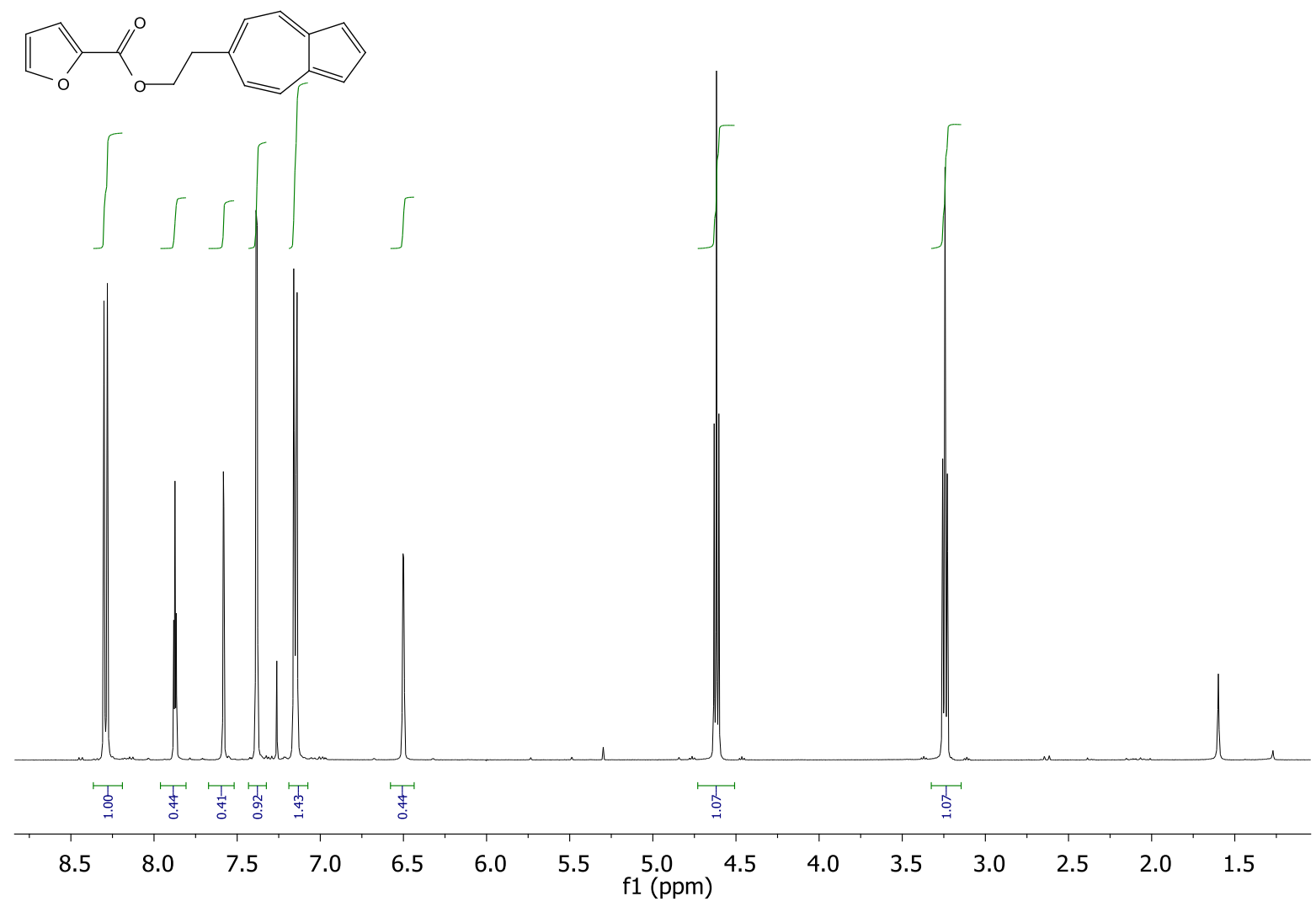

|

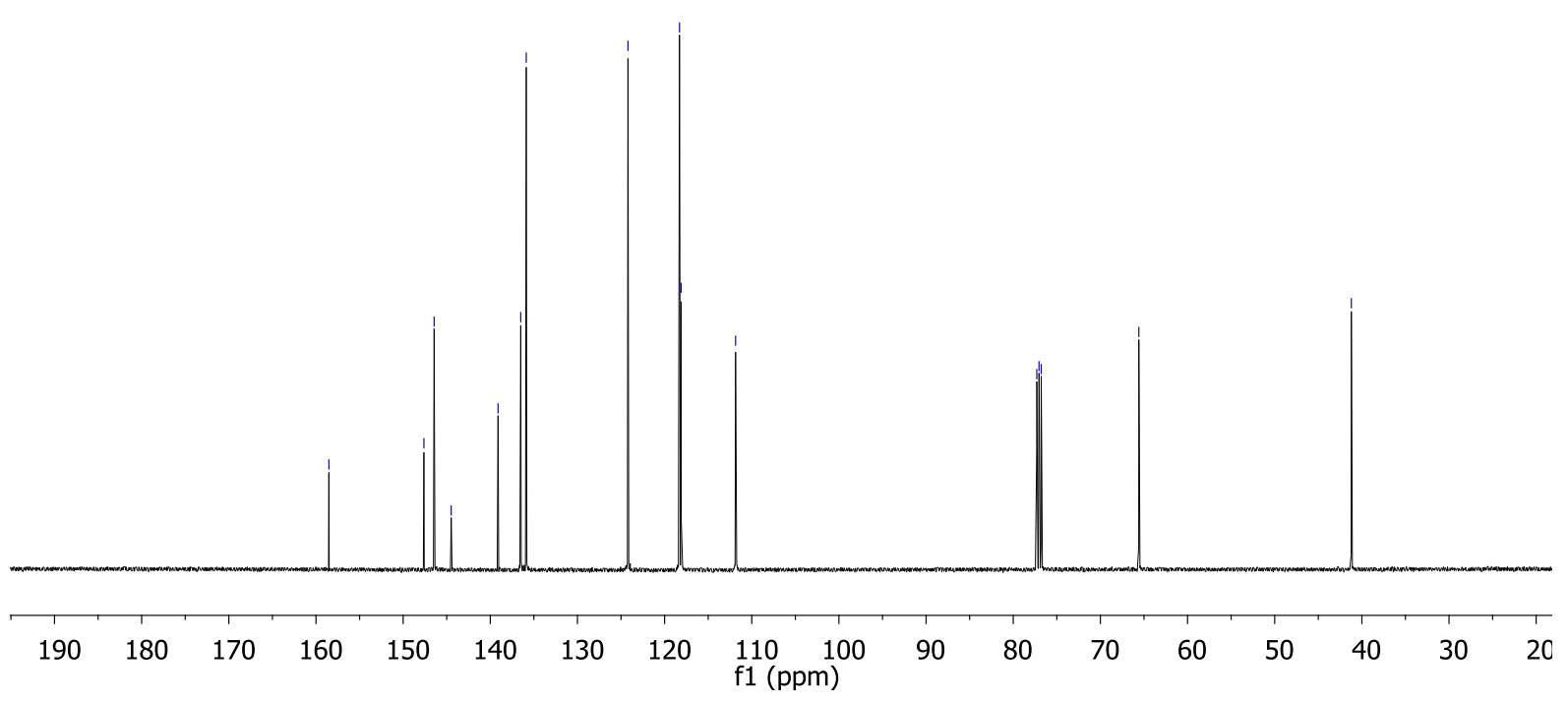


2-(Azulen-6-yl)ethyl 1-naphthoate (Azul-1-naphthoate) (188)

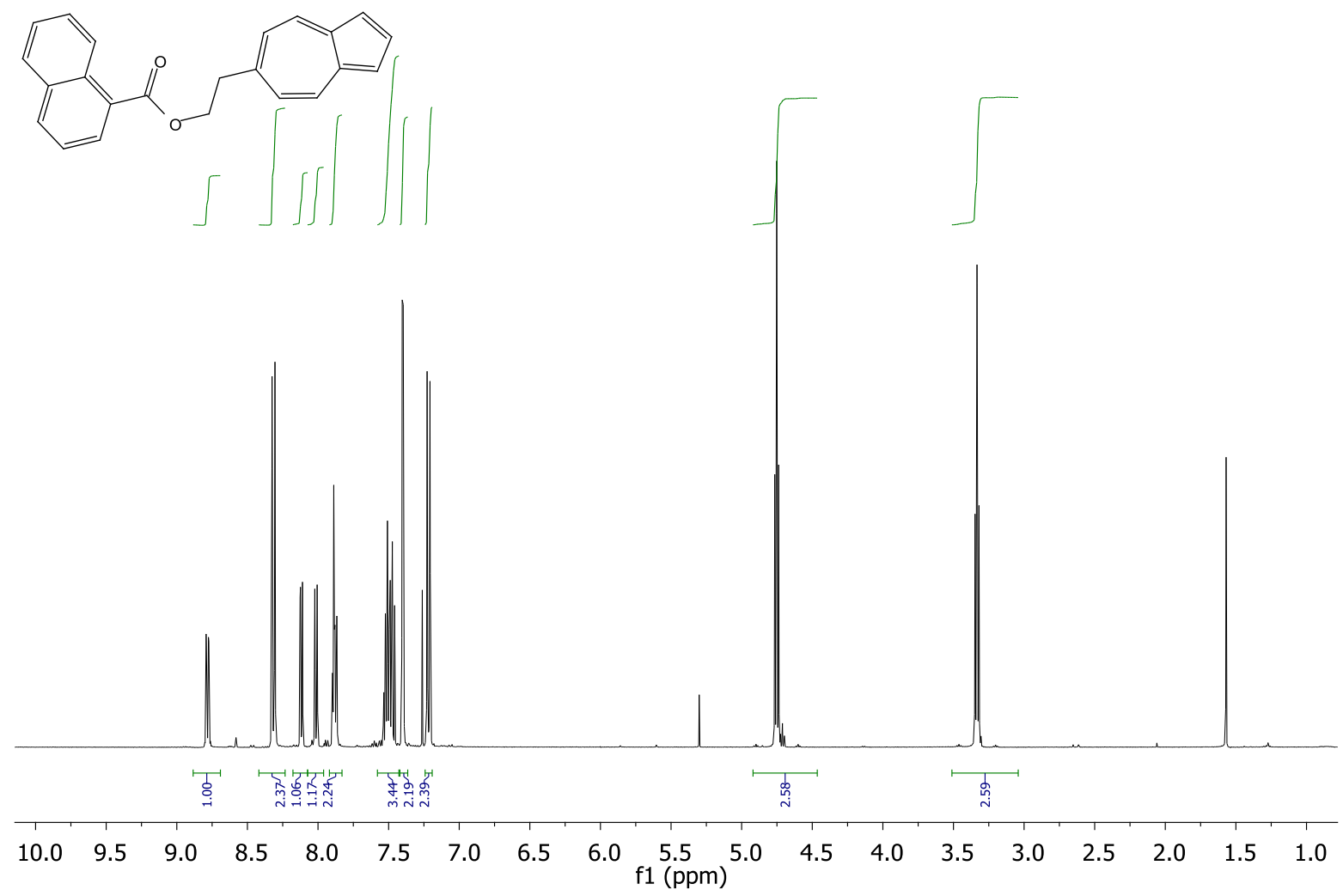

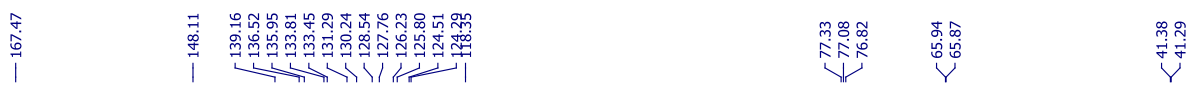

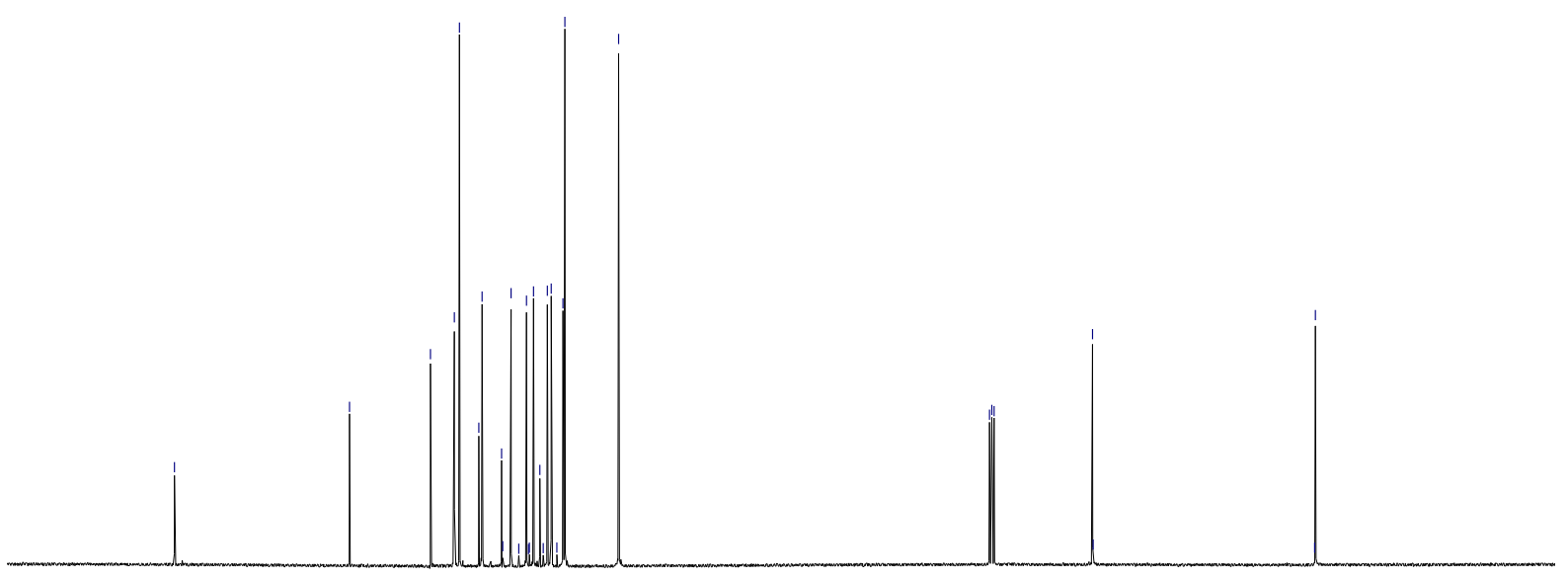

$\begin{array}{llllllllllllllll}180 & 170 & 160 & 150 & 140 & 130 & 120 & 110 \underset{f 1(\mathrm{ppm})}{100} & 90 & 80 & 70 & 60 & 50 & 40 & 30 & 20\end{array}$ 
2-(Azulen-6-yl)ethyl cyclohexanecarboxylate

(Azul-cyclohexanecarboxylate) (183)
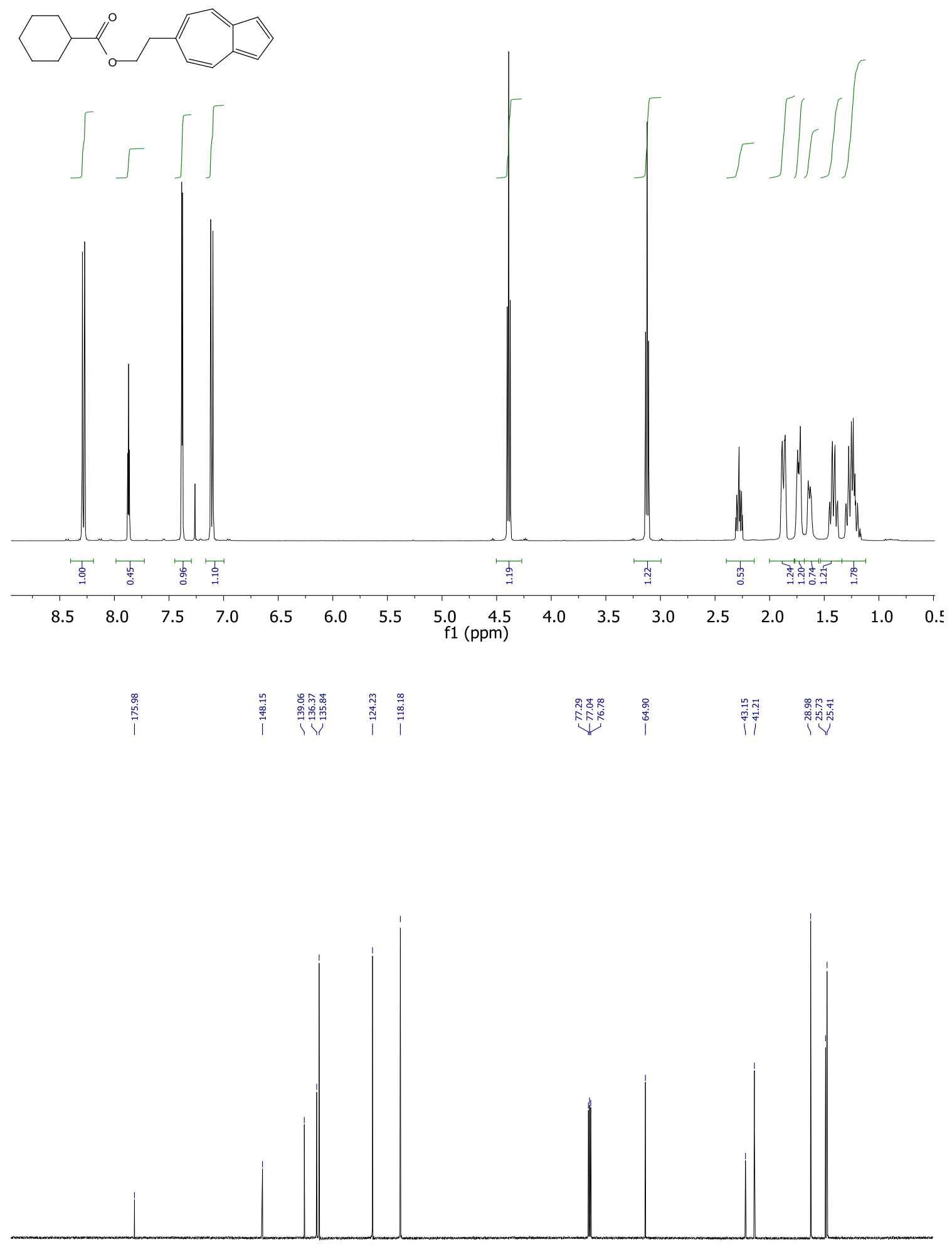

$\begin{array}{llllllllllllllllllll}200 & 190 & 180 & 170 & 160 & 150 & 140 & 130 & 120 & 110 & 100 & 90 & 80 & 70 & 60 & 50 & 40 & 30 & 20 & 10\end{array}$ 
2-(Azulen-6-yl)ethyl cyclobutanecarboxylate

(Azul-cyclobutanecarboxylate) (184)
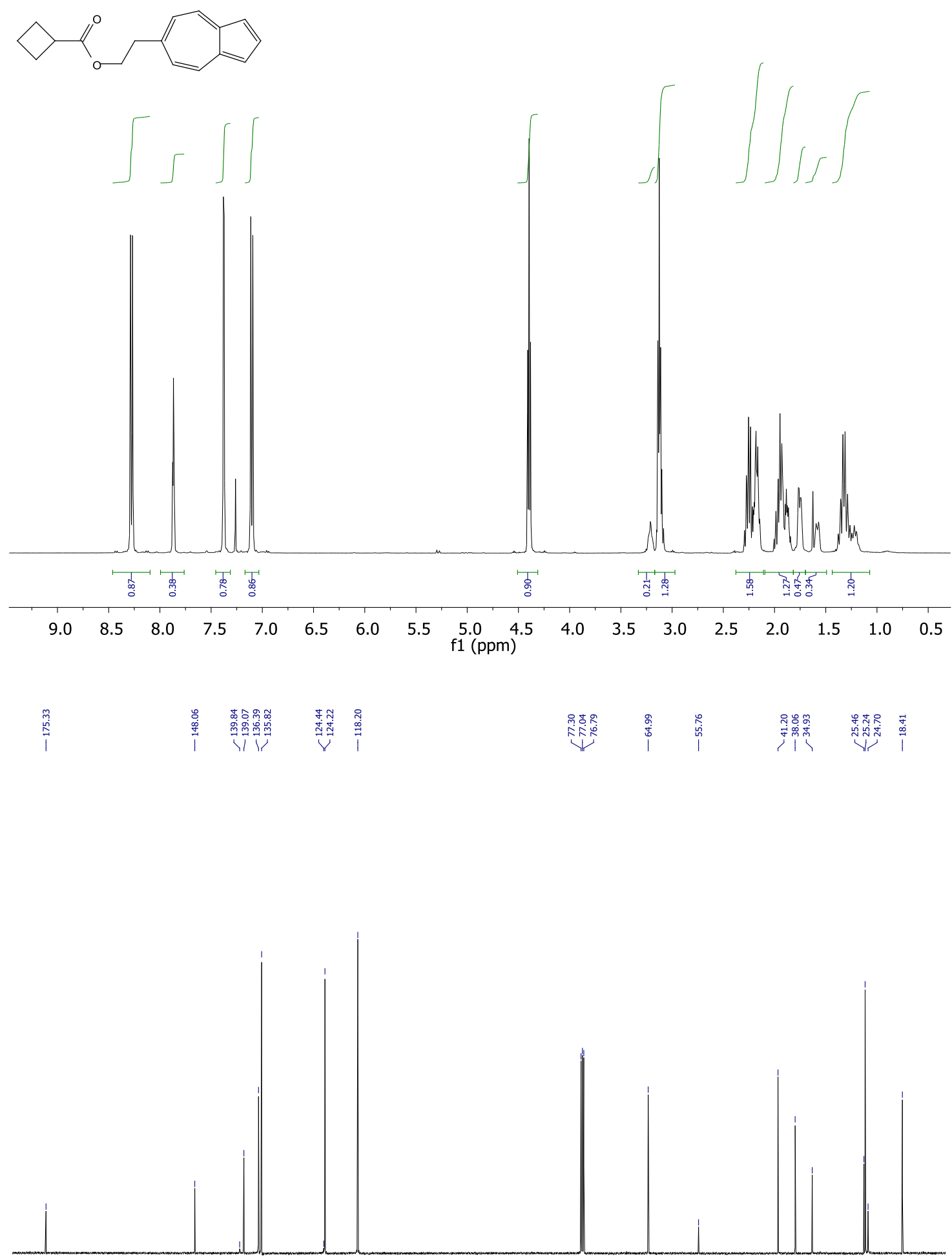

180

$\begin{array}{llllllll}170 & 160 & 150 & 140 & 130 & 120 & 110 & \begin{array}{l}100 \\ \mathrm{f} 1(\mathrm{ppm})\end{array}\end{array}$

$\begin{array}{lllllll}80 & 70 & 60 & 50 & 40 & 30 & 20\end{array}$ 
2-(Azulen-6-yl)ethyl azulene-1-carboxylate (Azul-azulenecarboxylate) (186)

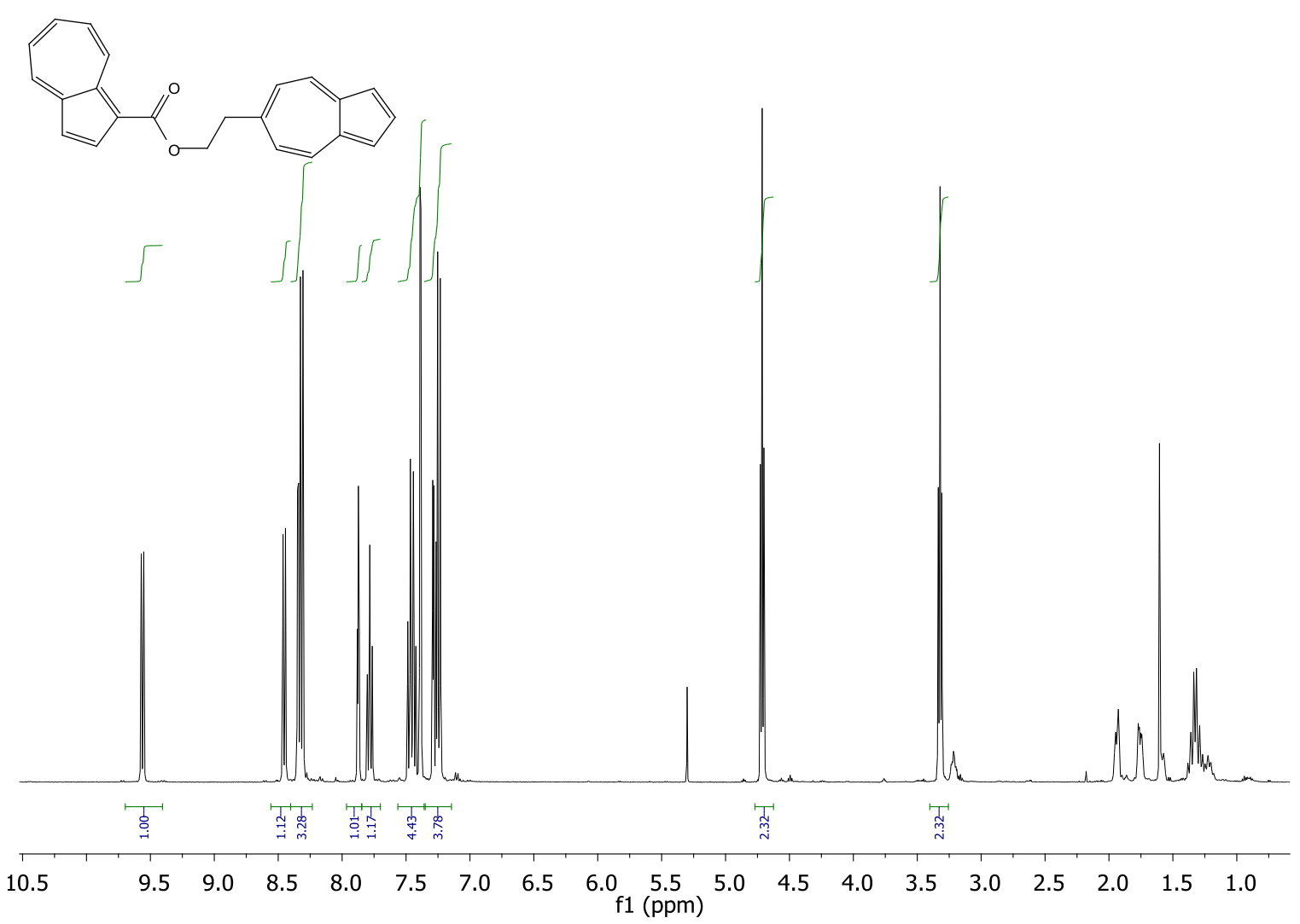

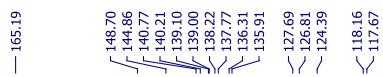
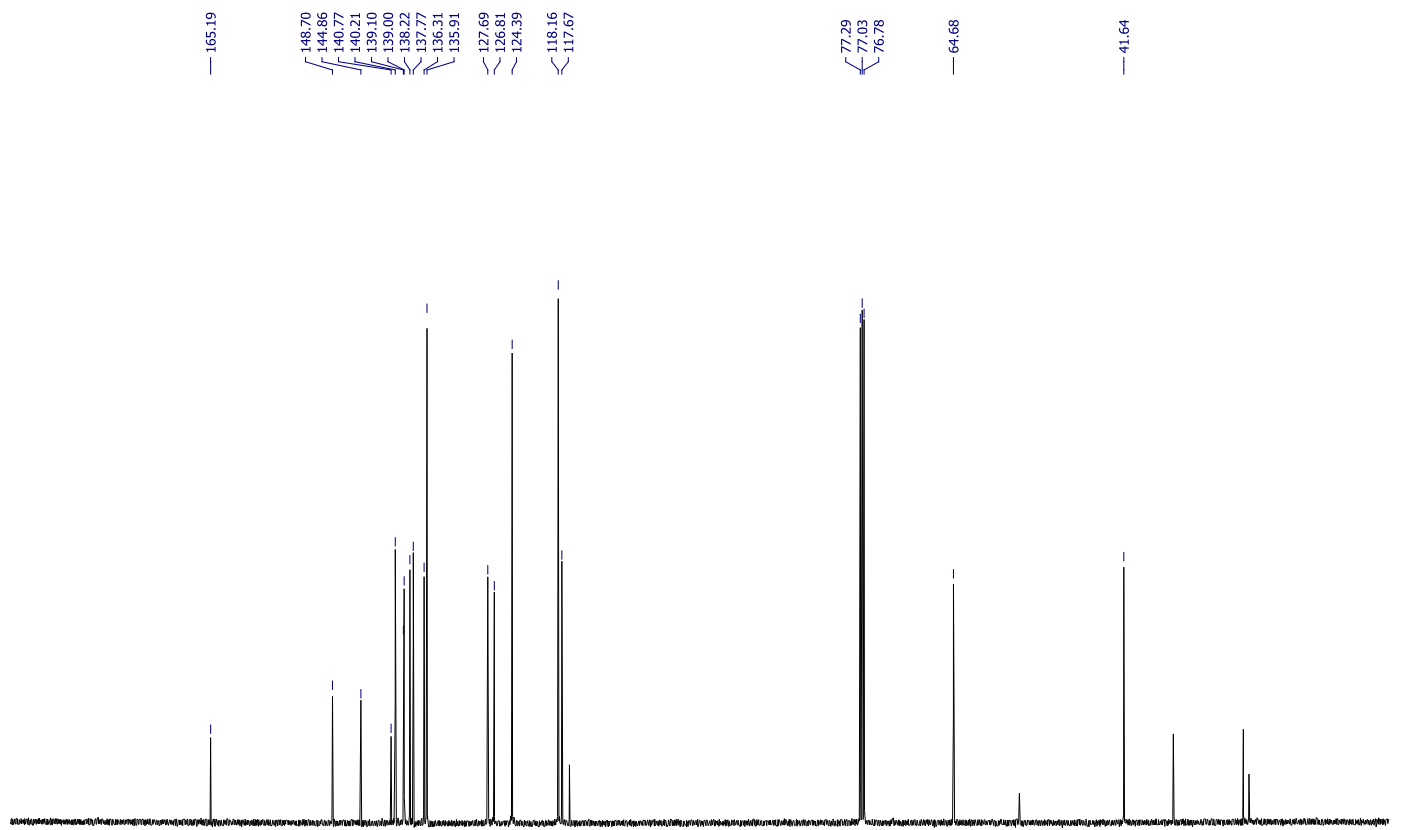

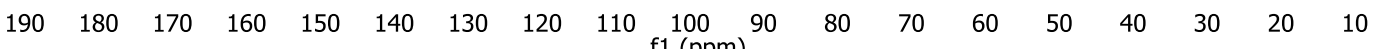


2-(Azulen-6-yl)ethyl 3,5-dinitrobenzoate (Azul-3,5-dinitrobenzoate) (187)
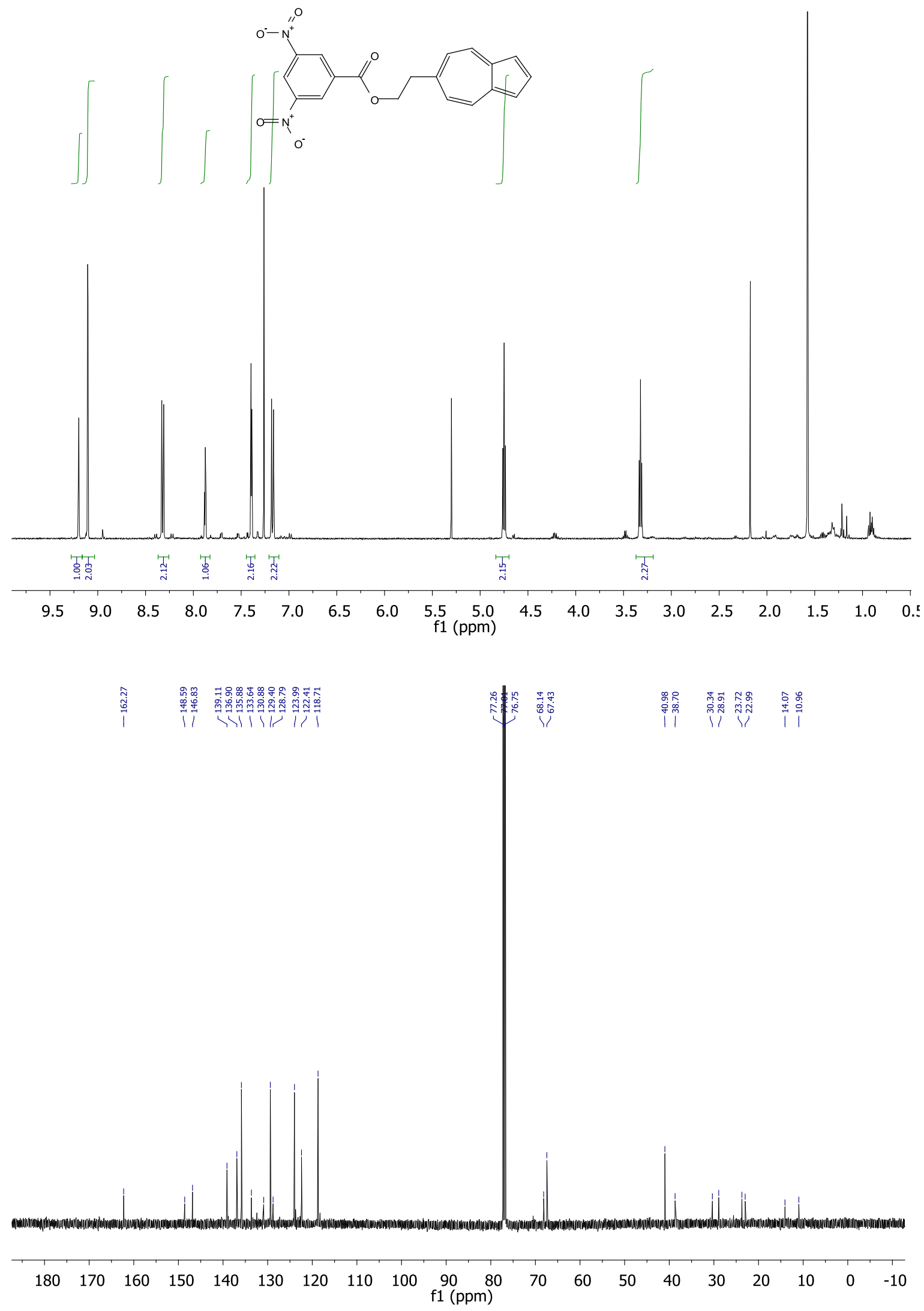
$N$-fluorenylmethyloxycarbonyl glycine 2-(azulen-6-yl)ethyl ester (FMOC-Gly-OAzul) (189)
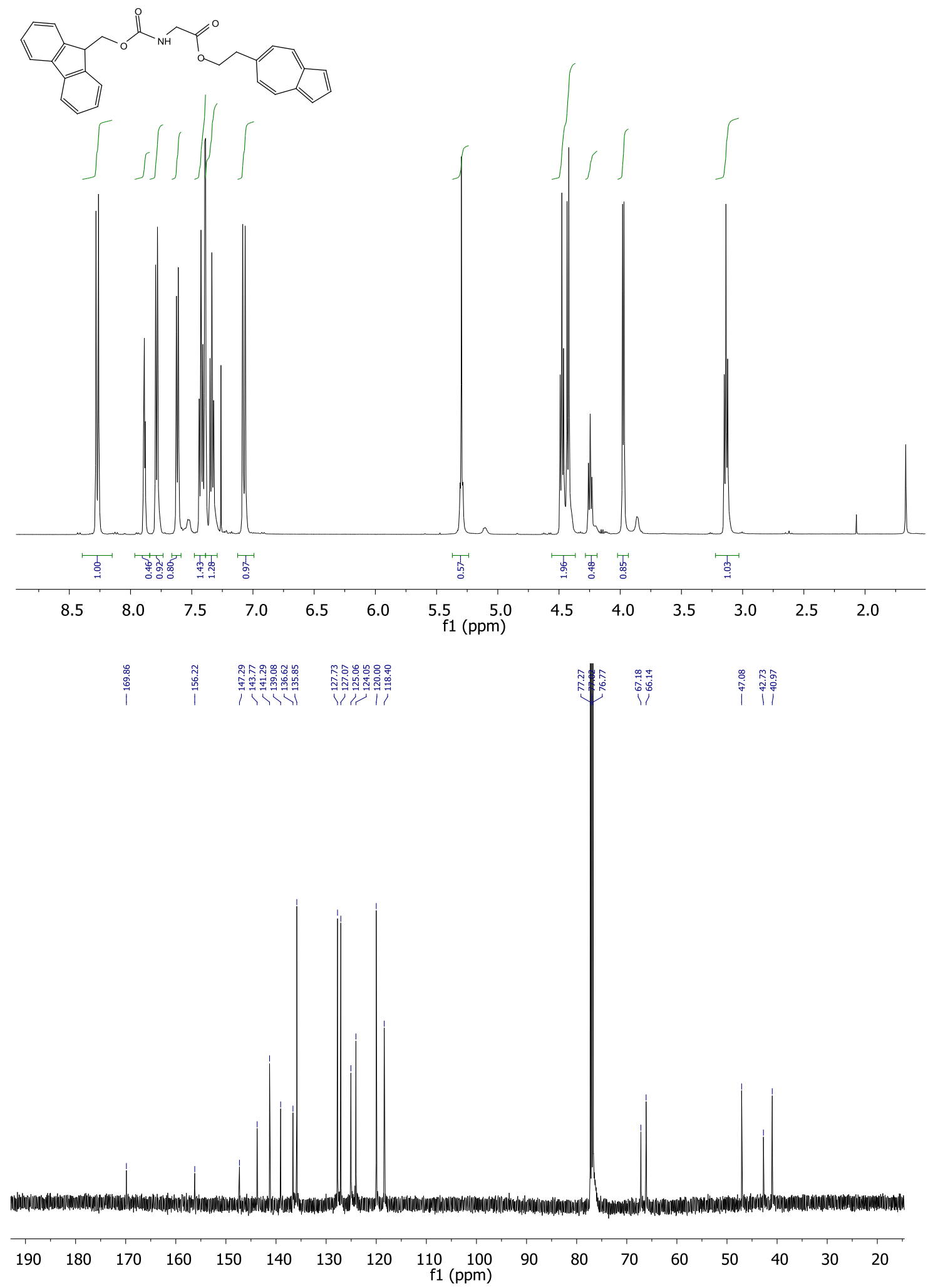


\section{6-Vinylazulene (94)}
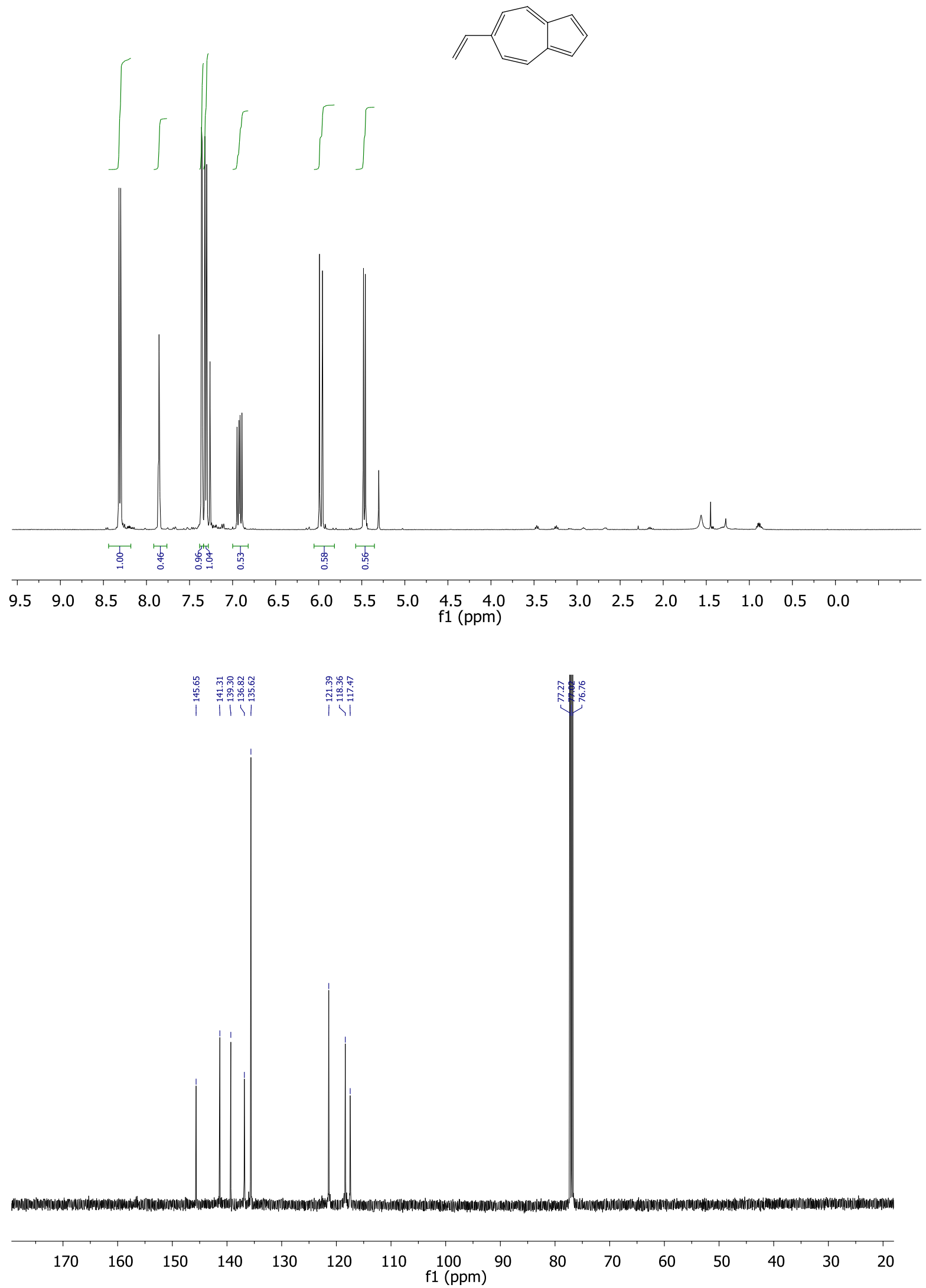
2-(1-Nitroazulen-6-yl)ethyl (E)-cinnamate (197)
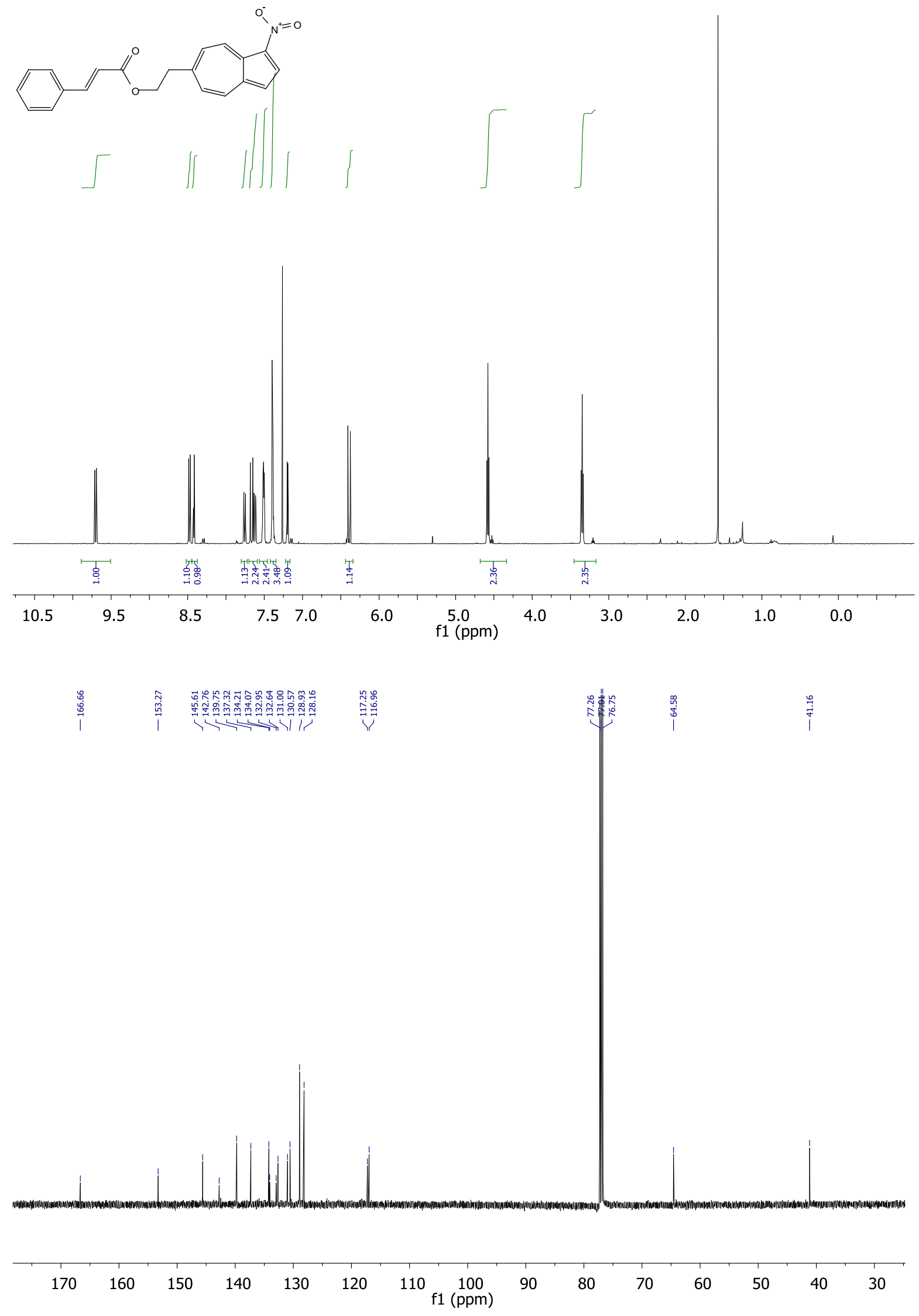
2-(1-(2-methoxy-2-oxoacetyl)azulen-6-yl)ethyl (E)-cinnamate (205)

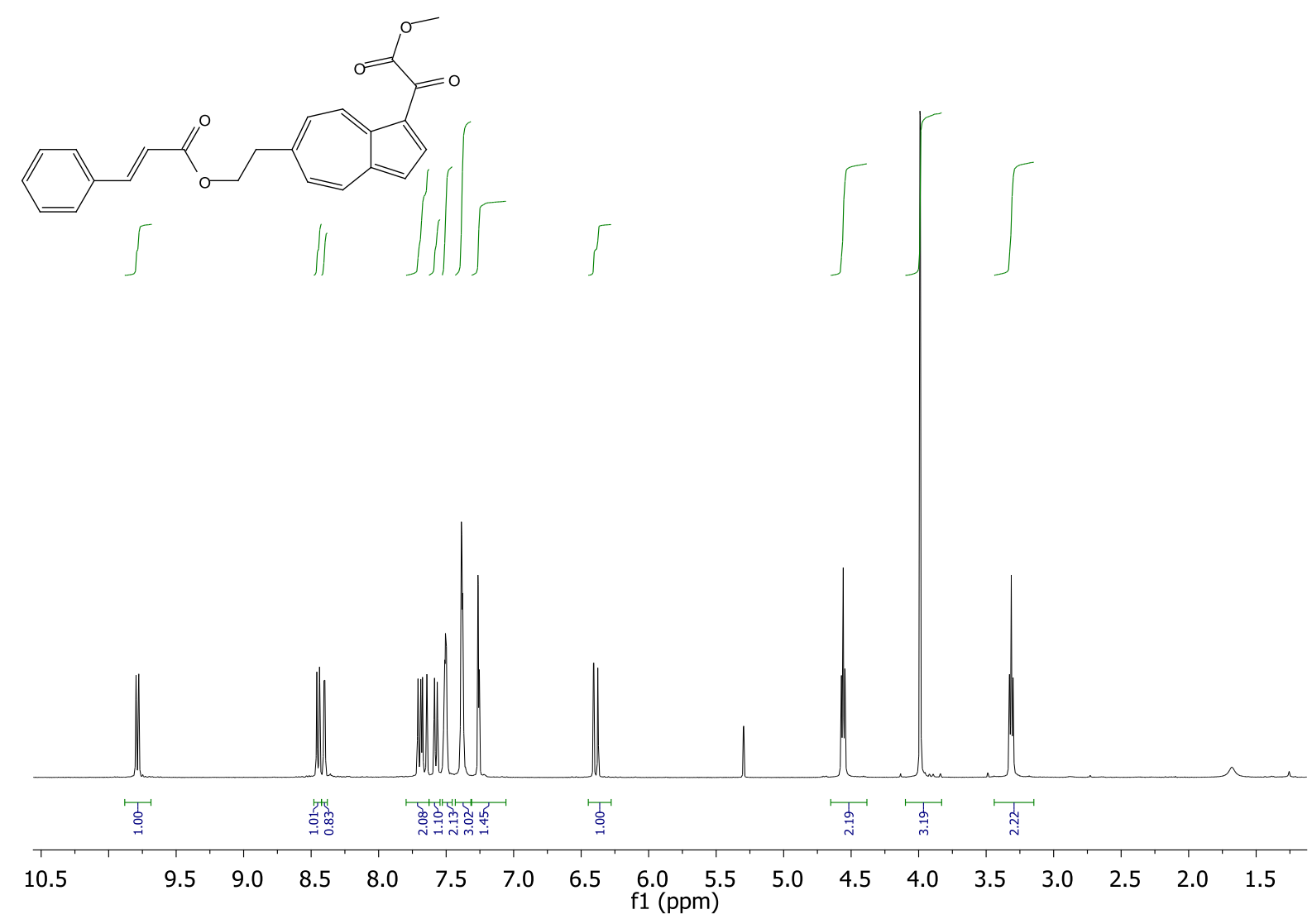

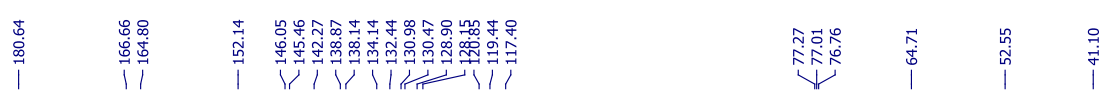

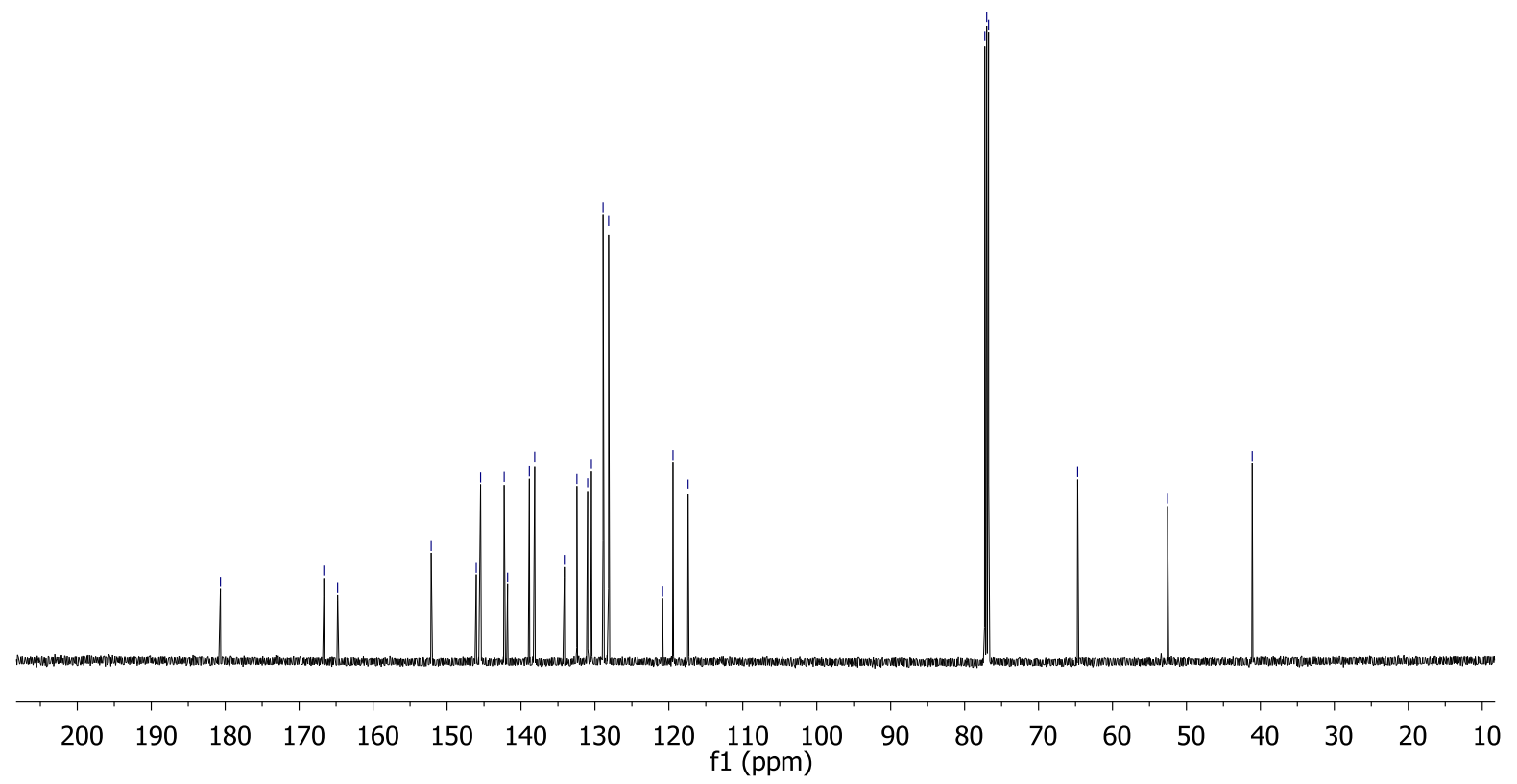


$\mathrm{N}$-fluorenylmethyloxycarbonyl glycine oxoacetyl)azulen-6-yl)ethyl ester (222)
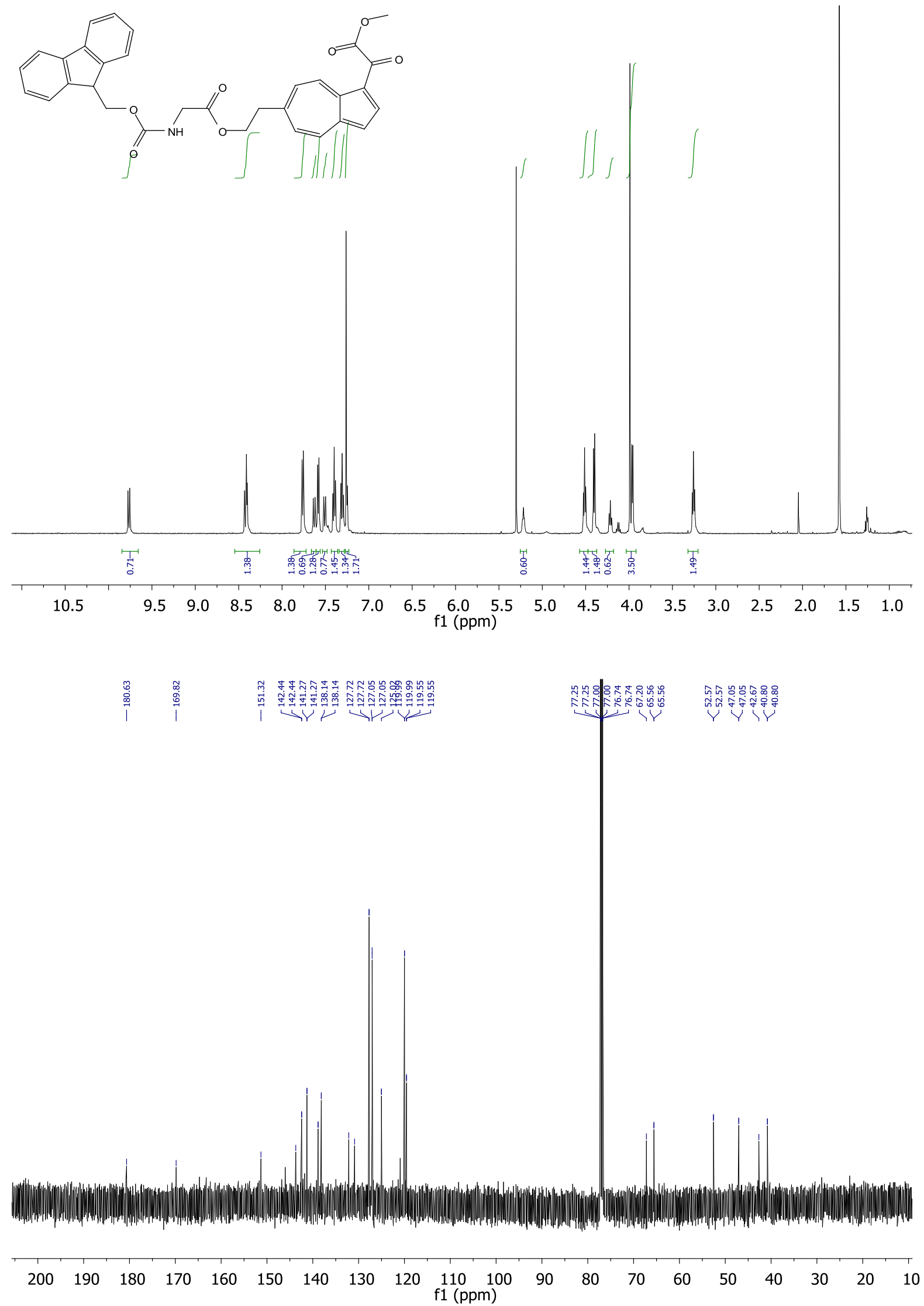
2-(Azulen-6-yl)ethyl 1H-imidazole-1-carboxylate (176)
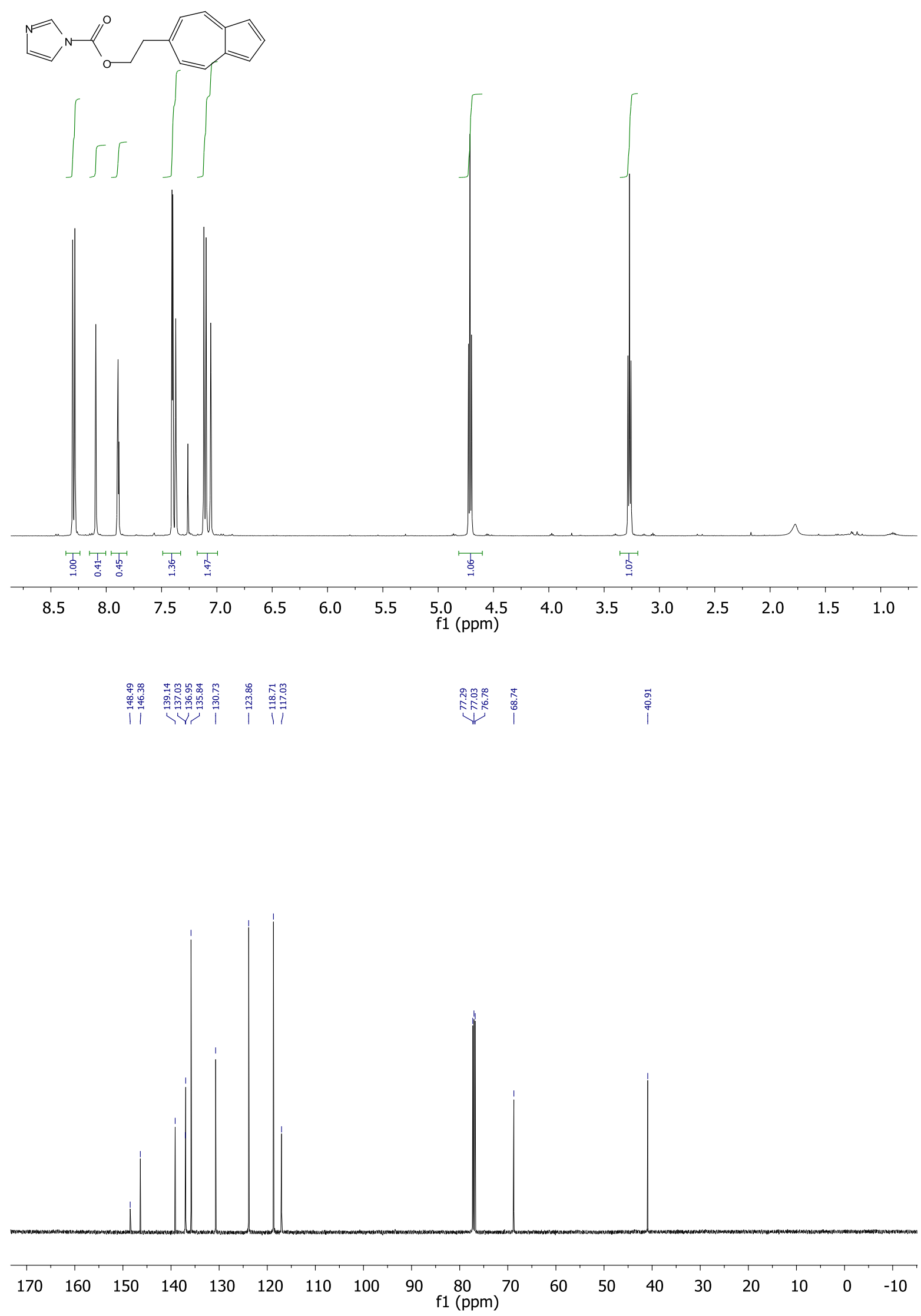
6-(2-Oxyethyl)azulene cyclohexanemethyl carbonate (AzulOCcyclohexanemethanol) (191)

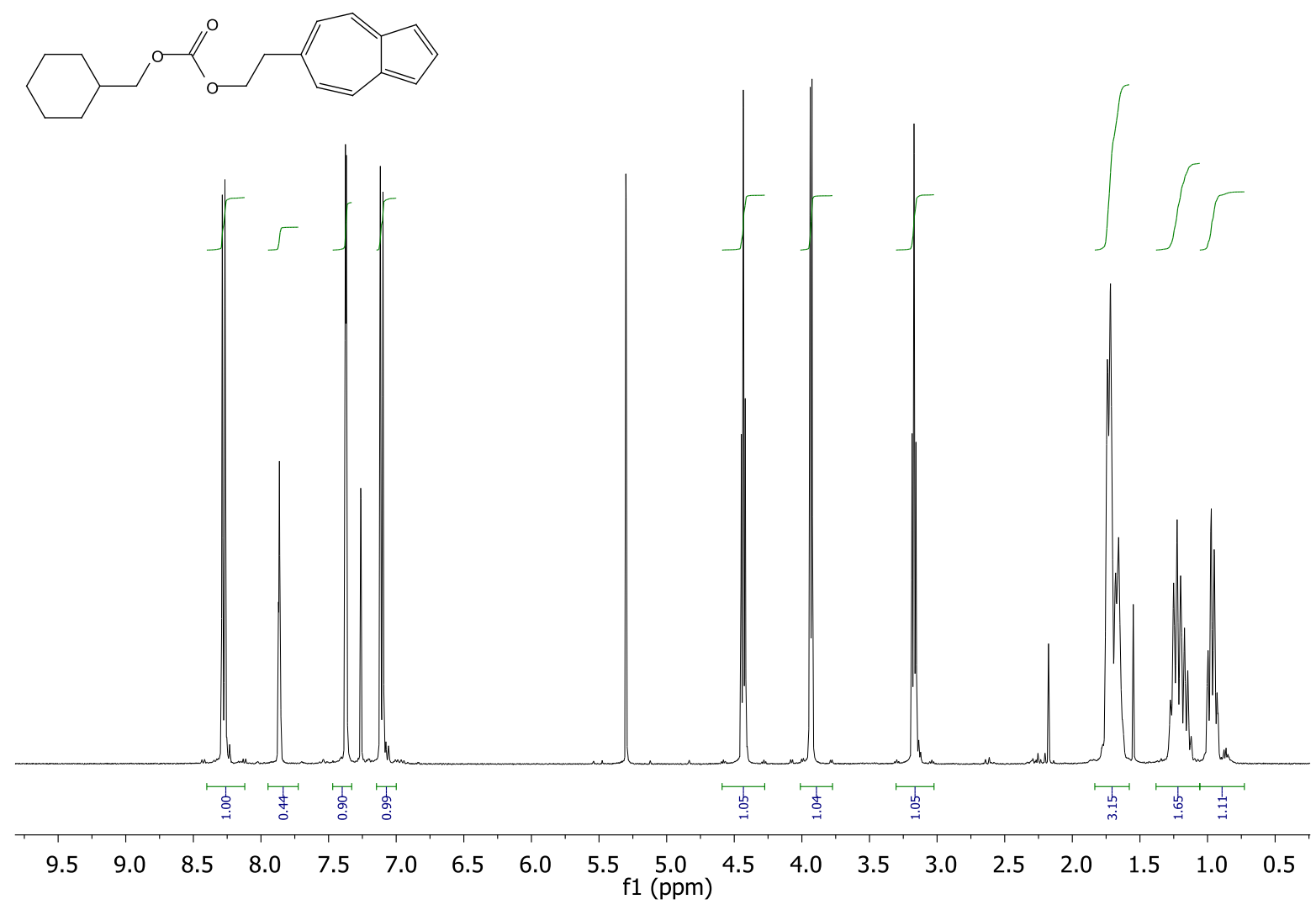

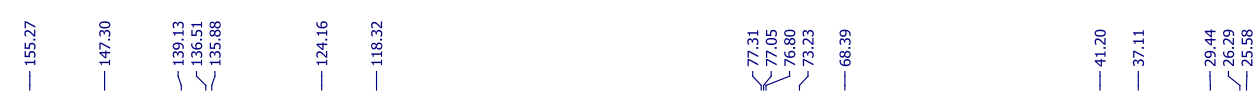

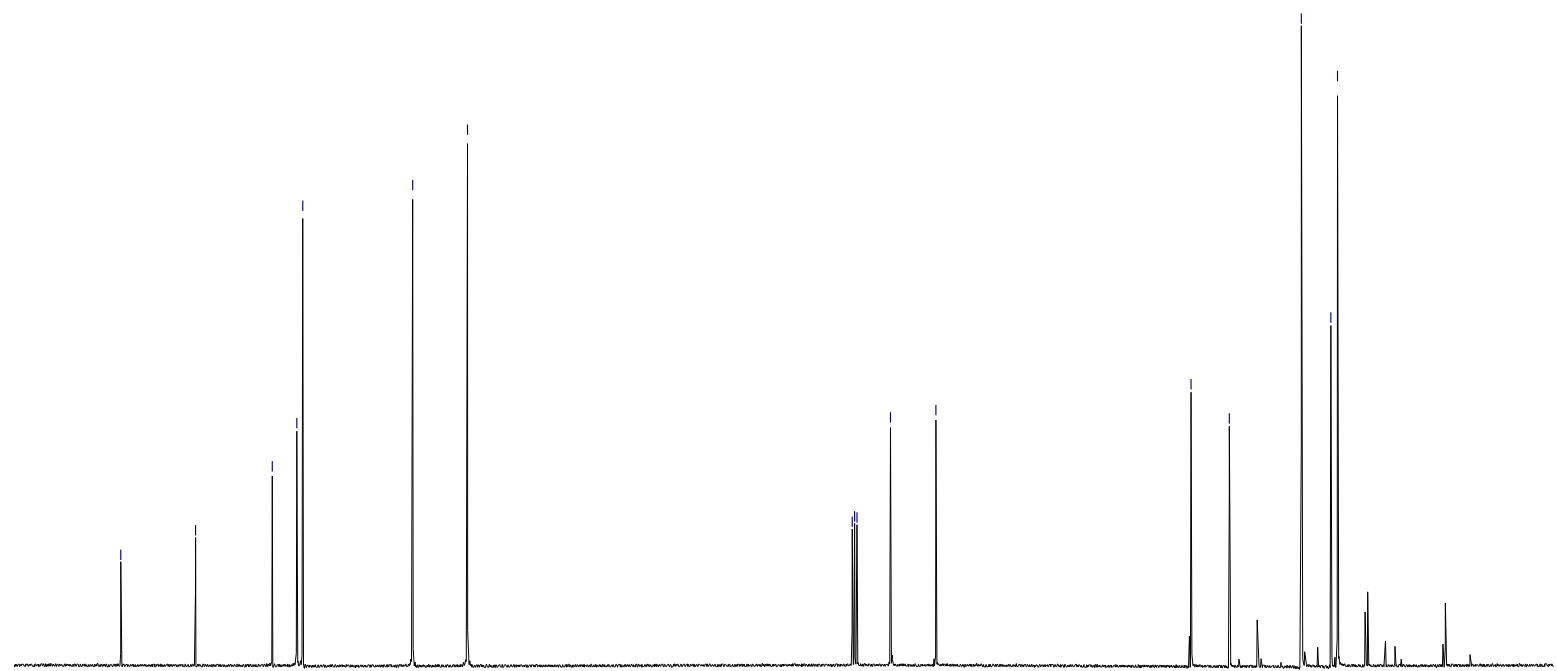

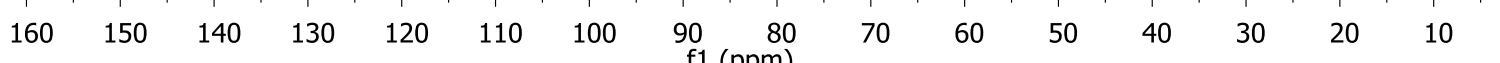


2-(Azulen-6-yl)ethyl cyclohexylcarbamate (194)

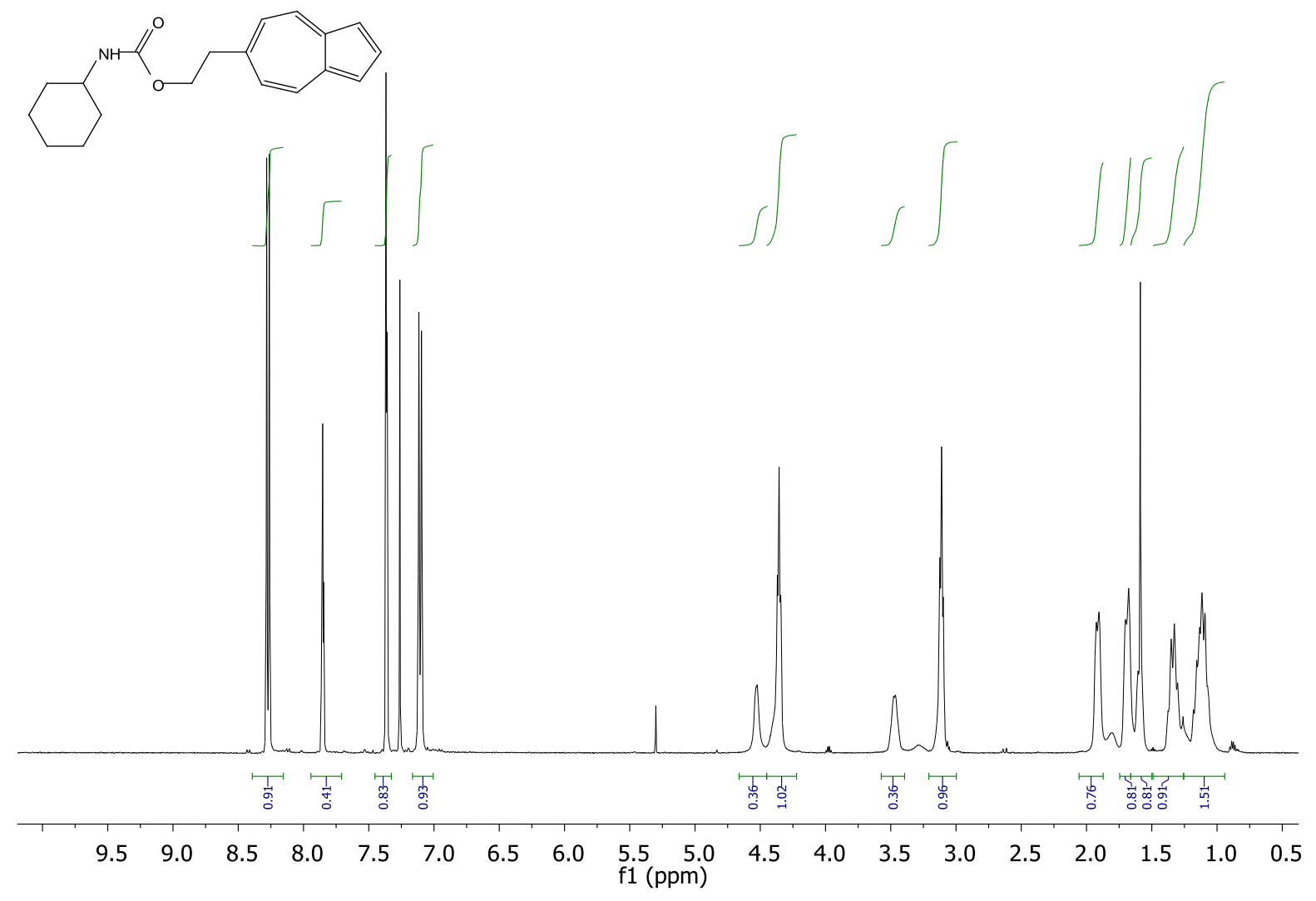

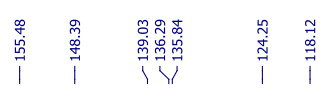

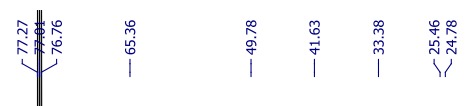

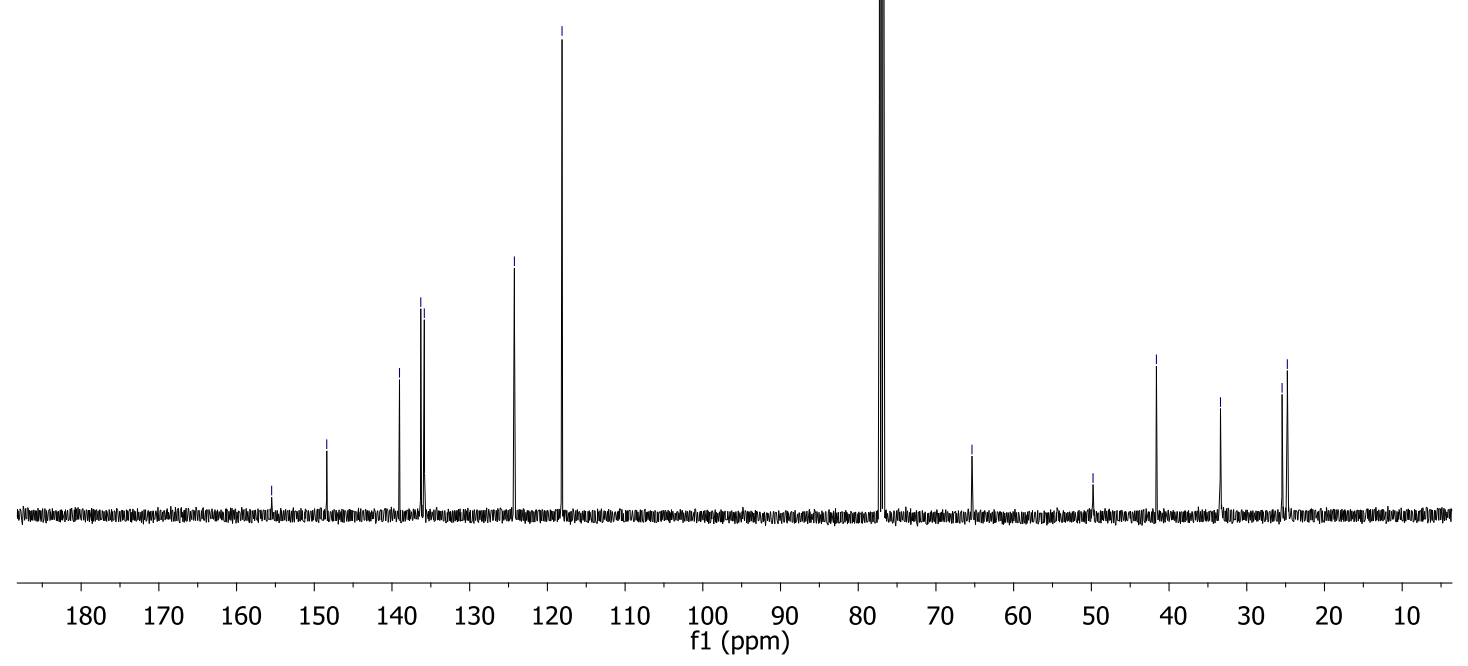




\section{6-(2-Oxyethyl)azulene cetyl carbonate (193)}
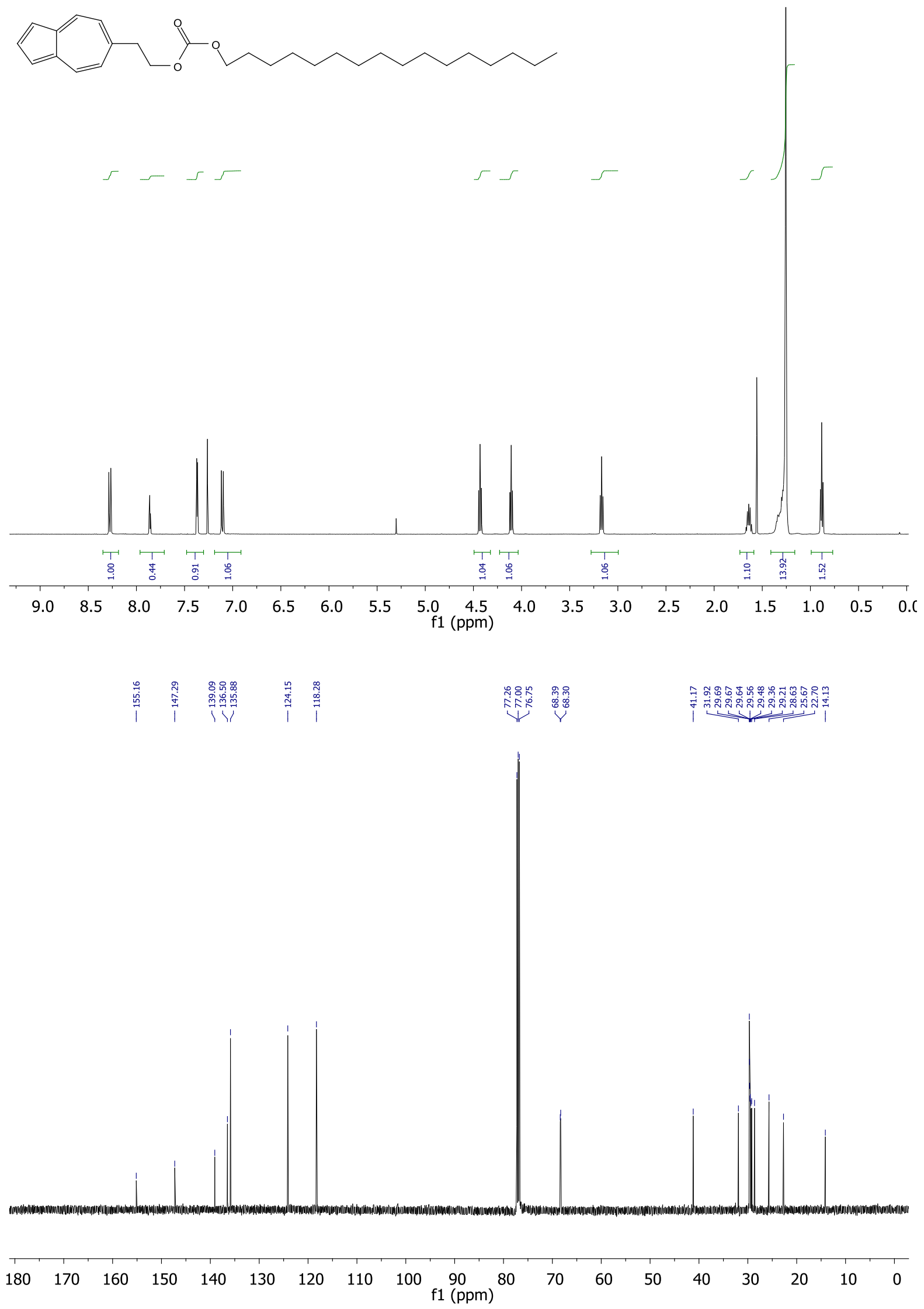


\section{Appendix 2. ${ }^{1} \mathrm{H}$ NMR raw data for kinetics study of 3-nitroazulene menthyl ester 136 saponification}
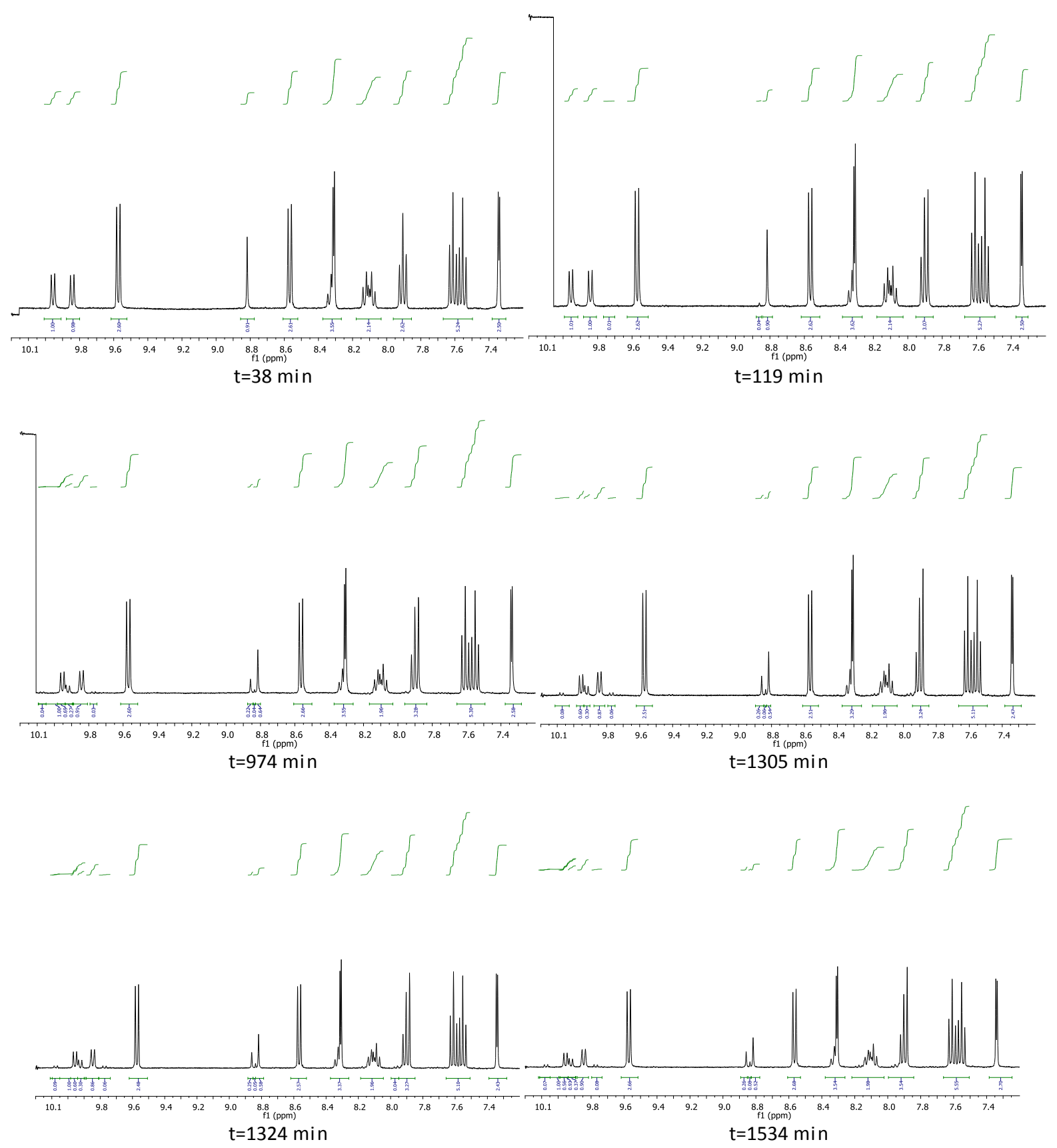

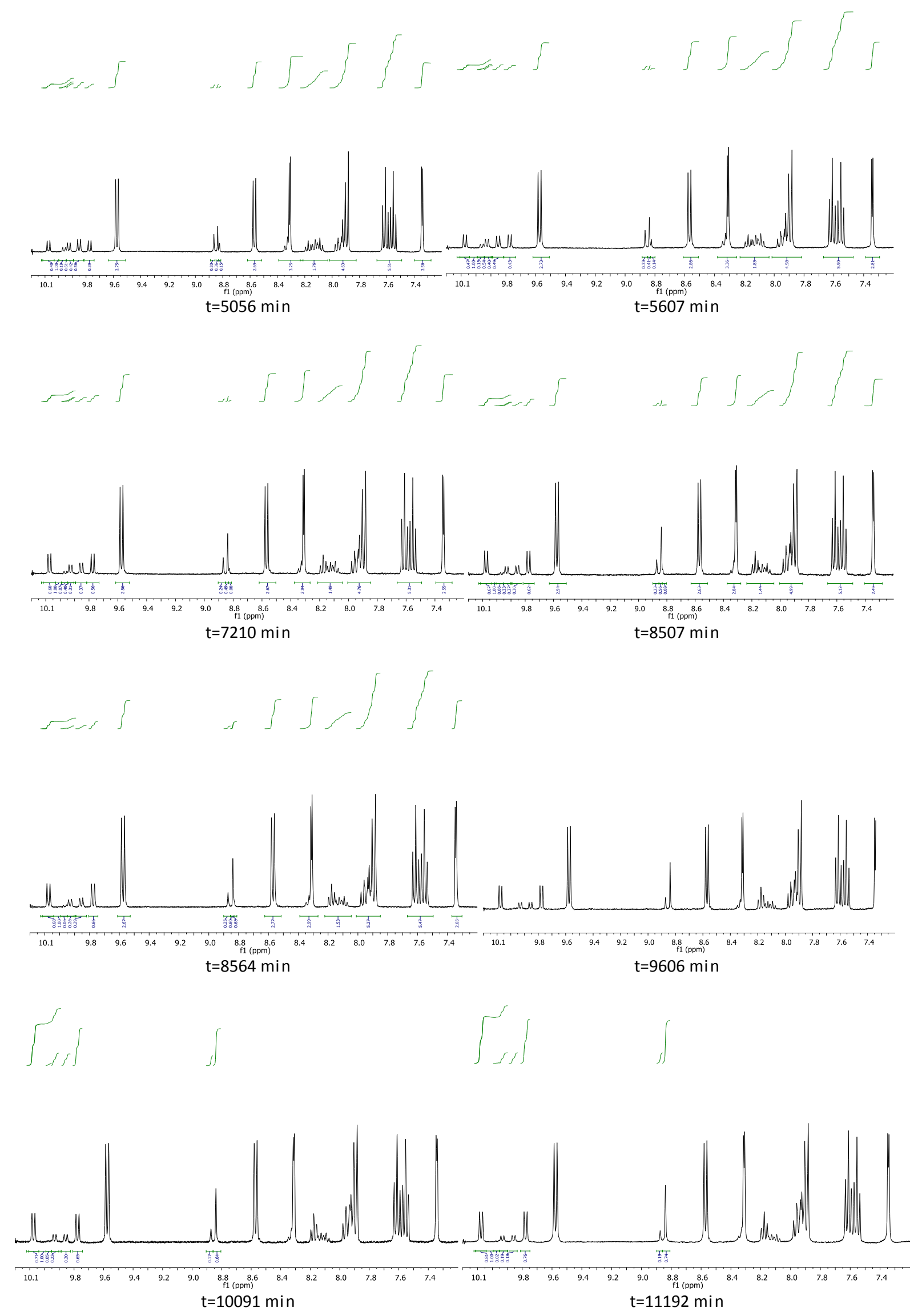

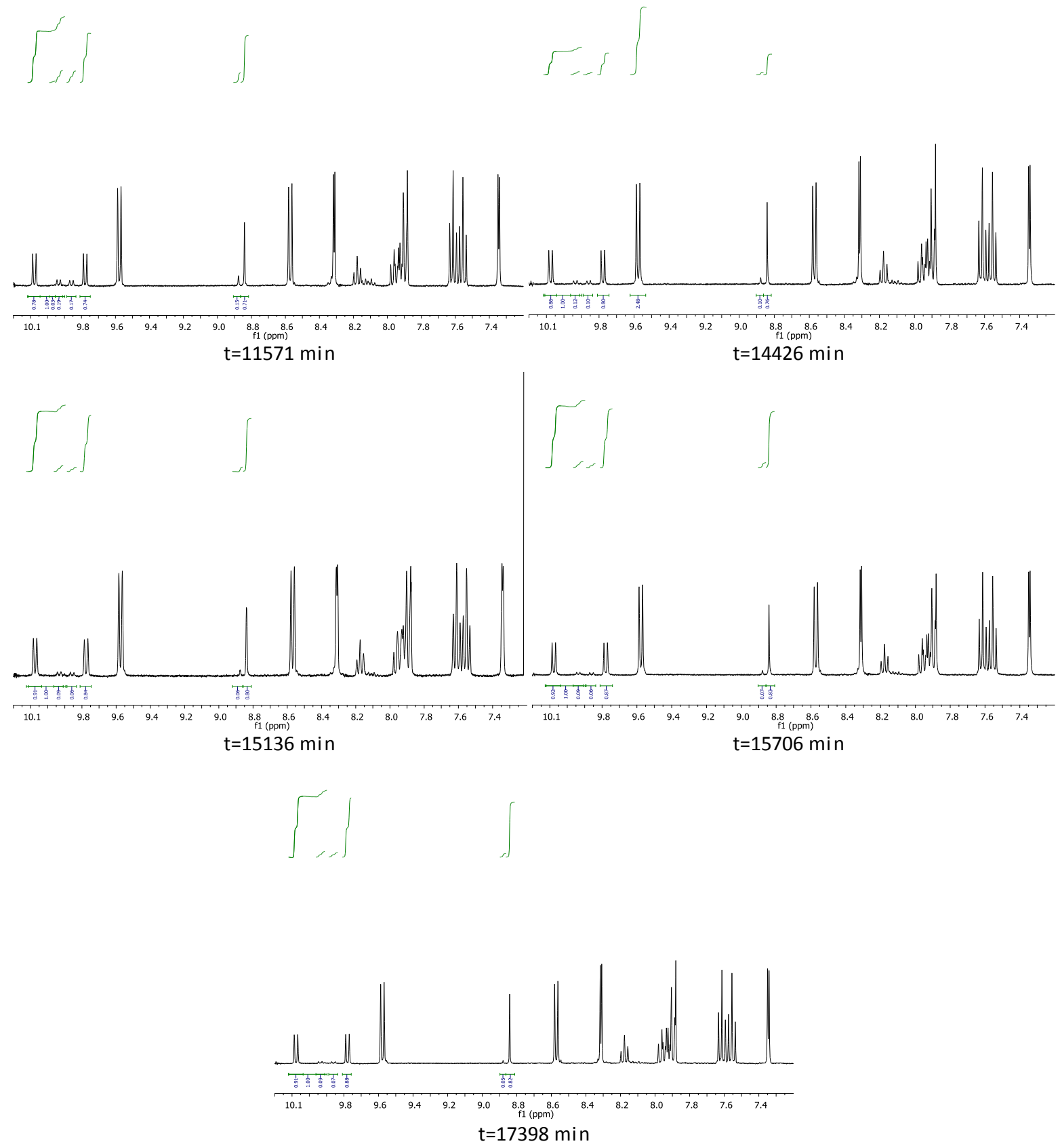


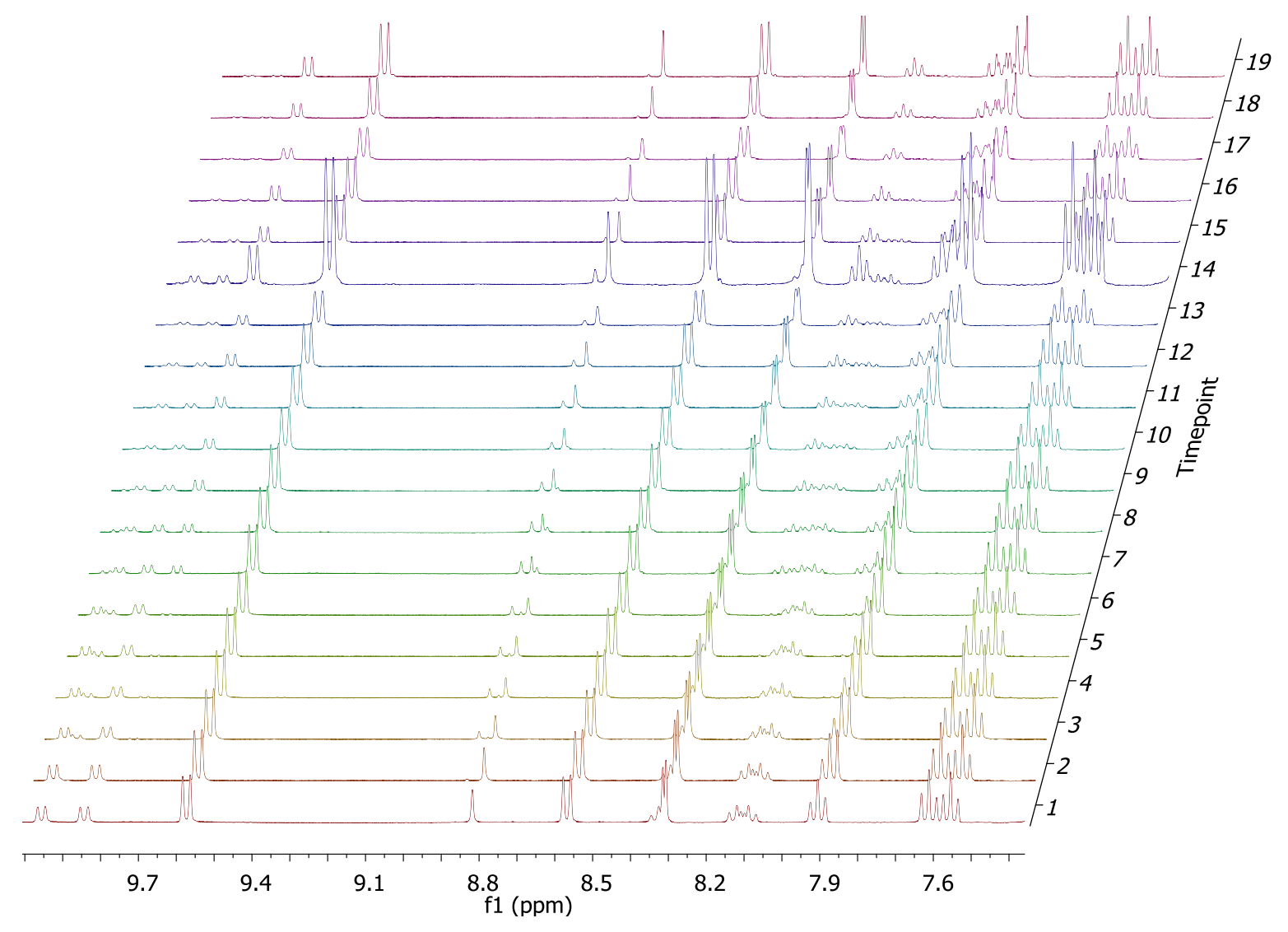

